June 1998

DOE/EIA-0340(97)/1

\title{
Petroleum Supply Annual 1997 Volume 1
}

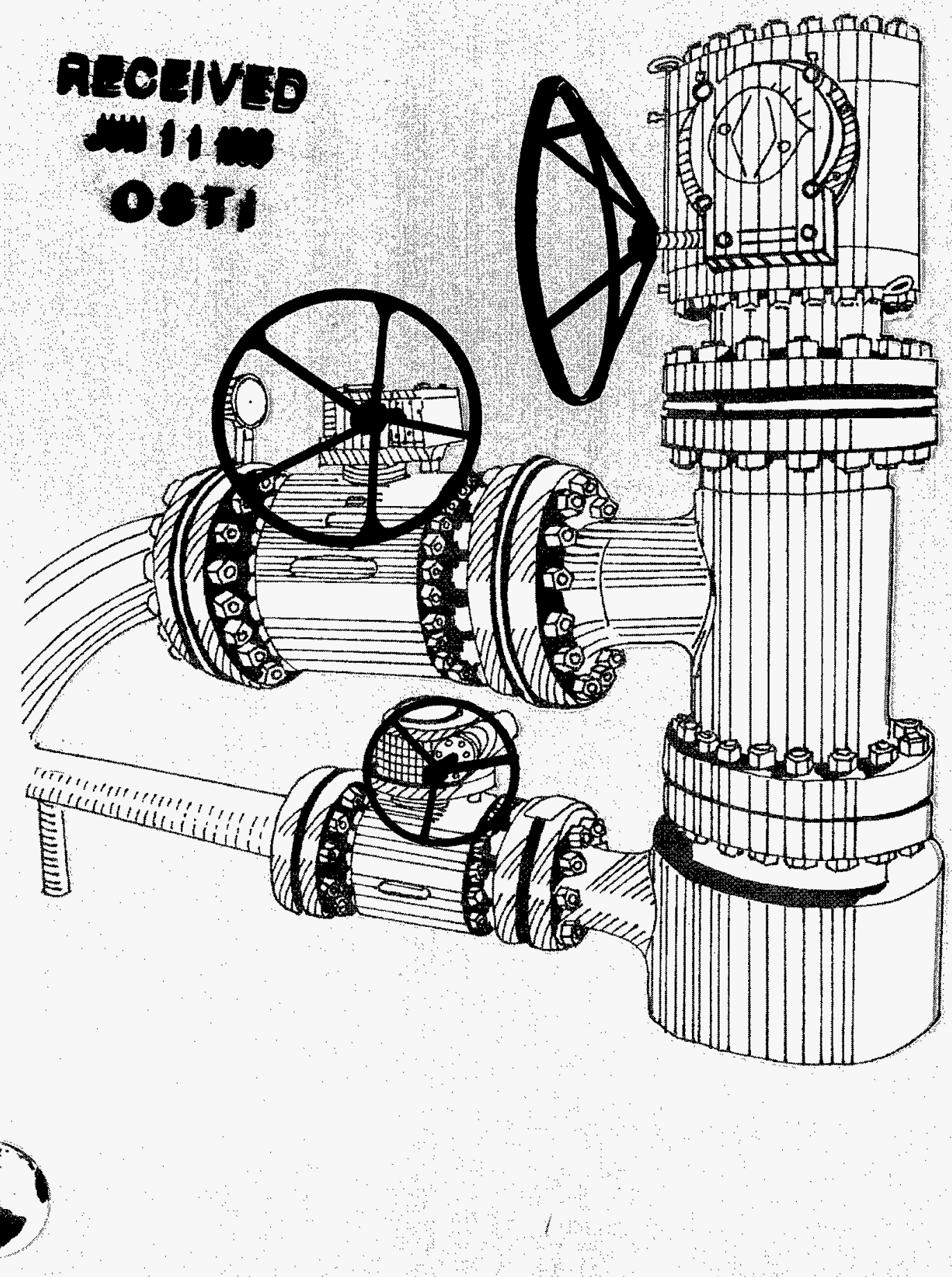

Energy

Administration 
This publication and other Energy Information Administration (EIA) publications may be purchased from the Superintendent of Documents, U.S. Government Printing Office.

Recent publications may be purchased from:

Superintendent of Documents
U.S. Government Printing Office
P.O. Box 371954
Pittsburgh, PA 15250-7954
(202) 512-1800
(202) 512-2250 (FAX)
8:00 a.m. to 4:30 p.m., eastern time, M-F

Older publications may be purchased from:

\author{
National Technical Information Service \\ U.S. Department of Commerce \\ 5285 Port Royal Road \\ Springfield, Virginia 22161 \\ (703) $487-4650$ \\ (703) $321-8547$ (FAX)
}

Complimentary subscriptions and single issues are available to certain groups of subscribers, such as public and academic libraries, Federal, State, local, and foreign governments, EIA survey respondents, and the media. For further information, and for answers to questions on energy statistics, please contact EIA's National Energy Information Center. Address, telephone numbers, and hours are as follows:

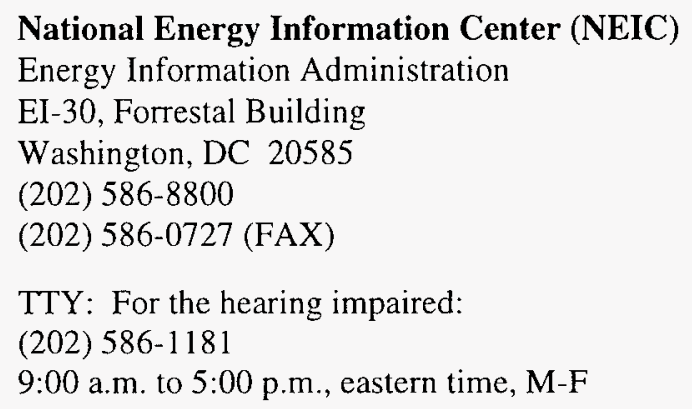

Internet Addresses:

E-mail: infoctr@eia.doe.gov

World Wide Web Site: http://www.eia.doe.gov

FTP Site: ftp://ftp.eia.doe.gov

Internet Site Services - offer nearly all EIA publications. Users can view and download selected pages or entire reports, search for information, download EIA data and analysis applications, and find out about new EIA information products and services.

EIA's CD-ROM, Energy InfoDisc, contains most EIA publications and major energy database applications. The Energy InfoDisc, produced quarterly, is available for a fee from STAT-USA, Department of Commence, 1-800-STAT-USA.

We thank the following for the use of their photographs and illustrations in this report.

Standard Oil Co., page 1 (courtesy of the American Petroleum Institute).

Phillips 66 Co., page 31 (courtesy of Phillips 66 Company).

Chevron U.S.A. Inc., page 79 (courtesy of the American Petroleum Institute).

Texaco Inc., page 121 (courtesy of Texaco Inc.).

Standard Oil Co., page 125 (courtesy of the American Petroleum Institute).

Texaco Inc., page 157 (courtesy of the American Petroleum Institute).

Atlantic Richfield Co., page 161 (courtesy of the American Petroleum Institute).

Released for printing: June 1, 1998

Question concerning the contents of this report should be directed as indicated on page $\mathrm{v}$.

On The Cover: Artist's rendition of a wellhead at Bryan Mound in Texas which is part of the Strategic Petroleum Reserve program. This program develops underground storage areas to hold emergency supplies of petroleum. Since 1976, the Department of Energy has been involved in a major facilities development program to stockpile crude oil. The Strategic Petroleum Reserve has four underground crude oil storage sites in salt domes. These sites are organized into three distribution systems and connected by DOE pipelines to commercial crude oil pipeline networks and marine terminals for drawdown and distribution.

Description above based on information provided by the Energy Technology Visuals Collection, Department of Energy. 


\section{DISCLAIMER}

This report was prepared as an account of work sponsored by an agency of the United States Government. Neither the United States Government nor any agency thereof, nor any of their employees, makes any warranty, express or implied, or assumes any legal liability or responsibility for the accuracy, completeness, or usefulness of any information, apparatus, product, or process disclosed, or represents that its use would not infringe privately owned rights. Reference herein to any specific commercial product, process, or service by trade name, trademark, manufacturer, or otherwise does not necessarily constitute or imply its endorsement, recommendation, or favoring by the United States Government or any agency thereof. The views and opinions of authors expressed herein do not necessarily state or refiect those of the United States Government or any agency thereof. 


\title{
Petroleum Supply Annual 1997
}

\author{
Volume 1
}

\section{June 1998}

\section{Energy Information Administration}

Office of Oil and Gas

U.S. Department of Energy

Washington, DC 20585

DISTRIBUTION OF THIS OOCUMENT IS UNLIMITEO

This report was prepared by the Energy Information Administration, the independent statistical and analytical agency within the Department of Energy. The information contained herein should not be construed as advocating or reflecting any policy position of the Department of Energy or any other organization. 


\section{Data Available Electronically}

Data from the Weekly Petroleum Status Report, Winter Fuels Report, and the Petroleum Supply Monthly publications as well as data from other sources are available electronically on the Energy Information Administration's Electronic Publication Bulletin (EPUB) Board, and the Comprehensive Oil and Gas Information Source (COGIS). The schedule for data release is as follows:

\begin{tabular}{l|l|l|}
\hline Publications/Sources & Platform & Information \\
\hline
\end{tabular}

\section{Weekly Petroleum Status Report}

Wednesday 9:00 a.m. (weekly)

Wednesday 5:00 p.m. 6th-12th (monthly)

Thursday by Noon (weekly)

Thursday by Noon 7th-13th (monthly)
EPUB/WWW

EPUB/WWW

COGIS

COGIS

Winter Fuels Report (October through March)

Wednesday 5:00 p.m. (weekly)

EPUB/WWW

Thursday by Noon (weekly)

All tables and highlights

All tables and highlights

Propane Data (April through September)

Second Wednesday of the

EPUB/WWW

month (9:00 a.m.)

\section{Petroleum Supply Monthly}

23rd-26th (monthly)

EPUB/WWW

23rd-26th (monthly)

COGIS

Petroleum Supply Annual

WWW

\section{Oxygenate Data}

15 working days after the report month

EPUB/WWW

Table H1 (Petroleum Supply Summary) and all Summary Statistics and Detailed Statistics Tables

Table H1 (Petroleum Supply Summary), and all Summary Statistics and Detailed Statistics Tables

All tables and data bases

Table D1 U.S. Summary Table D2 (Fuel Ethanol Production/Stocks) and Table D3 (MTBE Production/Stocks) Table D4 (MTBE Merchant and Captive)

\section{Imports Data}

7th-10th (preliminary)

EPUB/WWW

23rd-26th (final)

Import data by company from the Form EIA-814, "Monthly Imports Report" 


\section{Electronic Publishing System (EPUB) \\ User Instructions}

EPUB is an electronic publishing system maintained by the Energy Information Administration of the U.S. Department of Energy. EPUB allows the general public to electronically access selected energy data from many of EIA's statistical reports. The system is a menu-driven, bulletin board type system with extensive online help capabilities that can be accessed free of charge 24 hours a day by using a terminal or PC with an asynchronous modem. (EPUB will be taken down briefly every night at midnight for backup.)

\section{CONFIGURING YOUR PC SOFTWARE}

PC users must provide the following information to their communications software in order to successfully access the EPUB system. Consult your communications software documentation for information on how to correctly configure your software.

Communication Parameters:

BAUD RATE: Up to $28,800 \mathrm{bps}$

DATA BITS: 8

STOP BITS: 1

PARITY: NONE

DUPLEX: FULL

TERMINAL TYPE: examples: ANSI, ANSI-BBS, VT100

\section{ACCESS PHONE NUMBER}

Once your communications software and/or hardware has been configured, you can access EPUB by dialing (202) 586-2557.

\section{USING EPUB}

When a connection to the system has been made, some users may find that the menu-driven instructions and the online help capabilities will provide enough information to effectively use EPUB. If needed, more extensive information may be found in the EPUB Users Guide, which is available online from the EPUB system or from:

National Energy Information Center, EI-231

Energy Information Administration

Forrestal Building, Room 1F-048

Washington, DC 20585

(202) 586-8800

Internet E-MAIL: infoctr@eia.doe.gov

Hours: 9:00 a.m. to 5:00 p.m. Eastern Time, Monday through Friday

Telecommunications device for the hearing-impaired only:

(202) 586-1181. Hours: 9:00 a.m. to 5:00 p.m. Eastern Time, Monday through Friday

\section{EPUB ASSISTANCE}

For communications or technical assistance, call (202) 586-8959, 8:00 a.m. to 5:00 p.m. Eastern Time, Monday through Friday.

For questions about the content of EPUB reports, call (202) 586-8800, 9:00 a.m. to 5:00 p.m. Eastern Time, Monday through Friday.

\section{EPUB PROVIDES STATISTICAL INFORMATION, AS WELL AS DATA FROM THE FOLLOWING EIA PUBLICATIONS:}

Heating fuel data, (April through September) updated the 2nd week of the month

\section{Oxygenate data, updated approximately 15 working days after the end of the report month}

Weekly Petroleum Status Report, updated on Wednesdays (Thursday in event of a holiday) at 9:00 a.m.

Petroleum Supply Monthly, updated between the 23rd and 26th of the month

Petroleum Marketing Monthly, updated by the 8th of the month

Winter Fuels Report, propane and distillate highlights and distillate data updated Wednesday at 5:00 p.m. All other data updated

Thursday at 5:00 p.m. (October through March)

Natural Gas Monthly, updated on the 20th of the month

Weekly Coal Production, updated on Fridays by 5:00 p.m.

Quarterly Coal Report, updated 60 days after the end of the quarter

Electric Power Monthly, updated the first week of the month

Monthly Energy Review, updated the last week of the month

Short Term Energy Outlook, updated 60 days after the end of the quarter 


\section{Comprehensive Oil and Gas Information Source}

The Comprehensive Oil and Gas Information Source (COGIS) is a project recently developed by the Energy Information Administration (EIA), in cooperation with the U.S. Department of Commerce in an effort to provide more timely information to its customers. COGIS offers the latest oil and gas data published by the EIA. Selected data series from the Petroleum Supply Monthly, the Petroleum Marketing Monthly, the Natural Gas Monthly, the Monthly Energy Review, the Weekly Petroleum Status Report, the Short Term Energy Outlook, and the Winter Fuels Report are available. In addition, COGIS offers timely analysis of major oil and gas trends, and weekly and monthly highlights of oil and gas activity.

Anyone with a workstation connected to an Internet node, or with a personal computer and modem, can have immediate access to oil and gas industry information.

For information, call EIA's National Energy Information Center, (202) 586-8800. To open an account, call the U.S. Department of Commerce, Office of Business Analysis, (202) 482-1986.

Current fee schedule is listed below.

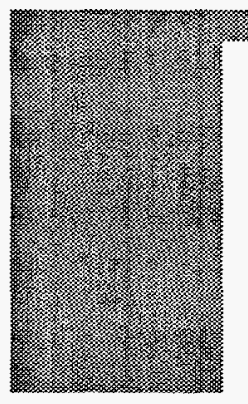

(1)

\section{Charge}

Annual Fee

Connect Charge Credit

Connect Charges (per minute based on eastern time)

Weekdays: 8:00 a.m. - noon

Noon - 6:00 p.m.

6:00 p.m. - 8:00 a.m.

(Also weekends and holidays)

Annual Flat Fee Option (cannot use account between 8:00 a.m. and noon)

Maximum 1 hour per day

Maximum 4 hours per day
Means Used to Access the EBB

\begin{tabular}{ccc}
\hline Up to 2400 Baud & 9600 Baud & Internet (telnet only) \\
$\$ 45.00$ & $\$ 45.00$ & $\$ 45.00$ \\
$\$ 20.00$ & $\$ 20.00$ & $\$ 20.00$
\end{tabular}

$\$ 0.20$

$\$ 0.40$

$\$ 0.25$

$\$ 0.15$

$\$ 0.10$

$\$ 0.40$

$\$ 0.25$

$\$ 0.10$ 


\section{Contacts}

The Petroleum Supply Annual is prepared by the Energy Information Administration, Office of Oil and Gas, Kenneth A. Vagts, Director.

Questions, comments, and general information concerning the contents of the Petroleum Supply Annual should be referred to the National Energy Information Center (202) 586-8800. Technical questions may be addressed to the following specialists:

\begin{tabular}{|c|c|c|}
\hline Immary Statistics. & Steve Patterson & (202) 586-5994 \\
\hline Supply and Disposition. & Steve Patterson & (202) 586-5994 \\
\hline Crude Oil Production... & David Hinton & (202) 586-2990 \\
\hline Natural Gas Processing. & David Hinton & (202) $586-2990$ \\
\hline Refinery Operations.. & Mike Conner & (202) 586-1795 \\
\hline Imports .. & Stacey Ungerleider & (202) $586-5130$ \\
\hline (1) & Steve Patterson & (202) $586-5994$ \\
\hline 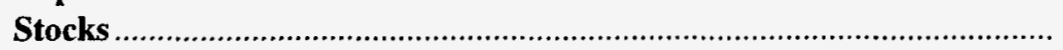 & Mike Conner & (202) 586-1795 \\
\hline (2) & Mike Conner & (202) 586-1795 \\
\hline 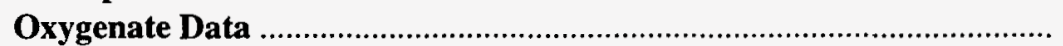 & Steve Patterson & (202) 586-5994 \\
\hline
\end{tabular}

Additional information on all energy statistics available from the Energy Information Administration may be obtained from the National Energy Information Center (202) 586-8800. 


\section{Preface}

The Petroleum Supply Annual (PSA) contains information on the supply and disposition of crude oil and petroleum products. The publication reflects data that were collected from the petroleum industry during 1997 through annual and monthly surveys. The PSA is divided into two volumes. This first volume contains three sections: Summary Statistics, Detailed Statistics, and Refinery Statistics; each with final annual data. The second volume contains final statistics for each month of 1997, and replaces data previously published in the Petroleum Supply Monthly (PSM). The tables in Volumes 1 and 2 are similarly numbered to facilitate comparison between them. Below is a description of each section in Volume 1 of the PSA.

\section{Summary Statistics}

This section contains a summary of the data presented each month in the PSM and in Volume 2 of the PSA. Graphs and tables are provided which show 16 years of data depicting the balance between supply, disposition and ending stocks for various commodities including crude oil, motor gasoline, distillate fuel oil, residual fuel oil, jet fuel, propane/propylene, and liquefied petroleum gases.

\section{Detailed Statistics}

The tables contained in this section provide 1997 detailed statistics on supply and disposition, refinery operations, imports and exports, stocks, and transportation of crude oil and petroleum products. In most cases, the statistics are presented for several geographic areas - the United States (50 States and the District of Columbia), five Petroleum Administration for Defense (PAD) Districts, and 12 Refining Districts. At the U.S. and PAD District level, the total volume and the daily rate of activities are presented.

\section{Refinery Statistics}

This section includes a list of refinery shutdowns and reactivations during 1997, refinery sales during 1997, and refinery fuel use of crude oil and petroleum products during 1997. Annual U.S. refinery capacity data collection and publication normally presented each year in this section has been moved to a biennial schedule (every other year). The next year refinery capacity data collection will occur is 1999, and will present refinery capacity data as of January 1, 1999.

\section{Appendices}

Three appendices are provided to assist in understanding and interpreting the data presented in this publication. Industry terminology and product definitions are listed alphabetically in the Glossary.

- Appendix A (District Descriptions and Maps)-Geographic aggregations of the 50 States and the District of Columbia into Refining Districts which make up the PAD Districts.

- Appendix B (Detailed Statistics Explanatory Notes) - Information describing data collection, sources, estimation methodology, data quality control procedures, modifications to reporting requirements and interpretation of tables.

- Appendix C (1996 Revised Crude Oil Production) -Updated monthly and annual crude oil production statistics received after the publication of the 1996 PSA. 


\section{Contents}

Tables

Summary Statistics

S1. Crude Oil and Petroleum Products Overview, 1981-Present .......................................................................................... 2

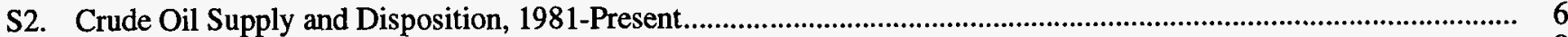

S3. Crude Oil and Petroleum Product Imports, 1981-Present ........................................................................................... 8

S4. Finished Motor Gasoline Supply and Disposition, 1981-Present ................................................................................. 17

S5. Distillate Fuel Oil Supply and Disposition, 1981-Present............................................................................................ 19

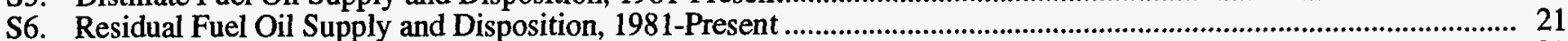

S7. Jet Fuel Supply and Disposition, 1981-Present ............................................................................................................ 23

S8. Propane/Propylene Supply and Disposition, 1981-Present .......................................................................................... 25

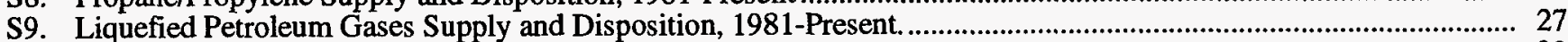

S10. Other Petroleum Products Supply and Disposition, 1981-Present ................................................................................. 28

Summary Statistics Table and Figure Sources................................................................................................................ 29.

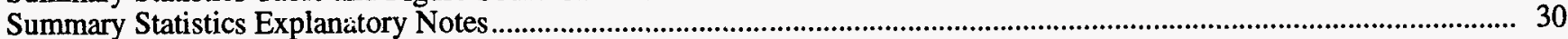

Detailed Statistics

National Statistics

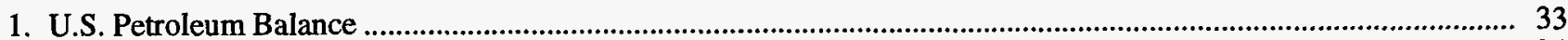

2. U.S. Supply, Disposition, and Ending Stocks of Crude Oil and Petroleum Products ................................................ 34

3. U.S. Daily Average Supply and Disposition of Crude Oil and Petroleum Products ................................................... 35

Supply and Disposition of Crude Oil and Petroleum Products

4. PAD District I …

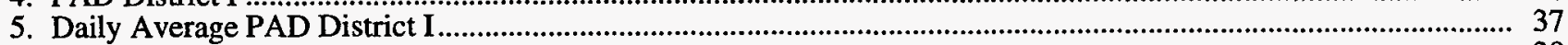

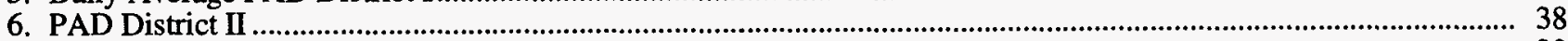

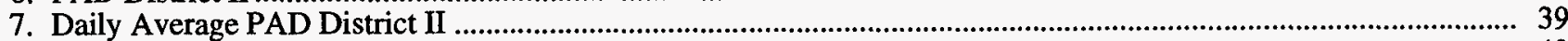

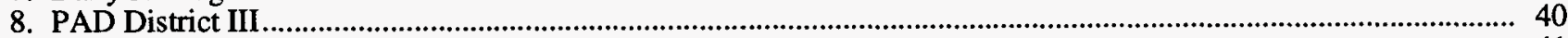

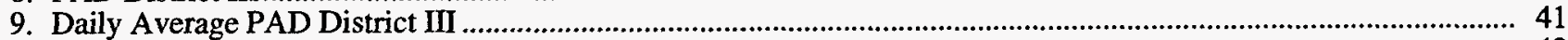

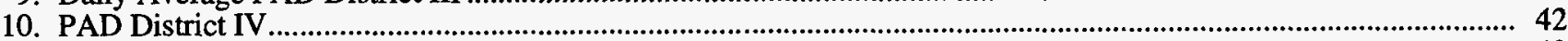

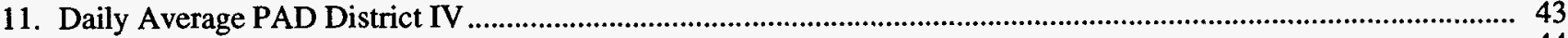

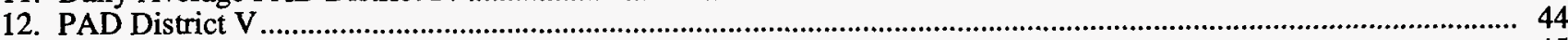

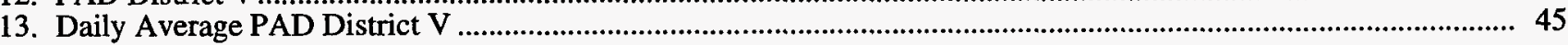

Production of Crude Oil

14. Production of Crude Oil by PAD District and State …...................................................................................... 46

Natural Gas Processing

15. Natural Gas Plant Net Production and Stocks of Petroleum Products by PAD and Refining Districts

\section{Refinery Operations}

16. Refinery Input of Crude Oil and Petroleum Products by PAD and Refining Districts............................................... 48

17. Refinery Net Production of Finished Petroleum Products by PAD and Refining Districts ......................................... 50

18. Refinery Stocks of Crude Oil and Petroleum Products by PAD and Refining Districts .......................................... 52

19. Percent Refinery Yield of Petroleum Products by PAD and Refining Districts ....................................................... 54

\section{Imports of Crude Oil and Petroleum Products}

PAD District

20. Imports of Crude Oil and Petroleum Products

\section{Country of Origin}

21. United States.

22. PAD District I

23. PAD District II

24. PAD District III

25. PAD Districts IV and V

64

State of Entry

26. Imports of Residual Fuel Oil by Sulfur Content 
Tables

Exports of Crude Oil and Petroleum Products

27. Exports of Crude Oil and Petroleum Products by PAD District.

28. Exports of Crude Oil and Petroleum Products by Destination.

Net Imports

29. Net Imports of Crude Oil and Petroleum Products into the United States by Country

Stocks

30. Stocks of Crude Oil and Petroleum Products by PAD District 71

31. Refinery, Bulk Terminal, and Natural Gas Plant Stocks of Selected Petroleum Products by PAD District and State

Movements of Crude Oil and Petroleum Products

32. Movements of Crude Oil and Petroleum Products by Pipeline, Tanker, and Barge Between PAD Districts

33. Movements of Crude Oil and Petroleum Products by Pipeline Between PAD Districts. 75

34. Movements of Crude Oil and Petroleum Products by Tanker and Barge Between PAD Districts

35. Net Movements of Crude Oil and Petroleum Products by Pipeline, Tanker, and Barge Between PAD Districts

\section{Refinery Statistics}

36. Refinery Fuel Use and Losses by PAD Dsitrict, 1997

37. Shutdown and Reactivated Refineries During 1997

38. Refinery Sales, March 1997 through February 1999

\section{Illustrations}

S1. Petroleum Overview, 1981-Present.

S2. Petroleum Products Supplied, 1981-Present

S3. Crude Oil Supply and Disposition, 1981-Present

S4. Crude Oil Ending Stocks, 1981-Present.

S5. Finished Motor Gasoline Supply and Disposition, 1981-Present.

S6. Motor Gasoline Ending Stocks, 1981-Present .

S7. Distillate Fuel Oil Supply and Disposition, 1981-Present

S8. Distillate Fuel Oil Ending Stocks, 1981-Present ...

S9. Residual Fuel Oil Supply and Disposition, 1981-Present

S10. Residual Fuel Oil Ending Stocks, 1981-Present

S11. Jet Fuel Supply and Disposition, 1981-Present

S12. Jet Fuel Ending Stocks, 1981-Present.

S13. Propane/Propylene Supply and Disposition, 1981-Present

S14. Propane/Propylene Ending Stocks, 1981-Present

S15. Liquefied Petroleum Gases Supply and Disposition, 1981-Present

S16. Liquefied Petroleum Gases Ending Stocks, 1981-Present

Appendices

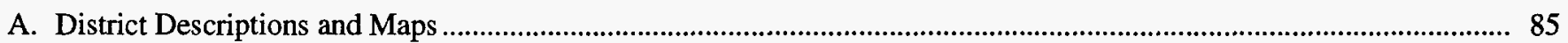

B. Explanatory Notes

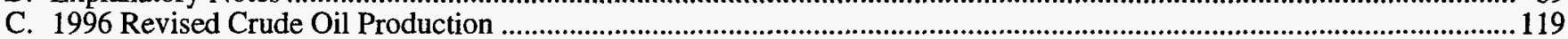

Glossary

Definitions of Petroleum Products and Other Terms 


\section{Summary \\ Statistics}

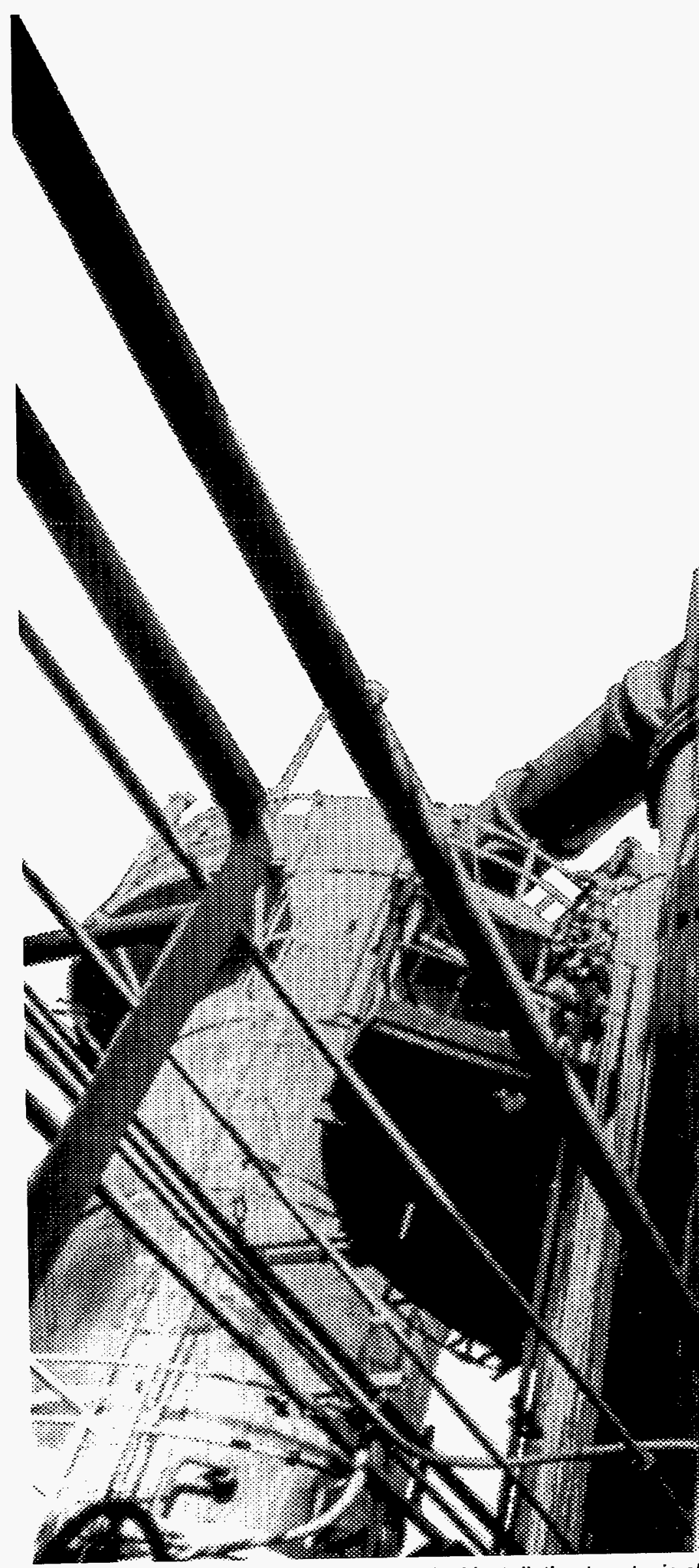


Table S1. Crude Oil and Petroleum Products Overview, 1981 - Present (Thousand Barrels per Day, Except Where Noted)

\begin{tabular}{|c|c|c|c|c|c|c|c|c|}
\hline & \multirow[b]{2}{*}{ Year/Month } & \multicolumn{3}{|c|}{ Field Production } & \multicolumn{2}{|c|}{ Stock Change ${ }^{a}$} & \multirow[b]{2}{*}{$\begin{array}{l}\text { Petroleum } \\
\text { Products } \\
\text { Supplied }\end{array}$} & \multirow{2}{*}{\begin{tabular}{|c|}
$\begin{array}{c}\text { Ending Stocks } \\
\text { (Million Barrels) }\end{array}$ \\
$\begin{array}{c}\text { Crude Oil } \\
\text { Cetroleum and } \\
\text { Petrom } \\
\text { Products }\end{array}$
\end{tabular}} \\
\hline & & $\begin{array}{c}\text { Total } \\
\text { Domestic }^{c}\end{array}$ & $\begin{array}{c}\text { Crude } \\
\text { Oil }\end{array}$ & $\begin{array}{l}\text { Natural } \\
\text { Gas Plant } \\
\text { Liquids }\end{array}$ & $\begin{array}{c}\text { Cruge } \\
\text { Oil }^{d}\end{array}$ & $\begin{array}{l}\text { Petroleum } \\
\text { Products }\end{array}$ & & \\
\hline $\begin{array}{l}1981 \\
1982 \\
1983 \\
1984 \\
1985 \\
1986 \\
1987 \\
1988 \\
1989 \\
1990 \\
1991 \\
1992 \\
1993 \\
1994\end{array}$ & 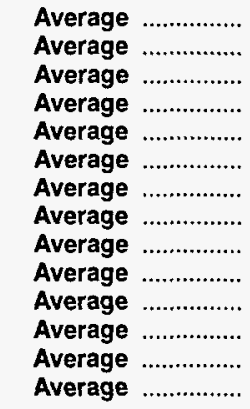 & $\begin{array}{r}10,230 \\
10,252 \\
10,299 \\
10,554 \\
10,636 \\
10,289 \\
10,008 \\
9,818 \\
9,219 \\
8,994 \\
9,168 \\
8,996 \\
8,836 \\
8,645\end{array}$ & $\begin{array}{l}8,572 \\
8,649 \\
8,688 \\
8,879 \\
8,971 \\
8,680 \\
8,349 \\
8,140 \\
7,613 \\
7,355 \\
7,417 \\
7,171 \\
6,847 \\
6,662\end{array}$ & $\begin{array}{l}1,609 \\
1,550 \\
1,559 \\
1,630 \\
1,609 \\
1,551 \\
1,595 \\
1,625 \\
1,546 \\
1,559 \\
1,659 \\
1,697 \\
1,736 \\
1,727\end{array}$ & $\begin{array}{r}9290 \\
136 \\
9214 \\
199 \\
50 \\
78 \\
128 \\
1 \\
86 \\
-35 \\
-42 \\
-1 \\
81 \\
18\end{array}$ & $\begin{array}{r}9-130 \\
-283 \\
g-234 \\
81 \\
-153 \\
124 \\
-87 \\
-29 \\
-129 \\
142 \\
32 \\
-68 \\
70 \\
-2\end{array}$ & $\begin{array}{l}16,058 \\
15,296 \\
15,231 \\
15,726 \\
15,726 \\
16,281 \\
16,665 \\
17,283 \\
17,325 \\
16,988 \\
16,714 \\
17,033 \\
17,237 \\
17,718\end{array}$ & $\begin{array}{r}1,484 \\
91,430 \\
1,454 \\
1,556 \\
1,519 \\
1,593 \\
1,607 \\
1,597 \\
1,581 \\
1,621 \\
1,617 \\
91,592 \\
1,647 \\
1,653\end{array}$ \\
\hline \multicolumn{2}{|c|}{ 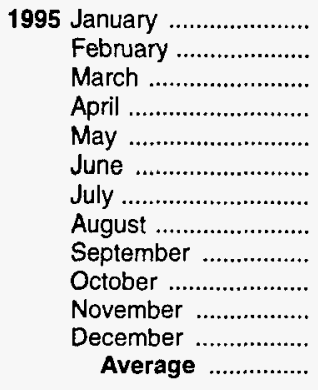 } & $\begin{array}{l}8,764 \\
8,935 \\
8,619 \\
8,720 \\
8,729 \\
8,607 \\
8,500 \\
8,498 \\
8,467 \\
8,501 \\
8,662 \\
8,533 \\
8,626\end{array}$ & $\begin{array}{l}6,682 \\
6,794 \\
6,600 \\
6,604 \\
6,629 \\
6,579 \\
6,449 \\
6,447 \\
6,416 \\
6,421 \\
6,585 \\
6,530 \\
6,560\end{array}$ & $\begin{array}{l}1,787 \\
1,780 \\
1,776 \\
1,794 \\
1,790 \\
1,740 \\
1,751 \\
1,730 \\
1,757 \\
1,757 \\
1,797 \\
1,691 \\
1,762\end{array}$ & $\begin{array}{r}-219 \\
-49 \\
336 \\
-101 \\
-132 \\
-148 \\
-397 \\
-253 \\
-64 \\
168 \\
263 \\
-505 \\
-93\end{array}$ & $\begin{array}{r}g-84 \\
-1,225 \\
-552 \\
114 \\
464 \\
57 \\
897 \\
-73 \\
243 \\
-589 \\
-352 \\
-822 \\
-153\end{array}$ & $\begin{array}{l}17,219 \\
18,279 \\
17,484 \\
17,142 \\
17,293 \\
18,131 \\
17,147 \\
18,044 \\
18,026 \\
17,651 \\
17,979 \\
18,366 \\
17,725\end{array}$ & $\begin{array}{r}91,643 \\
1,608 \\
1,601 \\
1,601 \\
1,612 \\
1,609 \\
1,624 \\
1,614 \\
1,620 \\
1,607 \\
1,604 \\
1,563 \\
-\end{array}$ \\
\hline \multicolumn{2}{|c|}{ 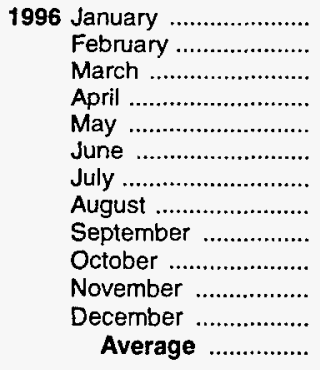 } & $\begin{array}{l}8,564 \\
8,558 \\
8,718 \\
8,597 \\
8,502 \\
8,550 \\
8,486 \\
8,535 \\
8,623 \\
8,685 \\
8,730 \\
8,738 \\
8,607\end{array}$ & $\begin{array}{l}6,495 \\
6,577 \\
6,571 \\
6,444 \\
6,394 \\
6,458 \\
6,338 \\
6,360 \\
6,482 \\
6,481 \\
6,476 \\
6,506 \\
6,465\end{array}$ & $\begin{array}{l}1,716 \\
1,680 \\
1,814 \\
1,845 \\
1,806 \\
1,833 \\
1,829 \\
1,858 \\
1,872 \\
1,912 \\
1,915 \\
1,876 \\
1,830\end{array}$ & $\begin{array}{r}-8 \\
-63 \\
-132 \\
29 \\
2 \\
305 \\
-244 \\
-19 \\
-499 \\
186 \\
-414 \\
-627 \\
-124\end{array}$ & $\begin{array}{r}-592 \\
-1,454 \\
-464 \\
633 \\
576 \\
593 \\
358 \\
-130 \\
701 \\
-630 \\
-117 \\
165 \\
-28\end{array}$ & $\begin{array}{l}18,261 \\
18,620 \\
18,301 \\
17,885 \\
17,957 \\
18,107 \\
18,211 \\
18,658 \\
17,655 \\
19,171 \\
18,535 \\
18,334 \\
18,309\end{array}$ & $\begin{array}{r}1,544 \\
1,500 \\
1,482 \\
1,502 \\
1,520 \\
1,546 \\
1,550 \\
1,545 \\
1,551 \\
1,538 \\
1,522 \\
1,507 \\
-\end{array}$ \\
\hline \multicolumn{2}{|c|}{ 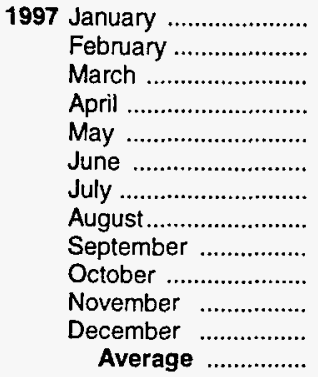 } & $\begin{array}{l}8,470 \\
8,708 \\
8,646 \\
8,604 \\
8,633 \\
8,610 \\
8,608 \\
8,535 \\
8,679 \\
8,624 \\
8,565 \\
8,662 \\
8,611\end{array}$ & $\begin{array}{l}6,402 \\
6,514 \\
6,452 \\
6,441 \\
6,474 \\
6,442 \\
6,409 \\
6,347 \\
6,486 \\
6,467 \\
6,459 \\
6,531 \\
6,452\end{array}$ & $\begin{array}{l}1,782 \\
1,867 \\
1,876 \\
1,824 \\
1,822 \\
1,827 \\
1,821 \\
1,831 \\
1,845 \\
1,813 \\
1,728 \\
1,773 \\
1,817\end{array}$ & $\begin{array}{r}462 \\
-122 \\
520 \\
197 \\
230 \\
-199 \\
-343 \\
-283 \\
95 \\
393 \\
252 \\
-608 \\
51\end{array}$ & $\begin{array}{r}-679 \\
-557 \\
444 \\
4 \\
1,172 \\
658 \\
-167 \\
643 \\
642 \\
-214 \\
-195 \\
-675 \\
93\end{array}$ & $\begin{array}{l}18,554 \\
18,398 \\
17,863 \\
18,559 \\
18,293 \\
18,617 \\
19,107 \\
18,565 \\
18,562 \\
19,071 \\
18,578 \\
19,250 \\
18,620\end{array}$ & $\begin{array}{r}1,501 \\
1,482 \\
1,512 \\
1,518 \\
1,561 \\
1,575 \\
1,559 \\
1,570 \\
1,592 \\
1,598 \\
1,600 \\
1,560 \\
-\end{array}$ \\
\hline
\end{tabular}

a A negative number indicates a decrease in stocks and a positive number indicates an increase.

b Stocks are totals as of end of period.

c Includes crude oil, natural gas plant liquids, and other liquids. Beginning in 1993, fuel ethanol blended into finished motor gasoline and oxygenate production from merchant MTBE plants are also included.

$\mathrm{d}$ includes stocks located in the Strategic Petroleum Reserve.

Includes crude oil for storage in the Strategic Petroleum Reserve.

f Net Imports equal Imports minus Exports.

$g$ In January 1981 and 1983, numerous respondents were added to surveys affecting stocks reported and stock change calculations. Stock changes are calculated using new basis stock levels. Bulk terminal and pipeline stocks of oxygenates were added beginning in January 1993 . See Summary Statistics Explanatory Note 2.

Footnotes continued on following page. 
Table S1. Crude Oil and Petroleum Products Overview, 1981 - Present (Continued) (Thousand Barrels per Day, Except Where Noted)

\begin{tabular}{|c|c|c|c|c|c|c|c|c|}
\hline & \multirow[b]{2}{*}{ Year/Month } & \multicolumn{3}{|c|}{ Imports } & \multicolumn{3}{|c|}{ Exports } & \multirow[b]{2}{*}{$\begin{array}{c}\text { Net } \\
\text { Imports }^{f}\end{array}$} \\
\hline & & Total & $\begin{array}{c}\text { Crude } \\
\text { Oile }^{e}\end{array}$ & $\begin{array}{l}\text { Petroleum } \\
\text { Products }\end{array}$ & Total & $\begin{array}{c}\text { Crude } \\
\text { Oil }\end{array}$ & $\begin{array}{l}\text { Petroleum } \\
\text { Products }\end{array}$ & \\
\hline $\begin{array}{l}1981 \\
1982 \\
1983 \\
1984 \\
1985 \\
1986 \\
1987 \\
1988 \\
1989 \\
1990 \\
1991 \\
1992 \\
1993 \\
1994\end{array}$ & $\begin{array}{l}\text { Average } \\
\text { Average } \ldots \ldots \ldots \ldots \ldots \\
\text { Average } \ldots \ldots \ldots \ldots \ldots \\
\text { Average } \ldots \ldots \ldots \ldots \ldots \\
\text { Average } \ldots \ldots \ldots \ldots \ldots \\
\text { Average } \ldots \ldots \ldots \ldots \ldots \\
\text { Average } \\
\text { Average } \\
\text { Average } \\
\text { Average }\end{array}$ & $\begin{array}{l}5,996 \\
5,113 \\
5,051 \\
5,437 \\
5,067 \\
6,224 \\
6,678 \\
7,402 \\
8,061 \\
8,018 \\
7,627 \\
7,888 \\
8,620 \\
8,996\end{array}$ & $\begin{array}{l}4,396 \\
3,488 \\
3,329 \\
3,426 \\
3,201 \\
4,178 \\
4,674 \\
5,107 \\
5,843 \\
5,894 \\
5,782 \\
6,083 \\
6,787 \\
7,063\end{array}$ & $\begin{array}{l}1,599 \\
1,625 \\
1,722 \\
2,011 \\
1,866 \\
2,045 \\
2,004 \\
2,295 \\
2,217 \\
2,123 \\
1,844 \\
1,805 \\
1,833 \\
1,933\end{array}$ & $\begin{array}{r}595 \\
815 \\
739 \\
722 \\
781 \\
785 \\
764 \\
815 \\
859 \\
857 \\
1,001 \\
950 \\
1,003 \\
942\end{array}$ & $\begin{array}{r}228 \\
236 \\
164 \\
181 \\
204 \\
154 \\
151 \\
155 \\
142 \\
109 \\
116 \\
89 \\
98 \\
99\end{array}$ & $\begin{array}{l}367 \\
579 \\
575 \\
541 \\
577 \\
631 \\
613 \\
661 \\
717 \\
748 \\
885 \\
861 \\
904 \\
843\end{array}$ & $\begin{array}{l}5,401 \\
4,298 \\
4,312 \\
4,715 \\
4,286 \\
5,439 \\
5,914 \\
6,587 \\
7,202 \\
7,161 \\
6,626 \\
6,938 \\
7,618 \\
8,054\end{array}$ \\
\hline $1995 \mathrm{~J}$ & 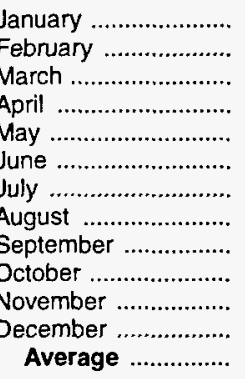 & $\begin{array}{l}8,015 \\
8,345 \\
9,006 \\
8,465 \\
8,709 \\
9,558 \\
8,863 \\
9,061 \\
9,736 \\
8,577 \\
9,074 \\
8,612 \\
8,835\end{array}$ & $\begin{array}{l}6,505 \\
6,546 \\
7,391 \\
7,038 \\
7,325 \\
7,927 \\
7,265 \\
7,437 \\
8,007 \\
7,075 \\
7,302 \\
6,916 \\
\mathbf{7 , 2 3 0}\end{array}$ & $\begin{array}{l}1,509 \\
1,799 \\
1,615 \\
1,427 \\
1,384 \\
1,631 \\
1,598 \\
1,624 \\
1,729 \\
1,502 \\
1,772 \\
1,696 \\
1,605\end{array}$ & $\begin{array}{r}978 \\
1,062 \\
948 \\
998 \\
876 \\
919 \\
895 \\
821 \\
805 \\
962 \\
1,002 \\
1,135 \\
949\end{array}$ & $\begin{array}{r}113 \\
95 \\
68 \\
155 \\
73 \\
101 \\
103 \\
61 \\
74 \\
50 \\
118 \\
127 \\
95\end{array}$ & $\begin{array}{r}865 \\
967 \\
880 \\
842 \\
803 \\
818 \\
792 \\
759 \\
731 \\
912 \\
884 \\
1,008 \\
855\end{array}$ & $\begin{array}{l}7,037 \\
7,283 \\
8,059 \\
7,467 \\
7,832 \\
8,639 \\
7,969 \\
8,240 \\
8,930 \\
7,615 \\
8,072 \\
7,477 \\
7,886\end{array}$ \\
\hline $1996 \mathrm{~J}$ & 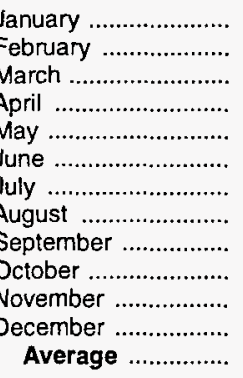 & $\begin{array}{r}9,364 \\
8,390 \\
9,092 \\
9,429 \\
10,007 \\
9,938 \\
9,820 \\
9,986 \\
9,142 \\
9,837 \\
9,244 \\
9,417 \\
9,478\end{array}$ & $\begin{array}{l}7,303 \\
6,612 \\
7,215 \\
7,371 \\
8,029 \\
7,958 \\
7,800 \\
8,041 \\
7,353 \\
7,701 \\
7,344 \\
7,307 \\
7,508\end{array}$ & $\begin{array}{l}2,061 \\
1,778 \\
1,877 \\
2,058 \\
1,977 \\
1,980 \\
2,020 \\
1,944 \\
1,789 \\
2,136 \\
1,900 \\
2,110 \\
1,971\end{array}$ & $\begin{array}{r}1,070 \\
1,048 \\
867 \\
976 \\
891 \\
895 \\
945 \\
896 \\
1,104 \\
1,045 \\
1,024 \\
1,013 \\
981\end{array}$ & $\begin{array}{r}89 \\
92 \\
94 \\
148 \\
37 \\
130 \\
139 \\
44 \\
147 \\
134 \\
172 \\
96 \\
110\end{array}$ & $\begin{array}{l}981 \\
956 \\
773 \\
828 \\
854 \\
766 \\
806 \\
852 \\
957 \\
911 \\
852 \\
917 \\
871\end{array}$ & $\begin{array}{l}8,294 \\
7,342 \\
8,225 \\
8,453 \\
9,116 \\
9,043 \\
8,876 \\
9,090 \\
8,038 \\
8,792 \\
8,220 \\
8,404 \\
8,498\end{array}$ \\
\hline $1997 \mathrm{~J}$ & 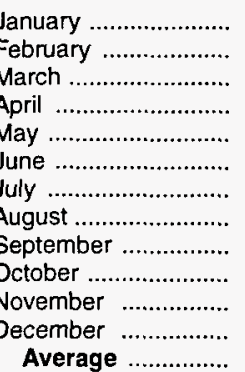 & $\begin{array}{r}9,763 \\
9,561 \\
9,833 \\
10,114 \\
10,818 \\
10,736 \\
10,008 \\
10,465 \\
10,537 \\
10,792 \\
9,948 \\
9,328 \\
10,162\end{array}$ & $\begin{array}{l}7,492 \\
7,434 \\
7,754 \\
7,987 \\
8,653 \\
8,759 \\
8,178 \\
8,621 \\
8,840 \\
8,927 \\
8,366 \\
7,653 \\
8,225\end{array}$ & $\begin{array}{l}2,271 \\
2,127 \\
2,079 \\
2,127 \\
2,165 \\
1,978 \\
1,830 \\
1,844 \\
1,697 \\
1,865 \\
1,582 \\
1,675 \\
1,936\end{array}$ & $\begin{array}{r}1,038 \\
1,017 \\
933 \\
937 \\
876 \\
955 \\
1,012 \\
1,074 \\
9997 \\
1,066 \\
934 \\
1,197 \\
1,003\end{array}$ & $\begin{array}{r}141 \\
229 \\
136 \\
92 \\
26 \\
57 \\
70 \\
110 \\
122 \\
152 \\
32 \\
131 \\
108\end{array}$ & $\begin{array}{r}897 \\
787 \\
796 \\
845 \\
851 \\
898 \\
942 \\
964 \\
875 \\
914 \\
901 \\
1.066 \\
896\end{array}$ & $\begin{array}{l}8,725 \\
8,544 \\
8,900 \\
9,177 \\
9,941 \\
9,782 \\
8,996 \\
9,390 \\
9,540 \\
9,726 \\
9,014 \\
8,130 \\
9,158\end{array}$ \\
\hline
\end{tabular}

Footnotes continued.

Notes: - Crude oil includes lease condensate. - Geographic coverage is the 50 States and the District of Columbia. - Totals may not equal sum of components due to independent rounding.

Source: See Summary Statistics Table and Figure Sources. 
Figure S1. Petroleum Overview, 1981 - Present

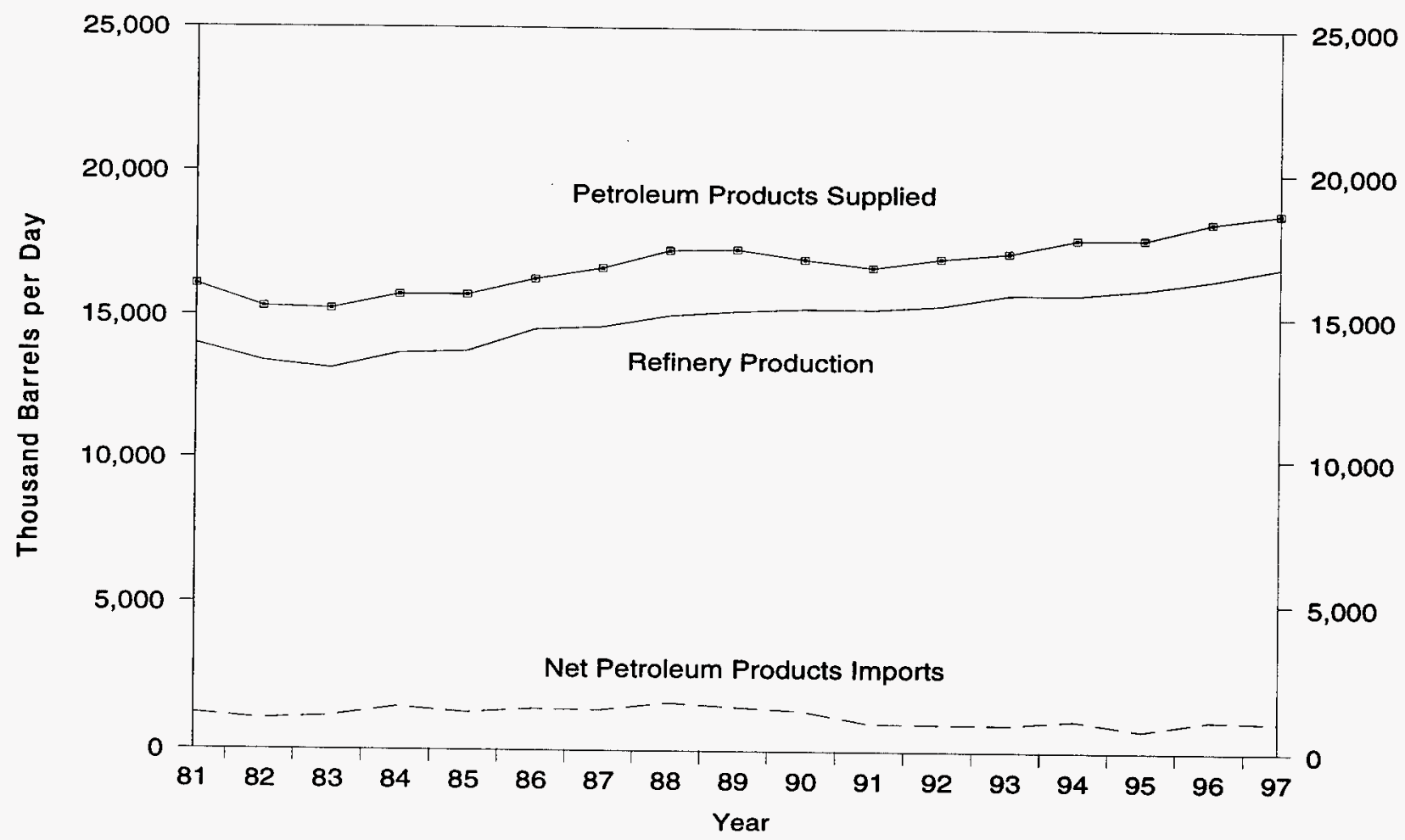

Source: Energy Information Administration, Petroleum Supply Annual, Table S1. See Summary Statistics Table and Figure Sources.

Figure S2. Petroleum Products Supplied, 1981 - Present

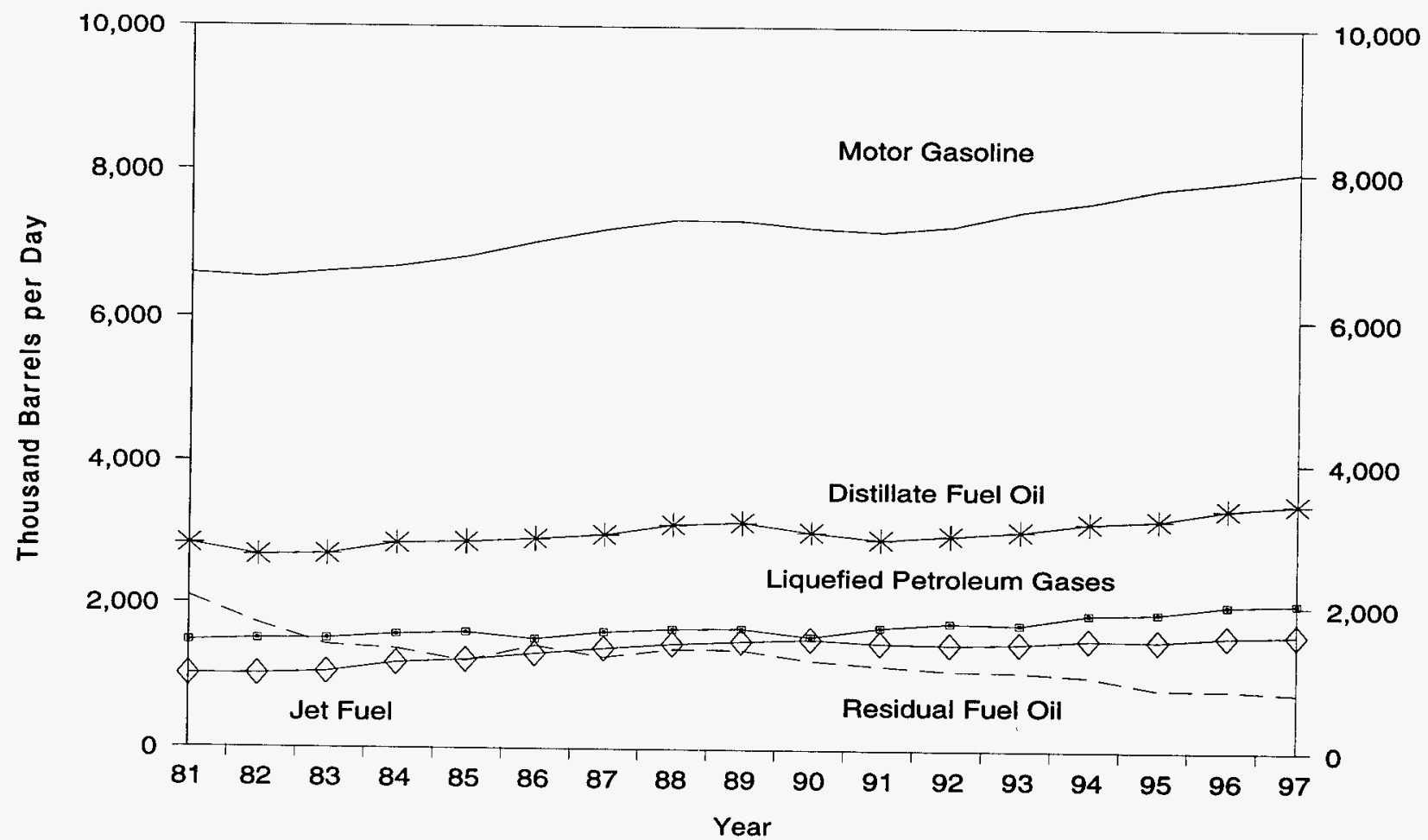

Source: Energy Information Administration, Petroleum Supply Annual, Tables S4 - S8. See Summary Statistics Table and Figure Sources. 
Figure S3. Crude Oil Supply and Disposition, 1981 - Present

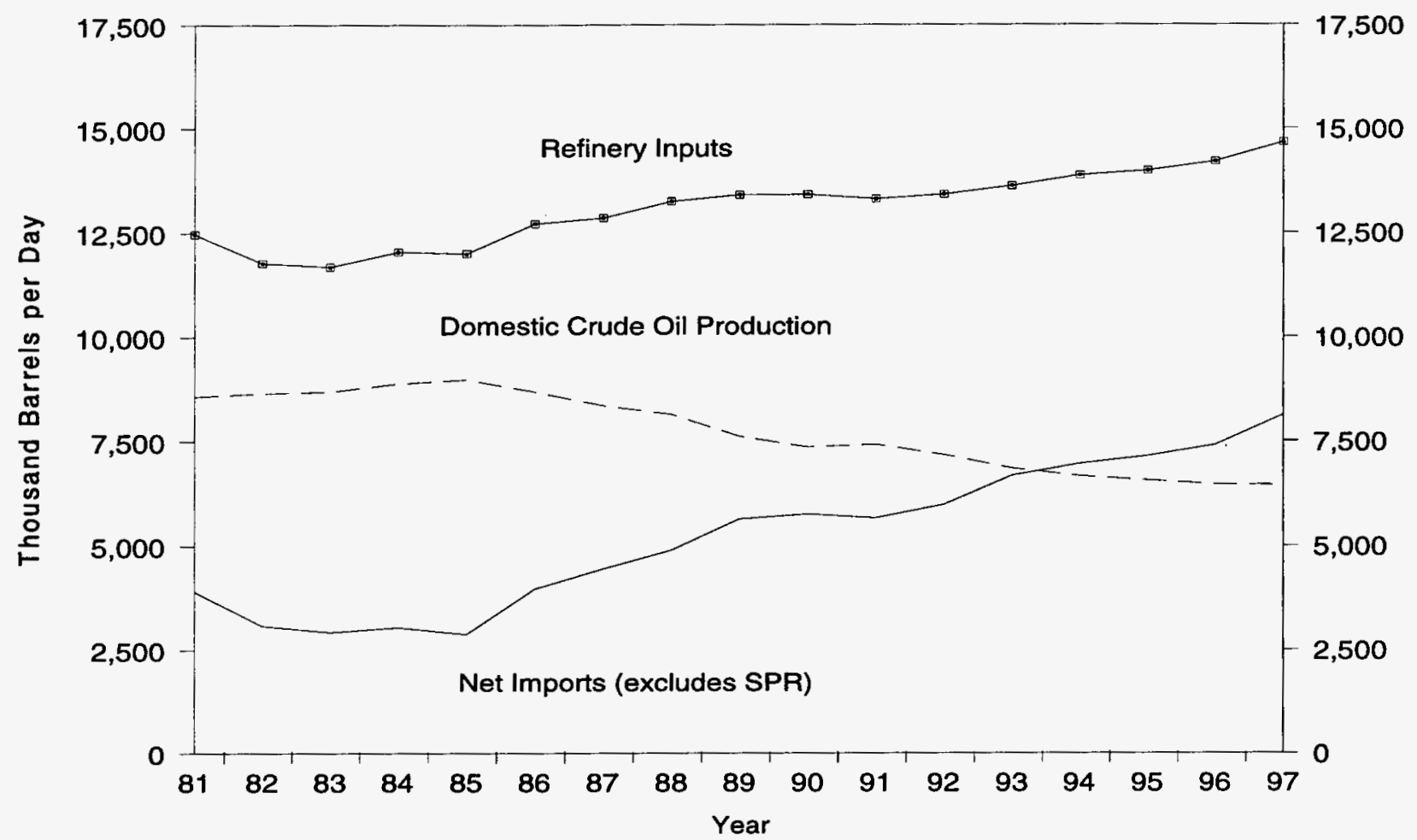

Source: Energy Information Administration, Petroleum Supply Annual, Table S2. See Summary Statistics Table and Figure Sources.

\section{Figure S4. Crude Oil Ending Stocks, ${ }^{1} 1981$ - Present}

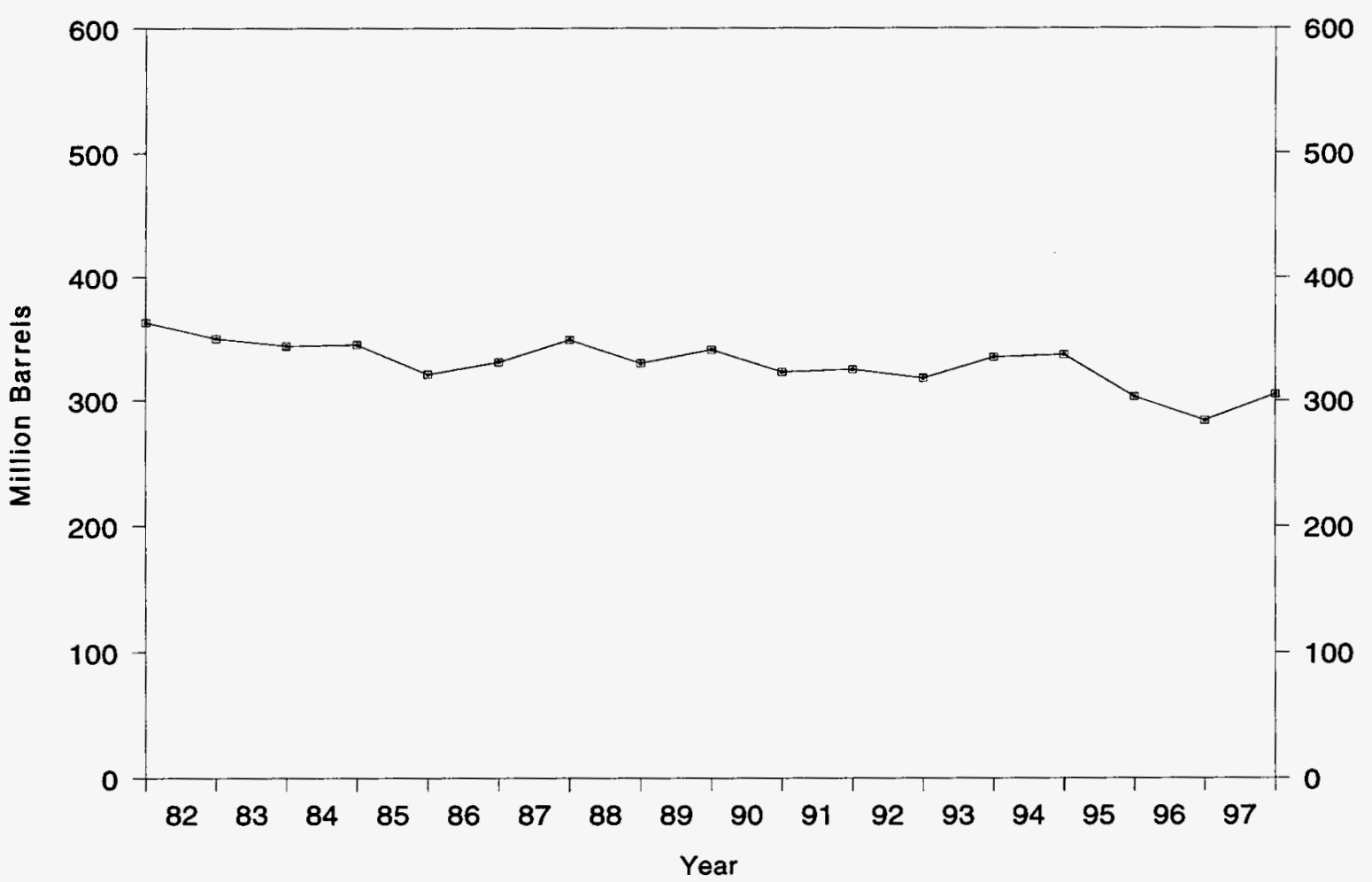

${ }^{1}$ Excludes stocks held in the Strategic Petroleum Reserve (SPR).

Source: Energy Information Administration, Petroleum Supply Annual, Table S2. See Summary Statistics Table and Figure Sources. 
Table S2. Crude Oil Supply and Disposition, 1981 - Present (Thousand Barrels per Day, Except Where Noted)

\begin{tabular}{|c|c|c|c|c|c|c|c|c|}
\hline & \multirow[b]{3}{*}{ Year/Month } & \multicolumn{6}{|c|}{ Supply } & \multirow{3}{*}{$\begin{array}{c}\text { Disposition } \\
\text { Crude } \\
\text { Losses }\end{array}$} \\
\hline & & \multicolumn{2}{|c|}{ Field Production } & \multicolumn{3}{|c|}{ Imports } & \multirow{2}{*}{$\begin{array}{c}\text { Unaccounted } \\
\text { for Crude } \\
\text { Oil }^{c}\end{array}$} & \\
\hline & & $\begin{array}{c}\text { Total } \\
\text { Domestic }\end{array}$ & Alaskan & Total & SPR & Other & & \\
\hline $\begin{array}{l}1981 \\
1982 \\
1983 \\
1984 \\
1985 \\
1986 \\
1987 \\
1988 \\
1989 \\
1990 \\
1991 \\
1992 \\
1993 \\
1994\end{array}$ & 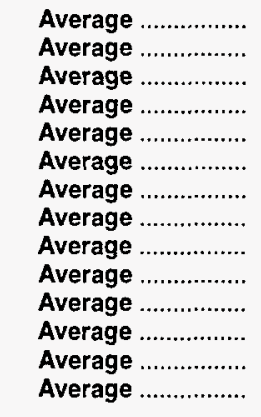 & $\begin{array}{l}8,572 \\
8,649 \\
8,688 \\
8,879 \\
8,971 \\
8,680 \\
8,349 \\
8,140 \\
7,613 \\
7,355 \\
7,417 \\
7,171 \\
6,847 \\
6,662\end{array}$ & $\begin{array}{l}1,609 \\
1,696 \\
1,714 \\
1,722 \\
1,825 \\
1,867 \\
1,962 \\
2,017 \\
1,874 \\
1,773 \\
1,798 \\
1,714 \\
1,582 \\
1,559\end{array}$ & $\begin{array}{l}4,396 \\
3,488 \\
3,329 \\
3,426 \\
3,201 \\
4,178 \\
4,674 \\
5,107 \\
5,843 \\
5,894 \\
5,782 \\
6,083 \\
6,787 \\
7,063\end{array}$ & $\begin{array}{r}256 \\
165 \\
234 \\
197 \\
118 \\
48 \\
73 \\
51 \\
56 \\
27 \\
0 \\
10 \\
15 \\
12\end{array}$ & $\begin{array}{l}4,141 \\
3,323 \\
3,096 \\
3,229 \\
3,083 \\
4,130 \\
4,601 \\
5,055 \\
5,787 \\
5,867 \\
5,782 \\
6,073 \\
6,772 \\
7,051\end{array}$ & $\begin{array}{r}83 \\
71 \\
114 \\
185 \\
145 \\
139 \\
145 \\
196 \\
200 \\
258 \\
195 \\
258 \\
168 \\
266\end{array}$ & $\begin{array}{r}5 \\
3 \\
2 \\
2 \\
1 \\
(s) \\
(s) \\
(s) \\
(s) \\
(s) \\
(s) \\
(s) \\
(s) \\
(s)\end{array}$ \\
\hline 1995 & 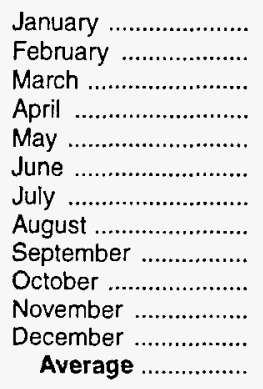 & $\begin{array}{l}6,682 \\
6,794 \\
6,600 \\
6,604 \\
6,629 \\
6,579 \\
6,449 \\
6,447 \\
6,416 \\
6,421 \\
6,585 \\
6,530 \\
6,560\end{array}$ & $\begin{array}{l}1,575 \\
1,578 \\
1,525 \\
1,511 \\
1,518 \\
1,484 \\
1,401 \\
1,432 \\
1,377 \\
1,475 \\
1,472 \\
1,466 \\
1,484\end{array}$ & $\begin{array}{l}6,505 \\
6,546 \\
7,391 \\
7,038 \\
7,325 \\
7,927 \\
7,265 \\
7,437 \\
8,007 \\
7,075 \\
7,302 \\
6,916 \\
7,230\end{array}$ & $\begin{array}{l}0 \\
0 \\
0 \\
0 \\
0 \\
0 \\
0 \\
0 \\
0 \\
0 \\
0 \\
0 \\
0\end{array}$ & $\begin{array}{l}6,505 \\
6,546 \\
7,391 \\
7,038 \\
7,325 \\
7,927 \\
7,265 \\
7,437 \\
8,007 \\
7,075 \\
7,302 \\
6,916 \\
7,230\end{array}$ & $\begin{array}{r}318 \\
78 \\
-101 \\
237 \\
296 \\
6 \\
402 \\
207 \\
-5 \\
328 \\
334 \\
193 \\
193\end{array}$ & $\begin{array}{r}(s) \\
0 \\
(s) \\
0 \\
0 \\
0 \\
0 \\
(s) \\
0 \\
(s) \\
0 \\
0 \\
(s)\end{array}$ \\
\hline 1996 & 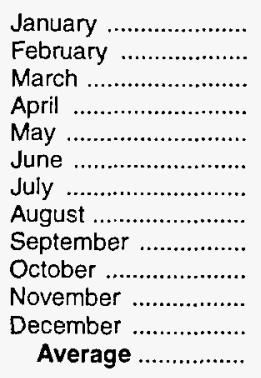 & $\begin{array}{l}6,495 \\
6,577 \\
6,571 \\
6,444 \\
6,394 \\
6,458 \\
6,338 \\
6,360 \\
6,482 \\
6,481 \\
6,476 \\
6,506 \\
6,465\end{array}$ & $\begin{array}{l}1,444 \\
1,482 \\
1,454 \\
1,367 \\
1,341 \\
1,419 \\
1,317 \\
1,327 \\
1,401 \\
1,379 \\
1,403 \\
1,392 \\
1,393\end{array}$ & $\begin{array}{l}7,303 \\
6,612 \\
7,215 \\
7,371 \\
8,029 \\
7,958 \\
7,800 \\
8,041 \\
7,353 \\
7,701 \\
7,344 \\
7,307 \\
7,508\end{array}$ & $\begin{array}{l}0 \\
0 \\
0 \\
0 \\
0 \\
0 \\
0 \\
0 \\
0 \\
0 \\
0 \\
0 \\
0\end{array}$ & $\begin{array}{l}7,303 \\
6,612 \\
7,215 \\
7,371 \\
8,029 \\
7,958 \\
7,800 \\
8,041 \\
7,353 \\
7,701 \\
7,344 \\
7,307 \\
7,508\end{array}$ & $\begin{array}{r}20 \\
413 \\
-25 \\
665 \\
61 \\
594 \\
121 \\
54 \\
303 \\
420 \\
148 \\
-153 \\
215\end{array}$ & $\begin{array}{r}0 \\
0 \\
0 \\
(s) \\
0 \\
0 \\
(s) \\
0 \\
0 \\
0 \\
0 \\
0 \\
\text { (s) }\end{array}$ \\
\hline 1997 & 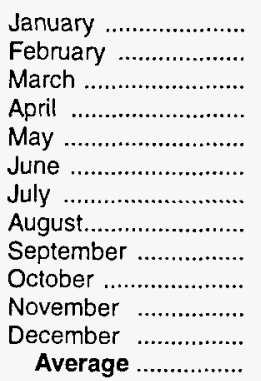 & $\begin{array}{l}6,402 \\
6,514 \\
6,452 \\
6,441 \\
6,474 \\
6,442 \\
6,409 \\
6,347 \\
6,486 \\
6,467 \\
6,459 \\
6,531 \\
6,452\end{array}$ & $\begin{array}{l}1,380 \\
1,384 \\
1,331 \\
1,330 \\
1,303 \\
1,260 \\
1,238 \\
1,200 \\
1,276 \\
1,286 \\
1,278 \\
1,290 \\
1,296\end{array}$ & $\begin{array}{l}7,492 \\
7,434 \\
7,754 \\
7,987 \\
8,653 \\
8,759 \\
8,178 \\
8,621 \\
8,840 \\
8,927 \\
8,366 \\
7,653 \\
8,225\end{array}$ & $\begin{array}{l}0 \\
0 \\
0 \\
0 \\
0 \\
0 \\
0 \\
0 \\
0 \\
0 \\
0 \\
0 \\
0\end{array}$ & $\begin{array}{l}7,492 \\
7,434 \\
7,754 \\
7,987 \\
8,653 \\
8,759 \\
8,178 \\
8,621 \\
8,840 \\
8,927 \\
8,366 \\
7,653 \\
8,225\end{array}$ & $\begin{array}{r}378 \\
-350 \\
501 \\
167 \\
257 \\
-170 \\
136 \\
130 \\
199 \\
5 \\
164 \\
267 \\
145\end{array}$ & $\begin{array}{l}0 \\
0 \\
0 \\
0 \\
0 \\
0 \\
0 \\
0 \\
0 \\
0 \\
0 \\
0 \\
0\end{array}$ \\
\hline
\end{tabular}

\footnotetext{
a Stocks are totals as of end of period.

b A negative number indicates a decrease in stocks and a positive number indicates an increase.

c Unaccounted for crude oil represents the difference between the supply and disposition of crude oil.

d Previously published as crude used directly.

e Stock changes are calculated using new basis stock levels. See Summary Statistics Explanatory Note 2.

Footnotes continued on following page.
} 
Table S2. Crude Oil Supply and Disposition, 1981 - Present (Continued)

(Thousand Barrels per Day, Except Where Noted)

\begin{tabular}{|c|c|c|c|c|c|c|c|c|c|}
\hline & \multirow[b]{3}{*}{ Year/Month } & \multicolumn{5}{|c|}{ Disposition } & \multirow{2}{*}{\multicolumn{3}{|c|}{$\begin{array}{l}\text { Ending Stocks } \\
\text { (Million Barrels) }\end{array}$}} \\
\hline & & \multicolumn{2}{|c|}{ Stock Change ${ }^{b}$} & \multirow[b]{2}{*}{$\begin{array}{l}\text { Refinery } \\
\text { Inputs }\end{array}$} & \multirow[b]{2}{*}{ Exports } & \multirow[b]{2}{*}{$\begin{array}{l}\text { Product } \\
\text { Supplied }\end{array}$} & & & \\
\hline & & SPR & Other & & & & Total & SPR & $\begin{array}{c}\text { Other } \\
\text { Primary }\end{array}$ \\
\hline $\begin{array}{l}1981 \\
1982 \\
1983 \\
1984 \\
1985 \\
1986 \\
1987 \\
1988 \\
1989 \\
1990 \\
1991 \\
1992 \\
1993 \\
1994\end{array}$ & 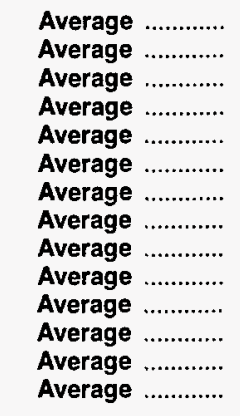 & $\begin{array}{r}336 \\
174 \\
234 \\
195 \\
117 \\
50 \\
80 \\
52 \\
56 \\
16 \\
-47 \\
17 \\
34 \\
13\end{array}$ & $\begin{array}{r}e-46 \\
-38 \\
\theta_{-20} \\
4 \\
-67 \\
28 \\
49 \\
-51 \\
30 \\
-51 \\
5 \\
-18 \\
47 \\
5\end{array}$ & $\begin{array}{l}12,470 \\
11,774 \\
11,685 \\
12,044 \\
12,002 \\
12,716 \\
12,854 \\
13,246 \\
13,401 \\
13,409 \\
13,301 \\
13,411 \\
13,613 \\
13,866\end{array}$ & $\begin{array}{r}228 \\
236 \\
164 \\
181 \\
204 \\
154 \\
151 \\
155 \\
142 \\
109 \\
116 \\
89 \\
98 \\
99\end{array}$ & $\begin{array}{r}d_{58} \\
d_{59} \\
66 \\
64 \\
60 \\
49 \\
34 \\
40 \\
28 \\
24 \\
18 \\
13 \\
10 \\
9\end{array}$ & $\begin{array}{l}594 \\
e_{644} \\
723 \\
796 \\
814 \\
843 \\
890 \\
890 \\
921 \\
908 \\
893 \\
893 \\
922 \\
929\end{array}$ & $\begin{array}{l}230 \\
294 \\
379 \\
451 \\
493 \\
512 \\
541 \\
560 \\
580 \\
586 \\
569 \\
575 \\
587 \\
592\end{array}$ & $\begin{array}{r}363 \\
\mathbf{0}^{350} \\
\mathbf{3 4 4} \\
\mathbf{3 4 5} \\
\mathbf{3 2 1} \\
\mathbf{3 3 1} \\
\mathbf{3 4 9} \\
\mathbf{3 3 0} \\
\mathbf{3 4 1} \\
\mathbf{3 2 3} \\
\mathbf{3 2 5} \\
\mathbf{3 1 8} \\
\mathbf{3 3 5} \\
\mathbf{3 3 7}\end{array}$ \\
\hline 1995 & 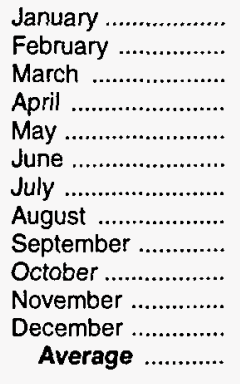 & $\begin{array}{l}(s) \\
(s) \\
(s) \\
(s) \\
(s) \\
(s) \\
(s) \\
(s) \\
(s) \\
(s) \\
-1 \\
(s) \\
(s)\end{array}$ & $\begin{array}{r}-219 \\
-49 \\
336 \\
-101 \\
-132 \\
-148 \\
-397 \\
-253 \\
-63 \\
169 \\
264 \\
-505 \\
-93\end{array}$ & $\begin{array}{l}13,604 \\
13,365 \\
13,480 \\
13,817 \\
14,303 \\
14,553 \\
14,403 \\
14,276 \\
14,402 \\
13,598 \\
13,833 \\
14,011 \\
13,973\end{array}$ & $\begin{array}{r}113 \\
95 \\
68 \\
155 \\
73 \\
101 \\
103 \\
61 \\
74 \\
50 \\
118 \\
127 \\
95\end{array}$ & $\begin{array}{l}7 \\
8 \\
7 \\
7 \\
7 \\
5 \\
7 \\
6 \\
6 \\
8 \\
7 \\
6 \\
7\end{array}$ & $\begin{array}{r}922 \\
921 \\
931 \\
928 \\
924 \\
920 \\
907 \\
899 \\
898 \\
903 \\
911 \\
895 \\
-\end{array}$ & $\begin{array}{r}592 \\
592 \\
592 \\
592 \\
592 \\
592 \\
592 \\
592 \\
592 \\
592 \\
592 \\
592 \\
-\end{array}$ & $\begin{array}{r}330 \\
329 \\
339 \\
336 \\
332 \\
328 \\
316 \\
308 \\
306 \\
311 \\
319 \\
303 \\
-\end{array}$ \\
\hline 1996 & 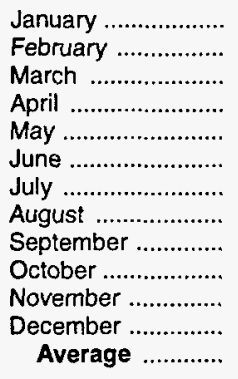 & $\begin{array}{r}(s) \\
(s) \\
-80 \\
-88 \\
-22 \\
-45 \\
-50 \\
-172 \\
-130 \\
-1 \\
-127 \\
-129 \\
-71\end{array}$ & $\begin{array}{r}-8 \\
-62 \\
-52 \\
117 \\
24 \\
350 \\
-194 \\
153 \\
-368 \\
187 \\
-288 \\
-498 \\
-53\end{array}$ & $\begin{array}{l}13,728 \\
13,564 \\
13,793 \\
14,295 \\
14,439 \\
14,569 \\
14,359 \\
14,424 \\
14,484 \\
14,277 \\
14,204 \\
14,185 \\
14,195\end{array}$ & $\begin{array}{r}89 \\
92 \\
94 \\
148 \\
37 \\
130 \\
139 \\
44 \\
147 \\
134 \\
172 \\
96 \\
110\end{array}$ & $\begin{array}{r}11 \\
8 \\
7 \\
6 \\
7 \\
6 \\
5 \\
6 \\
6 \\
5 \\
5 \\
6 \\
6\end{array}$ & $\begin{array}{r}895 \\
893 \\
889 \\
890 \\
890 \\
899 \\
891 \\
891 \\
876 \\
882 \\
869 \\
850 \\
-\end{array}$ & $\begin{array}{r}592 \\
592 \\
589 \\
586 \\
586 \\
584 \\
583 \\
578 \\
574 \\
574 \\
570 \\
566 \\
-\end{array}$ & $\begin{array}{r}303 \\
301 \\
300 \\
303 \\
304 \\
314 \\
308 \\
313 \\
302 \\
308 \\
299 \\
284 \\
-\end{array}$ \\
\hline 1997 & 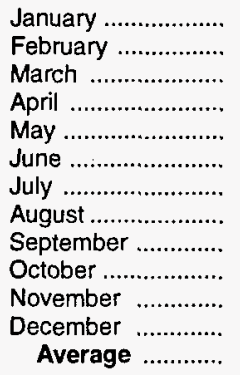 & $\begin{array}{l}-75 \\
(s) \\
(s) \\
(s) \\
(s) \\
(s) \\
(s) \\
(s) \\
(s) \\
(s) \\
(s) \\
(s) \\
-7\end{array}$ & $\begin{array}{r}537 \\
-121 \\
520 \\
197 \\
230 \\
-199 \\
-343 \\
-283 \\
95 \\
393 \\
252 \\
-607 \\
57\end{array}$ & $\begin{array}{l}13,664 \\
13,485 \\
14,047 \\
14,303 \\
15,123 \\
15,170 \\
14,994 \\
15,271 \\
15,308 \\
14,854 \\
14,706 \\
14,928 \\
14,662\end{array}$ & $\begin{array}{r}141 \\
229 \\
136 \\
92 \\
26 \\
57 \\
70 \\
110 \\
122 \\
152 \\
32 \\
131 \\
108\end{array}$ & $\begin{array}{r}5 \\
6 \\
5 \\
3 \\
4 \\
2 \\
2 \\
(s) \\
(s) \\
0 \\
0 \\
0 \\
2\end{array}$ & $\begin{array}{r}864 \\
861 \\
877 \\
883 \\
890 \\
884 \\
873 \\
864 \\
867 \\
879 \\
887 \\
868 \\
-\end{array}$ & $\begin{array}{r}563 \\
563 \\
563 \\
563 \\
563 \\
563 \\
563 \\
563 \\
563 \\
563 \\
563 \\
563 \\
-\end{array}$ & $\begin{array}{r}301 \\
297 \\
313 \\
319 \\
326 \\
320 \\
310 \\
301 \\
304 \\
316 \\
324 \\
305 \\
-\end{array}$ \\
\hline
\end{tabular}

Footnotes continued.

SPR = Strategic Petroleum Reserve

(s)=Less than 500 barrels per day.

Notes: - Crude oil includes lease condensate. - Geographic coverage is the 50 States and the District of Columbia. - Totals may not equal sum of components due to independent rounding.

Source: See Summary Statistics Table and Figure Sources. 
Table S3. Crude Oil and Petroleum Product Imports, 1981 - Present (Thousand Barrels per Day)

\begin{tabular}{|c|c|c|c|c|c|c|c|c|c|}
\hline & \multirow{3}{*}{ Year/Month } & \multicolumn{8}{|c|}{ Imports from Arab-OPEC Sources } \\
\hline & & \multicolumn{2}{|c|}{ Algeria } & \multicolumn{2}{|c|}{ Iraq } & \multicolumn{2}{|c|}{ Kuwait $^{\mathbf{b}}$} & \multicolumn{2}{|c|}{ Libya } \\
\hline & & Total & Crude Oil & Total & Crude Oil & Total & Crude Oil & Total & Crude Oil \\
\hline $\begin{array}{l}1981 \\
1982 \\
1983 \\
1984 \\
1985 \\
1986 \\
1987 \\
1988 \\
1989 \\
1990 \\
1991 \\
1992 \\
1993 \\
1994\end{array}$ & 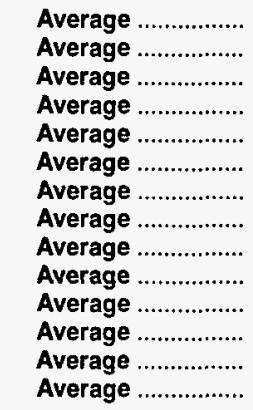 & $\begin{array}{l}311 \\
170 \\
240 \\
323 \\
187 \\
271 \\
295 \\
300 \\
269 \\
280 \\
253 \\
196 \\
220 \\
243\end{array}$ & $\begin{array}{r}261 \\
90 \\
176 \\
194 \\
84 \\
78 \\
115 \\
58 \\
60 \\
63 \\
44 \\
24 \\
24 \\
21\end{array}$ & $\begin{array}{r}\text { (s) } \\
3 \\
10 \\
12 \\
46 \\
81 \\
83 \\
345 \\
449 \\
518 \\
0 \\
0 \\
0 \\
0\end{array}$ & $\begin{array}{r}0 \\
3 \\
10 \\
12 \\
46 \\
81 \\
82 \\
343 \\
441 \\
514 \\
0 \\
0 \\
0 \\
0\end{array}$ & $\begin{array}{r}0 \\
5 \\
14 \\
36 \\
21 \\
68 \\
84 \\
92 \\
157 \\
86 \\
6 \\
51 \\
353 \\
312\end{array}$ & $\begin{array}{r}0 \\
2 \\
7 \\
24 \\
4 \\
28 \\
70 \\
80 \\
155 \\
79 \\
6 \\
39 \\
344 \\
307\end{array}$ & $\begin{array}{r}319 \\
26 \\
0 \\
1 \\
4 \\
0 \\
0 \\
0 \\
0 \\
0 \\
0 \\
0 \\
0 \\
0\end{array}$ & $\begin{array}{r}317 \\
23 \\
0 \\
0 \\
0 \\
0 \\
0 \\
0 \\
0 \\
0 \\
0 \\
0 \\
0 \\
0\end{array}$ \\
\hline 1995 & 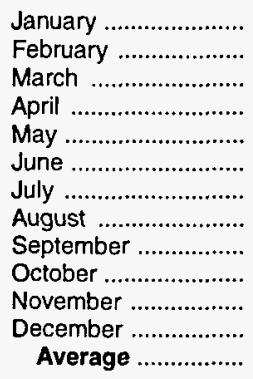 & $\begin{array}{l}153 \\
358 \\
196 \\
251 \\
163 \\
277 \\
257 \\
298 \\
250 \\
229 \\
241 \\
152 \\
234\end{array}$ & $\begin{array}{r}0 \\
64 \\
19 \\
31 \\
36 \\
39 \\
11 \\
65 \\
20 \\
39 \\
0 \\
0 \\
27\end{array}$ & $\begin{array}{l}0 \\
0 \\
0 \\
0 \\
0 \\
0 \\
0 \\
0 \\
0 \\
0 \\
0 \\
0 \\
0\end{array}$ & $\begin{array}{l}0 \\
0 \\
0 \\
0 \\
0 \\
0 \\
0 \\
0 \\
0 \\
0 \\
0 \\
0 \\
0\end{array}$ & $\begin{array}{l}130 \\
346 \\
252 \\
171 \\
208 \\
260 \\
195 \\
180 \\
187 \\
250 \\
238 \\
215 \\
218\end{array}$ & $\begin{array}{l}120 \\
324 \\
252 \\
164 \\
204 \\
259 \\
195 \\
175 \\
182 \\
244 \\
238 \\
215 \\
213\end{array}$ & $\begin{array}{l}0 \\
0 \\
0 \\
0 \\
0 \\
0 \\
0 \\
0 \\
0 \\
0 \\
0 \\
0 \\
0\end{array}$ & $\begin{array}{l}0 \\
0 \\
0 \\
0 \\
0 \\
0 \\
0 \\
0 \\
0 \\
0 \\
0 \\
0 \\
0\end{array}$ \\
\hline 1996 & 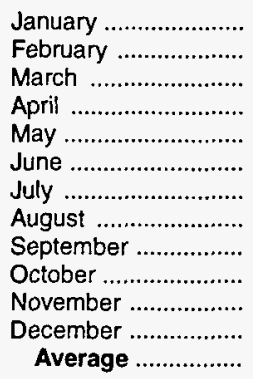 & $\begin{array}{l}313 \\
200 \\
241 \\
211 \\
340 \\
313 \\
305 \\
323 \\
186 \\
209 \\
214 \\
214 \\
256\end{array}$ & $\begin{array}{r}38 \\
16 \\
38 \\
2 \\
0 \\
0 \\
0 \\
0 \\
0 \\
0 \\
3 \\
0 \\
8\end{array}$ & $\begin{array}{r}0 \\
0 \\
0 \\
0 \\
0 \\
0 \\
0 \\
0 \\
0 \\
0 \\
0 \\
14 \\
1\end{array}$ & $\begin{array}{r}0 \\
0 \\
0 \\
0 \\
0 \\
0 \\
0 \\
0 \\
0 \\
0 \\
0 \\
14 \\
1\end{array}$ & $\begin{array}{l}148 \\
216 \\
127 \\
201 \\
230 \\
388 \\
266 \\
271 \\
236 \\
260 \\
228 \\
262 \\
236\end{array}$ & $\begin{array}{l}145 \\
216 \\
127 \\
201 \\
230 \\
388 \\
266 \\
266 \\
236 \\
260 \\
228 \\
262 \\
235\end{array}$ & $\begin{array}{l}0 \\
0 \\
0 \\
0 \\
0 \\
0 \\
0 \\
0 \\
0 \\
0 \\
0 \\
0 \\
0\end{array}$ & $\begin{array}{l}0 \\
0 \\
0 \\
0 \\
0 \\
0 \\
0 \\
0 \\
0 \\
0 \\
0 \\
0 \\
0\end{array}$ \\
\hline 1997 & $\begin{array}{l}\text { January } \\
\text { February } \\
\text { March ........................ } \\
\text { Apri! } \\
\text { May } \\
\text { June }\end{array}$ & $\begin{array}{l}282 \\
319 \\
309 \\
320 \\
290 \\
349 \\
291 \\
261 \\
259 \\
272 \\
267 \\
208 \\
285\end{array}$ & $\begin{array}{r}0 \\
0 \\
0 \\
23 \\
0 \\
0 \\
0 \\
4 \\
6 \\
3 \\
7 \\
28 \\
6\end{array}$ & $\begin{array}{r}0 \\
0 \\
35 \\
84 \\
102 \\
115 \\
88 \\
(s) \\
0 \\
177 \\
220 \\
240 \\
89\end{array}$ & $\begin{array}{r}0 \\
0 \\
35 \\
84 \\
102 \\
115 \\
88 \\
(s) \\
0 \\
177 \\
220 \\
240 \\
89\end{array}$ & $\begin{array}{l}209 \\
172 \\
315 \\
204 \\
128 \\
361 \\
331 \\
229 \\
322 \\
349 \\
220 \\
188 \\
253\end{array}$ & $\begin{array}{l}209 \\
172 \\
315 \\
204 \\
128 \\
361 \\
331 \\
229 \\
322 \\
349 \\
220 \\
188 \\
253\end{array}$ & $\begin{array}{l}0 \\
0 \\
0 \\
0 \\
0 \\
0 \\
0 \\
0 \\
0 \\
0 \\
0 \\
0 \\
0\end{array}$ & $\begin{array}{l}0 \\
0 \\
0 \\
0 \\
0 \\
0 \\
0 \\
0 \\
0 \\
0 \\
0 \\
0 \\
0\end{array}$ \\
\hline
\end{tabular}

See footnotes at end of table. 
Table S3. Crude Oil and Petroleum Product Imports, 1981 - Present (Continued) (Thousand Barrels per Day)

\begin{tabular}{|c|c|c|c|c|c|c|c|c|c|}
\hline & \multirow{3}{*}{ Year/Month } & \multicolumn{6}{|c|}{ Imports from Arab-OPEC Sources } & \multirow{2}{*}{\multicolumn{2}{|c|}{$\begin{array}{c}\text { Total } \\
\text { Arab } \\
\text { OPEC }\end{array}$}} \\
\hline & & \multicolumn{2}{|c|}{ Qatar } & \multicolumn{2}{|c|}{$\begin{array}{c}\text { Saudi } \\
\text { Arabia }^{b}\end{array}$} & \multicolumn{2}{|c|}{$\begin{array}{c}\text { United } \\
\text { Arab } \\
\text { Emirates }\end{array}$} & & \\
\hline & & Total & Crude Oil & Total & Crude Oil & Total & Crude Oil & Total & Crude Oil \\
\hline $\begin{array}{l}1981 \\
1982 \\
1983 \\
1984 \\
1985 \\
1986 \\
1987 \\
1988 \\
1989 \\
1990 \\
1991 \\
1992 \\
1993 \\
1994\end{array}$ & $\begin{array}{l}\text { Average } \ldots \ldots \ldots \ldots \\
\text { Average } \\
\text { Average } \ldots \ldots \ldots \ldots \\
\text { Average } \ldots \ldots \ldots \ldots \\
\text { Average } \ldots \ldots \ldots \ldots \\
\text { Average } \\
\text { Average }\end{array}$ & $\begin{array}{r}7 \\
7 \\
(5) \\
5 \\
(s) \\
13 \\
0 \\
0 \\
2 \\
4 \\
0 \\
1 \\
1 \\
0\end{array}$ & $\begin{array}{r}7 \\
7 \\
0 \\
4 \\
0 \\
12 \\
0 \\
0 \\
2 \\
4 \\
0 \\
0 \\
0 \\
0\end{array}$ & $\begin{array}{r}1,129 \\
552 \\
337 \\
325 \\
168 \\
685 \\
751 \\
1,073 \\
1,224 \\
1,339 \\
1,802 \\
1,720 \\
1,414 \\
1,402\end{array}$ & $\begin{array}{r}1,112 \\
530 \\
321 \\
309 \\
132 \\
618 \\
642 \\
911 \\
1,116 \\
1,195 \\
1,703 \\
1,597 \\
1,282 \\
1,297\end{array}$ & $\begin{array}{r}81 \\
92 \\
30 \\
117 \\
45 \\
44 \\
61 \\
29 \\
28 \\
17 \\
3 \\
6 \\
14 \\
13\end{array}$ & $\begin{array}{r}77 \\
81 \\
18 \\
90 \\
35 \\
38 \\
56 \\
23 \\
21 \\
9 \\
2 \\
0 \\
12 \\
11\end{array}$ & $\begin{array}{r}1,848 \\
854 \\
632 \\
819 \\
472 \\
1,162 \\
1,274 \\
1,839 \\
2,130 \\
2,244 \\
2,064 \\
1,974 \\
2,000 \\
1,970\end{array}$ & $\begin{array}{r}1,774 \\
736 \\
533 \\
634 \\
300 \\
854 \\
965 \\
1,415 \\
1,794 \\
1,864 \\
1,754 \\
1,660 \\
1,661 \\
1,636\end{array}$ \\
\hline 1995 & 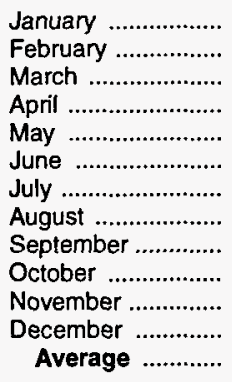 & $\begin{array}{l}0 \\
0 \\
0 \\
0 \\
0 \\
0 \\
0 \\
0 \\
0 \\
0 \\
0 \\
0 \\
0\end{array}$ & $\begin{array}{l}0 \\
0 \\
0 \\
0 \\
0 \\
0 \\
0 \\
0 \\
0 \\
0 \\
0 \\
0 \\
0\end{array}$ & $\begin{array}{l}1,309 \\
1,181 \\
1,535 \\
1,375 \\
1,281 \\
1,287 \\
1,265 \\
1,340 \\
1,474 \\
1,260 \\
1,429 \\
1,378 \\
1,344\end{array}$ & $\begin{array}{l}1,251 \\
1,134 \\
1,410 \\
1,321 \\
1,237 \\
1,221 \\
1,165 \\
1,245 \\
1,357 \\
1,181 \\
1,326 \\
1,263 \\
1,260\end{array}$ & $\begin{array}{r}20 \\
13 \\
0 \\
0 \\
0 \\
12 \\
0 \\
20 \\
29 \\
14 \\
10 \\
0 \\
10\end{array}$ & $\begin{array}{r}20 \\
13 \\
0 \\
0 \\
0 \\
1 \\
0 \\
20 \\
0 \\
0 \\
10 \\
0 \\
5\end{array}$ & $\begin{array}{l}1,613 \\
1,897 \\
1,983 \\
1,798 \\
1,653 \\
1,835 \\
1,716 \\
1,838 \\
1,941 \\
1,753 \\
1,918 \\
1,745 \\
1,806\end{array}$ & $\begin{array}{l}1,391 \\
1,535 \\
1,681 \\
1,516 \\
1,477 \\
1,520 \\
1,371 \\
1,505 \\
1,559 \\
1,464 \\
1,574 \\
1,478 \\
1,505\end{array}$ \\
\hline 1996 & 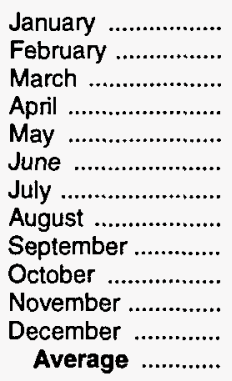 & $\begin{array}{l}0 \\
0 \\
0 \\
0 \\
0 \\
0 \\
0 \\
0 \\
0 \\
0 \\
0 \\
0 \\
0\end{array}$ & $\begin{array}{l}0 \\
0 \\
0 \\
0 \\
0 \\
0 \\
0 \\
0 \\
0 \\
0 \\
0 \\
0 \\
0\end{array}$ & $\begin{array}{l}1,398 \\
1,128 \\
1,422 \\
1,288 \\
1,518 \\
1,138 \\
1,548 \\
1,477 \\
1,355 \\
1,357 \\
1,297 \\
1,400 \\
1,363\end{array}$ & $\begin{array}{l}1,334 \\
1,053 \\
1,318 \\
1,200 \\
1,414 \\
1,035 \\
1,371 \\
1,333 \\
1,255 \\
1,209 \\
1,201 \\
1,236 \\
1,248\end{array}$ & $\begin{array}{r}0 \\
0 \\
0 \\
0 \\
0 \\
11 \\
4 \\
0 \\
0 \\
17 \\
0 \\
0 \\
3\end{array}$ & $\begin{array}{r}0 \\
0 \\
0 \\
0 \\
0 \\
11 \\
4 \\
0 \\
0 \\
17 \\
0 \\
0 \\
3\end{array}$ & $\begin{array}{l}1,859 \\
1,544 \\
1,790 \\
1,700 \\
2,087 \\
1,850 \\
2,123 \\
2,070 \\
1,777 \\
1,844 \\
1,738 \\
1,889 \\
1,859\end{array}$ & $\begin{array}{l}1,517 \\
1,285 \\
1,484 \\
1,403 \\
1,643 \\
1,433 \\
1,642 \\
1,599 \\
1,491 \\
1,486 \\
1,432 \\
1,511 \\
1,496\end{array}$ \\
\hline 1997 & 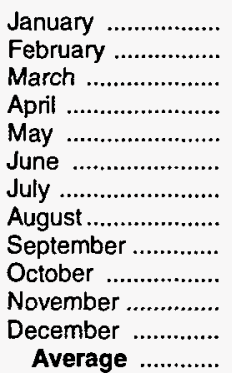 & $\begin{array}{r}0 \\
0 \\
0 \\
15 \\
0 \\
0 \\
0 \\
0 \\
0 \\
16 \\
0 \\
15 \\
4\end{array}$ & $\begin{array}{l}0 \\
0 \\
0 \\
0 \\
0 \\
0 \\
0 \\
0 \\
0 \\
0 \\
0 \\
0 \\
0\end{array}$ & $\begin{array}{l}1,344 \\
1,361 \\
1,292 \\
1,573 \\
1,475 \\
1,299 \\
1,313 \\
1,636 \\
1,599 \\
1,377 \\
1,308 \\
1,311 \\
1,407\end{array}$ & $\begin{array}{l}1,253 \\
1,250 \\
1,157 \\
1,408 \\
1,333 \\
1,174 \\
1,188 \\
1,516 \\
1,511 \\
1,282 \\
1,257 \\
1,192 \\
1,293\end{array}$ & $\begin{array}{r}0 \\
0 \\
0 \\
0 \\
0 \\
6 \\
14 \\
0 \\
0 \\
0 \\
0 \\
0 \\
2\end{array}$ & $\begin{array}{l}0 \\
0 \\
0 \\
0 \\
0 \\
0 \\
0 \\
0 \\
0 \\
0 \\
0 \\
0 \\
0\end{array}$ & $\begin{array}{l}1,835 \\
1,852 \\
1,950 \\
2,197 \\
1,996 \\
2,130 \\
2,037 \\
2,127 \\
2,180 \\
2,191 \\
2,015 \\
1,962 \\
2,040\end{array}$ & $\begin{array}{l}1,462 \\
1,421 \\
1,506 \\
1,720 \\
1,564 \\
1,650 \\
1,607 \\
1,750 \\
1,839 \\
1,812 \\
1,704 \\
1,649 \\
1,641\end{array}$ \\
\hline
\end{tabular}

See footnotes at end of table. 
Table S3. Crude Oil and Petroleum Product Imports, 1981 - Present (Continued) (Thousand Barrels per Day)

\begin{tabular}{|c|c|c|c|c|c|c|c|c|c|}
\hline & \multirow{3}{*}{ Year/Month } & \multicolumn{8}{|c|}{ Imports from Other-OPEC Sources } \\
\hline & & \multicolumn{2}{|c|}{ Ecuadore } & \multicolumn{2}{|c|}{ Cabon } & \multicolumn{2}{|c|}{ Indonesia } & \multicolumn{2}{|c|}{ Iran } \\
\hline & & Total & Crude Oil & Total & Crude Oil & Total & Crude Oil & Total & Crude Oil \\
\hline $\begin{array}{l}1981 \\
1982 \\
1983 \\
1984 \\
1985 \\
1986 \\
1987 \\
1988 \\
1989 \\
1990 \\
1991 \\
1992 \\
1993 \\
1994\end{array}$ & 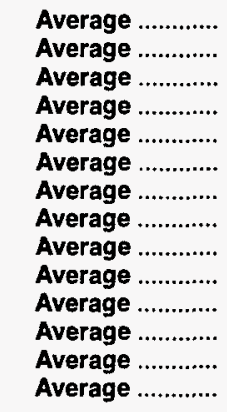 & $\begin{array}{l}48 \\
42 \\
61 \\
55 \\
67 \\
77 \\
29 \\
47 \\
89 \\
49 \\
63 \\
65 \\
81 \\
\text { (c) }\end{array}$ & $\begin{array}{l}38 \\
32 \\
56 \\
47 \\
56 \\
64 \\
23 \\
33 \\
80 \\
38 \\
53 \\
62 \\
78 \\
(c)\end{array}$ & $\begin{array}{r}35 \\
40 \\
59 \\
58 \\
52 \\
26 \\
35 \\
16 \\
50 \\
64 \\
84 \\
124 \\
152 \\
194\end{array}$ & $\begin{array}{r}35 \\
40 \\
59 \\
57 \\
51 \\
25 \\
35 \\
15 \\
49 \\
64 \\
84 \\
123 \\
151 \\
194 \\
\end{array}$ & $\begin{array}{r}366 \\
248 \\
338 \\
343 \\
314 \\
318 \\
285 \\
205 \\
183 \\
114 \\
111 \\
78 \\
81 \\
111\end{array}$ & $\begin{array}{r}318 \\
226 \\
315 \\
304 \\
292 \\
297 \\
262 \\
186 \\
158 \\
98 \\
102 \\
70 \\
65 \\
92\end{array}$ & $\begin{array}{r}0 \\
35 \\
48 \\
10 \\
27 \\
19 \\
98 \\
9(8) \\
0 \\
0 \\
32 \\
0 \\
0 \\
0\end{array}$ & $\begin{array}{r}0 \\
35 \\
48 \\
10 \\
27 \\
19 \\
98 \\
9(s) \\
0 \\
0 \\
32 \\
0 \\
0 \\
0\end{array}$ \\
\hline 1995 & 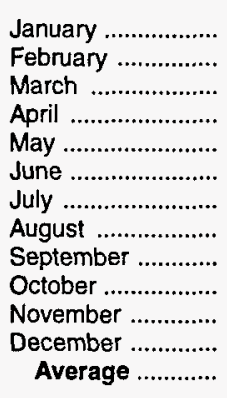 & $\begin{array}{l}\text { (c) } \\
\text { (c) } \\
\text { (c) } \\
\text { (c) } \\
\text { (c) } \\
\text { (c) } \\
\text { (c) } \\
\text { (c) } \\
\text { (c) } \\
\text { (c) } \\
\text { (c) } \\
\text { (c) }\end{array}$ & $\begin{array}{l}\text { (c) } \\
\text { (c) } \\
\text { (c) } \\
\text { (c) } \\
\text { (c) } \\
\text { (c) } \\
\text { (c) } \\
\text { (c) } \\
\text { (c) } \\
\text { (c) } \\
\text { (c) } \\
\text { (c) }\end{array}$ & $\begin{array}{l}\text { (d) } \\
\text { (d) } \\
\text { (d) } \\
\text { (d) } \\
\text { (d) } \\
\text { (d) } \\
\text { (d) } \\
\text { (d) } \\
\text { (d) } \\
\text { (d) } \\
\text { (d) } \\
\text { (d) } \\
\text { (d) }\end{array}$ & $\begin{array}{l}\text { (d) } \\
\text { (d) } \\
\text { (d) } \\
\text { (d) } \\
\text { (d) } \\
\text { (d) } \\
\text { (d) } \\
\text { (d) } \\
\text { (d) } \\
\text { (d) } \\
\text { (d) } \\
\text { (d) } \\
\text { (d) }\end{array}$ & $\begin{array}{r}38 \\
129 \\
51 \\
95 \\
65 \\
96 \\
104 \\
122 \\
94 \\
87 \\
107 \\
72 \\
88\end{array}$ & $\begin{array}{l}38 \\
87 \\
29 \\
87 \\
36 \\
51 \\
96 \\
95 \\
66 \\
68 \\
73 \\
41 \\
64\end{array}$ & $\begin{array}{l}0 \\
0 \\
0 \\
0 \\
0 \\
0 \\
0 \\
0 \\
0 \\
0 \\
0 \\
0 \\
0\end{array}$ & $\begin{array}{l}0 \\
0 \\
0 \\
0 \\
0 \\
0 \\
0 \\
0 \\
0 \\
0 \\
0 \\
0 \\
0\end{array}$ \\
\hline 1996 & 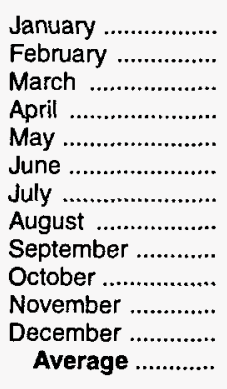 & $\begin{array}{l}\text { (c) } \\
\text { (c) } \\
\text { (c) } \\
\text { (c) } \\
\text { (c) } \\
\text { (c) } \\
\text { (c) } \\
\text { (c) } \\
\text { (c) } \\
\text { (c) } \\
\text { (c) } \\
\text { (c) } \\
\text { (c) }\end{array}$ & $\begin{array}{l}\text { (c) } \\
\text { (c) } \\
(c) \\
(c) \\
(c) \\
(c) \\
(c) \\
(c) \\
(c) \\
(c) \\
(c) \\
(c)\end{array}$ & $\begin{array}{l}\text { (d) } \\
\text { (d) } \\
\text { (d) } \\
\text { (d) } \\
\text { (d) } \\
\text { (d) } \\
\text { (d) } \\
\text { (d) } \\
\text { (d) } \\
\text { (d) } \\
\text { (d) } \\
\text { (d) } \\
\text { (d) }\end{array}$ & $\begin{array}{l}\text { (d) } \\
\text { (d) } \\
\text { (d) } \\
\text { (d) } \\
\text { (d) } \\
\text { (d) } \\
\text { (d) } \\
\text { (d) } \\
\text { (d) } \\
\text { (d) } \\
\text { (d) } \\
\text { (d) }\end{array}$ & $\begin{array}{r}52 \\
44 \\
58 \\
57 \\
49 \\
72 \\
56 \\
53 \\
26 \\
125 \\
36 \\
81 \\
59\end{array}$ & $\begin{array}{l}43 \\
43 \\
55 \\
57 \\
15 \\
65 \\
48 \\
49 \\
26 \\
82 \\
12 \\
32 \\
44\end{array}$ & $\begin{array}{l}0 \\
0 \\
0 \\
0 \\
0 \\
0 \\
0 \\
0 \\
0 \\
0 \\
0 \\
0 \\
0\end{array}$ & $\begin{array}{l}0 \\
0 \\
0 \\
0 \\
0 \\
0 \\
0 \\
0 \\
0 \\
0 \\
0 \\
0 \\
0\end{array}$ \\
\hline 1997 & 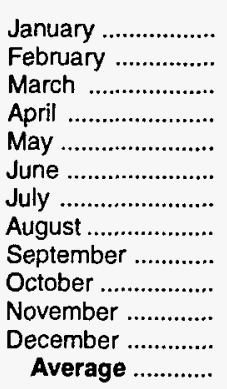 & $\begin{array}{l}\text { (c) } \\
\text { (c) } \\
\text { (c) } \\
\text { (c) } \\
\text { (c) } \\
\text { (c) } \\
\text { (c) } \\
(c) \\
(c) \\
(c) \\
\text { (c) } \\
\text { (c) } \\
\text { (c) }\end{array}$ & $\begin{array}{l}\text { (c) } \\
\text { (c) } \\
\text { (c) } \\
\text { (c) } \\
\text { (c) } \\
\text { (c) } \\
\text { (c) } \\
\text { (c) } \\
\text { (c) } \\
\text { (c) } \\
\text { (c) } \\
\text { (c) }\end{array}$ & $\begin{array}{l}\text { (d) } \\
\text { (d) } \\
\text { (d) } \\
\text { (d) } \\
\text { (d) } \\
\text { (d) } \\
\text { (d) } \\
\text { (d) } \\
\text { (d) } \\
\text { (d) } \\
\text { (d) } \\
\text { (d) } \\
\text { (d) }\end{array}$ & $\begin{array}{l}\text { (d) } \\
\text { (d) } \\
\text { (d) } \\
\text { (d) } \\
\text { (d) } \\
\text { (d) } \\
\text { (d) } \\
\text { (d) } \\
\text { (d) } \\
\text { (d) } \\
\text { (d) } \\
\text { (d) } \\
\text { (d) }\end{array}$ & $\begin{array}{l}55 \\
51 \\
18 \\
40 \\
86 \\
57 \\
73 \\
24 \\
90 \\
42 \\
79 \\
84 \\
58\end{array}$ & $\begin{array}{l}38 \\
39 \\
15 \\
32 \\
86 \\
50 \\
66 \\
21 \\
83 \\
42 \\
74 \\
68 \\
51\end{array}$ & $\begin{array}{l}0 \\
0 \\
0 \\
0 \\
0 \\
0 \\
0 \\
0 \\
0 \\
0 \\
0 \\
0 \\
0\end{array}$ & $\begin{array}{l}0 \\
0 \\
0 \\
0 \\
0 \\
0 \\
0 \\
0 \\
0 \\
0 \\
0 \\
0 \\
0\end{array}$ \\
\hline
\end{tabular}

See footnotes at end of table. 
Table S3. Crude Oil and Petroleum Product Imports, 1981 - Present (Continued) (Thousand Barrels per Day)

\begin{tabular}{|c|c|c|c|c|c|c|c|c|c|}
\hline & \multirow{3}{*}{ Year/Month } & \multicolumn{6}{|c|}{ Imports from Other-OPEC Sources } & \multirow{2}{*}{\multicolumn{2}{|c|}{$\begin{array}{c}\text { Total } \\
\text { OPEC }^{\mathrm{c}, \mathrm{d}}\end{array}$}} \\
\hline & & & & & ela & & & & \\
\hline & & Total & Crude Oil & Total & Crude Oil & Total & Crude Oil & Total & Crude Oil \\
\hline $\begin{array}{l}1981 \\
1982 \\
1983 \\
1984 \\
1985 \\
1986 \\
1987 \\
1988 \\
1989 \\
1990 \\
1991 \\
1992 \\
1993 \\
1994\end{array}$ & 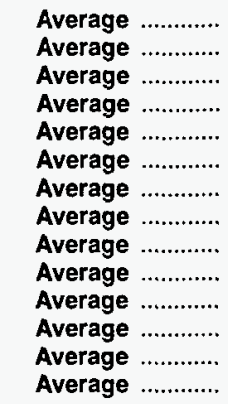 & $\begin{array}{l}620 \\
514 \\
302 \\
216 \\
293 \\
440 \\
535 \\
618 \\
815 \\
800 \\
703 \\
681 \\
740 \\
637\end{array}$ & $\begin{array}{l}611 \\
510 \\
301 \\
207 \\
280 \\
437 \\
529 \\
607 \\
800 \\
784 \\
683 \\
665 \\
722 \\
624\end{array}$ & $\begin{array}{r}406 \\
412 \\
422 \\
548 \\
605 \\
793 \\
804 \\
794 \\
873 \\
1,025 \\
1,035 \\
1,170 \\
1,300 \\
1,334 \\
\end{array}$ & $\begin{array}{r}147 \\
155 \\
164 \\
253 \\
306 \\
416 \\
488 \\
439 \\
495 \\
666 \\
668 \\
826 \\
1,010 \\
1,034\end{array}$ & $\begin{array}{l}1,476 \\
1,291 \\
1,231 \\
1,230 \\
1,358 \\
1,674 \\
1,787 \\
1,681 \\
2,010 \\
2,052 \\
2,028 \\
2,117 \\
2,354 \\
2,277\end{array}$ & $\begin{array}{r}1,149 \\
998 \\
944 \\
878 \\
1,012 \\
1,259 \\
1,435 \\
1,281 \\
1,582 \\
1,650 \\
1,622 \\
1,746 \\
2,026 \\
1,944\end{array}$ & $\begin{array}{l}3,323 \\
2,146 \\
1,862 \\
2,049 \\
1,830 \\
2,837 \\
3,060 \\
3,520 \\
4,140 \\
4,296 \\
4,092 \\
4,092 \\
4,354 \\
4,247\end{array}$ & $\begin{array}{l}2,922 \\
1,734 \\
1,477 \\
1,512 \\
1,312 \\
2,113 \\
2,400 \\
2,696 \\
3,376 \\
3,514 \\
3,377 \\
3,406 \\
3,687 \\
3,580\end{array}$ \\
\hline 1995 & 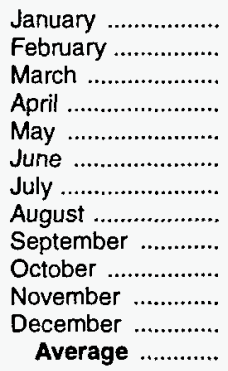 & $\begin{array}{l}625 \\
463 \\
687 \\
467 \\
603 \\
696 \\
696 \\
482 \\
851 \\
649 \\
646 \\
652 \\
627\end{array}$ & $\begin{array}{l}617 \\
463 \\
676 \\
458 \\
592 \\
696 \\
696 \\
463 \\
841 \\
649 \\
637 \\
652 \\
621\end{array}$ & $\begin{array}{l}1,442 \\
1,439 \\
1,499 \\
1,365 \\
1,480 \\
1,479 \\
1,536 \\
1,449 \\
1,655 \\
1,453 \\
1,507 \\
1,459 \\
1,480\end{array}$ & $\begin{array}{l}1,061 \\
1,083 \\
1,208 \\
1,083 \\
1,176 \\
1,209 \\
1,162 \\
1,162 \\
1,288 \\
1,159 \\
1,140 \\
1,074 \\
1,151\end{array}$ & $\begin{array}{l}2,105 \\
2,031 \\
2,236 \\
1,926 \\
2,148 \\
2,271 \\
2,336 \\
2,054 \\
2,600 \\
2,189 \\
2,260 \\
2,182 \\
2,196\end{array}$ & $\begin{array}{l}1,717 \\
1,633 \\
1,913 \\
1,628 \\
1,804 \\
1,956 \\
1,954 \\
1,719 \\
2,195 \\
1,876 \\
1,851 \\
1,767 \\
1,835\end{array}$ & $\begin{array}{l}3,718 \\
3,929 \\
4,220 \\
3,724 \\
3,801 \\
4,106 \\
4,052 \\
3,892 \\
4,541 \\
3,942 \\
4,178 \\
3,927 \\
4,002\end{array}$ & $\begin{array}{l}3,108 \\
3,168 \\
3,595 \\
3,144 \\
3,281 \\
3,476 \\
3,325 \\
3,225 \\
3,753 \\
3,340 \\
3,424 \\
3,245 \\
3,341\end{array}$ \\
\hline 1996 & 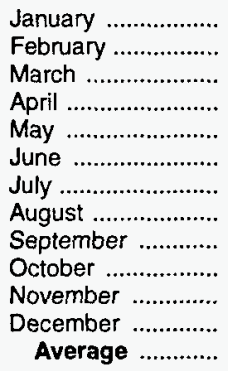 & $\begin{array}{l}690 \\
647 \\
594 \\
518 \\
705 \\
711 \\
750 \\
793 \\
694 \\
521 \\
465 \\
320 \\
617\end{array}$ & $\begin{array}{l}663 \\
639 \\
548 \\
497 \\
705 \\
697 \\
696 \\
785 \\
677 \\
488 \\
453 \\
298 \\
595\end{array}$ & $\begin{array}{l}1,518 \\
1,495 \\
1,719 \\
1,732 \\
1,700 \\
1,642 \\
1,690 \\
1,749 \\
1,708 \\
1,781 \\
1,728 \\
1,641 \\
1,676\end{array}$ & $\begin{array}{l}1,148 \\
1,166 \\
1,341 \\
1,288 \\
1,333 \\
1,236 \\
1,332 \\
1,431 \\
1,269 \\
1,448 \\
1,303 \\
1,324 \\
1,303\end{array}$ & $\begin{array}{l}2,261 \\
2,185 \\
2,371 \\
2,307 \\
2,454 \\
2,425 \\
2,496 \\
2,595 \\
2,428 \\
2,427 \\
2,229 \\
2,042 \\
2,353\end{array}$ & $\begin{array}{l}1,854 \\
1,849 \\
1,943 \\
1,842 \\
2,054 \\
1,999 \\
2,076 \\
2,265 \\
1,972 \\
2,019 \\
1,767 \\
1,654 \\
1,942\end{array}$ & $\begin{array}{l}4,120 \\
3,730 \\
4,161 \\
4,007 \\
4,541 \\
4,275 \\
4,619 \\
4,665 \\
4,204 \\
4,271 \\
3,967 \\
3,931 \\
4,211\end{array}$ & $\begin{array}{l}3,371 \\
3,133 \\
3,427 \\
3,245 \\
3,697 \\
3,432 \\
3,718 \\
3,865 \\
3,463 \\
3,504 \\
3,199 \\
3,166 \\
3,438\end{array}$ \\
\hline 1997 & 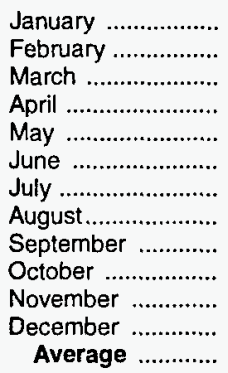 & $\begin{array}{l}548 \\
625 \\
542 \\
756 \\
992 \\
919 \\
580 \\
882 \\
769 \\
688 \\
649 \\
423 \\
698\end{array}$ & $\begin{array}{l}522 \\
620 \\
541 \\
747 \\
975 \\
919 \\
571 \\
866 \\
769 \\
675 \\
649 \\
423 \\
689\end{array}$ & $\begin{array}{l}1,641 \\
1,601 \\
1,769 \\
1,695 \\
1,927 \\
1,893 \\
1,738 \\
1,794 \\
1,822 \\
1,991 \\
1,689 \\
1,699 \\
1,773\end{array}$ & $\begin{array}{l}1,215 \\
1,262 \\
1,348 \\
1,319 \\
1,449 \\
1,508 \\
1,418 \\
1,394 \\
1,478 \\
1,605 \\
1,418 \\
1,304 \\
1,394\end{array}$ & $\begin{array}{l}2,243 \\
2,278 \\
2,329 \\
2,491 \\
3,005 \\
2,869 \\
2,391 \\
2,700 \\
2,680 \\
2,722 \\
2,416 \\
2,205 \\
2,529\end{array}$ & $\begin{array}{l}1,775 \\
1,920 \\
1,904 \\
2,098 \\
2,510 \\
2,478 \\
2,055 \\
2,280 \\
2,329 \\
2,323 \\
2,141 \\
1,795 \\
2,134\end{array}$ & $\begin{array}{l}4,078 \\
4,130 \\
4,279 \\
4,688 \\
5,001 \\
4,999 \\
4,429 \\
4,827 \\
4,860 \\
4,913 \\
4,431 \\
4,168 \\
4,569\end{array}$ & $\begin{array}{l}3,237 \\
3,341 \\
3,410 \\
3,818 \\
4,073 \\
4,128 \\
3,662 \\
4,030 \\
4,168 \\
4,134 \\
3,845 \\
3,444 \\
3,775\end{array}$ \\
\hline
\end{tabular}

See footnotes at end of table. 
Table S3. Crude Oil and Petroleum Product Imports, 1981 - Present (Continued) (Thousand Barrels per Day)

\begin{tabular}{|c|c|c|c|c|c|c|c|c|c|c|c|c|c|}
\hline & \multirow{3}{*}{ Year/Month } & \multicolumn{12}{|c|}{ Imports from Non-OPEC Sources ${ }^{a}$} \\
\hline & & \multicolumn{2}{|c|}{ Angola } & \multicolumn{2}{|c|}{ Australia } & \multicolumn{2}{|c|}{$\begin{array}{l}\text { Bahama } \\
\text { Islands }\end{array}$} & \multicolumn{2}{|c|}{ Brazil } & \multicolumn{2}{|c|}{ Canada } & \multicolumn{2}{|c|}{$\begin{array}{c}\text { China, } \\
\text { Peoples } \\
\text { Republic of }\end{array}$} \\
\hline & & Total & Crude Oil & Total & Crude Oil & Total & Crude Oil & Total & Crude Oil & Total & Crude Oil & Total & Crude Oil \\
\hline $\begin{array}{l}1981 \\
1982 \\
1983 \\
1984 \\
1985 \\
1986 \\
1987 \\
1988 \\
1989 \\
1990 \\
1991 \\
1992 \\
1993 \\
1994\end{array}$ & 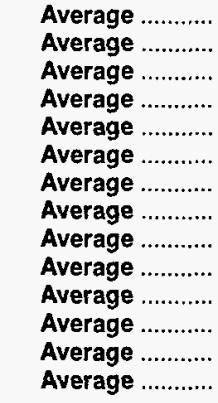 & $\begin{array}{r}49 \\
44 \\
78 \\
90 \\
110 \\
112 \\
192 \\
212 \\
284 \\
237 \\
254 \\
336 \\
336 \\
331\end{array}$ & $\begin{array}{r}45 \\
42 \\
71 \\
85 \\
104 \\
102 \\
180 \\
203 \\
279 \\
236 \\
254 \\
336 \\
336 \\
322 \\
\end{array}$ & $\begin{array}{r}5 \\
5 \\
4 \\
38 \\
37 \\
41 \\
58 \\
64 \\
36 \\
53 \\
26 \\
19 \\
19 \\
17\end{array}$ & $\begin{array}{r}0 \\
\text { (s) } \\
0 \\
25 \\
21 \\
30 \\
49 \\
59 \\
31 \\
47 \\
21 \\
17 \\
18 \\
16\end{array}$ & $\begin{array}{r}74 \\
65 \\
125 \\
88 \\
40 \\
37 \\
37 \\
32 \\
34 \\
37 \\
35 \\
36 \\
28 \\
29\end{array}$ & $\begin{array}{l}0 \\
0 \\
0 \\
0 \\
0 \\
0 \\
0 \\
0 \\
0 \\
0 \\
0 \\
0 \\
0 \\
0\end{array}$ & $\begin{array}{l}23 \\
47 \\
41 \\
60 \\
61 \\
50 \\
84 \\
98 \\
82 \\
49 \\
22 \\
20 \\
33 \\
31\end{array}$ & $\begin{array}{r}14 \\
19 \\
2 \\
(s) \\
0 \\
0 \\
0 \\
0 \\
0 \\
0 \\
0 \\
0 \\
0 \\
1\end{array}$ & \begin{tabular}{r|}
447 \\
482 \\
547 \\
630 \\
770 \\
807 \\
848 \\
999 \\
931 \\
934 \\
1,033 \\
1,069 \\
1,181 \\
1,272 \\
\end{tabular} & $\begin{array}{l}164 \\
214 \\
274 \\
341 \\
468 \\
570 \\
608 \\
681 \\
630 \\
643 \\
743 \\
797 \\
900 \\
983\end{array}$ & $\begin{array}{l}18 \\
40 \\
34 \\
46 \\
59 \\
90 \\
82 \\
88 \\
80 \\
80 \\
91 \\
90 \\
51 \\
65\end{array}$ & $\begin{array}{r}0 \\
8 \\
6 \\
15 \\
36 \\
68 \\
63 \\
82 \\
76 \\
77 \\
87 \\
84 \\
50 \\
64\end{array}$ \\
\hline 1995 & 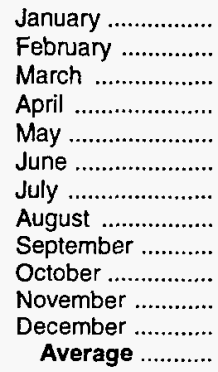 & $\begin{array}{l}273 \\
348 \\
427 \\
412 \\
419 \\
371 \\
295 \\
367 \\
444 \\
366 \\
318 \\
366 \\
367\end{array}$ & $\begin{array}{l}262 \\
335 \\
416 \\
402 \\
407 \\
358 \\
287 \\
355 \\
444 \\
366 \\
318 \\
366 \\
360\end{array}$ & $\begin{array}{r}21 \\
22 \\
0 \\
33 \\
21 \\
10 \\
42 \\
0 \\
0 \\
15 \\
(s) \\
23 \\
16\end{array}$ & $\begin{array}{r}21 \\
22 \\
0 \\
33 \\
21 \\
10 \\
42 \\
0 \\
0 \\
15 \\
0 \\
23 \\
16\end{array}$ & $\begin{array}{l}6 \\
8 \\
7 \\
0 \\
0 \\
0 \\
0 \\
0 \\
8 \\
0 \\
0 \\
0 \\
2\end{array}$ & $\begin{array}{l}0 \\
0 \\
0 \\
0 \\
0 \\
0 \\
0 \\
0 \\
0 \\
0 \\
0 \\
0 \\
0\end{array}$ & $\begin{array}{r}1 \\
0 \\
0 \\
0 \\
0 \\
0 \\
8 \\
9 \\
43 \\
9 \\
12 \\
12 \\
8\end{array}$ & $\begin{array}{l}0 \\
0 \\
0 \\
0 \\
0 \\
0 \\
0 \\
0 \\
0 \\
0 \\
0 \\
0 \\
0\end{array}$ & $\begin{array}{l}1,345 \\
1,311 \\
1,208 \\
1,243 \\
1,406 \\
1,420 \\
1,279 \\
1,345 \\
1,252 \\
1,300 \\
1,403 \\
1,471 \\
1,332\end{array}$ & $\begin{array}{r}1,011 \\
965 \\
891 \\
999 \\
1,167 \\
1,169 \\
1,028 \\
1,058 \\
959 \\
1,057 \\
1,069 \\
1,099 \\
1,040\end{array}$ & $\begin{array}{l}64 \\
21 \\
54 \\
65 \\
35 \\
26 \\
80 \\
40 \\
73 \\
40 \\
66 \\
73 \\
53\end{array}$ & $\begin{array}{l}62 \\
21 \\
54 \\
65 \\
35 \\
26 \\
80 \\
40 \\
73 \\
40 \\
66 \\
73 \\
53\end{array}$ \\
\hline 1996 & 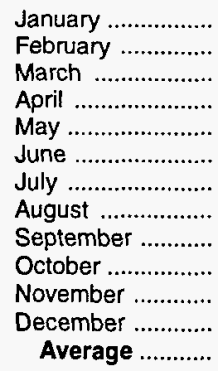 & $\begin{array}{l}312 \\
195 \\
257 \\
244 \\
403 \\
356 \\
292 \\
480 \\
391 \\
502 \\
353 \\
420 \\
351\end{array}$ & $\begin{array}{l}312 \\
195 \\
257 \\
233 \\
379 \\
356 \\
292 \\
456 \\
391 \\
485 \\
353 \\
405 \\
344\end{array}$ & $\begin{array}{r}21 \\
0 \\
0 \\
22 \\
22 \\
56 \\
11 \\
43 \\
47 \\
79 \\
35 \\
39 \\
31\end{array}$ & $\begin{array}{r}21 \\
0 \\
0 \\
22 \\
22 \\
47 \\
0 \\
43 \\
27 \\
65 \\
25 \\
21 \\
25\end{array}$ & $\begin{array}{r}0 \\
0 \\
12 \\
0 \\
0 \\
1 \\
0 \\
0 \\
0 \\
0 \\
0 \\
0 \\
1\end{array}$ & $\begin{array}{l}0 \\
0 \\
0 \\
0 \\
0 \\
0 \\
0 \\
0 \\
0 \\
0 \\
0 \\
0 \\
0\end{array}$ & $\begin{array}{r}1 \\
4 \\
1 \\
(s) \\
9 \\
10 \\
28 \\
38 \\
13 \\
1 \\
1 \\
3 \\
9\end{array}$ & $\begin{array}{l}0 \\
0 \\
0 \\
0 \\
0 \\
0 \\
0 \\
0 \\
0 \\
0 \\
0 \\
0 \\
0\end{array}$ & $\begin{array}{l}1,490 \\
1,413 \\
1,322 \\
1,427 \\
1,373 \\
1,395 \\
1,393 \\
1,393 \\
1,276 \\
1,407 \\
1,516 \\
1,675 \\
1,424\end{array}$ & $\begin{array}{l}1,117 \\
1,026 \\
1,001 \\
1,030 \\
1,056 \\
1,091 \\
1,093 \\
1,042 \\
1,000 \\
1,059 \\
1,151 \\
1,232 \\
1,075\end{array}$ & $\begin{array}{r}86 \\
42 \\
53 \\
18 \\
19 \\
37 \\
78 \\
73 \\
64 \\
36 \\
104 \\
78 \\
57\end{array}$ & $\begin{array}{r}86 \\
42 \\
53 \\
18 \\
19 \\
37 \\
78 \\
73 \\
64 \\
36 \\
104 \\
78 \\
57\end{array}$ \\
\hline 1997 & 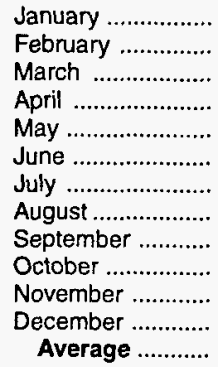 & $\begin{array}{l}485 \\
422 \\
467 \\
435 \\
374 \\
480 \\
416 \\
323 \\
428 \\
537 \\
480 \\
286 \\
427\end{array}$ & $\begin{array}{l}485 \\
422 \\
461 \\
422 \\
369 \\
480 \\
416 \\
323 \\
428 \\
537 \\
480 \\
286 \\
425\end{array}$ & $\begin{array}{r}21 \\
0 \\
37 \\
22 \\
61 \\
23 \\
77 \\
91 \\
67 \\
92 \\
23 \\
59 \\
48\end{array}$ & $\begin{array}{r}21 \\
0 \\
37 \\
22 \\
44 \\
23 \\
48 \\
60 \\
27 \\
53 \\
23 \\
14 \\
31\end{array}$ & $\begin{array}{r}0 \\
13 \\
0 \\
0 \\
0 \\
0 \\
0 \\
0 \\
0 \\
0 \\
0 \\
0 \\
1\end{array}$ & $\begin{array}{l}0 \\
0 \\
0 \\
0 \\
0 \\
0 \\
0 \\
0 \\
0 \\
0 \\
0 \\
0 \\
0\end{array}$ & $\begin{array}{r}1 \\
0 \\
4 \\
0 \\
0 \\
20 \\
21 \\
4 \\
3 \\
6 \\
2 \\
0 \\
5\end{array}$ & $\begin{array}{l}0 \\
0 \\
0 \\
0 \\
0 \\
0 \\
0 \\
0 \\
0 \\
0 \\
0 \\
0 \\
0\end{array}$ & $\begin{array}{l}1,571 \\
1,605 \\
1,508 \\
1,454 \\
1,571 \\
1,546 \\
1,547 \\
1,630 \\
1,577 \\
1,503 \\
1,559 \\
1,689 \\
1,563\end{array}$ & $\begin{array}{l}1,162 \\
1,155 \\
1,158 \\
1,063 \\
1,203 \\
1,184 \\
1,201 \\
1,275 \\
1,250 \\
1,175 \\
1,213 \\
1,333 \\
1,198\end{array}$ & $\begin{array}{r}84 \\
65 \\
120 \\
46 \\
21 \\
44 \\
0 \\
42 \\
49 \\
48 \\
22 \\
45 \\
49\end{array}$ & $\begin{array}{r}84 \\
65 \\
120 \\
46 \\
21 \\
44 \\
0 \\
42 \\
43 \\
47 \\
22 \\
45 \\
48\end{array}$ \\
\hline
\end{tabular}

See footnotes at end of table. 
Table S3. Crude Oil and Petroleum Product Imports, 1981 - Present (Continued) (Thousand Barrels per Day)

\begin{tabular}{|c|c|c|c|c|c|c|c|c|c|c|c|c|c|}
\hline & \multirow{3}{*}{ Year/Month } & \multicolumn{12}{|c|}{ Imports from Non-OPEC Sources ${ }^{a}$} \\
\hline & & \multicolumn{2}{|c|}{ Colombia } & \multicolumn{2}{|c|}{ Ecuador ${ }^{c}$} & \multicolumn{2}{|c|}{ Gabon $^{d}$} & \multicolumn{2}{|c|}{ Italy } & \multicolumn{2}{|c|}{ Malaysia } & \multicolumn{2}{|c|}{ Mexico } \\
\hline & & Total & Crude Oil & Total & Crude Ois & Total & Crude Oil & Total & Crude Oil & Total & Crude Oil & Total & Crude Oil \\
\hline $\begin{array}{l}1981 \\
1982 \\
1983 \\
1984 \\
1985 \\
1986 \\
1987 \\
1988 \\
1989 \\
1990 \\
1991 \\
1992 \\
1993 \\
1994\end{array}$ & $\begin{array}{c}\text { Average } \ldots \ldots \ldots . . \\
\text { Average } \ldots \ldots \ldots \ldots \\
\text { Average } \ldots \ldots \ldots \ldots \\
\text { Average } \ldots \ldots \ldots \ldots \\
\text { Average } \ldots \ldots \ldots \ldots \\
\text { Average } \\
\text { Average }\end{array}$ & $\begin{array}{r}1 \\
5 \\
10 \\
8 \\
23 \\
87 \\
148 \\
134 \\
172 \\
182 \\
163 \\
126 \\
171 \\
161\end{array}$ & $\begin{array}{r}0 \\
0 \\
0 \\
0 \\
0 \\
57 \\
115 \\
106 \\
136 \\
140 \\
123 \\
102 \\
141 \\
146\end{array}$ & $\begin{array}{l}\text { (c) } \\
\text { (c) } \\
\text { (c) } \\
\text { (c) } \\
\text { (c) } \\
\text { (c) } \\
\text { (c) } \\
(c) \\
\text { (c) } \\
(c) \\
\text { (c) } \\
\text { (c) } \\
\text { (c) } \\
91\end{array}$ & $\begin{array}{l}\text { (c) } \\
\text { (c) } \\
\text { (c) } \\
\text { (c) } \\
\text { (c) } \\
\text { (c) } \\
\text { (c) } \\
(c) \\
\text { (c) } \\
(c) \\
\text { (c) } \\
\text { (c) } \\
\text { (c) } \\
91\end{array}$ & $\begin{array}{l}\text { (d) } \\
\text { (d) } \\
\text { (d) } \\
\text { (d) } \\
\text { (d) } \\
\text { (d) } \\
\text { (d) } \\
\text { (d) } \\
\text { (d) } \\
\text { (d) } \\
\text { (d) } \\
\text { (d) } \\
\text { (d) } \\
\text { (d) }\end{array}$ & $\begin{array}{l}\text { (d) } \\
\text { (d) } \\
\text { (d) } \\
\text { (d) } \\
\text { (d) } \\
\text { (d) } \\
\text { (d) } \\
\text { (d) } \\
\text { (d) } \\
\text { (d) } \\
\text { (d) } \\
\text { (d) } \\
\text { (d) } \\
\text { (d) }\end{array}$ & $\begin{array}{l}11 \\
18 \\
18 \\
45 \\
60 \\
76 \\
54 \\
65 \\
34 \\
58 \\
47 \\
55 \\
31 \\
22\end{array}$ & $\begin{array}{r}0 \\
(s) \\
(s) \\
(s) \\
(s) \\
0 \\
1 \\
5 \\
3 \\
2 \\
3 \\
0 \\
0 \\
0\end{array}$ & $\begin{array}{r}36 \\
20 \\
4 \\
1 \\
3 \\
12 \\
13 \\
19 \\
39 \\
41 \\
24 \\
10 \\
11 \\
10\end{array}$ & $\begin{array}{r}33 \\
18 \\
3 \\
0 \\
1 \\
11 \\
12 \\
19 \\
39 \\
40 \\
24 \\
10 \\
10 \\
0\end{array}$ & $\begin{array}{l}522 \\
685 \\
826 \\
748 \\
816 \\
699 \\
655 \\
747 \\
767 \\
755 \\
807 \\
830 \\
919 \\
984\end{array}$ & $\begin{array}{l}469 \\
645 \\
766 \\
659 \\
715 \\
621 \\
602 \\
674 \\
716 \\
689 \\
759 \\
787 \\
863 \\
939\end{array}$ \\
\hline 1995 & 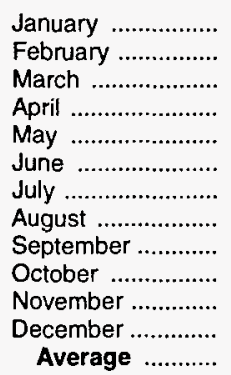 & $\begin{array}{l}223 \\
139 \\
239 \\
175 \\
171 \\
225 \\
223 \\
330 \\
252 \\
199 \\
240 \\
200 \\
219\end{array}$ & $\begin{array}{l}214 \\
129 \\
221 \\
175 \\
153 \\
202 \\
223 \\
311 \\
236 \\
190 \\
229 \\
190 \\
207\end{array}$ & $\begin{array}{r}130 \\
107 \\
104 \\
146 \\
116 \\
137 \\
87 \\
116 \\
61 \\
12 \\
102 \\
51 \\
97\end{array}$ & $\begin{array}{r}130 \\
107 \\
104 \\
146 \\
116 \\
137 \\
87 \\
104 \\
61 \\
12 \\
102 \\
51 \\
96\end{array}$ & $\begin{array}{l}193 \\
186 \\
159 \\
163 \\
206 \\
357 \\
311 \\
246 \\
216 \\
270 \\
271 \\
171 \\
229\end{array}$ & $\begin{array}{l}193 \\
186 \\
159 \\
163 \\
206 \\
357 \\
311 \\
246 \\
216 \\
270 \\
271 \\
171 \\
229\end{array}$ & $\begin{array}{r}4 \\
1 \\
8 \\
13 \\
0 \\
13 \\
4 \\
0 \\
0 \\
11 \\
4 \\
3 \\
5\end{array}$ & $\begin{array}{l}0 \\
0 \\
0 \\
0 \\
0 \\
0 \\
0 \\
0 \\
0 \\
0 \\
0 \\
0 \\
0\end{array}$ & $\begin{array}{r}21 \\
0 \\
0 \\
7 \\
0 \\
7 \\
0 \\
0 \\
14 \\
13 \\
16 \\
17 \\
8\end{array}$ & $\begin{array}{r}21 \\
0 \\
0 \\
0 \\
0 \\
0 \\
0 \\
0 \\
14 \\
5 \\
16 \\
11 \\
6\end{array}$ & $\begin{array}{r}925 \\
922 \\
1,006 \\
993 \\
1,118 \\
1,138 \\
1,188 \\
1,201 \\
1,311 \\
894 \\
1,114 \\
996 \\
1,068\end{array}$ & $\begin{array}{r}892 \\
890 \\
961 \\
963 \\
1,063 \\
1,076 \\
1,166 \\
1,172 \\
1,238 \\
854 \\
1,060 \\
978 \\
1,027\end{array}$ \\
\hline 1996 & 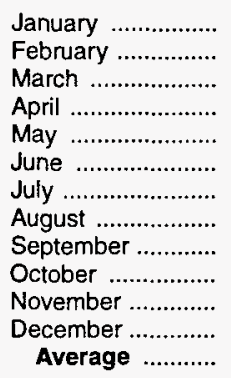 & $\begin{array}{l}186 \\
149 \\
262 \\
280 \\
263 \\
250 \\
204 \\
221 \\
213 \\
265 \\
267 \\
246 \\
234\end{array}$ & $\begin{array}{l}183 \\
139 \\
250 \\
280 \\
249 \\
247 \\
198 \\
217 \\
213 \\
252 \\
267 \\
218 \\
226\end{array}$ & $\begin{array}{r}126 \\
81 \\
131 \\
158 \\
100 \\
138 \\
113 \\
83 \\
48 \\
66 \\
111 \\
89 \\
104\end{array}$ & $\begin{array}{r}120 \\
81 \\
125 \\
143 \\
95 \\
133 \\
96 \\
71 \\
48 \\
60 \\
111 \\
72 \\
96\end{array}$ & $\begin{array}{l}171 \\
191 \\
154 \\
212 \\
154 \\
218 \\
191 \\
156 \\
104 \\
226 \\
253 \\
184 \\
184\end{array}$ & $\begin{array}{l}171 \\
191 \\
154 \\
212 \\
154 \\
218 \\
191 \\
156 \\
104 \\
226 \\
253 \\
184 \\
184\end{array}$ & $\begin{array}{r}2 \\
0 \\
13 \\
(s) \\
0 \\
16 \\
19 \\
8 \\
15 \\
4 \\
13 \\
8 \\
8\end{array}$ & $\begin{array}{l}0 \\
0 \\
0 \\
0 \\
0 \\
0 \\
0 \\
0 \\
0 \\
0 \\
0 \\
0 \\
0\end{array}$ & $\begin{array}{r}0 \\
24 \\
4 \\
0 \\
47 \\
19 \\
0 \\
5 \\
0 \\
31 \\
7 \\
0 \\
11\end{array}$ & $\begin{array}{r}0 \\
17 \\
0 \\
0 \\
40 \\
11 \\
0 \\
0 \\
0 \\
0 \\
0 \\
0 \\
6\end{array}$ & $\begin{array}{l}1,281 \\
1,083 \\
1,176 \\
1,303 \\
1,288 \\
1,351 \\
1,216 \\
1,157 \\
1,355 \\
1,213 \\
1,157 \\
1,346 \\
1,244\end{array}$ & $\begin{array}{l}1,245 \\
1,062 \\
1,165 \\
1,273 \\
1,222 \\
1,274 \\
1,186 \\
1,142 \\
1,306 \\
1,189 \\
1,110 \\
1,301 \\
1,207\end{array}$ \\
\hline 1997 & 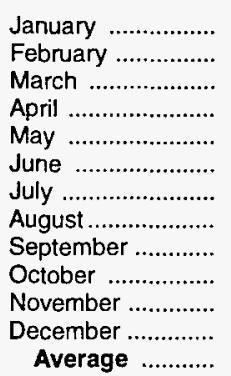 & $\begin{array}{l}227 \\
248 \\
260 \\
255 \\
272 \\
228 \\
235 \\
250 \\
289 \\
321 \\
322 \\
350 \\
271\end{array}$ & $\begin{array}{l}226 \\
248 \\
257 \\
255 \\
266 \\
228 \\
225 \\
250 \\
289 \\
321 \\
322 \\
350 \\
270\end{array}$ & $\begin{array}{r}112 \\
110 \\
148 \\
73 \\
109 \\
132 \\
122 \\
128 \\
143 \\
143 \\
91 \\
66 \\
115\end{array}$ & $\begin{array}{r}107 \\
110 \\
148 \\
73 \\
104 \\
132 \\
122 \\
128 \\
143 \\
143 \\
91 \\
66 \\
114\end{array}$ & $\begin{array}{r}62 \\
262 \\
217 \\
203 \\
210 \\
226 \\
335 \\
203 \\
271 \\
235 \\
256 \\
288 \\
230\end{array}$ & $\begin{array}{r}62 \\
262 \\
217 \\
203 \\
210 \\
226 \\
335 \\
203 \\
271 \\
235 \\
256 \\
288 \\
230\end{array}$ & $\begin{array}{r}8 \\
27 \\
5 \\
26 \\
9 \\
0 \\
0 \\
2 \\
0 \\
8 \\
0 \\
5 \\
7\end{array}$ & $\begin{array}{l}0 \\
0 \\
0 \\
0 \\
0 \\
0 \\
0 \\
0 \\
0 \\
0 \\
0 \\
0 \\
0\end{array}$ & $\begin{array}{r}32 \\
7 \\
33 \\
33 \\
9 \\
32 \\
28 \\
23 \\
37 \\
19 \\
8 \\
7 \\
23\end{array}$ & $\begin{array}{r}0 \\
7 \\
0 \\
0 \\
0 \\
24 \\
0 \\
15 \\
29 \\
19 \\
0 \\
0 \\
8\end{array}$ & $\begin{array}{l}1,324 \\
1,277 \\
1,310 \\
1,448 \\
1,429 \\
1,401 \\
1,366 \\
1,452 \\
1,410 \\
1,526 \\
1,460 \\
1,215 \\
1,385\end{array}$ & $\begin{array}{l}1,280 \\
1,241 \\
1,249 \\
1,416 \\
1,408 \\
1,382 \\
1,347 \\
1,448 \\
1,395 \\
1,500 \\
1,453 \\
1,192 \\
1,360\end{array}$ \\
\hline
\end{tabular}

See footnotes at end of table. 
Table S3. Crude Oil and Petroleum Product Imports, 1981 - Present (Continued) (Thousand Barrels per Day)

\begin{tabular}{|c|c|c|c|c|c|c|c|c|c|c|c|c|c|}
\hline & \multirow{3}{*}{ Year/Month } & \multicolumn{12}{|c|}{ Imports from Non-OPEC Sources ${ }^{a}$} \\
\hline & & \multicolumn{2}{|c|}{ Netheriands } & \multicolumn{2}{|c|}{$\begin{array}{l}\text { Netherlands } \\
\text { Antilles }\end{array}$} & \multicolumn{2}{|c|}{ Norway } & \multicolumn{2}{|c|}{$\begin{array}{c}\text { Puerto } \\
\text { Rico }\end{array}$} & \multicolumn{2}{|c|}{ Russia' } & \multicolumn{2}{|c|}{ Spain } \\
\hline & & Total & Crude Oil & Total & Crude Oil & Total & Crude Oil & Total & Crude Oil & Total & Crude Oil & Total & Crude Oil \\
\hline $\begin{array}{l}1981 \\
1982 \\
1983 \\
1984 \\
1985 \\
1986 \\
1987 \\
1988 \\
1989 \\
1990 \\
1991 \\
1992 \\
1993 \\
1994\end{array}$ & 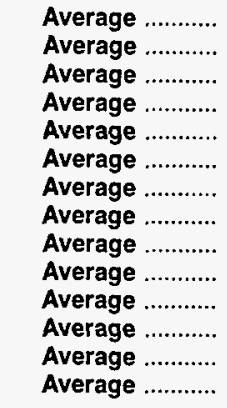 & $\begin{array}{l}30 \\
35 \\
65 \\
65 \\
58 \\
54 \\
60 \\
61 \\
49 \\
55 \\
29 \\
26 \\
10 \\
32\end{array}$ & $\begin{array}{l}(s) \\
(s) \\
3 \\
3 \\
0 \\
0 \\
0 \\
0 \\
0 \\
0 \\
0 \\
0 \\
0 \\
0\end{array}$ & $\begin{array}{r}197 \\
175 \\
189 \\
188 \\
40 \\
25 \\
29 \\
36 \\
42 \\
31 \\
81 \\
65 \\
82 \\
98\end{array}$ & $\begin{array}{l}0 \\
0 \\
0 \\
0 \\
0 \\
0 \\
0 \\
0 \\
0 \\
0 \\
0 \\
0 \\
0 \\
0\end{array}$ & $\begin{array}{r}119 \\
102 \\
66 \\
114 \\
32 \\
60 \\
80 \\
67 \\
138 \\
102 \\
82 \\
127 \\
142 \\
202\end{array}$ & $\begin{array}{r}114 \\
102 \\
65 \\
112 \\
31 \\
53 \\
70 \\
62 \\
127 \\
96 \\
74 \\
119 \\
137 \\
190\end{array}$ & $\begin{array}{l}62 \\
50 \\
40 \\
42 \\
28 \\
21 \\
21 \\
22 \\
32 \\
32 \\
27 \\
26 \\
29 \\
22\end{array}$ & $\begin{array}{l}0 \\
0 \\
0 \\
0 \\
0 \\
0 \\
0 \\
0 \\
0 \\
0 \\
0 \\
0 \\
0 \\
0\end{array}$ & $\begin{array}{r}5 \\
1 \\
1 \\
13 \\
8 \\
18 \\
11 \\
29 \\
48 \\
45 \\
29 \\
18 \\
55 \\
30\end{array}$ & $\begin{array}{r}(s) \\
0 \\
(s) \\
(s) \\
(s) \\
(s) \\
0 \\
0 \\
0 \\
1 \\
1 \\
5 \\
36 \\
27\end{array}$ & $\begin{array}{r}1 \\
3 \\
2 \\
11 \\
29 \\
53 \\
55 \\
68 \\
67 \\
47 \\
33 \\
32 \\
37 \\
37\end{array}$ & $\begin{array}{r}\text { (s) } \\
\text { (s) } \\
(\mathrm{s}) \\
0 \\
1 \\
0 \\
0 \\
0 \\
0 \\
0 \\
0 \\
0 \\
0 \\
0\end{array}$ \\
\hline 1995 & 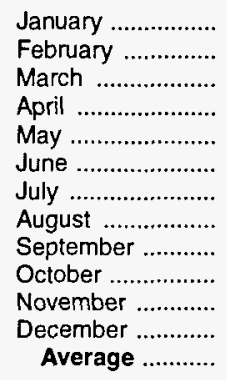 & $\begin{array}{r}0 \\
17 \\
21 \\
3 \\
24 \\
37 \\
9 \\
21 \\
0 \\
31 \\
20 \\
0 \\
15\end{array}$ & $\begin{array}{l}0 \\
0 \\
0 \\
0 \\
0 \\
0 \\
0 \\
0 \\
0 \\
0 \\
0 \\
0 \\
0\end{array}$ & $\begin{array}{r}60 \\
58 \\
68 \\
0 \\
86 \\
50 \\
65 \\
62 \\
33 \\
48 \\
69 \\
24 \\
52\end{array}$ & $\begin{array}{l}0 \\
0 \\
0 \\
0 \\
0 \\
0 \\
0 \\
0 \\
0 \\
0 \\
0 \\
0 \\
0\end{array}$ & $\begin{array}{l}195 \\
194 \\
241 \\
315 \\
292 \\
370 \\
263 \\
279 \\
364 \\
163 \\
255 \\
348 \\
273\end{array}$ & $\begin{array}{l}158 \\
164 \\
209 \\
291 \\
292 \\
370 \\
256 \\
264 \\
359 \\
163 \\
255 \\
316 \\
\mathbf{2 5 8}\end{array}$ & $\begin{array}{r}6 \\
7 \\
13 \\
9 \\
9 \\
19 \\
16 \\
17 \\
26 \\
12 \\
15 \\
27 \\
15 \\
15\end{array}$ & $\begin{array}{l}0 \\
0 \\
0 \\
0 \\
0 \\
0 \\
0 \\
0 \\
0 \\
0 \\
0 \\
0 \\
0\end{array}$ & $\begin{array}{r}0 \\
0 \\
0 \\
0 \\
12 \\
15 \\
41 \\
136 \\
50 \\
0 \\
28 \\
15 \\
25\end{array}$ & $\begin{array}{r}0 \\
0 \\
0 \\
0 \\
0 \\
0 \\
32 \\
98 \\
32 \\
0 \\
0 \\
0 \\
14\end{array}$ & $\begin{array}{r}7 \\
9 \\
16 \\
16 \\
25 \\
27 \\
10 \\
21 \\
27 \\
6 \\
16 \\
12 \\
16\end{array}$ & $\begin{array}{l}0 \\
0 \\
0 \\
7 \\
0 \\
0 \\
0 \\
0 \\
0 \\
0 \\
0 \\
5 \\
1\end{array}$ \\
\hline 1996 & 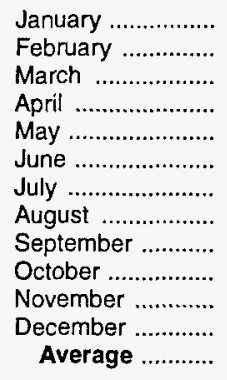 & $\begin{array}{r}16 \\
38 \\
35 \\
20 \\
9 \\
9 \\
26 \\
7 \\
14 \\
13 \\
24 \\
18 \\
14 \\
19\end{array}$ & $\begin{array}{l}0 \\
0 \\
0 \\
0 \\
0 \\
0 \\
0 \\
0 \\
0 \\
0 \\
0 \\
0 \\
0\end{array}$ & $\begin{array}{r}59 \\
101 \\
35 \\
50 \\
47 \\
52 \\
45 \\
53 \\
56 \\
97 \\
79 \\
98 \\
64\end{array}$ & $\begin{array}{l}0 \\
0 \\
0 \\
0 \\
0 \\
0 \\
0 \\
0 \\
0 \\
0 \\
0 \\
0 \\
0\end{array}$ & $\begin{array}{l}199 \\
236 \\
284 \\
375 \\
380 \\
434 \\
375 \\
369 \\
274 \\
389 \\
249 \\
187 \\
313\end{array}$ & $\begin{array}{l}178 \\
221 \\
264 \\
357 \\
364 \\
408 \\
359 \\
362 \\
254 \\
359 \\
220 \\
166 \\
293\end{array}$ & $\begin{array}{r}6 \\
17 \\
24 \\
17 \\
22 \\
25 \\
25 \\
33 \\
22 \\
14 \\
20 \\
18 \\
20\end{array}$ & $\begin{array}{l}0 \\
0 \\
0 \\
0 \\
0 \\
0 \\
0 \\
0 \\
0 \\
0 \\
0 \\
0 \\
0\end{array}$ & $\begin{array}{r}11 \\
14 \\
18 \\
0 \\
63 \\
14 \\
42 \\
32 \\
39 \\
42 \\
0 \\
26 \\
25\end{array}$ & $\begin{array}{r}0 \\
0 \\
0 \\
0 \\
63 \\
14 \\
33 \\
32 \\
37 \\
33 \\
0 \\
0 \\
18\end{array}$ & $\begin{array}{l}23 \\
23 \\
58 \\
36 \\
21 \\
12 \\
47 \\
21 \\
21 \\
34 \\
33 \\
13 \\
29\end{array}$ & $\begin{array}{r}0 \\
0 \\
0 \\
0 \\
0 \\
0 \\
10 \\
0 \\
0 \\
0 \\
0 \\
0 \\
1\end{array}$ \\
\hline 1997 & 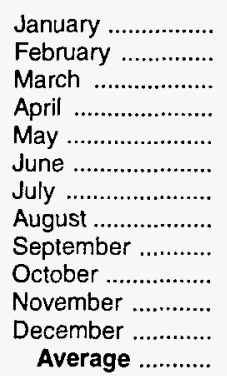 & $\begin{array}{r}40 \\
33 \\
40 \\
20 \\
13 \\
37 \\
5 \\
15 \\
54 \\
13 \\
28 \\
1 \\
25\end{array}$ & $\begin{array}{l}0 \\
0 \\
0 \\
0 \\
0 \\
0 \\
0 \\
0 \\
0 \\
0 \\
0 \\
0 \\
0\end{array}$ & $\begin{array}{r}94 \\
60 \\
102 \\
114 \\
116 \\
66 \\
61 \\
65 \\
71 \\
46 \\
33 \\
54 \\
74\end{array}$ & $\begin{array}{l}0 \\
0 \\
0 \\
0 \\
0 \\
0 \\
0 \\
0 \\
0 \\
0 \\
0 \\
0 \\
0\end{array}$ & $\begin{array}{l}244 \\
204 \\
295 \\
307 \\
388 \\
329 \\
386 \\
321 \\
285 \\
346 \\
316 \\
275 \\
309\end{array}$ & $\begin{array}{l}230 \\
179 \\
276 \\
294 \\
366 \\
318 \\
360 \\
320 \\
265 \\
312 \\
276 \\
249 \\
288\end{array}$ & $\begin{array}{r}18 \\
16 \\
7 \\
12 \\
21 \\
13 \\
24 \\
20 \\
14 \\
19 \\
23 \\
10 \\
16\end{array}$ & $\begin{array}{l}0 \\
0 \\
0 \\
0 \\
0 \\
0 \\
0 \\
0 \\
0 \\
0 \\
0 \\
0 \\
0\end{array}$ & $\begin{array}{r}21 \\
19 \\
13 \\
20 \\
0 \\
8 \\
9 \\
32 \\
0 \\
13 \\
21 \\
0 \\
13\end{array}$ & $\begin{array}{r}0 \\
0 \\
0 \\
0 \\
0 \\
0 \\
0 \\
19 \\
0 \\
6 \\
7 \\
0 \\
3\end{array}$ & $\begin{array}{r}31 \\
36 \\
6 \\
9 \\
23 \\
45 \\
6 \\
41 \\
21 \\
12 \\
19 \\
5 \\
21\end{array}$ & $\begin{array}{l}0 \\
0 \\
0 \\
0 \\
0 \\
0 \\
0 \\
0 \\
0 \\
0 \\
0 \\
0 \\
0\end{array}$ \\
\hline
\end{tabular}

See footnotes at end of table. 
Table S3. Crude Oil and Petroleum Product Imports, 1981 - Present (Continued) (Thousand Barrels per Day)

\begin{tabular}{|c|c|c|c|c|c|c|c|c|c|c|c|c|c|}
\hline & \multirow{3}{*}{ Year/Month } & \multicolumn{10}{|c|}{ Imports from Non-OPEC Sources ${ }^{a}$} & & \\
\hline & & \multicolumn{2}{|c|}{$\begin{array}{c}\text { Trinidad } \\
\text { and } \\
\text { Tobago }\end{array}$} & \multicolumn{2}{|c|}{$\begin{array}{c}\text { United } \\
\text { Kingdom }\end{array}$} & \multicolumn{2}{|c|}{$\begin{array}{c}\text { Virgin } \\
\text { Islands }\end{array}$} & \multicolumn{2}{|c|}{$\begin{array}{l}\text { Other } \\
\text { Non- } \\
\text { OPEC }\end{array}$} & \multicolumn{2}{|c|}{$\begin{array}{c}\text { Total } \\
\text { Non- } \\
\text { OPEC }\end{array}$} & \multicolumn{2}{|c|}{$\begin{array}{c}\text { Total } \\
\text { Imports }\end{array}$} \\
\hline & & Total & Crude Oil & Total & Crude Oil & Total & Crude Oil & Total & Crude Oil & Total & Crude Oil & Total & Crude Oil \\
\hline $\begin{array}{l}1981 \\
1982 \\
1983 \\
1984 \\
1985 \\
1986 \\
1987 \\
1988 \\
1989 \\
1990 \\
1991 \\
1992 \\
1993 \\
1994\end{array}$ & $\begin{array}{l}\text { Average } \ldots \ldots \ldots . . \\
\text { Average } \ldots \ldots \ldots \ldots \\
\text { Average } \ldots \ldots \ldots \ldots \\
\text { Average } \\
\text { Average } \ldots \ldots \ldots \ldots \\
\text { Average } \\
\text { Average }\end{array}$ & $\begin{array}{r}133 \\
112 \\
96 \\
94 \\
113 \\
125 \\
106 \\
97 \\
94 \\
96 \\
88 \\
95 \\
74 \\
77\end{array}$ & $\begin{array}{r}102 \\
92 \\
83 \\
87 \\
98 \\
93 \\
75 \\
71 \\
73 \\
76 \\
72 \\
70 \\
55 \\
62\end{array}$ & $\begin{array}{l}375 \\
456 \\
382 \\
402 \\
310 \\
350 \\
352 \\
315 \\
215 \\
189 \\
138 \\
230 \\
350 \\
458\end{array}$ & $\begin{array}{l}369 \\
441 \\
365 \\
378 \\
278 \\
317 \\
304 \\
254 \\
160 \\
155 \\
106 \\
200 \\
312 \\
396\end{array}$ & $\begin{array}{l}327 \\
316 \\
282 \\
294 \\
247 \\
244 \\
272 \\
242 \\
321 \\
282 \\
243 \\
249 \\
254 \\
328\end{array}$ & $\begin{array}{l}0 \\
0 \\
0 \\
0 \\
0 \\
0 \\
0 \\
0 \\
0 \\
0 \\
0 \\
0 \\
0 \\
0\end{array}$ & $\begin{array}{l}236 \\
306 \\
378 \\
411 \\
394 \\
426 \\
459 \\
487 \\
457 \\
417 \\
282 \\
335 \\
452 \\
450\end{array}$ & $\begin{array}{l}163 \\
174 \\
215 \\
210 \\
137 \\
144 \\
196 \\
196 \\
197 \\
180 \\
137 \\
149 \\
240 \\
239\end{array}$ & $\begin{array}{l}2,672 \\
2,968 \\
3,189 \\
3,388 \\
3,237 \\
3,387 \\
3,617 \\
3,882 \\
3,921 \\
3,721 \\
3,535 \\
3,796 \\
4,266 \\
4,749\end{array}$ & $\begin{array}{l}1,474 \\
1,754 \\
1,853 \\
1,914 \\
1,888 \\
2,065 \\
2,274 \\
2,411 \\
2,467 \\
2,381 \\
2,405 \\
2,676 \\
3,100 \\
3,483\end{array}$ & $\begin{array}{l}5,996 \\
5,113 \\
5,051 \\
5,437 \\
5,067 \\
6,224 \\
6,678 \\
7,402 \\
8,061 \\
8,018 \\
7,627 \\
7,888 \\
8,620 \\
8,996\end{array}$ & $\begin{array}{l}4,396 \\
3,488 \\
3,329 \\
3,426 \\
3,201 \\
4,178 \\
4,674 \\
5,107 \\
5,843 \\
5,894 \\
5,782 \\
6,083 \\
6,787 \\
7,063\end{array}$ \\
\hline 1995 & 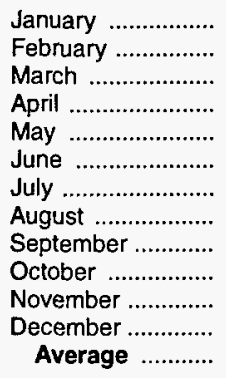 & $\begin{array}{l}91 \\
58 \\
70 \\
55 \\
61 \\
78 \\
73 \\
74 \\
73 \\
86 \\
61 \\
53 \\
70\end{array}$ & $\begin{array}{l}91 \\
58 \\
70 \\
55 \\
53 \\
74 \\
54 \\
53 \\
55 \\
70 \\
53 \\
53 \\
62\end{array}$ & $\begin{array}{l}240 \\
382 \\
663 \\
491 \\
405 \\
520 \\
137 \\
288 \\
427 \\
528 \\
284 \\
238 \\
383\end{array}$ & $\begin{array}{r}213 \\
359 \\
621 \\
450 \\
366 \\
418 \\
97 \\
249 \\
386 \\
479 \\
284 \\
177 \\
341\end{array}$ & $\begin{array}{l}283 \\
322 \\
298 \\
284 \\
203 \\
268 \\
240 \\
264 \\
223 \\
299 \\
317 \\
334 \\
278\end{array}$ & $\begin{array}{l}0 \\
0 \\
0 \\
0 \\
0 \\
0 \\
0 \\
0 \\
0 \\
0 \\
0 \\
0 \\
0\end{array}$ & $\begin{array}{l}209 \\
304 \\
183 \\
317 \\
286 \\
368 \\
441 \\
343 \\
312 \\
331 \\
273 \\
262 \\
302\end{array}$ & $\begin{array}{r}131 \\
143 \\
91 \\
143 \\
165 \\
253 \\
277 \\
261 \\
180 \\
214 \\
155 \\
156 \\
181\end{array}$ & $\begin{array}{l}4,297 \\
4,416 \\
4,787 \\
4,741 \\
4,907 \\
5,453 \\
4,812 \\
5,168 \\
5,194 \\
4,635 \\
4,896 \\
4,684 \\
\mathbf{4 , 8 3 3}\end{array}$ & $\begin{array}{l}3,397 \\
3,378 \\
3,797 \\
3,894 \\
4,044 \\
4,451 \\
3,940 \\
4,212 \\
4,254 \\
3,735 \\
3,878 \\
3,671 \\
3,889\end{array}$ & $\begin{array}{l}8,015 \\
8,345 \\
9,006 \\
8,465 \\
8,709 \\
9,558 \\
8,863 \\
9,061 \\
9,736 \\
8,577 \\
9,074 \\
8,612 \\
8,835\end{array}$ & $\begin{array}{l}6,505 \\
6,546 \\
7,391 \\
7,038 \\
7,325 \\
7,927 \\
7,265 \\
7,437 \\
8,007 \\
7,075 \\
7,302 \\
6,916 \\
7,230\end{array}$ \\
\hline 1996 & 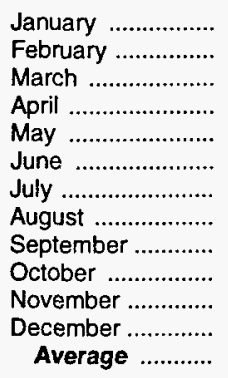 & $\begin{array}{l}92 \\
56 \\
63 \\
87 \\
97 \\
86 \\
70 \\
81 \\
51 \\
70 \\
96 \\
58 \\
76\end{array}$ & $\begin{array}{l}71 \\
56 \\
52 \\
55 \\
71 \\
54 \\
58 \\
59 \\
37 \\
55 \\
75 \\
54 \\
58\end{array}$ & $\begin{array}{l}364 \\
374 \\
346 \\
481 \\
421 \\
312 \\
244 \\
274 \\
165 \\
264 \\
199 \\
253 \\
308\end{array}$ & $\begin{array}{r}238 \\
280 \\
252 \\
347 \\
316 \\
234 \\
195 \\
177 \\
90 \\
136 \\
160 \\
167 \\
216\end{array}$ & $\begin{array}{l}390 \\
343 \\
311 \\
359 \\
298 \\
292 \\
344 \\
279 \\
268 \\
325 \\
253 \\
294 \\
313\end{array}$ & $\begin{array}{l}0 \\
0 \\
0 \\
0 \\
0 \\
0 \\
0 \\
0 \\
0 \\
0 \\
0 \\
0 \\
0\end{array}$ & $\begin{array}{l}406 \\
275 \\
373 \\
333 \\
429 \\
561 \\
456 \\
508 \\
502 \\
477 \\
513 \\
438 \\
440\end{array}$ & $\begin{array}{l}188 \\
169 \\
215 \\
157 \\
282 \\
402 \\
292 \\
348 \\
318 \\
240 \\
318 \\
245 \\
265\end{array}$ & $\begin{array}{l}5,244 \\
4,660 \\
4,932 \\
5,421 \\
5,465 \\
5,663 \\
5,201 \\
5,321 \\
4,938 \\
5,566 \\
5,277 \\
5,487 \\
\mathbf{5 , 2 6 7}\end{array}$ & $\begin{array}{l}3,932 \\
3,479 \\
3,788 \\
4,125 \\
4,332 \\
4,526 \\
4,082 \\
4,177 \\
3,891 \\
4,196 \\
4,145 \\
4,142 \\
4,070\end{array}$ & $\begin{array}{r}9,364 \\
8,390 \\
9,092 \\
9,429 \\
10,007 \\
9,938 \\
9,820 \\
9,986 \\
9,142 \\
9,837 \\
9,244 \\
9,417 \\
9,478\end{array}$ & $\begin{array}{l}7,303 \\
6,612 \\
7,215 \\
7,371 \\
8,029 \\
7,958 \\
7,800 \\
8,041 \\
7,353 \\
7,701 \\
7,344 \\
7,307 \\
7,508\end{array}$ \\
\hline 1997 & 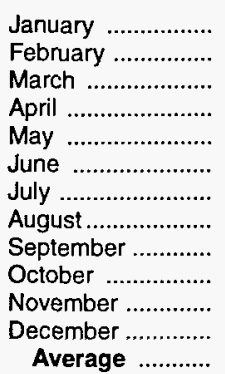 & $\begin{array}{l}74 \\
69 \\
56 \\
69 \\
70 \\
55 \\
62 \\
41 \\
66 \\
58 \\
65 \\
53 \\
61\end{array}$ & $\begin{array}{l}55 \\
61 \\
55 \\
62 \\
66 \\
55 \\
54 \\
37 \\
58 \\
55 \\
57 \\
53 \\
56\end{array}$ & $\begin{array}{l}400 \\
236 \\
236 \\
159 \\
261 \\
372 \\
198 \\
268 \\
166 \\
154 \\
127 \\
135 \\
226\end{array}$ & $\begin{array}{r}333 \\
172 \\
161 \\
70 \\
181 \\
311 \\
165 \\
220 \\
110 \\
119 \\
87 \\
98 \\
169\end{array}$ & $\begin{array}{l}335 \\
341 \\
254 \\
321 \\
300 \\
300 \\
310 \\
319 \\
248 \\
301 \\
260 \\
314 \\
300\end{array}$ & $\begin{array}{l}0 \\
0 \\
0 \\
0 \\
0 \\
0 \\
0 \\
0 \\
0 \\
0 \\
0 \\
0 \\
0\end{array}$ & $\begin{array}{l}502 \\
380 \\
437 \\
401 \\
558 \\
380 \\
370 \\
368 \\
476 \\
479 \\
403 \\
304 \\
422\end{array}$ & $\begin{array}{l}210 \\
170 \\
206 \\
242 \\
341 \\
225 \\
243 \\
251 \\
364 \\
271 \\
236 \\
235 \\
250\end{array}$ & $\begin{array}{l}5,685 \\
5,431 \\
5,554 \\
5,426 \\
5,817 \\
5,737 \\
5,579 \\
5,638 \\
5,677 \\
5,879 \\
5,517 \\
5,160 \\
\mathbf{5 , 5 9 3}\end{array}$ & $\begin{array}{l}4,255 \\
4,093 \\
4,344 \\
4,169 \\
4,579 \\
4,631 \\
4,515 \\
4,591 \\
4,672 \\
4,793 \\
4,521 \\
4,208 \\
4,450\end{array}$ & $\begin{array}{r}9,763 \\
9,561 \\
9,833 \\
10,114 \\
10,818 \\
10,736 \\
10,008 \\
10,465 \\
10,537 \\
10,792 \\
9,948 \\
9,328 \\
10,162\end{array}$ & $\begin{array}{l}7,492 \\
7,434 \\
7,754 \\
7,987 \\
8,653 \\
8,759 \\
8,178 \\
8,621 \\
8,840 \\
8,927 \\
8,366 \\
7,653 \\
8,225\end{array}$ \\
\hline
\end{tabular}

\footnotetext{
${ }^{a}$ Includes petroleum imported into the United States indirectly from members of the Organization of Petroleum Exporting Countries (OPEC) primarily from Caribbean and West European areas as petroleum products that were refined from crude oil produced by OPEC.

Imports from the Neutral Zone between Kuwait and Saudi Arabia are included in imports from Saudi Arabia.

${ }^{c}$ On December 31, 1992, Ecuador withdrew as a member of OPEC. As of January 1, 1994, imports of petroleum from Ecuador appear under imports Irom Non-OPEC Sources.

${ }^{d}$ On December 31, 1994, Gabon withdrew as a member of OPEC. As of January 1, 1995, imports of petroleum from Gabon appear under imports from Non-OPEC Sources.

${ }^{\ominus}$ Excludes petroleum imported into the United States indirectly from members of the Organization of Petroleum Exporting Countries (OPEC), primarily from Caribbean and West European areas, as petroleum products that were refined from crude oil produced by OPEC.

Imports from other States in the former U.S.S.R. may be included in imports from Russia for the years 1981 through 1992.

9 A small amount of Iranian crude oil entered the United States in January 1988 from the Virgin Islands. This oil originated in Iran and was exported to the Virgin Islands prior to the signing of Executive Order 12613 on October 29, 1987.

(s) = Less than 500 barrels per day.

Notes: - Geographic coverage is the 50 States and the District of Columbia. - Totals may not equal sum of components due to independent rounding

Source: See Summary Statistics Table and Figure Sources.
} 
Figure S5. Finished Motor Gasoline Supply and Disposition, 1981 - Present

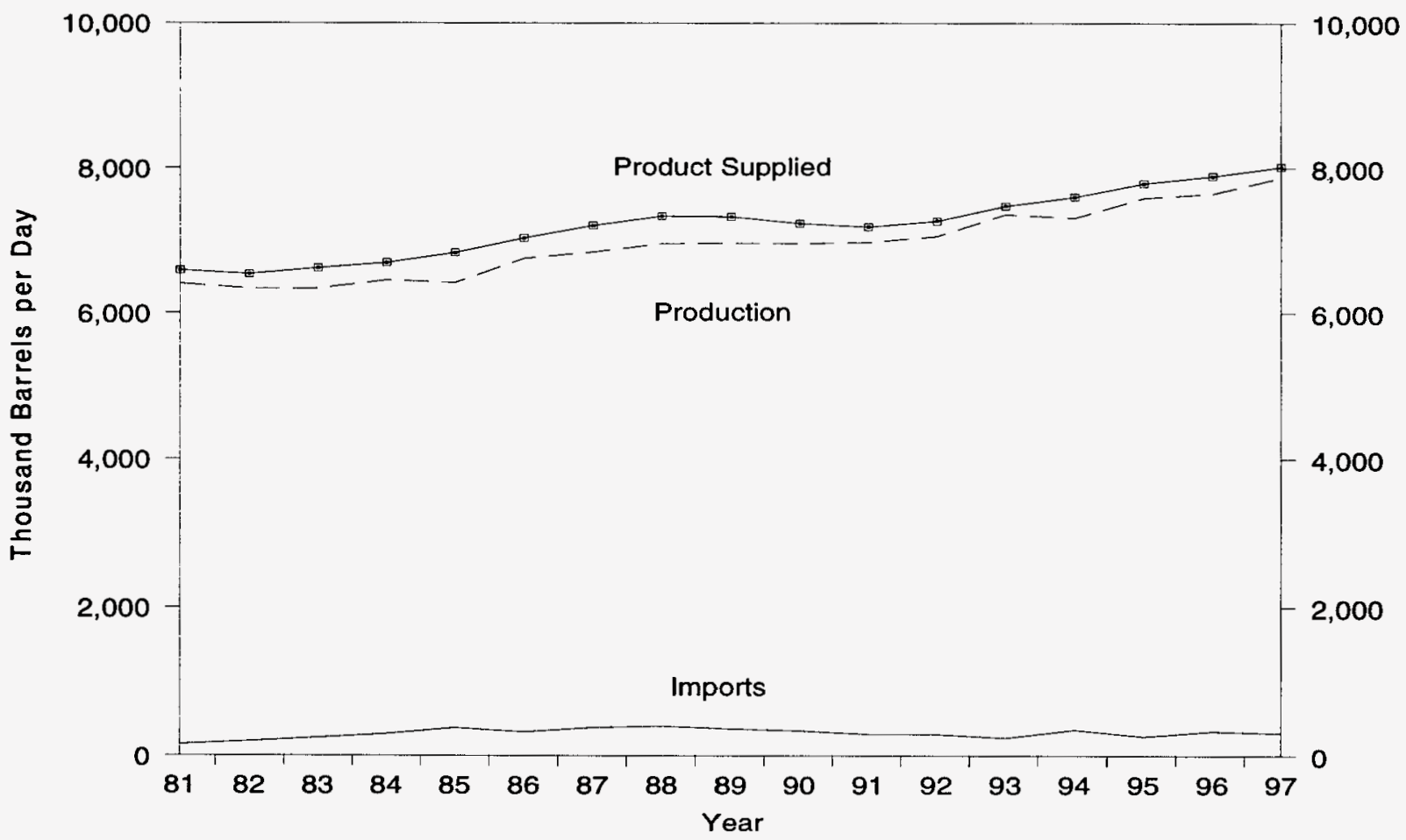

Source: Energy Information Administration, Petroleum Supply Annual, Table S4. See Summary Statistics Table and Figure Sources.

Figure S6. Motor Gasoline Ending Stocks, 1981 - Present

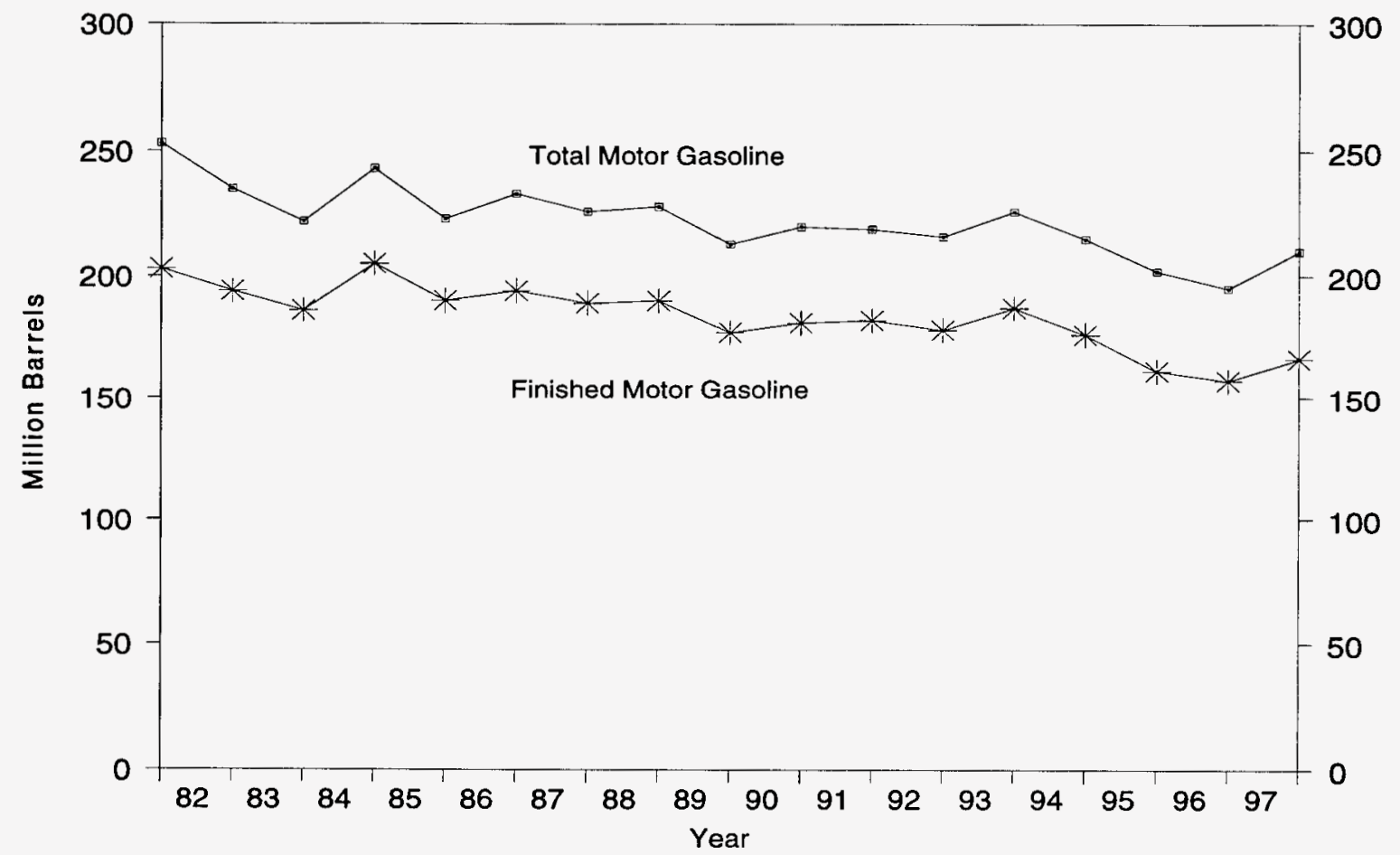

Note: Total motor gasoline includes motor gasoline blending components and finished motor gasoline.

Source: Energy Information Administration, Petroleum Supply Annual, Table S4. See Summary Statistics Table and Figure Sources. 
Table S4. Finished Motor Gasoline Supply and Disposition, 1981 - Present (Thousand Barrels per Day, Except Where Noted)

\begin{tabular}{|c|c|c|c|c|c|c|c|c|c|}
\hline & \multirow{3}{*}{ Year/Month } & \multicolumn{2}{|c|}{ Supply } & \multicolumn{3}{|c|}{ Disposition } & \multirow{2}{*}{\multicolumn{2}{|c|}{$\begin{array}{l}\begin{array}{l}\text { Ending Stocks } \\
\text { (Million Barrels) }\end{array} \\
\text { Motor Gasoline }\end{array}$}} & \multirow{3}{*}{\begin{tabular}{|c|}
$\begin{array}{c}\text { Ending Stocks } \\
\text { (Million Barrels) }\end{array}$ \\
\\
Oxygenates
\end{tabular}} \\
\hline & & \multirow[b]{2}{*}{$\begin{array}{c}\text { Total } \\
\text { Production }\end{array}$} & & \multirow[b]{2}{*}{$\begin{array}{c}\text { Stock } \\
\text { Change }\end{array}$} & \multirow[b]{2}{*}{ Exports } & \multirow[b]{2}{*}{$\begin{array}{l}\text { Product } \\
\text { Supplied }\end{array}$} & & & \\
\hline & & & & & & & Total $^{e}$ & Finished & \\
\hline $\begin{array}{l}1981 \\
1982 \\
1983 \\
1984 \\
1985 \\
1986 \\
1987 \\
1988 \\
1989 \\
1990 \\
1991 \\
1992 \\
1993 \\
1994\end{array}$ & 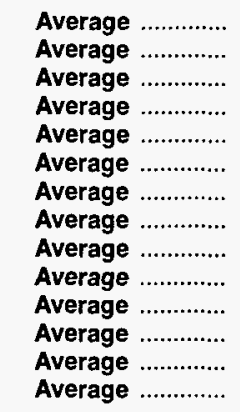 & $\begin{array}{l}6,405 \\
6,338 \\
6,340 \\
6,453 \\
6,419 \\
6,752 \\
6,841 \\
6,956 \\
6,963 \\
6,959 \\
6,975 \\
7,058 \\
7,360 \\
7,312\end{array}$ & $\begin{array}{l}157 \\
197 \\
247 \\
299 \\
381 \\
326 \\
384 \\
405 \\
369 \\
342 \\
297 \\
294 \\
247 \\
356\end{array}$ & $\begin{array}{r}f-28 \\
-25 \\
f-45 \\
54 \\
-41 \\
11 \\
-15 \\
3 \\
-35 \\
10 \\
3 \\
-11 \\
26 \\
-31\end{array}$ & $\begin{array}{r}2 \\
20 \\
10 \\
6 \\
10 \\
33 \\
35 \\
22 \\
39 \\
55 \\
82 \\
96 \\
105 \\
97\end{array}$ & $\begin{array}{l}6,588 \\
6,539 \\
6,622 \\
6,693 \\
6,831 \\
7,034 \\
7,206 \\
7,336 \\
7,328 \\
7,235 \\
7,188 \\
7,268 \\
7,476 \\
7,601\end{array}$ & $\begin{array}{r}253 \\
\\
235 \\
222 \\
243 \\
223 \\
233 \\
226 \\
228 \\
213 \\
220 \\
219 \\
216 \\
226 \\
215\end{array}$ & $\begin{array}{r}203 \\
194 \\
186 \\
205 \\
190 \\
194 \\
189 \\
190 \\
177 \\
181 \\
182 \\
178 \\
187 \\
176\end{array}$ & $\begin{array}{l}- \\
- \\
- \\
- \\
- \\
- \\
- \\
- \\
- \\
\overline{13} \\
17\end{array}$ \\
\hline 1995 & 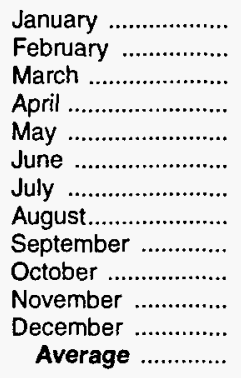 & $\begin{array}{l}7,303 \\
7,243 \\
7,168 \\
7,529 \\
7,678 \\
7,843 \\
7,747 \\
7,642 \\
7,785 \\
7,544 \\
7,739 \\
7,821 \\
7,588\end{array}$ & $\begin{array}{l}182 \\
223 \\
336 \\
235 \\
286 \\
347 \\
306 \\
280 \\
238 \\
253 \\
246 \\
244 \\
265\end{array}$ & $\begin{array}{r}221 \\
-99 \\
-391 \\
-26 \\
3 \\
-122 \\
80 \\
-367 \\
143 \\
-106 \\
1 \\
182 \\
-40\end{array}$ & $\begin{array}{r}100 \\
84 \\
107 \\
139 \\
67 \\
91 \\
86 \\
103 \\
94 \\
121 \\
118 \\
141 \\
104\end{array}$ & $\begin{array}{l}7,163 \\
7,481 \\
7,788 \\
7,651 \\
7,894 \\
8,220 \\
7,888 \\
8,187 \\
7,786 \\
7,781 \\
7,866 \\
7,742 \\
7,789\end{array}$ & $\begin{array}{r}227 \\
225 \\
211 \\
208 \\
208 \\
205 \\
207 \\
192 \\
199 \\
197 \\
196 \\
202 \\
-\end{array}$ & $\begin{array}{r}183 \\
180 \\
168 \\
167 \\
167 \\
163 \\
166 \\
155 \\
159 \\
156 \\
156 \\
161 \\
-\end{array}$ & $\begin{array}{l}16 \\
16 \\
15 \\
15 \\
15 \\
14 \\
15 \\
16 \\
15 \\
14 \\
11 \\
12 \\
-\end{array}$ \\
\hline 1996 & 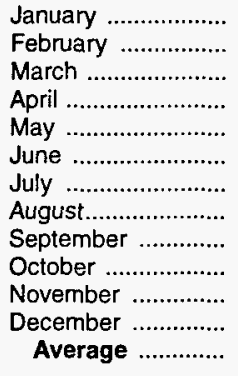 & $\begin{array}{l}7,370 \\
7,369 \\
7,289 \\
7,497 \\
7,804 \\
7,858 \\
7,924 \\
7,796 \\
7,606 \\
7,557 \\
7,864 \\
7,815 \\
7,647\end{array}$ & $\begin{array}{l}303 \\
293 \\
303 \\
501 \\
414 \\
393 \\
359 \\
346 \\
339 \\
253 \\
234 \\
298 \\
336\end{array}$ & $\begin{array}{r}240 \\
-10 \\
-327 \\
49 \\
66 \\
68 \\
-5 \\
-284 \\
215 \\
-396 \\
55 \\
202 \\
-12\end{array}$ & $\begin{array}{r}163 \\
72 \\
128 \\
77 \\
81 \\
95 \\
123 \\
82 \\
68 \\
113 \\
128 \\
117 \\
104\end{array}$ & $\begin{array}{l}7,271 \\
7,599 \\
7,792 \\
7,873 \\
8,071 \\
8,088 \\
8,165 \\
8,343 \\
7,662 \\
8,093 \\
7,915 \\
7,794 \\
7,891\end{array}$ & $\begin{array}{r}215 \\
214 \\
203 \\
203 \\
205 \\
205 \\
202 \\
191 \\
200 \\
189 \\
188 \\
195 \\
-\end{array}$ & $\begin{array}{r}169 \\
168 \\
158 \\
160 \\
162 \\
164 \\
164 \\
155 \\
161 \\
149 \\
151 \\
157 \\
-\end{array}$ & $\begin{array}{l}12 \\
12 \\
13 \\
13 \\
12 \\
11 \\
11 \\
12 \\
11 \\
11 \\
12 \\
13 \\
-\end{array}$ \\
\hline 1997 & 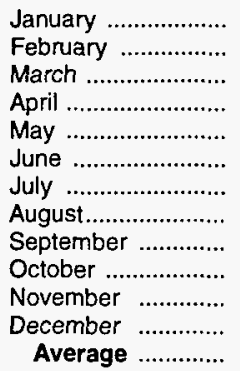 & $\begin{array}{l}7,307 \\
7,341 \\
7,302 \\
7,811 \\
8,081 \\
8,186 \\
7,954 \\
8,075 \\
8,158 \\
8,037 \\
7,999 \\
8,160 \\
7,870\end{array}$ & $\begin{array}{l}320 \\
324 \\
370 \\
300 \\
362 \\
387 \\
291 \\
292 \\
269 \\
291 \\
239 \\
265 \\
309\end{array}$ & $\begin{array}{r}250 \\
-114 \\
-247 \\
-70 \\
203 \\
189 \\
-414 \\
-41 \\
275 \\
1 \\
122 \\
154 \\
26\end{array}$ & $\begin{array}{r}75 \\
111 \\
123 \\
117 \\
101 \\
96 \\
164 \\
175 \\
130 \\
186 \\
151 \\
206 \\
137\end{array}$ & $\begin{array}{l}7,301 \\
7,668 \\
7,796 \\
8,064 \\
8,139 \\
8,288 \\
8,496 \\
8,233 \\
8,023 \\
8,141 \\
7,965 \\
8,065 \\
8,017\end{array}$ & $\begin{array}{r}208 \\
204 \\
200 \\
197 \\
202 \\
204 \\
190 \\
187 \\
198 \\
200 \\
203 \\
210 \\
-\end{array}$ & $\begin{array}{r}165 \\
162 \\
154 \\
152 \\
158 \\
164 \\
151 \\
150 \\
158 \\
158 \\
162 \\
166 \\
-\end{array}$ & $\begin{array}{r}13 \\
13 \\
14 \\
13 \\
13 \\
12 \\
13 \\
13 \\
13 \\
12 \\
12 \\
12 \\
-\end{array}$ \\
\hline
\end{tabular}

a Stocks are totals as of end of period.

b Beginning in 1993, motor gasoline production and product supplied includes blending of fuel ethanol and an adjustment to correct for the imbalance of motor gasoline blending components. Refer to Appendix B. Explanatory Note 10 for 1992 new basis product supplied.

c Beginning in 1981, excludes blending components.

d A negative number indicates a decrease in stocks and a positive number indicates an increase.

- Includes motor gasoline blending components but excludes stocks of oxygenates.

f In January 1981 and 1983 , numerous respondents were added to surveys affecting stocks reported and stock change calculations. Stock changes are calculated using new basis stock levels. See Summary Statistics Explanatory Note 2.

Notes: - Geographic coverage is the 50 States and the District of Columbia. - Totals may not equal sum of components due to independent rounding.

Source: See Summary Statistics Table and Figure Sources. 
Figure S7. Distillate Fuel Oil Supply and Disposition, 1981 - Present

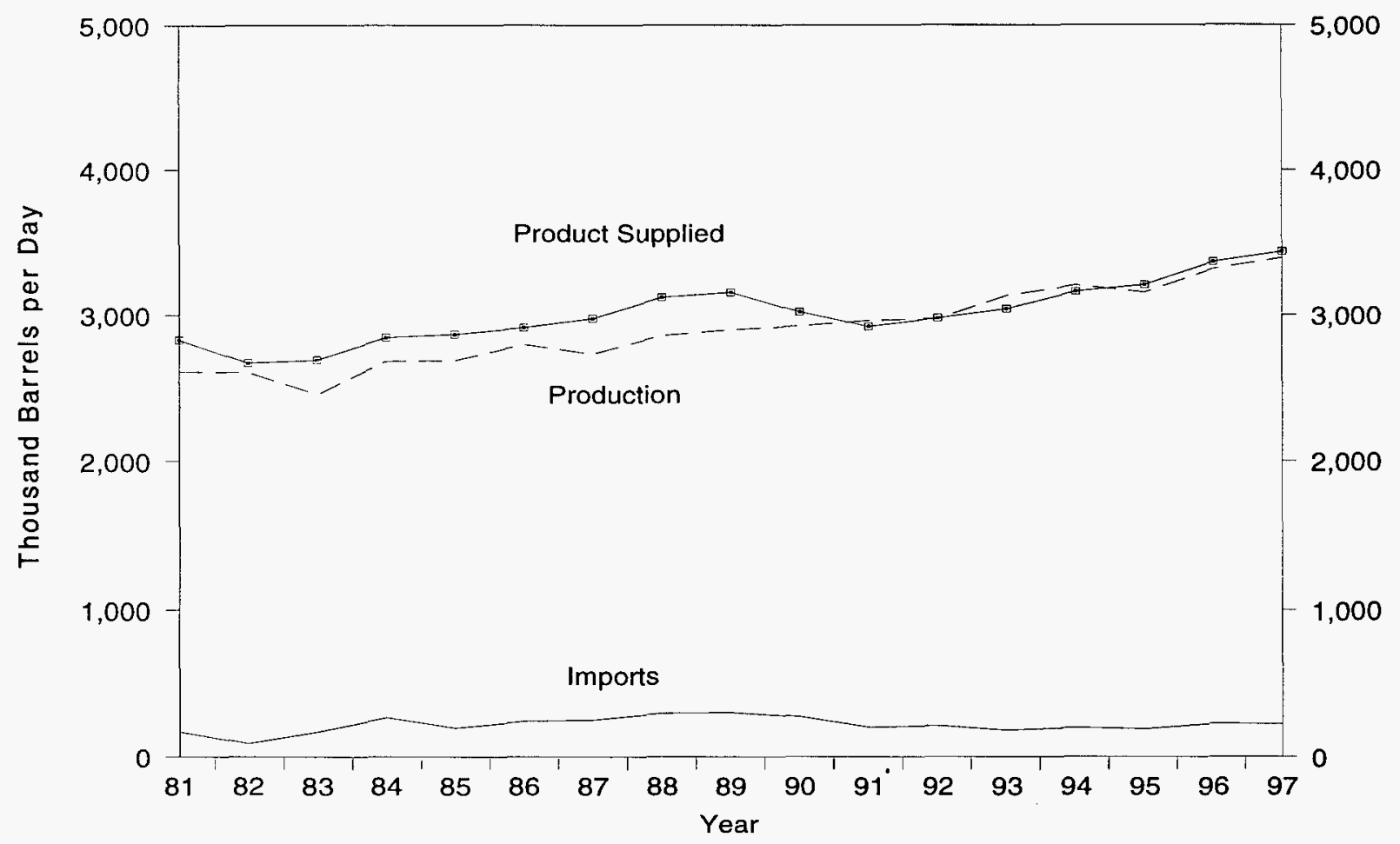

Source: Energy Information Administration, Petroleum Supply Annual, Table S5. See Summary Statistics Table and Figure Sources.

Figure S8. Distillate Fuel Oil Ending Stocks, 1981 - Present

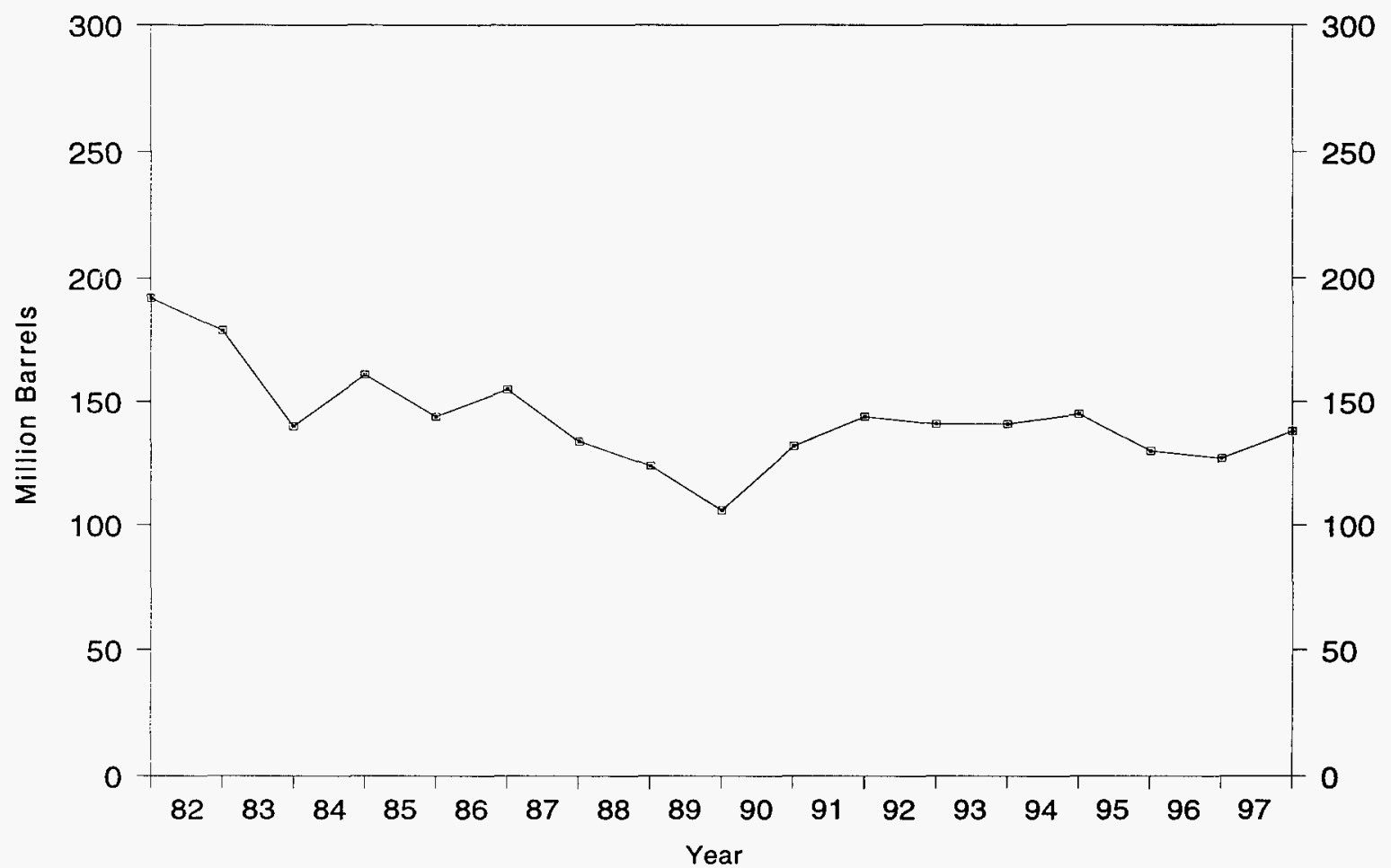

Source: Energy Information Administration, Petroleum Supply Annual, Table S5. See Summary Statistics Table and Figure Sources. 
Table S5. Distillate Fuel Oil Supply and Disposition, 1981 - Present (Thousand Barrels per Day, Except Where Noted)

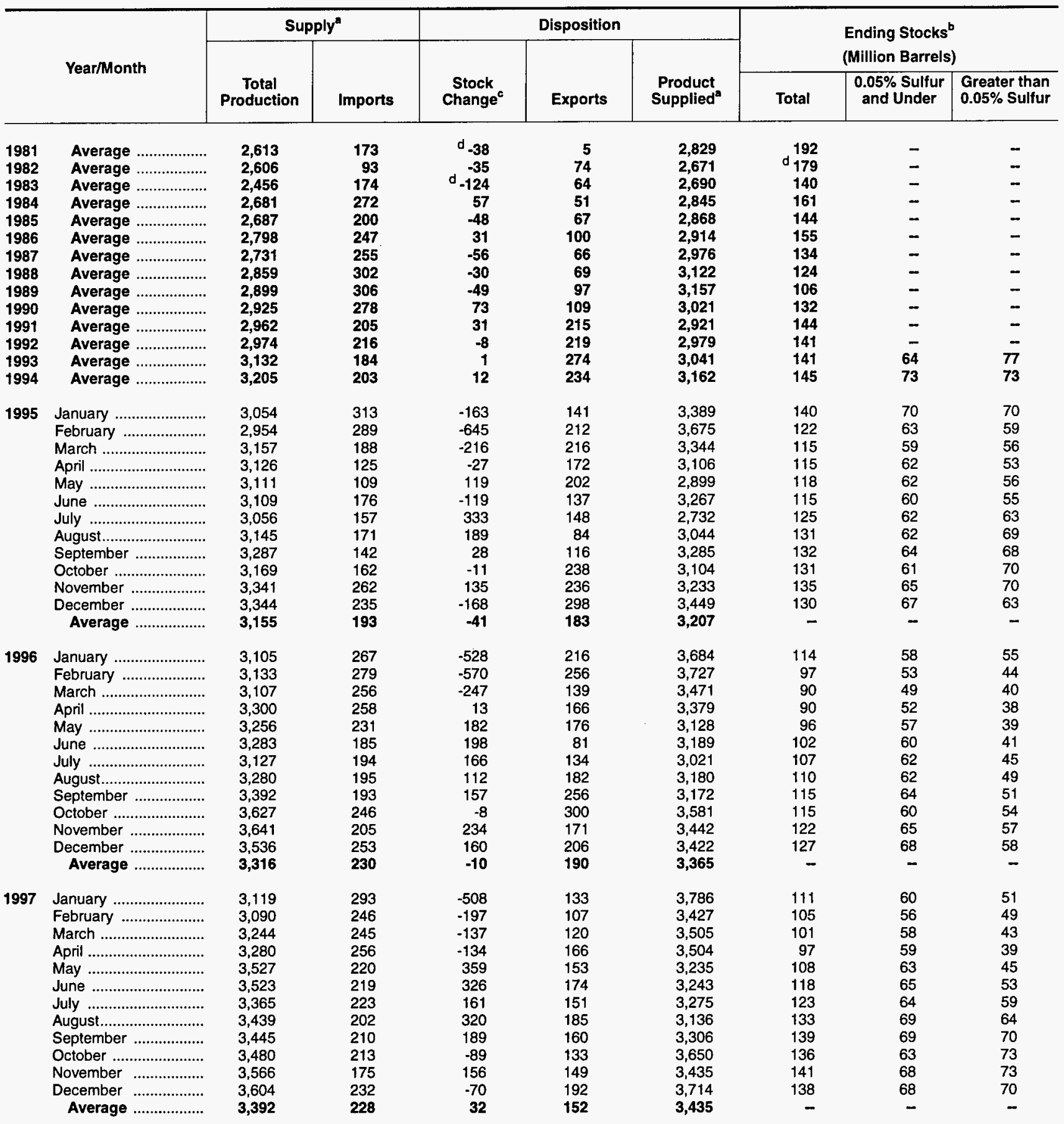

a Excludes 10,000 barrels per day in 1981 and 1982 previously published as crude used directly.

b Stocks are totals as of end of period.

c A negative number indicates a decrease in stocks and a positive number indicates an increase.

d In January 1981 and 1983, numerous respondents were added to surveys affecting stocks reported and stock change calculations. Stock changes are calculated using new stock basis stock levels. See Summary Statistics Explanatory Note 2.

Notes: - Geographic coverage is the $\mathbf{5 0}$ States and the District of Columbia. - Totals may not equal sum of components due to independent rounding. Source: See Summary Statistics Table and Figure Sources. 
Figure S9. Residual Fuel Oil Supply and Disposition, 1981 - Present

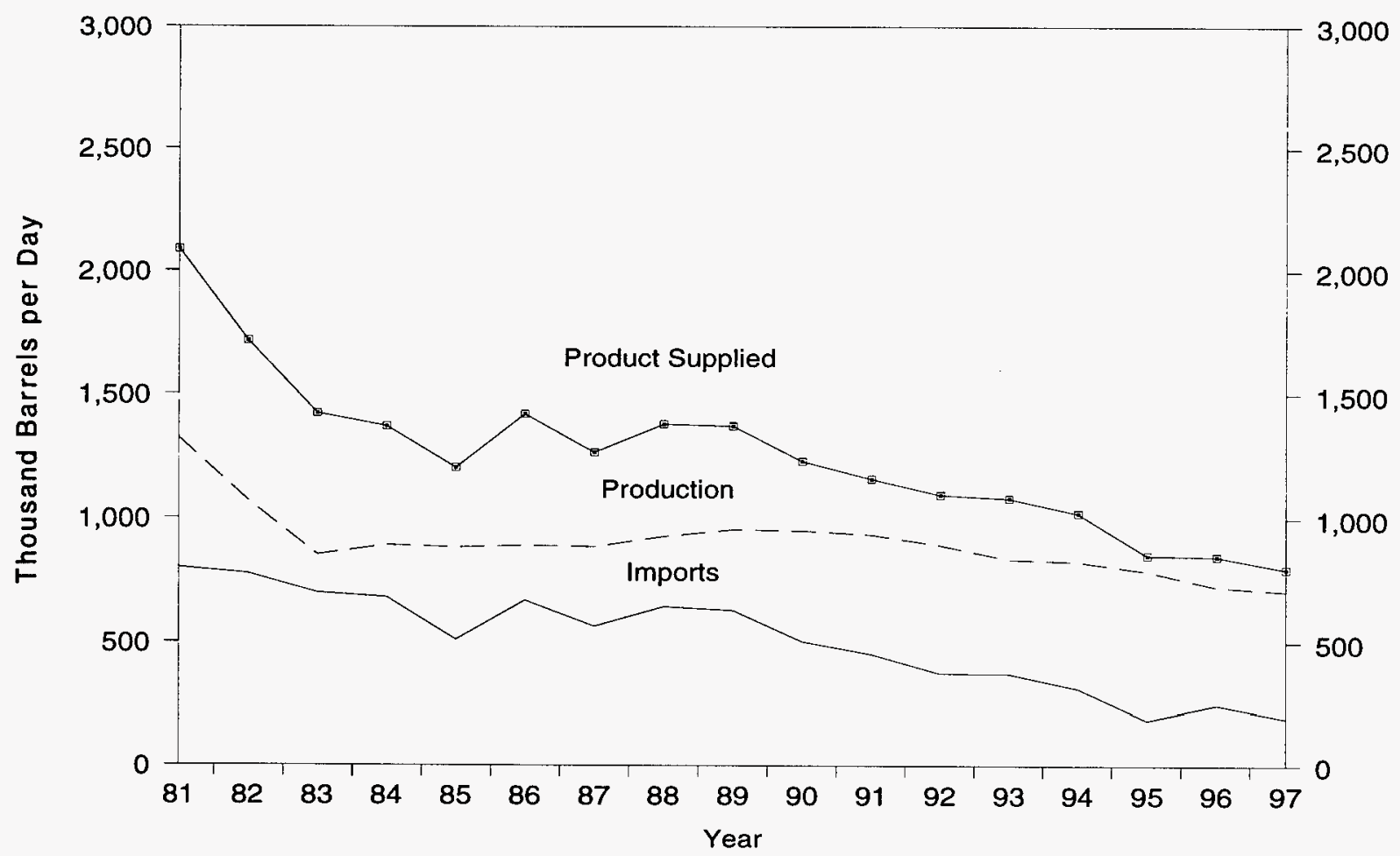

Source: Energy Information Administration, Petroleum Supply Annual, Table S6. See Summary Statistics Table and Figure Sources.

Figure S10. Residual Fuel Oil Ending Stocks, 1981 - Present

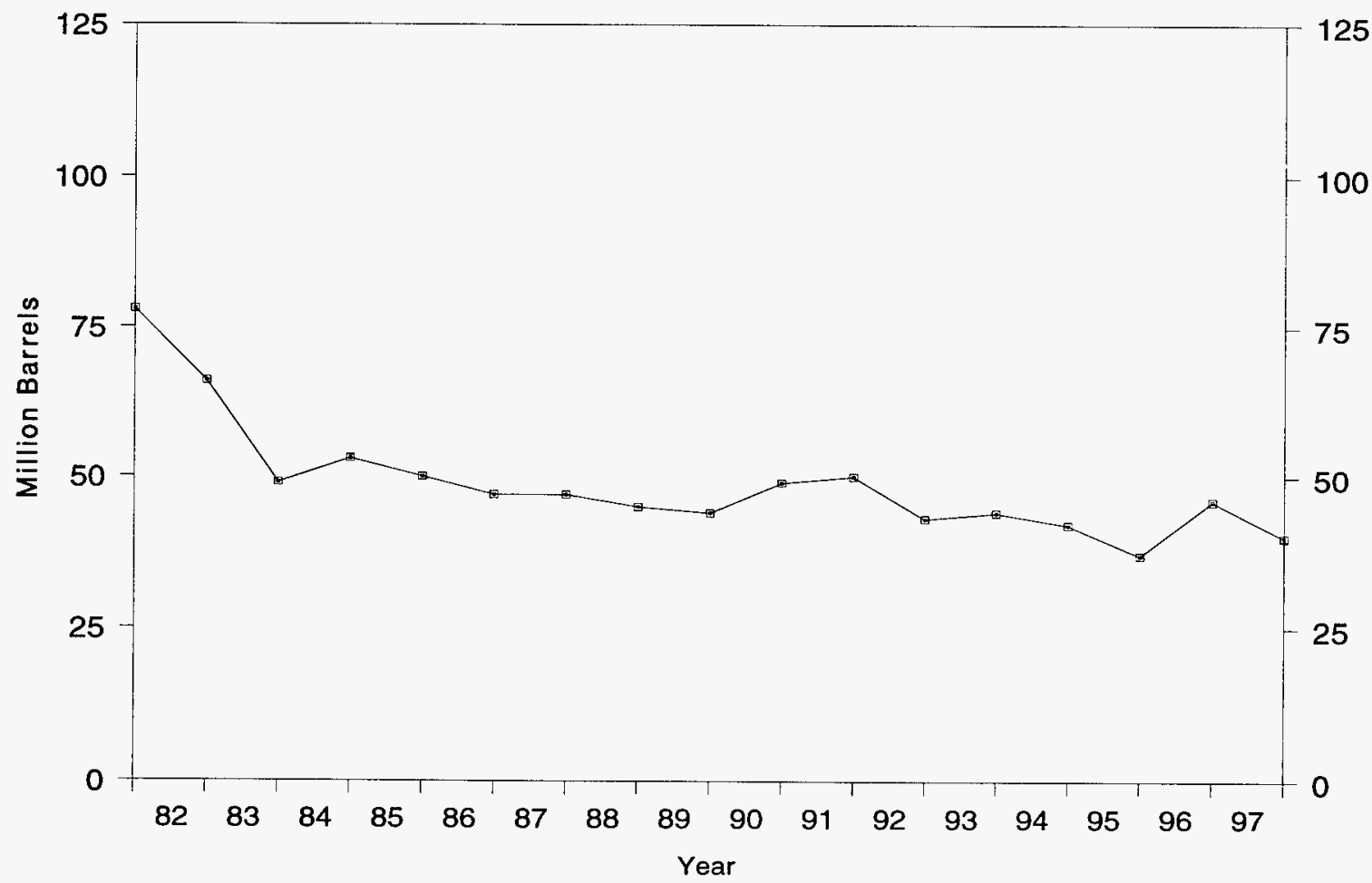

Source: Energy Information Administration, Petroleum Supply Annual, Table S6. See Summary Statistics Table and Figure Sources. 
Table S6. Residual Fuel Oil Supply and Disposition, 1981 - Present (Thousand Barrels per Day, Except Where Noted)

\begin{tabular}{|c|c|c|c|c|c|c|c|}
\hline \multirow{2}{*}{\multicolumn{2}{|c|}{ Year/Month }} & \multicolumn{2}{|c|}{ Supply } & \multicolumn{3}{|c|}{ Disposition } & \multirow[b]{2}{*}{$\begin{array}{c}\text { Ending } \\
\text { Stocks } \\
\text { (Million Barrels) }\end{array}$} \\
\hline & & $\begin{array}{c}\text { Total } \\
\text { Production }\end{array}$ & Imports & $\begin{array}{c}\text { Stock } \\
\text { Changeb }\end{array}$ & Exports & $\begin{array}{l}\text { Product } \\
\text { Supplied }^{\mathrm{a}}\end{array}$ & \\
\hline $\begin{array}{l}1981 \\
1982 \\
1983 \\
1984 \\
1985 \\
1986 \\
1987 \\
1988 \\
1989 \\
1990 \\
1991 \\
1992 \\
1993 \\
1994\end{array}$ & 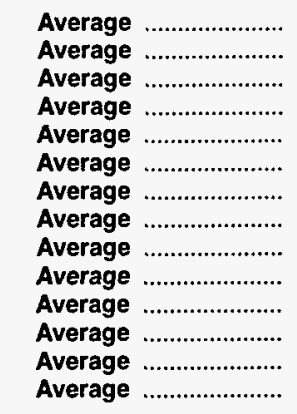 & $\begin{array}{r}1,321 \\
1,070 \\
852 \\
891 \\
882 \\
889 \\
885 \\
926 \\
954 \\
950 \\
934 \\
892 \\
835 \\
826\end{array}$ & $\begin{array}{l}800 \\
776 \\
699 \\
681 \\
510 \\
669 \\
565 \\
644 \\
629 \\
504 \\
453 \\
375 \\
373 \\
314\end{array}$ & $\begin{array}{r}d-37 \\
-32 \\
d-55 \\
12 \\
-7 \\
-8 \\
(s) \\
-8 \\
-2 \\
13 \\
4 \\
-20 \\
4 \\
-6\end{array}$ & $\begin{array}{l}118 \\
209 \\
185 \\
190 \\
197 \\
147 \\
186 \\
200 \\
215 \\
211 \\
226 \\
193 \\
123 \\
125\end{array}$ & $\begin{array}{l}2,088 \\
1,716 \\
1,421 \\
1,369 \\
1,202 \\
1,418 \\
1,264 \\
1,378 \\
1,370 \\
1,229 \\
1,158 \\
1,094 \\
1,080 \\
1,021\end{array}$ & $\begin{array}{r}78 \\
\text { d } 66 \\
49 \\
53 \\
50 \\
47 \\
47 \\
45 \\
44 \\
49 \\
50 \\
43 \\
44 \\
42\end{array}$ \\
\hline 1995 & 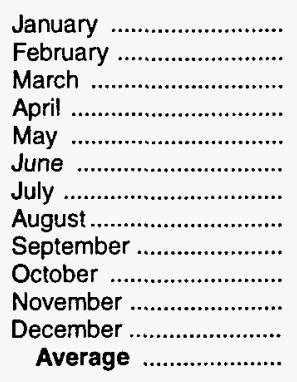 & $\begin{array}{l}903 \\
776 \\
778 \\
789 \\
748 \\
746 \\
797 \\
801 \\
811 \\
724 \\
705 \\
874 \\
788\end{array}$ & $\begin{array}{l}204 \\
225 \\
209 \\
128 \\
177 \\
184 \\
149 \\
177 \\
220 \\
131 \\
182 \\
257 \\
187\end{array}$ & $\begin{array}{r}56 \\
-246 \\
35 \\
-22 \\
48 \\
-87 \\
27 \\
36 \\
58 \\
-55 \\
-17 \\
-8 \\
-13\end{array}$ & $\begin{array}{r}203 \\
208 \\
154 \\
129 \\
115 \\
120 \\
164 \\
122 \\
124 \\
84 \\
111 \\
98 \\
136\end{array}$ & $\begin{array}{r}848 \\
1,040 \\
798 \\
810 \\
762 \\
896 \\
755 \\
820 \\
848 \\
825 \\
793 \\
1,040 \\
852\end{array}$ & $\begin{array}{l}44 \\
37 \\
38 \\
37 \\
39 \\
36 \\
37 \\
38 \\
40 \\
38 \\
37 \\
37 \\
-\end{array}$ \\
\hline 1996 & 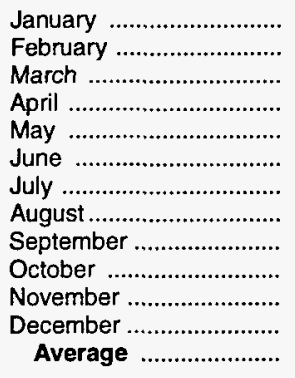 & $\begin{array}{l}799 \\
798 \\
700 \\
671 \\
732 \\
731 \\
646 \\
732 \\
713 \\
694 \\
714 \\
778 \\
726\end{array}$ & $\begin{array}{l}320 \\
222 \\
227 \\
237 \\
203 \\
168 \\
335 \\
227 \\
197 \\
260 \\
270 \\
307 \\
248\end{array}$ & $\begin{array}{r}-54 \\
-132 \\
-4 \\
69 \\
18 \\
21 \\
-3 \\
32 \\
68 \\
16 \\
139 \\
112 \\
24\end{array}$ & $\begin{array}{r}108 \\
114 \\
95 \\
96 \\
89 \\
144 \\
88 \\
56 \\
125 \\
104 \\
101 \\
102 \\
102\end{array}$ & $\begin{array}{r}1,064 \\
1,038 \\
836 \\
743 \\
827 \\
735 \\
896 \\
871 \\
717 \\
835 \\
744 \\
872 \\
848\end{array}$ & $\begin{array}{l}36 \\
32 \\
32 \\
34 \\
34 \\
35 \\
35 \\
36 \\
38 \\
38 \\
42 \\
46 \\
-\end{array}$ \\
\hline 1997 & 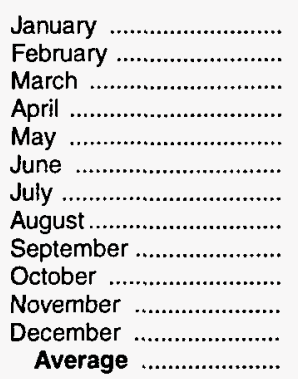 & $\begin{array}{l}801 \\
795 \\
638 \\
617 \\
618 \\
727 \\
643 \\
644 \\
687 \\
723 \\
789 \\
818 \\
708\end{array}$ & $\begin{array}{l}211 \\
253 \\
239 \\
250 \\
175 \\
168 \\
177 \\
187 \\
146 \\
158 \\
204 \\
167 \\
194\end{array}$ & $\begin{array}{r}-131 \\
-66 \\
46 \\
-29 \\
-44 \\
(s) \\
-119 \\
31 \\
-54 \\
41 \\
61 \\
83 \\
-15\end{array}$ & $\begin{array}{r}171 \\
137 \\
89 \\
105 \\
102 \\
130 \\
159 \\
80 \\
91 \\
133 \\
122 \\
120 \\
120\end{array}$ & $\begin{array}{l}972 \\
977 \\
742 \\
791 \\
736 \\
765 \\
781 \\
720 \\
797 \\
707 \\
809 \\
781 \\
797\end{array}$ & $\begin{array}{l}42 \\
40 \\
41 \\
41 \\
39 \\
39 \\
35 \\
36 \\
35 \\
36 \\
38 \\
40 \\
-\end{array}$ \\
\hline
\end{tabular}

a Excludes 48,000 barrels per day in 1981 and 1982 previously published as crude used directly.

b A negative number indicates a decrease in stocks and a positive number indicates an increase.

c Stocks are totals as of end of period.

d In January 1981 and 1983, numerous respondents were added to surveys affecting stocks reported and stock change calculations. Stock changes are calculated using new basis stock levels. See Summary Statistics Explanatory Note 2.

(s)=Less than 500 barrels per day.

Notes: - Geographic coverage is the 50 States and the District of Columbia. $\bullet$ Totals may not equal sum of components due to independent rounding.

Source: See Summary Statistics Table and Figure Sources. 
Figure S11. Jet Fuel Supply and Disposition, 1981 - Present

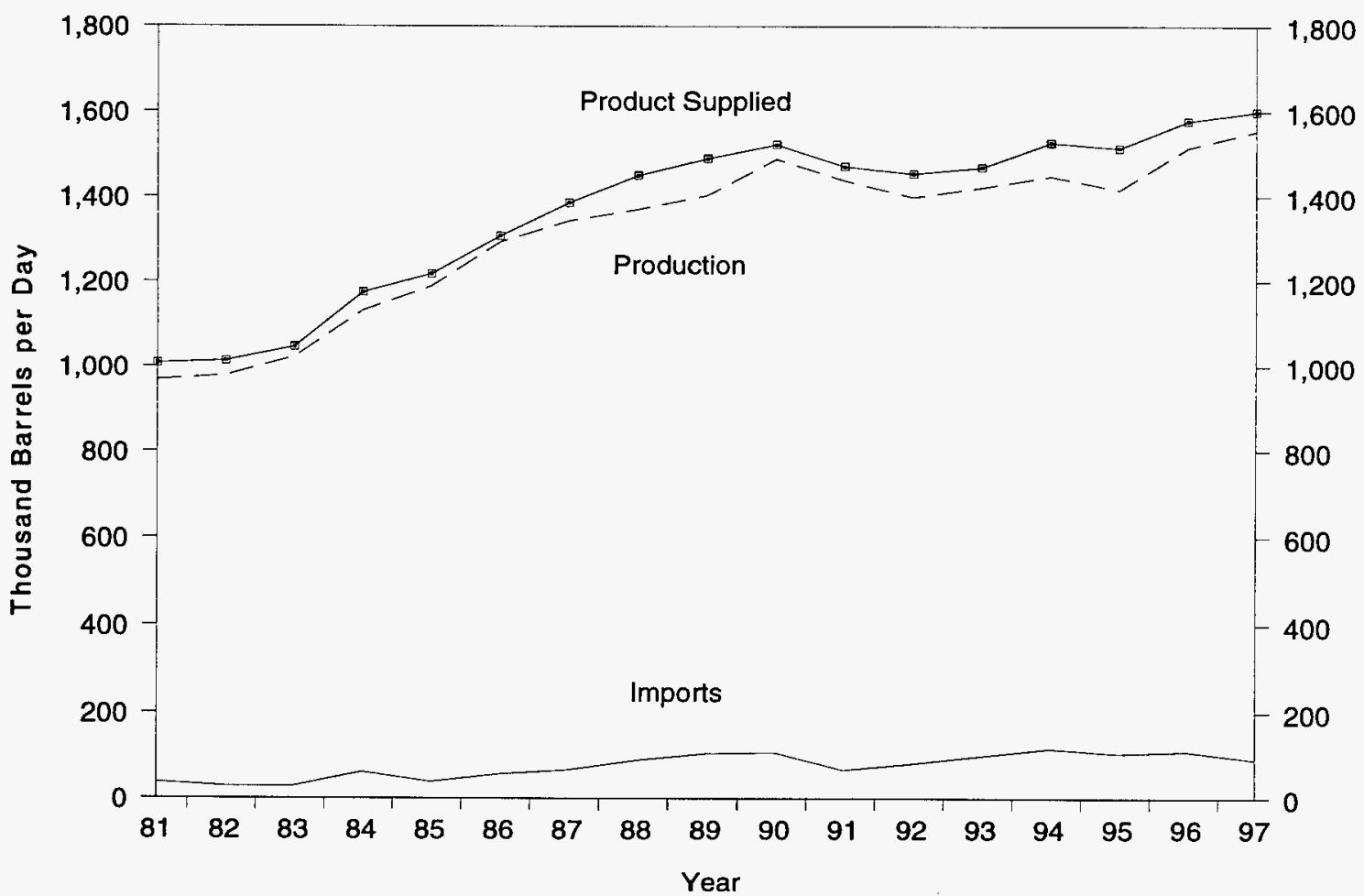

Source: Energy Information Administration, Petroleum Supply Annual, Table S7. See Summary Statistics Table and Figure Sources.

\section{Figure S12. Jet Fuel Ending Stocks, 1981 - Present}

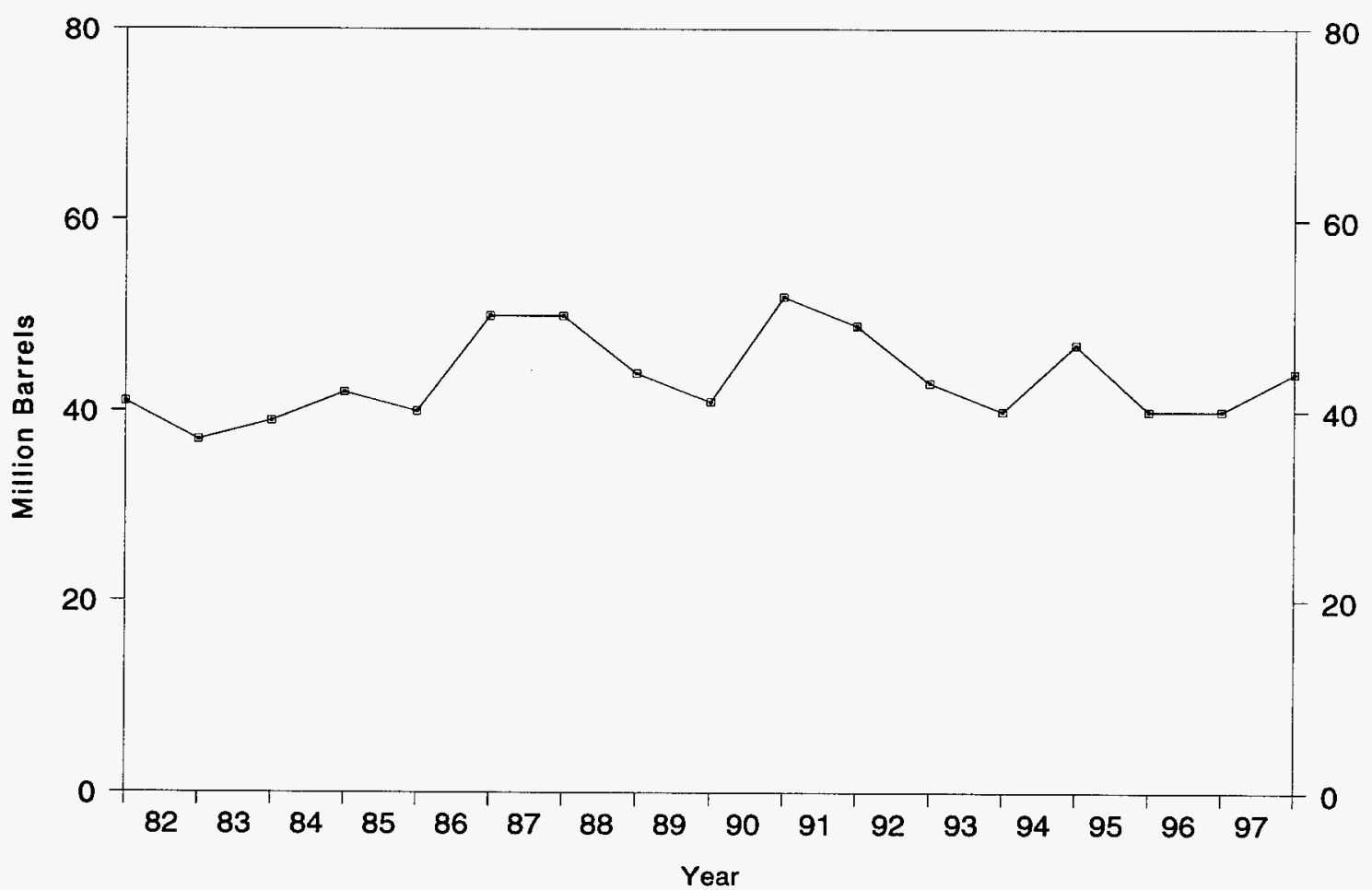

Source: Energy Information Adminstration, Petroleum Supply Annual, Table S7. See Summary Statistics Table and Figure Sources. 
Table S7. Jet Fuel Supply and Disposition, 1981 - Present (Thousand Barrels per Day, Except Where Noted)

\begin{tabular}{|c|c|c|c|c|c|c|c|c|c|c|}
\hline & \multirow[b]{3}{*}{ Year/Month } & \multicolumn{3}{|c|}{ Supply } & \multicolumn{4}{|c|}{ Disposition } & \multicolumn{2}{|c|}{$\begin{array}{l}\text { Ending Stocks } \\
\text { (Million Barrels) }\end{array}$} \\
\hline & & \multicolumn{2}{|c|}{ Production } & \multirow[b]{2}{*}{ Imports } & \multirow[b]{2}{*}{$\begin{array}{l}\text { Stock } \\
\text { Changeb }\end{array}$} & \multirow[b]{2}{*}{ Exports } & \multicolumn{2}{|c|}{ Product Supplied } & \multirow[b]{2}{*}{ Total } & \multirow[b]{2}{*}{$\begin{array}{c}\text { Kerosene } \\
\text { Type }\end{array}$} \\
\hline & & Total & Kerosene-Type & & & & Total & Kerosene-Type & & \\
\hline $\begin{array}{l}1981 \\
1982 \\
1983 \\
1984 \\
1985 \\
1986 \\
1987 \\
1988 \\
1989 \\
1990 \\
1991 \\
1992 \\
1993 \\
1994\end{array}$ & 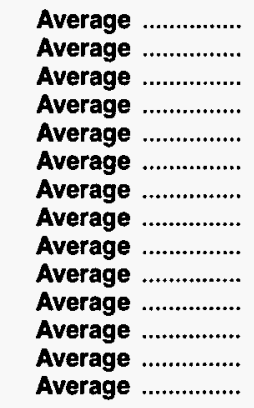 & $\begin{array}{r}968 \\
978 \\
1,022 \\
1,132 \\
1,189 \\
1,293 \\
1,343 \\
1,370 \\
1,403 \\
1,488 \\
1,438 \\
1,399 \\
1,422 \\
1,448\end{array}$ & $\begin{array}{r}775 \\
778 \\
817 \\
919 \\
983 \\
1,097 \\
1,138 \\
1,164 \\
1,197 \\
1,311 \\
1,274 \\
1,254 \\
1,309 \\
1,410\end{array}$ & $\begin{array}{r}38 \\
29 \\
29 \\
62 \\
39 \\
57 \\
67 \\
90 \\
106 \\
108 \\
67 \\
82 \\
100 \\
117\end{array}$ & $\begin{array}{r}c-4 \\
-12 \\
c(s) \\
9 \\
-4 \\
25 \\
(s) \\
-17 \\
-8 \\
31 \\
-9 \\
-16 \\
-7 \\
18\end{array}$ & $\begin{array}{r}2 \\
6 \\
6 \\
9 \\
13 \\
18 \\
24 \\
28 \\
27 \\
43 \\
43 \\
43 \\
59 \\
20\end{array}$ & $\begin{array}{l}1,007 \\
1,013 \\
1,046 \\
1,175 \\
1,218 \\
1,307 \\
1,385 \\
1,449 \\
1,489 \\
1,522 \\
1,471 \\
1,454 \\
1,469 \\
1,527\end{array}$ & $\begin{array}{r}809 \\
804 \\
839 \\
953 \\
1,005 \\
1,105 \\
1,181 \\
1,236 \\
1,284 \\
1,340 \\
1,296 \\
1,310 \\
1,357 \\
1,480\end{array}$ & $\begin{array}{r}\mathbf{4 1} \\
\mathbf{3 7} \\
\mathbf{3 9} \\
\mathbf{4 2} \\
\mathbf{4 0} \\
\mathbf{5 0} \\
50 \\
44 \\
\mathbf{4 1} \\
\mathbf{5 2} \\
\mathbf{4 9} \\
\mathbf{4 3} \\
\mathbf{4 0} \\
\mathbf{4 7}\end{array}$ & $\begin{array}{r}34 \\
c^{31} \\
32 \\
35 \\
34 \\
43 \\
42 \\
38 \\
34 \\
46 \\
44 \\
39 \\
38 \\
46\end{array}$ \\
\hline 1995 & 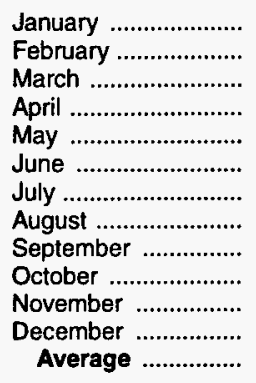 & $\begin{array}{l}1,412 \\
1,375 \\
1,281 \\
1,326 \\
1,367 \\
1,412 \\
1,458 \\
1,427 \\
1,465 \\
1,426 \\
1,496 \\
1,542 \\
1,416\end{array}$ & $\begin{array}{l}1,402 \\
1,366 \\
1,272 \\
1,317 \\
1,354 \\
1,398 \\
1,444 \\
1,418 \\
1,459 \\
1,422 \\
1,493 \\
1,538 \\
1,407\end{array}$ & $\begin{array}{r}79 \\
123 \\
99 \\
82 \\
104 \\
99 \\
97 \\
82 \\
155 \\
99 \\
164 \\
89 \\
106\end{array}$ & $\begin{array}{r}-84 \\
-43 \\
-115 \\
-12 \\
-35 \\
67 \\
23 \\
-23 \\
44 \\
-54 \\
64 \\
-51 \\
-19\end{array}$ & $\begin{array}{r}33 \\
21 \\
17 \\
5 \\
18 \\
11 \\
27 \\
21 \\
20 \\
57 \\
13 \\
63 \\
26\end{array}$ & $\begin{array}{l}1,542 \\
1,520 \\
1,478 \\
1,414 \\
1,487 \\
1,433 \\
1,505 \\
1,511 \\
1,557 \\
1,521 \\
1,584 \\
1,619 \\
1,514\end{array}$ & $\begin{array}{l}1,525 \\
1,514 \\
1,464 \\
1,402 \\
1,478 \\
1,393 \\
1,469 \\
1,505 \\
1,500 \\
1,518 \\
1,578 \\
1,618 \\
1,497\end{array}$ & $\begin{array}{l}44 \\
43 \\
39 \\
39 \\
38 \\
40 \\
41 \\
40 \\
41 \\
40 \\
42 \\
40 \\
-\end{array}$ & $\begin{array}{r}43 \\
42 \\
39 \\
38 \\
37 \\
39 \\
40 \\
39 \\
41 \\
39 \\
41 \\
39 \\
-\end{array}$ \\
\hline 1996 & 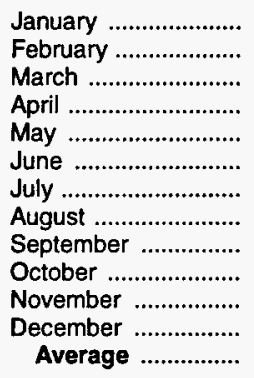 & $\begin{array}{l}1,596 \\
1,499 \\
1,470 \\
1,466 \\
1,419 \\
1,514 \\
1,496 \\
1,510 \\
1,650 \\
1,485 \\
1,501 \\
1,575 \\
1,515\end{array}$ & $\begin{array}{l}1,593 \\
1,495 \\
1,468 \\
1,464 \\
1,418 \\
1,512 \\
1,493 \\
1,507 \\
1,647 \\
1,484 \\
1,500 \\
1,574 \\
1,513\end{array}$ & $\begin{array}{r}89 \\
100 \\
105 \\
113 \\
122 \\
127 \\
89 \\
104 \\
159 \\
126 \\
87 \\
110 \\
111\end{array}$ & $\begin{array}{r}-49 \\
-129 \\
-24 \\
51 \\
39 \\
71 \\
-14 \\
-2 \\
152 \\
-55 \\
-45 \\
(s) \\
(s)\end{array}$ & $\begin{array}{r}111 \\
67 \\
59 \\
11 \\
13 \\
11 \\
27 \\
34 \\
51 \\
35 \\
45 \\
115 \\
48\end{array}$ & $\begin{array}{l}1,624 \\
1,661 \\
1,541 \\
1,517 \\
1,489 \\
1,558 \\
1,572 \\
1,582 \\
1,606 \\
1,631 \\
1,588 \\
1,570 \\
1,578\end{array}$ & $\begin{array}{l}1,607 \\
1,658 \\
1,547 \\
1,515 \\
1,467 \\
1,556 \\
1,569 \\
1,580 \\
1,604 \\
1,636 \\
1,588 \\
1,573 \\
1,575\end{array}$ & $\begin{array}{l}38 \\
35 \\
34 \\
36 \\
37 \\
39 \\
38 \\
38 \\
43 \\
41 \\
40 \\
40 \\
-\end{array}$ & $\begin{array}{l}38 \\
35 \\
34 \\
35 \\
37 \\
39 \\
38 \\
38 \\
43 \\
41 \\
40 \\
40 \\
-\end{array}$ \\
\hline 1997 & 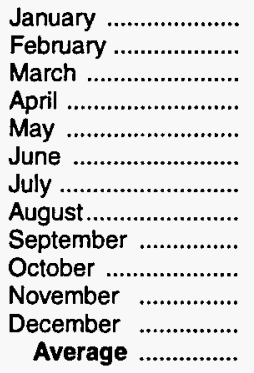 & $\begin{array}{l}1,491 \\
1,511 \\
1,488 \\
1,493 \\
1,515 \\
1,581 \\
1,619 \\
1,580 \\
1,593 \\
1,581 \\
1,609 \\
1,588 \\
1,554\end{array}$ & $\begin{array}{l}1,491 \\
1,510 \\
1,487 \\
1,492 \\
1,514 \\
1,580 \\
1,618 \\
1,579 \\
1,592 \\
1,580 \\
1,608 \\
1,588 \\
1,554\end{array}$ & $\begin{array}{r}100 \\
116 \\
106 \\
98 \\
91 \\
108 \\
86 \\
103 \\
87 \\
77 \\
55 \\
63 \\
91\end{array}$ & $\begin{array}{r}-101 \\
31 \\
55 \\
11 \\
46 \\
77 \\
-14 \\
7 \\
78 \\
19 \\
8 \\
-75 \\
11\end{array}$ & $\begin{array}{r}78 \\
23 \\
11 \\
21 \\
9 \\
38 \\
33 \\
27 \\
16 \\
40 \\
44 \\
78 \\
35\end{array}$ & $\begin{array}{l}1,615 \\
1,572 \\
1,529 \\
1,559 \\
1,551 \\
1,574 \\
1,685 \\
1,648 \\
1,586 \\
1,599 \\
1,612 \\
1,647 \\
1,599\end{array}$ & $\begin{array}{l}1,614 \\
1,571 \\
1,528 \\
1,558 \\
1,551 \\
1,573 \\
1,685 \\
1,648 \\
1,585 \\
1,599 \\
1,612 \\
1,647 \\
1,598\end{array}$ & $\begin{array}{r}37 \\
38 \\
39 \\
40 \\
41 \\
43 \\
43 \\
43 \\
46 \\
46 \\
46 \\
44 \\
-\end{array}$ & $\begin{array}{l}37 \\
38 \\
39 \\
40 \\
41 \\
43 \\
43 \\
43 \\
46 \\
46 \\
46 \\
44 \\
-\end{array}$ \\
\hline
\end{tabular}

a Stocks are totals as of end of period.

A negative number indicates a decrease in stocks and a positive number indicates an increase.

c In January 1981, and 1983, a new stock basis was established affecting stocks reported and stock change calculations. Stock changes are calculated using new basis stock levels. See Summary Statistics Explanatory Note 2.

$(s)=$ Less than 500 barrels per day.

Notes: - Geographic coverage is the 50 States and the District of Columbia. - Totals may not equal sum of components due to independent rounding.

Source: See Summary Statistics Table and Figure Sources. 
Figure S13. Propane/Propylene Supply and Disposition, 1981 - Present

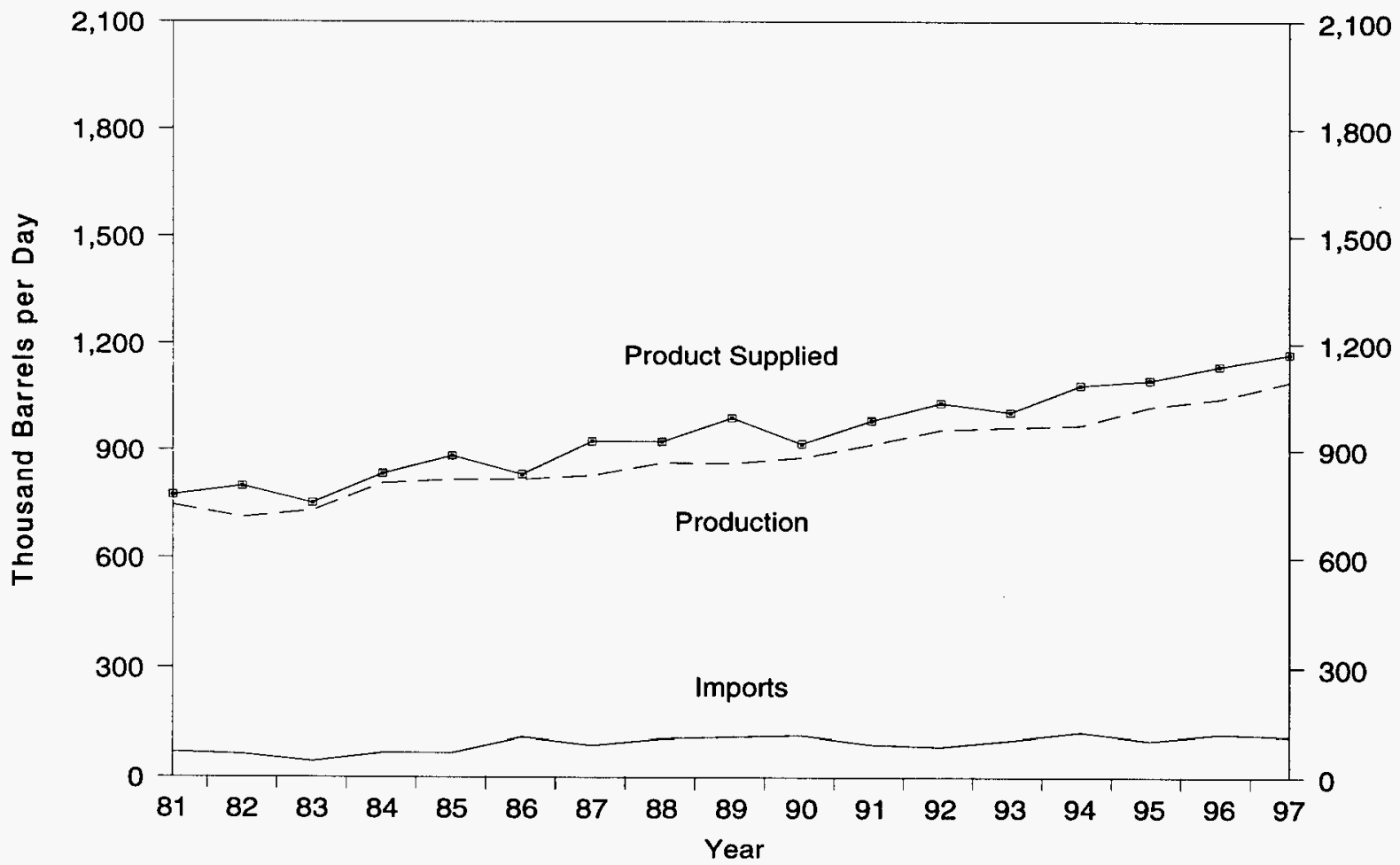

Source: Energy Information Administration, Petroleum Supply Annual, Table S8. See Summary Statistics Table and Figure Sources.

Figure S14. Propane/Propylene Ending Stocks, 1981 - Present

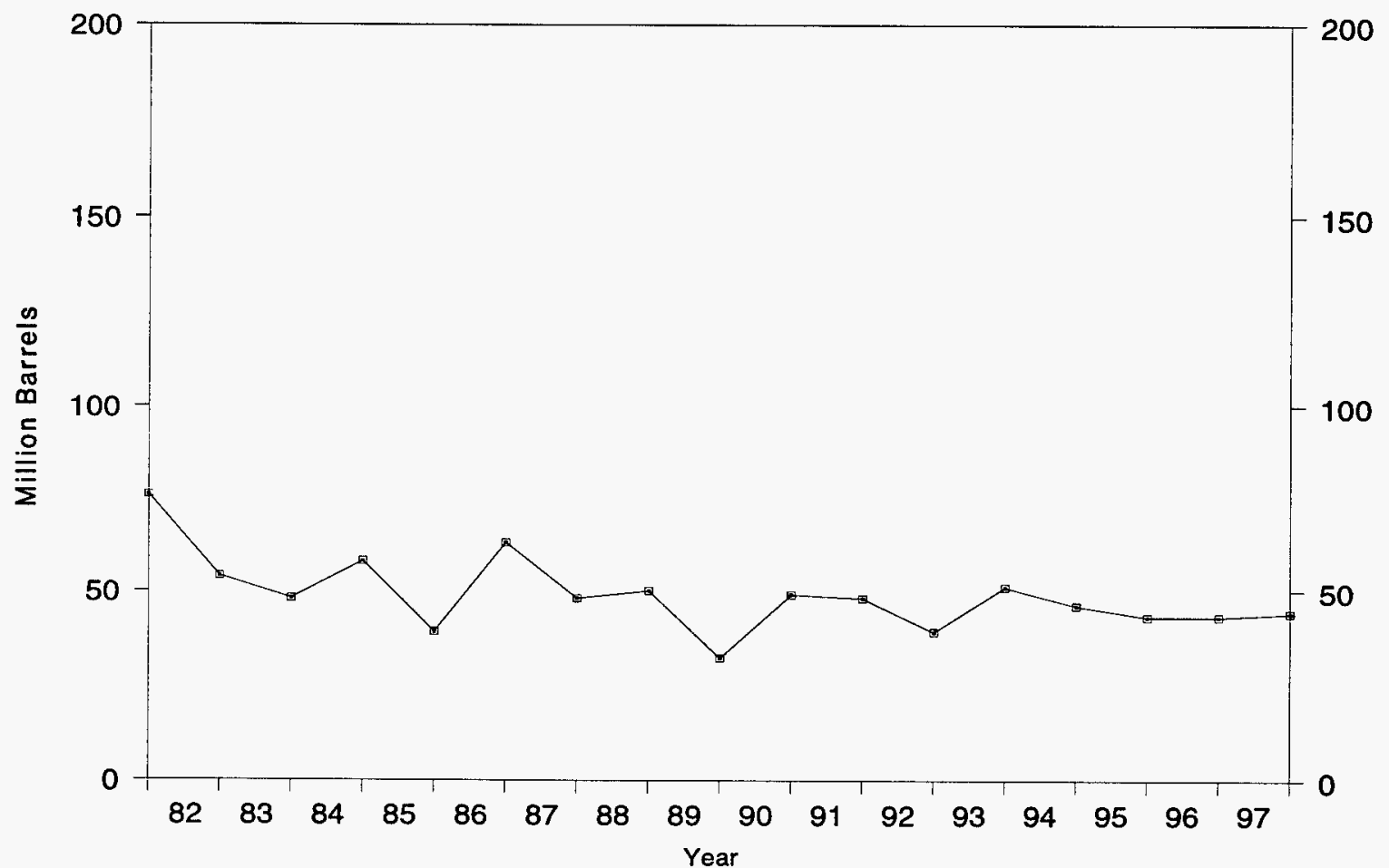

Source: Energy Information Administration, Petroleum Supply Annual, Table S8. See Summary Statistics Table and Figure Sources. 
Table S8. Propane/Propylene Supply and Disposition, 1981 - Present (Thousand Barrels per Day, Except Where Noted)

\begin{tabular}{|c|c|c|c|c|c|c|c|c|}
\hline & \multirow[b]{2}{*}{ Year/Month } & \multicolumn{2}{|c|}{ Supply } & \multicolumn{4}{|c|}{ Disposition } & \multirow[b]{2}{*}{$\begin{array}{c}\text { Ending } \\
\text { Stocks } \\
\text { (Miltion Barrels) }\end{array}$} \\
\hline & & $\begin{array}{c}\text { Total } \\
\text { Production }\end{array}$ & Imports & $\begin{array}{c}\text { Stock } \\
\text { Change }\end{array}$ & $\begin{array}{l}\text { Refinery } \\
\text { Inputs }\end{array}$ & Exports & $\begin{array}{l}\text { Product } \\
\text { Supplied }\end{array}$ & \\
\hline $\begin{array}{l}1981 \\
1982 \\
1983 \\
1984 \\
1985 \\
1986 \\
1987 \\
1988 \\
1989 \\
1990 \\
1991 \\
1992 \\
1993 \\
1994\end{array}$ & 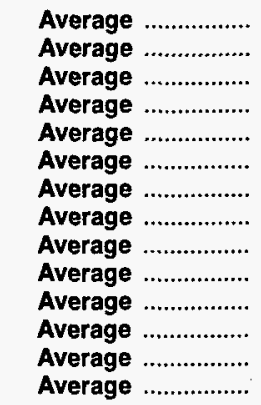 & $\begin{array}{l}745 \\
711 \\
730 \\
806 \\
816 \\
817 \\
828 \\
863 \\
862 \\
878 \\
915 \\
956 \\
963 \\
969\end{array}$ & $\begin{array}{r}70 \\
63 \\
44 \\
67 \\
67 \\
110 \\
88 \\
106 \\
111 \\
115 \\
91 \\
85 \\
103 \\
124\end{array}$ & $\begin{array}{r}c 18 \\
-59 \\
c^{c}-24 \\
c 7 \\
-50 \\
64 \\
-41 \\
7 \\
-52 \\
48 \\
-3 \\
-24 \\
34 \\
-13\end{array}$ & $\begin{array}{r}5 \\
4 \\
4 \\
4 \\
3 \\
4 \\
8 \\
8 \\
11 \\
(s) \\
(s) \\
(s) \\
\text { (s) } \\
0\end{array}$ & $\begin{array}{l}18 \\
31 \\
43 \\
30 \\
48 \\
28 \\
24 \\
31 \\
24 \\
28 \\
28 \\
33 \\
26 \\
24\end{array}$ & $\begin{array}{r}773 \\
798 \\
751 \\
833 \\
883 \\
831 \\
924 \\
923 \\
990 \\
917 \\
982 \\
1,032 \\
1,006 \\
1,082\end{array}$ & $\begin{array}{r}76 \\
c 54 \\
c 58 \\
c^{6} \\
58 \\
39 \\
63 \\
48 \\
50 \\
32 \\
49 \\
48 \\
39 \\
51 \\
46\end{array}$ \\
\hline 1995 & 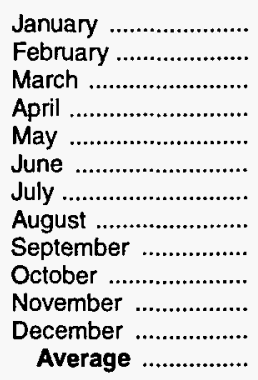 & $\begin{array}{r}1,007 \\
985 \\
1,017 \\
1,040 \\
1,046 \\
1,042 \\
1,011 \\
1,008 \\
1,022 \\
999 \\
1,045 \\
1,033 \\
1,021\end{array}$ & $\begin{array}{r}108 \\
94 \\
90 \\
107 \\
73 \\
114 \\
75 \\
107 \\
146 \\
98 \\
76 \\
135 \\
102\end{array}$ & $\begin{array}{r}-349 \\
-362 \\
14 \\
157 \\
209 \\
188 \\
236 \\
187 \\
45 \\
-22 \\
-160 \\
-285 \\
-10\end{array}$ & $\begin{array}{l}0 \\
0 \\
0 \\
0 \\
0 \\
0 \\
0 \\
0 \\
0 \\
0 \\
0 \\
0 \\
0\end{array}$ & $\begin{array}{r}55 \\
100 \\
39 \\
31 \\
29 \\
27 \\
27 \\
24 \\
25 \\
30 \\
37 \\
31 \\
38\end{array}$ & $\begin{array}{r}1,409 \\
1,341 \\
1,055 \\
958 \\
882 \\
941 \\
823 \\
905 \\
1,098 \\
1,090 \\
1,243 \\
1,422 \\
1,096\end{array}$ & $\begin{array}{l}36 \\
26 \\
26 \\
31 \\
37 \\
43 \\
50 \\
56 \\
57 \\
57 \\
52 \\
43 \\
-\end{array}$ \\
\hline 1996 & 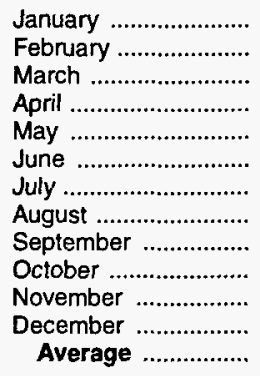 & $\begin{array}{r}995 \\
1,001 \\
1,043 \\
1,047 \\
1,048 \\
1,031 \\
1,043 \\
1,051 \\
1,057 \\
1,058 \\
1,063 \\
1,093 \\
1,044\end{array}$ & $\begin{array}{r}151 \\
106 \\
116 \\
78 \\
104 \\
122 \\
114 \\
126 \\
95 \\
151 \\
147 \\
122 \\
119\end{array}$ & $\begin{array}{r}-353 \\
-347 \\
-1 \\
114 \\
209 \\
293 \\
188 \\
83 \\
97 \\
-37 \\
-148 \\
-106 \\
(s)\end{array}$ & $\begin{array}{l}0 \\
0 \\
0 \\
0 \\
0 \\
0 \\
0 \\
0 \\
0 \\
0 \\
0 \\
0 \\
0\end{array}$ & $\begin{array}{l}30 \\
39 \\
25 \\
31 \\
21 \\
21 \\
29 \\
24 \\
21 \\
29 \\
34 \\
31 \\
28\end{array}$ & $\begin{array}{r}1,468 \\
1,415 \\
1,135 \\
981 \\
922 \\
839 \\
940 \\
1,069 \\
1,034 \\
1,218 \\
1,324 \\
1,289 \\
1,136\end{array}$ & $\begin{array}{r}32 \\
22 \\
22 \\
25 \\
32 \\
41 \\
46 \\
49 \\
52 \\
51 \\
46 \\
43 \\
-\end{array}$ \\
\hline 1997 & 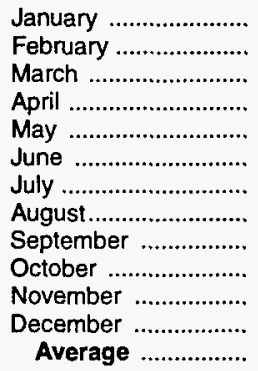 & $\begin{array}{l}1,039 \\
1,044 \\
1,059 \\
1,112 \\
1,114 \\
1,110 \\
1,083 \\
1,095 \\
1,110 \\
1,110 \\
1,099 \\
1,127 \\
1,092\end{array}$ & $\begin{array}{r}149 \\
126 \\
114 \\
109 \\
92 \\
88 \\
87 \\
108 \\
89 \\
122 \\
114 \\
159 \\
113\end{array}$ & $\begin{array}{r}-340 \\
-276 \\
92 \\
150 \\
252 \\
250 \\
231 \\
172 \\
30 \\
17 \\
-223 \\
-342 \\
3\end{array}$ & $\begin{array}{l}0 \\
0 \\
0 \\
0 \\
0 \\
0 \\
0 \\
0 \\
0 \\
0 \\
0 \\
0 \\
0\end{array}$ & $\begin{array}{l}28 \\
42 \\
40 \\
32 \\
23 \\
31 \\
24 \\
24 \\
16 \\
29 \\
48 \\
53 \\
32\end{array}$ & $\begin{array}{r}1,501 \\
1,404 \\
1,041 \\
1,039 \\
930 \\
916 \\
916 \\
1,007 \\
1,152 \\
1,185 \\
1,388 \\
1,576 \\
1,170\end{array}$ & $\begin{array}{l}32 \\
25 \\
28 \\
32 \\
40 \\
47 \\
55 \\
60 \\
61 \\
61 \\
55 \\
44 \\
-\end{array}$ \\
\hline
\end{tabular}

A negative number indicates a decrease in stocks and a positive number indicates an increase.

b tocks are totals as of end of period.

c In January 1981, 1983, and 1984, a new stock basis was established affecting stocks reported and stock change calculations. Stock changes are calculated using new basis stock levels. See Summary Statistics Explanatory Note 2.

(s) = Less than 500 barrels per day.

Notes: - Geographic coverage is the 50 States and the District of Columbia. $\bullet$ Totals may not equal sum of components due to independent rounding.

Source: See Summary Statistics Table and Figure Sources. 
Figure S15. Liquefied Petroleum Gases Supply and Disposition, 1981 - Present

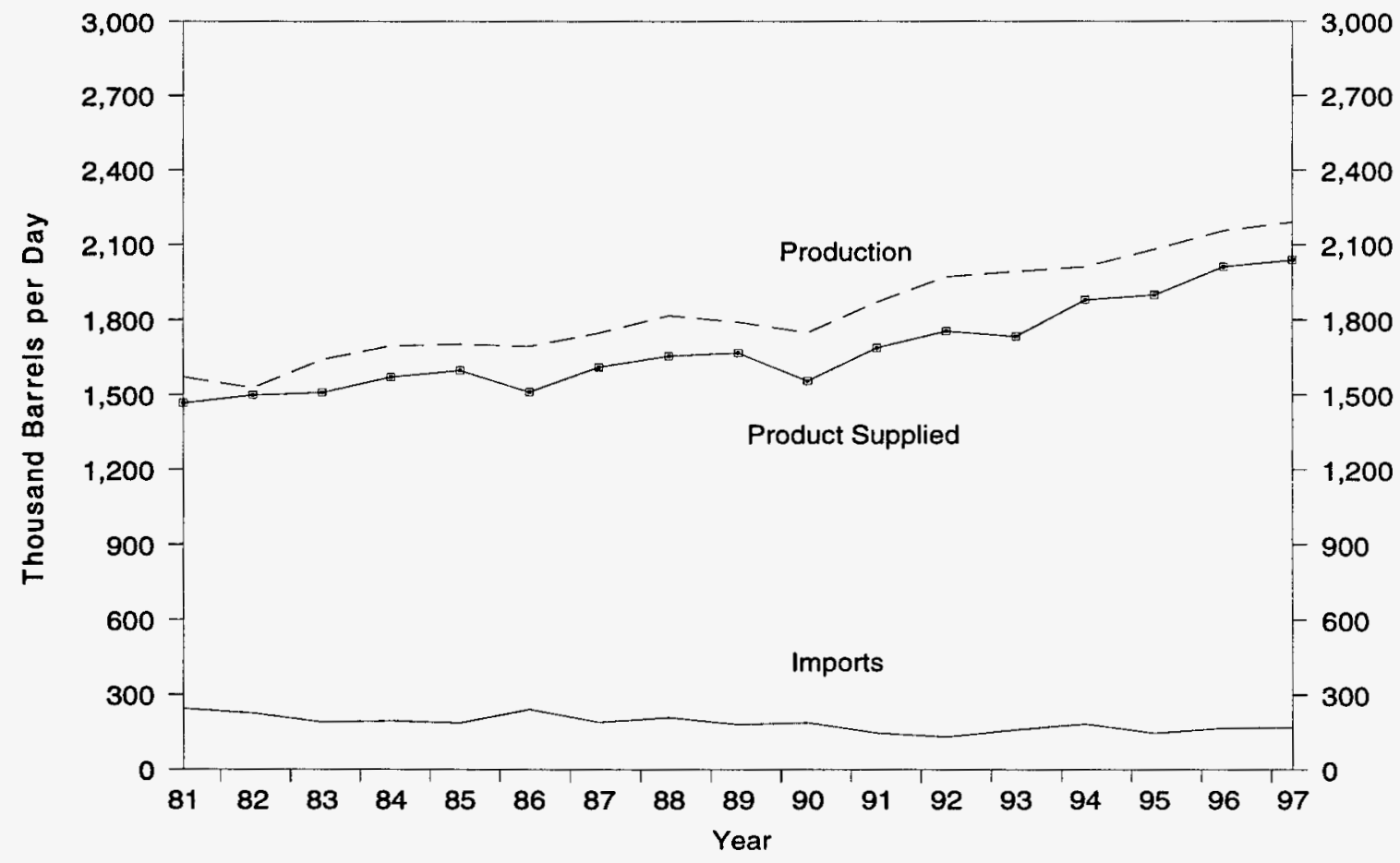

Source: Energy Information Administration, Petroleum Supply Annual, Table S9. See Summary Statistics Table and Figure Sources.

Figure S16. Liquefied Petroleum Gases Ending Stocks, 1981 - Present

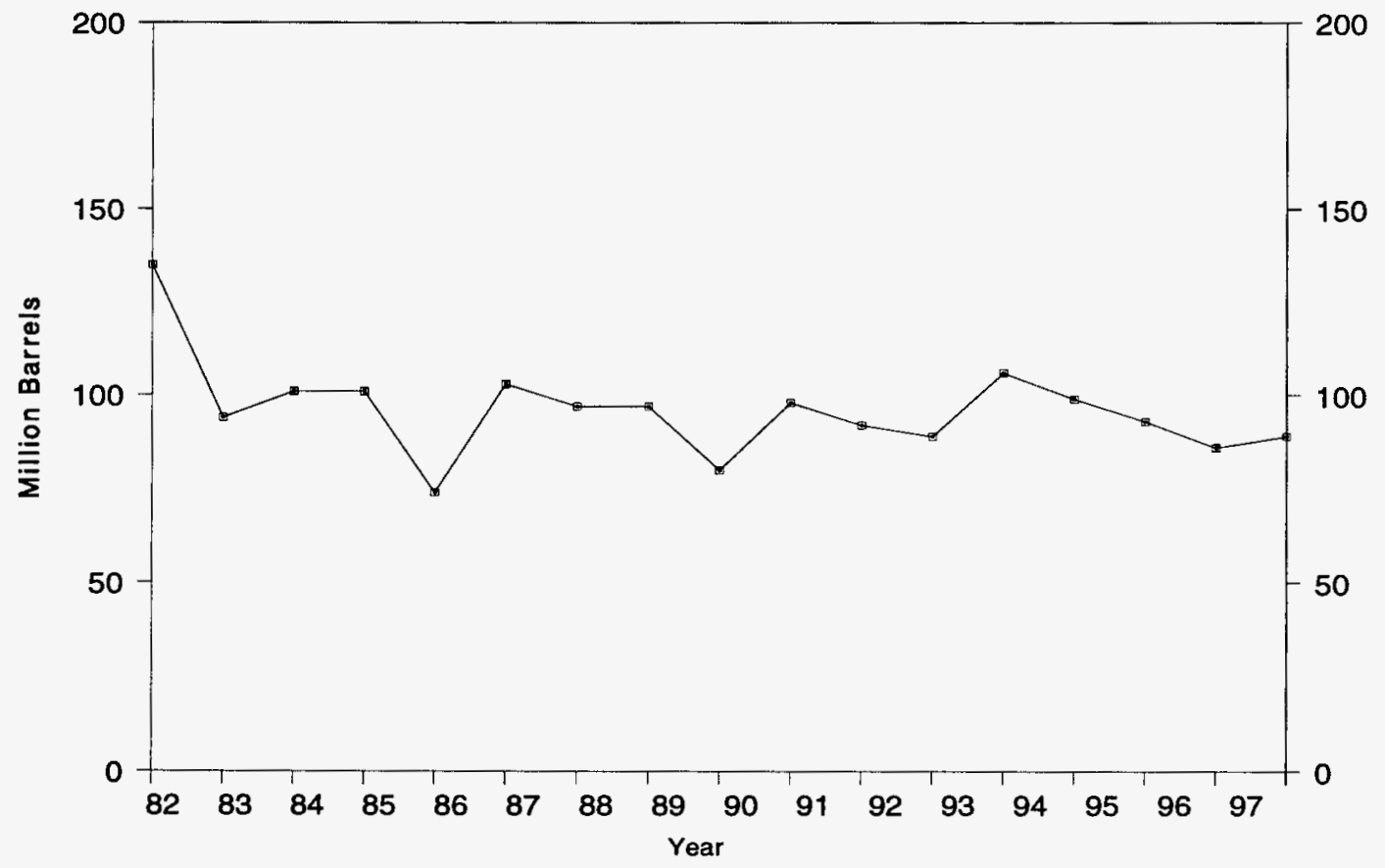

Source: Energy Information Administration, Petroleum Supply Annual, Table S9. See Summary Statistics Table and Figure Sources. 
Table S9. Liquefied Petroleum Gases Supply and Disposition, 1981 - Present (Thousand Barrels per Day, Except Where Noted)

\begin{tabular}{|c|c|c|c|c|c|c|c|c|}
\hline & \multirow[b]{2}{*}{ YearMonth } & \multicolumn{2}{|c|}{ Supply } & \multicolumn{4}{|c|}{ Disposition } & \multirow[b]{2}{*}{$\begin{array}{c}\text { Ending } \\
\text { Stocks } \\
\text { (Million Barrels) }\end{array}$} \\
\hline & & $\begin{array}{c}\text { Total } \\
\text { Production }\end{array}$ & Imports & $\begin{array}{c}\text { Stock } \\
\text { Change }^{a}\end{array}$ & $\begin{array}{l}\text { Refinery } \\
\text { Inputs }\end{array}$ & Exports & $\begin{array}{l}\text { Product } \\
\text { Supplied }\end{array}$ & \\
\hline $\begin{array}{l}1981 \\
1982 \\
1983 \\
1984 \\
1985 \\
1986 \\
1987 \\
1988 \\
1989 \\
1990 \\
1991 \\
1992 \\
1993 \\
1994\end{array}$ & 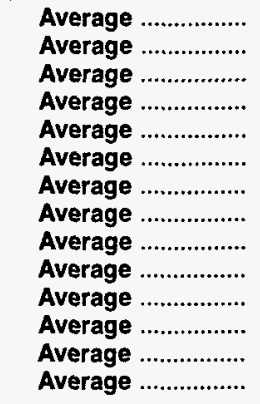 & $\begin{array}{l}1,571 \\
1,528 \\
1,642 \\
1,697 \\
1,704 \\
1,695 \\
1,748 \\
1,817 \\
1,791 \\
1,749 \\
1,871 \\
1,972 \\
1,993 \\
2,012\end{array}$ & $\begin{array}{l}244 \\
226 \\
190 \\
195 \\
187 \\
242 \\
190 \\
209 \\
181 \\
188 \\
147 \\
131 \\
160 \\
183\end{array}$ & $\begin{array}{r}c 18 \\
-111 \\
c-4 \\
c-19 \\
-75 \\
80 \\
-15 \\
1 \\
-47 \\
48 \\
-15 \\
-10 \\
49 \\
-19\end{array}$ & $\begin{array}{l}289 \\
300 \\
253 \\
291 \\
304 \\
302 \\
304 \\
321 \\
315 \\
293 \\
304 \\
309 \\
327 \\
296\end{array}$ & $\begin{array}{l}42 \\
65 \\
73 \\
48 \\
62 \\
42 \\
38 \\
49 \\
35 \\
40 \\
41 \\
49 \\
43 \\
38\end{array}$ & $\begin{array}{l}1,466 \\
1,499 \\
1,509 \\
1,572 \\
1,599 \\
1,512 \\
1,612 \\
1,656 \\
1,668 \\
1,556 \\
1,689 \\
1,755 \\
1,734 \\
1,880\end{array}$ & $\begin{array}{r}135 \\
c 94 \\
c \quad 101 \\
101 \\
74 \\
103 \\
97 \\
97 \\
80 \\
98 \\
92 \\
89 \\
106 \\
99\end{array}$ \\
\hline 1995 & 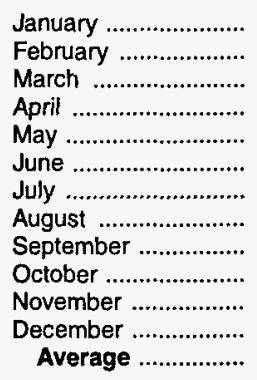 & $\begin{array}{l}1,952 \\
1,969 \\
2,126 \\
2,259 \\
2,269 \\
2,233 \\
2,203 \\
2,178 \\
2,038 \\
1,940 \\
1,943 \\
1,865 \\
2,082\end{array}$ & $\begin{array}{l}172 \\
134 \\
111 \\
147 \\
115 \\
174 \\
124 \\
169 \\
195 \\
130 \\
115 \\
169 \\
146\end{array}$ & $\begin{array}{r}-527 \\
-463 \\
170 \\
307 \\
403 \\
448 \\
488 \\
343 \\
14 \\
-245 \\
-500 \\
-680 \\
-17\end{array}$ & $\begin{array}{l}363 \\
306 \\
247 \\
216 \\
211 \\
198 \\
217 \\
217 \\
300 \\
358 \\
407 \\
424 \\
289\end{array}$ & $\begin{array}{r}64 \\
122 \\
57 \\
43 \\
62 \\
55 \\
41 \\
57 \\
29 \\
35 \\
63 \\
67 \\
58\end{array}$ & $\begin{array}{l}2,225 \\
2,138 \\
1,763 \\
1,841 \\
1,709 \\
1,705 \\
1,581 \\
1,730 \\
1,890 \\
1,921 \\
2,087 \\
2,223 \\
1,899\end{array}$ & $\begin{array}{r}83 \\
70 \\
75 \\
85 \\
97 \\
111 \\
126 \\
136 \\
137 \\
129 \\
114 \\
93 \\
-\end{array}$ \\
\hline 1996 & 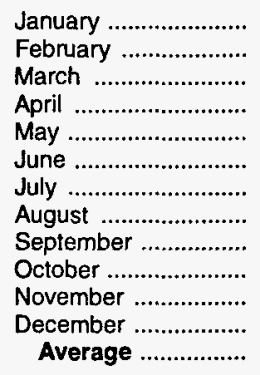 & $\begin{array}{l}1,906 \\
1,912 \\
2,181 \\
2,305 \\
2,287 \\
2,285 \\
2,264 \\
2,271 \\
2,194 \\
2,133 \\
2,041 \\
2,086 \\
2,156\end{array}$ & $\begin{array}{l}208 \\
138 \\
165 \\
122 \\
156 \\
184 \\
182 \\
166 \\
150 \\
183 \\
177 \\
159 \\
166\end{array}$ & $\begin{array}{r}-649 \\
-596 \\
15 \\
279 \\
315 \\
439 \\
385 \\
321 \\
165 \\
-103 \\
-466 \\
-352 \\
-19\end{array}$ & $\begin{array}{l}419 \\
320 \\
246 \\
226 \\
215 \\
211 \\
201 \\
201 \\
260 \\
309 \\
377 \\
355 \\
278\end{array}$ & $\begin{array}{l}49 \\
60 \\
38 \\
56 \\
67 \\
36 \\
72 \\
50 \\
47 \\
37 \\
41 \\
56 \\
51\end{array}$ & $\begin{array}{l}2,295 \\
2,267 \\
2,047 \\
1,867 \\
1,846 \\
1,783 \\
1,787 \\
1,864 \\
1,871 \\
2,073 \\
2,265 \\
2,186 \\
2,012 \\
\end{array}$ & $\begin{array}{r}73 \\
56 \\
56 \\
65 \\
74 \\
87 \\
99 \\
109 \\
114 \\
111 \\
97 \\
86 \\
-\end{array}$ \\
\hline 1997 & 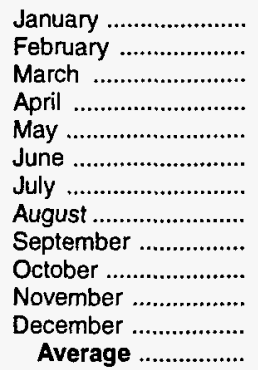 & $\begin{array}{l}2,009 \\
2,072 \\
2,210 \\
2,355 \\
2,364 \\
2,369 \\
2,331 \\
2,348 \\
2,196 \\
2,074 \\
1,926 \\
2,020 \\
2,190\end{array}$ & $\begin{array}{l}193 \\
178 \\
163 \\
169 \\
161 \\
160 \\
151 \\
175 \\
150 \\
168 \\
155 \\
205 \\
169\end{array}$ & $\begin{array}{r}-543 \\
-450 \\
214 \\
349 \\
481 \\
534 \\
433 \\
408 \\
54 \\
-100 \\
-535 \\
-770 \\
9\end{array}$ & $\begin{array}{l}344 \\
321 \\
244 \\
211 \\
200 \\
203 \\
195 \\
190 \\
247 \\
302 \\
345 \\
354 \\
263\end{array}$ & $\begin{array}{l}36 \\
78 \\
62 \\
41 \\
40 \\
43 \\
56 \\
37 \\
29 \\
42 \\
66 \\
74 \\
50\end{array}$ & $\begin{array}{l}2,365 \\
2,301 \\
1,854 \\
1,923 \\
1,804 \\
1,748 \\
1,798 \\
1,888 \\
2,017 \\
1,998 \\
2,206 \\
2,567 \\
2,038\end{array}$ & $\begin{array}{r}69 \\
57 \\
63 \\
74 \\
89 \\
105 \\
118 \\
131 \\
133 \\
129 \\
113 \\
89 \\
-\end{array}$ \\
\hline
\end{tabular}

a A negative number indicates a decrease in stocks and a positive number indicates an increase.

b Stocks are totals as of end of period.

c In January 1981, 1983, and 1984, a new stock basis was established affecting stocks reported and stock change calculations. Stock changes are calculated using new basis stock levels. See Summary Statistics Explanatory Note 2.

Notes: - Liquefied petroleum gases includes ethane/ethylene, propane/propylene, normal butane/butylene, and isobutane/isobutylene. Beginning in January 1984, unfractionated stream is reported by individual product. • Geographic coverage is the 50 States and the District of Columbia. - Totals may not equal sum of components due to independent rounding.

Source: See Summary Statistics Table and Figure Sources. 
Table S10.Other Petroleum Products Supply and Disposition, 1981 - Present (Thousand Barrels per Day, Except Where Noted)

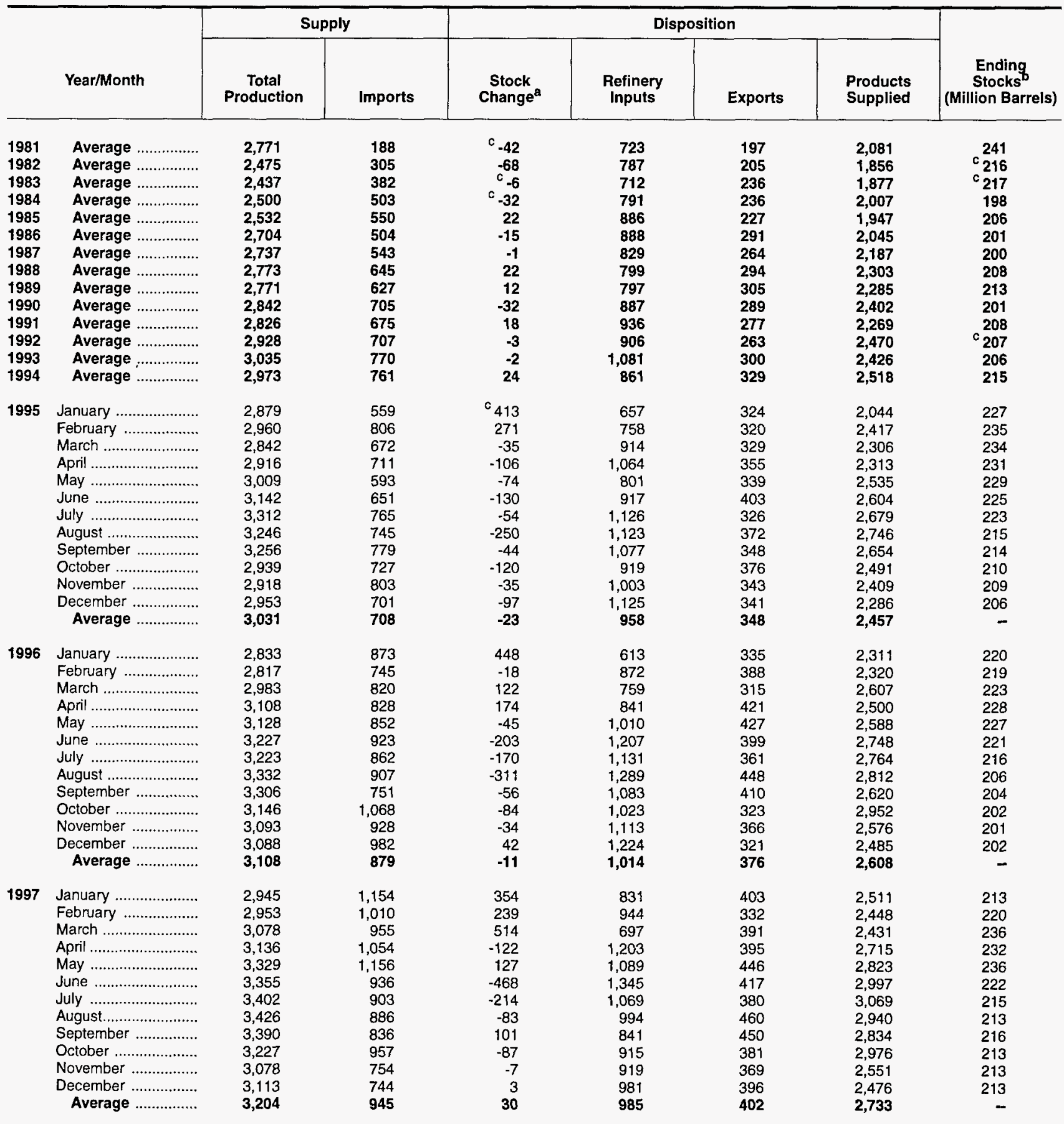

a A negative number indicates a decrease in stocks and a positive number indicates an increase.

b Stocks are totals as of end of period.

c In January 1981, 1983, and 1984, a new stock basis was established affecting stocks reported and stock change calculations. Stock changes are calculated using new basis stock levels. Bulk terminal and pipeline stocks of oxygenates were added beginning in January 1993 . See Summary Statistics Explanatory Note 2.

Notes: - Other petroleum products includes pentanes plus, other hydrocarbons and oxygenates, unfinished oils, gasoline blending components and all finished petroleum products except finished motor gasoline, distillate fuel oil, residual fuel oil, jet fuel, and liquefied petroleum gases, and crude oil product supplied. - Geographic coverage is the 50 States and the District of Columbia. - Totals may not equal sum of components due to independent rounding.

Source: See Summary Statistics Table and Figure Sources. 


\section{Summary Statistics Tables and Figures Sources}

Information about petroleum supply and disposition at the National level are presented in the Summary Statistics tables. Industry terminology and product definitions are listed alphabetically in the Glossary.

The data presented in these tables are from several sources and represent different levels of timeliness and data finality.

- U.S. Department of Energy, Energy Information Administration (EIA), Petroleum Supply Annual (1981 through 1997).

- Data on crude oil production are reported to the EIA by State government agencies. Data on crude oil production for Federal offshore areas are reported to the EIA by the Minerals Management Service of the U.S. Department of the Interior and the Conservation Committee of California Oil Producers. Crude oil production data for 1997 reflect data received as of April 1998. Data for 1997 received after April will be published as an appendix in the following year's Petroleum Supply Annual.

- Data on exports of crude oil and petroleum products are received from the U.S. Bureau of the Census. Export statistics reflect exports of domestic and foreign merchandise from the United States (the 50 States and the District of Columbia) to foreign countries and U.S. possessions. 


\section{Summary Statistics Explanatory Notes}

The following notes are provided to assist in understanding and interpreting the data presented in the Summary Statistics section of this publication.

\section{Note 1. Domestic Crude Oil Production}

The Energy Information Administration (EIA) collects monthly crude oil production data on an ongoing basis. Data on crude oil production for States are reported to the EIA by State government agencies. Data on crude oil production for Federal offshore areas are reported to the EIA by the Minerals Management Service of the U.S. Department of the Interior and the Conservation Committee of California Oil and Gas Producers.

Currently, all except two crude oil producing States (New York and Ohio) report production on a monthly basis. These four States report crude oil on an annual basis. Estimates of monthly crude oil production for these four States are made by the EIA using data reported on Form EIA-182, "Dornestic Crude Oil First Purchase Report."

After the end of each calendar year, the monthly crude oil production estimates are updated using annual reports from various State agencies, the Mineral Management Service, and the Conservation Committee of California Oil and Gas Producers. The EIA incorporates production data into its Crude Oil Production System (COPS) as the data are received from the reporting agencies. Tables S1 and S2 present the 1997 crude oil production data received by the EIA as of April 1998. Crude oil production data for 1997 received after April 1998 will be published later as an appendix in the following year's Petroleum Supply Annual (PSA). Table C1 of this publication presents the 1996 crude oil production a year after it was published in the PSA 1996.

\section{Note 2. Frames Maintenance}

In January 1981 and 1983, numerous respondents were added to bulk terminal and pipeline surveys affecting subsequent stocks reported and stock change calculations. Using the expanded coverage (new basis), the end-of-year stocks, in million barrels, would have been as listed below.

- Crude Oil: 1982- 645 (Total) and 351 (Other Primary).

- Crude Oil and Petroleum Products: 1980- 1,425; and 1982- 1,461 .

- Motor Gasoline: 1980- 263 (Total) and 214 (Finished); 1982- 244 (Total) and 202 (Finished).

- $\quad$ Distillate Fuel Oil: 1980- 205; and 1982- 186.
- $\quad$ Residual Fuel Oil: 1980-91; and 1982- 69.

- Jet Fuel: 1980- 42 (Total) and 36 (Kerosene-type); and 1982-39 (Total) and 32 (Kerosene-type).

- Propane/Propylene: 1980- 69; and 1982- 57.

- Liquefied Petroleum Gases: 1980- 128; and 1982-102.

- Other Petroleum Products: 1980- 207; and 1982-219.

Stock change calculations beginning in 1981 and 1983 were made using new basis stock levels.

Stocks of Alaskan crude oil in-transit were included for the first time in January 1981. The major impact of this change is on the reporting of stock change calculations. Using the expanded coverage (new basis), 1980 end-of-year crude oil stocks would have been 488 million barrels (Total) and 380 million barrels (Other Primary).

Beginning with January 1984, natural gas liquids supply and disposition data were collected on a component basis rather than a product basis. This change affected stocks reported and stock change calculations. Under the new basis, end-of-year 1983 stocks would have been:

- Propane/Propylene: 1983- 55.

- $\quad$ Liquefied Petroleum Gases: 1983- 108.

- Other Petroleum Products: 1983- 210.

In response to changes in the Clean Air Act Amendments of 1990 requiring that all gasoline sold in carbon monoxide nonattainment areas have an oxygen content of 2.7 percent (by weight) during winter months, the Energy Information Administration (EIA) conducted a frame identifier survey in 1991 of companies that produce, blend, store, or import oxygenates. The purpose of this survey was to (1) identify all U.S. producers, blenders, storers, and importers of oxygenates; and (2) collect supply and blending data for 1990 and end of 1990 inventory data on those oxygenates blended into motor gasoline. A summary of the results from the identification survey were published in the Weekly Petroleum Status Report dated February 12, 1992 and in the February 1992 issue of the Petroleum Supply Monthly.

In order to continue to provide relevant information about U.S. and regional gasoline supply, the EIA conducted a second frame identifier survey of these companies during 1992. As a result, a number of respondents were added to the monthly surveys effective in January 1993: 19 blenders, 25 stock holders, and 8 importers. This change did not affect stocks reported and therefore did not cause a new basis stock level to be calculated. 


\section{Detailed Statistics}

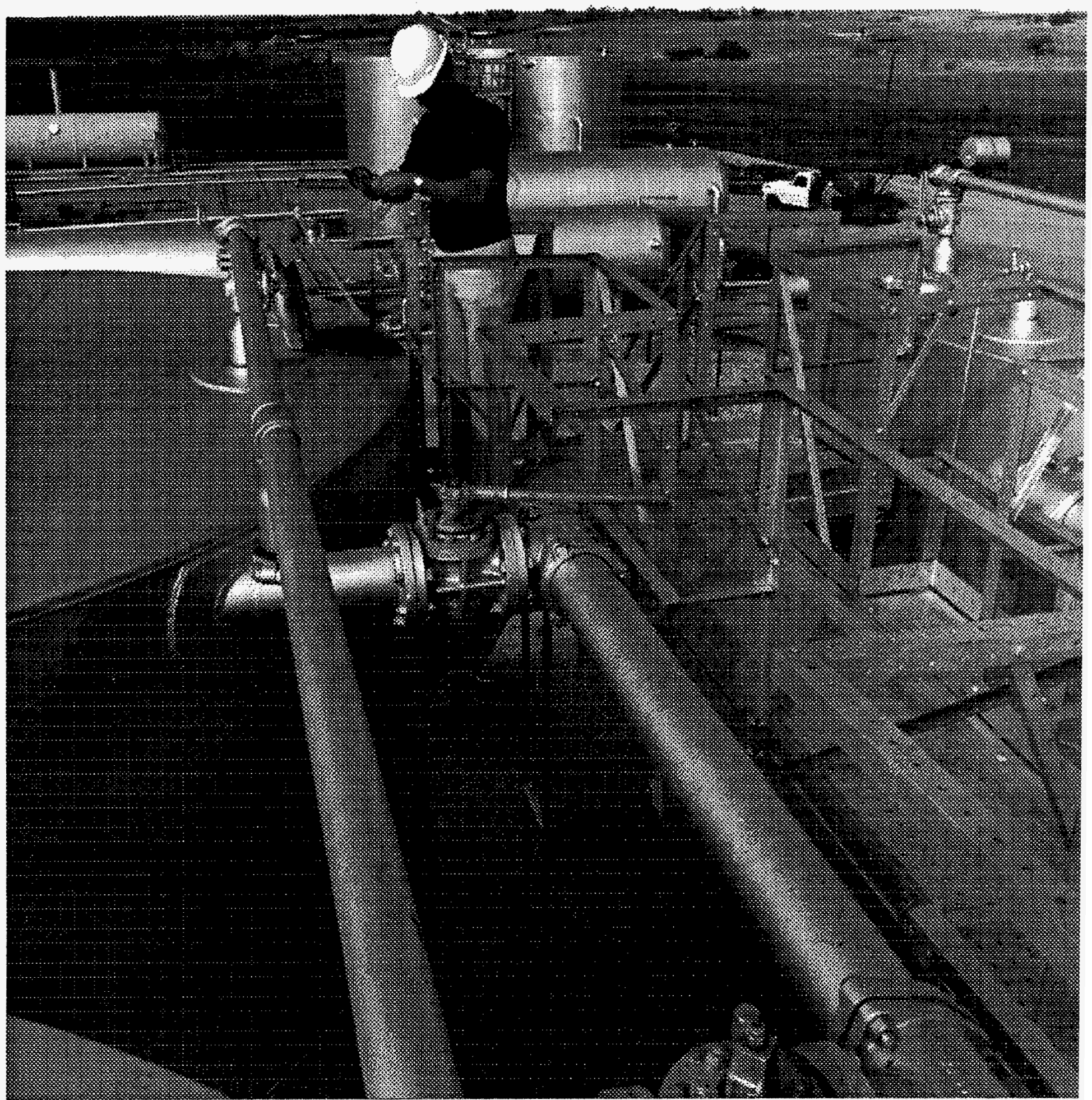




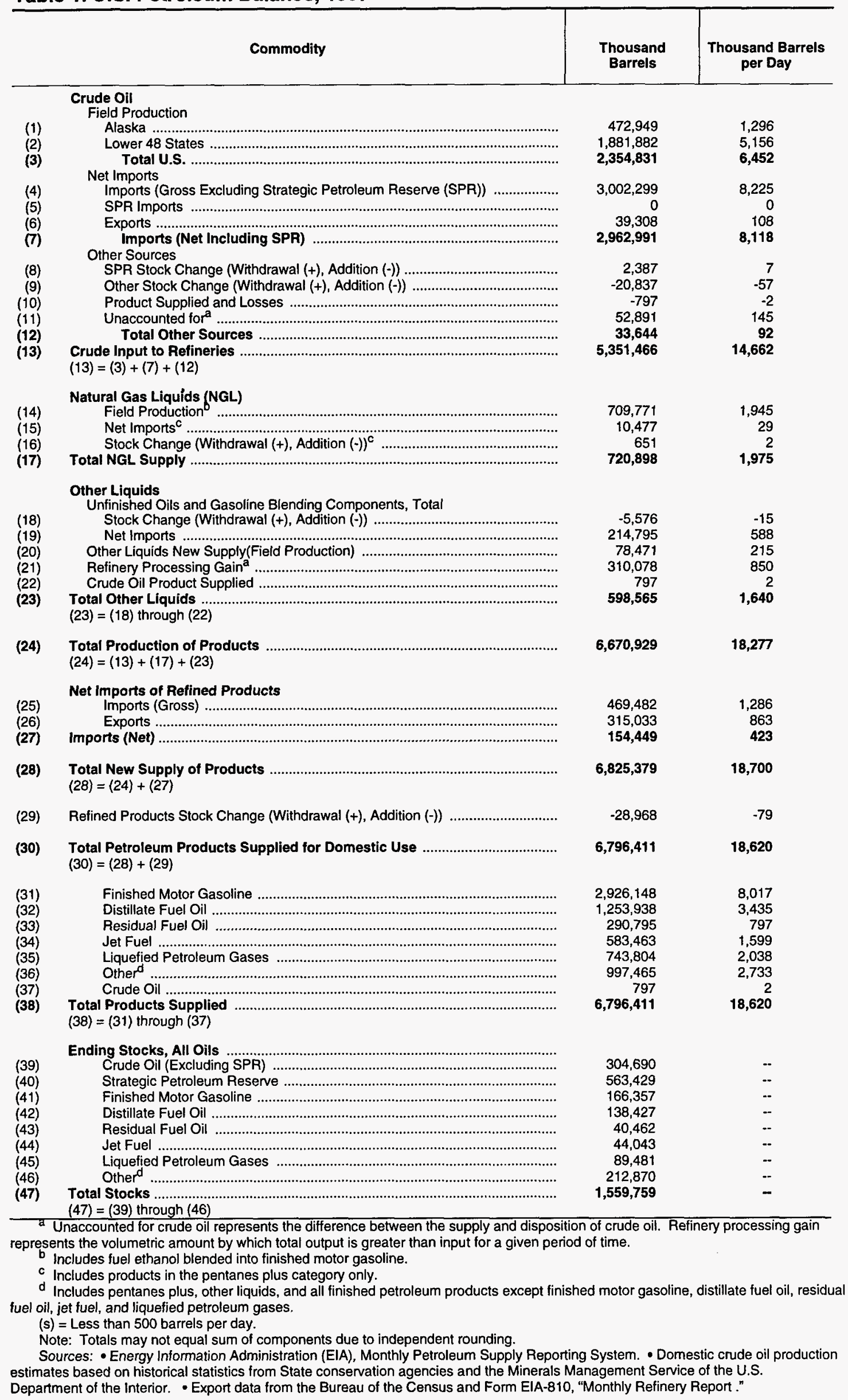


Table 2. U.S. Supply, Disposition, and Ending Stocks of Crude OiI and Petroleum Products, 1997 (Thousand Barrels)

\begin{tabular}{|c|c|c|c|c|c|c|c|c|c|c|}
\hline \multirow[b]{2}{*}{ Commodity } & \multicolumn{4}{|c|}{ Supply } & \multicolumn{5}{|c|}{ Disposition } & \multirow[b]{2}{*}{$\begin{array}{l}\text { Ending } \\
\text { Stocks }\end{array}$} \\
\hline & $\begin{array}{c}\text { Field } \\
\text { Production }\end{array}$ & $\begin{array}{c}\text { Refinery } \\
\text { Production }\end{array}$ & Imports & $\begin{array}{c}\text { Unaccounted } \\
\text { For Crude } \\
\text { Oil }^{\mathbf{a}}\end{array}$ & $\begin{array}{c}\text { Stock } \\
\text { Change }^{b}\end{array}$ & $\begin{array}{c}\text { Crude } \\
\text { Losses }\end{array}$ & $\begin{array}{l}\text { Refinery } \\
\text { Inputs }\end{array}$ & Exports & $\begin{array}{l}\text { Products } \\
\text { Supplied }^{6}\end{array}$ & \\
\hline 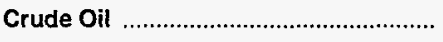 & $2,354,831$ & - & $3,002,299$ & 52,891 & 18,450 & $\mathbf{0}$ & $5,351,466$ & 39,308 & 797 & 868,119 \\
\hline Natural Gas Liquids and LRGs ........... & 663,266 & 252,168 & 74,831 & - & 2,617 & - & 151,769 & 20,882 & 814,997 & 95,196 \\
\hline 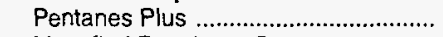 & 116,016 & - & 13,129 & -- & -651 & -- & 55,950 & 2,652 & 71,194 & 5,715 \\
\hline Liquefied Petroleum Gases ................. & 547,250 & 252,168 & 61,702 & -- & 3,268 & -. & 95,819 & 18,229 & 743,804 & 89,481 \\
\hline 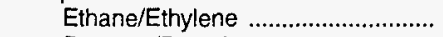 & 232,617 & 9,673 & 5,922 & -- & 1,388 & -- & 0 & 0 & 246,824 & 18,907 \\
\hline 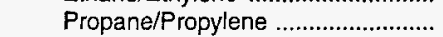 & 192,538 & 206,094 & 41,271 &.- & 1,037 &.- & 0 & 11,861 & 427,005 & 44,045 \\
\hline Normal Butane/Butylene ................ & 52,434 & 32,202 & 8,775 & -- & 381 & -- & 50,472 & 6,368 & 36,190 & 18,373 \\
\hline Isobutane/Isobutylene ..................... & 69,661 & 4,199 & 5,734 & $\cdot-$ & 462 & 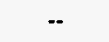 & 45,347 & 0 & 33,785 & 8,156 \\
\hline Other Liquids . & 78,471 & - & 224,060 & - & 5,576 & - & 303,557 & 9,265 & $-15,867$ & 144,817 \\
\hline Other Hydrocarbons/Oxygenates ...... & 106,879 & - & 22,098 & -- & -639 & -- & 125,265 & 4,351 & 0 & 12,493 \\
\hline Unfinished Oils ..................................... & -- & - & 128,814 & -. & 895 & -. & 145,580 & 0 & $-17,661$ & 88,755 \\
\hline Motor Gasoline Blend. Comp. ............. & $-28,409$ & - & 73,148 & - & 5,423 & -- & 34,403 & 4,913 & 0 & 43,418 \\
\hline Aviation Gasoline Blend. Comp. ........... & +- & - & 0 & -- & -103 & -- & $-1,691$ & 0 & 1,794 & 151 \\
\hline Finished Petroleum Products .............. & 46,505 & $5,864,702$ & 407,780 & - & 25,700 & - & - & 296,804 & $5,996,483$ & 451,627 \\
\hline Finished Motor Gasoline ...................... & 46,505 & $2,826,051$ & 112,837 & -- & 9,367 & -- & -- & 49,878 & $2,926,148$ & 166,357 \\
\hline Reformulated ...................................... & -- & 878,200 & 58,641 & -- & 5,100 & - & $\cdots$ & 79 & 931,662 & 42,931 \\
\hline 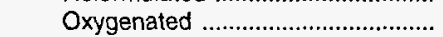 & 180,960 & 33,246 & 0 & -- & -505 &.- & -. & 341 & 214,370 & 1,082 \\
\hline 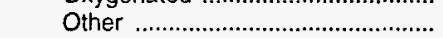 & $-134,455$ & $1,914,605$ & 54,196 & - & 4,772 & -- &.- & 49,457 & $1,780,117$ & 122,344 \\
\hline Finished Aviation Gasoline ................. & $-{ }_{1}$ & 7,248 & 41 & - & -575 & -- & -- & 0 & 7,864 & 1,697 \\
\hline 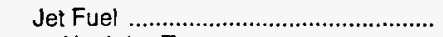 & -- & 567,295 & 33,109 & - & 4,178 & -- & $\cdots$ & 12,763 & 583,463 & 44,043 \\
\hline 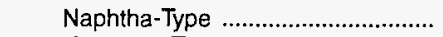 & - & 241 & 0 & - & -52 & -- & -- & 76 & 217 & 34 \\
\hline 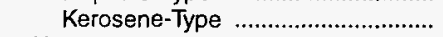 & - & 567,054 & 33,109 & - & 4,230 & - & -- & 12,687 & 583,246 & 44,009 \\
\hline Kerosene & - & 23,887 & 570 & -- & 273 & -- &.- & 138 & 24,046 & 7,294 \\
\hline Distillate Fuel Oil .................................... & -- & $1,238,041$ & 83,102 & -- & 11,698 & -. & -- & 55,507 & $1,253,938$ & 138,427 \\
\hline 0.05 percent sulfur and under ........ & - & 789,287 & 37,599 & -- & -302 & - & - & 15,884 & 811,304 & 68,085 \\
\hline Greater than 0.05 percent sulfur ... & -. & 448,754 & 45,503 & -- & 12,000 & -- & -- & 39,623 & 442,634 & 70,342 \\
\hline 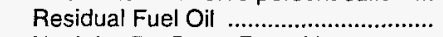 & - & 258,290 & 70,829 & - & $-5,458$ & -- & -- & 43,782 & 290,795 & 40,462 \\
\hline Naphtha For Petro. Feed. Use .............. & - & 83,560 & 18,681 & - & 35 & -- & - & 0 & 102,206 & 1,808 \\
\hline Other Oils For Petro. Feed. Use .......... & -- & 79,539 & 69,086 & -- & 772 & -- & -- & 0 & 147,853 & 2,199 \\
\hline 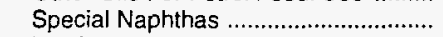 & -- & 19,191 & 2,709 & - & 281 & -- & - & 7,849 & 13,770 & 2,171 \\
\hline 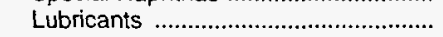 & - & 65,899 & 4,026 & -. & 215 & -. & -- & 11,275 & 58,435 & 12,889 \\
\hline Waxes & -- & 8,372 & 441 & - & -80 & - & -- & 993 & 7,900 & 834 \\
\hline Petroleum Coke &.- & 251,619 & 386 & -- & 2,757 & -- & -- & 111,615 & 137,633 & 9,437 \\
\hline Asphalt and Road Oil .......................... & -- & 177,019 & 11,862 & - & 1,619 & .. & - & 2,879 & 184,383 & 22,102 \\
\hline 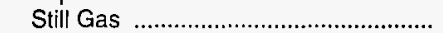 & -- & 241,184 & 0 & -- & 0 & .- & -- & 0 & 241,184 & 0 \\
\hline Miscellaneous Products ...................... & - & 17,507 & 101 & - & 618 & - & -- & 125 & 16,865 & 1,907 \\
\hline Total & $3,143,073$ & $6,116,870$ & $3,708,970$ & 52,891 & 52,343 & 0 & $5,806,792$ & 366,258 & $6,796,411$ & $1,559,759$ \\
\hline
\end{tabular}

a Unaccounted for crude oil represents the difference between the supply and disposition of crude oil.

b A negative number indicates a decrease in stocks and a positive number indicates an increase in stocks.

c Products supplied is equal to field production, plus refinery production, plus imports, plus unaccounted for crude oil, minus stock change, minus crude losses, minus refinery inputs, minus exports.

LRG = Liquefied Refinery Gas.

Note: Totals may not equal sum of components due to independent rounding.

Sources: Energy Information Administration (EIA) Forms ElA-810, "Monthly Refinery Report," ElA-811, "Monthly Bulk Terminal Report," EIA-812, "Monthly Product Pipeline Report," EIA-813, "Monthly Crude Oil Report," EIA-814, "Monthly Imports Report," EIA-816, "Monthly Natural Gas Liquids Report," EIA-817, "Monthly Tanker and Barge Movement Report," and EIA-819M, "Monthly Oxygenate Telephone Report." Domestic crude oil production from State conservation agencies and the Minerals Management Service of the U.S. Department of the Interior. Export data from the Bureau of the Census and Form ElA-810, "Monthly Refinery Report." 
Table 3. U.S. Daily Average Supply and Disposition of Crude Oil and Petroleum Products, 1997 (Thousand Barrels per Day)

\begin{tabular}{|c|c|c|c|c|c|c|c|c|c|}
\hline \multirow[b]{2}{*}{ Commodity } & \multicolumn{4}{|c|}{ Supply } & \multicolumn{5}{|c|}{ Disposition } \\
\hline & $\begin{array}{c}\text { Field } \\
\text { Production }\end{array}$ & $\begin{array}{c}\text { Refinery } \\
\text { Production }\end{array}$ & Imports & $\begin{array}{c}\text { Unaccounted } \\
\text { For Crude } \\
\text { Oil }^{\mathrm{a}}\end{array}$ & $\begin{array}{c}\text { Stock } \\
\text { Changeb }\end{array}$ & $\begin{array}{l}\text { Crude } \\
\text { Losses }\end{array}$ & $\begin{array}{l}\text { Refinery } \\
\text { Inputs }\end{array}$ & Exports & $\begin{array}{l}\text { Products } \\
\text { Supplied }^{c}\end{array}$ \\
\hline Crude Oil & 6,452 & - & 8,225 & 145 & 51 & $\mathbf{0}$ & 14,662 & 108 & 2 \\
\hline 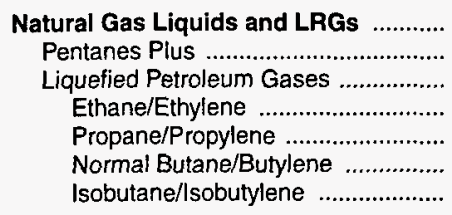 & $\begin{array}{r}1,817 \\
318 \\
1,499 \\
637 \\
528 \\
144 \\
191\end{array}$ & $\begin{array}{r}691 \\
-- \\
691 \\
27 \\
565 \\
88 \\
12\end{array}$ & $\begin{array}{r}205 \\
36 \\
169 \\
16 \\
113 \\
24 \\
16\end{array}$ & $\begin{array}{l}- \\
- \\
- \\
- \\
- \\
- \\
-\end{array}$ & $\begin{array}{r}7 \\
-2 \\
9 \\
4 \\
3 \\
1 \\
1\end{array}$ & $\begin{array}{l}- \\
- \\
- \\
- \\
- \\
- \\
-\end{array}$ & $\begin{array}{r}416 \\
153 \\
263 \\
0 \\
0 \\
138 \\
124\end{array}$ & $\begin{array}{r}57 \\
7 \\
50 \\
0 \\
32 \\
17 \\
0\end{array}$ & $\begin{array}{r}2,233 \\
195 \\
2,038 \\
676 \\
1,170 \\
99 \\
93\end{array}$ \\
\hline $\begin{array}{l}\text { Other Liquids ................................... } \\
\text { Other Hydrocarbons/Oxygenates ...... } \\
\text { Unfinished Oils .................................. } \\
\text { Motor Gasoline Blend. Comp. ............. } \\
\text { Aviation Gasoline Blend. Comp. ......... }\end{array}$ & $\begin{array}{r}215 \\
293 \\
-- \\
-78 \\
--\end{array}$ & $\begin{array}{l}-- \\
-- \\
-- \\
--\end{array}$ & $\begin{array}{r}614 \\
61 \\
353 \\
200 \\
0\end{array}$ & $\begin{array}{l}- \\
-- \\
-- \\
- \\
-\end{array}$ & $\begin{array}{r}15 \\
-2 \\
2 \\
15 \\
(s)\end{array}$ & $\begin{array}{l}- \\
-- \\
-- \\
-\end{array}$ & $\begin{array}{r}832 \\
343 \\
399 \\
94 \\
-5\end{array}$ & $\begin{array}{r}25 \\
12 \\
0 \\
13 \\
0\end{array}$ & $\begin{array}{r}-43 \\
0 \\
-48 \\
0 \\
5\end{array}$ \\
\hline 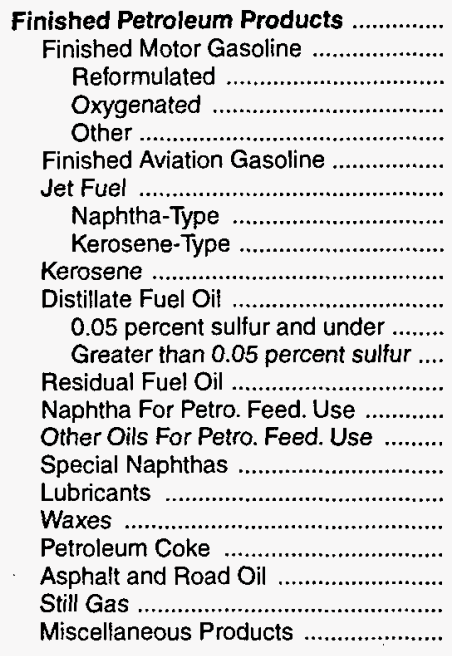 & $\begin{array}{r}127 \\
127 \\
-. \\
496 \\
-368 \\
. . \\
-. \\
-- \\
-. \\
-- \\
-. \\
-. \\
-. \\
-. \\
-. \\
-. \\
-- \\
-. \\
-- \\
-. \\
-. \\
-. \\
.-\end{array}$ & $\begin{array}{r}16,068 \\
7,743 \\
2,406 \\
91 \\
5,245 \\
20 \\
1,554 \\
1 \\
1,554 \\
65 \\
3,392 \\
2,162 \\
1,229 \\
708 \\
229 \\
218 \\
53 \\
181 \\
23 \\
689 \\
485 \\
661 \\
48\end{array}$ & $\begin{array}{r}1,117 \\
309 \\
161 \\
0 \\
148 \\
(s) \\
91 \\
0 \\
91 \\
2 \\
228 \\
103 \\
125 \\
194 \\
51 \\
189 \\
7 \\
11 \\
1 \\
1 \\
32 \\
0 \\
(s)\end{array}$ & $\begin{array}{l}-- \\
-. \\
-- \\
.- \\
-- \\
-- \\
-- \\
-- \\
- \\
- \\
-- \\
-- \\
- \\
- \\
-- \\
- \\
- \\
- \\
- \\
- \\
- \\
-\end{array}$ & $\begin{array}{r}70 \\
26 \\
14 \\
-1 \\
13 \\
-2 \\
11 \\
(s) \\
12 \\
1 \\
32 \\
-1 \\
33 \\
-15 \\
(s) \\
2 \\
1 \\
1 \\
(s) \\
8 \\
4 \\
0 \\
2\end{array}$ & $\begin{array}{l}-. \\
-. \\
-. \\
-. \\
-. \\
-. \\
-- \\
-. \\
- \\
- \\
-- \\
-- \\
-- \\
- \\
-- \\
- \\
- \\
- \\
- \\
- \\
- \\
-\end{array}$ & 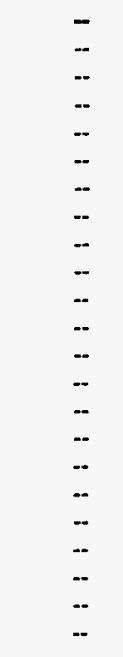 & $\begin{array}{r}813 \\
137 \\
(s) \\
1 \\
135 \\
0 \\
35 \\
(s) \\
35 \\
(s) \\
152 \\
44 \\
109 \\
120 \\
0 \\
0 \\
22 \\
31 \\
3 \\
306 \\
8 \\
0 \\
(s)\end{array}$ & $\begin{array}{r}16,429 \\
8,017 \\
2,552 \\
587 \\
4,877 \\
22 \\
1,599 \\
1 \\
1,598 \\
66 \\
3,435 \\
2,223 \\
1,213 \\
797 \\
280 \\
405 \\
38 \\
160 \\
22 \\
377 \\
505 \\
661 \\
46\end{array}$ \\
\hline 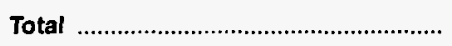 & 8,611 & 16,759 & 10,162 & 145 & 143 & 0 & 15,909 & 1,003 & 18,620 \\
\hline
\end{tabular}

a Unaccounted for crude oil represents the difference between the supply and dispositjon of crude oil.

b A negative number indicates a decrease in stocks and a positive number indicates an increase in stocks.

c Products supplied is equal to field production, plus refinery production, plus imports, plus unaccounted for crude oil, minus stock change, minus crude losses, minus refinery inputs, minus exports.

(s) = Less than 500 barrels per day.

LRG $=$ Liquefied Refinery Gas.

Note: Totals may not equal sum of components due to independent rounding

Sources: Energy Information Administration (EIA) Forms EIA-810, "Monthly Refinery Report," EIA-811, "Monthly Bulk Terminal Report," EIA-812, "Monthly Product Pipeline Report," EIA-813, "Monthly Crude Oil Report," EIA-814, "Monthly Imports Report," EIA-816, "Monthly Natural Gas Liquids Report," EIA-817, "Monthly Tanker and Barge Movement Report," and EIA-819M, "Monthly Oxygenate Telephone Report". Domestic crude oil production from State conservation agencies and the Minerals Management Service of the U.S. Department of the Interior. Export data from the Bureau of the Census and Form EIA-810, "Monthly Refinery Report." 
Table 4. PAD District I-Supply, Disposition, and Ending Stocks of Crude Oil and Petroleum Products, 1997 (Thousand Barrels)

\begin{tabular}{|c|c|c|c|c|c|c|c|c|c|c|c|}
\hline \multirow[b]{2}{*}{ Commodity } & \multicolumn{5}{|c|}{ Supply } & \multicolumn{5}{|c|}{ Disposition } & \multirow[b]{2}{*}{$\begin{array}{l}\text { Ending } \\
\text { Stocks }\end{array}$} \\
\hline & $\begin{array}{c}\text { Field } \\
\text { Production }\end{array}$ & $\begin{array}{c}\text { Refinery } \\
\text { Production }\end{array}$ & $\begin{array}{c}\text { Imports by } \\
\text { PAD } \\
\text { District } \\
\text { of Entry }{ }^{a}\end{array}$ & $\begin{array}{c}\text { Unac- } \\
\text { counted } \\
\text { For } \\
\text { Crude Oit }\end{array}$ & $\begin{array}{c}\text { Net } \\
\text { Receipts }\end{array}$ & $\begin{array}{c}\text { Stock } \\
\text { Change }\end{array}$ & $\begin{array}{c}\text { Crude } \\
\text { Losses }\end{array}$ & $\begin{array}{c}\text { Refinery } \\
\text { Inputs }\end{array}$ & Exports & $\begin{array}{l}\text { Products } \\
\text { Supplied }^{\mathrm{d}}\end{array}$ & \\
\hline 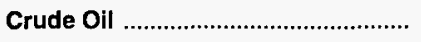 & 9,494 & - & 527,113 & 5,153 & $-2,953$ & $-2,564$ & 0 & 541,371 & 1 & 0 & 10,973 \\
\hline Natural Gas Liquids and LRGs ....... & 9,446 & 16,993 & 8,649 & -- & 41,435 & 53 & -- & 1,519 & 741 & 74,210 & 6,092 \\
\hline 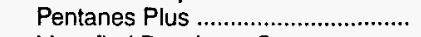 & 1,003 & 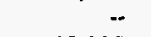 & 0 & -- & 0 & -18 & -- & 0 & 25 & 996 & 12 \\
\hline Liquefied Petroleum Gases ............. & 8,443 & 16,993 & 8,649 & $-\cdot$ & 41,435 & 71 & -- & 1,519 & 716 & 73,214 & 6,080 \\
\hline 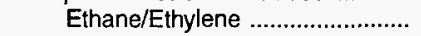 & 2,915 & 0 & 0 & -- & 0 & -1 & -. & 0 & 0 & 2,916 & 0 \\
\hline 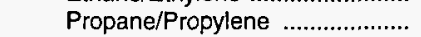 & 3,769 & 18,444 & 8,187 & - & 40,198 & -573 & - & 0 & 447 & 70,724 & 4,305 \\
\hline 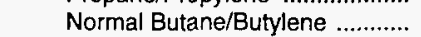 & 1,317 & -788 & 297 & -- & 976 & 422 & -- & 701 & 269 & 410 & 1,369 \\
\hline Isobutane/Isobutylene ................ & 442 & -663 & 165 & -- & 261 & 223 & -- & 818 & 0 & -836 & 406 \\
\hline Other Liquids .. & 8,196 & - & 88,627 & - & 5,385 & 513 & - & 121,292 & 345 & $-19,942$ & 18,817 \\
\hline Other Hydrocarbons/Oxygenates .. & 19,975 & - & 5,271 & -- & 0 & 360 & -- & 24,846 & 40 & 0 & 2,236 \\
\hline 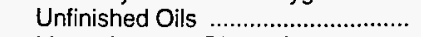 & - & - & 13,014 & -- & -60 & 341 & -- & 34,346 & 0 & $-21,733$ & 10,106 \\
\hline Motor Gasoline Blend. Comp. ......... & $-11,779$ & - & 70,342 & -- & 5,445 & -74 & -- & 63,777 & 305 & 0 & 6,396 \\
\hline Aviation Gasoline Blend. Comp. .... & - & - & 0 & $\cdots$ & 0 & -114 & -- & $-1,677$ & 0 & 1,791 & 79 \\
\hline Finished Petroleum Products .......... & 12,684 & 671,139 & 298,219 & - & $1,015,797$ & 15,070 & - & - & 12,320 & $1,970,449$ & 151,053 \\
\hline Finished Motor Gasoline ................. & 12,684 & 350,130 & 107,656 & .. & 571,105 & 5,772 & -. & -. & 1,188 & $1,034,615$ & 50,787 \\
\hline 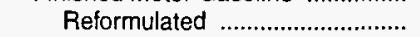 & .. & 224,012 & 56,352 & -- & 120,357 & 2,209 & -- & -- & (s) & 398,512 & 19,462 \\
\hline 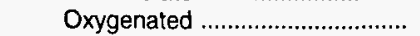 & 9,048 & 8 & 0 & -- & 1,604 & -78 & -- & - & 1 & 10,737 & 280 \\
\hline Other & 3,636 & 126,110 & 51,304 & -- & 449,144 & 3,641 & -. & -- & 1,186 & 625,367 & 31,045 \\
\hline Finished Aviation Gasoline .............. & -. & 39 & 3 & -. & 723 & -589 & -- & -- & 0 & 1,354 & 228 \\
\hline 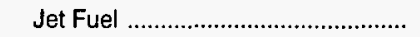 & -. & 33,116 & 30,877 & -- & 161,036 & 2,078 & -- & -- & 1,015 & 221,936 & 11,756 \\
\hline 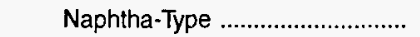 & -- & 0 & 0 & -- & 0 & 0 & -- & -- & 31 & -31 & 0 \\
\hline 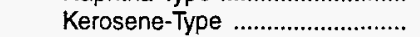 & -- & 33,116 & 30,877 & -- & 161,036 & 2,078 & - & -. & 983 & 221,968 & 11,756 \\
\hline 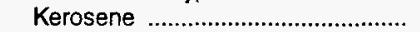 & -- & 3,822 & 495 & -. & 2,834 & 104 & -- & -- & 19 & 7,028 & 4,576 \\
\hline Distillate Fuel Oil ............................... & - & 154,771 & 76,162 & -. & 250,452 & 12,542 & -- & - & 2,345 & 466,498 & 59,932 \\
\hline 0.05 percent sulfur and under ... & .. & 54,063 & 34,050 & -- & 144,010 & -416 & -- & -- & 191 & 232,348 & 18,663 \\
\hline Greater than 0.05 percent sulfur & - & 100,708 & $42,+12$ & -. & 106,442 & 12,958 & -- & -. & 2,154 & 234,150 & 41,269 \\
\hline 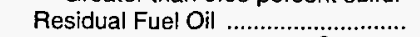 & -- & 46,418 & 64,352 & -- & 16,425 & $-5,033$ & -- & -- & 1,340 & 130,888 & 16,747 \\
\hline 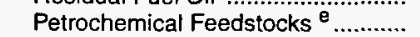 & -. & 5,417 & 2,044 & -- & .724 & 97 & - & -- & 0 & 6,640 & 478 \\
\hline 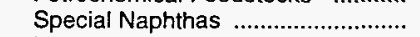 & -- & 715 & 1,976 & -- & 1,124 & -2 & -- & -- & 167 & 3,650 & 116 \\
\hline 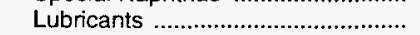 & -- & 6,765 & 3,563 & -- & 8,313 & 22 & -- & -- & 1,744 & 16,875 & 2,441 \\
\hline Waxes & -- & 571 & 256 & -- & 0 & -167 & -- & - & 311 & 683 & 45 \\
\hline Petroleum Coke & -. & 18,011 & 0 & -- & 0 & -206 & -. & -. & 3,915 & 14,302 & 267 \\
\hline Asphalt and Road Oil ....................... & -. & 28,867 & 10,835 & -- & 4,509 & 467 & - & -- & 225 & 43,519 & 3,591 \\
\hline Still Gas & -- & 21,716 & 0 & -. & 0 & 0 & - & -. & 0 & 21,716 & 0 \\
\hline Miscellaneous Products ................... & -- & 781 & 0 & -. & 0 & -15 & $\ldots$ & .. & 52 & 744 & 89 \\
\hline Total & 39,820 & 688,132 & 922,608 & 5,153 & $1,059,664$ & 13,072 & $\mathbf{0}$ & 664,182 & 13,407 & $2,024,716$ & 186,935 \\
\hline
\end{tabular}

\footnotetext{
a Represents the PAD District in which the material entered the United States and not necessarily where the crude oil or product is processed and/or consumed.

b Unaccounted for crude oil represents the difference between the supply and disposition of crude oil.

c A negative number indicates a decrease in stocks and a positive number indicates an increase in stocks.

d Products supplied is equal to field production, plus refinery production, plus imports, plus unaccounted for crude oil, plus net receipts, minus stock change, minus crude losses, minus refinery inputs, minus exports.

$\mathrm{e}$ Includes naphtha less than $401^{\circ} \mathrm{F}$ endpoint and other oils equal to or greater than $401^{\circ} \mathrm{F}$ endpoint.

(s) = Less than 500 barrels.

LRG $=$ Liquefied Refinery Gas.

Note: Totals may not equal sum of components due to independent rounding.

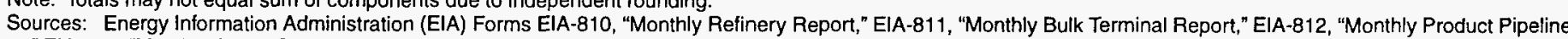
Report," EIA-813, "Monthly Crude Oil Report," EIA-814, "Monthly Imports Report," EIA-816, "Monthly Natural Gas Liquids Report," EIA-817, "Monthly Tanker and Barge Movement Report," and EIA-819M, "Monthly Oxygenate Telephone Report". Domestic crude oil production from State conservation agencies and the Minerals Management Service of the U.S. Department of the Interior. Export data from the Bureau of the Census and Form EIA-810, "Monthly Refinery Report."
} 
Table 5. PAD District I-Daily Average Supply and Disposition of Crude Oil and Petroleum Products, 1997 (Thousand Barrels per Day)

\begin{tabular}{|c|c|c|c|c|c|c|c|c|c|c|}
\hline \multirow[b]{2}{*}{ Commodity } & \multicolumn{5}{|c|}{ Supply } & \multicolumn{5}{|c|}{ Disposition } \\
\hline & $\begin{array}{c}\text { Field } \\
\text { Production }\end{array}$ & $\begin{array}{c}\text { Refinery } \\
\text { Production }\end{array}$ & $\begin{array}{c}\text { Imports by } \\
\text { PAD } \\
\text { District } \\
\text { of Entry }^{\mathrm{a}}\end{array}$ & $\begin{array}{l}\text { Unac- } \\
\text { counted } \\
\text { For } \\
\text { Crude Oil } \\
\end{array}$ & $\begin{array}{c}\begin{array}{c}\text { Net } \\
\text { Receipts }\end{array} \\
\end{array}$ & $\begin{array}{c}\text { Stock } \\
\text { Change }^{c}\end{array}$ & $\begin{array}{c}\text { Crude } \\
\text { Losses }\end{array}$ & $\begin{array}{c}\text { Refinery } \\
\text { Inputs }\end{array}$ & Exports & $\begin{array}{l}\text { Products } \\
\text { Supplied }\end{array}$ \\
\hline 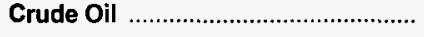 & 26 & - & 1,444 & 14 & -8 & -7 & 0 & 1,483 & (s) & 0 \\
\hline Natural Gas Liquids and LRGs ....... & 26 & 47 & 24 & - & 114 & (s) & - & 4 & 2 & 203 \\
\hline 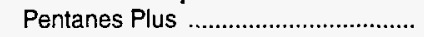 & 3 & -- & 0 & $-\cdot$ & 0 & (s) & -- & 0 & (s) & 3 \\
\hline Liquetied Petroleum Gases ............... & 23 & 47 & 24 & - & 114 & (s) & -- & 4 & 2 & 201 \\
\hline 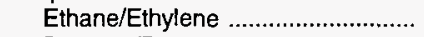 & 8 & 0 & 0 & -- & 0 & (s) & -. & 0 & 0 & 8 \\
\hline Propane/Propylene ........................... & 10 & 51 & 22 & -- & 110 & -2 & -- & 0 & 1 & 194 \\
\hline Normal Butane/Butylene ............... & 4 & -2 & 1 & -- & 3 & 1 & -- & 2 & 1 & 1 \\
\hline 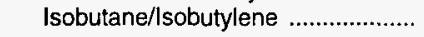 & 1 & -2 & (s) & - & 1 & 1 & -. & 2 & 0 & -2 \\
\hline 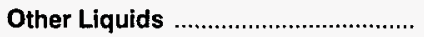 & 22 & - & 243 & - & 15 & 1 & - & 332 & 1 & -55 \\
\hline Other Hydrocarbons/Oxygenates .... & 55 & -- & 14 & -- & 0 & 1 & -- & 68 & (s) & 0 \\
\hline 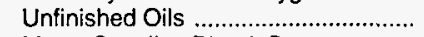 & - & -- & 36 & -- & (s) & 1 & $\cdots$ & 94 & 0 & -60 \\
\hline Motor Gasoline Blend. Comp. ........... & -32 & -- & 193 & -- & 15 & (s) & -- & 175 & 1 & 0 \\
\hline Aviation Gasoline Blend. Comp. ........ & - & -- & 0 & -- & 0 & (s) & -- & -5 & 0 & 5 \\
\hline Finished Petroleum Products ......... & 35 & 1,839 & 817 & - & 2,783 & 41 & - & - & 34 & 5,398 \\
\hline Finished Motor Gasoline ................... & 35 & 959 & 295 & $-\cdot$ & 1,565 & 16 & -- & -- & 3 & 2,835 \\
\hline 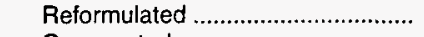 & -- & 614 & 154 & $\cdot-$ & 330 & 6 & -- & -- & (s) & 1,092 \\
\hline Oxygenated ...................................... & 25 & (s) & 0 & -- & 4 & (s) & -- & -- & (s) & 29 \\
\hline 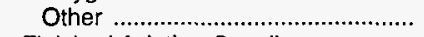 & 10 & 346 & 141 & -- & 1,231 & 10 & -- & -- & 3 & 1,713 \\
\hline Finished Aviation Gasoline ............... & -- & (s) & (s) & -. & 2 & -2 & -- & -- & 0 & 4 \\
\hline Jet Fuel & -- & 91 & 85 & -- & $44 \overline{1}$ & 6 & 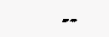 & -. & 3 & 608 \\
\hline 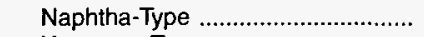 & -- & 0 & 0 & -- & 0 & 0 & -- & $-\cdot$ & (s) & (s) \\
\hline 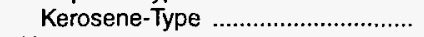 & -- & 91 & 85 & - & 441 & 6 & -. & -- & 3 & 608 \\
\hline 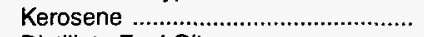 & -- & 10 & 1 & -- & 8 & (s) & - & -- & (s) & 19 \\
\hline Distillate Fuel Oil ................................. & -. & 424 & 209 & -- & 686 & 34 & -- & -- & 6 & 1,278 \\
\hline 0.05 percent sulfur and under ....... & -- & 148 & 93 & .. & 395 & -1 & - & -- & 1 & 637 \\
\hline Greater than 0.05 percent sulfur ... & 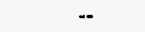 & 276 & 115 & -- & 292 & 36 & -- & -- & 6 & 642 \\
\hline 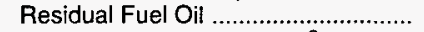 & -. & 127 & 176 & -- & 45 & -14 & -- & -- & 4 & 359 \\
\hline Petrochemical Feedstocks ${ }^{e}$............... & -- & 15 & 6 & -- & -2 & (s) & -- & -- & 0 & 18 \\
\hline 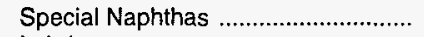 & -- & 2 & 5 & - & 3 & (s) & - & -- & (s) & 10 \\
\hline 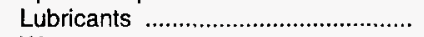 & -- & 19 & 10 & -- & 23 & (s) & $\cdots$ &.- & 5 & 46 \\
\hline 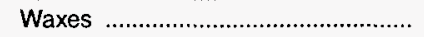 & $-\cdot$ & 2 & 1 & - & 0 & (s) & -. & -- & 1 & 2 \\
\hline 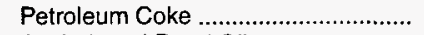 & -- & 49 & 0 & -- & 0 & -1 & -- & -- & 11 & 39 \\
\hline Asphalt and Road Oil ....................... & -- & 79 & 30 & - & 12 & 1 & - & -- & 1 & 119 \\
\hline 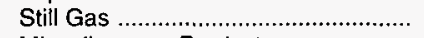 & -- & 59 & 0 & -- & 0 & 0 & -- & -- & 0 & 59 \\
\hline Miscellaneous Products .................... & - & 2 & 0 & -- & 0 & (s) & -- & -- & (s) & 2 \\
\hline Total & 109 & 1,885 & 2,528 & 14 & 2,903 & 36 & 0 & 1,820 & 37 & 5,547 \\
\hline
\end{tabular}

a Represents the PAD District in which the material entered the United States and not necessarily where the crude oil or product is processed and/or consumed.

b Unaccounted for crude oil represents the difference between the supply and disposition of crude oil.

c A negative number indicates a decrease in stocks and a positive number indicates an increase in stocks.

d Products supplied is equal to field production, plus refinery production, plus imports, plus unaccounted for crude oil, plus net receipts, minus stock change, minus crude losses, minus refinery inputs, minus exports.

- Includes naphtha less than $401^{\circ} \mathrm{F}$ endpoint and other oils equal to or greater than $401^{\circ} \mathrm{F}$ endpoint.

(s) = Less than 500 barrels per day.

LRG = Liquefied Refinery Gas.

Note: Totals may not equal sum of components due to independent rounding.

Sources: Energy Information Administration (EIA) Forms EIA-810, "Monthly Refinery Report," EIA-811, "Monthly Bulk Terminal Report," EIA-812, "Monthly Product Pipeline Report," EIA-813, "Monthly Crude Oil Report," EIA-814, "Monthly Imports Report," EIA-816, "Monthly Natural Gas Liquids Report," EIA-817, "Monthly Tanker and Barge Movement Report," and EIA-819M, "Monthly Oxygenate Telephone Report". Domestic crude oil production from State conservation agencies and the Minerals Management Service of the U.S. Department of the Interior. Export data from the Bureau of the Census and Form EIA-810, "Monthly Refinery Report." 
Table 6. PAD District II-Supply, Disposition, and Ending Stocks of Crude Oil and Petroleum Products, 1997 (Thousand Barrels)

\begin{tabular}{|c|c|c|c|c|c|c|c|c|c|c|c|}
\hline \multirow{2}{*}{ Commodity } & \multicolumn{5}{|c|}{ Supply } & \multicolumn{5}{|c|}{ Disposition } & \multirow[b]{2}{*}{$\begin{array}{l}\text { Ending } \\
\text { Stocks }\end{array}$} \\
\hline & $\begin{array}{c}\text { Field } \\
\text { Production }\end{array}$ & $\begin{array}{c}\text { Refinery } \\
\text { Production }\end{array}$ & $\begin{array}{c}\text { Imports by } \\
\text { PAD } \\
\text { District } \\
\text { of Entry }^{a}\end{array}$ & $\begin{array}{l}\text { Unac- } \\
\text { counted } \\
\text { For } \\
\text { Crude Oil } \\
\end{array}$ & $\begin{array}{c}\text { Net } \\
\text { Receipts }\end{array}$ & $\begin{array}{c}\text { Stock } \\
\text { Change } \\
\end{array}$ & $\begin{array}{c}\text { Crude } \\
\text { Losses } \\
\end{array}$ & $\begin{array}{c}\text { Refinery } \\
\text { Inputs }\end{array}$ & Exports & $\begin{array}{l}\text { Products } \\
\text { Supplied }^{d}\end{array}$ & \\
\hline Crude Oil . & . 204,365 & - & 310,905 & $-3,112$ & 731,769 & 10,166 & 0 & $1,222,785$ & 10,975 & 0 & 73,581 \\
\hline 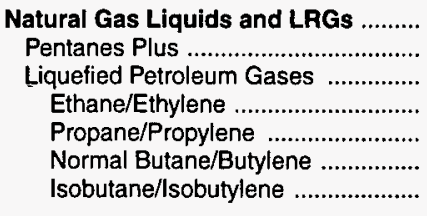 & $\begin{array}{r}109,286 \\
15,471 \\
93,815 \\
33,358 \\
\\
39,876 \\
12,854 \\
\\
7,727\end{array}$ & $\begin{array}{r}47,811 \\
- \\
47,811 \\
0 \\
44,853 \\
2,042 \\
916\end{array}$ & $\begin{array}{r}31,757 \\
412 \\
31,345 \\
138 \\
24,982 \\
3,621 \\
2,604\end{array}$ & $\begin{array}{l}- \\
- \\
- \\
- \\
- \\
- \\
-\end{array}$ & $\begin{array}{r}2,524 \\
7,413 \\
-4,889 \\
-23,040 \\
15,991 \\
-1,086 \\
3,246\end{array}$ & $\begin{array}{r}3,107 \\
-150 \\
3,257 \\
-491 \\
4,539 \\
-779 \\
-12\end{array}$ & $\begin{array}{l}- \\
- \\
- \\
-- \\
- \\
- \\
-\end{array}$ & $\begin{array}{r}32,684 \\
10,302 \\
22,382 \\
0 \\
0 \\
11,597 \\
10,785\end{array}$ & $\begin{array}{r}5,088 \\
2,457 \\
2,631 \\
0 \\
901 \\
1,729 \\
0\end{array}$ & $\begin{array}{r}150,499 \\
10,687 \\
139,812 \\
10,947 \\
120,262 \\
4,884 \\
3,720\end{array}$ & $\begin{array}{r}29,519 \\
1,774 \\
27,745 \\
2,978 \\
17,972 \\
4,802 \\
1,993\end{array}$ \\
\hline $\begin{array}{l}\text { Other Liquids ................................. } \\
\text { Other Hydrocarons/Oxygenates ..... } \\
\text { Unfinished Oils ................................. } \\
\text { Motor Gasoline Blend. Comp. ............ } \\
\text { Aviation Gasoline Blend. Comp. ......... }\end{array}$ & $\begin{array}{r}-13,819 \\
13,150 \\
- \\
-26,969 \\
-\end{array}$ & $\begin{array}{l}- \\
-- \\
-- \\
-- \\
-\end{array}$ & $\begin{array}{r}93 \\
0 \\
48 \\
45 \\
0\end{array}$ & $\begin{array}{l}- \\
- \\
- \\
- \\
-\end{array}$ & $\begin{array}{r}21,838 \\
0 \\
710 \\
21,128 \\
0\end{array}$ & $\begin{array}{r}2,541 \\
291 \\
475 \\
1,767 \\
8\end{array}$ & $\begin{array}{l}- \\
- \\
- \\
- \\
-\end{array}$ & $\begin{array}{r}14,184 \\
12,852 \\
8,912 \\
-7,566 \\
-14\end{array}$ & $\begin{array}{r}10 \\
7 \\
0 \\
3 \\
0\end{array}$ & $\begin{array}{r}-8,623 \\
0 \\
-8,629 \\
0 \\
6\end{array}$ & $\begin{array}{r}24,786 \\
1,950 \\
12,309 \\
10,491 \\
36\end{array}$ \\
\hline 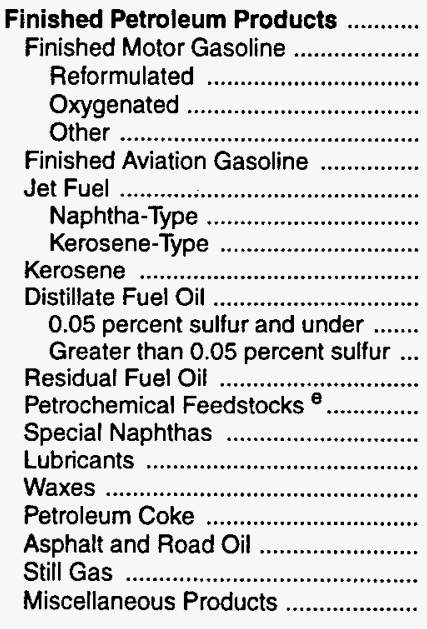 & 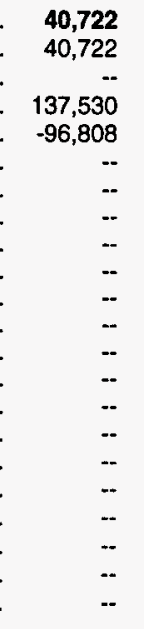 & $\begin{array}{r}1,283,540 \\
665,485 \\
87,471 \\
21,898 \\
556,116 \\
1,411 \\
78,800 \\
15 \\
78,785 \\
6,488 \\
307,243 \\
218,322 \\
88,921 \\
21,515 \\
15,926 \\
5,879 \\
8,311 \\
1,139 \\
50,490 \\
69,678 \\
47,778 \\
3,397\end{array}$ & $\begin{array}{r}4,671 \\
955 \\
0 \\
0 \\
955 \\
24 \\
75 \\
0 \\
75 \\
0 \\
1,632 \\
1,175 \\
457 \\
140 \\
402 \\
347 \\
259 \\
160 \\
0 \\
652 \\
0 \\
25\end{array}$ & $\begin{array}{l}- \\
- \\
-. \\
.- \\
- \\
- \\
- \\
-. \\
- \\
- \\
-. \\
- \\
- \\
- \\
- \\
- \\
- \\
- \\
- \\
-. \\
-.\end{array}$ & $\begin{array}{r}301,949 \\
176,176 \\
1,691 \\
-1,718 \\
176,203 \\
940 \\
41,026 \\
0 \\
41,026 \\
187 \\
79,350 \\
69,289 \\
10,061 \\
-3,131 \\
1,160 \\
651 \\
2,491 \\
0 \\
0 \\
3,099 \\
0 \\
0\end{array}$ & $\begin{array}{r}4,984 \\
827 \\
95 \\
-407 \\
1,139 \\
-44 \\
486 \\
-37 \\
523 \\
179 \\
-868 \\
-169 \\
-699 \\
694 \\
150 \\
250 \\
120 \\
-21 \\
1,691 \\
1,407 \\
0 \\
113\end{array}$ & 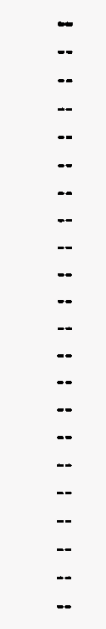 & $\begin{array}{l}-. \\
-. \\
-- \\
- \\
-- \\
- \\
- \\
- \\
- \\
- \\
- \\
- \\
-- \\
- \\
-- \\
-- \\
- \\
- \\
- \\
- \\
-\end{array}$ & $\begin{array}{r}6,533 \\
284 \\
0 \\
10 \\
274 \\
0 \\
38 \\
1 \\
36 \\
20 \\
552 \\
4 \\
548 \\
681 \\
0 \\
411 \\
686 \\
187 \\
1,640 \\
2,030 \\
0 \\
4\end{array}$ & $\begin{array}{r}1,619,365 \\
882,227 \\
89,067 \\
158,107 \\
635,053 \\
2,419 \\
119,377 \\
51 \\
119,327 \\
6,476 \\
388,541 \\
288,951 \\
99,590 \\
17,149 \\
17,338 \\
6,216 \\
10,255 \\
1,133 \\
47,159 \\
69,992 \\
47,778 \\
3,305\end{array}$ & $\begin{array}{r}103,433 \\
41,886 \\
1,195 \\
537 \\
40,154 \\
382 \\
9,145 \\
0 \\
9,145 \\
1,587 \\
31,226 \\
22,230 \\
8,996 \\
2,585 \\
363 \\
478 \\
1,735 \\
144 \\
3,214 \\
10,328 \\
0 \\
360\end{array}$ \\
\hline 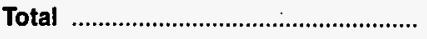 & 340,554 & $1,331,351$ & 347,426 & $-3,112$ & $1,058,080$ & 20,798 & 0 & $1,269,653$ & 22,607 & $1,761,241$ & 231,319 \\
\hline
\end{tabular}

a Represents the PAD District in which the material entered the United States and not necessarily where the crude oil or product is processed and/or consumed.

b Unaccounted for crude oil represents the difference between the supply and disposition of crude oil.

c A negative number indicates a decrease in stocks and a positive number indicates an increase in stocks.

d Products supplied is equal to field production, plus refinery production, plus imports, plus unaccounted for crude oil, plus net receipts, minus stock change, minus crude losses, minus refinery inputs, minus exports.

e Includes naphtha less than $401^{\circ} \mathrm{F}$ endpoint and other oils equal to or greater than $401^{\circ} \mathrm{F}$ endpoint

(s) = Less than 500 barrels.

LRG = Liquefied Refinery Gas.

Note: Totals may not equal sum of components due to independent rounding.

Sources: Energy Information Administration (EIA) Forms EIA-810, "Monthly Refinery Report," EIA-811, "Monthly Bulk Terminal Report," EIA-812, "Monthly Product

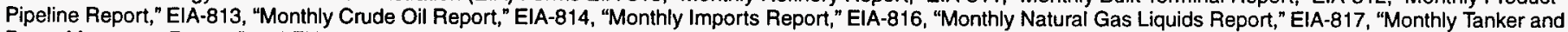
Barge Movement Report," and ElA-819M, "Monthly Oxygenate Telephone Report". Domestic crude oil production from State conservation agencies and the Minerals Management Service of the U.S. Department of the Interior. Export data from the Bureau of the Census and Form ElA-810, "Monthly Refinery Report." 
Table 7. PAD District II-Daily Average Supply and Disposition of Crude Oil and Petroleum Products, 1997 (Thousand Barrels per Day)

\begin{tabular}{|c|c|c|c|c|c|c|c|c|c|c|}
\hline \multirow[b]{2}{*}{ Commodity } & \multicolumn{5}{|c|}{ Supply } & \multicolumn{5}{|c|}{ Disposition } \\
\hline & $\begin{array}{c}\text { Field } \\
\text { Production }\end{array}$ & $\begin{array}{c}\text { Refinery } \\
\text { Production }\end{array}$ & $\begin{array}{c}\text { Imports by } \\
\text { PAD } \\
\text { District } \\
\text { of Entry }\end{array}$ & $\begin{array}{c}\text { Unac- } \\
\text { counted } \\
\text { For } \\
\text { Crude Oil } \\
\end{array}$ & $\begin{array}{c}\text { Net } \\
\text { Receipts }\end{array}$ & $\begin{array}{c}\text { Stock } \\
\text { Changec }\end{array}$ & $\begin{array}{l}\text { Crude } \\
\text { Losses }\end{array}$ & $\begin{array}{c}\text { Refinery } \\
\text { Inputs }\end{array}$ & Exports & $\begin{array}{l}\text { Products } \\
\text { Supplied d }\end{array}$ \\
\hline Crude Oil .. & 560 & - & 852 & -9 & 2,005 & 28 & 0 & 3,350 & 30 & 0 \\
\hline Natural Gas Liquids and LRGs ........ & 299 & 131 & 87 & - & 7 & 9 & - & 90 & 14 & 412 \\
\hline 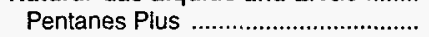 & 42 & - & 1 & - & 20 & (s) & -- & 28 & 7 & 29 \\
\hline Liquefied Petroleum Gases ............... & 257 & 131 & 86 & -- & -13 & 9 & -- & 61 & 7 & 383 \\
\hline Ethane/Ethylene & 91 & 0 & (s) & -. & -63 & -1 & -- & 0 & 0 & 30 \\
\hline 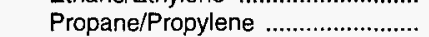 & 109 & 123 & 68 & -- & 44 & 12 & - & 0 & 2 & 329 \\
\hline Normal Butane/Butylene ................ & 35 & 6 & 10 & - & -3 & -2 & -- & 32 & 5 & 13 \\
\hline Isobutane/lsobutylene ...................... & 21 & 3 & 7 & -. & 9 & (s) & -- & 30 & 0 & 10 \\
\hline 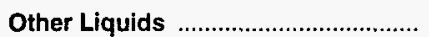 & -38 & - & (s) & - & 60 & 7 & - & 39 & (s) & -24 \\
\hline Other Hydrocarbons/Oxygenates .... & 36 & - & 0 & -- & 0 & 1 & -- & 35 & (s) & 0 \\
\hline 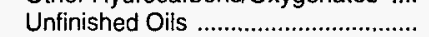 & -- & -. & (s) & -- & 2 & 1 & -- & 24 & 0 & -24 \\
\hline Motor Gasoline Blend. Comp. ........... & -74 & -- & (s) & -- & 58 & 5 & -- & -21 & (s) & 0 \\
\hline Aviation Gasoline Blend. Comp. ....... & $\cdots$ & - & 0 & - & 0 & (s) & - & (s) & 0 & (s) \\
\hline Finished Petroleum Products .......... & 112 & 3,517 & 13 & - & 827 & 14 & - & - & 18 & 4,437 \\
\hline Finished Motor Gasoline ....................... & 112 & 1,823 & 3 & -- & 483 & 2 & -- & -- & 1 & 2,417 \\
\hline 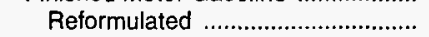 & - & 240 & 0 & -- & 5 & (s) & -- & -- & 0 & 244 \\
\hline 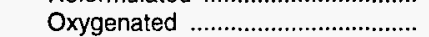 & 377 & 60 & 0 & -- & -5 & -1 & -- & -- & (s) & 433 \\
\hline Other & -265 & 1,524 & 3 & -- & 483 & 3 & - & -- & 1 & 1,740 \\
\hline Finished Aviation Gasoline ................ & -. & 4 & (s) & -. & 3 & (s) & -- & - & 0 & 7 \\
\hline Jet Fuel & -- & 216 & (s) & -- & 112 & 1 & -- & -- & (s) & 327 \\
\hline Naphtha-Type & - & (s) & 0 & - & 0 & (s) & - & -- & (s) & (s) \\
\hline 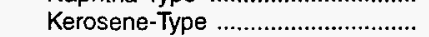 & - & 216 & (s) & -- & 112 & 1 & -- & -- & (s) & 327 \\
\hline 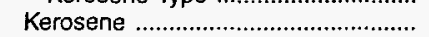 & - & 18 & 0 & -- & 1 & (s) & -- & -. & (s) & 18 \\
\hline 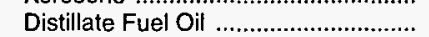 & - & 842 & 4 & -- & 217 & -2 & -- & - & 2 & 1,064 \\
\hline 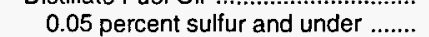 & -. & 598 & 3 & -- & 190 & $(s)$ & -. & -- & (s) & 792 \\
\hline Greater than 0.05 percent sulfur ... & -- & 244 & 1 & -- & 28 & -2 & -. & -- & 2 & 273 \\
\hline 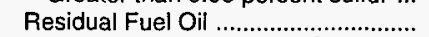 & -- & 59 & (s) & -- & -9 & 2 & - & -. & 2 & 47 \\
\hline 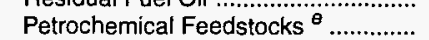 & - & 44 & 1 & .. & 3 & (s) & -- & - & 0 & 48 \\
\hline 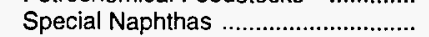 & -- & 16 & 1 &.- & 2 & 1 & -- & -- & 1 & 17 \\
\hline 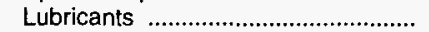 & -- & 23 & 1 & -- & 7 & (s) & -. & - & 2 & 28 \\
\hline Waxes & - & 3 & (s) & -- & 0 & (s) & -- & -- & 1 & 3 \\
\hline Petroleum Coke ................................. & -- & 138 & 0 & -- & 0 & 5 & -- & -- & 4 & 129 \\
\hline Asphalt and Road Oil ........................ & - & 191 & 2 & - & 8 & 4 & - & - & 6 & 192 \\
\hline 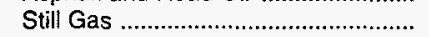 & -. & 131 & 0 & -- & 0 & 0 & -- & -. & 0 & 131 \\
\hline Miscellaneous Products .................... & - & 9 & (s) & - & 0 & (s) & -- & $-\cdot$ & (s) & 9 \\
\hline 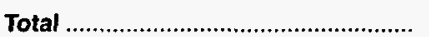 & 933 & 3,648 & 952 & -9 & 2,899 & 57 & $\mathbf{0}$ & 3,479 & 62 & 4,825 \\
\hline
\end{tabular}

a Represents the PAD District in which the material entered the United States and not necessarily where the crude oil or product is processed and/or consumed.

b Unaccounted for crude oil represents the difference between the supply and disposition of crude oil.

c A negative number indicates a decrease in stocks and a positive number indicates an increase in stocks.

Products supplied is equal to field production, plus refinery production, plus imports, plus unaccounted for crude oil, plus net receipts, minus stock change, minus crude losses, minus refinery inputs, minus exports.

e Includes naphtha less than $401^{\circ} \mathrm{F}$ endpoint and other oils equal to or greater than $401^{\circ} \mathrm{F}$ endpoint.

(s) = Less than 500 barrels per day.

LRG = Liquefied Refinery Gas.

Note: Totals may not equal sum of components due to independent rounding.

Sources: Energy Information Administration (EIA) Forms EIA-810, "Monthly Refinery Report," EIA-811, "Monthly Bulk Terminal Report," EIA-812, "Monthly Product Pipeline Report," EIA-813, "Monthly Crude Oil Report," EIA-814, "Monthly Imports Report," EIA-816, "Monthly Natural Gas Liquids Report," EIA-817, "Monthly Tanker and Barge Movement Report," and EIA-819M, "Monthly Oxygenate Telephone Report". Domestic crude oil production from State conservation agencies and the Minerals Management Service of the U.S. Department of the Interior. Export data from the Bureau of the Census and Form EIA-810, "Monthly Refinery Report." 
Table 8. PAD District III-Supply, Disposition, and Ending Stocks of Crude Oil and Petroleum Products, 1997 (Thousand Barrels)

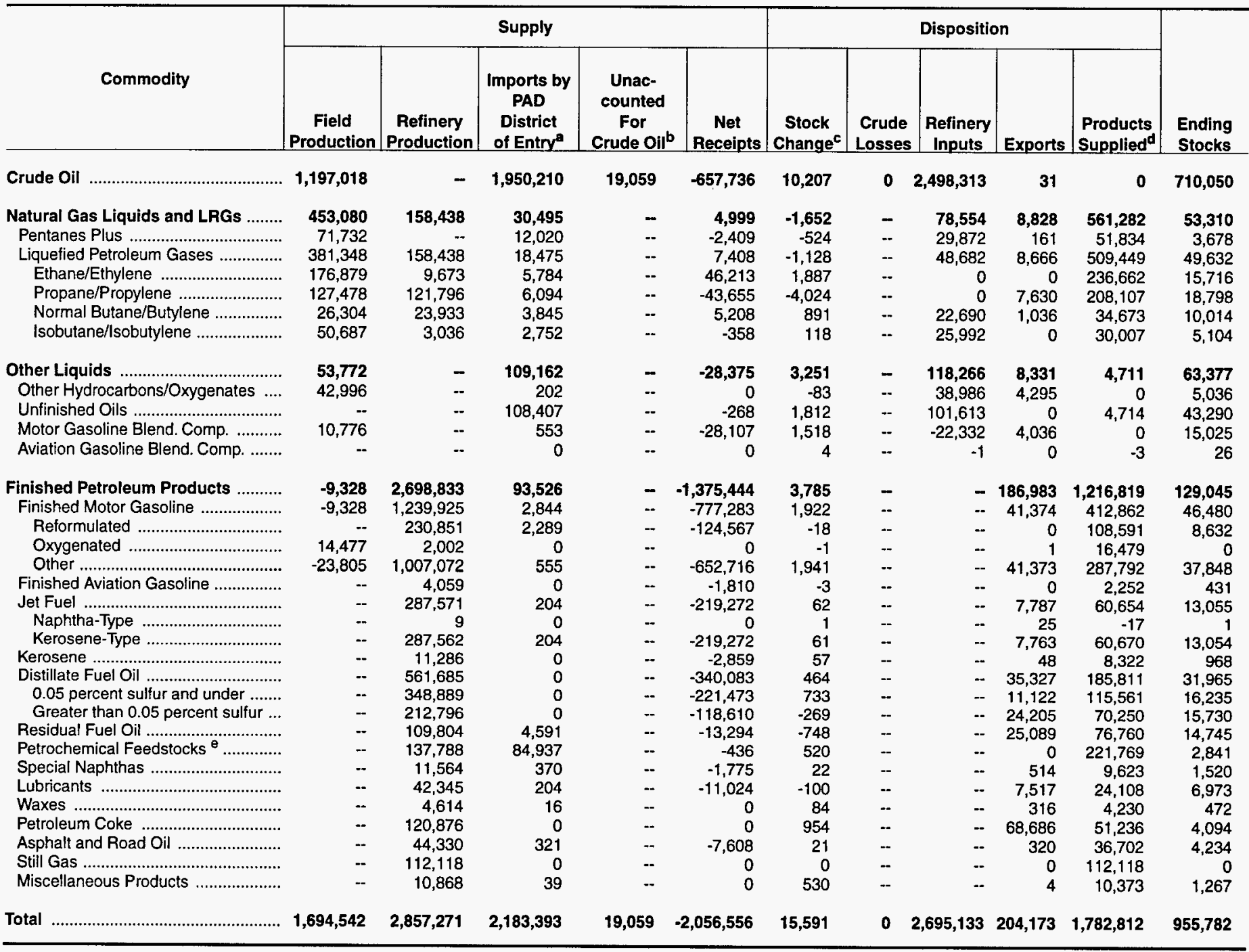

a Represents the PAD District in which the material entered the United States and not necessarily where the crude oil or product is processed and/or consumed.

b Unaccounted for crude oil represents the difference between the supply and disposition of crude oil.

c A negative number indicates a decrease in stocks and a positive number indicates an increase in stocks.

d Products supplied is equal to field production, plus refinery production, plus imports, plus unaccounted for crude oil, plus net receipts, minus stock change, minus crude losses, minus refinery inputs, minus exports.

Includes naphtha less than $401^{\circ} \mathrm{F}$ endpoint and other oils equal to or greater than $401^{\circ} \mathrm{F}$ endpoint.

(s) $=$ Less than 500 barrels.

LRG = Liquefied Refinery Gas.

Note: Totals may not equal sum of components due to independent rounding

Sources: Energy Information Administration (EIA) Forms EIA-810, "Monthly Refinery Report," EIA-811, "Monthly Bulk Terminal Report," ElA-812, "Monthly Product

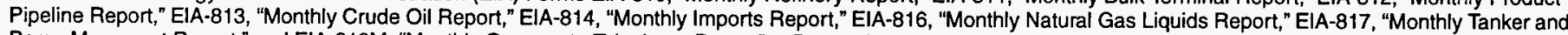
Barge Movement Report," and EIA-819M, "Monthly Oxygenate Telephone Report". Domestic crude oil production from State conservation agencies and the Minerals Management Service of the U.S. Department of the Interior. Export data from the Bureau of the Census and Form EIA-810, "Monthly Refinery Report." 
Table 9. PAD District III-Daily Average Supply and Disposition of Crude Oil and Petroleum Products, 1997 (Thousand Barrels per Day)

\begin{tabular}{|c|c|c|c|c|c|c|c|c|c|c|}
\hline \multirow[b]{2}{*}{ Commodity } & \multicolumn{5}{|c|}{ Supply } & \multicolumn{5}{|c|}{ Disposition } \\
\hline & $\begin{array}{c}\text { Field } \\
\text { Production }\end{array}$ & $\begin{array}{c}\text { Refinery } \\
\text { Production }\end{array}$ & $\begin{array}{c}\text { Imports by } \\
\text { PAD } \\
\text { District } \\
\text { of Entry }\end{array}$ & $\begin{array}{l}\text { Unac- } \\
\text { counted } \\
\text { For } \\
\text { Crude Oil }\end{array}$ & $\mid \begin{array}{c}\text { Net } \\
\text { Receipts }\end{array}$ & $\begin{array}{c}\text { Stock } \\
\text { Change }^{\mathbf{c}}\end{array}$ & $\begin{array}{c}\text { Crude } \\
\text { Losses }\end{array}$ & $\begin{array}{c}\text { Refinery } \\
\text { Inputs }\end{array}$ & Exports & $\begin{array}{l}\text { Products } \\
\text { Supplied }\end{array}$ \\
\hline 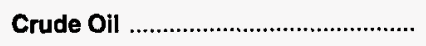 & 3,280 & - & 5,343 & 52 & $-1,802$ & 28 & 0 & 6,845 & (s) & 0 \\
\hline Natural Gas Liquids and LRGs ........ & 1,241 & 434 & 84 & - & 14 & -5 & - & 215 & 24 & 1,538 \\
\hline Pentanes Plus ................................. & 197 & -- & 33 & $-\cdot$ & -7 & -1 & -- & 82 & (s) & 142 \\
\hline Liquefied Petroleum Gases .............. & 1,045 & 434 & 51 & -- & 20 & -3 & -- & 133 & 24 & 1,396 \\
\hline 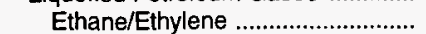 & 485 & 27 & 16 & -- & 127 & 5 & -- & 0 & 0 & 648 \\
\hline Propane/Propylene ........................ & 349 & 334 & 17 & -- & -120 & -11 & -- & 0 & 21 & 570 \\
\hline Normal Butane/Butylene ................ & 72 & 66 & 11 & -- & 14 & 2 & -. & 62 & 3 & 95 \\
\hline 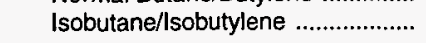 & 139 & 8 & 8 & -- & -1 & (s) & -- & 71 & 0 & 82 \\
\hline 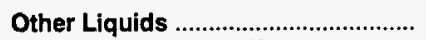 & 147 & - & 299 & - & .78 & 9 & - & 324 & 23 & 13 \\
\hline Other Hydrocarbons/Oxygenates .... & 118 & .. & 1 & -- & 0 & (s) & -. & 107 & 12 & 0 \\
\hline 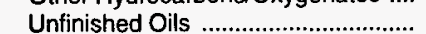 & -- & -- & 297 & -- & -1 & 5 & -- & 278 & 0 & 13 \\
\hline Motor Gasoline Blend. Comp. ............. & 30 & -- & 2 & -- & -77 & 4 & -. & -61 & 11. & 0 \\
\hline Aviation Gasoline Blend. Comp. ....... & - & -- & 0 & -- & 0 & (s) & -- & (s) & 0 & (s) \\
\hline Finished Petroleum Products ......... & -26 & 7,394 & 256 & -- & $-3,768$ & 10 & - & - & 512 & 3,334 \\
\hline Finished Motor Gasoline ....................... & -26 & 3,397 & 8 & -- & $-2,130$ & 5 & -. & -- & 113 & 1,131 \\
\hline Reformulated ………………...... & -- & 632 & 6 & -- & -341 & (s) & -- & -- & 0 & 298 \\
\hline 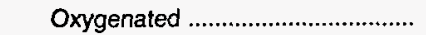 & 40 & 5 & 0 & -- & 0 & (s) & -- & -. & (s) & 45 \\
\hline Other & -65 & 2,759 & 2 & .- & $-1,788$ & 5 & .. & -- & 113 & 788 \\
\hline Finished Aviation Gasoline ................. & - & 11 & 0 & -- & -5 & (s) & -- & -. & 0 & 6 \\
\hline Jet Fuel & -- & 788 & 1 & -- & -601 & (s) & -. & -- & 21 & 166 \\
\hline Naphtha-Type ................................... & - & (s) & 0 & -- & 0 & (s) & -- & -- & (s) & (s) \\
\hline 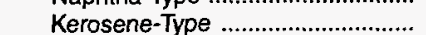 & -- & 788 & 1 & -- & -601 & (s) & -. & - & 21 & 166 \\
\hline 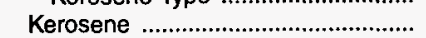 & -- & 31 & 0 & -- & -8 & (s) & -- & -- & (s) & 23 \\
\hline Distillate Fuel Oil ...................................... & -- & 1,539 & 0 & -- & -932 & 1 & -- & -- & 97 & 509 \\
\hline 0.05 percent sulfur and under ........ & .- & 956 & 0 & -- & -607 & 2 & -- & -- & 30 & 317 \\
\hline Greater than 0.05 percent sulfur .. & -- & 583 & 0 & -- & -325 & -1 & -- & -- & 66 & 192 \\
\hline 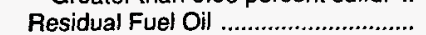 & -- & 301 & 13 & .- & -36 & -2 & -. & -. & 69 & 210 \\
\hline 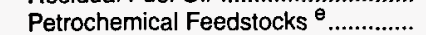 & .- & 378 & 233 & .- & -1 & 1 & -- & -- & 0 & 608 \\
\hline 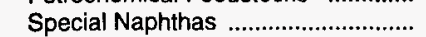 & - & 32 & 1 & -- & -5 & (s) & $\ldots$ & $\ldots$ & 1 & 26 \\
\hline 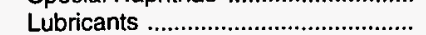 & .. & 116 & 1 & .- & -30 & (s) & -. & .- & 21 & 66 \\
\hline 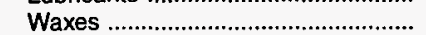 & -- & 13 & (s) & -- & 0 & (s) & -- & -- & 1 & 12 \\
\hline Petroleum Coke ..................................... & -- & 331 & 0 & -. & 0 & 3 & -- & -- & 188 & 140 \\
\hline Asphalt and Road Oil ............................. & .. & 121 & 1 & -- & -21 & (s) & -- & - & 1 & t01 \\
\hline 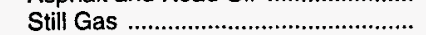 & .. & 307 & 0 & -- & 0 & 0 & -- & - & 0 & 307 \\
\hline Miscellaneous Products ........................ & - & 30 & (s) & -- & 0 & 1 & -- & - & (s) & 28 \\
\hline Total & 4,643 & 7,828 & 5,982 & 52 & $-5,634$ & 43 & 0 & 7,384 & 559 & 4,884 \\
\hline
\end{tabular}

a Represents the PAD District in which the material entered the United States and not necessarily where the crude oil or product is processed and/or consumed.

b Unaccounted for crude oil represents the difference between the supply and disposition of crude oil.

c A negative number indicates a decrease in stocks and a positive number indicates an increase in stocks.

Products supplied is equal to field production, plus refinery production, plus imports, plus unaccounted for crude oil, plus net receipts, minus stock change, minus crude losses, minus refinery inputs, minus exports.

Includes naphtha less than $401^{\circ} \mathrm{F}$ endpoint and other oils equal to or greater than $401^{\circ} \mathrm{F}$ endpoint.

(s) $=$ Less than 500 barrels per day.

LRG $=$ Liquefied Refinery Gas.

Note: Totals may not equal sum of components due to independent rounding.

Sources: Energy Information Administration (EIA) Forms EIA-810, "Monthly Refinery Report," EIA-811, "Monthly Bulk Terminal Report," EIA-812, "Monthly Product Pipeline Report," EIA-813, "Monthly Crude Oil Report," EIA-814, "Monthly Imports Report," EIA-816, "Monthly Natural Gas Liquids Report," ElA-817, "Monthly Tanker and Barge Movement Report," and EIA-819M," "Monthly Oxygenate Telephone Report". Domestic crude oil production from State conservation agencies and the Minerals Management Service of the U.S. Department of the Interior. Export data from the Bureau of the Census and Form EIA-810, "Monthly Refinery Report." 
Table 10. PAD District IV—Supply, Disposition, and Ending Stocks of Crude Oil and Petroleum Products, 1997 (Thousand Barrels)

\begin{tabular}{|c|c|c|c|c|c|c|c|c|c|c|c|}
\hline \multirow[b]{2}{*}{ Commodity } & \multicolumn{5}{|c|}{ Supply } & \multicolumn{5}{|c|}{ Disposition } & \multirow[b]{2}{*}{$\begin{array}{l}\text { Ending } \\
\text { Stocks }\end{array}$} \\
\hline & $\begin{array}{c}\text { Field } \\
\text { Production }\end{array}$ & $\begin{array}{c}\text { Refinery } \\
\text { Production }\end{array}$ & $\begin{array}{c}\text { Imports by } \\
\text { PAD } \\
\text { District } \\
\text { of Entry }\end{array}$ & $\begin{array}{c}\text { Unac- } \\
\text { counted } \\
\text { For } \\
\text { Crude Oil }\end{array}$ & $\begin{array}{c}\text { Net } \\
\text { Receipts }\end{array}$ & $\begin{array}{c}\text { Stock } \\
\text { Change }\end{array}$ & $\begin{array}{l}\text { Crude } \\
\text { Losses }\end{array}$ & $\begin{array}{c}\text { Refinery } \\
\text { Inputs }\end{array}$ & Exports & $\begin{array}{l}\text { Products } \\
\text { Supplied }\end{array}$ & \\
\hline 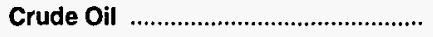 & 130,636 & - & 64,846 & 16,683 & $-35,545$ & 1,823 & 0 & 174,797 & 1 & 0 & 12,839 \\
\hline Natural Gas Liquids and LRGs ......... & 55,992 & 2,342 & 3,832 & - & $-48,958$ & 156 & - & 5,441 & 15 & 7,596 & 1,369 \\
\hline 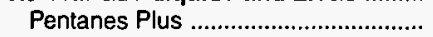 & 9,389 & - & 697 & -- & $-5,004$ & 57 & - & 1,260 & 8 & 3,757 & 227 \\
\hline Liquefied Petroleum Gases .............. & 46,603 & 2,342 & 3,135 & - & $-43,954$ & 99 & - & 4,181 & 7 & 3,839 & 1,142 \\
\hline 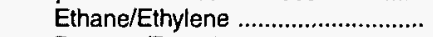 & 19,451 & 0 & 0 & -- & $-23,173$ & -7 & -- & 0 & 0 & $-3,715$ & 213 \\
\hline Propane/Propylene ........................... & 17,166 & 3,427 & 1,978 & -- & $-12,534$ & 86 & -- & 0 & 7 & 9,944 & 489 \\
\hline Normal Butane/Butylene ................. & 6,387 & -550 & 1,012 & - & $-5,098$ & 29 & - & 2,603 & (s) & -881 & 306 \\
\hline Isobutane/lsobutylene ........................ & 3,599 & -535 & 145 & -- & $-3,149$ & -9 & -- & 1,578 & 0 & $-1,509$ & 134 \\
\hline Other Liquids & 3,367 & - & 0 & - & 0 & 46 & - & 3,998 & 0 & -677 & 4,349 \\
\hline Other Hydrocarbons/Oxygenates ..... & 912 & -- & 0 & - & 0 & 65 & - & 847 & 0 & 0 & 252 \\
\hline Unfinished Oils & -. & -- & 0 & -- & 0 & 370 & -- & 307 & 0 & -677 & 2,208 \\
\hline Motor Gasoline Blend. Comp. ............. & 2,455 & -- & 0 & -- & 0 & -389 & - & 2,844 & 0 & 0 & 1,889 \\
\hline Aviation Gasoline Blend. Comp. ........ & - & - & 0 & - & 0 & 0 & -- & 0 & 0 & 0 & 0 \\
\hline Finished Petroleum Products ............ & $-2,093$ & 186,965 & 3,550 & - & 21,220 & 172 & - & - & 170 & 209,300 & 11,361 \\
\hline Finished Motor Gasoline ..................... & $-2,093$ & 92,581 & 234 & -- & 3,501 & 232 & -- & -- & 24 & 93,967 & 4,867 \\
\hline Reformulated .................................. & -- & 0 & 0 & $\ldots$ & 0 & 0 & -- & - & 0 & 0 & 0 \\
\hline 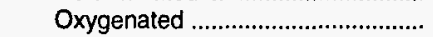 & 3,619 & 6,964 & 0 & -- & 114 & -16 & -- & -- & 18 & 10,695 & 264 \\
\hline Other & $-5,712$ & 85,617 & 234 & - & 3,387 & 248 & -. & -- & 5 & 83,272 & 4,603 \\
\hline Finished Aviation Gasoline ................ & -- & 163 & 5 & - & 147 & 17 & -- & -- & 0 & 298 & 41 \\
\hline 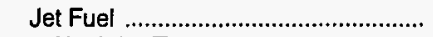 & -- & 9,546 & 0 & -- & 12,418 & 36 & - & - & 0 & 21,928 & 839 \\
\hline 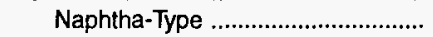 & -. & 0 & 0 & -- & 0 & -25 & -- & -- & 0 & 25 & 0 \\
\hline 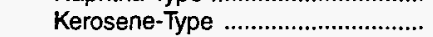 & -- & 9,546 & 0 & - & 12,418 & 61 & -. & - & 0 & 21,903 & 839 \\
\hline 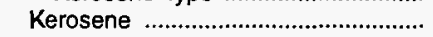 & -- & 884 & 0 & -- & -162 & -58 & -- & - & (s) & 780 & 67 \\
\hline 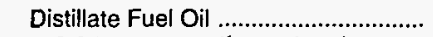 & -- & 50,277 & 3,256 & -- & 5,316 & -67 & -. & -- & (s) & 58,916 & 2,824 \\
\hline 0.05 percent sulfur and under ........ & -- & 40,798 & 643 & -- & 5,228 & -112 & -- & - & 0 & 46,781 & 2,344 \\
\hline Greater than 0.05 percent sulfur ... & -- & 9,479 & 2,613 & .. & 88 & 45 & -- &.- & (s) & 12,135 & 480 \\
\hline 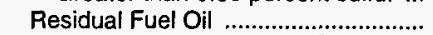 & - & 4,664 & 0 & -- & 0 & 167 & -- & - & 1 & 4,496 & 592 \\
\hline Petrochemical Feedstocks ${ }^{\ominus}$................... & -. & 234 & 0 & - & 0 & 1 & -- & - & 0 & 233 & 1 \\
\hline 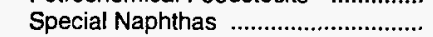 & -. & -1 & 1 & - & 0 & -1 & - & - & 3 & -2 & 0 \\
\hline 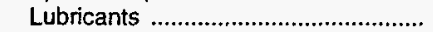 & -- & 0 & 0 & -- & 0 & 0 & - & -- & 81 & -81 & 0 \\
\hline Waxes & -- & 1,157 & 0 & - & 0 & 6 & -- & -- & 44 & 1,107 & 20 \\
\hline 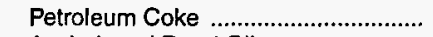 & -- & 5,414 & 0 & - & 0 & -82 & -- & - & 1 & 5,495 & 104 \\
\hline 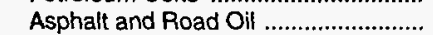 & - & 14,463 & 54 & -- & 0 & -74 & -- & -- & 16 & 14,575 & 1,992 \\
\hline 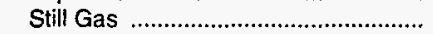 & - & 6,910 & 0 & -- & 0 & 0 & - & -- & 0 & 6,910 & 0 \\
\hline Miscellaneous Products .................... & -- & 673 & 0 & - & 0 & -5 & - & - & (s) & 678 & 14 \\
\hline Total & 187,902 & 189,307 & 72,228 & 16,683 & $-63,283$ & 2,197 & 0 & 184,236 & 186 & 216,219 & 29,918 \\
\hline
\end{tabular}

a Represents the PAD District in which the material entered the United States and not necessarily where the crude oil or product is processed and/or consumed.

b Unaccounted for crude oil represents the difference between the supply and disposition of crude oil.

c A negative number indicates a decrease in stocks and a positive number indicates an increase in stocks.

d Products supplied is equal to field production, plus refinery production, plus imports, plus unaccounted for crude oil, plus net receipts, minus stock change, minus crude losses, minus refinery inputs, minus exports.

Includes naphtha less than $401^{\circ} \mathrm{F}$ endpoint and other oils equal to or greater than $401^{\circ} \mathrm{F}$ endpoint.

(s) = Less than 500 barrels.

LRG = Liquefied Refinery Gas.

Note: Totals may not equal sum of components due to independent rounding.

Sources: Energy Information Administration (EIA) Forms EIA-810, "Monthly Refinery Report," EIA-811, "Monthly Bulk Terminal Report," EIA-812, "Monthly Product

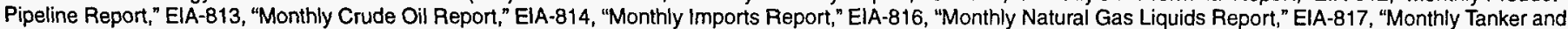
Barge Movement Report," and EIA-819M, "Monthly Oxygenate Telephone Report". Domestic crude oil production from State conservation agencies and the Minerals Management Service of the U.S. Department of the Interior. Export data from the Bureau of the Census and Form EIA-810, "Monthly Refinery Report." 
Table 11. PAD District IV-Daily Average Supply and Disposition of Crude Oil and Petroleum Products, 1997 (Thousand Barrels per Day)

\begin{tabular}{|c|c|c|c|c|c|c|c|c|c|c|}
\hline \multirow[b]{2}{*}{ Commodity } & \multicolumn{5}{|c|}{ Supply } & \multicolumn{5}{|c|}{ Disposition } \\
\hline & $\begin{array}{c}\text { Field } \\
\text { Production }\end{array}$ & \begin{tabular}{|c|} 
Refinery \\
Production
\end{tabular} & $\begin{array}{c}\text { Imports by } \\
\text { PAD } \\
\text { District } \\
\text { of Entry }\end{array}$ & $\begin{array}{l}\text { Unac- } \\
\text { counted } \\
\text { For } \\
\text { Crude Oil } \\
\end{array}$ & $\mid \begin{array}{c}\text { Net } \\
\text { Receipts }\end{array}$ & $\begin{array}{c}\text { Stock } \\
\text { Change }^{c} \\
\end{array}$ & $\begin{array}{c}\text { Crude } \\
\text { Losses }\end{array}$ & $\begin{array}{c}\text { Refinery } \\
\text { Inputs }\end{array}$ & Exports & $\begin{array}{l}\text { Products } \\
\text { Supplied }^{d}\end{array}$ \\
\hline Crude Oil & 358 & - & 178 & 46 & -97 & 5 & 0 & 479 & (s) & 0 \\
\hline 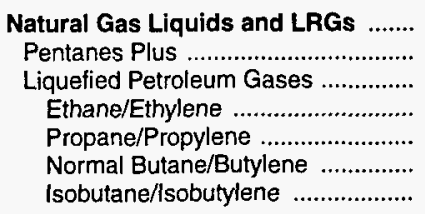 & $\begin{array}{r}153 \\
26 \\
128 \\
53 \\
47 \\
17 \\
10\end{array}$ & $\begin{array}{r}6 \\
-. \\
6 \\
0 \\
9 \\
-2 \\
-1\end{array}$ & $\begin{array}{r}10 \\
2 \\
9 \\
0 \\
5 \\
3 \\
(\mathrm{~s})\end{array}$ & $\begin{array}{l}- \\
- \\
- \\
- \\
- \\
-\end{array}$ & $\begin{array}{r}-134 \\
-14 \\
-120 \\
-63 \\
-34 \\
-14 \\
-9\end{array}$ & $\begin{array}{l}\text { (s) } \\
(s) \\
(s) \\
(s) \\
(s) \\
(s) \\
(s)\end{array}$ & $\begin{array}{l}- \\
- \\
- \\
- \\
- \\
- \\
-\end{array}$ & $\begin{array}{r}15 \\
3 \\
11 \\
0 \\
0 \\
7 \\
4\end{array}$ & $\begin{array}{l}\text { (s) } \\
(s) \\
(s) \\
0 \\
(s) \\
\text { (s) } \\
0\end{array}$ & $\begin{array}{r}21 \\
10 \\
11 \\
-10 \\
27 \\
-2 \\
-4\end{array}$ \\
\hline 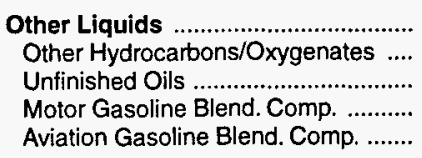 & $\begin{array}{l}9 \\
2 \\
- \\
7 \\
--\end{array}$ & $\begin{array}{l}- \\
- \\
- \\
- \\
-\end{array}$ & $\begin{array}{l}0 \\
0 \\
0 \\
0 \\
0\end{array}$ & $\begin{array}{l}- \\
- \\
- \\
-\end{array}$ & $\begin{array}{l}0 \\
0 \\
0 \\
0 \\
0\end{array}$ & $\begin{array}{r}(s) \\
(s) \\
1 \\
-1 \\
0\end{array}$ & $\begin{array}{l}- \\
- \\
- \\
- \\
-\end{array}$ & $\begin{array}{r}11 \\
2 \\
1 \\
8 \\
0\end{array}$ & $\begin{array}{l}0 \\
0 \\
0 \\
0 \\
0\end{array}$ & $\begin{array}{r}-2 \\
0 \\
-2 \\
0 \\
0\end{array}$ \\
\hline 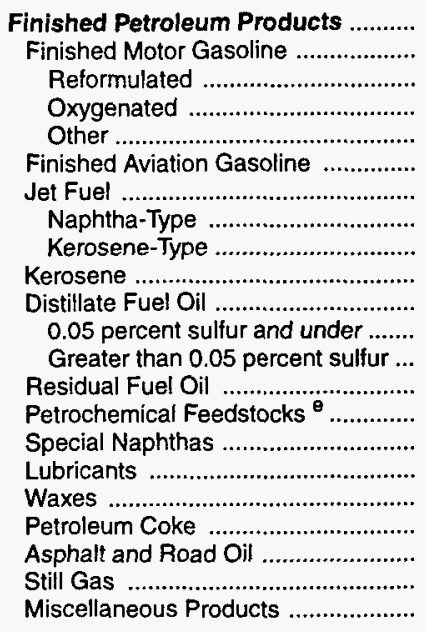 & $\begin{array}{r}-6 \\
-6 \\
- \\
10 \\
-16 \\
- \\
-- \\
-- \\
- \\
-- \\
- \\
- \\
- \\
- \\
- \\
- \\
- \\
- \\
- \\
- \\
- \\
- \\
-\end{array}$ & $\begin{array}{r}512 \\
254 \\
0 \\
19 \\
235 \\
(\mathbf{s}) \\
26 \\
0 \\
26 \\
2 \\
138 \\
112 \\
26 \\
13 \\
1 \\
1 \\
(s) \\
0 \\
3 \\
15 \\
40 \\
19 \\
2\end{array}$ & $\begin{array}{r}10 \\
1 \\
0 \\
0 \\
1 \\
(\mathbf{s}) \\
0 \\
0 \\
0 \\
0 \\
9 \\
2 \\
7 \\
0 \\
0 \\
(\mathrm{~s}) \\
0 \\
0 \\
0 \\
(\mathrm{~s}) \\
0 \\
0\end{array}$ & $\begin{array}{l}- \\
- \\
- \\
- \\
- \\
- \\
- \\
- \\
- \\
- \\
- \\
- \\
- \\
- \\
- \\
- \\
- \\
- \\
- \\
- \\
- \\
- \\
-\end{array}$ & $\begin{array}{r}58 \\
10 \\
0 \\
\text { (s) } \\
9 \\
(s) \\
34 \\
0 \\
34 \\
(s) \\
15 \\
14 \\
(s) \\
0 \\
0 \\
0 \\
0 \\
0 \\
0 \\
0 \\
0 \\
0\end{array}$ & $\begin{array}{r}\text { (s) } \\
1 \\
0 \\
\text { (s) } \\
1 \\
\text { (s) } \\
\text { (s) } \\
\text { (s) } \\
\text { (s) } \\
\text { (s) } \\
(\mathrm{s}) \\
(\mathrm{s}) \\
\text { (s) } \\
\text { (s) } \\
\text { (s) } \\
\text { (s) } \\
0 \\
\text { (s) } \\
\text { (s) } \\
\text { (s) } \\
0 \\
\text { (s) }\end{array}$ & $\begin{array}{l}- \\
- \\
-- \\
- \\
- \\
- \\
- \\
- \\
- \\
- \\
-- \\
- \\
- \\
- \\
- \\
- \\
- \\
- \\
- \\
- \\
- \\
- \\
-\end{array}$ & $\begin{array}{l}- \\
- \\
- \\
- \\
- \\
- \\
- \\
- \\
- \\
-- \\
-- \\
-. \\
-- \\
-. \\
- \\
- \\
- \\
- \\
- \\
- \\
-- \\
-- \\
-- \\
-.\end{array}$ & $\begin{array}{r}\text { (s) } \\
\text { (s) } \\
0 \\
(\mathrm{~s}) \\
(\mathrm{s}) \\
0 \\
0 \\
0 \\
0 \\
(\mathrm{~s}) \\
(\mathrm{s}) \\
0 \\
(\mathrm{~s}) \\
(\mathrm{s}) \\
0 \\
(\mathrm{~s}) \\
(\mathrm{s}) \\
(\mathrm{s}) \\
(\mathrm{s}) \\
\text { (s) } \\
0 \\
\text { (s) }\end{array}$ & $\begin{array}{r}573 \\
257 \\
0 \\
29 \\
228 \\
1 \\
60 \\
(\mathrm{~s}) \\
60 \\
2 \\
161 \\
128 \\
33 \\
12 \\
1 \\
\text { (s) } \\
\text { (s) } \\
3 \\
15 \\
40 \\
19 \\
2\end{array}$ \\
\hline Total & 515 & 519 & 198 & 46 & -173 & 6 & 0 & 505 & 1 & 592 \\
\hline
\end{tabular}

a Represents the PAD District in which the material entered the United States and not necessarily where the crude oil or product is processed and/or consumed.

b Unaccounted for crude oil represents the difference between the supply and disposition of crude oil.

c A negative number indicates a decrease in stocks and a positive number indicates an increase in stocks.

Products supplied is equal to field production, plus refinery production, plus imports, plus unaccounted for crude oil, plus net receipts, minus stock change, minus crude losses, minus refinery inputs, minus exports.

Includes naphtha less than $401^{\circ} \mathrm{F}$ endpoint and other oils equal to or greater than $401^{\circ} \mathrm{F}$ endpoint.

(s) = Less than 500 barrels per day.

LRG = Liquefied Refinery Gas.

Note: Totals may not equal sum of components due to independent rounding.

Sources: Energy Information Administration (EIA) Forms EIA-810, "Monthly Refinery Report," EIA-811, "Monthly Bulk Terminal Report," EIA-812, "Monthly Product Pipeline Report," EIA-813, "Monthly Crude Oil Report," EIA-814, "Monthly Imports Report," EIA-816, "Monthly Natural Gas Liquids Report," EIA-817, "Monthly Tanker and Barge Movement Report," and EIA-819M, "Monthly Oxygenate Telephone Report". Domestic crude oil production from State conservation agencies and the Minerals Management Service of the U.S. Department of the Interior. Export data from the Bureau of the Census and Form EIA-810, "Monthly Refinery Report." 
Table 12. PAD District V—Supply, Disposition, and Ending Stocks of Crude Oil and Petroleum Products, 1997 (Thousand Barrels)

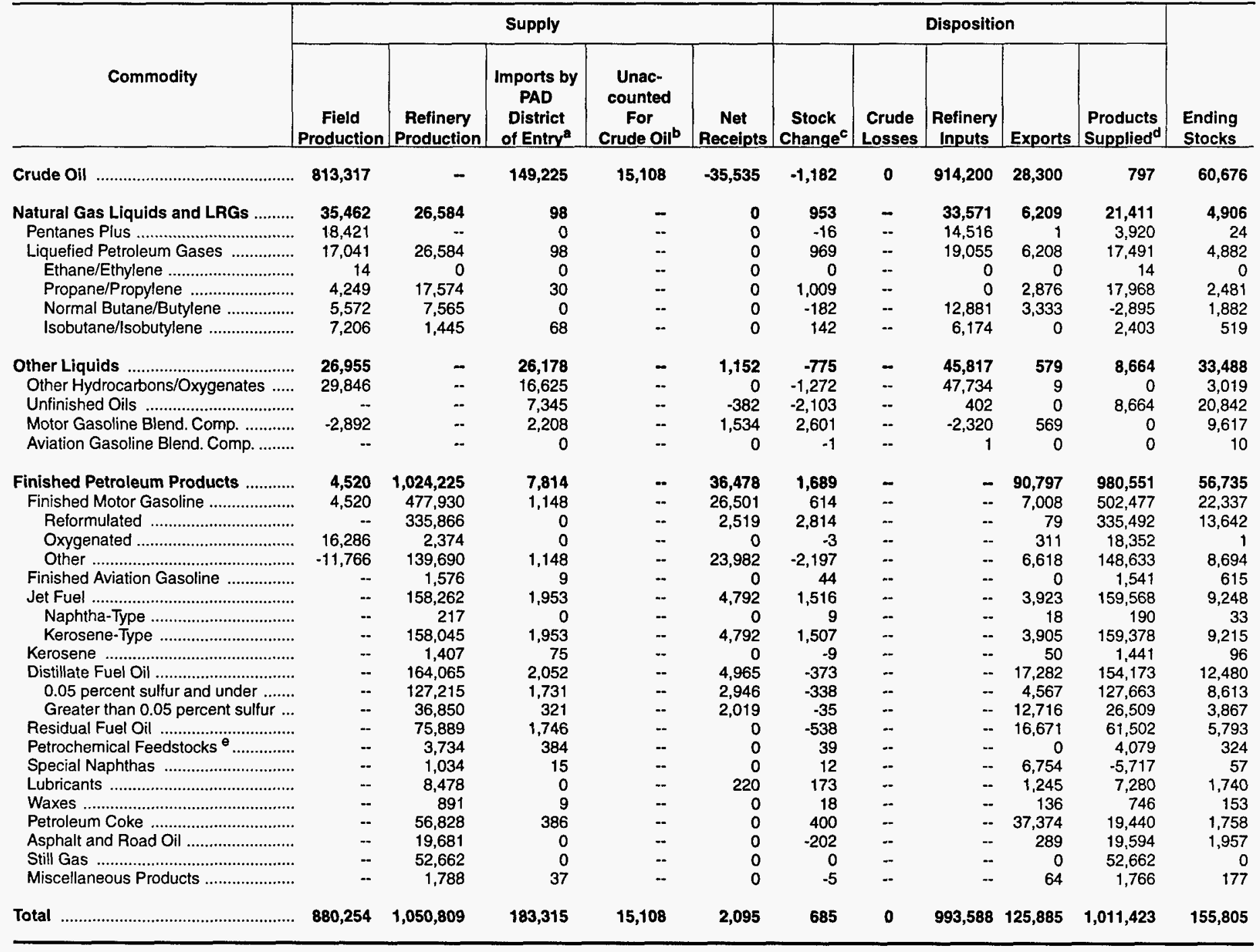

a Represents the PAD District in which the material entered the United States and not necessarily where the crude oil or product is processed and/or consumed.

b Unaccounted for crude oil represents the difference between the supply and disposition of crude oil.

c A negative number indicates a decrease in stocks and a positive number indicates an increase in stocks.

d Products supplied is equal to field production, plus refinery production, plus imports, plus unaccounted for crude oil, plus net receipts, minus stock change, minus crude losses, minus refinery inputs, minus exports.

Includes naphtha less than $401^{\circ} \mathrm{F}$ endpoint and other oils equal to or greater than $401^{\circ} \mathrm{F}$ endpoint.

(s) = Less than 500 barrels.

LRG = Liquefied Refinery Gas.

Note: Totals may not equal sum of components due to independent rounding.

Sources: Energy Information Administration (EIA) Forms EIA-810, "Monthly Refinery Report," EIA-811, "Monthly Bulk Terminal Report," EIA-812, "Monthly Product

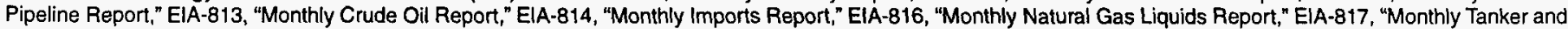
Barge Movement Report," and EIA-819M, "Monthly Oxygenate Telephone Report". Domestic crude oil production from State conservation agencies and the Minerals Management Service of the U.S. Department of the Interior. Export data from the Bureau of the Census and Form EIA-810, "Monthly Refinery Report." 
Table 13. PAD District V - Daily Average Supply and Disposition of Crude Oil and Petroleum Products, 1997 (Thousand Barrels per Day)

\begin{tabular}{|c|c|c|c|c|c|c|c|c|c|c|}
\hline \multirow[b]{2}{*}{ Commodity } & \multicolumn{5}{|c|}{ Supply } & \multicolumn{5}{|c|}{ Disposition } \\
\hline & \begin{tabular}{c|} 
Field \\
Production
\end{tabular} & $\begin{array}{c}\text { Refinery } \\
\text { Production }\end{array}$ & $\begin{array}{l}\text { Imports by } \\
\text { PAD } \\
\text { District } \\
\text { of Entry }\end{array}$ & $\begin{array}{l}\text { Unac- } \\
\text { counted } \\
\text { For } \\
\text { Crude Oil } \\
\end{array}$ & $\begin{array}{c}\text { Net } \\
\text { Receipts }\end{array}$ & $\begin{array}{c}\text { Stock } \\
\text { Change }\end{array}$ & $\begin{array}{l}\text { Crude } \\
\text { Losses }\end{array}$ & $\begin{array}{c}\text { Refinery } \\
\text { Inputs }\end{array}$ & Exports & $\begin{array}{l}\text { Products } \\
\text { Supplied }^{d}\end{array}$ \\
\hline 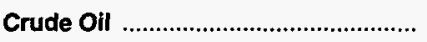 & 2,228 & - & 409 & 41 & -97 & -3 & 0 & 2,505 & 78 & 2 \\
\hline 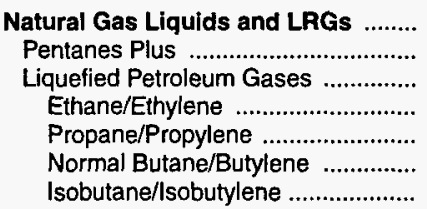 & $\begin{array}{l}97 \\
50 \\
47 \\
(s) \\
12 \\
15 \\
20\end{array}$ & $\begin{array}{r}73 \\
-- \\
73 \\
0 \\
48 \\
21 \\
4\end{array}$ & $\begin{array}{r}\text { (s) } \\
0 \\
(s) \\
0 \\
(s) \\
0 \\
(s)\end{array}$ & $\begin{array}{l}-- \\
-- \\
- \\
- \\
-- \\
-- \\
--\end{array}$ & $\begin{array}{l}0 \\
0 \\
0 \\
0 \\
0 \\
0 \\
0\end{array}$ & $\begin{array}{r}3 \\
(s) \\
3 \\
0 \\
3 \\
(s) \\
(s)\end{array}$ & $\begin{array}{l}- \\
-- \\
-- \\
- \\
- \\
-- \\
-\end{array}$ & $\begin{array}{r}92 \\
40 \\
52 \\
0 \\
0 \\
35 \\
17\end{array}$ & $\begin{array}{r}17 \\
\text { (s) } \\
17 \\
0 \\
8 \\
9 \\
0\end{array}$ & $\begin{array}{r}59 \\
11 \\
48 \\
(s) \\
49 \\
-8 \\
7\end{array}$ \\
\hline $\begin{array}{l}\text { Other Liquids ................................ } \\
\text { Other Hydrocarbons/Oxygenates ...... } \\
\text { Unfinished Oils ................................ } \\
\text { Motor Gasoline Blend. Comp. ............ } \\
\text { Aviation Gasoline Blend. Comp. ....... }\end{array}$ & $\begin{array}{r}74 \\
82 \\
-. \\
-8 \\
--\end{array}$ & $\begin{array}{l}- \\
- \\
- \\
- \\
-\end{array}$ & $\begin{array}{r}72 \\
46 \\
20 \\
6 \\
0\end{array}$ & $\begin{array}{l}- \\
- \\
- \\
-- \\
-\end{array}$ & $\begin{array}{r}3 \\
0 \\
-1 \\
4 \\
0\end{array}$ & $\begin{array}{r}-2 \\
-3 \\
-6 \\
7 \\
(s)\end{array}$ & $\begin{array}{l}- \\
-- \\
- \\
- \\
--\end{array}$ & $\begin{array}{r}126 \\
131 \\
1 \\
-6 \\
(s)\end{array}$ & $\begin{array}{r}2 \\
\text { (s) } \\
0 \\
2 \\
0\end{array}$ & $\begin{array}{r}24 \\
0 \\
24 \\
0 \\
0\end{array}$ \\
\hline 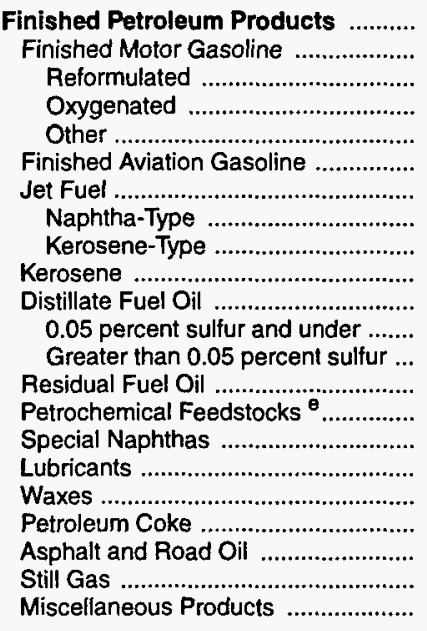 & $\begin{array}{r}12 \\
12 \\
- \\
45 \\
-32 \\
- \\
-. \\
-. \\
-- \\
-. \\
-- \\
-. \\
- \\
-- \\
- \\
-- \\
- \\
-- \\
-- \\
-- \\
-- \\
-\end{array}$ & $\begin{array}{r}2,806 \\
1,309 \\
920 \\
7 \\
383 \\
4 \\
434 \\
1 \\
433 \\
4 \\
449 \\
349 \\
101 \\
208 \\
10 \\
3 \\
23 \\
2 \\
156 \\
54 \\
144 \\
5\end{array}$ & $\begin{array}{r}21 \\
3 \\
0 \\
0 \\
3 \\
(s) \\
5 \\
0 \\
5 \\
(\mathrm{~s}) \\
6 \\
5 \\
1 \\
5 \\
1 \\
(\mathrm{~s}) \\
0 \\
\text { (s) } \\
1 \\
0 \\
0 \\
\text { (s) }\end{array}$ & $\begin{array}{l}- \\
- \\
- \\
- \\
- \\
- \\
-- \\
- \\
- \\
- \\
- \\
- \\
- \\
-- \\
- \\
- \\
- \\
- \\
- \\
- \\
- \\
- \\
-\end{array}$ & $\begin{array}{r}100 \\
73 \\
7 \\
0 \\
66 \\
0 \\
13 \\
0 \\
13 \\
0 \\
14 \\
8 \\
6 \\
0 \\
0 \\
0 \\
1 \\
0 \\
0 \\
0 \\
0 \\
0\end{array}$ & $\begin{array}{r}5 \\
2 \\
8 \\
(s) \\
-6 \\
(s) \\
4 \\
(s) \\
4 \\
(s) \\
-1 \\
-1 \\
(s) \\
-1 \\
(s) \\
(s) \\
(s) \\
(s) \\
1 \\
-1 \\
0 \\
(s)\end{array}$ & $\begin{array}{l}- \\
-- \\
- \\
-- \\
- \\
-- \\
-- \\
- \\
- \\
- \\
- \\
- \\
-- \\
-- \\
-- \\
-- \\
- \\
- \\
- \\
- \\
-\end{array}$ & $\begin{array}{l}-. \\
-- \\
-- \\
-- \\
-- \\
-- \\
- \\
-- \\
- \\
- \\
- \\
- \\
- \\
- \\
- \\
- \\
- \\
- \\
- \\
- \\
- \\
- \\
-\end{array}$ & $\begin{array}{r}249 \\
19 \\
(s) \\
1 \\
18 \\
0 \\
11 \\
(s) \\
11 \\
(s) \\
47 \\
13 \\
35 \\
46 \\
0 \\
19 \\
3 \\
(s) \\
102 \\
1 \\
0 \\
(s)\end{array}$ & $\begin{array}{r}2,686 \\
1,377 \\
919 \\
50 \\
407 \\
4 \\
437 \\
1 \\
437 \\
4 \\
422 \\
350 \\
73 \\
168 \\
11 \\
-16 \\
20 \\
2 \\
53 \\
54 \\
144 \\
5\end{array}$ \\
\hline Total & 2,412 & 2,879 & 502 & 41 & 6 & 2 & 0 & 2,722 & 345 & 2,771 \\
\hline
\end{tabular}

a Represents the PAD District in which the material entered the United States and not necessarily where the crude oil or product is processed and/or consumed.

b Unaccounted for crude oil represents the difference between the supply and disposition of crude oil.

c A negative number indicates a decrease in stocks and a positive number indicates an increase in stocks.

d Products supplied is equal to field production, plus refinery production, plus imports, plus unaccounted for crude oil, plus net receipts, minus stock change, minus crude losses, minus refinery inputs, minus exports.

- Includes naphtha less than $401^{\circ} \mathrm{F}$ endpoint and other oils equal to or greater than $401^{\circ} \mathrm{F}$ endpoint.

(s) = Less than 500 barrels per day.

LRG = Liquefied Refinery Gas.

Note: Totals may not equal sum of components due to independent rounding.

Sources: Energy Information Administration (EIA) Forms EIA-810, "Monthly Refinery Report," EIA-811, "Monthly Bulk Terminal Report," EIA-812, "Monthly Product Pipeline Report," EIA-813, "Monthly Crude Oil Report," EIA-814, "Monthly Imports Report," EIA-816, "Monthly Natural Gas Liquids Report," EIA-817, "Monthly Tanker and Barge Movement Report," and EIA-819M, "Monthly Oxygenate Telephone Report". Domestic crude oil production from State conservation agencies and the Minerals Management Service of the U.S. Department of the Interior. Export data from the Bureau of the Census and Form ElA-810, "Monthly Refinery Report." 
Table 14. Production of Crude Oil by PAD District and State, 1997 (Thousand Barrels)

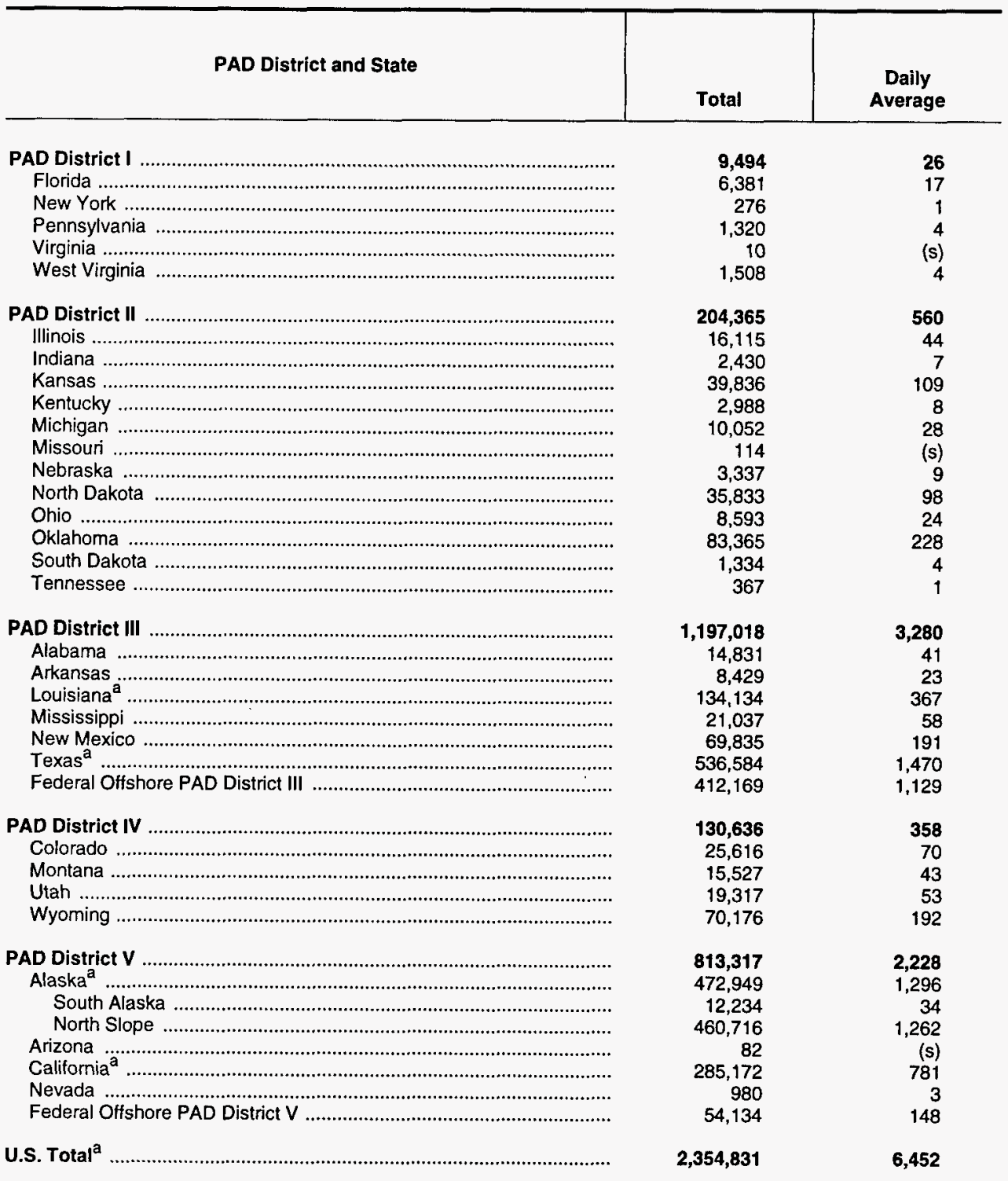

a Includes the following offshore production (thousand barrels): Alaska: State - 90,100; California: State - 21,495; Louisiana: State - 22,736; Texas: State -1,060; U.S. Total, including Federal offshore - 601,693.

$(s)=$ Less than 500 barrels or less than 500 barrels per day.

Note: - Crude oil production data for Alabama, Arkansas, Califomia, Colorado, Federal Ofíshore PAD District III Illinois, Indiana, Kansas, Kentucky, Michigan, Mississippi, Montana, Nebraska, New Mexico, New York, Ohio, Oklahoma, South Dakota, Texas, Utah, and Wyoming are changed from those reported in the Petroleum Supply Monthly during 1997. Crude oil production data for Arkansas, Federal Offshore PAD District III, Kansas, Montana, New Mexico, and Utah were estimated based on first purchaser monthly crude oil volumes collected on Form EIA-182, "Domestic Crude Oil First Purchase Report." - Annual crude oil production for New York and Ohio was prorated by month based on first purchaser monthly crude oil volumes collected on Form EIA-182. - A final revision to the State data for 1998 will appear in the 1998 Petroleum Supply Annual. - Totals may not equal sum of components due to independent rounding.

Sources: State government agencies, U.S. Department of the Interior, Minerals Management Service and the Conservation Committee of California Oil and Gas Producers.

Revised 1996 crude oil production statistics are available in Appendix C. 
Table 15. Natural Gas Plant Net Production and Stocks of Petroleum Products by PAD and Refining Districts, 1997

(Thousand Barrels)

\begin{tabular}{|c|c|c|c|c|c|c|c|}
\hline \multirow[b]{2}{*}{ Commodity } & \multicolumn{3}{|c|}{ PAD District I } & \multicolumn{4}{|c|}{ PAD District II } \\
\hline & $\begin{array}{l}\text { East } \\
\text { Coast }\end{array}$ & $\begin{array}{c}\text { Appalachian } \\
\text { No. } 1\end{array}$ & Total & Ind., Ill., Ky. & $\begin{array}{c}\text { Minn., Wis., } \\
\text { N. Dak., S. Dak. }\end{array}$ & $\begin{array}{c}\text { Okla., Kans., } \\
\text { Mo. }\end{array}$ & Total \\
\hline & \multicolumn{7}{|c|}{ Net Production } \\
\hline 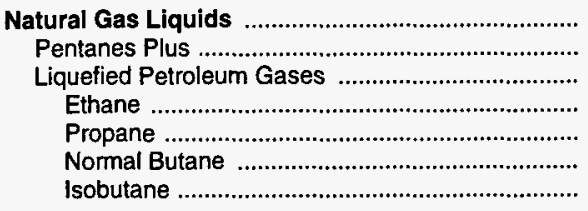 & $\begin{array}{r}1,596 \\
146 \\
1,450 \\
575 \\
534 \\
341 \\
0\end{array}$ & $\begin{array}{r}7,850 \\
857 \\
6,993 \\
2,340 \\
3,235 \\
976 \\
442\end{array}$ & $\begin{array}{r}9,446 \\
1,003 \\
8,443 \\
2,915 \\
3,769 \\
1,317 \\
442\end{array}$ & $\begin{array}{r}6,686 \\
1,147 \\
5,539 \\
1,405 \\
2,527 \\
866 \\
741\end{array}$ & $\begin{array}{r}3,887 \\
1,026 \\
2,861 \\
0 \\
1,771 \\
1,090 \\
0\end{array}$ & $\begin{array}{r}98,713 \\
13,298 \\
85,415 \\
31,953 \\
35,578 \\
10,898 \\
6,986\end{array}$ & $\begin{array}{r}109,286 \\
15,471 \\
93,815 \\
33,358 \\
39,876 \\
12,854 \\
7,727\end{array}$ \\
\hline & \multicolumn{7}{|c|}{ Stocks } \\
\hline 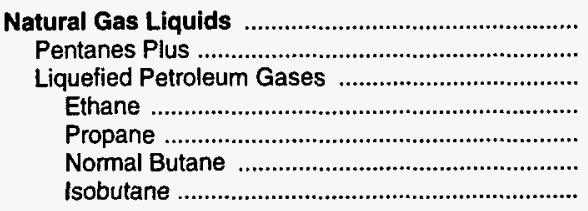 & $\begin{array}{l}8 \\
0 \\
8 \\
0 \\
3 \\
5 \\
0\end{array}$ & $\begin{array}{r}35 \\
3 \\
32 \\
0 \\
25 \\
4 \\
3\end{array}$ & $\begin{array}{r}43 \\
3 \\
40 \\
0 \\
28 \\
9 \\
3\end{array}$ & $\begin{array}{r}102 \\
13 \\
89 \\
17 \\
39 \\
15 \\
18\end{array}$ & $\begin{array}{r}41 \\
9 \\
32 \\
0 \\
21 \\
11 \\
0\end{array}$ & $\begin{array}{r}1,217 \\
202 \\
1,015 \\
150 \\
464 \\
275 \\
126\end{array}$ & $\begin{array}{r}1,360 \\
224 \\
1,136 \\
167 \\
524 \\
301 \\
144\end{array}$ \\
\hline
\end{tabular}

\begin{tabular}{|c|c|c|c|c|c|c|c|c|c|}
\hline \multirow{3}{*}{ Commodity } & \multicolumn{6}{|c|}{ PAD District Ill } & \multirow{2}{*}{$\begin{array}{l}\text { PAD Dist. } \\
\text { IV }\end{array}$} & \multirow{2}{*}{$\begin{array}{c}\text { PAD Dist. } \\
\text { v }\end{array}$} & \multirow{3}{*}{ U.S. } \\
\hline & \multirow{2}{*}{$\begin{array}{l}\text { Texas } \\
\text { Inland }\end{array}$} & \multirow{2}{*}{$\begin{array}{c}\text { Texas } \\
\text { Gulf } \\
\text { Coast }\end{array}$} & \multirow{2}{*}{$\begin{array}{c}\text { La. } \\
\text { Coast }\end{array}$} & \multirow{2}{*}{$\begin{array}{c}\text { N. La., } \\
\text { Ark. }\end{array}$} & \multirow{2}{*}{$\begin{array}{l}\text { New } \\
\text { Mexico }\end{array}$} & \multirow[b]{2}{*}{ Total } & & & \\
\hline & & & & & & & Rocky Mt. & West Coast & \\
\hline
\end{tabular}

Net Production

\begin{tabular}{|c|c|c|c|c|c|c|c|c|c|}
\hline \multirow[b]{2}{*}{ 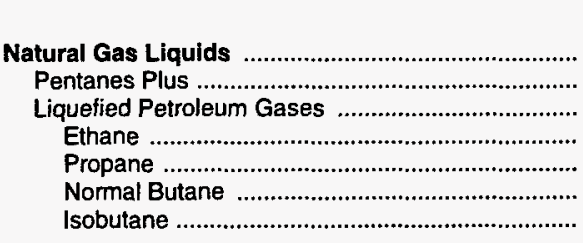 } & \\
\hline & $\begin{array}{r}220,787 \\
36,496 \\
184,291 \\
83,067 \\
63,638 \\
26,259 \\
11,327\end{array}$ & $\begin{array}{r}47,294 \\
6,897 \\
40,397 \\
22,304 \\
11,480 \\
-18,738 \\
25,351\end{array}$ & $\begin{array}{r}102,290 \\
17,497 \\
84,793 \\
35,856 \\
29,361 \\
9,984 \\
9,592\end{array}$ & $\begin{array}{r}8,298 \\
2,604 \\
5,694 \\
927 \\
2,518 \\
1,488 \\
761\end{array}$ & $\begin{array}{r}74,411 \\
8,238 \\
66,173 \\
34,725 \\
20,481 \\
7,311 \\
3,656\end{array}$ & $\begin{array}{r}453,080 \\
71,732 \\
381,348 \\
176,879 \\
127,478 \\
26,304 \\
50,687\end{array}$ & $\begin{array}{r}\mathbf{5 5 , 9 9 2} \\
9,389 \\
46,603 \\
19,451 \\
17,166 \\
6,387 \\
3,599\end{array}$ & $\begin{array}{r}35,462 \\
18,421 \\
17,041 \\
14 \\
4,249 \\
5,572 \\
7,206\end{array}$ & $\begin{array}{r}663,266 \\
116,016 \\
547,250 \\
232,617 \\
192,538 \\
52,434 \\
69,661\end{array}$ \\
\hline & \multicolumn{9}{|c|}{ Stocks } \\
\hline 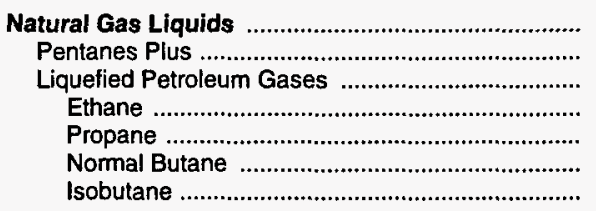 & $\begin{array}{r}214 \\
95 \\
119 \\
8 \\
71 \\
30 \\
10\end{array}$ & $\begin{array}{r}346 \\
126 \\
220 \\
49 \\
36 \\
67 \\
68\end{array}$ & $\begin{array}{r}835 \\
231 \\
604 \\
0 \\
59 \\
290 \\
255\end{array}$ & $\begin{array}{r}65 \\
27 \\
38 \\
0 \\
18 \\
12 \\
8\end{array}$ & $\begin{array}{r}97 \\
4 \\
93 \\
0 \\
60 \\
24 \\
9\end{array}$ & $\begin{array}{r}1,557 \\
483 \\
1,074 \\
57 \\
244 \\
423 \\
350\end{array}$ & $\begin{array}{r}309 \\
150 \\
159 \\
2 \\
92 \\
51 \\
14\end{array}$ & $\begin{array}{r}159 \\
20 \\
139 \\
0 \\
96 \\
15 \\
28\end{array}$ & $\begin{array}{r}3,428 \\
880 \\
2,548 \\
226 \\
984 \\
799 \\
539\end{array}$ \\
\hline
\end{tabular}

Note: - Stocks are reported as of the end of December. - Refer to Appendix A for Refining District descriptions.

Source: Energy Information Administration (EIA) Form EIA-816, "Monthly Natural Gas Liquids Report." 
Table 16. Refinery Input of Crude Oil and Petroleum Products by PAD and Refining Districts, 1997

(Thousand Barrels, Except Where Noted)

\begin{tabular}{|c|c|c|c|c|c|c|c|}
\hline \multirow{2}{*}{ Commodity } & \multicolumn{3}{|c|}{ PAD District I } & \multicolumn{4}{|c|}{ PAD District II } \\
\hline & $\begin{array}{l}\text { East } \\
\text { Coast }\end{array}$ & $\begin{array}{c}\text { Appalachian } \\
\text { No. } 1\end{array}$ & Total & Ind., III., Ky. & Minn., Wis., & $\begin{array}{c}\text { Okla., Kans., } \\
\text { Mo. }\end{array}$ & Total \\
\hline 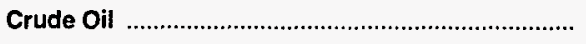 & 508,755 & 32,616 & 541,371 & 823,332 & 154,808 & 244,645 & $1,222,785$ \\
\hline 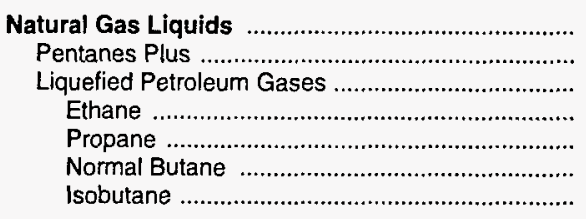 & $\begin{array}{r}1,519 \\
0 \\
1,519 \\
0 \\
0 \\
701 \\
818\end{array}$ & $\begin{array}{l}0 \\
0 \\
0 \\
0 \\
0 \\
0 \\
0\end{array}$ & $\begin{array}{r}1,519 \\
0 \\
1,519 \\
0 \\
0 \\
701 \\
818\end{array}$ & $\begin{array}{r}18,846 \\
2,438 \\
16,408 \\
0 \\
0 \\
8,432 \\
7,976\end{array}$ & $\begin{array}{r}2,669 \\
1,065 \\
1,604 \\
0 \\
0 \\
859 \\
745\end{array}$ & $\begin{array}{r}11,169 \\
6,799 \\
4,370 \\
0 \\
0 \\
2,306 \\
2,064\end{array}$ & $\begin{array}{r}32,684 \\
10,302 \\
22,382 \\
0 \\
0 \\
11,597 \\
10,785\end{array}$ \\
\hline $\begin{array}{l}\text { Other Liquids } \\
\text { Other Hydrocarbons/Hydrogen/Oxygenates } \\
\text { Other Hydrocabons/Hydrogen } \\
\text { Oxygenates } \\
\text { Fuel Ethanol } \\
\text { Methanol } \\
\text { MTBE } \\
\text { Other Oxygenates }{ }^{a} \\
\text { Unfinished Oils (net) } \\
\text { Motor Gasoline Blend. Comp. (net) } \\
\text { Aviation Gasoline Blend. Comp. (net) }\end{array}$ & $\begin{array}{r}120,841 \\
24,838 \\
14 \\
W \\
W \\
W \\
W \\
W \\
33,890 \\
63,790 \\
-1,677\end{array}$ & $\begin{array}{r}451 \\
8 \\
0 \\
W \\
W \\
W \\
W \\
W \\
456 \\
-13 \\
0\end{array}$ & $\begin{array}{r}121,292 \\
24,846 \\
14 \\
24,832 \\
W \\
W \\
23,584 \\
W \\
34,346 \\
63,777 \\
-1,677\end{array}$ & $\begin{array}{r}18,965 \\
9,229 \\
410 \\
8,819 \\
W \\
W \\
W \\
W \\
19,130 \\
-9,380 \\
-14\end{array}$ & $\begin{array}{r}3,660 \\
2,477 \\
0 \\
2,477 \\
W \\
W \\
W \\
W \\
-167 \\
1,350 \\
0\end{array}$ & $\begin{array}{r}-8,441 \\
1,146 \\
335 \\
811 \\
W \\
W \\
W \\
W \\
-10,051 \\
464 \\
0\end{array}$ & $\begin{array}{r}14,184 \\
12,852 \\
745 \\
12,107 \\
10,222 \\
W \\
W \\
W \\
8,912 \\
-7,566 \\
-14\end{array}$ \\
\hline 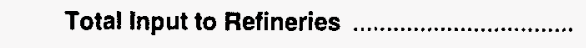 & 631,115 & 33,067 & 664,182 & 861,143 & 161,137 & 247,373 & $1,269,653$ \\
\hline 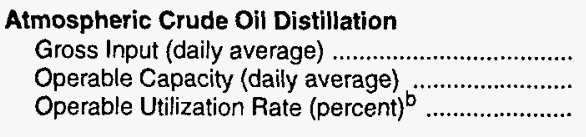 & $\begin{array}{r}1,361 \\
1,516 \\
89.8\end{array}$ & $\begin{array}{r}90 \\
97 \\
92.1\end{array}$ & $\begin{array}{r}1,450 \\
1,614 \\
89.9\end{array}$ & $\begin{array}{r}2,310 \\
2,341 \\
98.7\end{array}$ & $\begin{array}{r}425 \\
413 \\
102.8\end{array}$ & $\begin{array}{r}677 \\
695 \\
97.4\end{array}$ & $\begin{array}{r}3,411 \\
3,449 \\
98.9\end{array}$ \\
\hline $\begin{array}{l}\text { Downstream Processing } \\
\text { Fresh Feed Input (daily average) } \\
\text { Catalytic Cracking } \\
\text { Catalytic Hydrocracking } \\
\text { Delayed and Fluid Coking }\end{array}$ & $\begin{array}{r}592 \\
41 \\
80\end{array}$ & $\begin{array}{r}16 \\
2 \\
0\end{array}$ & $\begin{array}{r}608 \\
43 \\
80\end{array}$ & $\begin{array}{l}811 \\
133 \\
185\end{array}$ & $\begin{array}{r}134 \\
0 \\
64\end{array}$ & $\begin{array}{r}192 \\
5 \\
71\end{array}$ & $\begin{array}{r}1,137 \\
138 \\
320\end{array}$ \\
\hline $\begin{array}{l}\text { Crude Oil Qualities } \\
\text { Sulfur Content, Weighted Average (percent) ......... } \\
\text { API Gravity, Weighted Average (degrees) ............. }\end{array}$ & $\begin{array}{r}0.94 \\
32.93\end{array}$ & $\begin{array}{r}0.96 \\
34.62\end{array}$ & $\begin{array}{r}0.94 \\
33.03\end{array}$ & $\begin{array}{r}1.17 \\
33.32\end{array}$ & $\begin{array}{r}2.20 \\
29.19\end{array}$ & $\begin{array}{r}0.80 \\
35.91\end{array}$ & $\begin{array}{r}1.23 \\
33.31\end{array}$ \\
\hline $\begin{array}{l}\text { Operable Capacity (daily average) } \\
\text { Operating }\end{array}$ & $\begin{array}{r}1,516 \\
1,387 \\
129\end{array}$ & $\begin{array}{r}97 \\
97 \\
0\end{array}$ & $\begin{array}{r}1,614 \\
1,485 \\
129\end{array}$ & $\begin{array}{r}2,341 \\
2,341 \\
0\end{array}$ & $\begin{array}{r}413 \\
413 \\
0\end{array}$ & $\begin{array}{r}695 \\
695 \\
0\end{array}$ & $\begin{array}{r}3,449 \\
3,449 \\
0\end{array}$ \\
\hline 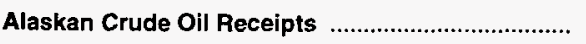 & 0 & $\mathbf{0}$ & 0 & 3,112 & 0 & $\mathbf{0}$ & 3,112 \\
\hline
\end{tabular}

See foothotes at end of table. 
Table 16. Refinery Input of Crude Oil and Petroleum Products by PAD and Refining Districts, 1997 (Continued) (Thousand Barrels, Except Where Noted)

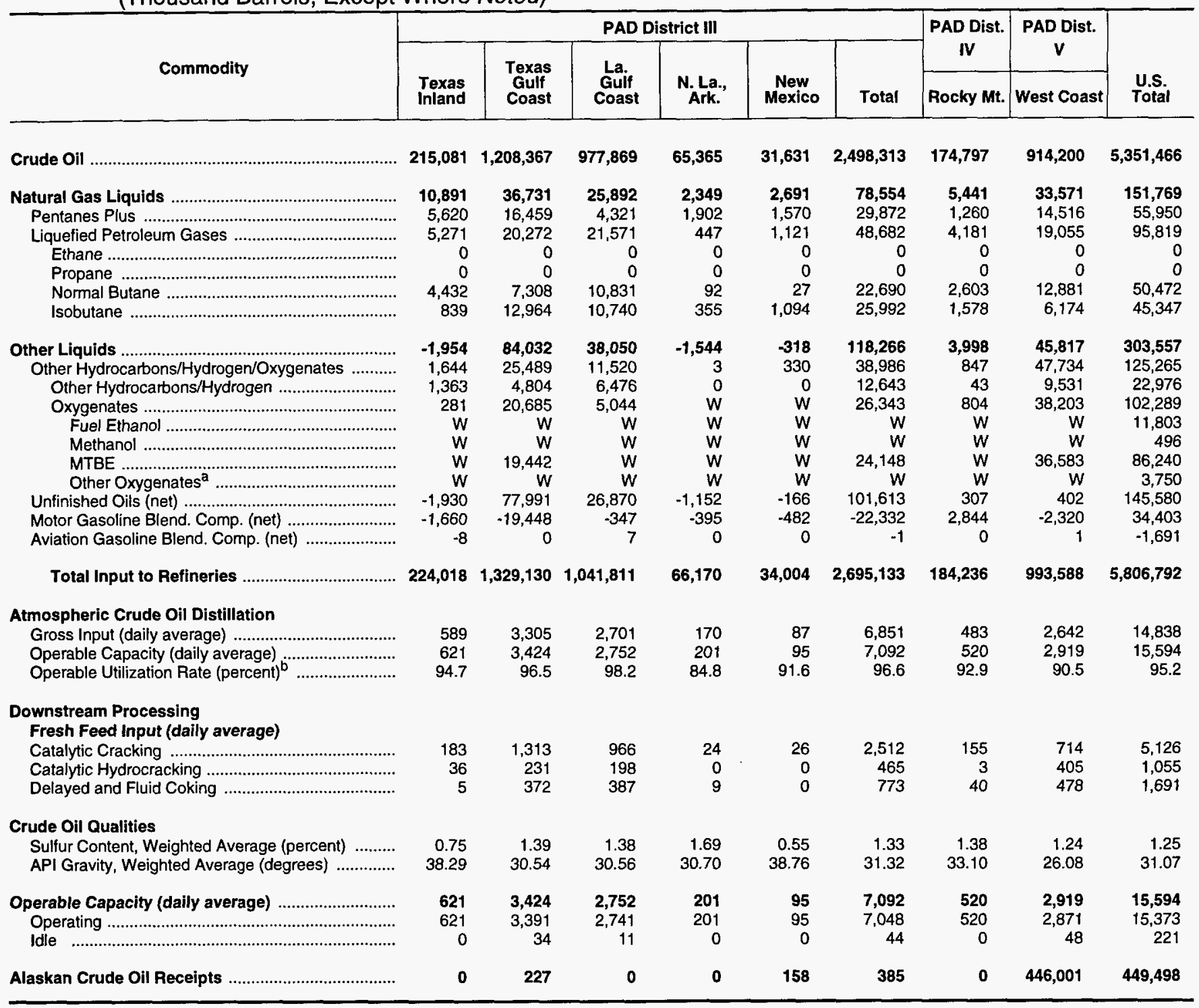

a Includes ethyl tertiary butyl ether (ETBE), tertiary amyl methyl ether (TAME), tertiary butyl alcohol (TBA), and other aliphatic alcohols and ethers intended for motor gasoline blending (e.g., isopropyl ether (IPE) or n-propanol).

b Represents gross input divided by operable capacity.

$W=$ Witheld to avoid disclosure of individual company data.

Note: - Totals may not equal sum of components due to independent rounding. - Refer to Appendix A for Refining District descriptions.

Source: Energy Information Administration (EIA) Form EIA-810, "Monthly Refinery Report." 
Table 17. Refinery Net Production of Finished Petroleum Products by PAD and Refining Districts, 1997

(Thousand Barrels)

\begin{tabular}{|c|c|c|c|c|c|c|c|}
\hline \multirow[b]{2}{*}{ Commodity } & \multicolumn{3}{|c|}{ PAD District I } & \multicolumn{4}{|c|}{ PAD District \| } \\
\hline & $\begin{array}{l}\text { East } \\
\text { Coast }\end{array}$ & $\begin{array}{c}\text { Appalachian } \\
\text { No. } 1\end{array}$ & Total & Ind., III., Ky. & $\begin{array}{c}\text { Minn., Wis., } \\
\text { N. Dak., } \\
\text { S. Dak. }\end{array}$ & $\begin{array}{c}\text { Okla., Kans., } \\
\text { Mo. }\end{array}$ & Total \\
\hline 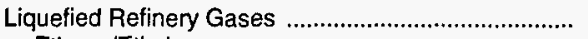 & 16,433 & 560 & 16,993 & 37,285 & 3,543 & 6,983 & 47,811 \\
\hline 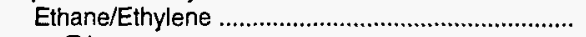 & 0 & 0 & 0 & 0 & 0 & 0 & 0 \\
\hline Ethane & $W$ & $W$ & $w$ & W & W & W & $W$ \\
\hline 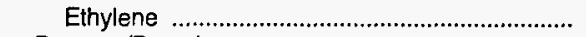 & W & $W$ & $\mathbf{w}$ & $\mathbf{W}$ & W & W & W \\
\hline 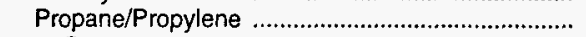 & 17,986 & 458 & 18,444 & 33,513 & 3,997 & 7,343 & 44,853 \\
\hline Propane . & W & W & W & 25,565 & W & $W$ & W \\
\hline 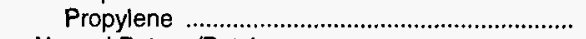 & W & W & $\mathbf{W}$ & 7,948 & W & W & W \\
\hline 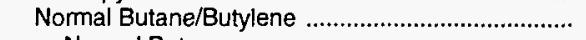 & -924 & 136 &.-788 & 2,435 & -371 & -22 & 2,042 \\
\hline 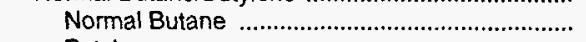 & W & $W$ & W & $W$ & $w$ & $\bar{W}$ & W \\
\hline Butylene & W & W & W & W & W & W & W \\
\hline 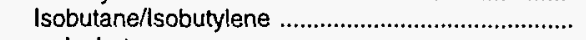 & -629 & -34 & -663 & 1,337 & -83 & -338 & 916 \\
\hline Isobutane & W & W & W & $W$ & $W$ & W & W \\
\hline Isobutylene & $\mathbf{W}$ & W & W & $\ddot{W}$ & W & $W$ & W \\
\hline Finished Motor Gasoline & 337,783 & 12,347 & 350,130 & 452,178 & 84,888 & 128,419 & 665,485 \\
\hline Reformulated & 224,012 & 0 & 224,012 & 78,303 & 9,168 & 0 & 87,471 \\
\hline 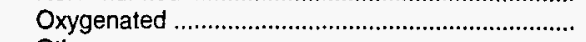 & 8 & 0 & 8 & 6,518 & 15,096 & 284 & 21,898 \\
\hline Other & 113,763 & 12,347 & 126,110 & 367,357 & 60,624 & 128,135 & 556,116 \\
\hline 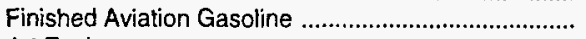 & 39 & 0 & 39 & 705 & 370 & 336 & 1,411 \\
\hline Jet Fuel & 32,735 & 381 & 33,116 & 54,583 & 11,394 & 12,823 & 78,800 \\
\hline Naphtha-Type & 0 & 0 & 0 & 15 & 0 & 0 & 15 \\
\hline Kerosene-Type & 32,735 & 381 & 33,116 & 54,568 & 11,394 & 12,823 & 78,785 \\
\hline 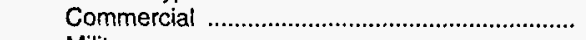 & 32,735 & 287 & 33,022 & 52,152 & 10,478 & 11,243 & 73,873 \\
\hline Military & 0 & 94 & 94 & 2,416 & 916 & 1,580 & 4,912 \\
\hline Kerosene & 2,880 & 942 & 3,822 & 4,633 & 729 & 1,126 & 6,488 \\
\hline 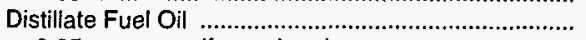 & 146,058 & 8,713 & 154,771 & 194,254 & 38,642 & 74,347 & 307,243 \\
\hline 0.05 percent sulfur and under ...................................... & 46,952 & 7,111 & 54,063 & 133,706 & 29,657 & 54,959 & 218,322 \\
\hline 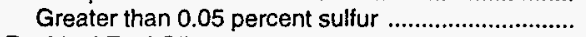 & 99,106 & 1,602 & 100,708 & 60,548 & 8,985 & 19,388 & 88,921 \\
\hline 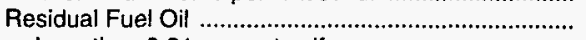 & 45,494 & 924 & 46,418 & 16,442 & 4,023 & 1,050 & 21,515 \\
\hline 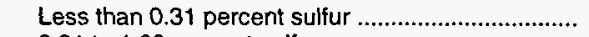 & 16,383 & 411 & 16,794 & 49 & 0 & 0 & 49 \\
\hline 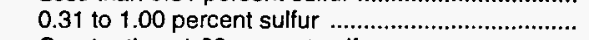 & 24,621 & 513 & 25,134 & 4,054 & 0 & 12 & 4,066 \\
\hline 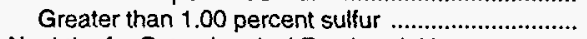 & 4,490 & 0 & 4,490 & 12,339 & 4,023 & 1,038 & 17,400 \\
\hline Naphtha for Petrochemical Feedstock Use ................. & 5,417 & 0 & 5,417 & 6,770 & 0 & 302 & 7,072 \\
\hline Other Oils for Petrochemical Feedstock Use .............. & 0 & 0 & 0 & 8,087 & 0 & 767 & 8,854 \\
\hline 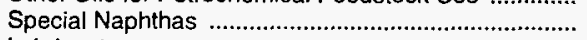 & 414 & 301 & 715 & 5,039 & 0 & 840 & 5,879 \\
\hline Lubricants & 4,189 & 2,576 & 6,765 & 5,362 & 0 & 2,949 & 8,311 \\
\hline 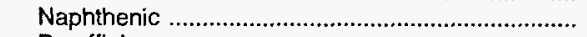 & 0 & 0 & 0 & 0 & 0 & 0 & 0 \\
\hline 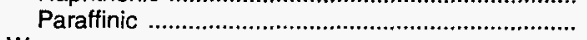 & 4,189 & 2,576 & 6,765 & 5,362 & 0 & 2,949 & 8,311 \\
\hline Waxes & 0 & 571 & 571 & 644 & 0 & 495 & 1,139 \\
\hline Petroleum Coke & $\uparrow 7,716$ & 295 & 18,011 & 31,499 & 9,561 & 9,430 & 50,490 \\
\hline 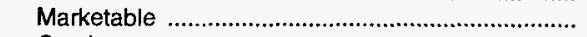 & 7,014 & 0 & 7,014 & 18,450 & 7,559 & 7,107 & 33,116 \\
\hline 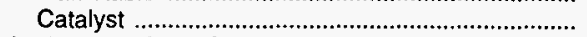 & 10,702 & 295 & 10,997 & 13,049 & 2,002 & 2,323 & 17,374 \\
\hline 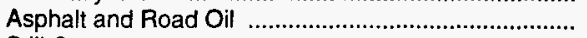 & 24,392 & 4,475 & 28,867 & 49,217 & 12,551 & 7,910 & 69,678 \\
\hline Still Gas & 20,712 & 1,004 & 21,716 & 33,164 & 5,355 & 9,259 & 47,778 \\
\hline 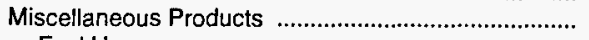 & 305 & 476 & 781 & 1,930 & 889 & 578 & 3,397 \\
\hline 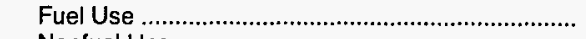 & 0 & 0 & 0 & & 0 & 0 & 0 \\
\hline 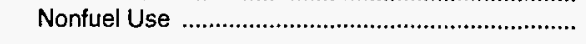 & 305 & 476 & 781 & 1,930 & 889 & 578 & 3,397 \\
\hline Total & 654,567 & 33,565 & 688,132 & 901,792 & 171,945 & 257,614 & $1,331,351$ \\
\hline 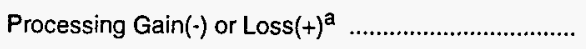 & $-23,452$ & -498 & $-23,950$ & $-40,649$ & $-10,808$ & $-10,241$ & $-61,698$ \\
\hline
\end{tabular}

See footnotes at end of table. 
Table 17. Refinery Net Production of Finished Petroleum Products by PAD and Refining Districts, 1997 (Continued)

(Thousand Barrels)

\begin{tabular}{|c|c|c|c|c|c|c|c|c|c|}
\hline \multirow[b]{2}{*}{ Commodity } & \multicolumn{6}{|c|}{ PAD District III } & \multirow{2}{*}{\begin{tabular}{|c|}
$\begin{array}{c}\text { PAD Dist. } \\
\text { IV }\end{array}$ \\
Rocky Mt.
\end{tabular}} & \multirow{2}{*}{\begin{tabular}{|c|}
$\begin{array}{c}\text { PAD Dist. } \\
V\end{array}$ \\
West Coast
\end{tabular}} & \multirow[b]{2}{*}{$\begin{array}{l}\text { U.S. } \\
\text { Total }\end{array}$} \\
\hline & $\begin{array}{l}\text { Texas } \\
\text { Inland }\end{array}$ & $\begin{array}{l}\text { Texas } \\
\text { Gulf } \\
\text { Coast }\end{array}$ & $\begin{array}{l}\text { La. } \\
\text { Gulf } \\
\text { Coast }\end{array}$ & $\begin{array}{l}\text { N. La., } \\
\text { Ark. }\end{array}$ & $\begin{array}{c}\text { New } \\
\text { Mexico }\end{array}$ & Total & & & \\
\hline Liquefied Refinery Gases ...... & 10,634 & 91,344 & 54,938 & 629 & 893 & 158,438 & 2,342 & 26,584 & 252,168 \\
\hline Ethane/Ethylene ................ & 383 & 7,506 & 1,784 & 0 & 0 & 9,673 & 0 & 0 & 9,673 \\
\hline 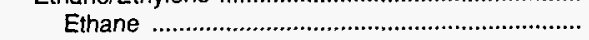 & W & W & $W$ & $\mathbf{W}$ & W & $W$ & W & $w$ & 7,843 \\
\hline Ethylene & $W$ & $W$ & $\ddot{w}$ & $\ddot{W}$ & W & $w$ & W & $w$ & 1,830 \\
\hline Propane/Propylene & 7,908 & 66,781 & 45,515 & 935 & 657 & 121,796 & 3,427 & 17,574 & 206,094 \\
\hline Propane & W & 30,578 & 28,515 & W & W & 65,396 & $W$ & W & 133,557 \\
\hline 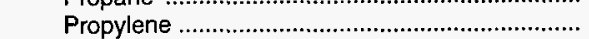 & W & 36,203 & 17,000 & W & W & 56,400 & W & W & 72,537 \\
\hline Nomal Butane/Butylene .... & 2,714 & 14,690 & 6,522 & -195 & 202 & 23,933 & -550 & 7,565 & 32,202 \\
\hline 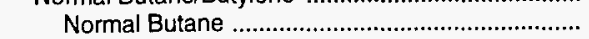 & $W$ & W & $W$ & W & W & W & W & W & 31,985 \\
\hline Butylene & W & W & W & W & $W$ & W & W & W & 217 \\
\hline Isobutane/Isobutylene & -371 & 2,367 & 1,117 & -111 & 34 & 3,036 & -535 & 1,445 & 4,199 \\
\hline Isobutane & $W$ & W & $W$ & $w$ & $w$ & $\dot{W}$ & W & W & 3,039 \\
\hline 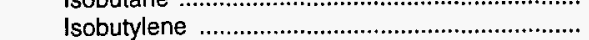 & $w$ & $w$ & W & W & $W$ & $w$ & W & W & 1,160 \\
\hline 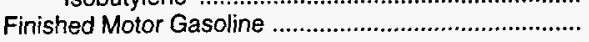 & 119,296 & 604,215 & 479,197 & 18,126 & 19,091 & $1,239,925$ & 92,581 & 477,930 & $2,826,051$ \\
\hline Reformulated & 8,019 & 170,599 & 52,233 & 0 & 0 & 230,851 & 0 & 335,866 & 878,200 \\
\hline (2) & 388 & 0 & 324 & 0 & 1,290 & 2,002 & 6,964 & 2,374 & 33,246 \\
\hline 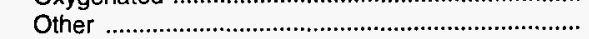 & 110,889 & 433,616 & 426,640 & 18,126 & 17,801 & $1,007,072$ & 85,617 & 139,690 & $1,914,605$ \\
\hline 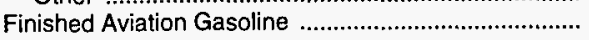 & 1,444 & 1,609 & 1,006 & 0 & 0 & 4,059 & 163 & 1,576 & 7,248 \\
\hline Jet Fuel & 20,210 & 124,658 & 137,169 & 3,224 & 2,310 & 287,571 & 9,546 & 158,262 & 567,295 \\
\hline 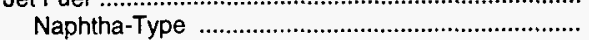 & 9 & 0 & 0 & 0 & 0 & 9 & 0 & 217 & 241 \\
\hline 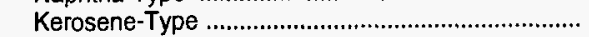 & 20,201 & 124,658 & 137,169 & 3,224 & 2,310 & 287,562 & 9,546 & 158,045 & 567,054 \\
\hline Commercial & 14,366 & 112,729 & 128,967 & 2,495 & 0 & 258,557 & 7,826 & 141,274 & 514,552 \\
\hline 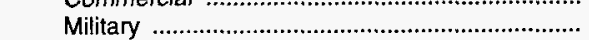 & 5,835 & 11,929 & 8,202 & 729 & 2,310 & 29,005 & 1,720 & 16,771 & 52,502 \\
\hline 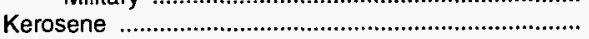 & 72 & 8,407 & 2,081 & 719 & 7 & 11,286 & 884 & 1,407 & 23,887 \\
\hline 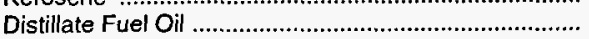 & 53,732 & 265,778 & 218,206 & 15,318 & 8,651 & 561,685 & 50,277 & 164,065 & $1,238,041$ \\
\hline 0.05 percent sulfur and under & 39,748 & 184,405 & 108,689 & 7,748 & 8,299 & 348,889 & 40,798 & 127,215 & 789,287 \\
\hline 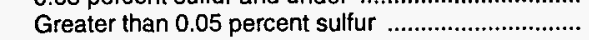 & 13,984 & 81,373 & 109,517 & 7,570 & 352 & 212,796 & 9,479 & 36,850 & 448,754 \\
\hline Residual Fuel Oil & 3,506 & 57,598 & 45,880 & 2,548 & 272 & 109,804 & 4,664 & 75,889 & 258,290 \\
\hline Less than 0.31 percent sulfur & 1,395 & 91 & 4,381 & 0 & 0 & 5,867 & 959 & 2,421 & 26,090 \\
\hline 0.31 to 1.00 percent sulfur & 1,354 & 13,092 & 8,573 & 2,244 & 272 & 25,535 & 1,257 & 19,342 & 75,334 \\
\hline Greater than 1.00 percent sulfur & 757 & 44,415 & 32,926 & 304 & 0 & 78,402 & 2,448 & 54,126 & 156,866 \\
\hline Naphtha for Petrochemical Feedstock Use .................. & 1,302 & 57,704 & 10,761 & 0 & 12 & 69,779 & 0 & 1,292 & 83,560 \\
\hline Other Oils for Petrochemical Feedstock Use ............... & 1,704 & 37,252 & 29,053 & 0 & 0 & 68,009 & 234 & 2,442 & 79,539 \\
\hline Special Naphthas & 1,146 & 7,125 & 1,662 & 1,631 & 0 & 11,564 & -1 & 1,034 & 19,191 \\
\hline 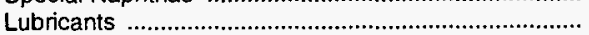 & $W$ & 20,541 & W & W & W & 42,345 & 0 & 8,478 & 65,899 \\
\hline 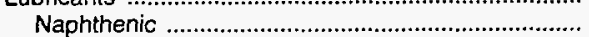 & W & 4,275 & W & w & W & 10,762 & 0 & 3,622 & 14,384 \\
\hline 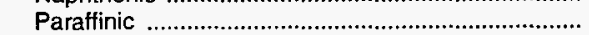 & $\mathbf{W}$ & 16,266 & $\dddot{W}$ & $\ddot{w}$ & $\ddot{W}$ & 31,583 & 0 & 4,856 & 51,515 \\
\hline Waxes & 55 & 2,500 & 1,046 & 1,013 & 0 & 4,614 & 1,157 & 891 & 8,372 \\
\hline Petroleum Coke ..... & 3,491 & 64,247 & 51,982 & 916 & 240 & 120,876 & 5,414 & 56,828 & 251,619 \\
\hline . & 375 & 41,374 & 38,180 & 680 & 0 & 80,609 & 3,082 & 43,504 & 167,325 \\
\hline 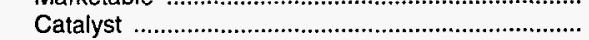 & 3,116 & 22,873 & 13,802 & 236 & 240 & 40,267 & 2,332 & 13,324 & 84,294 \\
\hline Asphalt and Road Oil. & 6,237 & 11,646 & 12,098 & 12,775 & 1,574 & 44,330 & 14,463 & 19,681 & 177,019 \\
\hline Still Gas & 8,945 & 60,772 & 39,384 & 2,083 & 934 & 112,118 & 6,910 & 52,662 & 241,184 \\
\hline Miscellaneous Products & 760 & 4,522 & 5,586 & 0 & 0 & 10,868 & 673 & 1,788 & 17,507 \\
\hline Fuel Use & 238 & & 2,131 & 0 & 0 & 2,369 & 0 & -321 & 2,048 \\
\hline 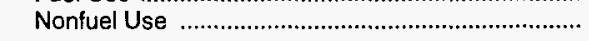 & 522 & 4,522 & 3,455 & 0 & 0 & 8,499 & 673 & 2,109 & 15,459 \\
\hline Total & 233,074 & $1,419,918$ & $1,103,611$ & 66,684 & 33,984 & $2,857,271$ & 189,307 & $1,050,809$ & $6,116,870$ \\
\hline Processing Gain(-) or Loss $(+)^{a}$. & $-9,056$ & $-90,788$ & $-61,800$ & -514 & 20 & $-162,138$ & $-5,071$ & $-57,221$ & $-310,078$ \\
\hline
\end{tabular}

a Represents the arithmetic difference between input and production.

$W=$ Withheld to avoid disclosure of individual company data.

Note: Refer to Appendix A for refining District descriptions.

Source: Energy Information Administration (EIA) Form ElA-810, "Monthly Refinery Report." 
Table 18. Refinery Stocks of Crude Oil and Petroleum Products by PAD and Refining Districts, 1997

(Thousand Barrels)

\begin{tabular}{|c|c|c|c|c|c|c|c|}
\hline \multirow[b]{2}{*}{ Commodity } & \multicolumn{3}{|c|}{ PAD District I } & \multicolumn{4}{|c|}{ PAD District II } \\
\hline & $\begin{array}{c}\text { East } \\
\text { Coast }\end{array}$ & $\begin{array}{c}\text { Appalachian } \\
\text { No. } 1\end{array}$ & Total & Ind., III., Ky. & $\begin{array}{c}\text { Minn., Wis., } \\
\text { N. Dak., } \\
\text { S. Dak. }\end{array}$ & $\begin{array}{c}\text { Okla. Kans., } \\
\text { Mo. }\end{array}$ & Total \\
\hline Crude Oil . & 9,601 & 541 & 10,142 & 8,206 & 1,790 & 2,826 & 12,822 \\
\hline Petroleum Products & 53,974 & 2,602 & 56,576 & 37,830 & 10,195 & 12,775 & 60,800 \\
\hline 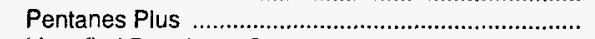 & 0 & 0 & 0 & 4 & 219 & 223 & 446 \\
\hline 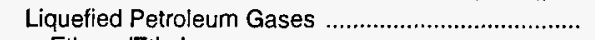 & 1,890 & 14 & 1,904 & 2,266 & 382 & 1,063 & 3,711 \\
\hline Ethane/Ethylene & 0 & 0 & 0 & 3 & 0 & 0 & 3 \\
\hline 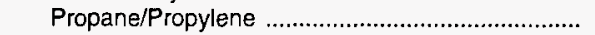 & 527 & 3 & 530 & 1,422 & 26 & 620 & 2,068 \\
\hline 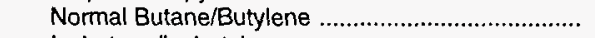 & 962 & 9 & 971 & 545 & 281 & 296 & 1,122 \\
\hline Isobutane/lsobutylene & 404 & 2 & 403 & 296 & 75 & 147 & 518 \\
\hline Other Hydrocarbons/Hydrogen/Oxygenates .............. & 2,010 & 7 & 2,017 & 336 & 129 & 57 & 522 \\
\hline 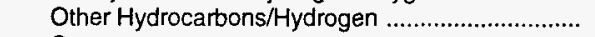 & 0 & 0 & 0 & 19 & 0 & 0 & 19 \\
\hline Oxygenates & $W$ & $W$ & 2,017 & 317 & 129 & 57 & 503 \\
\hline 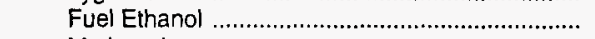 & $W$ & W & W & W & W & W & 302 \\
\hline 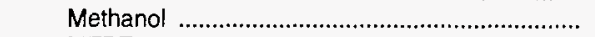 & W & W & W & W & W & $w$ & W \\
\hline MTBE & $W$ & W & 1,574 & W & $\ddot{W}$ & W & W \\
\hline 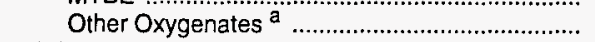 & $W$ & W & W & W & W & $W$ & W \\
\hline 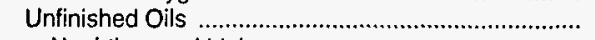 & 9,384 & 722 & 10,106 & 7,858 & 535 & 3,916 & 12,309 \\
\hline 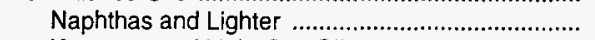 & 1,771 & 332 & 2,103 & 2,262 & 202 & 959 & 3,423 \\
\hline 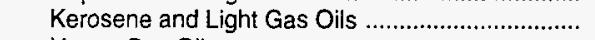 & 2,784 & 4 & 2,788 & 1,325 & 66 & 253 & 1,644 \\
\hline 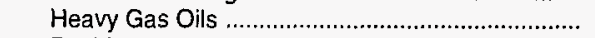 & 3,805 & 332 & 4,137 & 2,405 & 259 & 1,817 & 4,481 \\
\hline 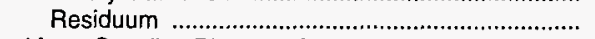 & 1,024 & 54 & 1,078 & 1,866 & 8 & 887 & 2,761 \\
\hline Motor Gasoline Blending Components ...................... & 6,071 & 38 & 6,109 & 6,225 & 1,204 & 1,122 & 8,551 \\
\hline Aviation Gasoline Blending Components .................... & 79 & 0 & 79 & 36 & 0 & 0 & 36 \\
\hline 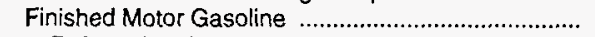 & 9,266 & 303 & 9,569 & 5,724 & 1,199 & 2,057 & 8,980 \\
\hline Reformulated & 5,038 & 0 & 5,038 & 311 & 0 & 0 & 311 \\
\hline Oxygenated & 0 & 9 & 9 & 113 & 291 & 0 & 404 \\
\hline 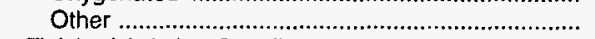 & 4,228 & 294 & 4,522 & 5,300 & 908 & 2,057 & 8,265 \\
\hline 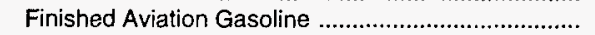 & 41 & 0 & 41 & 42 & 35 & 44 & 121 \\
\hline Jet Fuel & 1,517 & 25 & 1,542 & 2,528 & 130 & 459 & 3,117 \\
\hline 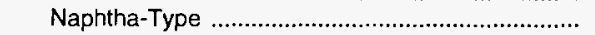 & 0 & 0 & 0 & 0 & 0 & 0 & 0 \\
\hline Kerosene-Type & 1,517 & 25 & 1,542 & 2,528 & 130 & 459 & 3,117 \\
\hline 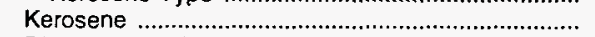 & 936 & 45 & 981 & 298 & 175 & 117 & 590 \\
\hline Distillate Fuel Oil & 15,732 & 317 & 16,049 & 5,733 & 1,942 & 1,799 & 9,474 \\
\hline 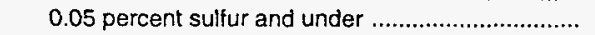 & 2,408 & 287 & 2,695 & 3,503 & 1,159 & 1,205 & 5,867 \\
\hline 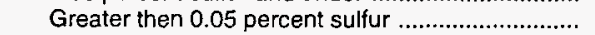 & 13,324 & 30 & 13,354 & 2,230 & 783 & 594 & 3,607 \\
\hline 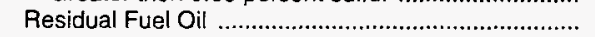 & 5,039 & 59 & 5,098 & 1,277 & 378 & 101 & 1,756 \\
\hline 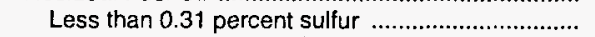 & 1,452 & 39 & 1,491 & 0 & 0 & 0 & 0 \\
\hline 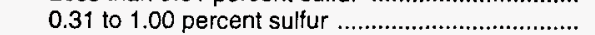 & 2,119 & 20 & 2,139 & 225 & 0 & 1 & 226 \\
\hline 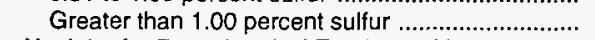 & 1,468 & 0 & 1,468 & 1,052 & 378 & 100 & 1,530 \\
\hline Naphtha for Petrochemical Feedstock Use ................ & 478 & 0 & 478 & 144 & 0 & 3 & 147 \\
\hline Other Oils for Petrochemical Feedstock Use ............. & 0 & 0 & 0 & 216 & 0 & 0 & 216 \\
\hline Special Naphthas & 52 & 34 & 86 & 453 & 0 & 25 & 478 \\
\hline Lubricants & 424 & 275 & 699 & 789 & 0 & 0 & 789 \\
\hline Waxes & 0 & 45 & 45 & 102 & 0 & 42 & 144 \\
\hline Petroleum Coke (Marketable) & 267 & 0 & 267 & 768 & 2,175 & 271 & 3,214 \\
\hline 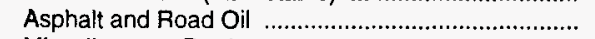 & 783 & 679 & 1,462 & 2,933 & 1,675 & 1,454 & 6,062 \\
\hline 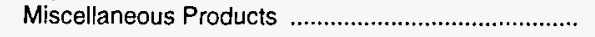 & 5 & 39 & 44 & 98 & 17 & 22 & 137 \\
\hline 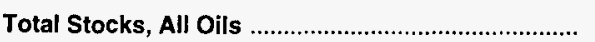 & 63,575 & 3,143 & 66,718 & 46,036 & 11,985 & 15,601 & 73,622 \\
\hline
\end{tabular}

See footnotes at end of table. 
Table 18. Refinery Stocks of Crude Oil and Petroleum Products by PAD and Refining Districts, 1997 (Continued)

(Thousand Barrels)

\begin{tabular}{|c|c|c|c|c|c|c|c|c|c|}
\hline \multirow{3}{*}{ Commodity } & \multicolumn{6}{|c|}{ PAD District III } & \multirow{3}{*}{\begin{tabular}{|c|}
$\begin{array}{c}\text { PAD Dist. } \\
\text { IV }\end{array}$ \\
Rocky Mt.
\end{tabular}} & \multirow{3}{*}{\begin{tabular}{|c|}
$\begin{array}{c}\text { PAD Dist. } \\
V\end{array}$ \\
West Coast
\end{tabular}} & \multirow[b]{3}{*}{$\begin{array}{l}\text { U.S. } \\
\text { Total }\end{array}$} \\
\hline & \multirow[b]{2}{*}{$\begin{array}{l}\text { Texas } \\
\text { Inland }\end{array}$} & \multirow{2}{*}{$\begin{array}{l}\text { Texas } \\
\text { Gulf } \\
\text { Coast }\end{array}$} & \multirow{2}{*}{$\begin{array}{c}\text { La. } \\
\text { Guif } \\
\text { Coast }\end{array}$} & \multirow[b]{2}{*}{$\begin{array}{l}\text { N. La., } \\
\text { Ark. }\end{array}$} & \multirow[b]{2}{*}{$\begin{array}{c}\text { New } \\
\text { Mexico }\end{array}$} & \multirow[b]{2}{*}{ Total } & & & \\
\hline & & & & & & & & & \\
\hline Crude Oil ....... & 887 & 25,748 & 17,250 & 1,095 & 406 & 45,386 & 2,266 & 20,047 & 90,663 \\
\hline Petroleum Products & 11,162 & 65,253 & 52,164 & 4,410 & 1,832 & 134,821 & 11,603 & 66,431 & 330,231 \\
\hline Pentanes Plus ......... & 121 & 69 & 23 & 12 & 11 & 236 & 10 & 0 & 692 \\
\hline Liquefied Petroleum Gases ........................................ & 2,095 & 2,926 & 3,139 & 46 & 75 & 8,281 & 378 & 1,167 & 15,441 \\
\hline 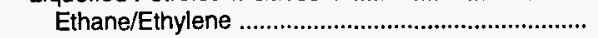 & 45 & 644 & 0 & 0 & 0 & 689 & 0 & 0 & 692 \\
\hline Propane/Propylene ………….................................. & 1,059 & 922 & 775 & 6 & 6 & 2,768 & 117 & 231 & 5,714 \\
\hline Normal Butane/Butylene ............................................ & 601 & 845 & 1,774 & 16 & 42 & 3,278 & 180 & 585 & 6,136 \\
\hline Isobutane/Isobutylene ............................................... & 390 & 515 & 590 & 24 & 27 & 1,546 & 81 & 351 & 2,899 \\
\hline Other Hydrocarbons/HydrogenOXxygenates .............. & 28 & 1,376 & 797 & 8 & 4 & 2,213 & 85 & 2,318 & 7,155 \\
\hline 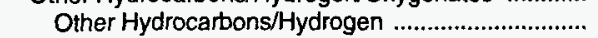 & 0 & 0 & 1 & 0 & 0 & 1 & 0 & 13 & 33 \\
\hline 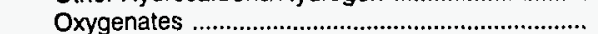 & 28 & 1,376 & 796 & W & w & 2,212 & 85 & 2,305 & 7,122 \\
\hline Fuel Ethanol ......................................................... & W & W & w & W & w & W & W & W & 424 \\
\hline 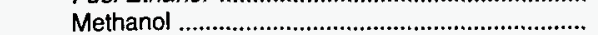 & $w$ & $\mathbf{w}$ & $w$ & w & w & w & W & W & 753 \\
\hline 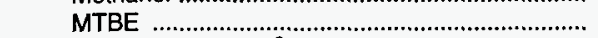 & w & 1,093 & W & W & w & 1,815 & w & 2,281 & 5,849 \\
\hline Other Oxygenates a .... & $w$ & w & $w$ & $\mathbf{w}$ & w & w & w & w & 96 \\
\hline Unfinished Oils ................... & 2,849 & 22,092 & 16,690 & 957 & 702 & 43,290 & 2,208 & 20,842 & 88,755 \\
\hline 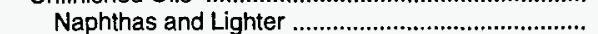 & 1,038 & 5,716 & 3,764 & 206 & 197 & 10,921 & 353 & 3,633 & 20,433 \\
\hline 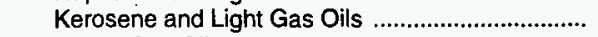 & 322 & 3,054 & 2,384 & 305 & 93 & 6,158 & 416 & 4,505 & 15,511 \\
\hline Heavy Gas Oils ................................................ & 1,142 & 7,674 & 7,212 & 414 & 412 & 16,854 & 863 & 9,681 & 36,016 \\
\hline 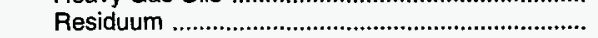 & 347 & 5,648 & 3,330 & 32 & 0 & 9,357 & 576 & 3,023 & 16,795 \\
\hline Motor Gasoline Blending Components ......................... & 1,348 & 6,435 & 5,528 & 172 & 355 & 13,838 & 1,889 & 8,248 & 38,635 \\
\hline Aviation Gasoline Blending Components ....................... & 11 & 0 & 15 & 0 & 0 & 26 & 0 & 10 & 151 \\
\hline 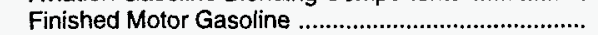 & 1,735 & 9,007 & 7,366 & 230 & 159 & 18,497 & 2,287 & 12,424 & 51,757 \\
\hline Reformulated & 33 & 2,714 & 406 & 0 & 0 & 3,153 & 0 & 8,051 & 16,553 \\
\hline Oxygenated ……………………………………….. & 0 & & 0 & 0 & 0 & 0 & 115 & 0 & 528 \\
\hline Other & 1,702 & 6,293 & 6,960 & 230 & 159 & 15,344 & 2,172 & 4,373 & 34,676 \\
\hline 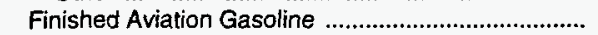 & 70 & 145 & 162 & 0 & 0 & 377 & 29 & 340 & 908 \\
\hline Jet Fuel & 498 & 3,361 & 3,051 & 86 & 58 & 7,054 & 402 & 5,121 & 17,236 \\
\hline 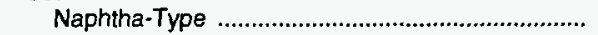 & 1 & 0 & 0 & 0 & 0 & 1 & 0 & 33 & 34 \\
\hline 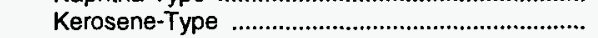 & 497 & 3,361 & 3,051 & 86 & 58 & 7,053 & 402 & 5,088 & 17,202 \\
\hline 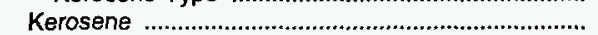 & 18 & 228 & 213 & 32 & 14 & 505 & 40 & 84 & 2,200 \\
\hline Distillate Fuel Oil .. & 1,151 & 8,304 & 5,185 & 516 & 197 & 15,353 & 1,668 & 6,453 & 48,997 \\
\hline 0.05 percent sulfur and under & 591 & 3,658 & 2,145 & 227 & 144 & 6,765 & 1,292 & 4,470 & 21,089 \\
\hline Greater then 0.05 percent sulfur ...................................... & 560 & 4,646 & 3,040 & 289 & 53 & 8,588 & 376 & 1,983 & 27,908 \\
\hline 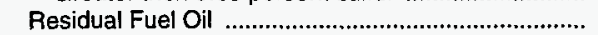 & 226 & 3,657 & 2,556 & 169 & 23 & 6,631 & 592 & 4,174 & 18,251 \\
\hline Less than 0.31 percent sulfur ............................................. & 25 & 6 & 53 & 0 & 0 & 84 & 23 & 522 & 2,120 \\
\hline 0.31 to 1.00 percent sulfur ....................................... & 92 & 714 & 491 & 113 & 23 & 1,433 & 399 & 715 & 4,912 \\
\hline 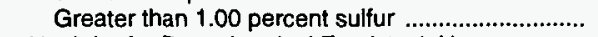 & 109 & 2,937 & 2,012 & 56 & 0 & 5,114 & 170 & 2,937 & 11,219 \\
\hline Naphtha for Petrochemical Feedstock Use ................... & 28 & 593 & 375 & 0 & 27 & 1,023 & 0 & 160 & 1,808 \\
\hline Other Oils for Petrochemical Feedstock Use .............. & 71 & 1,173 & 574 & 0 & 0 & 1,818 & 1 & 164 & 2,199 \\
\hline 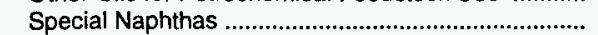 & 84 & 1,075 & 51 & 86 & 0 & 1,296 & 0 & 57 & 1,917 \\
\hline 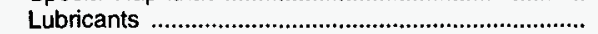 & 21 & 2,599 & 1,996 & 855 & 0 & 5,471 & 0 & 1,195 & 8,154 \\
\hline 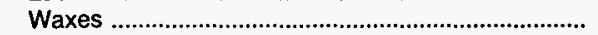 & 4 & 252 & 189 & 27 & 0 & 472 & 20 & 153 & 834 \\
\hline 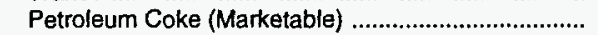 & 0 & 1,309 & 2,785 & 0 & 0 & 4,094 & 104 & 1,758 & 9,437 \\
\hline Asphalt and Road Oil .............................................. & 785 & 536 & 785 & 1,214 & 207 & 3,527 & 1,890 & 1,651 & 14,592 \\
\hline 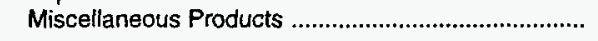 & 19 & 116 & 684 & 0 & 0 & 819 & 0 & 112 & 1,112 \\
\hline Total Stocks, All Oils & 12,049 & 91,001 & 69,414 & 5,505 & 2,238 & 180,207 & 13,869 & 86,478 & 420,894 \\
\hline
\end{tabular}

a Includes ethyl tertiary butyl ether (ETBE), tertiary amyl methyl ether (TAME), tertiary butyl alcohol (TBA), and other aliphatic alcohols and ethers intended for motor gasoline blending (e.g., isopropyl ether (IPE) or n-propanol).

$W=$ Withheld to avoid disclosure of individual company data.

Notes: - Stocks are reported as of the end of December. - Refer to Appendix A for Refining District descriptions.

Source: Energy Information Administration (EIA) Form EIA-810, "Monthly Refinery Report." 
Table 19. Percent Refinery Yield of Petroleum Products by PAD and Refining Districts, ${ }^{a}$

1997

\begin{tabular}{|c|c|c|c|c|c|c|c|}
\hline \multirow[b]{2}{*}{ Commodity } & \multicolumn{3}{|c|}{ PAD District I } & \multicolumn{4}{|c|}{ PAD District II } \\
\hline & $\begin{array}{l}\text { East } \\
\text { Coast }\end{array}$ & $\begin{array}{c}\text { Appalachian } \\
\text { No. } 1\end{array}$ & Total & Ind., III., Ky. & $\begin{array}{c}\text { Minn., Wis., } \\
\text { N. Dak., } \\
\text { S. Dak. }\end{array}$ & $\begin{array}{c}\text { Okla., Kans., } \\
\text { Mo. }\end{array}$ & Total \\
\hline 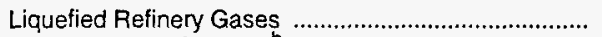 & 3.0 & 1.7 & 3.0 & 4.4 & 2.3 & 3.0 & 3.9 \\
\hline Finished Motor Gasoline ${ }^{b}$ & 45.6 & 37.3 & 45.2 & 51.5 & 50.7 & 49.3 & 50.9 \\
\hline Finished Aviation Gasoline ${ }^{c}$ & 0.3 & 0.0 & 0.3 & 0.1 & 0.2 & 0.1 & 0.1 \\
\hline Naphtha-Type Jet Fuel ......... & 0.0 & 0.0 & 0.0 & 0.0 & 0.0 & 0.0 & 0.0 \\
\hline 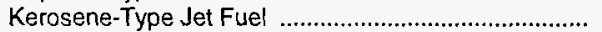 & 6.0 & 1.2 & 5.8 & 6.5 & 7.4 & 5.5 & 6.4 \\
\hline 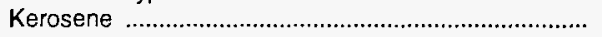 & 0.5 & 2.8 & 0.7 & 0.5 & 0.5 & 0.5 & 0.5 \\
\hline 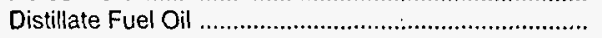 & 26.9 & 26.3 & 26.9 & 23.1 & 25.0 . & 31.7 & 24.9 \\
\hline 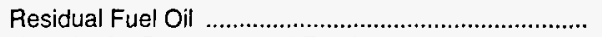 & 8.4 & 2.8 & 8.1 & 2.0 & 2.6 & 0.4 & 1.7 \\
\hline Naphtha for Petrochemical Feedstock Use ................. & 1.0 & 0.0 & 0.9 & 0.8 & 0.0 & 0.1 & 0.6 \\
\hline Other Oils for Petrochemical Feedstock Use ............... & 0.0 & 0.0 & 0.0 & 1.0 & 0.0 & 0.3 & 0.7 \\
\hline 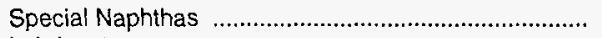 & 0.1 & 0.9 & 0.1 & 0.6 & 0.0 & 0.4 & 0.5 \\
\hline 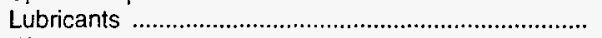 & 0.8 & 7.8 & 1.2 & 0.6 & 0.0 & 1.3 & 0.7 \\
\hline Waxes & 0.0 & 1.7 & 0.1 & 0.1 & 0.0 & 0.2 & 0.1 \\
\hline Petroleum Coke & 3.3 & 0.9 & 3.1 & 3.7 & 6.2 & 4.0 & 4.1 \\
\hline 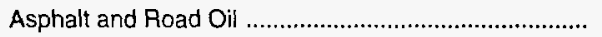 & 4.5 & 13.5 & 5.0 & 5.8 & 8.1 & 3.4 & 5.7 \\
\hline 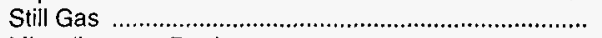 & 3.8 & 3.0 & 3.8 & 3.9 & 3.5 & 3.9 & 3.9 \\
\hline 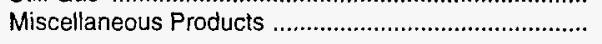 & 0.1 & 1.4 & 0.1 & 0.2 & 0.6 & 0.2 & 0.3 \\
\hline Processing Gain(-) or Loss $(+)^{d}$. & -4.3 & -1.5 & -4.2 & -4.8 & -7.0 & -4.4 & -5.0 \\
\hline
\end{tabular}

\begin{tabular}{|c|c|c|c|c|c|c|c|c|c|}
\hline \multirow{3}{*}{ Commodity } & \multicolumn{6}{|c|}{ PAD District III } & \multirow{3}{*}{\begin{tabular}{|c|}
$\begin{array}{c}\text { PAD Dist. } \\
\text { IV }\end{array}$ \\
Rocky Mt.
\end{tabular}} & \multirow{3}{*}{\begin{tabular}{|c|}
$\begin{array}{c}\text { PAD Dist. } \\
\mathbf{V}\end{array}$ \\
West Coast
\end{tabular}} & \multirow[b]{3}{*}{$\begin{array}{l}\text { U.S. } \\
\text { Total }\end{array}$} \\
\hline & \multirow{2}{*}{$\begin{array}{l}\text { Texas } \\
\text { Inland }\end{array}$} & \multirow{2}{*}{$\begin{array}{l}\text { Texas } \\
\text { Gulf } \\
\text { Coast }\end{array}$} & \multirow{2}{*}{$\begin{array}{c}\text { La. } \\
\text { Guif } \\
\text { Coast }\end{array}$} & \multirow[b]{2}{*}{$\begin{array}{c}\text { N. La., } \\
\text { Ark. }\end{array}$} & \multirow{2}{*}{$\begin{array}{c}\text { New } \\
\text { Mexico }\end{array}$} & \multirow[b]{2}{*}{ Total } & & & \\
\hline & & & & & & & & & \\
\hline 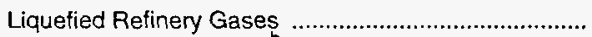 & 5.0 & 7.1 & 5.5 & 1.0 & 2.8 & 6.1 & 1.3 & 2.9 & 4.6 \\
\hline Finished Motor Gasoline ${ }^{b}$ & 50.9 & 43.6 & 44.0 & 25.2 & 52.6 & 44.0 & 47.7 & 43.6 & 45.7 \\
\hline 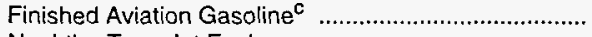 & 0.7 & 0.1 & 0.1 & 0.0 & 0.0 & 0.2 & 0.1 & 0.2 & 0.2 \\
\hline Naphtha-Type Jet Fuel & 0.0 & 0.0 & 0.0 & 0.0 & 0.0 & 0.0 & 0.0 & 0.0 & 0.0 \\
\hline 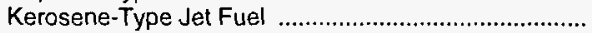 & 9.5 & 9.7 & 13.7 & 5.0 & 7.3 & 11.1 & 5.5 & 17.3 & 10.3 \\
\hline Kerosene & 0.0 & 0.7 & 0.2 & 1.1 & 0.0 & 0.4 & 0.5 & 0.2 & 0.4 \\
\hline 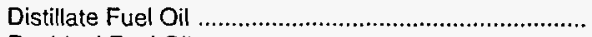 & 25.2 & 20.7 & 21.7 & 23.9 & 27.5 & 21.6 & 28.7 & 17.9 & 22.5 \\
\hline 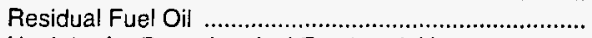 & 1.6 & 4.5 & 4.6 & 4.0 & 0.9 & 4.2 & 2.7 & 8.3 & 4.7 \\
\hline Naphtha for Petrochemical Feedstock Use .................. & 0.6 & 4.5 & 1.1 & 0.0 & 0.0 & 2.7 & 0.0 & 0.1 & 1.5 \\
\hline Other Oils for Petrochemical Feedstock Use ............... & 0.8 & 2.9 & 2.9 & 0.0 & 0.0 & 2.6 & 0.1 & 0.3 & 1.4 \\
\hline 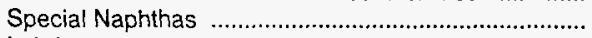 & 0.5 & 0.6 & 0.2 & 2.5 & 0.0 & 0.4 & 0.0 & 0.1 & 0.3 \\
\hline 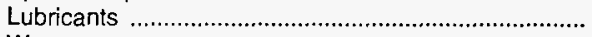 & 0.3 & 1.6 & 1.3 & 12.0 & 0.0 & 1.6 & 0.0 & 0.9 & 1.2 \\
\hline 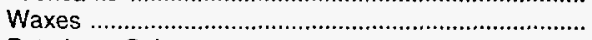 & 0.0 & 0.2 & 0.1 & 1.6 & 0.0 & 0.2 & 0.7 & 0.1 & 0.2 \\
\hline Petroleum Coke & 1.6 & 5.0 & 5.2 & 1.4 & 0.8 & 4.6 & 3.1 & 6.2 & 4.6 \\
\hline Asphalt and Road Oil & 2.9 & 0.9 & 1.2 & 19.9 & 5.0 & 1.7 & 8.3 & 2.2 & 3.2 \\
\hline Still Gas & 4.2 & 4.7 & 3.9 & 3.2 & 3.0 & 4.3 & 3.9 & 5.8 & 4.4 \\
\hline 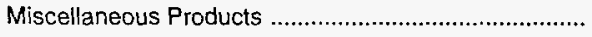 & 0.4 & 0.4 & 0.6 & 0.0 & 0.0 & 0.4 & 0.4 & 0.2 & 0.3 \\
\hline Processing Gain(-) or Loss $(t)^{d}$. & -4.2 & -7.1 & -6.2 & -0.8 & 0.1 & -6.2 & -2.9 & -6.3 & -5.6 \\
\hline
\end{tabular}

a Based on crude oil input and net reruns of unfinished oils.

b Based on total finished motor gasoline output minus net input of motor gasoline blending components, minus input of natural gas plant liquids, other hydrocarbons and oxygenates.

c Based on finished aviation gasoline output minus net input of aviation gasoline blending components.

Represents the difference between input and production.

Notes: - Totals may not equal sum of components due to independent rounding. - Refer to Appendix A for Refining District descriptions.

Sources: Calculated from data on Tables 16 and 17. 
Table 20. Imports of Crude Oil and Petroleum Products by PAD District, 1997 (Thousand Barrels)

\begin{tabular}{|c|c|c|c|c|c|c|c|}
\hline \multirow{2}{*}{ Commodity } & \multicolumn{6}{|c|}{ Petroleum Administration for Defense Districts } & \multirow[b]{2}{*}{$\begin{array}{c}\text { Daily } \\
\text { Average }\end{array}$} \\
\hline & $\mathbf{I}$ & ॥ & III & IV & $\mathbf{V}$ & $\begin{array}{l}\text { U.S. } \\
\text { Total }\end{array}$ & \\
\hline Crude Oil ${ }^{\mathrm{a}, \mathrm{b}}$ & 528,144 & 596,411 & $1,683,236$ & 45,283 & 149,225 & $3,002,299$ & 8,225 \\
\hline 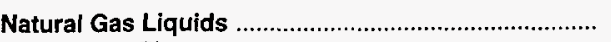 & 8,649 & 31,757 & 30,495 & 3,832 & 98 & 74,831 & 205 \\
\hline 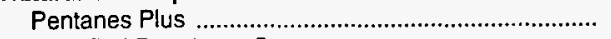 & 0 & 412 & 12,020 & 697 & 0 & 13,129 & 36 \\
\hline 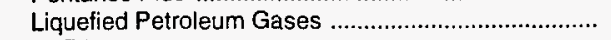 & 8,649 & 31,345 & 18,475 & 3,135 & 98 & 61,702 & 169 \\
\hline 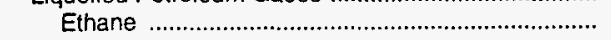 & 0 & 0 & 5,784 & 0 & 0 & 5,784 & 16 \\
\hline 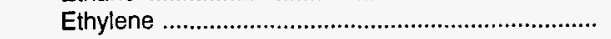 & 0 & 138 & 0 & 0 & 0 & 138 & (s) \\
\hline Propane & 8,187 & 22,852 & 5,880 & 1,978 & 30 & 38,927 & 107 \\
\hline Propylene & 0 & 2,130 & 214 & 0 & 0 & 2,344 & 6 \\
\hline 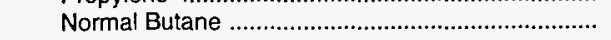 & 297 & 3,621 & 3,845 & 1,012 & 0 & 8,775 & 24 \\
\hline Butylene & 0 & 0 & 0 & 0 & 0 & 0 & 0 \\
\hline Isobutane & 165 & 2,604 & 2,752 & 145 & 68 & 5,734 & 16 \\
\hline 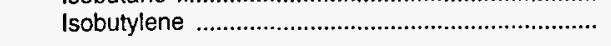 & 0 & 0 & 0 & 0 & 0 & 0 & 0 \\
\hline Other Liquids & 88,627 & 93 & 109,162 & 0 & 26,178 & 224,060 & 614 \\
\hline Other Hydrocarbons/Hydrogen/Oxygenates ................ & 5,271 & 0 & 202 & 0 & 16,625 & 22,098 & 61 \\
\hline 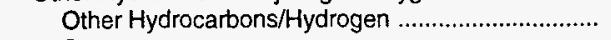 & 0 & 0 & 0 & 0 & 0 & 0 & 0 \\
\hline Oxygenates & 5,271 & 0 & 202 & 0 & 16,625 & 22,098 & 61 \\
\hline 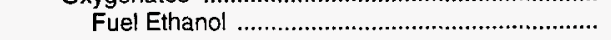 & 0 & 0 & 0 & 0 & 85 & 85 & (s) \\
\hline MTBE & 5,271 & 0 & 44 & 0 & 16,540 & 21,855 & 60 \\
\hline (2) & 0 & 0 & 158 & 0 & 0 & 158 & (s) \\
\hline Unfinished Oils ${ }^{2}$ & 13,014 & 48 & 108,407 & 0 & 7,345 & 128,814 & 353 \\
\hline Naphthas and Lighter & 2,244 & 48 & 17,486 & 0 & 650 & 20,428 & 56 \\
\hline 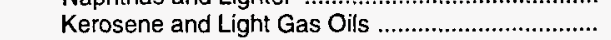 & 0 & 0 & 0 & 0 & 0 & 0 & 0 \\
\hline 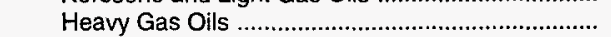 & 10,650 & 0 & 49,443 & 0 & 206 & 60,299 & 165 \\
\hline Residuum & 120 & 0 & 41,478 & 0 & 6,489 & 48,087 & 132 \\
\hline Motor Gasoline Blending Components ...................... & 70,342 & 45 & 553 & 0 & 2,208 & 73,148 & 200 \\
\hline 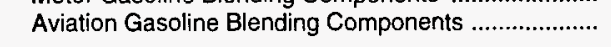 & 0 & 0 & 0 & 0 & 0 & 0 & 0 \\
\hline 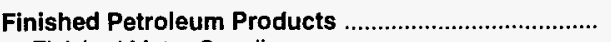 & 298,219 & 4,671 & 93,526 & 3,550 & 7,814 & 407,780 & 1,117 \\
\hline 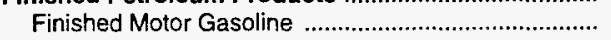 & 107,656 & 955 & 2,844 & 234 & 1,148 & 112,837 & 309 \\
\hline 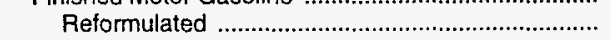 & 56,352 & 0 & 2,289 & 0 & 0 & 58,641 & 161 \\
\hline Oxygenated & 0 & 0 & 0 & 0 & 0 & 0 & 0 \\
\hline Other & 51,304 & 955 & 555 & 234 & 1,148 & 54,196 & 148 \\
\hline 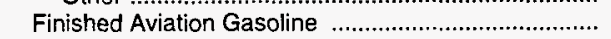 & 3 & 24 & 0 & 5 & 9 & 41 & (s) \\
\hline Jet Fuel & 30,877 & 75 & 204 & 0 & 1,953 & 33,109 & 91 \\
\hline Naphtha-Type & 0 & 0 & 0 & 0 & 0 & 0 & 0 \\
\hline 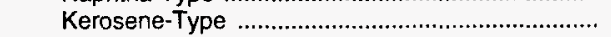 & 30,877 & 75 & 204 & 0 & 1,953 & 33,109 & 91 \\
\hline 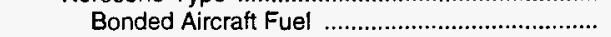 & 19,040 & 0 & 0 & 0 & 163 & 19,203 & 53 \\
\hline Other & 11,837 & 75 & 204 & 0 & 1,790 & 13,906 & 38 \\
\hline Kerosene & 495 & 0 & 0 & 0 & 75 & 570 & 2 \\
\hline 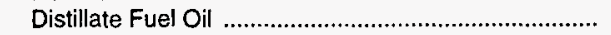 & 76,162 & 1,632 & 0 & 3,256 & 2,052 & 83,102 & 228 \\
\hline 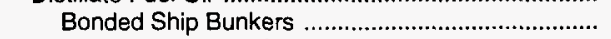 & 0 & 0 & 0 & 19 & 318 & 337 & 1 \\
\hline 0.05 percent sulfur and under & 0 & 0 & 0 & 19 & 0 & 19 & (s) \\
\hline 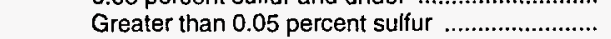 & 0 & 0 & 0 & 0 & 318 & 318 & 1 \\
\hline Other & 76,162 & 1,632 & 0 & 3,237 & 1,734 & 82,765 & 227 \\
\hline 0.05 percent sulfur and under & 34,050 & 1,175 & 0 & 624 & 1,731 & 37,580 & 103 \\
\hline Greater than 0.05 percent sulfur & 42,112 & 457 & 0 & 2,613 & 3 & 45,185 & 124 \\
\hline Residua! Fuel Oil & 64,352 & 140 & 4,591 & 0 & 1,746 & 70,829 & 194 \\
\hline Bonded Ship Bunkers ........................................ & 0 & 0 & 0 & 0 & 0 & 0 & 0 \\
\hline 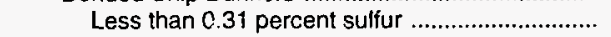 & 0 & 0 & 0 & 0 & 0 & 0 & 0 \\
\hline 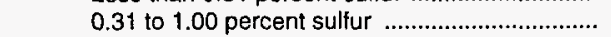 & 0 & 0 & 0 & 0 & 0 & 0 & 0 \\
\hline 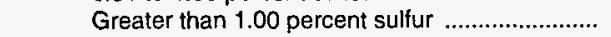 & 0 & 0 & 0 & 0 & 0 & 0 & 0 \\
\hline Other & 64,352 & 140 & 4,591 & 0 & 1,746 & 70,829 & 194 \\
\hline 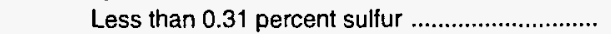 & 14,775 & 99 & 469 & 0 & 1,581 & 16,924 & 46 \\
\hline 0.31 to 1.00 percent sulfur & 11,578 & 0 & 1,223 & 0 & 0 & 12,801 & 35 \\
\hline 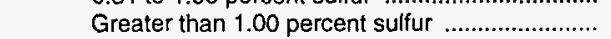 & 37,999 & 41 & 2,899 & 0 & 165 & 41,104 & 113 \\
\hline 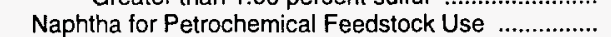 & 2,044 & 402 & 16,199 & 0 & 36 & 18,681 & 51 \\
\hline 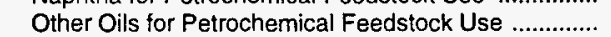 & 0 & 0 & 68,738 & 0 & 348 & 69,086 & 189 \\
\hline Special Naphthas & 1,976 & 347 & 370 & 1 & 15 & 2,709 & 7 \\
\hline 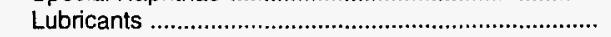 & 3,563 & 259 & 204 & 0 & 0 & 4,026 & 11 \\
\hline Waxes & 256 & 160 & 16 & 0 & 9 & 441 & 1 \\
\hline Petroleum Coke & 0 & 0 & 0 & 0 & 386 & 386 & 1 \\
\hline 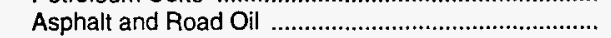 & 10,835 & 652 & 321 & 54 & 0 & 11,862 & 32 \\
\hline 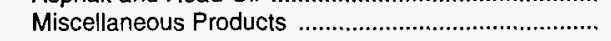 & 0 & 25 & 39 & 0 & 37 & 101 & (s) \\
\hline (n) & 923,639 & 632,932 & $1,916,419$ & 52,665 & 183,315 & $3,708,970$ & 10,162 \\
\hline
\end{tabular}

\footnotetext{
${ }^{a}$ Crude oil and unfinished oils are reported by the PAD District in which they are to be processed; all other products are reported by the PAD District of entry.

b Includes crude oil imported for storage in the Strategic Petroleum Reserve.

c Includes ethyl tertiary butyl ether (ETBE), tertiary amyl methyl ether (TAME), tertiary butyl alcohol (TBA), and other aliphatic alcohols and ethers intended for motor gasoline blending (e.g., isopropyl ether (IPE) or n-propanol).

(s) = Less than 500 barrels per day.

Note: Totals may not equal sum of components due to independent rounding.

Sources: Energy Information Administration (EIA) Form EIA-814, "Monthly Imports Report."
} 
Table 21. Imports of Crude Oil and Petroleum Products into the United States by Country of Origin, ${ }^{\mathrm{a}} 1997$

(Thousand Barrels)

\begin{tabular}{|c|c|c|c|c|c|c|c|c|c|c|}
\hline Country of Origin & $\begin{array}{c}\text { Crude } \\
\text { Oil }^{\mathbf{b}}\end{array}$ & $\begin{array}{c}\text { Liquefied } \\
\text { Petroleum } \\
\text { Gases } \\
\end{array}$ & $\begin{array}{c}\text { Unfinished } \\
\text { Oils }\end{array}$ & $\begin{array}{c}\text { Gasoline } \\
\text { Blending } \\
\text { Compo- } \\
\text { nents } \\
\end{array}$ & $\begin{array}{c}\text { Finished } \\
\text { Motor } \\
\text { Gasoline }\end{array}$ & Jet Fuel & $\begin{array}{l}\text { Distillate } \\
\text { Fuel Oil }\end{array}$ & $\begin{array}{l}\text { Residual } \\
\text { Fuel Oil }\end{array}$ & Kerosene & $\begin{array}{c}\text { Special } \\
\text { Naphthas }\end{array}$ \\
\hline Arab OPEC & 599,047 & 11,322 & 34,809 & 882 & 10,709 & 0 & 447 & 12,083 & 63 & 0 \\
\hline Algeria & 2,195 & 11,322 & 16,395 & 222 & 0 & 0 & 381 & 9,365 & 0 & 0 \\
\hline 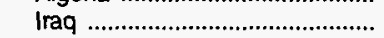 & 32,530 & 0 & 0 & 0 & 0 & 0 & 0 & 0 & 0 & 0 \\
\hline 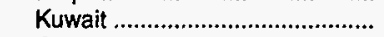 & 92,229 & 0 & 0 & 0 & 0 & 0 & 0 & 0 & 0 & 0 \\
\hline 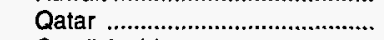 & 0 & 0 & 0 & 0 & 0 & 0 & 0 & 0 & 0 & 0 \\
\hline 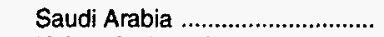 & 472,093 & 0 & 17,798 & 660 & 10,709 & 0 & 66 & 2,718 & 63 & 0 \\
\hline United Arab Emirates .................. & 0 & 0 & 616 & 0 & 0 & 0 & 0 & 0 & 0 & 0 \\
\hline Other OPEC & 778,995 & 4,145 & 35,555 & 19,041 & 15,880 & 14,251 & 18,723 & 21,540 & 7 & 0 \\
\hline 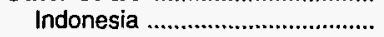 & 18,657 & 0 & 961 & 0 & 0 & 0 & 0 & 1,663 & 0 & 0 \\
\hline 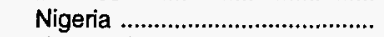 & 251,608 & 0 & 1,160 & 515 & 0 & 0 & 0 & 1,308 & 0 & 0 \\
\hline 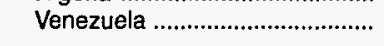 & 508,730 & 4,145 & 33,434 & 18,526 & 15,880 & 14,251 & 18,723 & 18,569 & 7 & 0 \\
\hline 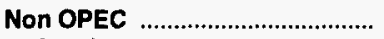 & $1,624,257$ & 46,235 & $\mathbf{5 8 , 4 5 0}$ & 53,225 & 86,248 & 18,858 & 63,932 & 37,206 & 500 & 2,709 \\
\hline Angola & 155,298 & 0 & 349 & 0 & 0 & 0 & 0 & 0 & 0 & 0 \\
\hline 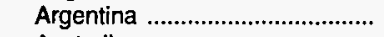 & 25,053 & 0 & 614 & 1,088 & 0 & 0 & 189 & 370 & 0 & 0 \\
\hline 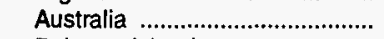 & 11,405 & 0 & 0 & 0 & 0 & 0 & 0 & 0 & 0 & 0 \\
\hline 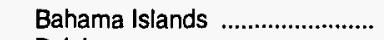 & 0 & 0 & 350 & 0 & 0 & 0 & 0 & 0 & 0 & 0 \\
\hline 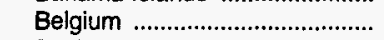 & 0 & 0 & 5,337 & 3,562 & 911 & 0 & 0 & 484 & 0 & 0 \\
\hline 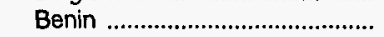 & 440 & 0 & 0 & 0 & 0 & 0 & 0 & 0 & 0 & 0 \\
\hline 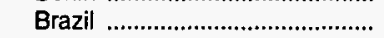 & 0 & 133 & 0 & 917 & 490 & 0 & 0 & 77 & 0 & 66 \\
\hline 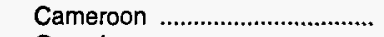 & 0 & 0 & 0 & 0 & 0 & 0 & 0 & 1,675 & 0 & 0 \\
\hline 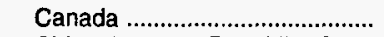 & 437,396 & 44,847 & 2,084 & 2,651 & 28,148 & 1,159 & 30,505 & 8,957 & 425 & 2,643 \\
\hline China, People's Republic of ..... & 17,612 & 0 & 0 & 10 & 222 & 0 & 0 & 0 & 0 & 0 \\
\hline Colombia & 98,461 & 0 & 161 & 0 & 0 & 0 & 0 & 456 & 0 & 0 \\
\hline 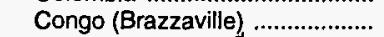 & 17,178 & 0 & 0 & 0 & 0 & 0 & 0 & 0 & 0 & 0 \\
\hline Congo (Kinshasa) ${ }^{d}$ & 7,599 & 0 & 0 & 0 & 0 & 0 & 0 & 0 & 0 & 0 \\
\hline 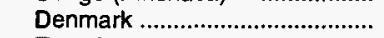 & 226 & 0 & 0 & 0 & 0 & 0 & 0 & 0 & 0 & 0 \\
\hline 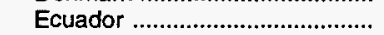 & 41,567 & 0 & 140 & 0 & 0 & 0 & 0 & 172 & 0 & 0 \\
\hline 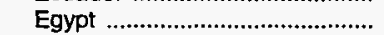 & 12,989 & 0 & 100 & 0 & 0 & 0 & 0 & 0 & 0 & 0 \\
\hline France & 0 & 0 & 3,310 & 3,678 & 1,424 & 0 & 0 & 210 & 0 & 0 \\
\hline Gabon & 84,109 & 0 & 0 & 0 & 0 & 0 & 0 & 0 & 0 & 0 \\
\hline Germany, FR & 0 & 0 & 903 & 828 & 397 & 0 & 0 & 631 & 0 & 0 \\
\hline 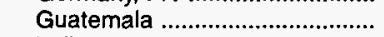 & 6,121 & 0 & 0 & 0 & 0 & 0 & 0 & 0 & 0 & 0 \\
\hline 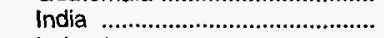 & 0 & 0 & 233 & 0 & 0 & 0 & 0 & 0 & 0 & 0 \\
\hline 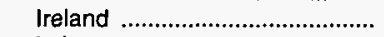 & 0 & 0 & 0 & 0 & 0 & 0 & 0 & 342 & 0 & 0 \\
\hline Italy & 0 & 0 & 51 & 1,718 & 905 & 0 & 0 & 0 & 0 & 0 \\
\hline 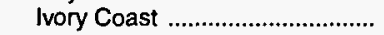 & 0 & 0 & 499 & 0 & 0 & 0 & 0 & 120 & 0 & 0 \\
\hline Japan & 0 & 0 & 0 & 227 & 0 & 121 & 0 & 0 & 0 & 0 \\
\hline Korea, Republic of ...................... & 0 & 0 & 365 & 0 & 0 & 768 & 0 & 0 & 0 & 0 \\
\hline 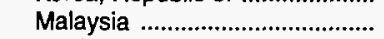 & 2,850 & 0 & 2,276 & 0 & 0 & 0 & 106 & 627 & 0 & 0 \\
\hline 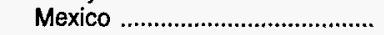 & 496,275 & 197 & 0 & 1,709 & 0 & 205 & 0 & 0 & 0 & 0 \\
\hline 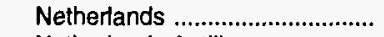 & 0 & 0 & 3,147 & 2,953 & 1,256 & 0 & 0 & 201 & 0 & 0 \\
\hline Netherlands Antilles .................. & 0 & 0 & 11,818 & 1,485 & 1,012 & 8,542 & 0 & 787 & 0 & 0 \\
\hline 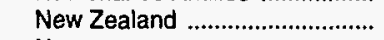 & 0 & 0 & 0 & 0 & 0 & 0 & 0 & 0 & 0 & 0 \\
\hline 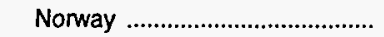 & 105,048 & 1,058 & 2,170 & 120 & 1,918 & 0 & 0 & 430 & 0 & 0 \\
\hline Oman & 1,511 & 0 & 2,430 & 0 & 0 & 0 & 0 & 0 & 0 & 0 \\
\hline 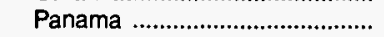 & 0 & 0 & 0 & 0 & 0 & 0 & 0 & 135 & 0 & 0 \\
\hline 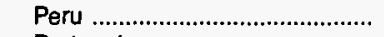 & 11,195 & 0 & 260 & 0 & 141 & 0 & 0 & 0 & 0 & 0 \\
\hline 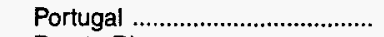 & 0 & 0 & 0 & 1,115 & 3,069 & 0 & 0 & 0 & 0 & 0 \\
\hline 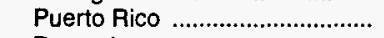 & 0 & 0 & 0 & 0 & 175 & 0 & 0 & 0 & 0 & 0 \\
\hline 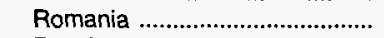 & 0 & 0 & 514 & 2,369 & 61 & 0 & 0 & 0 & 0 & 0 \\
\hline Russia & 955 & 0 & 671 & 2,249 & 173 & 0 & 330 & 347 & 0 & 0 \\
\hline Singapore & 0 & 0 & 3,685 & 0 & 0 & 0 & 0 & 0 & 0 & 0 \\
\hline Spain & 0 & 0 & 3,001 & 2,025 & 860 & 0 & 0 & 710 & 0 & 0 \\
\hline 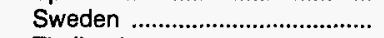 & 0 & 0 & 938 & 949 & 309 & 0 & 0 & 421 & 0 & 0 \\
\hline 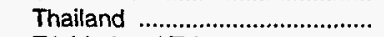 & 0 & 0 & 0 & 0 & 459 & 0 & 0 & 0 & 0 & 0 \\
\hline Trinidad and Tobago .................... & 20,300 & 0 & 349 & 1,271 & 0 & 0 & 0 & 234 & 0 & 0 \\
\hline 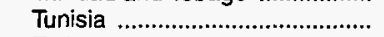 & 0 & 0 & 0 & 0 & 0 & 0 & 0 & 198 & 0 & 0 \\
\hline 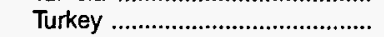 & 0 & 0 & 230 & 0 & 0 & 0 & 0 & 0 & 0 & 0 \\
\hline 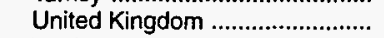 & 61,700 & 0 & 540 & 14,268 & 4,613 & 0 & 6 & 1,434 & 0 & 0 \\
\hline 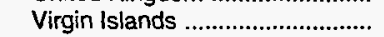 & 0 & 0 & 10,410 & 2,416 & 38,885 & 8,063 & 32,715 & 16,545 & 75 & 0 \\
\hline Yemen & 0 & 0 & 0 & 0 & 0 & 0 & 0 & 304 & 0 & 0 \\
\hline 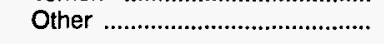 & 8,969 & 0 & 1,515 & 5,617 & 820 & 0 & 81 & 1,339 & 0 & 0 \\
\hline 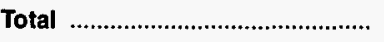 & $3,002,299$ & 61,702 & 128,814 & 73,148 & 112,837 & 33,109 & 83,102 & 70,829 & 570 & 2,709 \\
\hline Persian Gulf ${ }^{\mathbf{e}}$............................... & 596,852 & 0 & 18,414 & 660 & 10,709 & 0 & 66 & 2,718 & 63 & 0 \\
\hline
\end{tabular}

See footnotes at end of table. 
Table 21. Imports of Crude Oil and Petroleum Products into the United States by Country of Origin, 1997 (Continued)

(Thousand Barrels)

\begin{tabular}{|c|c|c|c|c|c|c|c|c|c|c|}
\hline \multirow[b]{2}{*}{ Country of Origin } & \multirow[b]{2}{*}{$\begin{array}{c}\text { Naphtha for } \\
\text { Petrochemical } \\
\text { Feedstock } \\
\text { Use }\end{array}$} & \multirow[b]{2}{*}{$\begin{array}{c}\text { Other Oils for } \\
\text { Petrochemical } \\
\text { Feedstock } \\
\text { Use }\end{array}$} & \multirow[b]{2}{*}{ Lubricants } & \multirow[b]{2}{*}{$\begin{array}{c}\text { Asphalt and } \\
\text { Road Oil }\end{array}$} & \multirow[b]{2}{*}{$\begin{array}{c}\text { Other } \\
\text { Products }\end{array}$} & \multirow[b]{2}{*}{\begin{tabular}{|c} 
Total \\
Products
\end{tabular}} & \multirow[b]{2}{*}{$\begin{array}{c}\text { Total } \\
\text { Crude Oil } \\
\text { and } \\
\text { Products }\end{array}$} & \multicolumn{3}{|c|}{ Daily Average } \\
\hline & & & & & & & & $\begin{array}{c}\text { Crude } \\
\text { Oil } \\
\end{array}$ & Products & Total \\
\hline Arab OPEC ....... & 2,727 & 51,293 & 0 & 0 & 21,176 & 145,511 & 744,558 & 1,641 & 399 & 2,040 \\
\hline Algeria & 2,727 & 49,891 & 0 & 0 & 11,590 & 101,893 & 104,088 & 6 & 279 & 285 \\
\hline 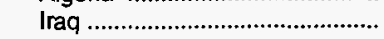 & 0 & 0 & 0 & 0 & 0 & 0 & 32,530 & 89 & 0 & 89 \\
\hline 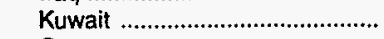 & 0 & 0 & 0 & 0 & 0 & 0 & 92,229 & 253 & 0 & 253 \\
\hline 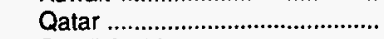 & 0 & 1,402 & 0 & 0 & 0 & 1,402 & 1,402 & 0 & 4 & 4 \\
\hline Saudi Arabia ................................. & 0 & 0 & 0 & 0 & 9,586 & 41,600 & 513,693 & 1,293 & 114 & 1,407 \\
\hline United Arab Emirates ................ & 0 & 0 & 0 & 0 & 0 & 616 & 616 & 0 & 2 & 2 \\
\hline Other OPEC & 4,610 & 309 & $\mathbf{0}$ & 5,869 & 4,042 & 143,972 & 922,967 & 2,134 & 394 & 2,529 \\
\hline Indonesia & 0 & 0 & 0 & 0 & 0 & 2,624 & 21,281 & 51 & 7 & 58 \\
\hline Nigeria ........................................ & 0 & 0 & 0 & 0 & 0 & 2,983 & 254,591 & 689 & 8 & 698 \\
\hline 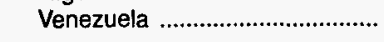 & 4,610 & 309 & 0 & 5,869 & 4,042 & 138,365 & 647,095 & 1,394 & 379 & 1,773 \\
\hline Non OPEC & 11,344 & 17,484 & 4,026 & 5,993 & 10,978 & 417,188 & $2,041,445$ & 4,450 & 1,143 & 5,593 \\
\hline 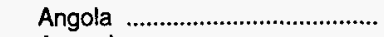 & 376 & 0 & 0 & 0 & 0 & 725 & 156,023 & 425 & 2 & 427 \\
\hline 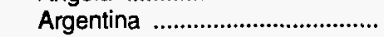 & 211 & 0 & 0 & 0 & 0 & 2,472 & 27,525 & 69 & 7 & 75 \\
\hline 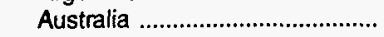 & 0 & 6,216 & 0 & 0 & 0 & 6,216 & 17,621 & 31 & 17 & 48 \\
\hline 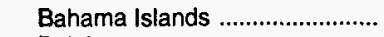 & 0 & 0 & 0 & 0 & 0 & 350 & 350 & 0 & 1 & 1 \\
\hline 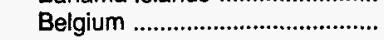 & 367 & 0 & 0 & 0 & 0 & 10,661 & 10,661 & 0 & 29 & 29 \\
\hline 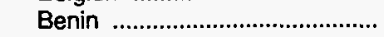 & 0 & 0 & 0 & 0 & 0 & 0 & 440 & 1 & 0 & 1 \\
\hline 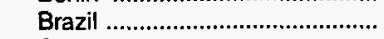 & 62 & 0 & 0 & 0 & 97 & 1,842 & 1,842 & 0 & 5 & 5 \\
\hline 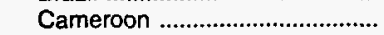 & 0 & 0 & 0 & 0 & 0 & 1,675 & 1,675 & 0 & 5 & 5 \\
\hline 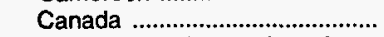 & 1,106 & 348 & 731 & 3,046 & 6,556 & 133,206 & 570,602 & 1,198 & 365 & 1,563 \\
\hline China, People's Republic of ....... & 0 & 0 & 0 & 0 & 0 & 232 & 17,844 & 48 & 1 & 49 \\
\hline 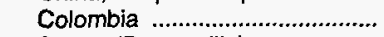 & 0 & 0 & 0 & 0 & 0 & 617 & 99,078 & 270 & 2 & 271 \\
\hline Congo (Brazzaville) ..................... & 0 & 0 & 0 & 0 & 0 & 0 & 17,178 & 47 & 0 & 47 \\
\hline Congo (Kinshasa) & 0 & 0 & 0 & 0 & 0 & 0 & 7,599 & 21 & 0 & 21 \\
\hline 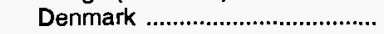 & 0 & 0 & 0 & 0 & 0 & 0 & 226 & 1 & 0 & 1 \\
\hline 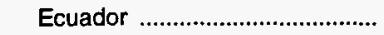 & 0 & 0 & 0 & 0 & 0 & 312 & 41,879 & 114 & 1 & 115 \\
\hline 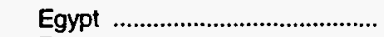 & 255 & 228 & 0 & 0 & 0 & 583 & 13,572 & 36 & 2 & 37 \\
\hline 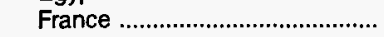 & 66 & 0 & 35 & 0 & 1,464 & 10,187 & 10,187 & 0 & 28 & 28 \\
\hline 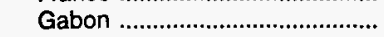 & 0 & 0 & 0 & 0 & 0 & 0 & 84,109 & 230 & 0 & 230 \\
\hline 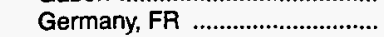 & 302 & 0 & 0 & 0 & 66 & 3,127 & 3,127 & 0 & 9 & 9 \\
\hline 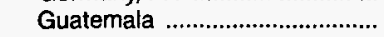 & 0 & 0 & 0 & 0 & 0 & 0 & 6,121 & 17 & 0 & 17 \\
\hline India & 380 & 1,091 & 0 & 0 & 0 & 1,704 & 1,704 & 0 & 5 & 5 \\
\hline 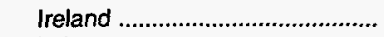 & 0 & 0 & 0 & 0 & 0 & 342 & 342 & 0 & 1 & 1 \\
\hline Italy & 21 & 0 & 0 & 0 & 0 & 2,695 & 2,695 & 0 & 7 & 7 \\
\hline 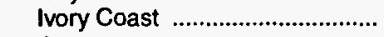 & 0 & 0 & 0 & 0 & 0 & 619 & 619 & 0 & 2 & 2 \\
\hline 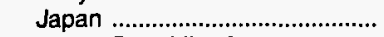 & 33 & 0 & 0 & 0 & 55 & 436 & 436 & 0 & 1 & 1 \\
\hline Korea, Republic of ......................... & 122 & 0 & 0 & 0 & 244 & 1,499 & 1,499 & 0 & 4 & 4 \\
\hline 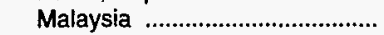 & 0 & 1,872 & 0 & 0 & 483 & 5,364 & 8,214 & 8 & 15 & 23 \\
\hline 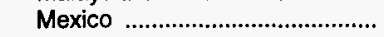 & 3,413 & 2,032 & 169 & 1,601 & 9 & 9,335 & 505,610 & 1,360 & 26 & 1,385 \\
\hline 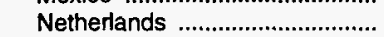 & 596 & 0 & 0 & 0 & 852 & 9,005 & 9,005 & 0 & 25 & 25 \\
\hline Netherlands Antilles ................... & 647 & 2,372 & 0 & 241 & 0 & 26,904 & 26,904 & 0 & 74 & 74 \\
\hline 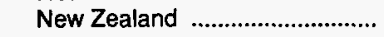 & 0 & 498 & 0 & 0 & 0 & 498 & 498 & 0 & 1 & 1 \\
\hline 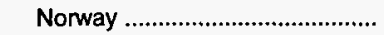 & 0 & 1,989 & 0 & 0 & 0 & 7,685 & 112,733 & 288 & 21 & 309 \\
\hline Oman & 0 & 0 & 0 & 0 & 0 & 2,430 & 3,941 & 4 & 7 & 11 \\
\hline 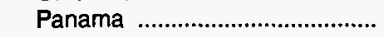 & 0 & 0 & 0 & 0 & 0 & 135 & 135 & 0 & (s) & (s) \\
\hline Peru & 0 & 0 & 0 & 0 & 0 & 401 & 11,596 & 31 & 1 & 32 \\
\hline 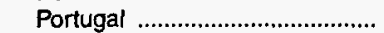 & 0 & 0 & 0 & 0 & 53 & 4,237 & 4,237 & 0 & 12 & 12 \\
\hline 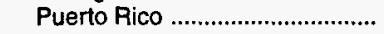 & 2,755 & 0 & 3,091 & 0 & 0 & 6,021 & 6,021 & 0 & 16 & 16 \\
\hline 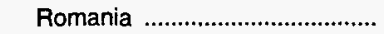 & 0 & 0 & 0 & 0 & 0 & 2,944 & 2,944 & 0 & 8 & 8 \\
\hline 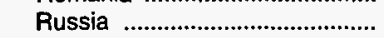 & 0 & 0 & 0 & 0 & 0 & 3,770 & 4,725 & 3 & 10 & 13 \\
\hline 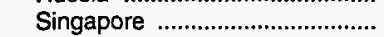 & 0 & 0 & 0 & 0 & 632 & 4,317 & 4,317 & 0 & 12 & 12 \\
\hline Spain & 22 & 0 & 0 & 1,105 & 0 & 7,723 & 7,723 & 0 & 21 & 21 \\
\hline 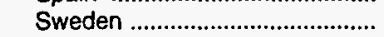 & 0 & 0 & 0 & 0 & 0 & 2,617 & 2,617 & 0 & 7 & 7 \\
\hline 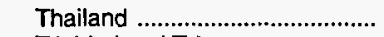 & 29 & 0 & 0 & 0 & 0 & 488 & 488 & 0 & 1 & 1 \\
\hline Trinidad and Tobago ..................... & 113 & 135 & 0 & 0 & 0 & 2,102 & 22,402 & 56 & 6 & 61 \\
\hline 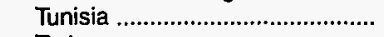 & 241 & 0 & 0 & 0 & 0 & 439 & 439 & 0 & 1 & 1 \\
\hline 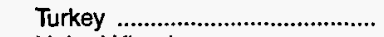 & 0 & 0 & 0 & 0 & 0 & 230 & 230 & 0 & 1 & 1 \\
\hline 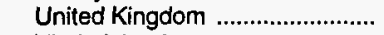 & 0 & 0 & 0 & 0 & 0 & 20,861 & 82,561 & 169 & 57 & 226 \\
\hline 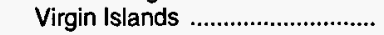 & 110 & 0 & 0 & 0 & 312 & 109,531 & 109,531 & 0 & 300 & 300 \\
\hline Yemen & 0 & 0 & 0 & 0 & 0 & 304 & 304 & 0 & 1 & 1 \\
\hline 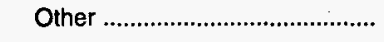 & 117 & 703 & 0 & 0 & 155 & 10,347 & 19,316 & 25 & 28 & 53 \\
\hline 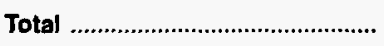 & 18,681 & 69,086 & 4,026 & 11,862 & 36,196 & 706,671 & $3,708,970$ & 8,225 & 1,936 & 10,162 \\
\hline 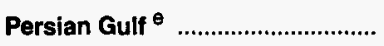 & 0 & 1,402 & 0 & 0 & 9,586 & 43,618 & 640,470 & 1,635 & 120 & 1,755 \\
\hline
\end{tabular}

a Crude oil and unfinished oils are reported by the PAD District in which they are to be processed; all other products are reported by the PAD District of entry.

b Includes crude oil imported for storage in the Strategic Petroleum Reserve.

c Includes aviation gasoline, aviation gasoline blending components, miscellaneous products, other hydrocarbons and oxygenates, pentanes plus, petroleum coke, and waxes.

d. Formenty Zaire.

- Includes Bahrain, Iran, Iraq, Kuwait, Qatar, Saudi Arabia, and United Arab Emirates.

(s) = Less than 500 barrels per day.

Note: Totals may not equal sum of components due to independent rounding.

Source: Energy Information Administration (EIA) Form EIA-814, "Monthly Imports Report." 
Table 22. PAD District I-Imports of Crude Oil and Petroleum Products by Country of Origin, ${ }^{a} 1997$

(Thousand Barrels)

\begin{tabular}{|c|c|c|c|c|c|c|c|c|c|c|}
\hline Country of Origin & $\begin{array}{c}\text { Crude } \\
\text { Oil }^{\mathbf{b}}\end{array}$ & $\begin{array}{c}\text { Liquefied } \\
\text { Petroleum } \\
\text { Gases }\end{array}$ & $\begin{array}{c}\begin{array}{c}\text { Unfinished } \\
\text { Oils }\end{array} \\
\end{array}$ & $\begin{array}{c}\text { Gasoline } \\
\text { Blending } \\
\text { Compo- } \\
\text { nents }\end{array}$ & $\begin{array}{l}\text { Finished } \\
\text { Motor } \\
\text { Gasoline }\end{array}$ & Jet Fuel & $\begin{array}{l}\text { Distillate } \\
\text { Fuel Oil }\end{array}$ & $\begin{array}{l}\text { Residual } \\
\text { Fuel Oil }\end{array}$ & Kerosene & $\begin{array}{c}\text { Special } \\
\text { Naphthas }\end{array}$ \\
\hline 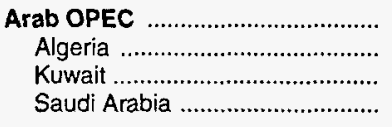 & $\begin{array}{r}56,284 \\
605 \\
243 \\
55,436\end{array}$ & $\begin{array}{r}4,347 \\
4,347 \\
0 \\
0\end{array}$ & $\begin{array}{l}0 \\
0 \\
0 \\
0\end{array}$ & $\begin{array}{r}882 \\
222 \\
0 \\
660\end{array}$ & $\begin{array}{r}10,709 \\
0 \\
0 \\
10,709\end{array}$ & $\begin{array}{l}0 \\
0 \\
0 \\
0\end{array}$ & $\begin{array}{r}447 \\
381 \\
0 \\
66\end{array}$ & $\begin{array}{r}9,277 \\
9,023 \\
0 \\
254\end{array}$ & $\begin{array}{l}0 \\
0 \\
0 \\
0\end{array}$ & $\begin{array}{l}0 \\
0 \\
0 \\
0\end{array}$ \\
\hline 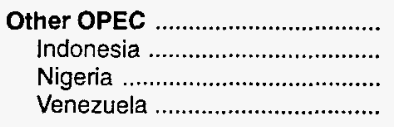 & $\begin{array}{r}169,019 \\
0 \\
104,384 \\
64,635\end{array}$ & $\begin{array}{r}251 \\
0 \\
0 \\
251\end{array}$ & $\begin{array}{r}364 \\
0 \\
0 \\
364\end{array}$ & $\begin{array}{r}18,503 \\
0 \\
441 \\
18,062\end{array}$ & $\begin{array}{r}15,880 \\
0 \\
0 \\
15,880\end{array}$ & $\begin{array}{r}13,976 \\
0 \\
0 \\
13,976\end{array}$ & $\begin{array}{r}18,723 \\
0 \\
0 \\
18,723\end{array}$ & $\begin{array}{r}19,929 \\
709 \\
1,215 \\
18,005\end{array}$ & $\begin{array}{l}7 \\
0 \\
0 \\
7\end{array}$ & $\begin{array}{l}0 \\
0 \\
0 \\
0\end{array}$ \\
\hline 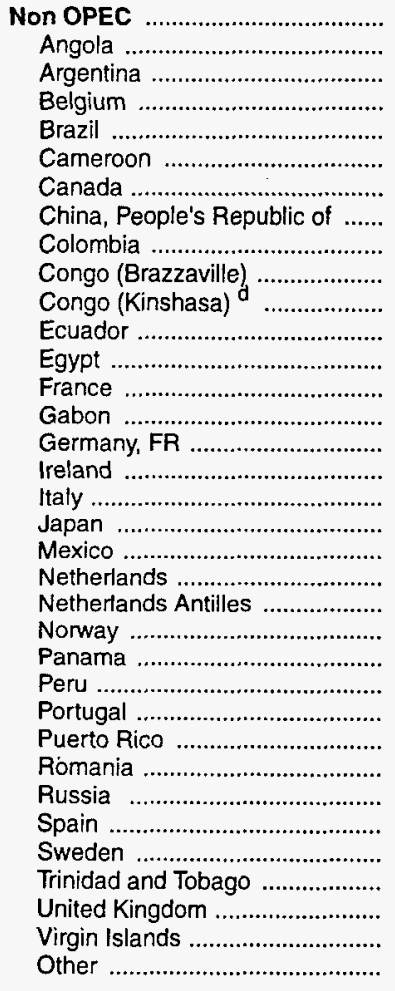 & $\begin{array}{r}302,841 \\
82,860 \\
2,562 \\
0 \\
0 \\
0 \\
22,996 \\
2,535 \\
19,348 \\
6,081 \\
3,522 \\
12,942 \\
10,509 \\
0 \\
38,550 \\
0 \\
0 \\
0 \\
0 \\
8,423 \\
0 \\
0 \\
68,978 \\
0 \\
714 \\
0 \\
0 \\
0 \\
0 \\
0 \\
0 \\
0 \\
22,474 \\
0 \\
347\end{array}$ & $\begin{array}{r}4,051 \\
0 \\
0 \\
0 \\
0 \\
0 \\
2,993 \\
0 \\
0 \\
0 \\
0 \\
0 \\
0 \\
0 \\
0 \\
0 \\
0 \\
0 \\
0 \\
0 \\
0 \\
0 \\
1,058 \\
0 \\
0 \\
0 \\
0 \\
0 \\
0 \\
0 \\
0 \\
0 \\
0 \\
0 \\
0\end{array}$ & $\begin{array}{r}12,650 \\
0 \\
0 \\
391 \\
0 \\
0 \\
277 \\
0 \\
0 \\
0 \\
0 \\
0 \\
0 \\
0 \\
0 \\
0 \\
0 \\
51 \\
0 \\
0 \\
0 \\
1,288 \\
0 \\
0 \\
0 \\
0 \\
0 \\
514 \\
439 \\
0 \\
0 \\
0 \\
0 \\
9,690 \\
0\end{array}$ & $\begin{array}{r}50,957 \\
0 \\
1,088 \\
3,562 \\
902 \\
0 \\
2,606 \\
0 \\
0 \\
0 \\
0 \\
0 \\
0 \\
3,678 \\
0 \\
828 \\
0 \\
1,718 \\
0 \\
1,709 \\
2,841 \\
1,485 \\
120 \\
0 \\
0 \\
1,115 \\
0 \\
2,369 \\
2,249 \\
2,025 \\
949 \\
1,271 \\
14,268 \\
2,416 \\
3,758\end{array}$ & $\begin{array}{r}81,067 \\
0 \\
0 \\
911 \\
490 \\
0 \\
26,781 \\
0 \\
0 \\
0 \\
0 \\
0 \\
0 \\
1,424 \\
0 \\
397 \\
0 \\
905 \\
0 \\
0 \\
1,256 \\
1,012 \\
1,918 \\
0 \\
141 \\
225 \\
175 \\
61 \\
173 \\
860 \\
309 \\
0 \\
4,613 \\
38,596 \\
820\end{array}$ & $\begin{array}{r}16,901 \\
0 \\
0 \\
0 \\
0 \\
0 \\
1,132 \\
0 \\
0 \\
0 \\
0 \\
0 \\
0 \\
0 \\
0 \\
0 \\
0 \\
0 \\
0 \\
0 \\
0 \\
8,401 \\
0 \\
0 \\
0 \\
0 \\
0 \\
0 \\
0 \\
0 \\
0 \\
0 \\
0 \\
7,368 \\
0\end{array}$ & $\begin{array}{r}56,992 \\
0 \\
189 \\
0 \\
0 \\
0 \\
24,831 \\
0 \\
0 \\
0 \\
0 \\
0 \\
0 \\
0 \\
0 \\
0 \\
0 \\
0 \\
0 \\
0 \\
0 \\
0 \\
0 \\
0 \\
0 \\
0 \\
0 \\
0 \\
330 \\
0 \\
0 \\
0 \\
6 \\
31,555 \\
81\end{array}$ & $\begin{array}{r}35,146 \\
0 \\
370 \\
484 \\
77 \\
1,040 \\
8,817 \\
0 \\
456 \\
0 \\
0 \\
172 \\
0 \\
210 \\
0 \\
631 \\
342 \\
0 \\
0 \\
0 \\
201 \\
787 \\
430 \\
135 \\
0 \\
0 \\
0 \\
0 \\
347 \\
710 \\
421 \\
234 \\
1,434 \\
16,545 \\
1,303\end{array}$ & $\begin{array}{r}488 \\
0 \\
0 \\
0 \\
0 \\
0 \\
413 \\
0 \\
0 \\
0 \\
0 \\
0 \\
0 \\
0 \\
0 \\
0 \\
0 \\
0 \\
0 \\
0 \\
0 \\
0 \\
0 \\
0 \\
0 \\
0 \\
0 \\
0 \\
0 \\
0 \\
0 \\
0 \\
0 \\
0 \\
0 \\
0 \\
0\end{array}$ & $\begin{array}{r}1,976 \\
0 \\
0 \\
0 \\
0 \\
0 \\
1,976 \\
0 \\
0 \\
0 \\
0 \\
0 \\
0 \\
0 \\
0 \\
0 \\
0 \\
0 \\
0 \\
0 \\
0 \\
0 \\
0 \\
0 \\
0 \\
0 \\
0 \\
0 \\
0 \\
0 \\
0 \\
0 \\
0 \\
0 \\
0\end{array}$ \\
\hline Total & 528,144 & 8,649 & 13,014 & 70,342 & 107,656 & 30,877 & 76,162 & 64,352 & 495 & 1,976 \\
\hline Persian Gulf $^{\ominus}$.............................. & 55,679 & $\mathbf{0}$ & $\mathbf{0}$ & 660 & 10,709 & $\mathbf{0}$ & 66 & 254 & 0 & 0 \\
\hline
\end{tabular}

See footnotes at end of table. 
Table 22. PAD District I-Imports of Crude Oil and Petroleum Products by Country of Origin, ${ }^{a} 1997$ (Continued) (Thousand Barrels)

\begin{tabular}{|c|c|c|c|c|c|c|c|c|c|c|}
\hline \multirow[b]{2}{*}{ Country of Origin } & \multirow[b]{2}{*}{$\begin{array}{c}\text { Naphtha for } \\
\text { Petrochemical } \\
\text { Feedstock } \\
\text { Use }\end{array}$} & \multirow[b]{2}{*}{$\begin{array}{c}\text { Other Oils for } \\
\text { Petrochemical } \\
\text { Feedstock } \\
\text { Use }\end{array}$} & \multirow[b]{2}{*}{ Lubricants } & \multirow[b]{2}{*}{$\begin{array}{c}\text { Asphalt and } \\
\text { Road Oil }\end{array}$} & \multirow[b]{2}{*}{$\begin{array}{c}\text { Other } \\
\text { Products }\end{array}$} & \multirow[b]{2}{*}{$\begin{array}{c}\text { Total } \\
\text { Products }\end{array}$} & \multirow[b]{2}{*}{$\begin{array}{c}\text { Total } \\
\text { Crude Oil } \\
\text { and } \\
\text { Products }\end{array}$} & \multicolumn{3}{|c|}{ Daily Average } \\
\hline & & & & & & & & $\begin{array}{c}\text { Crude } \\
\text { Oil } \\
\end{array}$ & Products & Total \\
\hline Arab OPEC & $\mathbf{0}$ & $\mathbf{0}$ & 0 & $\mathbf{0}$ & 1,014 & 26,676 & 82,960 & 154 & 73 & 227 \\
\hline Algeria & 0 & 0 & 0 & 0 & 0 & 13,973 & 14,578 & 2 & 38 & 40 \\
\hline 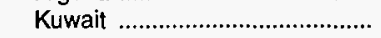 & 0 & 0 & 0 & 0 & 0 & 0 & 243 & 1 & 0 & 1 \\
\hline Saudi Arabia .................................. & 0 & 0 & 0 & 0 & 1,014 & 12,703 & 68,139 & 152 & 35 & 187 \\
\hline Other OPEC & 0 & 0 & 0 & 5,548 & 1,794 & 94,975 & 263,994 & 463 & 260 & 723 \\
\hline 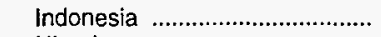 & 0 & 0 & 0 & 0 & 0 & 709 & 709 & 0 & 2 & 2 \\
\hline 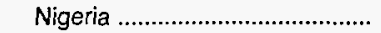 & 0 & 0 & 0 & 0 & 0 & 1,656 & 106,040 & 286 & 5 & 291 \\
\hline 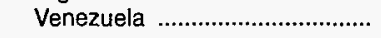 & 0 & 0 & 0 & 5,548 & 1,794 & 92,610 & 157,245 & 177 & 254 & 431 \\
\hline 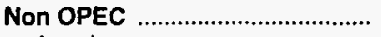 & 2,044 & 0 & 3,563 & 5,287 & 2,722 & 273,844 & 576,685 & 830 & 750 & 1,580 \\
\hline 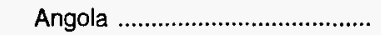 & 0 & 0 & 0 & 0 & 0 & 0 & 82,860 & 227 & 0 & 227 \\
\hline 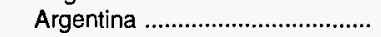 & 0 & 0 & 0 & 0 & 0 & 1,647 & 4,209 & 7 & 5 & 12 \\
\hline 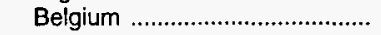 & 0 & 0 & 0 & 0 & 0 & 5,348 & 5,348 & 0 & 15 & 15 \\
\hline 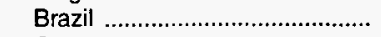 & 0 & 0 & 0 & 0 & 53 & 1,522 & 1,522 & 0 & 4 & 4 \\
\hline 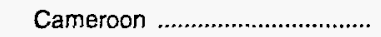 & 0 & 0 & 0 & 0 & 0 & 1,040 & 1,040 & 0 & 3 & 3 \\
\hline 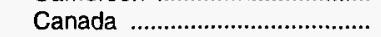 & 90 & 0 & 472 & 2,340 & 126 & 72,854 & 95,850 & 63 & 200 & 263 \\
\hline China, People's Republic of ...... & 0 & 0 & 0 & 0 & 0 & 0 & 2,535 & 7 & 0 & 7 \\
\hline 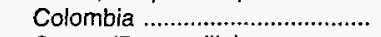 & 0 & 0 & 0 & 0 & 0 & 456 & 19,804 & 53 & 1 & 54 \\
\hline Congo (Brazzaville) .................... & 0 & 0 & 0 & 0 & 0 & 0 & 6,081 & 17 & 0 & 17 \\
\hline Congo (Kinshasa) & 0 & 0 & 0 & 0 & 0 & 0 & 3,522 & 10 & 0 & 10 \\
\hline 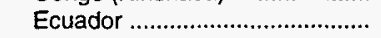 & 0 & 0 & 0 & 0 & 0 & 172 & 13,114 & 35 & (s) & 36 \\
\hline 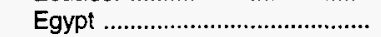 & 0 & 0 & 0 & 0 & 0 & 0 & 10,509 & 29 & 0 & 29 \\
\hline 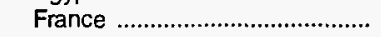 & 9 & 0 & 0 & 0 & 1,306 & 6,627 & 6,627 & 0 & 18 & 18 \\
\hline 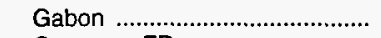 & 0 & 0 & 0 & 0 & 0 & 0 & 38,550 & 106 & 0 & 106 \\
\hline 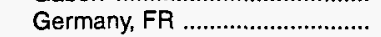 & 0 & 0 & 0 & 0 & 63 & 1,919 & 1,919 & 0 & 5 & 5 \\
\hline 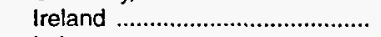 & 0 & 0 & 0 & 0 & 0 & 342 & 342 & 0 & 1 & 1 \\
\hline Italy & 0 & 0 & 0 & 0 & 0 & 2,674 & 2,674 & 0 & 7 & 7 \\
\hline 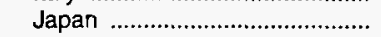 & 8 & 0 & 0 & 0 & 16 & 24 & 24 & 0 & (s) & (s) \\
\hline Mexico & 0 & 0 & 0 & 1,601 & 0 & 3,310 & 11,733 & 23 & 9 & 32 \\
\hline 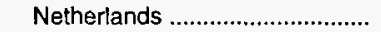 & 0 & 0 & 0 & 0 & 739 & 5,037 & 5,037 & 0 & 14 & 14 \\
\hline Netherlands Antil!es .................... & 0 & 0 & 0 & 241 & 0 & 13,214 & 13,214 & 0 & 36 & 36 \\
\hline 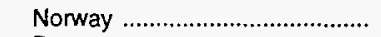 & 0 & 0 & 0 & 0 & 0 & 3,526 & 72,504 & 189 & 10 & 199 \\
\hline 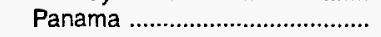 & 0 & 0 & 0 & 0 & 0 & 135 & 135 & 0 & (s) & (s) \\
\hline Peru & 0 & 0 & 0 & 0 & 0 & 141 & 855 & 2 & (s) & 2 \\
\hline 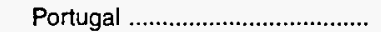 & 0 & 0 & 0 & 0 & 53 & 1,393 & 1,393 & 0 & 4 & 4 \\
\hline 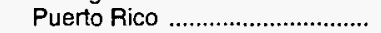 & 1,937 & 0 & 3,091 & 0 & 0 & 5,203 & 5,203 & 0 & 14 & 14 \\
\hline 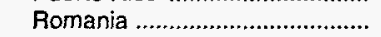 & 0 & 0 & 0 & 0 & 0 & 2,944 & 2,944 & 0 & 8 & 8 \\
\hline Russia & 0 & 0 & 0 & 0 & 0 & 3,538 & 3,538 & 0 & 10 & 10 \\
\hline Spain & 0 & 0 & 0 & 1,105 & 0 & 4,700 & 4,700 & 0 & 13 & 13 \\
\hline Sweden & 0 & 0 & 0 & 0 & 0 & 1,679 & 1,679 & 0 & 5 & 5 \\
\hline Trinidad and Tobago ................... & 0 & 0 & 0 & 0 & 0 & 1,505 & 1,505 & 0 & 4 & 4 \\
\hline 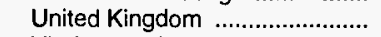 & 0 & 0 & 0 & 0 & 0 & 20,321 & 42,795 & 62 & 56 & 117 \\
\hline 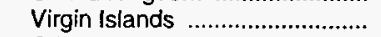 & 0 & 0 & 0 & 0 & 312 & 106,557 & 106,557 & 0 & 292 & 292 \\
\hline 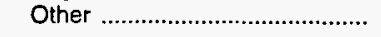 & 0 & 0 & 0 & 0 & 54 & 6,016 & 6,363 & 1 & 16 & 17 \\
\hline Total & 2,044 & $\mathbf{0}$ & 3,563 & 10,835 & 5,530 & 395,495 & 923,639 & 1,447 & 1,084 & 2,531 \\
\hline Persian Gulf ${ }^{{ }^{\mathrm{a}}}$................................ & 0 & 0 & 0 & $\mathbf{0}$ & 1,014 & 12,703 & 68,382 & 153 & 35 & 187 \\
\hline
\end{tabular}

a Crude oil and unfinished oils are reported by the PAD District in which they are to be processed; all other products are reported by the PAD District of entry.

Includes crude oil imported for storage in the Strategic Petroleum Reserve.

c Includes aviation gasoline, aviation gasoline blending components, miscellaneous products, other hydrocarbons and oxygenates, pentanes plus, petroleum coke, and waxes.

Formerly Zaire

- Includes Bahrain, Iran, Iraq, Kuwait, Qatar, Saudi Arabia, and United Arab Emirates.

(s) = Less than 500 barrels per day

Note: Totals may not equal sum of components due to independent rounding.

Source: Energy Information Administration (EIA) Form EIA-814, "Monthly Imports Report." 
Table 23. PAD District II-Imports of Crude Oil and Petroleum Products by Country of Origin, ${ }^{a} 1997$

(Thousand Barrels)

\begin{tabular}{|c|c|c|c|c|c|c|c|c|c|c|}
\hline Country of Origin & $\begin{array}{c}\text { Crude } \\
\text { Oil }^{\mathrm{b}}\end{array}$ & $\begin{array}{c}\text { Liquefied } \\
\text { Petroleum } \\
\text { Gases } \\
\end{array}$ & $\begin{array}{c}\text { Unfinished } \\
\text { Oils }\end{array}$ & $\begin{array}{c}\text { Gasoline } \\
\text { Blending } \\
\text { Compo- } \\
\text { nents }\end{array}$ & $\begin{array}{l}\text { Finished } \\
\text { Motor } \\
\text { Gasoline }\end{array}$ & Jet Fuel & $\begin{array}{c}\text { Distillate } \\
\text { Fuel Oil }\end{array}$ & $\begin{array}{c}\text { Residual } \\
\text { Fuel Oil } \\
\end{array}$ & Kerosene & $\begin{array}{c}\text { Special } \\
\text { Naphthas }\end{array}$ \\
\hline $\begin{array}{l}\text { Arab OPEC } \\
\text { Iraq } \\
\text { Kuwait } \\
\text { Saudi Arabia }\end{array}$ & $\begin{array}{r}62,012 \\
2,521 \\
17,518 \\
41,973\end{array}$ & $\begin{array}{l}0 \\
0 \\
0 \\
0\end{array}$ & $\begin{array}{l}0 \\
0 \\
0 \\
0\end{array}$ & $\begin{array}{l}0 \\
0 \\
0 \\
0\end{array}$ & $\begin{array}{l}0 \\
0 \\
0 \\
0\end{array}$ & $\begin{array}{l}0 \\
0 \\
0 \\
0\end{array}$ & $\begin{array}{l}0 \\
0 \\
0 \\
0\end{array}$ & $\begin{array}{l}0 \\
0 \\
0 \\
0\end{array}$ & $\begin{array}{l}0 \\
0 \\
0 \\
0\end{array}$ & $\begin{array}{l}0 \\
0 \\
0 \\
0\end{array}$ \\
\hline $\begin{array}{l}\text { Other OPEC } \\
\quad \text { Nigeria } \\
\text { Venezuela }\end{array}$ & $\begin{array}{l}96,742 \\
38,978 \\
57,764\end{array}$ & $\begin{array}{l}0 \\
0 \\
0\end{array}$ & $\begin{array}{l}0 \\
0 \\
0\end{array}$ & $\begin{array}{l}0 \\
0 \\
0\end{array}$ & $\begin{array}{l}0 \\
0 \\
0\end{array}$ & $\begin{array}{l}0 \\
0 \\
0\end{array}$ & $\begin{array}{l}0 \\
0 \\
0\end{array}$ & $\begin{array}{l}0 \\
0 \\
0\end{array}$ & $\begin{array}{l}0 \\
0 \\
0\end{array}$ & $\begin{array}{l}0 \\
0 \\
0\end{array}$ \\
\hline 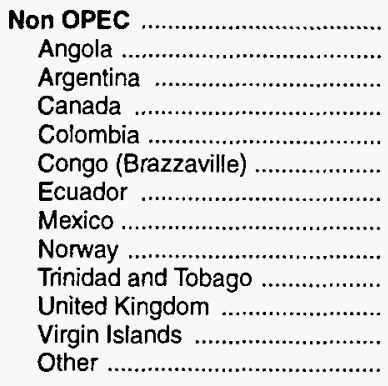 & $\begin{array}{r}437,657 \\
19,620 \\
2,640 \\
330,416 \\
24,059 \\
1,522 \\
6,185 \\
38,738 \\
5,550 \\
3,122 \\
5,297 \\
0 \\
508\end{array}$ & $\begin{array}{r}31,345 \\
0 \\
0 \\
31,345 \\
0 \\
0 \\
0 \\
0 \\
0 \\
0 \\
0 \\
0 \\
0\end{array}$ & $\begin{array}{r}48 \\
0 \\
0 \\
48 \\
0 \\
0 \\
0 \\
0 \\
0 \\
0 \\
0 \\
0 \\
0\end{array}$ & $\begin{array}{r}45 \\
0 \\
0 \\
45 \\
0 \\
0 \\
0 \\
0 \\
0 \\
0 \\
0 \\
0 \\
0\end{array}$ & $\begin{array}{r}955 \\
0 \\
0 \\
955 \\
0 \\
0 \\
0 \\
0 \\
0 \\
0 \\
0 \\
0 \\
0\end{array}$ & $\begin{array}{r}75 \\
0 \\
0 \\
0 \\
0 \\
0 \\
0 \\
0 \\
0 \\
0 \\
0 \\
75 \\
0\end{array}$ & $\begin{array}{r}1,632 \\
0 \\
0 \\
1,632 \\
0 \\
0 \\
0 \\
0 \\
0 \\
0 \\
0 \\
0 \\
0\end{array}$ & $\begin{array}{r}140 \\
0 \\
0 \\
140 \\
0 \\
0 \\
0 \\
0 \\
0 \\
0 \\
0 \\
0 \\
0\end{array}$ & $\begin{array}{l}0 \\
0 \\
0 \\
0 \\
0 \\
0 \\
0 \\
0 \\
0 \\
0 \\
0 \\
0 \\
0\end{array}$ & $\begin{array}{r}347 \\
0 \\
0 \\
347 \\
0 \\
0 \\
0 \\
0 \\
0 \\
0 \\
0 \\
0 \\
0\end{array}$ \\
\hline Total & 596,411 & 31,345 & 48 & 45 & 955 & 75 & 1,632 & 140 & 0 & 347 \\
\hline Persian Gulf ${ }^{\ominus}$ & 62,012 & 0 & 0 & $\mathbf{0}$ & $\mathbf{0}$ & $\mathbf{0}$ & 0 & 0 & 0 & 0 \\
\hline
\end{tabular}

See footnotes at end of table. 
Table 23. PAD District II-Imports of Crude Oil and Petroleum Products by Country of Origin, ${ }^{a} 1997$ (Continued) (Thousand Barrels)

\begin{tabular}{|c|c|c|c|c|c|c|c|c|c|c|}
\hline \multirow[b]{2}{*}{ Country of Origin } & \multirow[b]{2}{*}{$\begin{array}{c}\text { Naphtha for } \\
\text { Petrochemical } \\
\text { Feedstock } \\
\text { Use }\end{array}$} & \multirow[b]{2}{*}{$\begin{array}{c}\text { Other Oils for } \\
\text { Petrochemical } \\
\text { Feedstock } \\
\text { Use }\end{array}$} & \multirow[b]{2}{*}{ Lubricants } & \multirow[b]{2}{*}{$\begin{array}{c}\text { Asphalt and } \\
\text { Road Oil }\end{array}$} & \multirow[b]{2}{*}{$\begin{array}{c}\text { Other } \\
\text { Products }\end{array}$} & \multirow[b]{2}{*}{$\begin{array}{c}\text { Total } \\
\text { Products }\end{array}$} & \multirow[b]{2}{*}{$\begin{array}{l}\text { Total } \\
\text { Crude Oil } \\
\text { and } \\
\text { Products }\end{array}$} & \multicolumn{3}{|c|}{ Daily Average } \\
\hline & & & & & & & & $\begin{array}{c}\text { Crude } \\
\text { Oil }\end{array}$ & Products & Total \\
\hline Arab OPEC & 0 & 0 & 0 & 0 & 0 & 0 & 62,012 & 170 & $\mathbf{0}$ & 170 \\
\hline Iraq & 0 & 0 & 0 & 0 & 0 & 0 & 2,521 & 7 & 0 & 7 \\
\hline 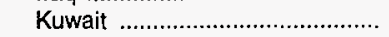 & 0 & 0 & 0 & 0 & 0 & 0 & 17,518 & 48 & 0 & 48 \\
\hline 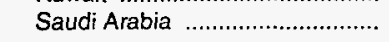 & 0 & 0 & 0 & 0 & 0 & 0 & 41,973 & 115 & 0 & 115 \\
\hline Other OPEC & 0 & 0 & 0 & 0 & 0 & 0 & 96,742 & 265 & 0 & 265 \\
\hline 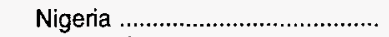 & 0 & 0 & 0 & 0 & 0 & 0 & 38,978 & 107 & 0 & 107 \\
\hline 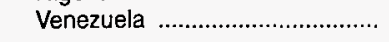 & 0 & 0 & 0 & 0 & 0 & 0 & 57,764 & 158 & 0 & 158 \\
\hline Non OPEC & 402 & 0 & 259 & 652 & 621 & 36,521 & 474,178 & 1,199 & 100 & 1,299 \\
\hline 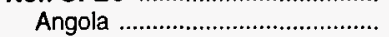 & 0 & 0 & 0 & 0 & 0 & 0 & 19,620 & 54 & 0 & 54 \\
\hline 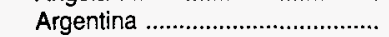 & 0 & 0 & 0 & 0 & 0 & 0 & 2,640 & 7 & 0 & 7 \\
\hline 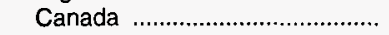 & 402 & 0 & 259 & 652 & 618 & 36,443 & 366,859 & 905 & 100 & 1,005 \\
\hline 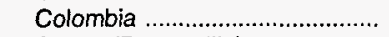 & 0 & 0 & 0 & 0 & 0 & 0 & 24,059 & 66 & 0 & 66 \\
\hline Congo (Brazzaville) .................... & 0 & 0 & 0 & 0 & 0 & 0 & 1,522 & 4 & 0 & 4 \\
\hline 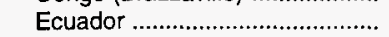 & 0 & 0 & 0 & 0 & 0 & 0 & 6,185 & 17 & 0 & 17 \\
\hline Mexico & 0 & 0 & 0 & 0 & 0 & 0 & 38,738 & 106 & 0 & 106 \\
\hline 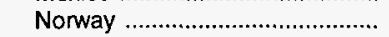 & 0 & 0 & 0 & 0 & 0 & 0 & 5,550 & 15 & 0 & 15 \\
\hline Trinidad and Tobago ..................... & 0 & 0 & 0 & 0 & 0 & 0 & 3,122 & 9 & 0 & 9 \\
\hline United Kingdom ......................... & 0 & 0 & 0 & 0 & 0 & 0 & 5,297 & 15 & 0 & 15 \\
\hline Virgin Islands ............................. & 0 & 0 & 0 & 0 & 0 & 75 & 75 & 0 & (s) & (s) \\
\hline Other & 0 & 0 & 0 & 0 & 3 & 3 & 511 & 1 & (s) & 1 \\
\hline 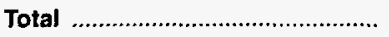 & 402 & 0 & 259 & 652 & 621 & 36,521 & 632,932 & 1,634 & 100 & 1,734 \\
\hline Persian Gulf ${ }^{e}$ & 0 & 0 & 0 & 0 & 0 & 0 & 62,012 & 170 & 0 & 170 \\
\hline
\end{tabular}

a Crude oil and unfinished oils are reported by the PAD District in which they are to be processed; all other products are reported by the PAD District of entry.

b Includes crude oil imported for storage in the Strategic Petroleum Reserve.

c Includes aviation gasoline, aviation gasoline blending components, miscellaneous products, other hydrocarbons and oxygenates, pentanes plus, petroleum coke, and waxes.

d Formerly Zaire.

- Includes Bahrain, Iran, Iraq, Kuwait, Qatar, Saudi Arabia, and United Arab Emirates.

(s) = Less than 500 barrels per day.

Note: Totals may not equal sum of components due to independent rounding.

Source: Energy Information Administration (EIA) Form ElA-814, "Monthly Imports Report." 
Table 24. PAD District III-Imports of Crude Oil and Petroleum Products by Country of Origin, ${ }^{a} 1997$

(Thousand Barrels)

\begin{tabular}{|c|c|c|c|c|c|c|c|c|c|c|}
\hline Country of Origin & $\begin{array}{c}\text { Crude } \\
\text { Oil }^{\mathbf{b}}\end{array}$ & $\begin{array}{c}\text { Liquefied } \\
\text { Petroleum } \\
\text { Gases }\end{array}$ & $\begin{array}{c}\text { Unfinished } \\
\text { Oils }\end{array}$ & $\begin{array}{c}\text { Gasoline } \\
\text { Blending } \\
\text { Compo- } \\
\text { nents }\end{array}$ & $\begin{array}{l}\text { Finished } \\
\text { Motor } \\
\text { Gasoline }\end{array}$ & Jet Fuel & $\begin{array}{l}\text { Distillate } \\
\text { Fuel Oil }\end{array}$ & $\begin{array}{c}\text { Residual } \\
\text { Fuel Oil } \\
\end{array}$ & Kerosene & $\begin{array}{c}\text { Special } \\
\text { Naphthas }\end{array}$ \\
\hline Arab OPEC & 454,202 & 6,975 & 34,809 & 0 & 0 & 0 & 0 & 2,806 & 0 & 0 \\
\hline Algeria & 1,590 & 6,975 & 16,395 & 0 & 0 & 0 & 0 & 342 & 0 & 0 \\
\hline Iraq & 17,102 & 0 & 0 & 0 & 0 & 0 & 0 & 0 & 0 & 0 \\
\hline Kuwait ....................................... & 66,112 & 0 & 0 & 0 & 0 & 0 & 0 & 0 & 0 & 0 \\
\hline Qatar ........................................... & & 0 & 0 & 0 & 0 & 0 & 0 & 0 & 0 & 0 \\
\hline 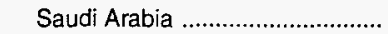 & 369,398 & 0 & 17,798 & 0 & 0 & 0 & 0 & 2,464 & 0 & 0 \\
\hline United Arab Emirates ..................... & 0 & 0 & 616 & 0 & 0 & 0 & 0 & 0 & 0 & 0 \\
\hline Other OPEC & 488,605 & 3,894 & 34,094 & 538 & 0 & $\mathbf{0}$ & 0 & 492 & 0 & 0 \\
\hline 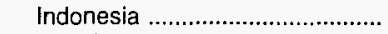 & 982 & 0 & 528 & 0 & 0 & 0 & 0 & 0 & 0 & 0 \\
\hline Nigeria & 108,246 & 0 & 1,160 & 74 & 0 & 0 & 0 & 93 & 0 & 0 \\
\hline Venezuela & 379,377 & 3,894 & 32,406 & 464 & 0 & 0 & 0 & 399 & 0 & 0 \\
\hline Non OPEC & 740,429 & 7,606 & 39,504 & 15 & 2,844 & 204 & $\mathbf{0}$ & 1,293 & 0 & 370 \\
\hline Angola & 52,818 & 0 & 349 & 0 & 0 & 0 & 0 & 0 & 0 & 0 \\
\hline Argentina & 10,295 & 0 & 614 & 0 & 0 & 0 & 0 & 0 & 0 & 0 \\
\hline Australia & 0 & 0 & 0 & 0 & 0 & 0 & 0 & 0 & 0 & 0 \\
\hline Bahama Islands & 0 & 0 & 350 & 0 & 0 & 0 & 0 & 0 & 0 & 0 \\
\hline Belgium & 0 & 0 & 4,946 & 0 & 0 & 0 & 0 & 0 & 0 & 0 \\
\hline 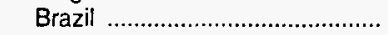 & 0 & 133 & 0 & 15 & 0 & 0 & 0 & 0 & 0 & 66 \\
\hline 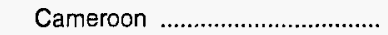 & 0 & 0 & 0 & 0 & 0 & 0 & 0 & 635 & 0 & 0 \\
\hline Canada ............................................. & 896 & 7,276 & 1,692 & 0 & 0 & 0 & 0 & 0 & 0 & 304 \\
\hline China, People's Republic of ....... & 6,938 & 0 & 0 & 0 & 0 & 0 & 0 & 0 & 0 & 0 \\
\hline 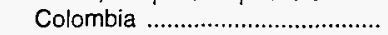 & 52,347 & 0 & 161 & 0 & 0 & 0 & 0 & 0 & 0 & 0 \\
\hline Congo (Brazzaville) .......................... & 9,575 & 0 & 0 & 0 & 0 & 0 & 0 & 0 & 0 & 0 \\
\hline Congo (Kinshasa) ${ }^{d}$ & 4,077 & 0 & 0 & 0 & 0 & 0 & 0 & 0 & 0 & 0 \\
\hline 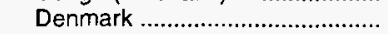 & 226 & 0 & 0 & 0 & 0 & 0 & 0 & 0 & 0 & 0 \\
\hline 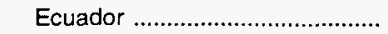 & 12,888 & 0 & 140 & 0 & 0 & 0 & 0 & 0 & 0 & 0 \\
\hline 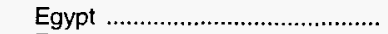 & 2,480 & 0 & 100 & 0 & 0 & 0 & 0 & 0 & 0 & 0 \\
\hline 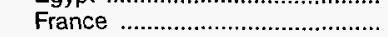 & 0 & 0 & 3,310 & 0 & 0 & 0 & 0 & 0 & 0 & 0 \\
\hline 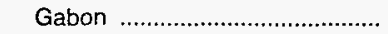 & 45,559 & 0 & 0 & 0 & 0 & 0 & 0 & 0 & 0 & 0 \\
\hline 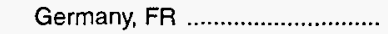 & 0 & 0 & 903 & 0 & 0 & 0 & 0 & 0 & 0 & 0 \\
\hline 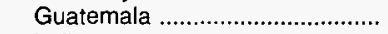 & 6,121 & 0 & 0 & 0 & 0 & 0 & 0 & 0 & 0 & 0 \\
\hline 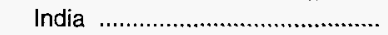 & 0 & 0 & 233 & 0 & 0 & 0 & 0 & 0 & 0 & 0 \\
\hline italy & 0 & 0 & 0 & 0 & 0 & 0 & 0 & 0 & 0 & 0 \\
\hline 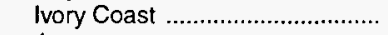 & 0 & 0 & 499 & 0 & 0 & 0 & 0 & 120 & 0 & 0 \\
\hline Japan & 0 & 0 & 0 & 0 & 0 & 0 & 0 & 0 & 0 & 0 \\
\hline Korea, Republic of ........................ & 0 & 0 & 365 & 0 & 0 & 0 & 0 & 0 & 0 & 0 \\
\hline Malaysia & 1,622 & 0 & 0 & 0 & 0 & 0 & 0 & 0 & 0 & 0 \\
\hline 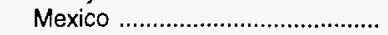 & 444,110 & 197 & 0 & 0 & 0 & 204 & 0 & 0 & 0 & 0 \\
\hline Netherlands & 0 & 0 & 3,147 & 0 & 0 & 0 & 0 & 0 & 0 & 0 \\
\hline Netherlands Antilles & 0 & 0 & 10,184 & 0 & 0 & 0 & 0 & 0 & 0 & 0 \\
\hline 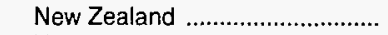 & 0 & 0 & 0 & 0 & 0 & 0 & 0 & 0 & 0 & 0 \\
\hline 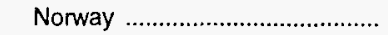 & 30,520 & 0 & 2,170 & 0 & 0 & 0 & 0 & 0 & 0 & 0 \\
\hline Oman & 0 & 0 & 2,430 & 0 & 0 & 0 & 0 & 0 & 0 & 0 \\
\hline 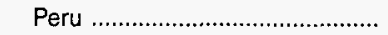 & 4,919 & 0 & 260 & 0 & 0 & 0 & 0 & 0 & 0 & 0 \\
\hline 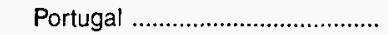 & 0 & 0 & 0 & 0 & 2,844 & 0 & 0 & 0 & 0 & 0 \\
\hline 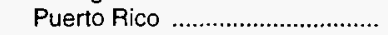 & 0 & 0 & 0 & 0 & 0 & 0 & 0 & 0 & 0 & 0 \\
\hline 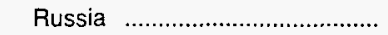 & 955 & 0 & 232 & 0 & 0 & 0 & 0 & 0 & 0 & 0 \\
\hline Singapore & 0 & 0 & 408 & 0 & 0 & 0 & 0 & 0 & 0 & 0 \\
\hline Spain & 0 & 0 & 2,719 & 0 & 0 & 0 & 0 & 0 & 0 & 0 \\
\hline 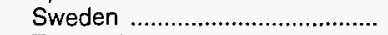 & 0 & 0 & 938 & 0 & 0 & 0 & 0 & 0 & 0 & 0 \\
\hline Thailand & 0 & 0 & 0 & 0 & 0 & 0 & 0 & 0 & 0 & 0 \\
\hline Trinidad and Tobago ............................ & 17,178 & 0 & 349 & 0 & 0 & 0 & 0 & 0 & 0 & 0 \\
\hline Tunisia & 0 & 0 & 0 & 0 & 0 & 0 & 0 & 198 & 0 & 0 \\
\hline 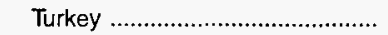 & 0 & 0 & 230 & 0 & 0 & 0 & 0 & 0 & 0 & 0 \\
\hline United Kingdom ……...................... & 33,929 & 0 & 540 & 0 & 0 & 0 & 0 & 0 & 0 & 0 \\
\hline 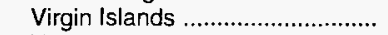 & 0 & 0 & 720 & 0 & 0 & 0 & 0 & 0 & 0 & 0 \\
\hline Yemen & 0 & 0 & 0 & 0 & 0 & 0 & 0 & 304 & 0 & 0 \\
\hline Other & 2,536 & 0 & 1,515 & 0 & 0 & 0 & 0 & 36 & 0 & 0 \\
\hline Total & $1,683,236$ & 18,475 & 108,407 & 553 & 2,844 & 204 & 0 & 4,591 & 0 & 370 \\
\hline Persian Gulf ${ }^{e}$ & 452,612 & 0 & 18,414 & 0 & 0 & 0 & 0 & 2,464 & 0 & 0 \\
\hline
\end{tabular}

See footnotes at end of table. 
Table 24. PAD District III-Imports of Crude Oil and Petroleum Products by Country of Origin, ${ }^{\mathrm{a}} 1997$ (Continued) (Thousand Barrels)

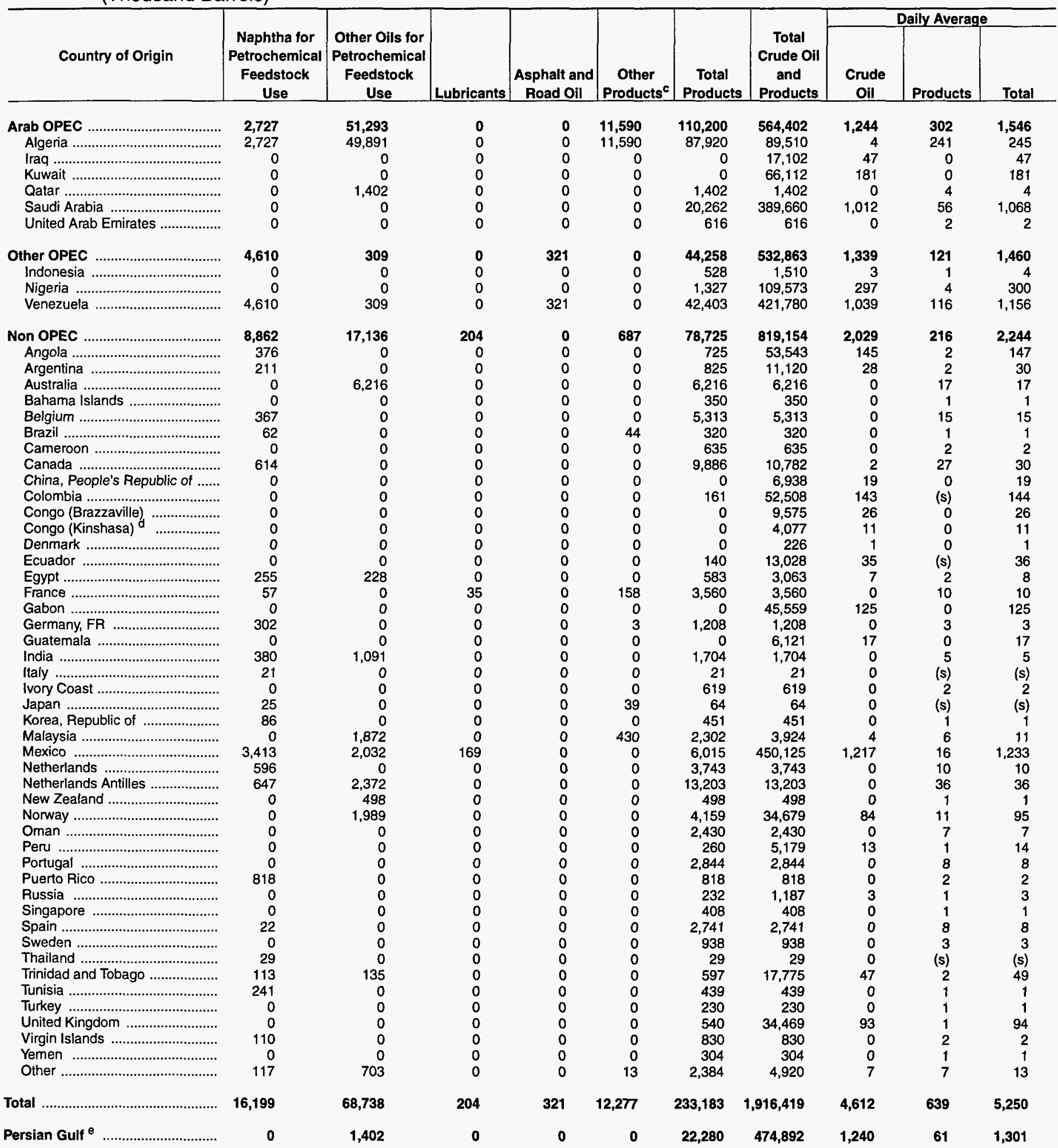

Crude oil and unfinished oils are reported by the PAD District in which they are to be processed; all other products are reported by the PAD District of entry.

b Includes crude oil imported for storage in the Strategic Petroleum Reserve.

c Includes aviation gasoline, aviation gasoline blending components, miscellaneous products, other hydrocarbons and oxygenates, pentanes plus, petroleum coke, and waxes.

d Formerty Zaire.

- Includes Bahrain, Iran, Iraq, Kuwait, Qatar, Saudi Arabia, and United Arab Emirates.

$(s)=$ Less than 500 barrels per day.

Note: Totals may not equal sum of components due to independent rounding.

Source: Energy Information Administration (EIA) Form EIA-814, "Monthly Imports Report." 
Table 25. PAD Districts IV and V-Imports of Crude Oil and Petroleum Products by Country of Origin, ${ }^{a} 1997$

(Thousand Barrels)

\begin{tabular}{|c|c|c|c|c|c|c|c|c|c|c|}
\hline Country of Origin & $\begin{array}{c}\text { Crude } \\
\text { Oil }^{\mathrm{b}}\end{array}$ & $\begin{array}{c}\text { Liquefied } \\
\text { Petroleum } \\
\text { Gases }\end{array}$ & $\begin{array}{c}\text { Unfinished } \\
\text { Oils }\end{array}$ & $\begin{array}{c}\text { Gasoline } \\
\text { Blending } \\
\text { Compo- } \\
\text { nents } \\
\end{array}$ & $\begin{array}{l}\text { Finished } \\
\text { Motor } \\
\text { Gasoline }\end{array}$ & Jet Fuel & $\begin{array}{c}\text { Distillate } \\
\text { Fuel Oil }\end{array}$ & $\begin{array}{c}\text { Residual } \\
\text { Fuel Oil }\end{array}$ & Kerosene & $\begin{array}{c}\text { Special } \\
\text { Naphthas }\end{array}$ \\
\hline & \multicolumn{10}{|c|}{ PAD District IV } \\
\hline $\begin{array}{l}\text { Non OPEC } \\
\text { Canada }\end{array}$ & $\begin{array}{l}45,283 \\
45,283\end{array}$ & $\begin{array}{l}3,135 \\
3,135\end{array}$ & $\begin{array}{l}0 \\
0\end{array}$ & $\begin{array}{l}0 \\
0\end{array}$ & $\begin{array}{l}234 \\
234\end{array}$ & $\begin{array}{l}0 \\
0\end{array}$ & $\begin{array}{l}3,256 \\
3,256\end{array}$ & $\begin{array}{l}0 \\
0\end{array}$ & $\begin{array}{l}0 \\
0\end{array}$ & $\begin{array}{l}1 \\
1\end{array}$ \\
\hline Total & 45,283 & 3,135 & 0 & 0 & 234 & 0 & 3,256 & $\mathbf{0}$ & 0 & 1 \\
\hline
\end{tabular}

PAD District $V$

\begin{tabular}{|c|c|c|c|c|c|c|c|c|c|c|}
\hline Arab OPEC & 26,549 & $\mathbf{0}$ & $\mathbf{0}$ & 0 & 0 & 0 & 0 & 0 & 63 & $\mathbf{0}$ \\
\hline Iraq & 12,907 & 0 & 0 & 0 & 0 & 0 & 0 & 0 & 0 & 0 \\
\hline 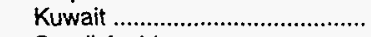 & 8,356 & 0 & 0 & 0 & 0 & 0 & 0 & 0 & 0 & 0 \\
\hline 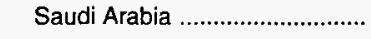 & 5,286 & 0 & 0 & 0 & 0 & 0 & 0 & 0 & 63 & 0 \\
\hline 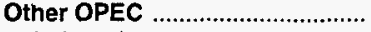 & 24,629 & 0 & 1,097 & 0 & $\mathbf{0}$ & 275 & 0 & 1,119 & $\mathbf{0}$ & $\mathbf{0}$ \\
\hline 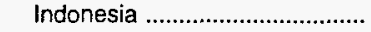 & 17,675 & 0 & 433 & 0 & 0 & 0 & 0 & 954 & 0 & 0 \\
\hline 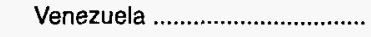 & 6,954 & 0 & 664 & 0 & 0 & 275 & 0 & 165 & 0 & 0 \\
\hline 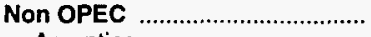 & 98,047 & 98 & 6,248 & 2,208 & 1,148 & 1,678 & 2,052 & 627 & 12 & 15 \\
\hline 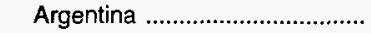 & 9,556 & 0 & 0 & 0 & 0 & 0 & 0 & 0 & 0 & 0 \\
\hline 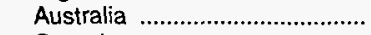 & 11,405 & 0 & 0 & 0 & 0 & 0 & 0 & 0 & 0 & 0 \\
\hline 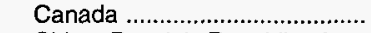 & 37,805 & 98 & 67 & 0 & 178 & 27 & 786 & 0 & 12 & 15 \\
\hline China, People's Republic of .... & 8,139 & 0 & 0 & 10 & 222 & 0 & 0 & 0 & 0 & 0 \\
\hline 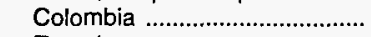 & 2,707 & 0 & 0 & 0 & 0 & 0 & 0 & 0 & 0 & 0 \\
\hline 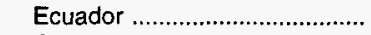 & 9,552 & 0 & 0 & 0 & 0 & 0 & 0 & 0 & 0 & 0 \\
\hline Japan & 0 & 0 & 0 & 227 & 0 & 121 & 0 & 0 & 0 & 0 \\
\hline Korea, Republic of ...................... & 0 & 0 & 0 & 0 & 0 & 768 & 0 & 0 & 0 & 0 \\
\hline 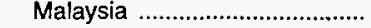 & 1,228 & 0 & 2,276 & 0 & 0 & 0 & 106 & 627 & 0 & 0 \\
\hline 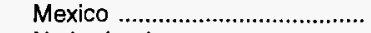 & 5,004 & 0 & 0 & 0 & 0 & 1 & 0 & 0 & 0 & 0 \\
\hline 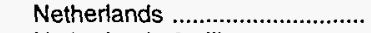 & 0 & 0 & 0 & 112 & 0 & 0 & 0 & 0 & 0 & 0 \\
\hline Netherlands Antilles ................. & 0 & 0 & 346 & 0 & 0 & 141 & 0 & 0 & 0 & 0 \\
\hline Oman & 1,511 & 0 & 0 & 0 & 0 & 0 & 0 & 0 & 0 & 0 \\
\hline Peru & 5,562 & 0 & 0 & 0 & 0 & 0 & 0 & 0 & 0 & 0 \\
\hline 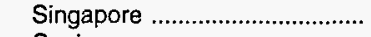 & 0 & 0 & 3,277 & 0 & 0 & 0 & 0 & 0 & 0 & 0 \\
\hline 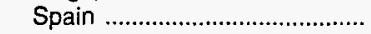 & 0 & 0 & 282 & 0 & 0 & 0 & 0 & 0 & 0 & 0 \\
\hline 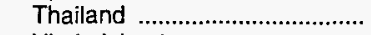 & 0 & 0 & 0 & 0 & 459 & 0 & 0 & 0 & 0 & 0 \\
\hline 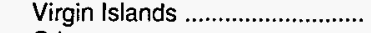 & 0 & 0 & 0 & 0 & 289 & 620 & 1,160 & 0 & 0 & 0 \\
\hline 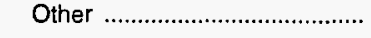 & 5,578 & 0 & 0 & 1,859 & 0 & 0 & 0 & 0 & 0 & 0 \\
\hline (1) & 149,225 & 98 & 7,345 & 2,208 & 1,148 & 1,953 & 2,052 & 1,746 & 75 & 15 \\
\hline Persian Gulf ${ }^{\ominus}$ & 26,549 & 0 & 0 & 0 & 0 & 0 & $\mathbf{0}$ & 0 & 63 & $\mathbf{0}$ \\
\hline
\end{tabular}


Table 25. PAD Districts IV and V-Imports of Crude Oil and Petroleum Products by Country of Origin, ${ }^{\mathrm{a}} 1997$ (Continued)

(Thousand Barrels)

\begin{tabular}{c|c|c|c|c|c|c|c|c|}
\hline Country of Origin & $\begin{array}{c}\text { Naphtha for } \\
\text { Petrochemical } \\
\text { Feedstock } \\
\text { Use }\end{array}$ & $\begin{array}{c}\text { Other Oils for } \\
\text { Petrochemical } \\
\text { Feedstock } \\
\text { Use }\end{array}$ & $\begin{array}{c}\text { Lubricants } \\
\text { Asphalt and } \\
\text { Road Oil }\end{array}$ & $\begin{array}{c}\text { Other } \\
\text { Products }\end{array}$ & $\begin{array}{c}\text { Total } \\
\text { Products }\end{array}$ & $\begin{array}{c}\text { Total } \\
\text { Crude Oil } \\
\text { and } \\
\text { Products }\end{array}$ & $\begin{array}{c}\text { Daily Average } \\
\text { Crude } \\
\text { Oil }\end{array}$ & $\begin{array}{c}\text { Products } \\
\text { Prodal }\end{array}$ \\
\hline
\end{tabular}

PAD District IV

\begin{tabular}{|c|c|c|c|c|c|c|c|c|c|c|}
\hline 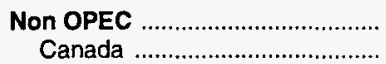 & $\begin{array}{l}0 \\
0\end{array}$ & $\begin{array}{l}0 \\
0\end{array}$ & $\begin{array}{l}0 \\
0\end{array}$ & $\begin{array}{l}54 \\
54\end{array}$ & $\begin{array}{l}702 \\
702\end{array}$ & $\begin{array}{l}7,382 \\
7,382\end{array}$ & $\begin{array}{l}52,665 \\
52,665\end{array}$ & $\begin{array}{l}124 \\
124\end{array}$ & $\begin{array}{l}20 \\
20\end{array}$ & $\begin{array}{l}144 \\
144\end{array}$ \\
\hline Total & 0 & 0 & 0 & 54 & 702 & 7,382 & 52,665 & 124 & 20 & 144 \\
\hline
\end{tabular}

PAD District V

\begin{tabular}{|c|c|c|c|c|c|c|c|c|c|c|}
\hline Arab OPEC & $\mathbf{0}$ & 0 & 0 & 0 & 8,572 & 8,635 & 35,184 & 73 & 24 & 96 \\
\hline Iraq & 0 & 0 & 0 & 0 & 0 & 0 & 12,907 & 35 & 0 & 35 \\
\hline 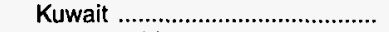 & 0 & 0 & 0 & 0 & 0 & 0 & 8,356 & 23 & 0 & 23 \\
\hline Saudi Arabia ................................... & 0 & 0 & 0 & 0 & 8,572 & 8,635 & 13,921 & 14 & 24 & 38 \\
\hline Other OPEC & $\mathbf{0}$ & 0 & 0 & 0 & 2,248 & 4,739 & 29,368 & 67 & 13 & 80 \\
\hline 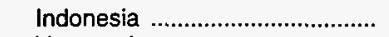 & 0 & 0 & 0 & 0 & 0 & 1,387 & 19,062 & 48 & 4 & 52 \\
\hline 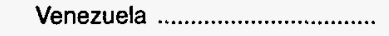 & 0 & 0 & 0 & 0 & 2,248 & 3,352 & 10,306 & 19 & 9 & 28 \\
\hline 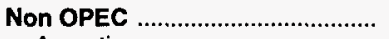 & 36 & 348 & 0 & 0 & 6,246 & 20,716 & 118,763 & 269 & 57 & 325 \\
\hline Argentina & 0 & 0 & 0 & 0 & 0 & 0 & 9,556 & 26 & 0 & 26 \\
\hline 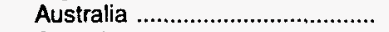 & 0 & 0 & 0 & 0 & 0 & 0 & 11,405 & 31 & 0 & 31 \\
\hline Canada & 0 & 348 & 0 & 0 & 5,110 & 6,641 & 44,446 & 104 & 18 & 122 \\
\hline China, People's Republic of ....... & 0 & 0 & 0 & 0 & 0 & 232 & 8,371 & 22 & 1 & 23 \\
\hline 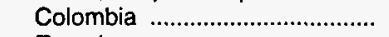 & 0 & 0 & 0 & 0 & 0 & 0 & 2,707 & 7 & 0 & 7 \\
\hline 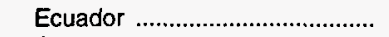 & 0 & 0 & 0 & 0 & 0 & 0 & 9,552 & 26 & 0 & 26 \\
\hline 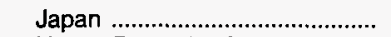 & 0 & 0 & 0 & 0 & 0 & 348 & 348 & 0 & 1 & 1 \\
\hline Korea, Republic of .......................... & 36 & 0 & 0 & 0 & 244 & 1,048 & 1,048 & 0 & 3 & 3 \\
\hline 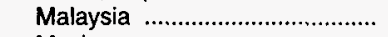 & 0 & 0 & 0 & 0 & 53 & 3,062 & 4,290 & 3 & 8 & 12 \\
\hline 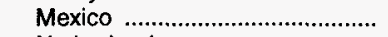 & 0 & 0 & 0 & 0 & 9 & 10 & 5,014 & 14 & (s) & 14 \\
\hline 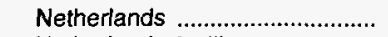 & 0 & 0 & 0 & 0 & 113 & 225 & 225 & 0 & 1 & 1 \\
\hline Netherlands Antilles .................... & 0 & 0 & 0 & 0 & 0 & 487 & 487 & 0 & 1 & 1 \\
\hline Oman & 0 & 0 & 0 & 0 & 0 & 0 & 1,511 & 4 & 0 & 4 \\
\hline 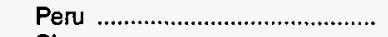 & 0 & 0 & 0 & 0 & 0 & 0 & 5,562 & 15 & 0 & 15 \\
\hline Singapore & 0 & 0 & 0 & 0 & 632 & 3,909 & 3,909 & 0 & 11 & 11 \\
\hline 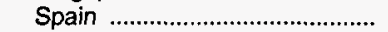 & 0 & 0 & 0 & 0 & 0 & 282 & 282 & 0 & 1 & 1 \\
\hline 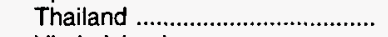 & 0 & 0 & 0 & 0 & 0 & 459 & 459 & 0 & 1 & 1 \\
\hline Virgin Islands ............................... & 0 & 0 & 0 & 0 & 0 & 2,069 & 2,069 & 0 & 6 & 6 \\
\hline Other $\ldots \ldots \ldots \ldots \ldots \ldots \ldots \ldots \ldots \ldots$ & 0 & 0 & 0 & 0 & 85 & 1,944 & 7,522 & 15 & 5 & 21 \\
\hline Total & 36 & 348 & 0 & 0 & 17,066 & 34,090 & 183,315 & 409 & 93 & 502 \\
\hline Persian Gulf ${ }^{\ominus}$ & $\mathbf{0}$ & $\mathbf{0}$ & 0 & $\mathbf{0}$ & 8,572 & 8,635 & 35,184 & 73 & 24 & 96 \\
\hline
\end{tabular}

\footnotetext{
a Crude oil and unfinished oils are reported by the PAD District in which they are to be processed; all other products are reported by the PAD District of entry.

b Includes crude oil imported for storage in the Strategic Petroleum Reserve.

c Includes aviation gasoline, aviation gasoline blending components, miscellaneous products, other hydrocarbons and oxygenates, pentanes plus, petroleum coke, and waxes.

Formerly Zaire.

Includes Bahrain, Iran, Iraq, Kuwait, Qatar, Saudi Arabia, and United Arab Emirates.

(s) = Less than 500 barrels per day.

Note: Totals may not equal sum of components due to independent rounding.

Source: Energy Information Administration (EIA) Form EIA-814, "Monthly Imports Report."
} 
Table 26. Imports of Residual Fuel Oil by Sulfur Content and by PAD District and State of Entry, 1997 (Thousand Barrels)

\begin{tabular}{|c|c|c|c|c|}
\hline \multirow[b]{2}{*}{ PAD District and State of Entry } & \multicolumn{4}{|c|}{ Residual Fuel Oil } \\
\hline & $\begin{array}{l}\text { Less than } \\
0.31 \% \\
\text { Sulfur }\end{array}$ & $\begin{array}{l}0.31 \text { to } \\
1.00 \% \\
\text { Sulfur }\end{array}$ & $\begin{array}{l}\text { Greater } \\
\text { than } 1.00 \% \\
\text { Sulfur }\end{array}$ & Total \\
\hline $\begin{array}{l}\text { PAD District I } \\
\text { Connecticut } \\
\text { Delaware } \\
\text { Georgida } \\
\text { Maine } \\
\text { Maryland } \\
\text { Massachusetts } \\
\text { New Hampshire Jersey } \\
\text { New York } \\
\text { Penth Carolina } \\
\text { South Carolina } \\
\text { Vermont } \\
\text { Virginia }\end{array}$ & $\begin{array}{r}14,775 \\
0 \\
0 \\
305 \\
0 \\
788 \\
0 \\
40 \\
0 \\
7,506 \\
5,684 \\
0 \\
452 \\
0 \\
0 \\
0\end{array}$ & $\begin{array}{r}11,578 \\
0 \\
116 \\
0 \\
0 \\
0 \\
1,038 \\
1,927 \\
0 \\
3,737 \\
3,000 \\
0 \\
869 \\
263 \\
5 \\
623\end{array}$ & $\begin{array}{r}37,999 \\
91 \\
1,829 \\
7,390 \\
1,528 \\
2,824 \\
730 \\
735 \\
97 \\
7,900 \\
3,857 \\
4,411 \\
1,980 \\
2,271 \\
16 \\
2,340\end{array}$ & $\begin{array}{r}64,352 \\
91 \\
1,945 \\
7,695 \\
1,528 \\
3,612 \\
1,768 \\
2,702 \\
97 \\
19,143 \\
12,541 \\
4,411 \\
3,301 \\
2,534 \\
21 \\
2,963\end{array}$ \\
\hline PAD District II & $\begin{array}{l}99 \\
21 \\
78\end{array}$ & $\begin{array}{l}0 \\
0 \\
0\end{array}$ & $\begin{array}{r}41 \\
0 \\
41\end{array}$ & $\begin{array}{r}140 \\
21 \\
119\end{array}$ \\
\hline PAD District III & $\begin{array}{r}469 \\
469 \\
0 \\
0\end{array}$ & $\begin{array}{r}1,223 \\
1,223 \\
0 \\
0\end{array}$ & $\begin{array}{r}2,899 \\
226 \\
2,272 \\
401\end{array}$ & $\begin{array}{r}4,591 \\
1,918 \\
2,272 \\
401\end{array}$ \\
\hline $\begin{array}{l}\text { PAD District V } \\
\text { Califomia } \\
\text { Hawaii }\end{array}$ & $\begin{array}{r}1,581 \\
724 \\
857\end{array}$ & $\begin{array}{l}0 \\
0 \\
0\end{array}$ & $\begin{array}{r}165 \\
165 \\
0\end{array}$ & $\begin{array}{r}1,746 \\
889 \\
857\end{array}$ \\
\hline U.S. Total & 16,924 & 12,801 & 41,104 & 70,829 \\
\hline
\end{tabular}

Source: Energy Information Administration (EIA) Form EIA-814, "Monthly Imports Report." 
Table 27. Exports of Crude Oil and Petroleum Products by PAD District, 1997 (Thousand Barrels)

\begin{tabular}{|c|c|c|c|c|c|c|c|}
\hline \multirow[b]{2}{*}{ Commodity } & \multicolumn{6}{|c|}{ Petroleum Administration for Defense Districts } & \multirow[b]{2}{*}{$\begin{array}{c}\text { Daily } \\
\text { Average }\end{array}$} \\
\hline & $\mathbf{I}$ & II & III & IV & $\mathbf{V}$ & U.S. & \\
\hline Crude Oil $^{\mathrm{a}}$ & 1 & 10,975 & 31 & 1 & 28,300 & 39,308 & 108 \\
\hline 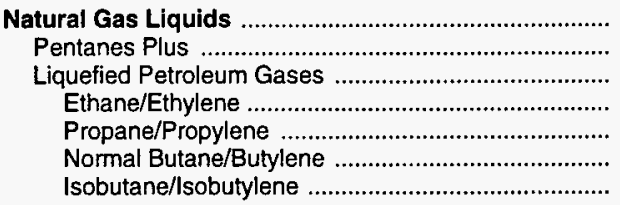 & $\begin{array}{r}741 \\
25 \\
716 \\
0 \\
447 \\
269 \\
0\end{array}$ & $\begin{array}{r}5,088 \\
2,457 \\
2,631 \\
0 \\
901 \\
1,729 \\
0\end{array}$ & $\begin{array}{r}8,828 \\
161 \\
8,666 \\
0 \\
7,630 \\
1,036 \\
0\end{array}$ & $\begin{array}{r}15 \\
8 \\
7 \\
0 \\
7 \\
(s) \\
0\end{array}$ & $\begin{array}{r}6,209 \\
1 \\
6,208 \\
0 \\
2,876 \\
3,333 \\
0\end{array}$ & $\begin{array}{r}20,882 \\
2,652 \\
18,229 \\
0 \\
11,861 \\
6,368 \\
0\end{array}$ & $\begin{array}{r}57 \\
7 \\
50 \\
0 \\
32 \\
17 \\
0\end{array}$ \\
\hline 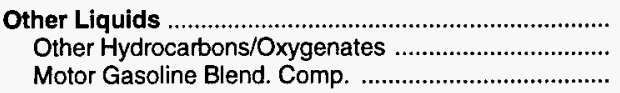 & $\begin{array}{r}345 \\
40 \\
305\end{array}$ & $\begin{array}{r}10 \\
7 \\
3\end{array}$ & $\begin{array}{l}8,331 \\
4,295 \\
4,036\end{array}$ & $\begin{array}{l}0 \\
0 \\
0\end{array}$ & $\begin{array}{r}579 \\
9 \\
569\end{array}$ & $\begin{array}{l}9,265 \\
4,351 \\
4,913\end{array}$ & $\begin{array}{l}25 \\
12 \\
13\end{array}$ \\
\hline 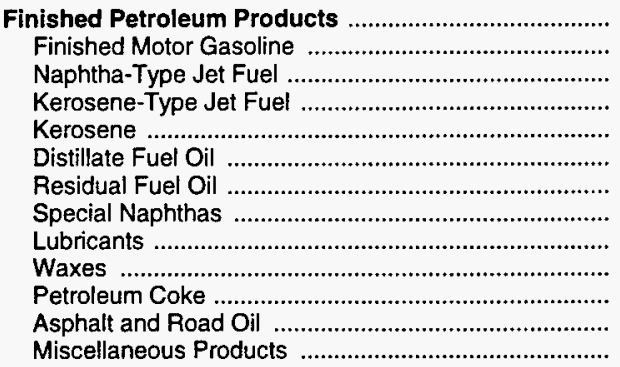 & $\begin{array}{r}12,320 \\
1,188 \\
31 \\
983 \\
19 \\
2,345 \\
1,340 \\
167 \\
1,744 \\
311 \\
3,915 \\
225 \\
52\end{array}$ & $\begin{array}{r}6,533 \\
284 \\
1 \\
36 \\
20 \\
552 \\
681 \\
411 \\
686 \\
187 \\
1,640 \\
2,030 \\
4\end{array}$ & $\begin{array}{r}186,983 \\
41,374 \\
25 \\
7,763 \\
48 \\
35,327 \\
25,089 \\
514 \\
7,517 \\
316 \\
68,686 \\
320 \\
4\end{array}$ & $\begin{array}{r}170 \\
24 \\
0 \\
0 \\
(s) \\
(s) \\
1 \\
3 \\
81 \\
44 \\
1 \\
16 \\
\text { (s) }\end{array}$ & $\begin{array}{r}90,797 \\
7,008 \\
18 \\
3,905 \\
50 \\
17,282 \\
16,671 \\
6,754 \\
1,245 \\
136 \\
37,374 \\
289 \\
64\end{array}$ & $\begin{array}{r}296,804 \\
49,878 \\
76 \\
12,687 \\
138 \\
55,507 \\
43,782 \\
7,849 \\
11,275 \\
993 \\
111,615 \\
2,879 \\
125\end{array}$ & $\begin{array}{r}813 \\
137 \\
(s) \\
35 \\
(s) \\
152 \\
120 \\
22 \\
31 \\
3 \\
306 \\
8 \\
(s)\end{array}$ \\
\hline Total & 13,407 & 22,607 & 204,173 & 186 & 125,885 & 366,258 & 1,003 \\
\hline
\end{tabular}

a Crude oil exports are restricted to: (1) crude oil derived from fields under the State waters of Alaska's Cook Inlet; (2) certain domestically produced crude oil destined for Canada; and (3) shipments to U.S. territories, and California crude oil to Pacific Rim countries. On December 6, 1991, the U.S. Department of Commerce approved a license to export 25,000 barrels per day of California heavy crude oil (less than 20 degrees API gravity) to Pacific Rim countries for one year. (s) = Less than 500 barrels or less than 500 barrels per day.

Note: Totals may not equal sum of components due to independent rounding.

Sources: Energy Information Administration (EIA) Form EIA-810, "Monthly Refinery Report" and the U.S. Bureau of the Census 
Table 28. Exports of Crude Oil and Petroleum Products by Destination, 1997 (Thousand Barrels)

\begin{tabular}{|c|c|c|c|c|c|c|c|c|}
\hline Destination & $\begin{array}{c}\text { Crude } \\
\text { Oil }^{\mathbf{a}} \\
\end{array}$ & $\begin{array}{c}\text { Pentanes } \\
\text { Plus }\end{array}$ & $\begin{array}{c}\text { Liquefied } \\
\text { Petroleum } \\
\text { Gases }\end{array}$ & $\begin{array}{l}\text { Finished } \\
\text { Motor } \\
\text { Gasoline }\end{array}$ & Jet Fuel & Kerosene & $\begin{array}{c}\text { Distillate Fuel } \\
\text { Oil } \\
\end{array}$ & $\begin{array}{l}\text { Residual } \\
\text { Fuel Oil }\end{array}$ \\
\hline Argentina & 0 & 0 & 2 & 0 & 206 & 0 & 802 & 0 \\
\hline Australia & 0 & 0 & 14 & 4 & 0 & 1 & 20 & 2 \\
\hline 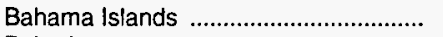 & 0 & (s) & 132 & 288 & 107 & 1 & 1,311 & $53 \overline{2}$ \\
\hline 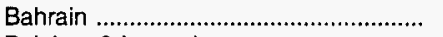 & 0 & 0 & 0 & 0 & 0 & 0 & (s) & 0 \\
\hline Belgium \& Luxembourg ............................ & 0 & 0 & 3 & 1 & 0 & 0 & 11 & 399 \\
\hline 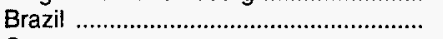 & 0 & 0 & 1 & 0 & 1,686 & 0 & 2,460 & 0 \\
\hline 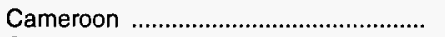 & 0 & 0 & 0 & 1 & 0 & 0 & 1 & 0 \\
\hline Canada & 11,009 & 2,480 & 2,871 & 3,098 & 4,317 & 43 & 3,358 & 5,872 \\
\hline Chile & 0 & 0 & 186 & 1,646 & 98 & (s) & 2,767 & 151 \\
\hline China, People's Republic of ........................ & 5,470 & 0 & 772 & 0 & 0 & 3 & 2,591 & 565 \\
\hline 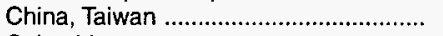 & 2,570 & 0 & 1 & 783 & (s) & (s) & 530 & 61 \\
\hline 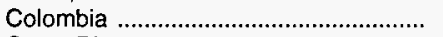 & 0 & 0 & 294 & 3,756 & 2 & (s) & 9 & 0 \\
\hline 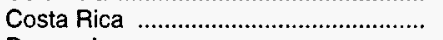 & 0 & 0 & $(\mathrm{~s})$ & 126 & 20 & 0 & 416 & 159 \\
\hline Denmark & 0 & 0 & 0 & 0 & 0 & (s) & 8 & 0 \\
\hline Dominican Republic ................................ & 0 & 5 & 487 & 88 & 0 & 0 & 333 & 558 \\
\hline 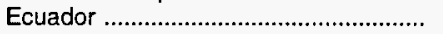 & 0 & 0 & 56 & 655 & 35 & 2 & 2,533 & 0 \\
\hline 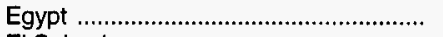 & 0 & 0 & 0 & 0 & 0 & 0 & (s) & 0 \\
\hline 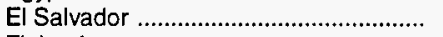 & 0 & 3 & 280 & 642 & 45 & 0 & 1,084 & 115 \\
\hline 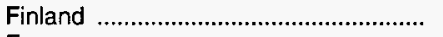 & 0 & 0 & 0 & 0 & 438 & 0 & 249 & 0 \\
\hline 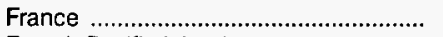 & 0 & 0 & 136 & 0 & (s) & 0 & 306 & 178 \\
\hline French Pacific Islands ............................. & 0 & (s) & 0 & 0 & 0 & 0 & 350 & 0 \\
\hline Germany, FR & 0 & 0 & 0 & (s) & 0 & 0 & 18 & 0 \\
\hline 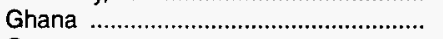 & 0 & 0 & 0 & 0 & 0 & 0 & 0 & 0 \\
\hline Greece & 0 & 0 & (s) & 0 & 0 & 0 & 4 & 0 \\
\hline 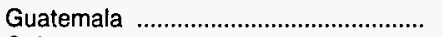 & 0 & 0 & 26 & $1,96 \dagger$ & 214 & 10 & 1,233 & 627 \\
\hline Guinea & 0 & 0 & 0 & 0 & (s) & 0 & 1 & 0 \\
\hline 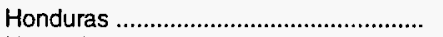 & 0 & 0 & 24 & 1,097 & 288 & 0 & 2,218 & 682 \\
\hline 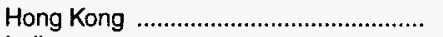 & 0 & (s) & (s) & 0 & 3 & (s) & 340 & 266 \\
\hline 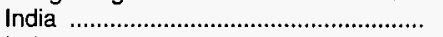 & 0 & 0 & 0 & 0 & 0 & 0 & 436 & 0 \\
\hline 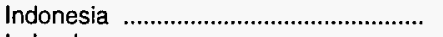 & 0 & 0 & 0 & 0 & 0 & 3 & 4 & 0 \\
\hline 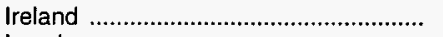 & 0 & 0 & 0 & 0 & 0 & 0 & 4 & (s) \\
\hline Israel & 0 & 0 & 4 & (s) & 1,526 & 0 & 389 & 1 \\
\hline Italy & 0 & 0 & 3 & (s) & 0 & 0 & 303 & 272 \\
\hline Jamaica & 0 & 0 & 172 & 1 & 75 & (s) & 4 & 8,686 \\
\hline 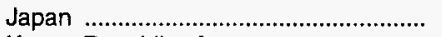 & 4,026 & 0 & 261 & 225 & 887 & 1 & 1,260 & 646 \\
\hline 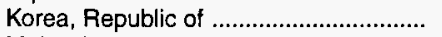 & 12,209 & 0 & 783 & (s) & 190 & 15 & 2,694 & 482 \\
\hline 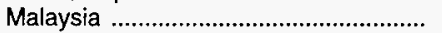 & 0 & 0 & (s) & (s) & 0 & 0 & 21 & 0 \\
\hline 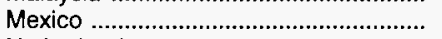 & 6 & (s) & 10,887 & 32,746 & 51 & 28 & 7,779 & 13,515 \\
\hline 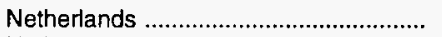 & 0 & 0 & 0 & 0 & 101 & 0 & 4,880 & 398 \\
\hline Netherlands Antilles .............................. & 0 & 0 & 0 & 384 & 0 & 0 & 1,592 & 924 \\
\hline 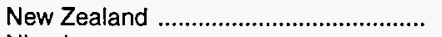 & 0 & 0 & (s) & 169 & (s) & 0 & 3 & (s) \\
\hline 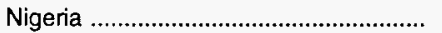 & 0 & 0 & 0 & 0 & 1,154 & 0 & 213 & 0 \\
\hline 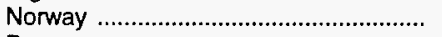 & 0 & 0 & 3 & 0 & 0 & 0 & 1 & (s) \\
\hline 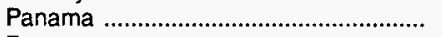 & 0 & 161 & 190 & 196 & 231 & 0 & 3,245 & 3,211 \\
\hline 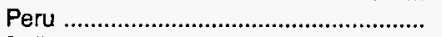 & 0 & 0 & 1 & 110 & 165 & 0 & 19 & 0 \\
\hline 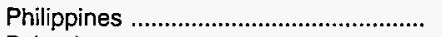 & 0 & 0 & 0 & 0 & 0 & 0 & 410 & 0 \\
\hline Poland & 0 & 0 & 0 & 0 & 0 & 0 & (s) & 0 \\
\hline 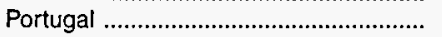 & 0 & 0 & 188 & 0 & 0 & 0 & (s) & 0 \\
\hline 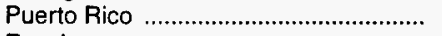 & 0 & 0 & 8 & 521 & 3 & 0 & 882 & 10 \\
\hline 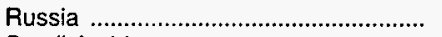 & 0 & 0 & (s) & 411 & 0 & 0 & 556 & 4 \\
\hline 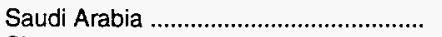 & 0 & 0 & 2 & 0 & 0 & 0 & 54 & 0 \\
\hline Singapore & 0 & 0 & 1 & 0 & 0 & 0 & 5,335 & 3,966 \\
\hline 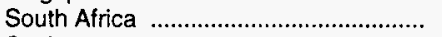 & 0 & 0 & (s) & 0 & 0 & 0 & 1 & (s) \\
\hline Spain & 0 & 0 & 310 & 0 & (s) & 0 & 321 & (s) \\
\hline 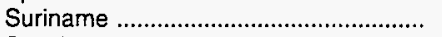 & 0 & 0 & 0 & 0 & 0 & 0 & 0 & 0 \\
\hline 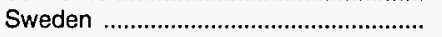 & 0 & 1 & 0 & 1 & 0 & 5 & 7 & 0 \\
\hline 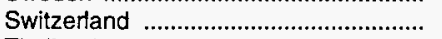 & 0 & 0 & 0 & 0 & 0 & 0 & 1 & 0 \\
\hline 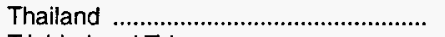 & 0 & 0 & 0 & 0 & 0 & 0 & 274 & 162 \\
\hline Trinidad and Tobago ................................... & 0 & 0 & 4 & 1 & 0 & 0 & 5 & 1 \\
\hline 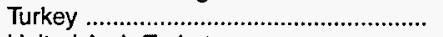 & 0 & 0 & (s) & 0 & 0 & 0 & 403 & 0 \\
\hline United Arab Emirates .................................. & 0 & 0 & 1 & 0 & 0 & 0 & 346 & 0 \\
\hline 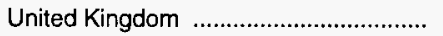 & 0 & (s) & 26 & 3 & 794 & (s) & 29 & 35 \\
\hline 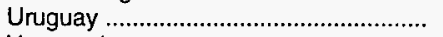 & 0 & 0 & 0 & 1 & 1 & 0 & 0 & 0 \\
\hline 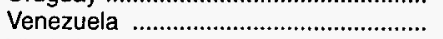 & 0 & 0 & 1 & 314 & 32 & 23 & 675 & 232 \\
\hline Virgin Islands & 3,956 & 0 & 0 & 0 & 0 & 0 & (s) & 0 \\
\hline 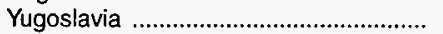 & 0 & 0 & 0 & 0 & 0 & 0 & 0 & 3 \\
\hline Other & 63 & 1 & 99 & 649 & 94 & 2 & 407 & 1,062 \\
\hline Total & 39,308 & 2,652 & 18,229 & 49,878 & 12,763 & 138 & 55,507 & 43,782 \\
\hline
\end{tabular}


Table 28. Exports of Crude Oil and Petroleum Products by Destination, 1997 (Continued) (Thousand Barrels)

\begin{tabular}{|c|c|c|c|c|c|c|c|c|}
\hline \multirow{2}{*}{ Destination } & \multirow[b]{2}{*}{$\begin{array}{c}\text { Special } \\
\text { Naphthas }\end{array}$} & \multirow[b]{2}{*}{ Lubricants } & \multirow[b]{2}{*}{ Waxes } & \multirow[b]{2}{*}{$\begin{array}{c}\text { Petroleum } \\
\text { Coke }\end{array}$} & \multirow{2}{*}{$\begin{array}{c}\text { Asphalt } \\
\text { and Road } \\
\text { Oil }\end{array}$} & \multirow[b]{2}{*}{$\begin{array}{c}\text { Other } \\
\text { Products }^{\text {b }}\end{array}$} & \multicolumn{2}{|c|}{ Crude Oil and Products } \\
\hline & & & & & & & Total & $\begin{array}{c}\text { Daily } \\
\text { Average }\end{array}$ \\
\hline Argentina & 4 & 129 & 7 & 53 & 3 & 1 & 1,206 & 3 \\
\hline Australia & 1 & 91 & 8 & 3,568 & 5 & (s) & 3,714 & 10 \\
\hline 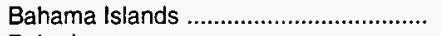 & (s) & 31 & 0 & 0 & 21 & (s) & 2,423 & 7 \\
\hline 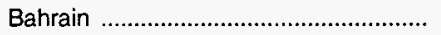 & 0 & 1 & 0 & 589 & (s) & 0 & 590 & 2 \\
\hline Belgium \& Luxembourg .............................. & 2 & 98 & 3 & 7,316 & 6 & 5 & 7,845 & 21 \\
\hline 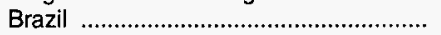 & 27 & 330 & 6 & 1,101 & 7 & 5 & 5,623 & 15 \\
\hline 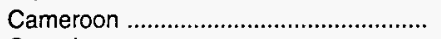 & 0 & (s) & 0 & 91 & 0 & 0 & 93 & (s) \\
\hline 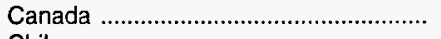 & 488 & 1,630 & 473 & 5,572 & 2,247 & 92 & 43,551 & 119 \\
\hline Chile & 6 & 231 & 3 & 656 & (s) & (s) & 5,745 & 16 \\
\hline China, People's Republic of .................... & 28 & 66 & 3 & 0 & 2 & (s) & 9,500 & 26 \\
\hline 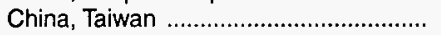 & 10 & 339 & 5 & 34 & 1 & 2 & 4,336 & 12 \\
\hline 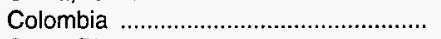 & 3 & 121 & 7 & 7 & 3 & 8 & 4,209 & 12 \\
\hline 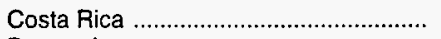 & 8 & 236 & 2 & (s) & (s) & 1 & 968 & 3 \\
\hline 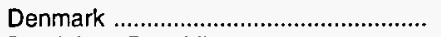 & 0 & 1 & 1 & 1,086 & 1 & (s) & 1,098 & 3 \\
\hline Dominican Republic .................................. & 10 & 178 & 1 & 163 & (s) & 21 & 1,845 & 5 \\
\hline 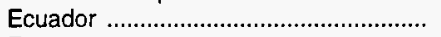 & (s) & 244 & (s) & 0 & (s) & 50 & 3,575 & 10 \\
\hline 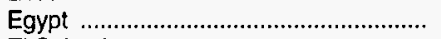 & 1 & 8 & (s) & (s) & 2 & 0 & 12 & (s) \\
\hline 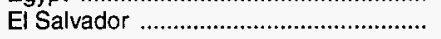 & 4 & 36 & 1 & 0 & 0 & 2 & 2,212 & 6 \\
\hline Finland & 0 & 9 & 0 & 0 & (s) & (s) & 697 & 2 \\
\hline 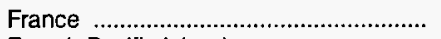 & 22 & 30 & 21 & 3,228 & 8 & 1 & 3,931 & 11 \\
\hline French Pacific Islands ............................. & 40 & 1 & 0 & 0 & 0 & 0 & 391 & 1 \\
\hline Germany, FR & 2 & 45 & 47 & 1,452 & 64 & 3 & 1,632 & 4 \\
\hline Ghana & 0 & 3 & 0 & 479 & 0 & 0 & 482 & 1 \\
\hline 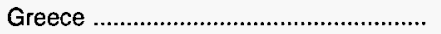 & (s) & 31 & (s) & 1,976 & 1 & (s) & 2,012 & 6 \\
\hline 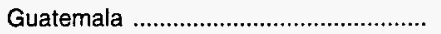 & 13 & 104 & 7 & 0 & 0 & 10 & 4,204 & 12 \\
\hline Guinea & 0 & 13 & 0 & 0 & 0 & 0 & 14 & (s) \\
\hline 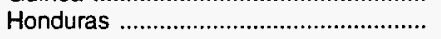 & 6 & 107 & 2 & 0 & 2 & (s) & 4,426 & 12 \\
\hline 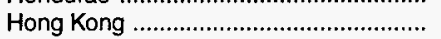 & 3 & 82 & 8 & 0 & 1 & 1 & 704 & 2 \\
\hline 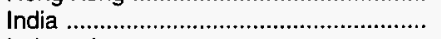 & 2 & 518 & 8 & 1,608 & 27 & 3 & 2,602 & 7 \\
\hline 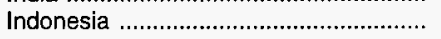 & 1 & 29 & 1 & 307 & 4 & 5 & 353 & 1 \\
\hline Ireland & 2 & 1 & 2 & 724 & 0 & 7 & 741 & 2 \\
\hline Israel & (s) & 31 & (s) & 1,263 & 1 & (s) & 3,215 & 9 \\
\hline Italy & 0 & 70 & 6 & 10,352 & 7 & 2 & 11,013 & 30 \\
\hline 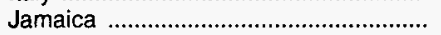 & 13 & 63 & 1 & 246 & 0 & 114 & 9,378 & 26 \\
\hline 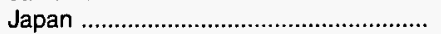 & 6,654 & 284 & 43 & 19,974 & 18 & 546 & 34,825 & 95 \\
\hline 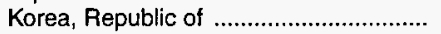 & 1 & 97 & 11 & 1,754 & 7 & 5 & 18,250 & 50 \\
\hline Malaysia & (s) & 39 & 2 & 4 & (s) & 2 & 70 & (s) \\
\hline Mexico & 76 & 2,029 & 269 & 2,669 & 217 & 5,375 & 75,646 & 207 \\
\hline 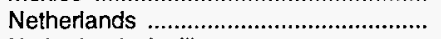 & 42 & 48 & 3 & 9,536 & 29 & 23 & 15,058 & 41 \\
\hline 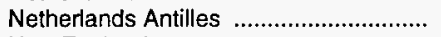 & 0 & 554 & 1 & 0 & 1 & 0 & 3,455 & 9 \\
\hline 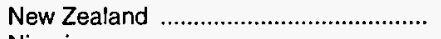 & (s) & 20 & (s) & 679 & (s) & (s) & 874 & 2 \\
\hline Nigeria & 0 & 149 & 0 & 0 & 1 & (s) & 1,517 & 4 \\
\hline 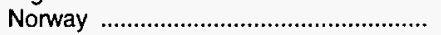 & (s) & 4 & (s) & 995 & (s) & (s) & 1,005 & 3 \\
\hline 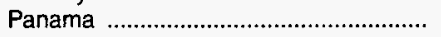 & 0 & 312 & 1 & (s) & 44 & 0 & 7,591 & 21 \\
\hline Peru & 1 & 46 & 2 & (s) & (s) & 1 & 345 & 1 \\
\hline Philippines ............................................. & (s) & 41 & 6 & 9 & 1 & 1 & 467 & 1 \\
\hline Poland & 0 & 2 & 0 & 0 & 0 & 0 & 2 & (s) \\
\hline 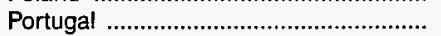 & 0 & 1 & (s) & 1,515 & (s) & (s) & 1,704 & 5 \\
\hline 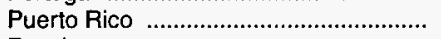 & 325 & 209 & 3 & 0 & (s) & 492 & 2,455 & 7 \\
\hline Russia & 1 & 72 & (s) & 0 & 0 & 0 & 1,045 & 3 \\
\hline Saudi Arabia & (s) & 14 & 1 & 183 & (s) & (s) & 256 & 1 \\
\hline 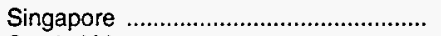 & 1 & 371 & 3 & 54 & 4 & 1 & 9,735 & 27 \\
\hline 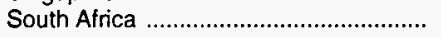 & (s) & 157 & 1 & 1,148 & 1 & (s) & 1,309 & 4 \\
\hline Spain & (s) & 150 & 4 & 14,482 & 3 & 0 & 15,270 & 42 \\
\hline Suriname & 0 & 7 & 0 & 0 & 0 & 0 & 7 & (s) \\
\hline Sweden & 0 & 13 & 2 & 495 & 0 & (s) & 524 & 1 \\
\hline 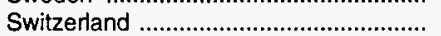 & 16 & 4 & (s) & 0 & 0 & (s) & 21 & (s) \\
\hline 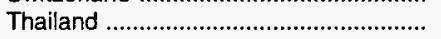 & 3 & 86 & 3 & 6 & 12 & 4 & 550 & 2 \\
\hline Trinidad and Tobago ............................... & 6 & 481 & 1 & (s) & 1 & 1 & 499 & 1 \\
\hline Turkey & (s) & 66 & (s) & 5,777 & 1 & 0 & 6,247 & 17 \\
\hline United Arab Emirates ............................ & 1 & 624 & (s) & 985 & 1 & (s) & 1,958 & 5 \\
\hline 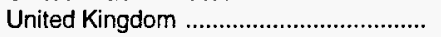 & 1 & 103 & 8 & 3,497 & 47 & 3 & 4,546 & 12 \\
\hline 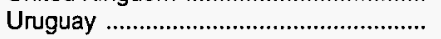 & 1 & 33 & (s) & 0 & (s) & (s) & 35 & (s) \\
\hline 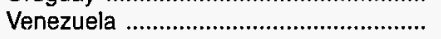 & 3 & 41 & 4 & 1,946 & 58 & 2,277 & 5,606 & 15 \\
\hline Virgin Islands & 0 & 1 & 0 & 0 & (s) & 296 & 4,254 & 12 \\
\hline 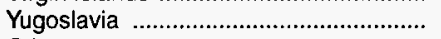 & 0 & 5 & (s) & 57 & 0 & 0 & 65 & (s) \\
\hline Other & 20 & 303 & 3 & 3,978 & 22 & 26 & 6,729 & 18 \\
\hline Total & 7,849 & 11,275 & 993 & 111,615 & 2,879 & 9,389 & 366,258 & 1,003 \\
\hline
\end{tabular}

a Crude oil exports are restricted to: (1) crude oil derived from fields under the State waters of Alaska's Cook Inlet; (2) certain domestically produced crude oil destined for Canada; and (3) shipments to U.S. territories, and California crude oil to Pacific Rim countries. On December 6, 1991, the U.S. Department of Commerce approved a license to export 25,000 barrels per day of California heavy crude oil (less than 20 degrees API gravity) to Pacific Rim countries for one year.

${ }^{b}$ Includes miscellaneous products, motor gasoline blending components, and other hydrocarbons and oxygenates.

(s) = Less than 500 barrels or less than 500 barrels per day.

Note: Totals may not equal sum of components due to independent rounding.

Sources: Energy Information Administration (EIA) Form EIA-810, "Monthly Refinery Report" and the U.S. Bureau of the Census. 
Table 29. Net Imports of Crude Oil and Petroleum Products into the United States by Country, 1997 (Thousand Barrels per Day)

\begin{tabular}{|c|c|c|c|c|c|c|c|c|c|c|c|}
\hline Country & $\begin{array}{c}\text { Crude } \\
\text { Oil }^{\mathbf{a}}\end{array}$ & \begin{tabular}{|c|} 
Liquefied \\
Petroleum \\
Gases
\end{tabular} & $\begin{array}{l}\text { Finished } \\
\text { Motor } \\
\text { Gasoline }\end{array}$ & Jet Fuel & $\begin{array}{c}\text { Distillate } \\
\text { Fuel Oil }\end{array}$ & $\begin{array}{c}\text { Residual } \\
\text { Fuel Oil }\end{array}$ & $\begin{array}{c}\text { Petroleum } \\
\text { Coke }\end{array}$ & Lubricants & $\begin{array}{c}\text { Other } \\
\text { Products }^{\mathbf{b}}\end{array}$ & \begin{tabular}{|c|} 
Total \\
Products \\
\end{tabular} & $\begin{array}{c}\text { Total } \\
\text { Crude Oil } \\
\text { and Products }\end{array}$ \\
\hline Arab OPEC & 1,641 & 31 & 29 & 0 & (s) & 33 & -3 & -2 & 304 & 393 & 2,034 \\
\hline Algeria & 6 & 31 & 0 & 0 & 1 & 26 & 0 & (s) & 221 & 279 & 285 \\
\hline Iraq & 89 & 0 & 0 & 0 & 0 & 0 & 0 & 0 & 0 & 0 & 89 \\
\hline 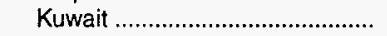 & 253 & (s) & 0 & 0 & (s) & 0 & (s) & (s) & (s) & (s) & 253 \\
\hline 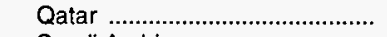 & 0 & 0 & 0 & 0 & 0 & 0 & 0 & (s) & 4 & 4 & 4 \\
\hline 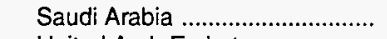 & 1,293 & (s) & 29 & 0 & (s) & 7 & -1 & (s) & 77 & 113 & 1,407 \\
\hline United Arab Emirates ................. & 0 & (s) & 0 & 0 & -1 & 0 & -3 & -2 & 2 & -4 & -4 \\
\hline Other OPEC & 2,134 & 11 & 43 & 36 & 49 & 58 & -6 & -1 & 184 & 374 & 2,508 \\
\hline 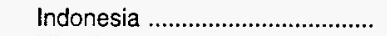 & 51 & 0 & 0 & 0 & (s) & 5 & -1 & (s) & 3 & 6 & 57 \\
\hline Nigeria ................................... & 689 & 0 & 0 & -3 & -1 & 4 & 0 & (s) & 5 & 4 & 693 \\
\hline 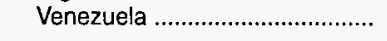 & 1,394 & 11 & 43 & 39 & 49 & 50 & -5 & (s) & 177 & 364 & 1,758 \\
\hline Non OPEC & 4,342 & 77 & 101 & 20 & 27 & -17 & -295 & -17 & 380 & 274 & 4,616 \\
\hline Angola & 425 & 0 & 0 & 0 & 0 & 0 & 0 & (s) & 2 & 2 & 427 \\
\hline Argentina .................................. & 69 & (s) & 0 & -1 & -2 & 1 & (s) & (s) & 5 & 3 & 72 \\
\hline 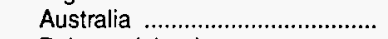 & 31 & (s) & (s) & 0 & $(s)$ & (s) & -10 & (s) & 17 & 7 & 38 \\
\hline Bahama Islands ......................... & 0 & (s) & -1 & (s) & -4 & -1 & 0 & (s) & 1 & -6 & -6 \\
\hline Belgium \& Luxembourg ............. & 0 & (s) & 2 & 0 & (s) & (s) & -20 & (s) & 25 & 8 & 8 \\
\hline 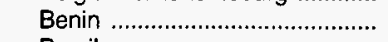 & 1 & 0 & $\overline{0}$ & 0 & 0 & 0 & 0 & 0 & 0 & 0 & 1 \\
\hline 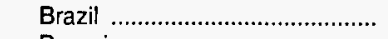 & 0 & (s) & 1 & -5 & -7 & (s) & -3 & -1 & 3 & -10 & -10 \\
\hline Brunei & 0 & 0 & 0 & 0 & (s) & 0 & 0 & (s) & 0 & (s) & (s) \\
\hline 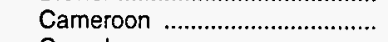 & 0 & 0 & (s) & 0 & (s) & 5 & (s) & (s) & 0 & 4 & 4 \\
\hline 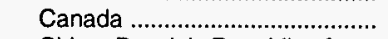 & 1,168 & 115 & 69 & -9 & 74 & 8 & -14 & -2 & 35 & 276 & 1,444 \\
\hline China, People's Republic of ...... & 33 & -2 & 1 & 0 & -7 & -2 & 0 & (s) & (s) & -10 & 23 \\
\hline 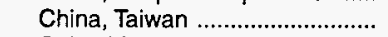 & -7 & (s) & -2 & (s) & -1 & $(\bar{s})$ & (s) & -1 & (s) & -5 & -12 \\
\hline 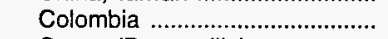 & 270 & -1 & -10 & (s) & (s) & 1 & (s) & (s) & (s) & -10 & 260 \\
\hline 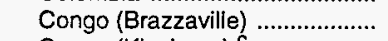 & 47 & 0 & 0 & 0 & 0 & 0 & 0 & (s) & 0 & (s) & 47 \\
\hline Congo (Kinshasa) ${ }^{c}$..................... & 21 & 0 & 0 & 0 & 0 & 0 & 0 & (s) & 0 & (s) & 21 \\
\hline Ecuador .................................... & 114 & (s) & -2 & (s) & -7 & (s) & 0 & -1 & (s) & -9 & 105 \\
\hline 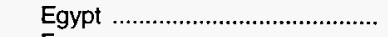 & 36 & 0 & 0 & 0 & (s) & 0 & (s) & (s) & 2 & 2 & 37 \\
\hline France & 0 & (s) & 4 & (s) & -1 & (s) & -9 & (s) & 23 & 17 & 17 \\
\hline Gabon & 230 & 0 & 0 & 0 & 0 & 0 & 0 & (s) & 0 & (s) & 230 \\
\hline 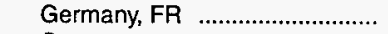 & 0 & 0 & 1 & 0 & (s) & 2 & -4 & (s) & 5 & 4 & 4 \\
\hline Greece & 0 & (s) & 0 & 0 & (s) & 0 & -5 & (s) & (s) & -6 & -6 \\
\hline 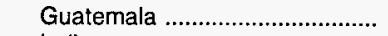 & 17 & (s) & -5 & -1 & -3 & -2 & 0 & (s) & (s) & -12 & 5 \\
\hline India & 0 & 0 & 0 & 0 & -1 & 0 & -4 & -1 & 5 & -2 & -2 \\
\hline 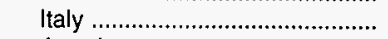 & 0 & (s) & 2 & 0 & -1 & -1 & -28 & (s) & 5 & -23 & -23 \\
\hline 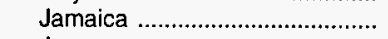 & 0 & (s) & (s) & (s) & (s) & -24 & -1 & (s) & (s) & -26 & -26 \\
\hline 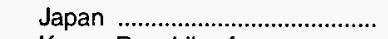 & -11 & -1 & -1 & -2 & -3 & -2 & -55 & -1 & -19 & .83 & -94 \\
\hline Korea, Republic of & -33 & -2 & (s) & 2 & -7 & -1 & -5 & (s) & 2 & -12 & -46 \\
\hline 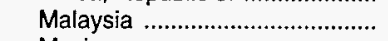 & 8 & (s) & (s) & 0 & (s) & 2 & (s) & (s) & 13 & 15 & 22 \\
\hline 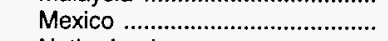 & 1,360 & -29 & -90 & (s) & -21 & -37 & -7 & -5 & 8 & -182 & 1,178 \\
\hline 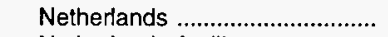 & 0 & 0 & 3 & (s) & -13 & -1 & -26 & (s) & 20 & -17 & -17 \\
\hline Netherlands Antilles ...................... & 0 & 0 & 2 & 23 & -4 & (s) & 0 & -2 & 45 & 64 & 64 \\
\hline Norway & 288 & 3 & 5 & 0 & (s) & 1 & -3 & (s) & 12 & 18 & 306 \\
\hline Oman & 4 & (s) & 0 & 0 & 0 & 0 & 0 & (s) & 7 & 7 & 11 \\
\hline 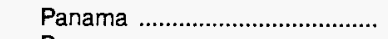 & 0 & -1 & -1 & -1 & -9 & -8 & (s) & -1 & -1 & -20 & -20 \\
\hline Peru & 31 & (s) & (s) & (s) & (s) & 0 & (s) & (s) & 1 & (s) & 31 \\
\hline 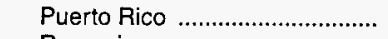 & 0 & (s) & -1 & (s) & -2 & (s) & 0 & 8 & 5 & 10 & 10 \\
\hline 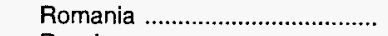 & 0 & 0 & (s) & 0 & (s) & -1 & -1 & $(s)$ & 8 & 6 & 6 \\
\hline Russia & 3 & (s) & -1 & 0 & -1 & 1 & 0 & (s) & 8 & 7 & 10 \\
\hline Syria & 0 & (s) & 0 & 0 & (s) & 0 & 0 & (s) & (s) & (s) & (s) \\
\hline Spain & 0 & -1 & 2 & (s) & -1 & 2 & -40 & (s) & 17 & -21 & -21 \\
\hline Sweden & 0 & 0 & $\overline{1}$ & 0 & (s) & 1 & -1 & (s) & 5 & 6 & 6 \\
\hline 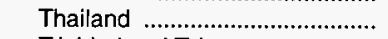 & 0 & 0 & 1 & 0 & -1 & (s) & (s) & (s) & (s) & (s) & (s) \\
\hline 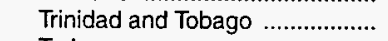 & 56 & (s) & (s) & 0 & (s) & 1 & (s) & -1 & 5 & 4 & 60 \\
\hline 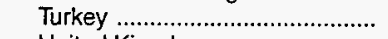 & 0 & (s) & 0 & 0 & -1 & 0 & -16 & (s) & 1 & -16 & -16 \\
\hline United Kingdom .......................... & 169 & (s) & 13 & -2 & (s) & 4 & -10 & (s) & 40 & 45 & 214 \\
\hline Virgin Islands & -11 & 0 & 107 & 22 & 90 & 45 & 0 & (s) & 36 & 299 & 288 \\
\hline Yemen & 0 & 0 & 0 & 0 & 0 & 1 & 0 & 0 & 0 & 1 & 1 \\
\hline 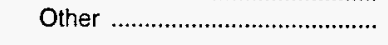 & 25 & -3 & -1 & -7 & -39 & -12 & -33 & -5 & 40 & -61 & -36 \\
\hline 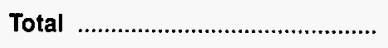 & 8,118 & 119 & 172 & 56 & 76 & 74 & -305 & -20 & 868 & 1,040 & 9,158 \\
\hline Persian Gulf ${ }^{d}$ & 1,635 & (s) & 29 & 0 & -1 & 7 & -5 & -2 & 83 & 112 & 1,747 \\
\hline
\end{tabular}

\footnotetext{
a Includes crude oil imported for storage in the Strategic Petroleum Reserve.
}

b Includes asphalt and road oil, aviation gasoline, aviation gasoline blending components, kerosene, miscellaneous products, motor gasoline blending

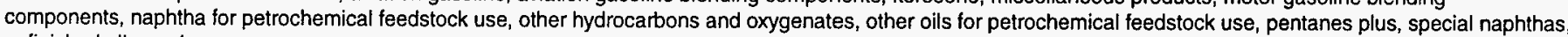
untinished oils, and waxes.

c Formerly Zaire.

d Includes Bahrain, Iran, Iraq, Kuwait, Qatar, Saudi Arabia, and United Arab Emirates.

(s) = Less than 500 barrels per day.

Note: Totals may not equal sum of components due to independent rounding.

Sources: Energy Information Administration (EIA) Forms EIA-810, "Monthly Refinery Report," and the U.S. Bureau of the Census. 
Table 30. Stocks of Crude Oil and Petroleum Products by PAD District, 1997 (Thousand Barrels)

\begin{tabular}{|c|c|c|c|c|c|c|}
\hline \multirow{2}{*}{ Commodity } & \multicolumn{5}{|c|}{ Petroleum Administration for Defense Districts } & \multirow[b]{2}{*}{$\begin{array}{l}\text { U. S. } \\
\text { Total }\end{array}$} \\
\hline & $\mathbf{I}$ & $\|$ & III & IV & $\mathbf{v}$ & \\
\hline 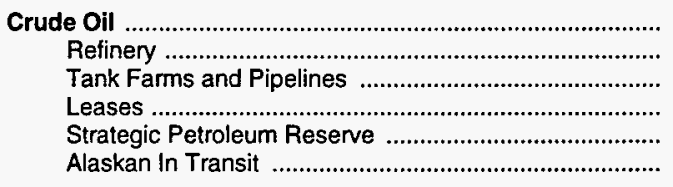 & $\begin{array}{r}10,973 \\
10,142 \\
811 \\
20 \\
0 \\
0\end{array}$ & $\begin{array}{r}73,581 \\
12,822 \\
59,677 \\
1,082 \\
0 \\
0\end{array}$ & $\begin{array}{r}710,050 \\
45,386 \\
87,480 \\
13,755 \\
563,429 \\
0\end{array}$ & $\begin{array}{r}12,839 \\
2,266 \\
9,698 \\
875 \\
0 \\
0\end{array}$ & $\begin{array}{r}60,676 \\
20,047 \\
30,914 \\
858 \\
0 \\
8,857\end{array}$ & $\begin{array}{r}868,119 \\
90,663 \\
188,580 \\
16,590 \\
563,429 \\
8,857\end{array}$ \\
\hline 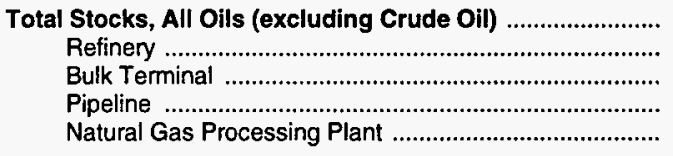 & $\begin{array}{r}175,962 \\
56,576 \\
89,804 \\
29,539 \\
43\end{array}$ & $\begin{array}{r}157,738 \\
60,800 \\
60,003 \\
35,575 \\
1,360\end{array}$ & $\begin{array}{r}245,732 \\
134,821 \\
62,365 \\
46,989 \\
1,557\end{array}$ & $\begin{array}{r}17,079 \\
11,603 \\
2,425 \\
2,742 \\
309\end{array}$ & $\begin{array}{r}95,129 \\
66,431 \\
21,917 \\
6,622 \\
159\end{array}$ & $\begin{array}{r}691,640 \\
330,231 \\
236,514 \\
121,467 \\
3,428\end{array}$ \\
\hline 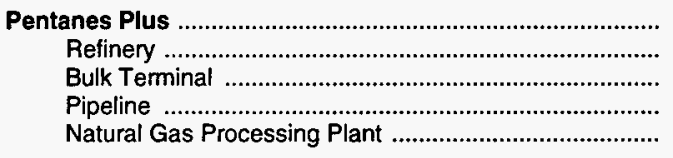 & $\begin{array}{r}12 \\
0 \\
9 \\
0 \\
3\end{array}$ & $\begin{array}{r}1,774 \\
446 \\
626 \\
478 \\
224\end{array}$ & $\begin{array}{r}3,678 \\
236 \\
1,999 \\
960 \\
483\end{array}$ & $\begin{array}{r}227 \\
10 \\
3 \\
64 \\
150\end{array}$ & $\begin{array}{r}24 \\
0 \\
4 \\
0 \\
20\end{array}$ & $\begin{array}{r}5,715 \\
692 \\
2,641 \\
1,502 \\
880\end{array}$ \\
\hline 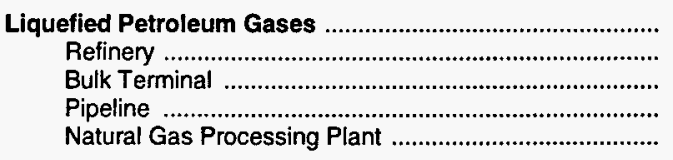 & $\begin{array}{r}6,080 \\
1,904 \\
2,181 \\
1,955 \\
40\end{array}$ & $\begin{array}{r}27,745 \\
3,711 \\
15,134 \\
7,764 \\
1,136\end{array}$ & $\begin{array}{r}49,632 \\
8,281 \\
28,912 \\
11,365 \\
1,074\end{array}$ & $\begin{array}{r}1,142 \\
378 \\
133 \\
472 \\
159\end{array}$ & $\begin{array}{r}4,882 \\
1,167 \\
3,576 \\
0 \\
139\end{array}$ & $\begin{array}{r}89,481 \\
15,441 \\
49,936 \\
21,556 \\
2,548\end{array}$ \\
\hline 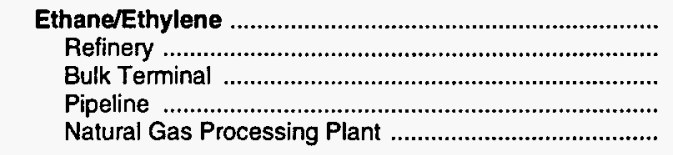 & $\begin{array}{l}0 \\
0 \\
0 \\
0 \\
0\end{array}$ & $\begin{array}{r}2,978 \\
3 \\
1,106 \\
1,702 \\
167\end{array}$ & $\begin{array}{r}15,716 \\
689 \\
11,767 \\
3,203 \\
57\end{array}$ & $\begin{array}{r}213 \\
0 \\
0 \\
211 \\
2\end{array}$ & $\begin{array}{l}0 \\
0 \\
0 \\
0 \\
0\end{array}$ & $\begin{array}{r}18,907 \\
692 \\
12,873 \\
5,116 \\
226\end{array}$ \\
\hline $\begin{array}{l}\text { Propane/Propylene } \\
\text { Refinery } \\
\text { Bulk Terminal } \\
\text { Pipeline } \\
\text { Natural Gas Processing Plant }\end{array}$ & $\begin{array}{r}4,305 \\
530 \\
1,866 \\
1,881 \\
28\end{array}$ & $\begin{array}{r}17,972 \\
2,068 \\
11,318 \\
4,062 \\
524\end{array}$ & $\begin{array}{r}18,798 \\
2,768 \\
10,369 \\
5,417 \\
244\end{array}$ & $\begin{array}{r}489 \\
117 \\
129 \\
151 \\
92\end{array}$ & $\begin{array}{r}2,481 \\
231 \\
2,154 \\
0 \\
96\end{array}$ & $\begin{array}{r}44,045 \\
5,714 \\
25,836 \\
11,511 \\
984\end{array}$ \\
\hline 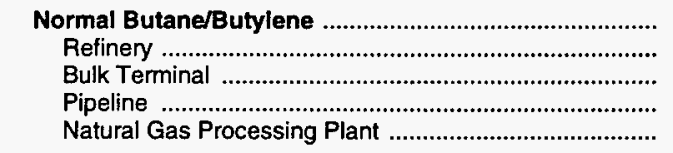 & $\begin{array}{r}1,369 \\
971 \\
315 \\
74 \\
9\end{array}$ & $\begin{array}{r}4,802 \\
1,122 \\
1,862 \\
1,517 \\
301\end{array}$ & $\begin{array}{r}10,014 \\
3,278 \\
4,251 \\
2,062 \\
423\end{array}$ & $\begin{array}{r}306 \\
180 \\
4 \\
71 \\
51\end{array}$ & $\begin{array}{r}1,882 \\
585 \\
1,282 \\
0 \\
15\end{array}$ & $\begin{array}{r}18,373 \\
6,136 \\
7,714 \\
3,724 \\
799\end{array}$ \\
\hline 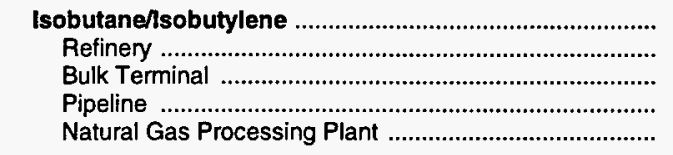 & $\begin{array}{r}406 \\
403 \\
0 \\
0 \\
3\end{array}$ & $\begin{array}{r}1,993 \\
518 \\
848 \\
483 \\
144\end{array}$ & $\begin{array}{r}5,104 \\
1,546 \\
2,525 \\
683 \\
350\end{array}$ & $\begin{array}{r}134 \\
81 \\
0 \\
39 \\
14\end{array}$ & $\begin{array}{r}519 \\
351 \\
140 \\
0 \\
28\end{array}$ & $\begin{array}{r}8,156 \\
2,899 \\
3,513 \\
1,205 \\
539\end{array}$ \\
\hline 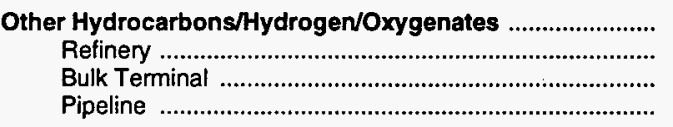 & $\begin{array}{r}2,236 \\
2,017 \\
219 \\
0\end{array}$ & $\begin{array}{r}1,950 \\
522 \\
1,394 \\
34\end{array}$ & $\begin{array}{r}5,036 \\
2,213 \\
2,488 \\
335\end{array}$ & $\begin{array}{r}252 \\
85 \\
151 \\
16\end{array}$ & $\begin{array}{r}3,019 \\
2,318 \\
555 \\
146\end{array}$ & $\begin{array}{r}12,493 \\
7,155 \\
4,807 \\
531\end{array}$ \\
\hline Other Hydrocarbons/Hydrogen & $\begin{array}{l}0 \\
0\end{array}$ & $\begin{array}{l}19 \\
19\end{array}$ & $\begin{array}{l}1 \\
1\end{array}$ & $\begin{array}{l}0 \\
0\end{array}$ & $\begin{array}{l}13 \\
13\end{array}$ & $\begin{array}{l}33 \\
33\end{array}$ \\
\hline $\begin{array}{l}\text { Fuel Ethanol } \\
\text { Refinery } \\
\text { Bulk Terminal a } \\
\text { Pipeline }\end{array}$ & $\begin{array}{r}142 \\
W \\
W \\
W\end{array}$ & $\begin{array}{r}1,696 \\
302 \\
W \\
W\end{array}$ & $\begin{array}{r}398 \\
W \\
W \\
W\end{array}$ & $\begin{array}{r}123 \\
W \\
W \\
W\end{array}$ & $\begin{array}{r}566 \\
W \\
W \\
W\end{array}$ & $\begin{array}{r}2,925 \\
424 \\
W \\
W\end{array}$ \\
\hline 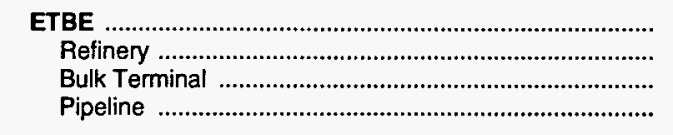 & $\begin{array}{l}\mathbf{w} \\
\mathbf{W} \\
\mathbf{W}\end{array}$ & $\begin{array}{l}w \\
w \\
w \\
W\end{array}$ & $\begin{array}{l}w \\
W \\
W\end{array}$ & $\begin{array}{l}W \\
W \\
W \\
W\end{array}$ & $\begin{array}{l}W \\
W \\
W \\
W\end{array}$ & $\begin{array}{l}W \\
W \\
W \\
W\end{array}$ \\
\hline $\begin{array}{l}\text { Methanol } \\
\text { Refinery }\end{array}$ & $\begin{array}{l}W \\
W\end{array}$ & $\mathbf{w}$ & $\begin{array}{l}w \\
W\end{array}$ & $\mathbf{W}$ & $\begin{array}{l}\mathbf{W} \\
\mathbf{W}\end{array}$ & $\begin{array}{l}753 \\
753\end{array}$ \\
\hline
\end{tabular}

See footnotes at end of table. 
Table 30. Stocks of Crude Oil and Petroleum Products by PAD District, 1997 (Continued) (Thousand Barrels)

\begin{tabular}{|c|c|c|c|c|c|c|}
\hline \multirow{2}{*}{ Commodity } & \multicolumn{5}{|c|}{ Petroleum Administration for Defense Districts } & \multirow{2}{*}{$\begin{array}{l}\text { U. S. } \\
\text { Total }\end{array}$} \\
\hline & 1 & II & III & IV & $\mathbf{v}$ & \\
\hline $\begin{array}{l}\text { MTBE } \\
\text { Refinery } \\
\text { Bulk Terminal } \\
\text { Pipeline }\end{array}$ & $\begin{array}{r}1,694 \\
1,574 \\
W \\
W\end{array}$ & $\begin{array}{l}W \\
W \\
W \\
W\end{array}$ & $\begin{array}{r}3,796 \\
1,815 \\
1,704 \\
277\end{array}$ & $\begin{array}{l}W \\
W \\
W \\
W\end{array}$ & $\begin{array}{r}2,431 \\
2,281 \\
25 \\
125\end{array}$ & $\begin{array}{r}8,229 \\
5,849 \\
1,944 \\
436\end{array}$ \\
\hline $\begin{array}{l}\text { Other Oxygenates }^{b} \\
\quad \text { Refinery } \\
\text { Bulk Terminal } \\
\text { Pipeline }\end{array}$ & $\begin{array}{l}w \\
W \\
W \\
W\end{array}$ & $\begin{array}{l}W \\
W \\
W \\
W\end{array}$ & $\begin{array}{l}\mathbf{w} \\
W \\
W \\
W\end{array}$ & $\begin{array}{l}W \\
W \\
W \\
W\end{array}$ & $\begin{array}{l}W \\
W \\
W \\
W\end{array}$ & $\begin{array}{l}W \\
W \\
W \\
W\end{array}$ \\
\hline $\begin{array}{l}\text { Unfinished Oils } \\
\text { Refinery }\end{array}$ & 10,106 & 12,309 & 43,290 & 2,208 & 20,842 & 88,755 \\
\hline 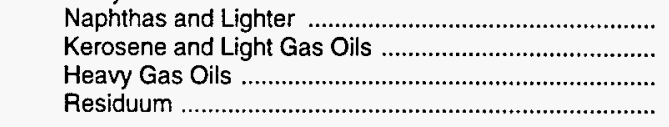 & $\begin{array}{l}2,103 \\
2,788 \\
4,137 \\
1,078\end{array}$ & $\begin{array}{l}3,423 \\
1,644 \\
4,481 \\
2,761\end{array}$ & $\begin{array}{r}10,921 \\
6,158 \\
16,854 \\
9,357\end{array}$ & $\begin{array}{l}353 \\
416 \\
863 \\
576\end{array}$ & $\begin{array}{l}3,633 \\
4,505 \\
9,681 \\
3,023\end{array}$ & $\begin{array}{l}20,433 \\
15,511 \\
36,016 \\
16,795\end{array}$ \\
\hline $\begin{array}{l}\text { Motor Gasoline Blending Components } \\
\text { Refinery } \\
\text { Bulk Terminal } \\
\text { Pipeline }\end{array}$ & $\begin{array}{r}6,396 \\
6,109 \\
287 \\
0\end{array}$ & $\begin{array}{r}10,491 \\
8,551 \\
556 \\
1,384\end{array}$ & $\begin{array}{r}15,025 \\
13,838 \\
632 \\
555\end{array}$ & $\begin{array}{r}1,889 \\
1,889 \\
0 \\
0\end{array}$ & $\begin{array}{r}9,617 \\
8,248 \\
285 \\
1,084\end{array}$ & $\begin{array}{r}43,418 \\
38,635 \\
1,760 \\
3,023\end{array}$ \\
\hline 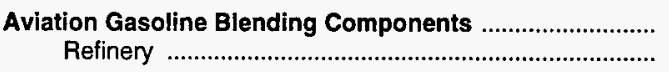 & $\begin{array}{l}79 \\
79\end{array}$ & $\begin{array}{l}36 \\
36\end{array}$ & $\begin{array}{l}26 \\
26\end{array}$ & $\begin{array}{l}0 \\
0\end{array}$ & $\begin{array}{l}10 \\
10\end{array}$ & $\begin{array}{l}151 \\
151\end{array}$ \\
\hline 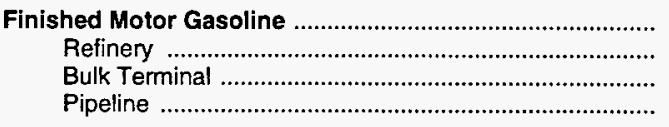 & $\begin{array}{r}50,787 \\
9,569 \\
26,842 \\
14,376\end{array}$ & $\begin{array}{r}41,886 \\
8,980 \\
19,127 \\
13,779\end{array}$ & $\begin{array}{r}\mathbf{4 6 , 4 8 0} \\
18,497 \\
9,508 \\
18,475\end{array}$ & $\begin{array}{l}4,867 \\
2,287 \\
1,132 \\
1,448\end{array}$ & $\begin{array}{r}22,337 \\
12,424 \\
7,610 \\
2,303\end{array}$ & $\begin{array}{r}166,357 \\
51,757 \\
64,219 \\
50,381\end{array}$ \\
\hline $\begin{array}{l}\text { Reformulated } \\
\text { Refinery } \\
\text { Bulk Terminal } \\
\text { Pipeline }\end{array}$ & $\begin{array}{r}19,462 \\
5,038 \\
10,285 \\
4,139\end{array}$ & $\begin{array}{r}1,195 \\
311 \\
639 \\
245\end{array}$ & $\begin{array}{l}8,632 \\
3,153 \\
1,880 \\
3,599\end{array}$ & $\begin{array}{l}0 \\
0 \\
0 \\
0\end{array}$ & $\begin{array}{r}13,642 \\
8,051 \\
4,017 \\
1,574\end{array}$ & $\begin{array}{r}42,931 \\
16,553 \\
16,821 \\
9,557\end{array}$ \\
\hline $\begin{array}{l}\text { Oxygenated } \\
\text { Refinery } \\
\text { Bulk Terminal } \\
\text { Pipeline }\end{array}$ & $\begin{array}{r}280 \\
9 \\
175 \\
96\end{array}$ & $\begin{array}{r}537 \\
404 \\
133 \\
0\end{array}$ & $\begin{array}{l}0 \\
0 \\
0 \\
0\end{array}$ & $\begin{array}{r}264 \\
115 \\
149 \\
0\end{array}$ & $\begin{array}{l}1 \\
0 \\
1 \\
0\end{array}$ & $\begin{array}{r}1,082 \\
528 \\
458 \\
96\end{array}$ \\
\hline $\begin{array}{l}\text { Other } \\
\text { Refinery } \\
\text { Bulk Terminal } \\
\text { Pipeline }\end{array}$ & $\begin{array}{r}31,045 \\
4,522 \\
16,382 \\
10,141\end{array}$ & $\begin{array}{r}40,154 \\
8,265 \\
18,355 \\
13,534\end{array}$ & $\begin{array}{r}37,848 \\
15,344 \\
7,628 \\
14,876\end{array}$ & $\begin{array}{r}4,603 \\
2,172 \\
983 \\
1,448\end{array}$ & $\begin{array}{r}8,694 \\
4,373 \\
3,592 \\
729\end{array}$ & $\begin{array}{r}122,344 \\
34,676 \\
46,940 \\
40,728\end{array}$ \\
\hline $\begin{array}{l}\text { Finished Aviation Gasoline } \\
\text { Refinery } \\
\text { Bulk Terminal } \\
\text { Pipeline }\end{array}$ & $\begin{array}{r}228 \\
41 \\
187 \\
0\end{array}$ & $\begin{array}{r}382 \\
121 \\
222 \\
39\end{array}$ & $\begin{array}{r}431 \\
377 \\
54 \\
0\end{array}$ & $\begin{array}{r}41 \\
29 \\
5 \\
7\end{array}$ & $\begin{array}{r}615 \\
340 \\
275 \\
0\end{array}$ & $\begin{array}{r}1,697 \\
908 \\
743 \\
46\end{array}$ \\
\hline 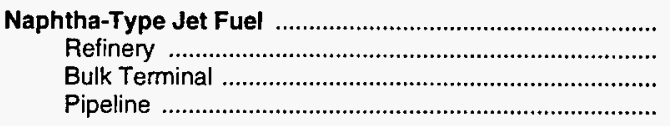 & $\begin{array}{l}0 \\
0 \\
0 \\
0\end{array}$ & $\begin{array}{l}0 \\
0 \\
0 \\
0\end{array}$ & $\begin{array}{l}1 \\
1 \\
0 \\
0\end{array}$ & $\begin{array}{l}0 \\
0 \\
0 \\
0\end{array}$ & $\begin{array}{r}33 \\
33 \\
0 \\
0\end{array}$ & $\begin{array}{r}34 \\
34 \\
0 \\
0\end{array}$ \\
\hline $\begin{array}{l}\text { Kerosene-Type Jet Fuel } \\
\text { Refinery } \\
\text { Bulk Terminal } \\
\text { Pipeline }\end{array}$ & $\begin{array}{r}11,756 \\
1,542 \\
5,541 \\
4,673\end{array}$ & $\begin{array}{l}9,145 \\
3,117 \\
2,150 \\
3,878\end{array}$ & $\begin{array}{r}13,054 \\
7,053 \\
1,763 \\
4,238\end{array}$ & $\begin{array}{l}839 \\
402 \\
206 \\
231\end{array}$ & $\begin{array}{l}9,215 \\
5,088 \\
2,823 \\
1,304\end{array}$ & $\begin{array}{l}\mathbf{4 4 , 0 0 9} \\
17,202 \\
12,483 \\
14,324\end{array}$ \\
\hline
\end{tabular}

See footnotes at end of table. 
Table 30. Stocks of Crude Oil and Petroleum Products by PAD District, 1997 (Continued) (Thousand Barrels)

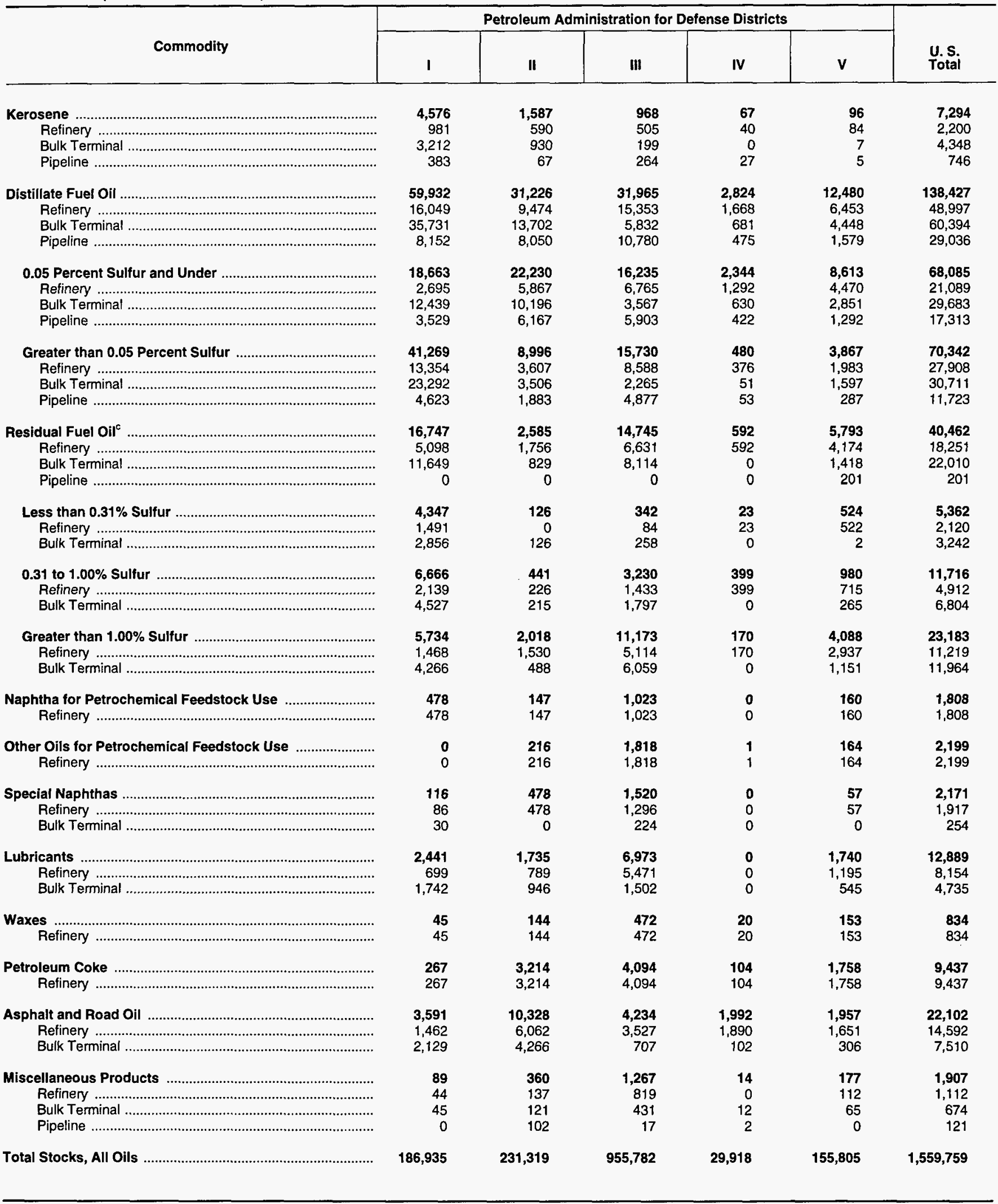

a Includes stocks held by producers.

b includes tertiary amyl methyl ether (TAME), tertiary butyl alcohol (TBA), and other aliphatic alcohols and ethers Intended for motor gasoline blending (e.g.. isopropyl ether (IPE) or n-propanol).

c Sulfur content not available for stocks held by pipelines.

$W=$ Withheld to avoid disclosure of individual company data

Note: Stocks are reported as of the end of December.

Sources: Energy Information Administration (EIA) Forms EIA-810, "Monthly Refinery Report," EIA-811, "Monthly Bulk Terminal Report," EIA-812, "Monthly Product Pipeline Report," EIA-813, "Monthly Crude Oil Report," and EIA-816, "Monthly Natural Gas Liquids Report." 
Table 31. Refinery, Bulk Terminal, and Natural Gas Plant Stocks of Selected Petroleum Products by PAD District and State, 1997 (Thousand Barrels)

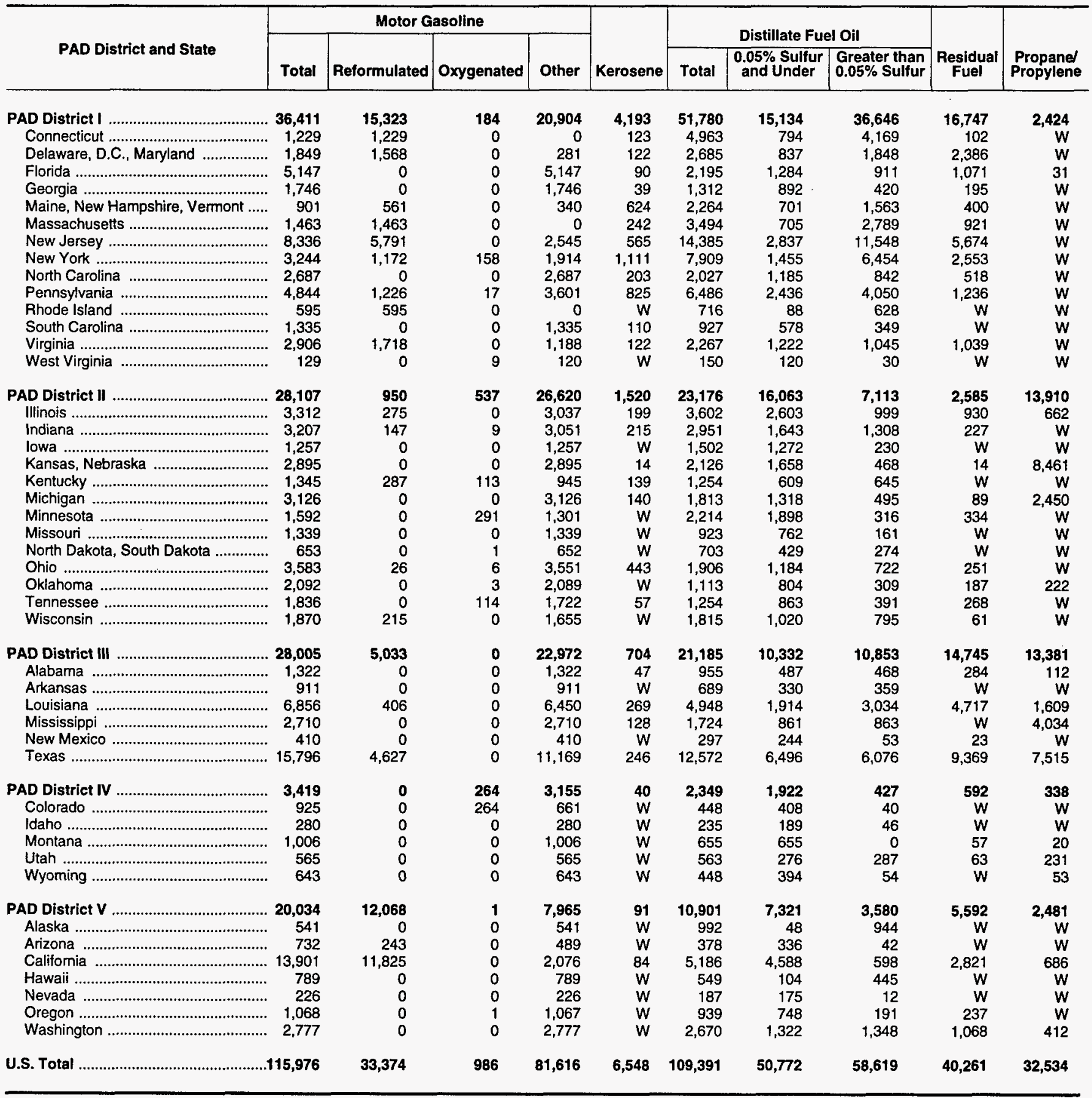

$W=$ Withheld to avoid disclosure of individual company data.

Notes: - Stocks are reported as of the end of December. - Totals may not equal sum of components due to independent rounding.

Sources: Energy Information Administration (EIA) Forms EIA-810, "Monthly Refinery Report," EIA-811, "Monthly Bulk Terminal Report," and EIA-816, "Monthly Natural Gas Liquids Report." 
Table 32. Movements of Crude Oil and Petroleum Products by Pipeline, Tanker, and Barge Between PAD Districts, 1997

(Thousand Barrels)

\begin{tabular}{|c|c|c|c|c|c|c|c|c|c|}
\hline \multirow{2}{*}{ Commodity } & \multicolumn{3}{|c|}{ From I to } & \multicolumn{4}{|c|}{ From II to } & \multicolumn{2}{|c|}{ From III to } \\
\hline & II & III & $\mathbf{V}$ & $\mathbf{I}$ & III & IV & V & 1 & II \\
\hline 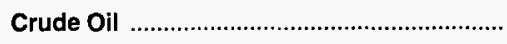 & 0 & 5,256 & 0 & 2,303 & 12,228 & 9,587 & 0 & 0 & 721,106 \\
\hline 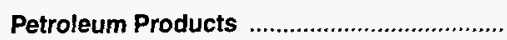 & 105,084 & 982 & $\mathbf{0}$ & 40,029 & 72,457 & 35,658 & 0 & $1,128,654$ & 343,616 \\
\hline 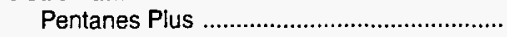 & 0 & 0 & 0 & 0 & 2,794 & 7 & 0 & 0 & 8,310 \\
\hline Liquefied Petroleum Gases ........................... & 40 & 0 & 0 & 12,853 & 54,125 & 1,033 & 0 & 28,622 & 47,785 \\
\hline 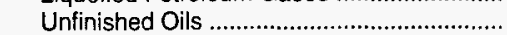 & 310 & 41 & 0 & 291 & 726 & 0 & 0 & 0 & 1,417 \\
\hline Motor Gasoline Blending Components ....... & 28 & 290 & 0 & 14 & 97 & 0 & 0 & 5,749 & 21,211 \\
\hline 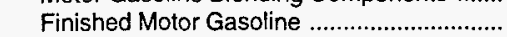 & 69,139 & 0 & 0 & 14,754 & 9,023 & 13,765 & 0 & 625,490 & 139,361 \\
\hline Reformulated & 25 & 0 & 0 & 0 & 6,450 & 0 & 0 & 120,382 & 8,116 \\
\hline Oxygenated & 15 & 0 & 0 & 1,619 & 0 & 114 & 0 & 0 & 0 \\
\hline Other & 69,099 & 0 & 0 & 13,135 & 2,573 & 13,651 & 0 & 505,108 & 131,245 \\
\hline Finished Aviation Gasoline .......................... & 0 & 0 & 0 & 0 & 0 & 147 & 0 & 723 & 1,087 \\
\hline Jet Fuel & 3,600 & 0 & 0 & 1,150 & 1 & 12,256 & 0 & 163,486 & 50,698 \\
\hline Naphtha-Type & 0 & 0 & 0 & 0 & 0 & 0 & 0 & 0 & 0 \\
\hline 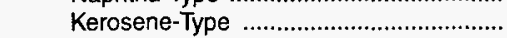 & 3,600 & 0 & 0 & 1,150 & 1 & 12,256 & 0 & 163,486 & 50,698 \\
\hline Kerosene & 294 & 0 & 0 & 504 & 0 & 0 & 0 & 2,624 & 235 \\
\hline 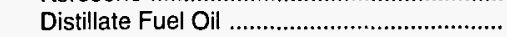 & 30,835 & 1 & 0 & 7,484 & 2,781 & 8,450 & 0 & 273,804 & 64,191 \\
\hline 0.05 percent sulfur and under & 24,030 & 1 & 0 & 2,784 & 2,488 & 8,306 & 0 & 165,257 & 55,798 \\
\hline Greater than 0.05 percent sulfur ............ & 6,805 & 0 & 0 & 4,700 & 293 & 144 & 0 & 108,547 & 8,393 \\
\hline Residual Fuel Oil & 12 & 414 & 0 & 636 & 2,598 & 0 & 0 & 16,215 & 91 \\
\hline 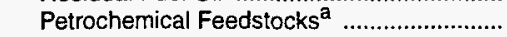 & 724 & 0 & 0 & 0 & 0 & 0 & 0 & 0 & 436 \\
\hline Special Naphthas & 0 & 33 & 0 & 10 & 45 & 0 & 0 & 1,147 & 706 \\
\hline 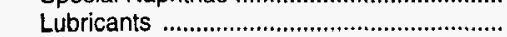 & 69 & 203 & 0 & 579 & 267 & 0 & 0 & 8,006 & 3,268 \\
\hline Waxes & 0 & 0 & 0 & 0 & 0 & 0 & 0 & 0 & 0 \\
\hline 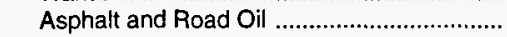 & 33 & 0 & 0 & 1,754 & 0 & 0 & 0 & 2,788 & 4,820 \\
\hline Miscellaneous Products ............................. & 0 & 0 & 0 & 0 & 0 & 0 & 0 & 0 & 0 \\
\hline Total & 105,084 & 6,238 & 0 & 42,332 & 84,685 & 45,245 & 0 & $1,128,654$ & $1,064,722$ \\
\hline
\end{tabular}

\begin{tabular}{|c|c|c|c|c|c|c|c|c|c|}
\hline \multirow{2}{*}{ Commodity } & \multicolumn{2}{|c|}{ From III to } & \multicolumn{3}{|c|}{ From IV to } & \multicolumn{4}{|c|}{ From V to } \\
\hline & IV & $\mathbf{v}$ & II & III & $\mathbf{v}$ & $\mathbf{I}$ & II & III & IV \\
\hline 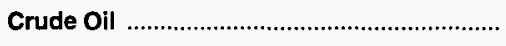 & 0 & 0 & 34,781 & 10,351 & 0 & 0 & 0 & 35,535 & 0 \\
\hline Petroleum Products ........ & 5,665 & 28,411 & 25,755 & 32,797 & 10,509 & 0 & 0 & 1,290 & 0 \\
\hline 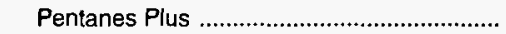 & & & 1,904 & 3,107 & 0 & 0 & 0 & 0 & 0 \\
\hline Liquefied Petroleum Gases .............................. & 0 & 0 & 15,297 & 29,690 & 0 & 0 & 0 & 0 & 0 \\
\hline 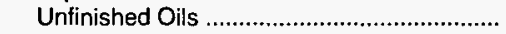 & 0 & 0 & 0 & 0 & 0 & 0 & 0 & 382 & 0 \\
\hline Motor Gasoline Blending Components ........ & 0 & 1,844 & 0 & 0 & 0 & 0 & 0 & 310 & 0 \\
\hline Finished Motor Gasoline ................................... & 4,302 & 17,391 & 5,218 & 0 & 9,348 & 0 & 0 & 238 & 0 \\
\hline 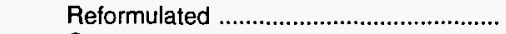 & 0 & 2,519 & 0 & 0 & 0 & 0 & 0 & 0 & 0 \\
\hline Oxygenated ……………………………....... & & 0 & 0 & 0 & 0 & 0 & 0 & 0 & 0 \\
\hline 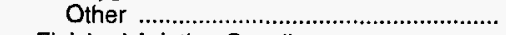 & 4,302 & 14,872 & 5,218 & 0 & 9,348 & 0 & 0 & 238 & 0 \\
\hline 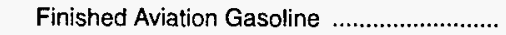 & 0 & 0 & 0 & 0 & 0 & 0 & 0 & 0 & 0 \\
\hline Jet Fuel & 1,011 & 4,172 & 135 & 0 & 714 & 0 & 0 & 94 & 0 \\
\hline 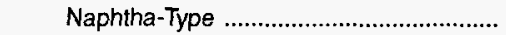 & 0 & 0 & 0 & 0 & 0 & 0 & 0 & 0 & 0 \\
\hline 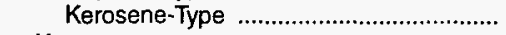 & 1,011 & 4,172 & 135 & 0 & 714 & 0 & 0 & 94 & 0 \\
\hline 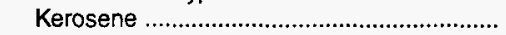 & 0 & 0 & 162 & 0 & 0 & 0 & 0 & 0 & 0 \\
\hline 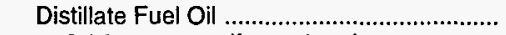 & 352 & 4,518 & 3,039 & 0 & 447 & 0 & 0 & 0 & 0 \\
\hline 0.05 percent sulfur and under ....................... & 352 & 2,555 & 3,039 & 0 & 391 & 0 & 0 & 0 & 0 \\
\hline Greater than 0.05 percent sulfur .............. & 0 & 1,963 & 0 & 0 & 56 & 0 & 0 & 0 & 0 \\
\hline Residual Fuel Oil ............................................ & 0 & 0 & 0 & 0 & 0 & 0 & 0 & 0 & 0 \\
\hline 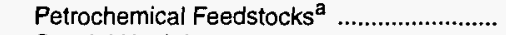 & 0 & 0 & 0 & 0 & 0 & 0 & 0 & 0 & 0 \\
\hline 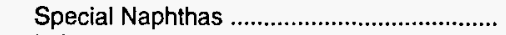 & 0 & 0 & 0 & 0 & 0 & 0 & 0 & 0 & 0 \\
\hline 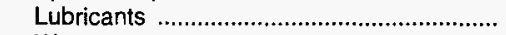 & 0 & 486 & 0 & 0 & 0 & 0 & 0 & 266 & 0 \\
\hline 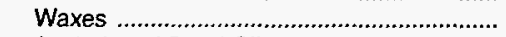 & 0 & 0 & 0 & 0 & 0 & 0 & 0 & 0 & 0 \\
\hline 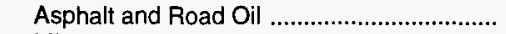 & 0 & 0 & 0 & 0 & 0 & 0 & 0 & 0 & 0 \\
\hline 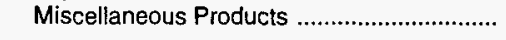 & 0 & 0 & 0 & 0 & 0 & 0 & 0 & 0 & 0 \\
\hline Total & 5,665 & 28,411 & 60,536 & 43,148 & 10,509 & 0 & 0 & 36,825 & 0 \\
\hline
\end{tabular}

a Includes naphtha less than $401^{\circ} \mathrm{F}$ endpoint and other oils equal to or greater than $401^{\circ} \mathrm{F}$ endpoint.

Sources: Energy Information Administration (ElA) Forms EIA-812, "Monthly Product Pipeline Report," EIA-813, "Monthly Crude Oil Report," and EIA-817, "Monthly Tanker and Barge Movement Report." 
Table 33. Movements of Crude Oil and Petroleum Products by Pipeline Between PAD Districts, 1997 (Thousand Barrels)

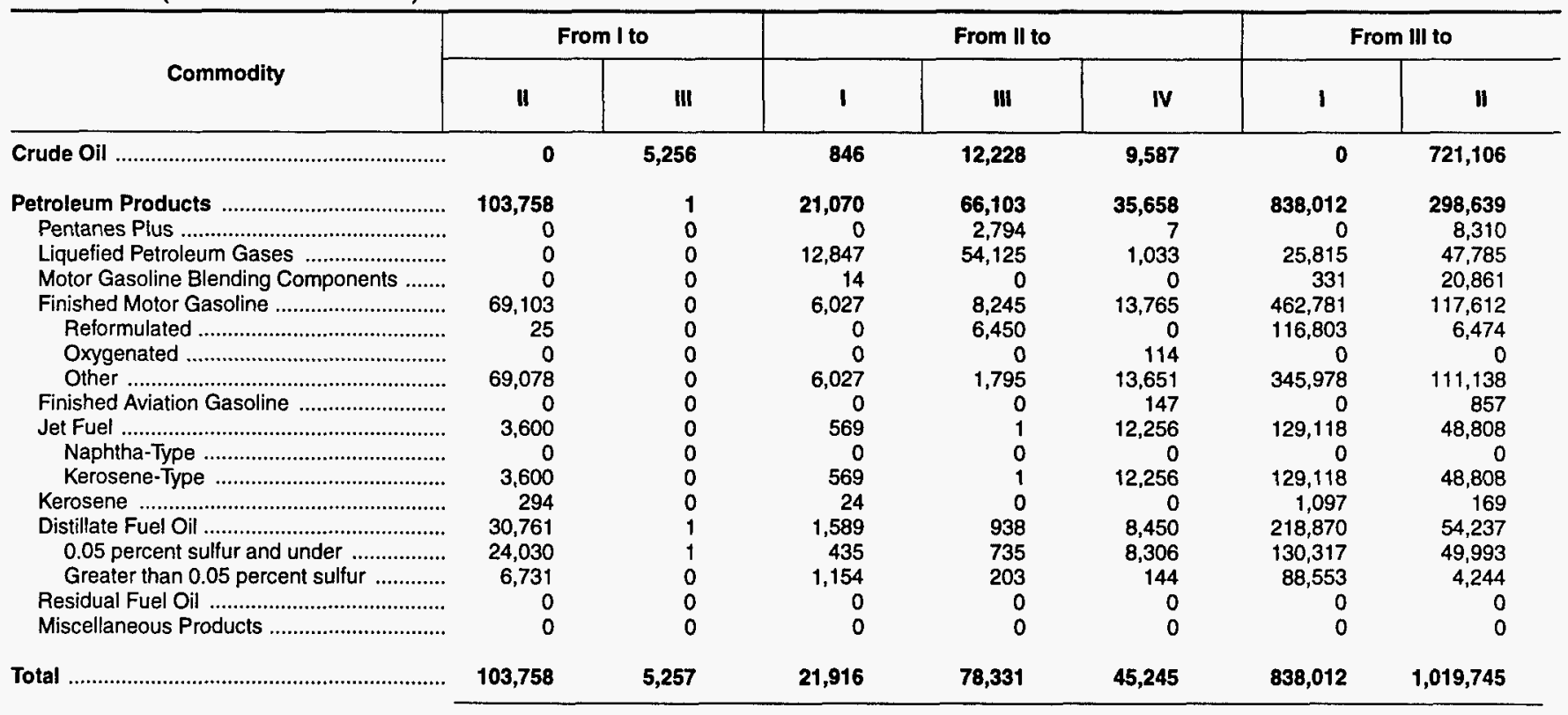

\begin{tabular}{|c|c|c|c|c|c|c|c|}
\hline \multirow{2}{*}{ Commodity } & \multicolumn{2}{|c|}{ From III to } & \multicolumn{3}{|c|}{ From IV to } & \multicolumn{2}{|c|}{ From V to } \\
\hline & IV & $\mathbf{v}$ & II & III & $\mathbf{v}$ & III & IV \\
\hline 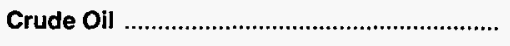 & 0 & 0 & 34,781 & 10,351 & 0 & 35,535 & 0 \\
\hline 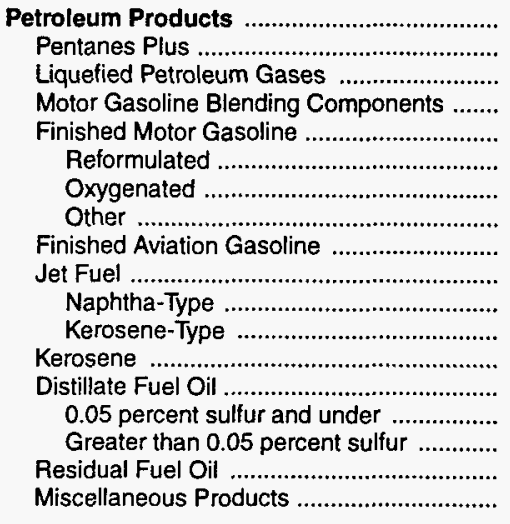 & $\begin{array}{r}5,665 \\
0 \\
0 \\
0 \\
4,302 \\
0 \\
0 \\
4,302 \\
0 \\
1,011 \\
0 \\
1,011 \\
0 \\
352 \\
352 \\
0 \\
0 \\
0\end{array}$ & $\begin{array}{r}25,704 \\
0 \\
0 \\
1,295 \\
16,340 \\
1,724 \\
0 \\
14,616 \\
0 \\
4,003 \\
0 \\
4,003 \\
0 \\
4,066 \\
2,555 \\
1,511 \\
0 \\
0\end{array}$ & $\begin{array}{r}25,755 \\
1,904 \\
15,297 \\
0 \\
5,218 \\
0 \\
0 \\
5,218 \\
0 \\
135 \\
0 \\
135 \\
162 \\
3,039 \\
3,039 \\
0 \\
0 \\
0\end{array}$ & $\begin{array}{r}32,797 \\
3,107 \\
29,690 \\
0 \\
0 \\
0 \\
0 \\
0 \\
0 \\
0 \\
0 \\
0 \\
0 \\
0 \\
0 \\
0 \\
0 \\
0\end{array}$ & $\begin{array}{r}10,509 \\
0 \\
0 \\
0 \\
9,348 \\
0 \\
0 \\
9,348 \\
0 \\
714 \\
0 \\
714 \\
0 \\
447 \\
391 \\
56 \\
0 \\
0\end{array}$ & $\begin{array}{l}0 \\
0 \\
0 \\
0 \\
0 \\
0 \\
0 \\
0 \\
0 \\
0 \\
0 \\
0 \\
0 \\
0 \\
0 \\
0 \\
0 \\
0\end{array}$ & $\begin{array}{l}0 \\
0 \\
0 \\
0 \\
0 \\
0 \\
0 \\
0 \\
0 \\
0 \\
0 \\
0 \\
0 \\
0 \\
0 \\
0 \\
0 \\
0\end{array}$ \\
\hline Total & 5,665 & 25,704 & 60,536 & 43,148 & 10,509 & 35,535 & 0 \\
\hline
\end{tabular}

Sources: Energy Information Administration (EIA) Forms EIA-812, "Monthly Product Pipeline Report," and EIA-813, Monthly Crude Oil Report." 
Table 34. Movements of Crude Oil and Petroleum Products by Tanker and Barge Between PAD Districts, 1997

(Thousand Barrels)

\begin{tabular}{|c|c|c|c|c|c|c|c|c|}
\hline \multirow[b]{2}{*}{ Commodity } & \multicolumn{3}{|c|}{ From I to } & \multicolumn{3}{|c|}{ From II to } & \multicolumn{2}{|c|}{ From lll to } \\
\hline & II & III & $\mathbf{v}$ & $\mathbf{I}$ & III & $\mathbf{v}$ & 1 & $\begin{array}{c}\text { New } \\
\text { England }\end{array}$ \\
\hline 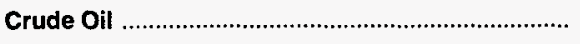 & 0 & 0 & $\mathbf{0}$ & 1,457 & 0 & 0 & 0 & $\mathbf{0}$ \\
\hline Petroleum Products & 1,326 & 981 & 0 & 18,959 & 6,354 & $\mathbf{0}$ & 290,642 & 9,379 \\
\hline 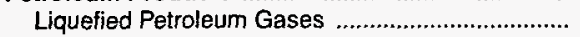 & 40 & 0 & 0 & 6 & 0 & 0 & 2,807 & 0 \\
\hline 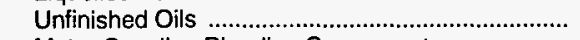 & 310 & 41 & 0 & 291 & 726 & 0 & 0 & 0 \\
\hline Motor Gasoline Blending Components ................... & 28 & 290 & 0 & 0 & 97 & 0 & 5,418 & 0 \\
\hline Finished Motor Gasoline & 36 & 0 & 0 & 8,727 & 778 & 0 & 162,709 & 2,315 \\
\hline Reformulated & 0 & 0 & 0 & 0 & 0 & 0 & 3,579 & 2,315 \\
\hline Oxygenated & 15 & 0 & 0 & 1,619 & 0 & 0 & 0 & 0 \\
\hline 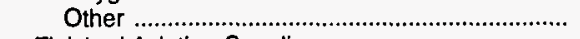 & 21 & 0 & 0 & 7,108 & 778 & 0 & 159,130 & 0 \\
\hline 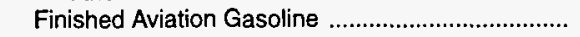 & 0 & 0 & 0 & 0 & 0 & 0 & 723 & 30 \\
\hline 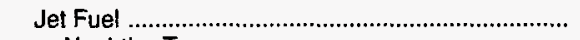 & 0 & 0 & 0 & 581 & 0 & 0 & 34,368 & 104 \\
\hline Naphtha-Type & 0 & 0 & 0 & 0 & 0 & 0 & 0 & 0 \\
\hline 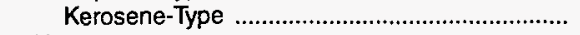 & 0 & 0 & 0 & 581 & 0 & 0 & 34,368 & 104 \\
\hline 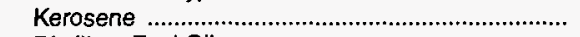 & 0 & 0 & 0 & 480 & 0 & 0 & 1,527 & 267 \\
\hline 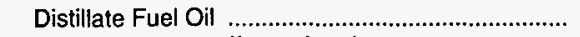 & 74 & 0 & 0 & 5,895 & 1,843 & 0 & 54,934 & 5,826 \\
\hline 0.05 percent sulfur and under ............................... & 0 & 0 & 0 & 2,349 & 1,753 & 0 & 34,940 & 1,105 \\
\hline Greater then 0.05 percent sulfur ........................... & 74 & 0 & 0 & 3,546 & 90 & 0 & 19,994 & 4,721 \\
\hline 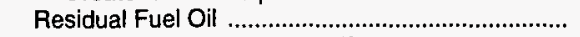 & 12 & 414 & 0 & 636 & 2,598 & 0 & 16,215 & 699 \\
\hline 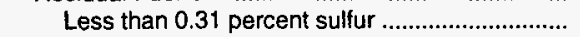 & 0 & 0 & 0 & 0 & 0 & 0 & 0 & 0 \\
\hline 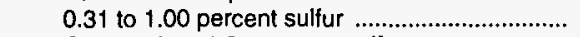 & 0 & 0 & 0 & 0 & 0 & 0 & 0 & 0 \\
\hline Greater than 1.00 percent sulfur .......................... & 12 & 414 & 0 & 636 & 2,598 & 0 & 16,215 & 699 \\
\hline 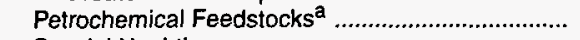 & 724 & 0 & 0 & 0 & 0 & 0 & 0 & 0 \\
\hline 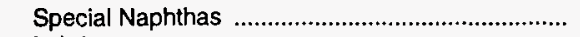 & 0 & 33 & 0 & 10 & 45 & 0 & 1,147 & 0 \\
\hline 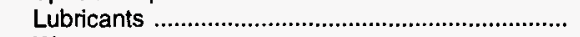 & 69 & 203 & 0 & 579 & 267 & 0 & 8,006 & 0 \\
\hline Waxes & 0 & 0 & 0 & 0 & 0 & 0 & $\mathbf{0}$ & 0 \\
\hline 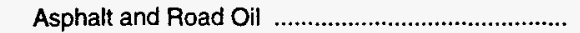 & 33 & 0 & 0 & 1,754 & 0 & 0 & 2,788 & 138 \\
\hline 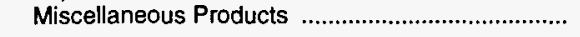 & 0 & 0 & 0 & 0 & 0 & 0 & 0 & 0 \\
\hline Total & 1,326 & 981 & 0 & 20,416 & 6,354 & 0 & 290,642 & 9,379 \\
\hline
\end{tabular}

\begin{tabular}{|c|c|c|c|c|c|c|c|}
\hline \multirow[b]{2}{*}{ Commodity } & \multicolumn{4}{|c|}{ From III to } & \multicolumn{3}{|c|}{ From $V$ to } \\
\hline & $\begin{array}{l}\text { Central } \\
\text { Atlantic }\end{array}$ & $\begin{array}{l}\text { Lower } \\
\text { Atlantic }\end{array}$ & II & $\mathbf{v}$ & $\mathbf{I}$ & II & III \\
\hline 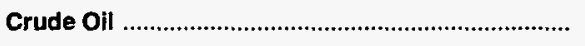 & 0 & 0 & 0 & 0 & 0 & 0 & 0 \\
\hline Petroleum Products & 18,971 & 262,292 & 44,977 & 2,707 & 0 & $\mathbf{0}$ & 1,290 \\
\hline 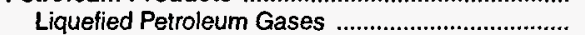 & 0 & 2,807 & 0 & 0 & 0 & 0 & 0 \\
\hline Unfinished Oils ......................................................... & 0 & 0 & 1,417 & 0 & 0 & 0 & 382 \\
\hline 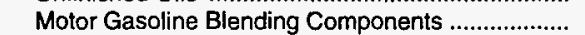 & 4,955 & 463 & 350 & 549 & 0 & 0 & 310 \\
\hline Finished Motor Gasoline ............................................. & 3,245 & 157,149 & 21,749 & 1,051 & 0 & 0 & 238 \\
\hline 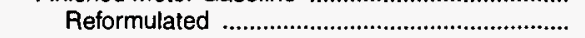 & 1,190 & 74 & 1,642 & 795 & 0 & 0 & 0 \\
\hline 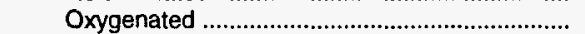 & 0 & 0 & 0 & 0 & 0 & 0 & 0 \\
\hline 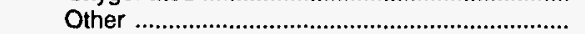 & 2,055 & 157,075 & 20,107 & 256 & 0 & 0 & 238 \\
\hline Finished Aviation Gasoline ................................... & 172 & 521 & 230 & 0 & 0 & 0 & 0 \\
\hline 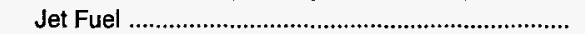 & 583 & 33,681 & 1,890 & 169 & 0 & 0 & 94 \\
\hline 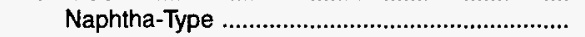 & 0 & 0 & 0 & 0 & 0 & 0 & 0 \\
\hline 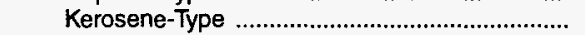 & 583 & 33,681 & 1,890 & 169 & 0 & 0 & 94 \\
\hline 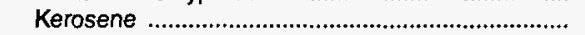 & 638 & 622 & 66 & 0 & 0 & 0 & 0 \\
\hline 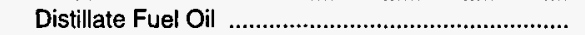 & 4,065 & 45,043 & 9,954 & 452 & 0 & 0 & 0 \\
\hline 0.05 percent sulfur and under ................................... & 1,798 & 32,037 & 5,805 & 0 & 0 & 0 & 0 \\
\hline Greater then 0.05 percent sulfur ............................. & 2,267 & 13,006 & 4,149 & 452 & 0 & 0 & 0 \\
\hline 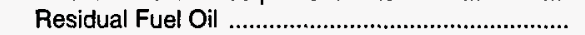 & 1,399 & 14,117 & 91 & 0 & 0 & 0 & 0 \\
\hline Less than 0.31 percent sulfur .................................... & 0 & 0 & 0 & 0 & 0 & 0 & 0 \\
\hline 0.31 to 1.00 percent sulfur & 0 & 0 & 0 & 0 & 0 & 0 & 0 \\
\hline Greater than 1.00 percent sulfur ............................. & 1,399 & 14,117 & 91 & 0 & 0 & 0 & 0 \\
\hline Petrochemical Feedstocks ${ }^{a}$ & 0 & 0 & 436 & 0 & 0 & 0 & 0 \\
\hline 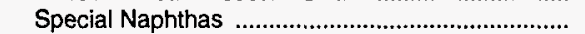 & 0 & 1,147 & 706 & 0 & 0 & 0 & 0 \\
\hline 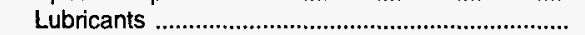 & 3,549 & 4,457 & 3,268 & 486 & 0 & 0 & 266 \\
\hline 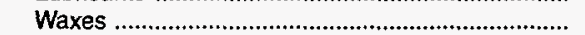 & 0 & 0 & 0 & 0 & 0 & 0 & 0 \\
\hline Asphalt and Road Oil ........................................ & 365 & 2,285 & 4,820 & 0 & 0 & 0 & 0 \\
\hline 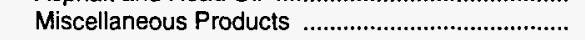 & 0 & 0 & 0 & 0 & 0 & 0 & 0 \\
\hline Total & 18,971 & 262,292 & 44,977 & 2,707 & 0 & 0 & 1,290 \\
\hline
\end{tabular}

a Includes naphtha less than $401^{\circ} \mathrm{F}$ endpoint and other oils equal to or greater than $401^{\circ} \mathrm{F}$ endpoint.

Source: Energy Information Administration (EIA) Form EIA-817, "Monthly Tanker and Barge Movement Report." 
Table 35. Net Movements of Crude Oil and Petroleum Products by Pipeline, Tanker, and Barge Between PAD Districts, 1997

(Thousand Barrels)

\begin{tabular}{|c|c|c|c|c|c|c|}
\hline \multirow{2}{*}{ Commodity } & \multicolumn{3}{|c|}{ PAD District I } & \multicolumn{3}{|c|}{ PAD District II } \\
\hline & Receipts & Shipments & Net Receipts & Receipts & Shipments & Net Receipts \\
\hline 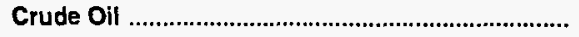 & 2,303 & 5,256 & $-2,953$ & 755,887 & 24,118 & 731,769 \\
\hline 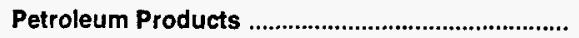 & $1,168,683$ & 106,066 & $1,062,617$ & 474,455 & 148,144 & 326,311 \\
\hline 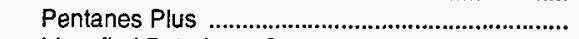 & 0 & 0 & 0 & 10,214 & 2,801 & 7,413 \\
\hline Liquefied Petroleum Gases ..................................... & 41,475 & 40 & 41,435 & 63,122 & 68,011 & $-4,889$ \\
\hline 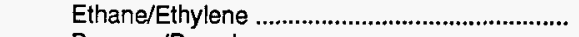 & & 0 & 0 & 8,989 & 32,029 & $-23,040$ \\
\hline 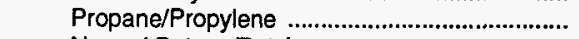 & 40,198 & 0 & 40,198 & 40,673 & 24,682 & 15,991 \\
\hline Normal Butane/Butylene ..................................... & 1,016 & 40 & 976 & 8,062 & 9,148 & $-1,086$ \\
\hline Isobutane/lsobutylene ............................................ & 261 & 0 & 261 & 5,398 & 2,152 & 3,246 \\
\hline 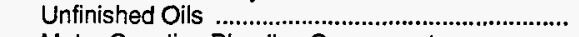 & 291 & 351 & -60 & 1,727 & 1,017 & 710 \\
\hline Motor Gasoline Blending Components .................. & 5,763 & 318 & 5,445 & 21,239 & 111 & 21,128 \\
\hline 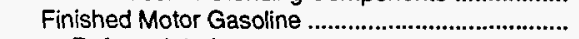 & 640,244 & 69,139 & 571,105 & 213,718 & 37,542 & 176,176 \\
\hline Reformulated & 120,382 & 25 & 120,357 & 8,141 & 6,450 & 1,691 \\
\hline 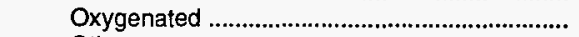 & 1,619 & 15 & 1,604 & 15 & 1,733 & $-1,718$ \\
\hline Other & 518,243 & 69,099 & 449,144 & 205,562 & 29,359 & 176,203 \\
\hline Finished Aviation Gasoline ...................................... & 723 & 0 & 723 & 1,087 & 147 & 940 \\
\hline Jet Fuel & 164,636 & 3,600 & 161,036 & 54,433 & 13,407 & 41,026 \\
\hline 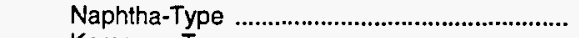 & 0 & 0 & 0 & 0 & 0 & 0 \\
\hline 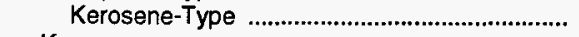 & 164,636 & 3,600 & 161,036 & 54,433 & 13,407 & 41,026 \\
\hline 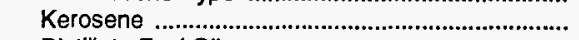 & 3,128 & 294 & 2,834 & 691 & 504 & 187 \\
\hline 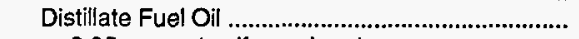 & 281,288 & 30,836 & 250,452 & 98,065 & 18,715 & 79,350 \\
\hline 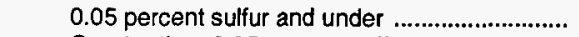 & 168,041 & 24,031 & 144,010 & 82,867 & 13,578 & 69,289 \\
\hline Greater than 0.05 percent sulfur ........................... & 113,247 & 6,805 & 106,442 & 15,198 & 5,137 & 10,061 \\
\hline 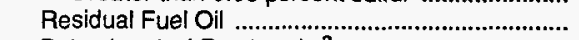 & 16,851 & 426 & 16,425 & 103 & 3,234 & $-3,131$ \\
\hline 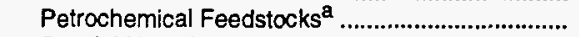 & & 724 & -724 & 1,160 & 0 & 1,160 \\
\hline Special Naphthas & 1,157 & 33 & 1,124 & 706 & 55 & 651 \\
\hline 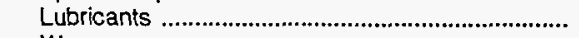 & 8,585 & 272 & 8,313 & 3,337 & 846 & 2,491 \\
\hline Waxes & 0 & 0 & 0 & 0 & 0 & 0 \\
\hline 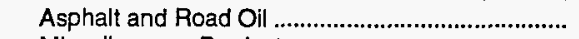 & 4,542 & 33 & 4,509 & 4,853 & 1,754 & 3,099 \\
\hline 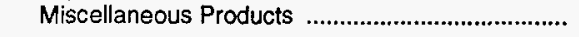 & 0 & 0 & 0 & 0 & 0 & 0 \\
\hline Total & $1,170,986$ & 111,322 & $1,059,664$ & $1,230,342$ & 172,262 & $1,058,080$ \\
\hline
\end{tabular}

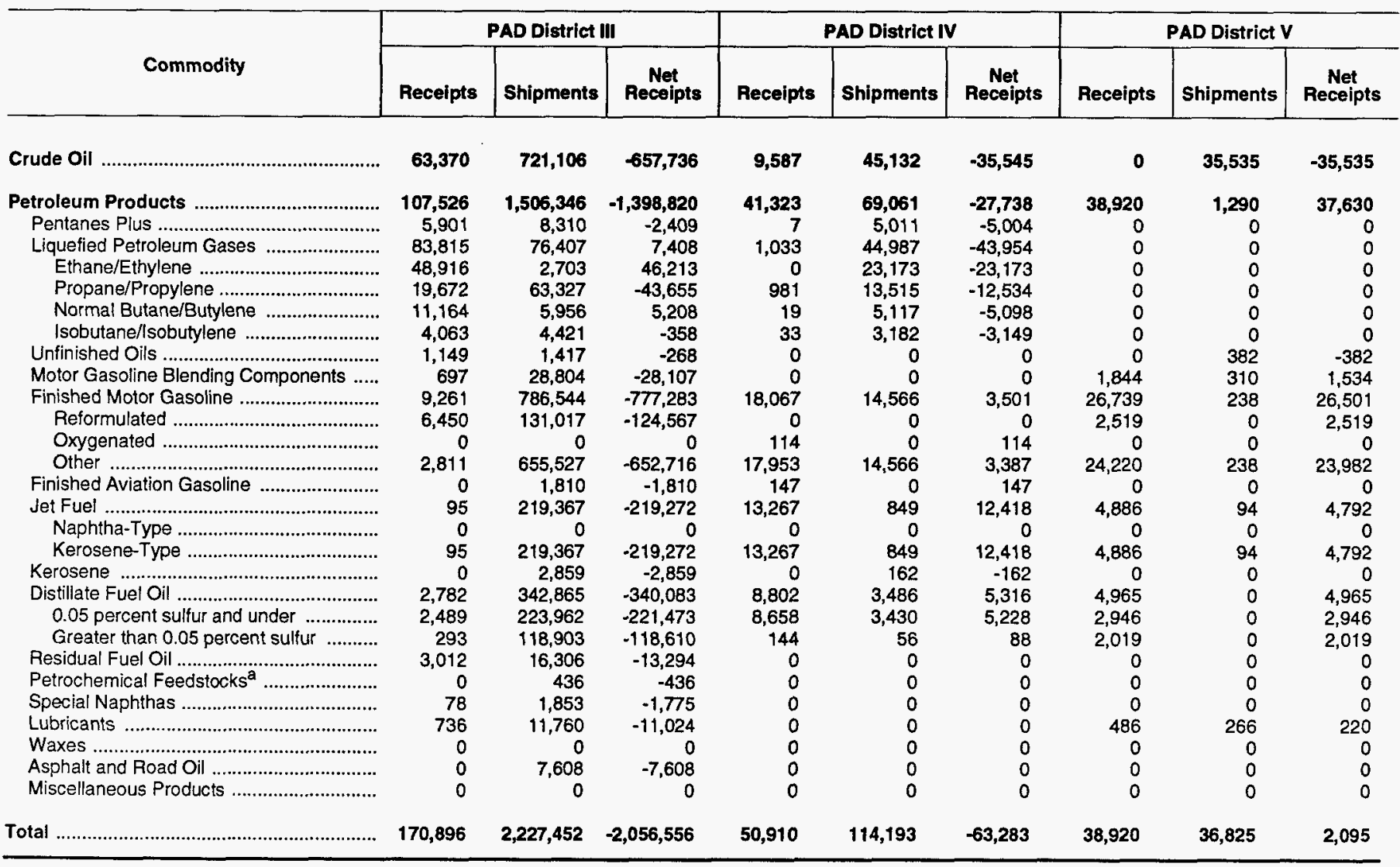

a Includes naphtha less than $401^{\circ} \mathrm{F}$ endpoint and other oils equal to or greater than $401^{\circ} \mathrm{F}$ endpoint.

Sources: Energy Information Administration (EIA) Forms EIA-812, "Monthly Product Pipeline Report," EIA-813, "Monthly Crude Oil Report," and EIA-817, "Monthly Tanker and Barge Movement Report," 


\section{Refinery Statistics}

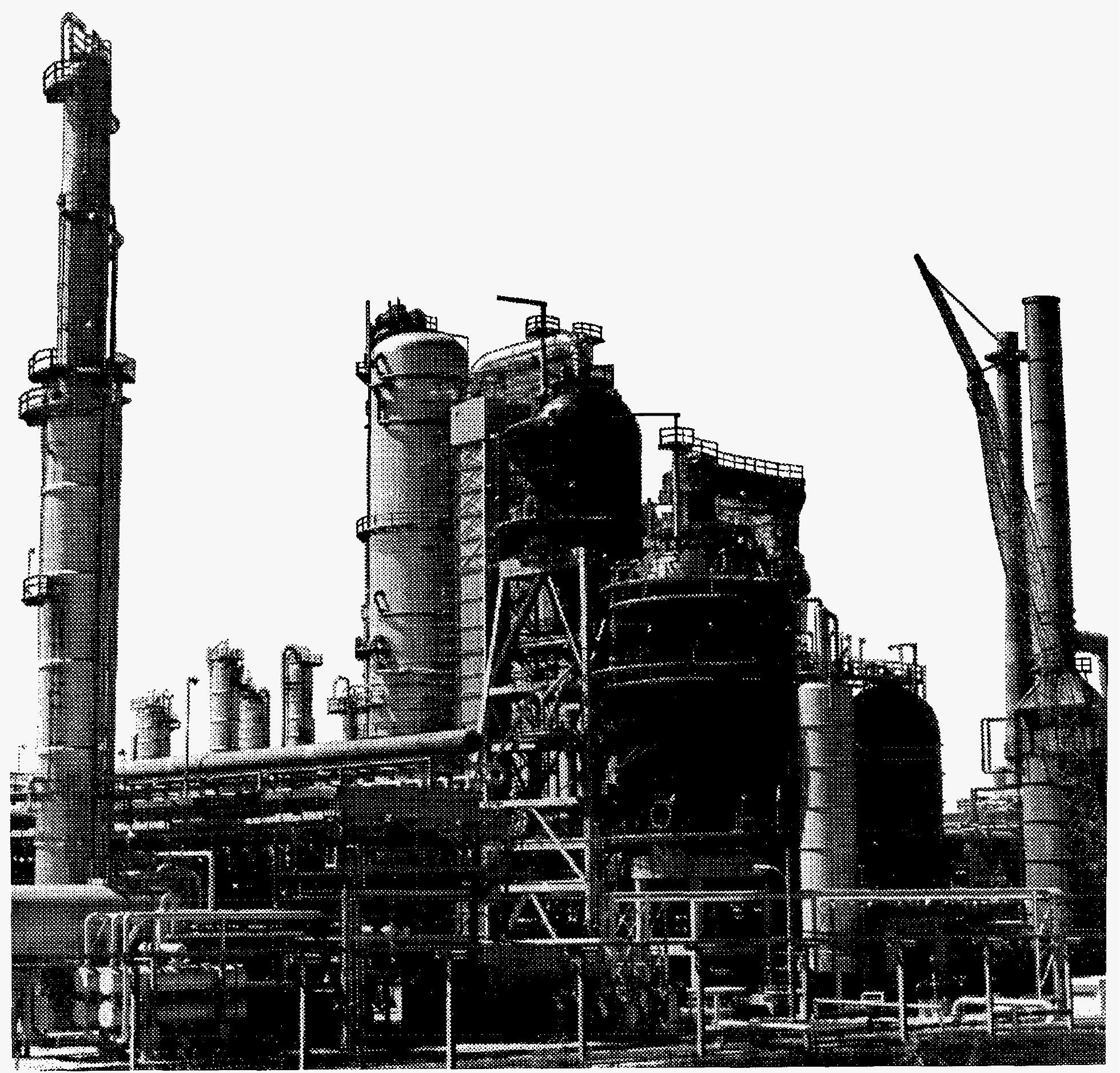

As part of the refining process, a catalytic cracking unit is used to increase the yield of gasoline from crude oil. 
Table 36. Refinery Fuel Use and Losses by PAD District, 1997 (Thousand Barrels)

\begin{tabular}{|c|c|c|c|c|c|c|}
\hline \multirow[b]{2}{*}{ Commodity } & \multicolumn{5}{|c|}{ PAD Districts } & \multirow{2}{*}{$\begin{array}{l}\text { United } \\
\text { States }\end{array}$} \\
\hline & $\mathbf{I}$ & II & III & IV & $\mathbf{v}$ & \\
\hline 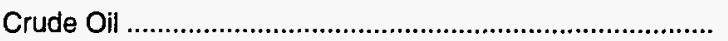 & 0 & 0 & 0 & 0 & 0 & 0 \\
\hline 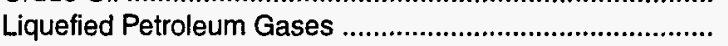 & 156 & 1,323 & 650 & 39 & 999 & 3,167 \\
\hline 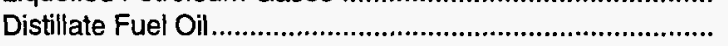 & 234 & 113 & 84 & 0 & 290 & 721 \\
\hline Residual Fuel Oil & 2,338 & 2,886 & 1,379 & 405 & 808 & 7,816 \\
\hline Still Gas & 18,490 & 46,926 & 118,216 & 6,782 & 48,751 & 239,165 \\
\hline 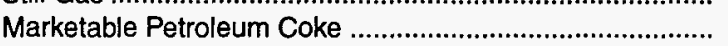 & 1,520 & 24 & 82 & 141 & 907 & 2,674 \\
\hline Catalyst Petroleum Coke & 10,997 & 17,374 & 40,267 & 2,332 & 13,324 & 84,294 \\
\hline Other Products & 159 & 1,539 & 3,347 & 694 & 941 & 6,680 \\
\hline
\end{tabular}

Note: Includes volumes used as fuel at refineries and all nonprocessing losses of crude oil and petroleum products (e.g., spills, fire losses, contamination, etc.). Other products includes miscellaneous products; finished motor gasoline; pentanes plus; unfinished oils, other hydrocarbons, hydrogen, and oxygenates; asphalt and road oil, motor gasoline blending components; lubricants; jet fuel; aviation gasoline; and special naphthas.

Source: Energy Information Administration (EIA), Form EIA-810, "Monthly Refinery Report."

Table 37. Shutdown and Reactivated Refineries During 1997

\begin{tabular}{c|c|c|c|c|c|}
\hline PAD District / Refinery & Location & $\begin{array}{c}\text { Total Atmospheric } \\
\text { Crude Oil } \\
\text { Distillation } \\
\text { Capacity (bbl/cd) }\end{array}$ & $\begin{array}{c}\text { Total Downstream } \\
\text { Charge Capacity } \\
\text { (bbl/sd) }\end{array}$ & $\begin{array}{c}\text { Date } \\
\text { Operable }\end{array}$ & $\begin{array}{c}\text { Date of Last } \\
\text { Operation }\end{array}$ \\
Shutdown \\
\hline
\end{tabular}

\section{SHUTDOWNS}

\section{PAD District III}

Gold Line Refg. LTD

Canal Refg. Co.

PAD District V

Pacific Refg. Co.

Lake Charles, LA
Church Point, LA

\section{7,100}

27,600

9,500

50,000

50,000

87,100
20,100

18,000

2,100

62,400

62,400

$01 / 68$

09/97

$12 / 78$

$01 / 52$

$05 / 97$

05/97

04/97

$06 / 97$

Total U.S. Shutdowns

REACTIVATIONS

\section{PAD District I}

Tosco Refining Company

Trainer, PA

Jennings, LA

Gold Line Refg. LTD

(Formerly Cas Refg. Co.)
82,500 
Table 38. Refinery Sales During 1997

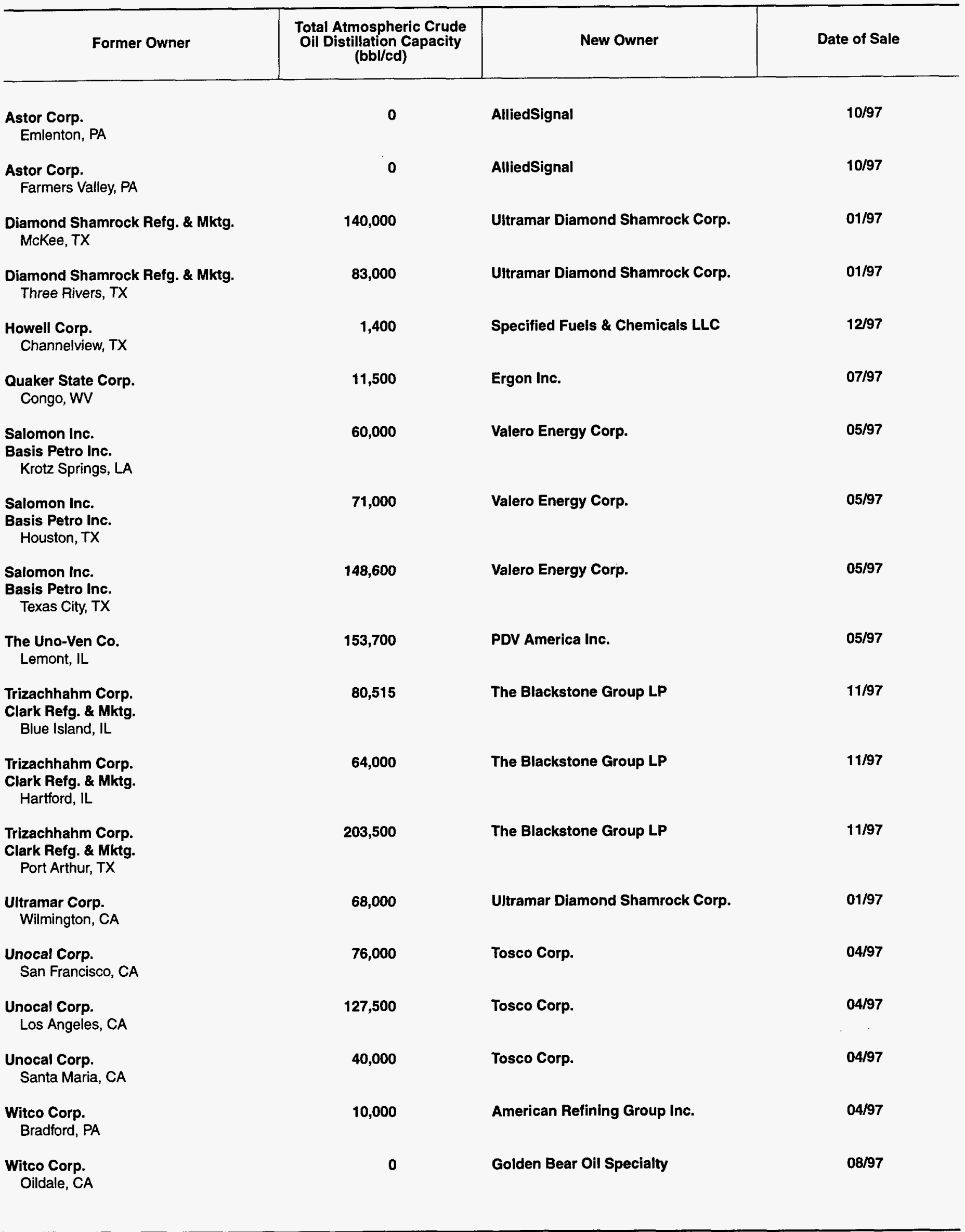




\title{
Appendix A
}

\author{
District \\ Descriptions \\ and Maps
}

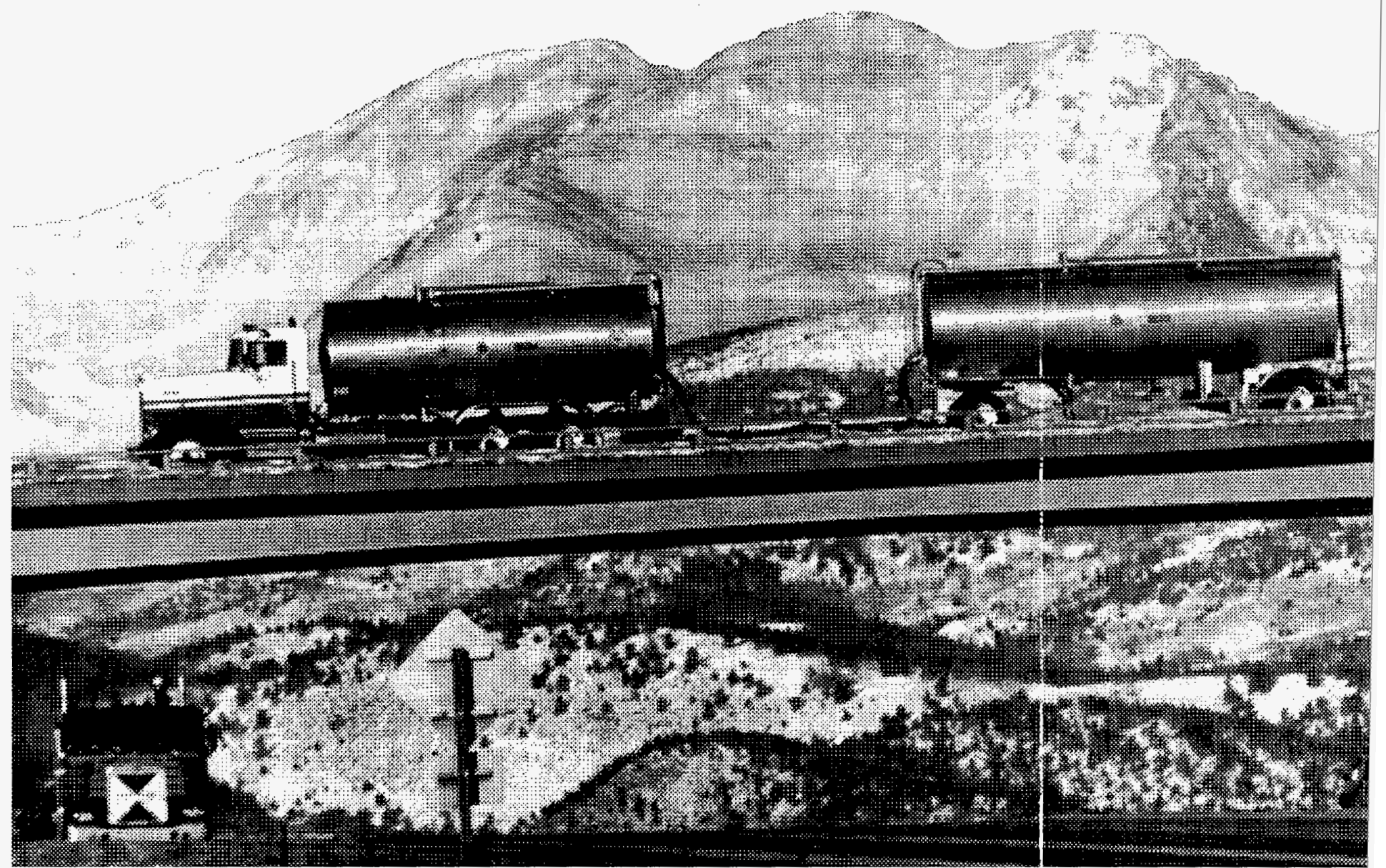




\section{District Descriptions and Maps}

The following are the Refining Districts which make up the Petroleum Administration for Defense (PAD) Districts.

\section{PAD District I}

East Coast: District of Columbia and the States of Maine, New Hampshire, Vermont, Massachusetts, Rhode Island, Connecticut, New Jersey, Delaware, Maryland, Virginia, North Carolina, South Carolina, Georgia, Florida, and the following counties of the State of New York: Cayuga, Tompkins, Chemung, and all counties east and north thereof. Also the following counties in the State of Pennsylvania: Bradford, Sullivan, Columbia, Montour, Northumberland, Dauphin, York, and all counties east thereof.

Appalachian No. 1: The State of West Virginia and those parts of the States of Pennsylvania and New York not included in the East Coast District.

\section{Sub-PAD District I}

New England: The States of Connecticut, Maine, Massachusetts, New Hampshire, Rhode Island and Vermont.

Central Atlantic: The District of Columbia and the States of Delaware, Maryland, New Jersey, New York, and Pennsylvania.

Lower Atlantic: The States of Florida, Georgia, North Carolina, South Carolina, Virginia and West Virginia.

\section{PAD District II}

Indiana-Illinois-Kentucky: The States of Indiana, Illinois, Kentucky, Tennessee, Michigan, and Ohio.

Minnesota-Wisconsin-North and South Dakota: The States of Minnesota, Wisconsin, North Dakota, and South Dakota.

Oklahoma-Kansas-Missouri: The States of Oklahoma, Kansas, Missouri, Nebraska, and Iowa.

\section{PAD District III}

Texas Inland: The State of Texas except the Texas Gulf Coast District.

Texas Gulf Coast: The following counties of the State of Texas: Newton, Orange, Jefferson, Jasper, Tyler, Hardin, Liberty, Chambers, Polk, San Jacinto, Montgomery, Harris, Galveston, Waller, Fort Bend, Brazoria, Wharton, Matagorda, Jackson, Victoria, Calhoun, Refugio, Aransas, San Patricio, Nueces, Kleberg, Kenedy, Willacy, and Cameron.

Louisiana Gulf Coast: The following Parishes of the State of Louisiana: Vernon, Rapides, Avoyelles, Pointe Coupee, West Feliciana, East Feliciana, Saint Helena, Tangipahoa, Washington, and all Parishes south thereof. Also the following counties of the State of Mississippi: Pearl River, Stone, George, Hancock, Harrison, and Jackson. Also the following counties of the State of Alabama: Mobile and Baldwin.

North Louisiana-Arkansas: The State of Arkansas and those parts of the States of Louisiana, Mississippi, and Alabama not included in the Louisiana Gulf Coast District.

New Mexico: The State of New Mexico.

\section{PAD District IV}

Rocky Mountain: The States of Montana, Idaho, Wyoming, Utah, and Colorado.

\section{PAD District V}

West Coast: The States of Washington, Oregon, California, Nevada, Arizona, Alaska, and Hawaii. 


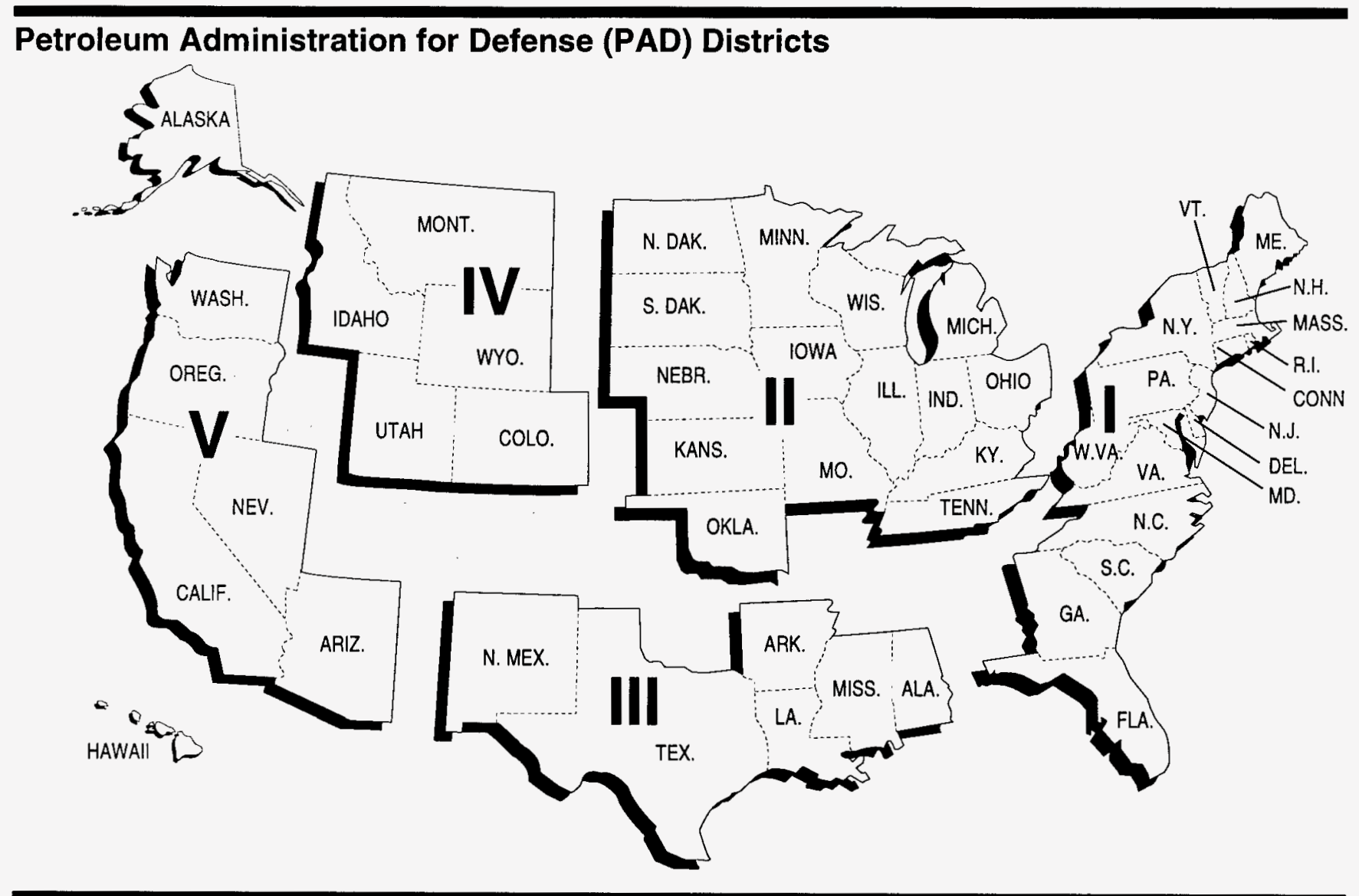

\section{Refining Districts}

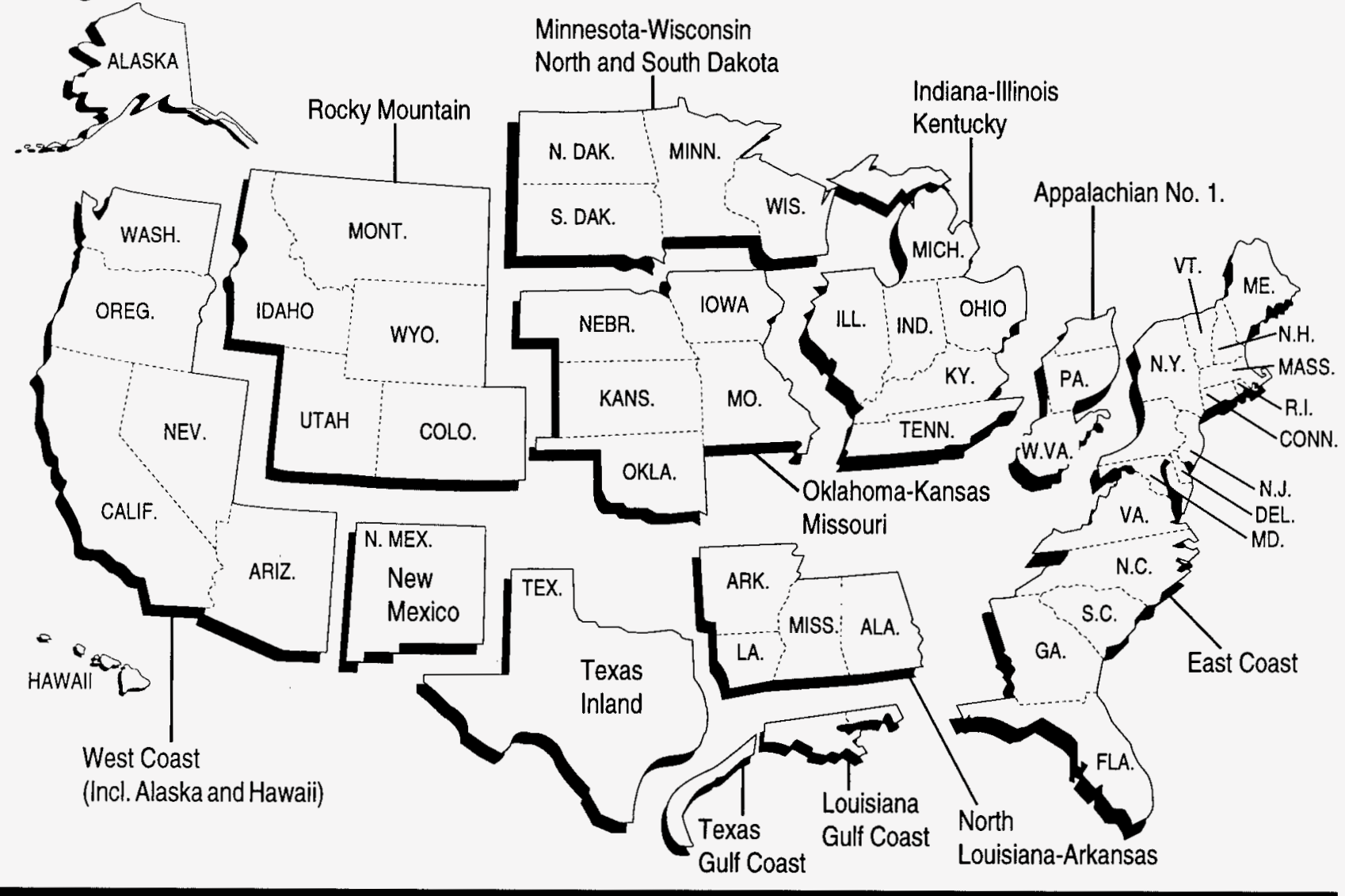




\section{Appendix B}

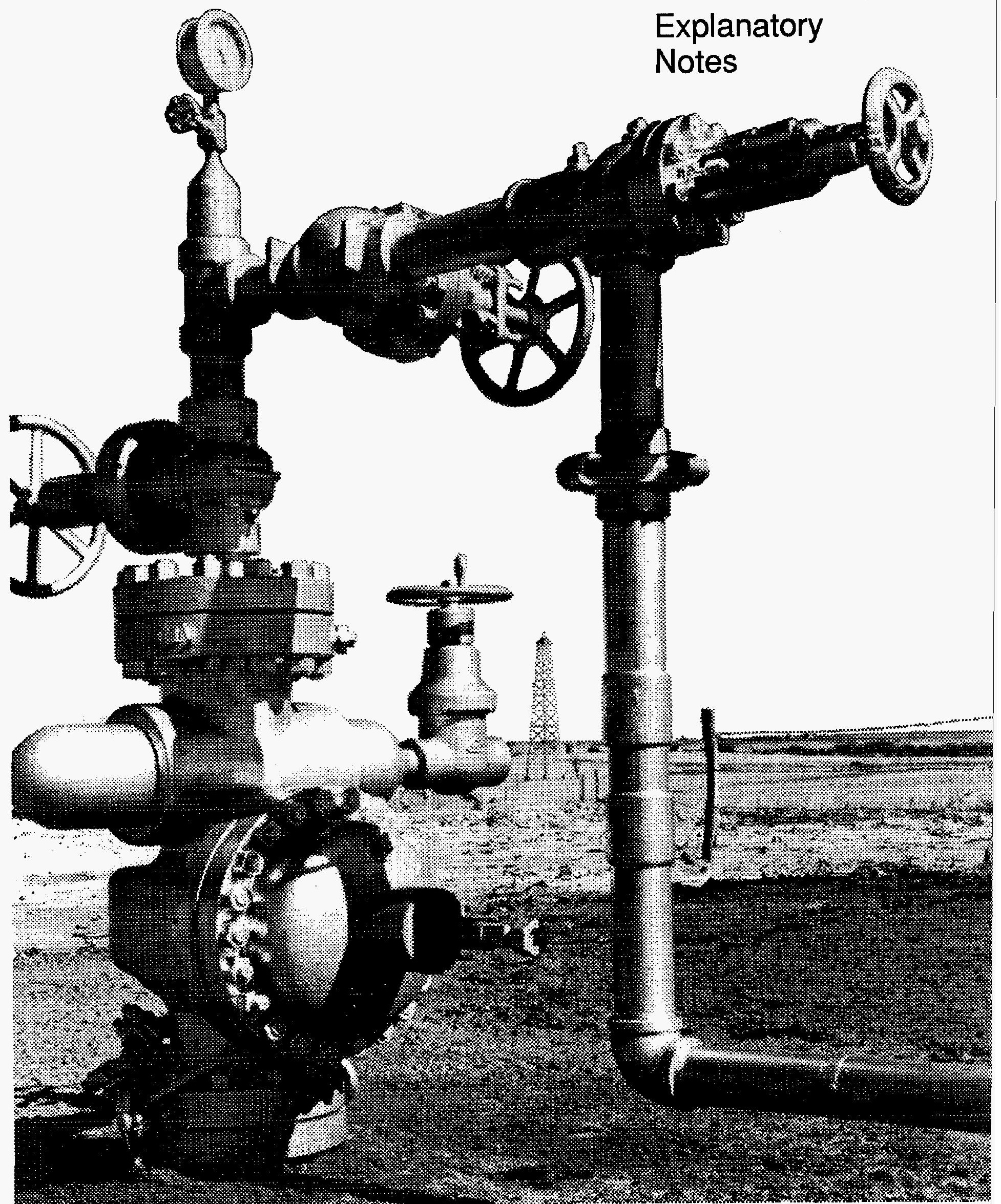




\section{Explanatory Notes}

The following Explanatory Notes are provided to assist in understanding and interpreting the data presented in this publication.

- Note 1. Petroleum Supply Reporting System

- Note 2. Monthly Petroleum Supply Reporting System

- Note 3. Form EIA-820: Biennial Refinery Report

- Note 4. Technical Notes for Detailed Statistics Tables

- Note 5. Domestic Crude Oil Production

- Note 6. Export Data

- Note 7. Quality Control and Data Revision

- Note 8. Frames Maintenance

- Note 9. Descriptive Monthly Statistics

- Note 10. Practical Limitations of Data Collection Efforts

- Note 11. 1981 Changes in the Petroleum Supply Reporting System

- Note 12. 1983 Changes in the Petroleum Supply Reporting System

- Note 13. 1984 Changes in the Petroleum Supply Reporting System

- Note 14. 1985 Changes in the Petroleum Supply Reporting System

- Note 15. 1986 Changes in the Petroleum Supply Reporting System

- Note 16. 1987 Changes in the Petroleum Supply Reporting System

- Note 17. 1989 Changes in the Petroleum Supply Reporting System

- Note 18. 1990 Changes in the Petroleum Supply Reporting System

- Note 19. 1993 Changes in the Petroleum Supply Reporting System

- Note 20. 1994 Changes in the Petroleum Supply Reporting System

- Note 21. 1995 Changes in the Petroleum Supply Reporting System

- Note 22. 1997 Changes in the Petroleum Supply Reporting System

- Note 23. Motor Gasoline Blending Plants

\section{Note 1. Petroleum Supply Reporting System}

The Petroleum Supply Reporting System (PSRS) represents a family of data collection survey forms, data processing systems, and publication systems that have been consolidated to achieve comparability and consistency throughout. The survey forms that comprise the PSRS are:

\begin{tabular}{ll} 
Form & \\
Number & Name \\
EIA-800 & "Weekly Refinery Report" \\
EIA-801 & "Weekly Bulk Terminal Report" \\
EIA-802 & "Weekly Product Pipeline Report" \\
EIA-803 & "Weekly Crude Oil Stocks Report" \\
EIA-804 & "Weekly Imports Report" \\
EIA-807 & "Propane Telephone Survey" \\
EIA-810 & "Monthly Refinery Report" \\
EIA-811 & "Monthly Bulk Terminal Report" \\
EIA-812 & "Monthly Product Pipeline Report" \\
EIA-813 & "Monthly Crude Oil Report" \\
EIA-814 & "Monthly Imports Report" \\
EIA-816 & "Monthly Natural Gas Liquids Report" \\
EIA-817 & "Monthly Tanker and Barge Movement \\
EIA -819M & "Meport" \\
EIA-820 & "Bonthly Oxygenate Telephone Report" \\
& \\
\hline
\end{tabular}

Forms EIA-800 through 804 comprise the Weekly Petroleum Supply Reporting System (WPSRS). A sample of all petroleum companies report weekly data to the Energy Information Administration (EIA) on crude oil and petroleum product stocks, refinery inputs and production, and crude oil and petroleum product imports. The sample of companies that report weekly is selected from the universe of companies that report on the comparable monthly surveys. Data collected from the WPSRS are used to develop estimates of the most current monthly quantities in the Summary Statistics section of the Petroleum Supply Monthly (PSM) and which appear in the Weekly Petroleum Status Report(WPSR).

The Form EIA-807, "Propane Telephone Survey," is used to collect data on production, stocks, and imports of pro- 
pane. These data are used to monitor the supply of propane and to report to the Congress and others on supplies when requested. Data are collected from a sample of respondents reporting on the Monthly Petroleum Supply Reporting System (MPSRS) surveys. Data are collected on a weekly basis during the heating season (October through March) and published in the Winter Fuels Report. During the non-heating season (April through September) data are collected on end-of-month stocks only. These data are published in the WPSR.

Forms EIA-810 through 814,816 , and 817 comprise the MPSRS. These surveys are used to collect detailed refinery/blender and natural gas plant operations data; refinery/blender, bulk terminal, oxygenate plant, natural gas plant and pipeline stocks data; crude oil and petroleum product imports data; and data on movements of petroleum products and crude oil between Petroleum Administration for Defense (PAD) Districts. A description of the MPSRS forms follows in Explanatory Note 2.

Data from these surveys are published in preliminary form in the PSM. They are published in final form in the Petroleum Supply Annual (PSA), Volumes 1 and 2.

Summary information on the revision error between preliminary and final data is published once a year in the PSM feature article entitled, "Accuracy of Petroleum Supply Data." The last article was published in the December 1997 issue and evaluated the accuracy of the data for 1996 compared with previous years.

The Form EIA-819M, "Monthly Oxygenate Telephone Report," is used to collect preliminary data on production and stocks of oxygenates by PAD District. These data are used to monitor the supply of oxygenates. Data are collected from a sample of respondents reporting on the MPSRS surveys and from a sample of fuel ethanol producers. Data are published in Appendix D of the PSM and also in the WPSR.

The Form EIA-819A, "Annual Oxygenate Capacity Report," was used to collect data on current and projected production capacity of oxygenates and annual production and end-of-year inventories of fuel ethanol. This survey, which was last conducted for January 1, 1995 and published in the Petroleum Supply Annual 1994, has been eliminated.

The Form EIA-820, "Biennial Refinery Report," is used to collect data on refinery fuel use and consumption of steam and electricity, refinery receipts of crude oil by method of transportation, operable capacity for atmospheric crude oil distillation units and downstream units, as well as production capacity and storage capacity for petroleum products. In 1996, this survey was moved to a biennial schedule (every other year). The survey was last conducted in January 1997. This survey is described in more detail in Explanatory Note 3.

\section{Note 2. Monthly Petroleum Supply Reporting System}

The Monthly Petroleum Supply Reporting System (MPSRS) was implemented in January 1983 as the result of an extensive effort by the Energy Information Administration (EIA) to integrate the collection and processing of petroleum supply data that had been collected on other survey forms for many years. The collection of monthly petroleum supply statistics began as early as 1918 when the U.S. Bureau of Mines began collecting data on refinery operations and crude oil stocks and movements. The collection systems were further expanded in 1925 to include natural gas plant liquids production and storage, imports of crude oil and petroleum products and storage and movement of petroleum products in 1959, and tanker and barge movements of crude oil and petroleum products in 1964. Since their inception, each survey has undergone numerous changes, but the MPSRS was the first effort to make them all consistent and comparable. The forms that comprise the MPSRS are:

\begin{tabular}{|ll|}
\hline Form & \\
Number & Name \\
EIA-810 & "Monthly Refinery Report" \\
EIA-811 & "Monthly Bulk Terminal Report" \\
EIA-812 & "Monthly Product Pipeline Report" \\
EIA-813 & "Monthly Crude Oil Report" \\
EIA-814 & "Monthly Imports Report" \\
EIA-816 & "Monthly Natural Gas Liquids Report" \\
EIA-817 & "Monthly Tanker and Barge Movement \\
& Report" \\
EIA-819M & "Monthly Oxygenate Telephone Report" \\
\hline
\end{tabular}

\section{Respondent Frame}

Form EIA-810, "Monthly Refinery Report" - Operators of all operating and idle petroleum refineries and blending plants located in the 50 States, the District of Columbia, Puerto Rico, the Virgin Islands, Guam and other U.S. possessions. Approximately 260 respondents report on the Form EIA-810.

Form EIA-811, "Monthly Bulk Terminal Report" - Every bulk terminal operating company located in the 50 States, the District of Columbia, Puerto Rico, the Virgin Islands, and other U.S. possessions. A bulk terminal is primarily used for storage and/or marketing of petroleum products and has a total bulk storage capacity of 50,000 barrels or more, and/or receives petroleum products by tanker, 
barge, or pipeline. Bulk terminal facilities associated with a product pipeline are included. Approximately 320 respondents report on the Form EIA-811.

Form EIA-812, "Monthly Product Pipeline Report" - All product pipeline companies that carry petroleum products (including interstate, intrastate, and intracompany pipelines) in the 50 States and the District of Columbia. Approximately 80 respondents report on the Form EIA-812.

Form EIA-813, "Monthly Crude Oil Report" - All companies which carry or store 1,000 barrels or more of crude oil. Included in this survey are gathering and trunk pipeline companies (including interstate, intrastate, and intracompany pipelines), crude oil producers, terminal operators, storers of crude oil (except refineries), and companies transporting Alaskan crude oil by water in the 50 States and the District of Columbia. Approximately 175 respondents report on the Form EIA-813.

Form EIA-814, "Monthly Imports Report" - All companies, including subsidiary or affiliated companies, that import crude oil or petroleum products (1) into the 50 States and the District of Columbia, (2) into Puerto Rico, the Virgin Islands and other U.S. possessions (Guam, Midway Islands, Wake Island, American Samoa, and Northern Mariana Islands), and (3) from Puerto Rico, the Virgin Islands and other U.S. possessions into the 50 States and the District of Columbia. Imports into Foreign Trade Zones located in the 50 States and the District of Columbia are considered imports into the 50 States and the District of Columbia and must be reported. A report is required only if there has been an import during the month unless the importer has been selected as part of a sample to report every month regardless of activity. Approximately 220 respondents report on the Form EIA-814.

Form EIA-816, "Monthly Natural Gas Liquids Report" -Operators of all facilities that extract liquid hydrocarbons from a natural gas stream (natural gas processing plant) and/or separate a liquid hydrocarbon stream into its component products (fractionator). Approximately 585 respondents report on the Form EIA-816.

Form EIA-817, "Monthly Tanker and Barge Movement Report" - All companies that have custody of crude oil or petroleum products transported by tanker or barge between Petroleum Administration for Defense (PAD) Districts or between the Panama Canal and the United States. For purposes of this report, custody is defined as physical possession of crude oil or petroleum products on a company-owned tanker or barge. Also, companies which lease vessels or contract for the movement of crude oil or petroleum products on a tanker or barge between PAD Districts or between the Panama Canal and the United States are considered to have custody. Approximately 40 respondents report on the Form EIA-817.

Form EIA-819M, "Monthly Oxygenate Telephone Report" - The sample of companies that report on the EIA$819 \mathrm{M}$ are selected from the universe of companies that report on the MPSRS surveys and from the universe of fuel ethanol producers who reported on the Form EIA819A, "Annual Oxygenate Capacity Report", in 1995. The universe consists of (1) operators of facilities that produce (manufacture or distill) oxygenates (including MTBE plants, petrochemical plants, and refineries that produce oxygenates as part of their operations); (2) operators of petroleum refineries; (3) operators of bulk terminals, bulk stations, blending plants, and other nonrefinery facilities that store and/or blend oxygenates; and (4) importers of oxygenates (importer of record) located in or importing oxygenates into the 50 States and the District of Columbia. Approximately 85 respondents report on the Form EIA-819M.

\section{Sampling}

The sampling procedure used for the survey Form EIA$819 \mathrm{M}$ is the cut-off method and is performed using software developed for EIA's Survey Methods Group. In the cut-off method, companies are ranked from largest to smallest on the basis of quantities reported (oxygenate production, oxygenate stocks, and oxygenate imports) during the previous year. Companies are chosen for the sample beginning with the largest and adding companies until the sample covers approximately 90 percent of the total for each oxygenate product and supply type by geographic region (PAD Districts I through V).

\section{Description of Survey Forms}

The Form EIA-810, "Monthly Refinery Report," is used to collect data on refinery input and capacity, sulfur content and API gravity of crude oil, and data on supply (beginning stocks, receipts, and production) and disposition (inputs, shipments, fuel use and losses, and ending stocks) of crude oil and refined products.

The Form EIA-811, "Monthly Bulk Terminal Report," is used to collect data on end-of-month stock levels of finished petroleum products by State in the custody of the bulk terminal company regardless of ownership. Leased tankage at other facilities is excluded. All domestic and foreign stocks held at bulk terminals and in-transit thereto, except those in-transit by pipeline are included. Petroleum products in-transit by pipeline are reported by pipeline operators on Form EIA-812, "Monthly Product Pipeline Report." 
The Form EIA-812, "Monthly Product Pipeline Report," is used to collect data on end-of-month stock levels and movements of petroleum products transported by pipeline. Intermediate movements for pipeline systems operating in more than two PAD Districts are included.

The Form EIA-813, "Monthly Crude Oil Report," is used to collect data on end-of-month stocks of crude oil held at pipeline and tank farms (associated with the pipelines) and terminals operated by the reporting company. Also, crude oil consumed by pipelines and on leases as pump fuel, boiler fuel, etc., is reported. Data are reported on a PAD District basis.

Total Alaskan crude oil stocks in-transit by water (including stocks held at transshipment terminals between Alaska and the continental United States) to the 50 States, the District of Columbia, Puerto Rico, and the Virgin Islands are also reported by the transporting company having custody of the stocks.

Inter-PAD District movements of crude oil by pipeline are collected by the shipping and receiving PAD District. Intermediate movements for pipeline systems operating in more than two PAD Districts are not included.

The Form EIA-814, "Monthly Imports Report," is used to collect data on imports of crude oil and petroleum products (1) into the 50 States and the District of Columbia, (2) into Puerto Rico, the Virgin Islands, and other U.S. possessions (Guam, Midway Islands, Wake Island, American Samoa, and Northern Mariana Islands), and (3) from Puerto Rico, the Virgin Islands, and other U.S. possessions into the 50 States and the District of Columbia. Imports into Foreign Trade Zones located in the 50 States and the District of Columbia are considered imports into the 50 States and the District of Columbia.

The type of commodity, port of entry, country of origin, quantity (thousand barrels), sulfur percent by weight, API gravity, and name and location of the processing or storage facility are reported. Sulfur percent by weight is requested for crude oil, crude oil burned as fuel, and residual fuel oil only. API gravity is requested for crude oil only. The name and location of the processing or storage facility is requested for crude oil, unfinished oils, other hydrocarbons/hydrogen/oxygenates, and blending components only.

The Form EIA-816, "Monthly Natural Gas Liquids Report," is used to collect data on the operations of natural gas processing plants and fractionators. Beginning and end-of-month stocks, receipts, inputs, production, shipments, and plant fuel use and losses during the month are collected from operators of natural gas processing plants. End-of-month stocks are collected from fractionators.
The Form EIA-817, "Monthly Tanker and Barge Movement Report," is used to collect data on the movements of crude oil and petroleum products between PAD Districts. Data are reported by shipping and receiving PAD District and sub-PAD District. Shipments to and from the Panama Canal are also included if the shipment was delivered to the Canal.

The Form EIA-819M, "Monthly Oxygenate Telephone Report," is used to collect data on production, stocks, and imports of oxygenates. Data on end-of-month stocks are reported on a custody basis regardless of ownership. Data are reported on a PAD District basis.

\section{Collection Methods}

Except for the EIA- 819M, survey forms for the MPSRS can be submitted by mail, facsimile, or electronic transmission. Completed forms are required to be postmarked by the 20th calendar day following the end of the report month. Data collection for the EIA-819M begins on the seventh working day of each month. Data are solicited by telephone or transmitted to the EIA by facsimile. Receipt of the reports are monitored using an automated respondent mailing list. Telephone follow-up calls are made to nonrespondents prior to the publication deadline.

\section{Response Rate}

The response rate is generally 98 to 100 percent. Chronic nonrespondents and late filing respondents are contacted in writing and reminded of their requirement to report. Companies that file late or fail to file are subject to criminal fines, civil penalties, and other sanctions as provided by Section 13(i) of the Federal Energy Administration (FEA) Act.

\section{Data Imputation}

Imputation is performed for companies that fail to file Forms EIA-810 through 813, 816, and 819M. For such companies, previous monthly values are used for current values. On the EIA-819M, data are aggregated for each geographic region. Estimation factors, which are derived from the previous year's data, are then applied to each cell to generate published estimates. Data for nonrespondents on the Forms EIA-814 and 817 are not imputed because these data series, by respondent, are highly variable.

\section{Confidentiality}

The Office of Legal Counsel of the Department of Justice concluded on March 20, 1991, that the Federal Energy Administration Act requires the Energy Information Administration to provide company-specific data to the Department of Justice, or to any Federal agency when 
requested for official use, which may include enforcement of Federal law. The information contained on this form may also be made available, upon request, to another component of the Department of Energy (DOE), to any Committee of Congress, the General Accounting Office, or other Congressional agencies authorized by law to receive such information. A court of competent jurisdiction may obtain this information in response to an order.

The information contained on Forms EIA-810 through $813,816,817$, and $819 \mathrm{M}$ are kept confidential and not disclosed to the public to the extent that they satisfy the criteria for exemption under the Freedom of Information Act (FOIA), 5 U.S.C. 552, the Department of Energy (DOE) regulations, 10 C.F.R. 1004.11, implementing the FOIA, and the Trade Secrets Act, 18 U.S.C. 1905. The information contained on Form EIA-814 are not considered confidential and historically has not been treated as such.

Upon receipt of a request for this information under the FOIA, the DOE shall make a final determination whether the information is exempt from disclosure in accordance with the procedures and criteria provided in the regulations. To assist us in this determination, respondents should demonstrate to the DOE that, for example, their information contains trade secrets or commercial or financial information whose release would be likely to cause substantial harm to their company's competitive position. A letter accompanying the submission that explains (on an element-by-element basis) the reasons why the information would be likely to cause the respondent substantial competitive harm if released to the public would aid in this determination. A new justification does not need to be provided each time information is submitted on the form, if the company has previously submitted a justification for that information and the justification has not changed. Company specific data are also provided to other DOE offices for the purpose of examining operations in the context of emergency response planning and actual emergencies.

The data collected on Forms EIA-810 through 814,816 , and 817 appear in EIA publications such as Petroleum Supply Monthly (PSM), Monthly Energy Review, Petroleum Supply Annual (PSA), and the Annual Energy Review.

Data on the breakdown between liquefied refinery gases and olefins and lubricants are suppressed on Table 16, "Refinery Net Production of Finished Petroleum Products by PAD and Refining Districts" to avoid disclosure of company identifiable data.

Statistics representing data aggregated from less than three companies or aggregated data representing 60 per- cent or more of a single company's data are suppressed on the PSA tables listed below. In addition, complementary suppression is performed to avoid any residual disclosure.

- Table 16, "Refinery Input of Crude Oil and Petroleum Products by PAD and Refining Districts," (inputs of oxygenates)

- Table 18, "Refinery Stocks of Crude Oil and Petroleum Products by PAD and Refining Districts," (stocks of oxygenates)

- Table 30, "Stocks of Crude Oil and Petroleum Products by PAD District," (stocks of oxygenates)

- Table 31, "Refinery, Bulk Terminal, and Natural Gas Plant Stocks of Selected Petroleum Products," (all products)

With the exception of the tables listed above, the tables in the PSA are not subject to statistical nondisclosure procedures. Thus, there may be some table cells which are based on data from only one or two respondents, or which are dominated by data from one or two large respondents. In these cases, it may be possible for a knowledgeable user of the data to make inferences about the data reported by a specific respondent.

\section{Note 3. Form EIA-820: Biennial Refinery Report}

Refinery capacity data collection was begun in 1918 by the Bureau of Mines, then in the Department of Commerce, and was operated on a voluntary basis until 1980 . In 1980, the mandatory Energy Information Administration (EIA) Form EIA-177, Capacity of Petroleum Refineries, was implemented. Information on refining capacity was expanded to include not only current year operations, two-year projections, and refinery input/production data. Working storage capacity data was also added to the form and product categories were added for total coverage. Information on refinery downstream facilities was expanded to include a breakdown of thermal operations and to add vacuum distillation, catalytic hydrorefining and hydrotreating. Production capacity was also added to include information on isomerization, alkylation, aromatics, asphalt/road oil, coking, lubricants and hydrogen.

In 1983, the form was revised to improve the consistency and quality of the data collected by the EIA and redesignated as Form EIA-820, "Annual Refinery Report." Two sections for data previously reported monthly were added: (1) refinery receipts of crude oil by method of transportation, and (2) fuels consumed for all purposes at refineries. Also, the second year projections on refining capacity were eliminated. As a result of a study conducted by the EIA evaluating motor gasoline data collected by the Federal Highway Administration (FHWA) and by the EIA, motor gasoline blending plants were included for the first 
time to the respondent frame in order to produce more accurate statistics on the production of motor gasoline.

In 1987, the form was revised to reduce respondent burden and to better reflect current refinery operations through updated terminology. Information on projected input/production of refinery processing facilities was deleted. Several categories under catalytic hydrotreating were combined: naphtha and reformer feeds were combined into a single category as well as residual fuel oil and other. Thermal cracking types, gas oil and "other" were also combined into a single category. Catalytic reforming types, conventional and bi-metallic were replaced with low and high pressure processing units. Two new categories were added: fuels solvent deasphalting was added to downstream charge capacity and sulfur recovery was added to production capacity.

In 1994, the form was revised to enable EIA to calculate utilization rates for certain downstream processing units and to reflect storage capacity of fuels mandated by the Clean Air Act Amendments of 1990. Additions to the form included calendar day downstream charge capacity for fluid and delayed coking, catalytic cracking, and catalytic hydrocracking. Also storage capacity categories for reformulated, oxygenated, and other finished motor gasoline were added, as well as oxygenate storage capacity and separate categories for high and low sulfur distillate fuel oil.

In 1995, motor gasoline blending plants were dropped from the survey frame, since by this time, the only section of the form that applied to them was working and shell storage capacity. Also in 1995, a decision was made to no longer collect storage capacity from shutdown refineries; therefore, these refineries were also eliminated from the survey frame.

In 1996, the survey was moved to a biennial schedule (every other year) and was renamed "Biennial Refinery Report". The survey was not conducted for January 1, 1998 .

In 1997, respondents were not required to submit data for crude oil and petroleum products consumed at refineries during 1996. These data are available from the Form EIA-810, "Monthly Refinery Report." The requirement to submit data for refinery consumption of natural gas, coal, and purchased steam and electricity on the Form EIA-820 remains.

\section{Respondent Frame}

The respondent frame consists of all operating and idle petroleum refineries (including new refineries under con-

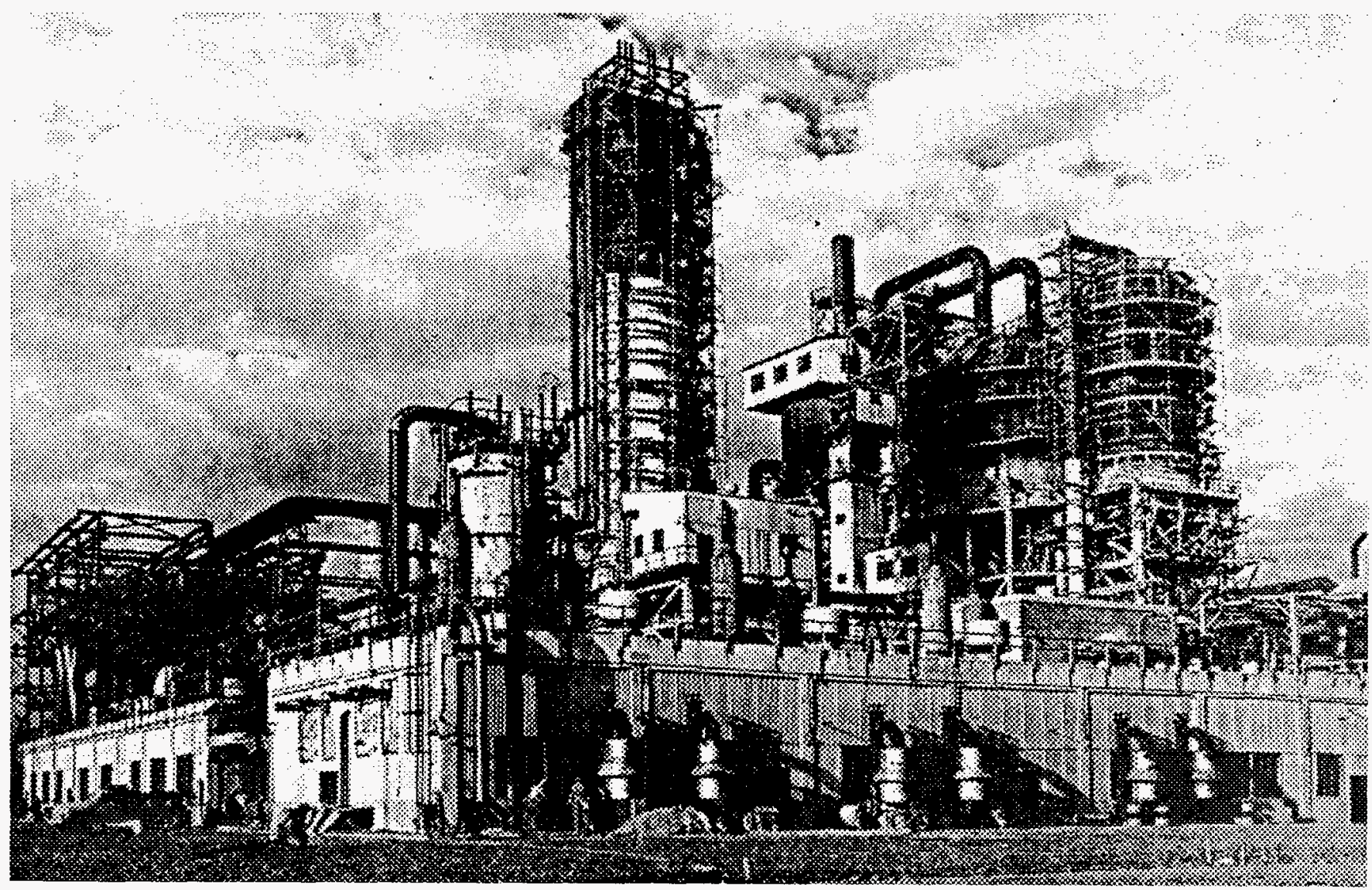

Refinery cat-cracker. 
struction), located in the 50 States, the District of Columbia, Puerto Rico, the Virgin Islands, Guam and other U.S. possessions. As of January 1, 1998, there were 162 refineries and 51 motor gasoline blending plants in the 50 States. A list of motor gasoline blending plants operating during 1997 is provided in Explanatory Note 23.

The respondent frame is maintained by monitoring the monthly Form EIA-810, "Monthly Refinery Report," and industry publications for changes and developments in the petroleum industry such as refinery sales, mergers and new operations.

\section{Description of Survey Form}

The Form EIA-820 is used to collect data on fuels consumed for all purposes at the refinery during the preceding year; refinery receipts of crude oil by method of transportation during the preceding year; current and next year projections for operable atmospheric crude oil distillation capacity, downstream charge capacity and production capacity; and current year working and shell storage capacity for crude oil and petroleum products at the refinery.

\section{Collection Methods}

The Form EIA-820 is sent to respondents in December. Survey forms can be submitted by mail or facsimile. Completed forms are required to be postmarked by the 15 th day of February of the current report year. Receipt of the reports is monitored using an automated respondent mailing list. Telephone follow-up calls are made to secure responses from those companies failing to report by February 15 th.

\section{Response Rate}

The response rate for the Form EIA-820 is normally very high. Data are estimated and non-compliance procedures are implemented for those companies still not reporting data by close-out for the report year. For the January 1, 1997 survey, there was one nonrespondent.

\section{Data Imputation}

Imputation is performed for companies that fail to file prior to the publication deadline. For the January 1, 1997 survey, there was one nonrespondent, and their total operable capacity is estimated to be about 0.1 percent of the U.S. total. When nonresponse occurs, values for these companies are imputed from data reported on the most recent year's Form EIA-820 and/or from data reported on Form EIA-810, "Monthly Refinery Report," for that company. For most surveyed items, the value imputed for nonrespondents is the value that company reported on the Form EIA-820 for the most recent year. For three catego- ries of information however, the imputed value is also based on their data from the Form EIA-810 as follows:

\section{Section 1: Fuel, Electricity, and Steam Consumed for all Purposes at Refineries}

Data for crude oil, distillate and residual fuel oil, liquefied petroleum gases, still gas, and marketable and catalyst petroleum coke are based upon data reported on the monthly Form EIA-810.

Estimates for natural gas, coal, electricity and steam are taken directly from data reported on the previous year's annual Form EIA-820.

\section{Section 2: Refinery Receipts of Crude Oil by Method of Transportation}

The imputation methodology for this section is based on data reported on both the monthly Form EIA-810 and the annual Form EIA-820. Annual refinery receipts of domestic and foreign crude oil for a nonrespondent are imputed by aggregating the values for the refinery on the monthly survey. These values are allocated to the method of transportation by using the percentages reported for the refinery in the previous year. The difference between the values reported on the two surveys by all respondents in 1997 is about 2.0 percent.

\section{Section 3: Operable and Storage Capacity as of January 1}

Operable atmospheric crude oil distillation capacity in barrels per calendar day is collected on the monthly Form EIA-810 as of the first day of each month and on the annual Form EIA-820 as of January 1. As part of the editing process for the Form EIA-820, these two values are compared. Companies are contacted and any discrepancies are resolved by the time of publication. Imputed values for operable atmospheric crude oil distillation capacity in barrels per calendar day are taken directly from the January Form EIA-810. A barrels per stream day capacity is then derived by dividing the reported barrels per calendar day capacity by .95 .

Current year and projected year data for downstream charge capacity, production capacity, and data for working and shell storage capacity are taken directly from the previous year's annual report.

\section{Confidentiality}

The Office of Legal Counsel of the Department of Justice concluded on March 20, 1991, that the Federal Energy Administration Act requires the Energy Information Administration to provide company-specific data to the Department of Justice, or to any other Federal agency when 
requested for official use, which may include enforcement of Federal law. The information contained on this form may also be made available, upon request, to another component of the Department of Energy (DOE), to any Committee of Congress, the General Accounting Office, or other Congressional agencies authorized by law to receive such information. A court of competent jurisdiction may obtain this information in response to an order.

Information on operable atmospheric crude oil distillation capacity, downstream charge capacity, and production capacity on Form EIA-820 are not considered as confidential, and historically have not been treated as such. Company identifiable data are published in the Petroleum Supply Annual (PSA) 1996, Volume 1, Tables 38, 39, and 40.

Other data on the Form EIA-820 are kept confidential and not disclosed to the public to the extent that it satisfies the criteria for exemption under the Freedom of Information Act (FOIA), 5 U.S.C.552, Department of Energy (DOE) regulations, 10 C.F.R.1004.11, implementing the FOIA, and the Trade Secrets Act, 18 U.S.C.1905.

Upon receipt of a request for this information under the FOIA, the DOE shall make a final determination whether the information is exempt from disclosure in accordance with the procedures and criteria provided in the regulations. To assist us in this determination, respondents should demonstrate to the DOE that, for example, their information contains trade secrets or commercial or financial information whose release would be likely to cause substantial harm to their company's competitive position. A letter accompanying the submission that explains (on an element-by-element basis) the reasons why the information would be likely to cause the respondent substantial competitive harm if released to the public would aid in this determination. A new justification does not need to be provided each time information is submitted on the form, if the company has previously submitted a justification for that information and the justification has not changed.

The data collected on Form EIA-820, "Biennial Refinery Report," is used to report aggregate statistics on and conduct analyses of the operation of U.S. petroleum refineries. The data appear in EIA publications such as PSA, and the Annual Energy Review. Company specific data are also provided to other DOE offices for the purpose of examining specific refinery operations in the context of emergency response planning and actual emergencies.

The tables pertaining to refinery receipts of crude oil by method of transportation and fuels consumed at the refinery published in the PSA are not subject to statistical nondisclosure procedures. Thus, there may be some table cells which are based on data from only one or two respon- dents, or which are dominated by data from one or two large respondents. In these cases, it may be possible for a knowledgeable user of the data to make inferences about the data reported by a specific respondent.

\section{Quality Control}

There are two types of errors usually associated with data produced from a survey - sampling errors and nonsampling errors. Because estimates from the Form EIA-820 survey are based on a complete census of the frame of petroleum refineries, there is no sampling error in the data presented in this report. The data, however, are subject to nonsampling errors. Nonsampling errors are those which can arise from: (1) the inability to obtain data from all companies in the frame or sample (nonresponse) and the method used to account for nonresponses; (2) definitional difficulties and/or improperly worded questions which lead to different interpretations; (3) mistakes in recording or coding the data obtained from respondents; and (4) other errors of collection, response, coverage, and estimation. Quality control procedures are employed in the collection and editing operations to minimize misrepresentation and misreporting. Nonresponse follow-up procedures are employed to reduce the number of nonrespondents, and procedures employed to impute missing data, introduce a minimal amount of error, given the relatively small volume of imputed data.

\section{Resubmissions}

Resubmissions are required whenever an error greater than 5 percent of the true value is discovered. In the event of a reporting error, company reports are updated after contact with the company and are followed up by corrected report resubmissions. Late submissions or resubmissions received after the publication date are entered into a "working" file. This file contains the most up-todate data for the Form EIA-820 and is used to edit next year's data.

\section{Note 4. Technical Notes for Detailed Statistics Tables}

The detailed statistics tables in the Petroleum Supply Annual provide complete supply and demand information for the previous year. The tables are organized to locate $\mathrm{Na}$ tional and Petroleum Administration for Defense (PAD) District summary data at the front followed by tables on crude oil and petroleum product production, import/export data, stocks information, and lastly, data on crude oil and petroleum product movements. To assist in the interpretation of these tables, the following technical notes are provided. Column and row headings are defined in the Glossary. 


\section{Supply}

Field Production - Field production is the sum of crude oil production, natural gas plant liquids production, other liquids production, and finished petroleum products production.

Crude oil production is an estimate based on data received from State conservation agencies and the Mineral Management Service of the U.S. Department of the Interior. Refer to Explanatory Note 5 for further details.

Field production of natural gas plant liquids is reported on Form EIA-816 and published on a net basis (i.e., production minus inputs) in this column.

Other liquids field production is calculated by forcing the product supplied to be zero: thereby backing into field production.

Field production of finished petroleum products is calculated by (1) adding the amount of fuel ethanol that has been blended into finished motor gasoline, and (2) plus $(+)$ or minus (-) the field production of motor gasoline blending components. Refer to Explanatory Note 10 for a further discussion of this calculation.

Negative field production of motor gasoline blending components represents an understatement for finished motor gasoline.

Negative field production of other finished motor gasoline represents an overstatement of other finished motor gasoline and an understatement of oxygenated motor gasoline.

Refinery Production - Published production of these products equal refinery production minus refinery input. Refinery production of other hydrocarbons, hydrogen and alcohol, unfinished oils, and motor and aviation gasoline blending components appear on a net basis under refinery input. Negative refinery production will occur when the amount of a product produced during the month is less than the amount of that same product that is reprocessed (input) or reclassified to become another product during the same month.

Unaccounted for Crude Oil - This column is a balancing item for crude oil. This data element represents the difference between crude oil supply and disposition. Crude oil supply is the sum of field production and imports. Crude oil disposition is the sum of stock change, losses, refinery inputs, exports, and products supplied. A positive result indicates that refiners and exporters reported use of more crude oil than was reported to have been available to them. (This occurs, for example, when imports are undercounted due to late reporting or other problems.) A negative result indicates that more crude oil was reported to have been supplied to refiners and exporters than they reported to have used.

\section{Disposition}

Stock Change - This column is calculated as the difference between the Ending Stocks column of this table and the Ending Stocks column of the prior year's publication. A negative number indicates a decrease in stocks and a positive number indicates an increase in stocks.

Crude Losses - The volume of crude oil reported by petroleum refineries as being lost in their operations. These losses are due to spills, contamination, fires, etc., as opposed to refining processing losses or gains.

Refinery Inputs - Refinery inputs of crude oil and intermediate materials (unfinished oils, gasoline blending components, other hydrocarbons and oxygenates, liquefied petroleum gases, and pentanes plus) that are processed at refineries to produce finished petroleum products.

Crude oil inputs represents total crude oil (domestic and foreign) input to atmospheric crude oil distillation units and other refinery processing units (i.e., catalytic cracking units, cokers).

Inputs of natural gas liquids are natural gas liquids received from natural gas plants for blending and processing. Published inputs of natural gas liquids are reported on a gross basis.

Inputs of unfinished oils, motor and aviation gasoline blending components, and other hydrocarbons and oxygenates are published on a net basis (i.e., refinery input minus refinery production).

Inputs of finished petroleum products are published on a net basis (i.e., refinery production minus refinery inputs) and displayed under the refinery production column.

Exports - Exports include crude oil shipments from the 50 States to Puerto Rico, and the Virgin Islands.

Products Supplied - Products supplied is equal to field production, plus refinery production, plus imports, plus unaccounted for crude oil, (plus net receipts on a PAD District basis), minus stock change, minus crude losses, minus refinery inputs, minus exports.

Products supplied indicates those quantities of petroleum products supplied for domestic consumption. Occasionally, the result for a product is negative because total disposition of the product exceeds total supply. Negative products supplied may occur for a number of reasons: (1) product reclassification has not been reported; (2) data 
were misreported or reported late; (3) in the case of calculations on a PAD District basis, the figure for net receipts was inaccurate because the coverage of interdistrict movements was incomplete; and (4) products such as gasoline blending components and unfinished oils have entered the primary supply channels with their production not having been reported, e.g., streams returned to refineries from petrochemical plants.

Product supplied for crude oil is the sum of crude oil burned on leases and by pipelines as fuel. Prior to January 1983, crude oil burned on leases and by pipelines as fuel were reported as either distillate or residual fuel oil and were included in product supplied for these products.

\section{Yields}

The refinery yield of finished motor gasoline is calculated by subtracting the inputs of pentanes plus, liquefied petroleum gases, other hydrocarbons/alcohol and motor gasoline blending components from the production of finished motor gasoline before dividing by the sum of crude oil input and unfinished oils input (net).

The refinery yield of finished aviation gasoline is calculated by subtracting the inputs of aviation gasoline blending components from the production of finished aviation gasoline before dividing by the sum of crude oil input and unfinished oils input (net).

Refinery yields for all products (except finished motor gasoline and finished aviation gasoline) are calculated by dividing the production for each product by the sum of crude oil input and unfinished oils input (net) reported in the U.S. total.

\section{Stocks}

Primary stocks of petroleum products do not include either secondary stocks held by dealers and jobbers or tertiary stocks held by consumers.

\section{Movements}

Movements of crude oil by pipeline between PAD Districts include trunk pipeline companies (interstate, intrastate, and intracompany pipelines). Intermediate movements for crude oil pipeline systems operating in more than two PAD Districts are not included.

Movements of petroleum products by pipeline between PAD Districts include trunk pipeline companies (interstate, intrastate and intracompany pipelines). Intermediate movements for product pipeline systems operating in more than two PAD Districts are included. For example, a shipment originating in PAD District 3, passing through PAD District 2 to PAD District 1 , is reported as a move- ment from PAD District 3 to PAD District 2 and also from PAD District 2 to PAD District 1.

Waterborne movements of crude oil and petroleum products between PAD Districts include all shipments of crude oil or petroleum products for which the transporter has custody at the time of shipment. Custody is defined as physical possession of crude oil or petroleum products on a company-owned tanker and barge.

\section{Note 5. Domestic Crude Oil Production}

The Energy Information Administration (EIA) collects monthly crude oil production data on an ongoing basis. Data on crude oil production for States are reported to the EIA by State government agencies. Data on crude oil production for Federal offshore areas are reported to the EIA by the Minerals Management Service of the U.S. Department of the Interior. Currently, all except two crude oil producing States (New York and Ohio) report production on a monthly basis. These two States report crude oil production on an annual basis. Estimates of monthly crude oil production for these two States are made by the EIA using data reported on Form EIA-182, "Domestic Crude Oil First Purchase Report."

After the end of each calendar year, the monthly crude oil production estimates are updated using annual reports from various State agencies, the Minerals Management Service and the Conservation Committee of California Oil and Gas Producers. The EIA incorporates production data into its Crude Oil Production System (COPS) as the data are received from the reporting agencies. EIA publications show portions of this database at specific points in time. Table 14 of this publication presents the 1997 crude oil production data received by the EIA as of April 1998. Crude oil production data for 1997 received after April 1998 will be published later as an appendix in the following year's Petroleum Supply Annual (PSA). Table Cl of this publication presents the 1996 crude oil production a year after it was published in the PSA 1996.

\section{Note 6. Export Data}

Each month the Energy Information Administration (EIA) receives magnetic tapes of aggregated export statistics from the U.S. Bureau of the Census (EM-522 and EM594).

Census export statistics used in the Petroleum Supply Annual reflect both government and nongovernment exports of domestic and foreign merchandise from the United States (the 50 States and the District of Columbia) to foreign countries and U.S. possessions, without regard to whether or not the exportation involves a commercial 
transaction. The following types of transactions are excluded from the statistics:

(1) Merchandise shipped in transit through the United States from one foreign country to another, when documented as such with U.S. Customs.

(2) Bunker fuels and other supplies and equipment for use on departing vessels, planes, or other carriers engaged in foreign trade.

\section{Source of Export Information}

The official U.S. export statistics are compiled by the U.S. Bureau of the Census. Exporters are required to file export documents with U.S. Customs officials (Customs Form 7525).

\section{Country and Area of Destination}

The country of destination is defined as the country of ultimate destination or the country where the goods are to be consumed, further processed, or manufactured, as known to the shipper at the time of exportation. If the shipper does not know the country of ultimate destination, the shipment is credited to the last country to which the shipper knows that the merchandise will be shipped in the same form as it was when exported.

\section{Note 7. Quality Control and Data Revision}

\section{Quality Control}

The Energy Information Administration (EIA) monitors the supply and disposition of crude oil, petroleum products, and natural gas liquids in the United States. Through a tracking system, the EIA provides insight into the activities of primary operators and distributors in the petroleum industry. The tracking system, known as the Petroleum Supply Reporting System (PSRS), consists of production, inputs, imports, inventories, movements, and other petroleum-related data collected on weekly, monthly, and annual surveys.

Survey forms are periodically reviewed for completeness, meaningfulness, and clarity. Modifications are made, when needed, to maintain efficient measure of the intended data items and to track product movement accurately throughout the industry. Through this process, the EIA can maintain consistency among forms, minimize respondent burden, and eliminate ambiguity.

\section{Sampling and Nonsampling Errors}

There are two types of errors usually associated with data produced from a survey — nonsampling errors and sampling errors. Because the estimates for the monthly surveys 810 through 813,816 , and 817 are based on a complete census of the frame, there is no sampling error in the data presented. The data, however, are subject to nonsampling errors. Nonsampling errors, sometimes referred to as biases, are those which can arise from a number of sources: (1) the inability to obtain data from all companies in the frame or sample (nonresponse and the method used to account for nonresponses, (2) definitional difficulties and/or improperly worded questions which lead to different interpretations. (3) mistakes in recording or coding the data obtained from respondents, and (4) other errors of collection, response, coverage, and estimation.

Response rates on the monthly surveys are very high. In general, response rates average above 95 percent for the weekly survey and above 98 percent for monthly surveys. Whenever survey responses are not received in time to be included in published statistics, the data are imputed. Although imputing for missing data may not eliminate the total error associated with nonresponse, it can serve to reduce the error. The data reported in the previous month are used as imputed values for missing data for all surveys except the Forms EIA-814, "Monthly Imports Report," and EIA-817, "Monthly Tanker and Barge Movement Report." There is no imputation procedure for these surveys because these data series, by respondent, are highly variable.

Response error is the major factor affecting the accuracy of PSRS data. Response, or reporting error, is the difference between the true value and the value reported on a survey form. Response error can occur for any number of reasons. For example, figures may be entered incorrectly when written on forms by the respondent, or errors may result from the misunderstanding of survey form instructions or definitions. Response error can also occur from the use of preliminary data when final data are not available. This can result in differences between published preliminary and final data. To help detect and minimize probable reporting errors, automated editing procedures are used to check current data for consistency with past data, as well as for internal consistency (e.g., totals equal to the sums of the parts), and to flag those data elements that fail edit criteria.

Errors can also be introduced during data processing. For example, while creating computer data files, key errors can occur in transcribing or coding the data; or information can be entered into the wrong cell. Using well designed edit criteria which examine orders of magnitude, 
cell position, and historical reporting patterns, many of these errors can be identified and corrected.

Monthly data are compared to weekly data on a regular basis. Discrepancies between weekly and monthly data are documented and respondents are called when discrepancies are either large (usually over 300 thousand barrels) or consistent (e.g., weekly data are always lower than monthly data). In addition, a comparison of the data collected on the PSRS with other similar data series from sources outside of the Petroleum Supply Division is performed each year. The results of this data comparison are published once a year in the Petroleum Supply Monthly (PSM) feature article, "Comparisons of Independent Petroleum Supply Statistics."

Sampling errors are those errors that occur when survey estimates are based on a sample rather than being derived from a complete census of the frame. The $819 \mathrm{M}$ data, which are based on sample estimates, serve as leading indicators of the PSRS monthly data for oxygenates. To assess the accuracy of the $819 \mathrm{M}$ statistics, data are compared with the monthly aggregate data for the EIA-810, 811 , and 812 surveys. Although monthly data are still subject to error, they have been thoroughly reviewed and edited, and are considered to be the most accurate data available.

\section{Data Revision}

Resubmissions are any changes to the originally submitted data that were either requested by the EIA or initiated by the respondent. Resubmissions are compared with the original submission and processed at the time of receipt. For Forms EIA-810 through 813, 816, and 817 the Resubmission Tracking System (RTS) is run after resubmissions have been processed for the month. The RTS enables the user to study major products and data series to see how company resubmissions impact published data on a month by month basis. During the processing year, a summary of the effect of these resubmissions to major series is provided in Appendix C of the PSM.

For the EIA-819M data, a determination is made on whether to process the resubmissions based on the magnitude of the revision. Cell entries on publication tables are marked with an " $R$ " for revised.

\section{Late Response}

Respondents who fail to respond within the prescribed time limit (25th day following the end of the report month) become nonrespondents for that particular report period and are contacted by phone to obtain the current month's data. Respondents who are chronically late (i.e., 3 con- secutive months) are notified by EIA either by letter or telephone.

\section{Nonresponse}

Follow-up action is taken when a company fails to respond adequately to data requests from the EIA. Preliminary attempts to gather delinquent reports are made by phone. Noncompliance form letters are sent to those companies that have not submitted reports and have not responded to data requests by phone.

\section{Note 8. Frames Maintenance}

The Petroleum Supply Division (PSD) maintains complete lists of respondents to its monthly surveys. Each survey has a list of companies and facilities required to submit petroleum activity data. This list is known as the survey frame. Frame maintenance procedures are used to monitor the status of petroleum companies and facilities currently contained in each survey frame as well as to identify new members to be added to the frame. As a result, all known petroleum supply organizations falling within the definition of "Who Must Submit" participate in the survey.

The activities for frames maintenance are conducted on a monthly and annual basis. Monthly frames maintenance procedures focus on examining several frequently published industry periodicals that report changes in status (births, deaths, sales, and acquisitions) of petroleum facilities producing, transporting, importing, and/or storing crude oil and petroleum products. These sources are augmented by articles in newspapers, letters from respondents indicating changes in status, and information received from survey systems operated by other offices. Survey managers review these sources to monitor changes in company operations and to develop lists of potential respondents. These activities assure coverage of the reporting universe and maintain accurate facility information on addresses and ownership.

Annual frames maintenance focuses on re-evaluating the "must submit" companies filing the Form EIA-814 and reviewing the sample frame for the Form EIA-819M, "Monthly Oxygenate Telephone Report."

To supplement the monthly and annual frames maintenance activities and to provide more comprehensive coverage, the PSD periodically conducts a comprehensive frames investigation. These investigations result in the reassessment and recompilation of the complete frame for each survey. The effort also includes the evaluation of the impact of potential frame changes on the historical time series data published from these respondents. The results 
of this frame study are usually implemented in January to provide a full year under the same frame.

\section{Changes in Survey Frames}

Beginning in January 1981, the Energy Information Administration (EIA) expanded its universe to include nonrefinery blenders; redefined motor gasoline into two categories (finished leaded and finished unleaded); and separated blending components from finished motor gasoline as a reporting category. Refer to Explanatory Note 11 for further discussion.

In January 1981, 1983, and 1984 numerous respondents were added to bulk terminal and pipeline surveys affecting subsequent stocks reported and stock change calculations. Table B1 displays the end-of-year stocks, in million barrels using the expanded coverage (new basis).

Beginning in January 1986, as a result of frames maintenance activities, 39 respondents were added to the monthly survey frames: 2 motor gasoline blenders, 30 bulk terminal operators, 3 pipeline operators, 3 crude oil stock holders, and 1 tanker and barge operator. Table B2 shows the impact of the data reported by the new respondents on published data for production and stocks of major petroleum products.

Also, beginning in January 1986, a major petroleum company consolidated production and stocks reporting for some of its facilities. Data previously reported separately on Form EIA-811, "Monthly Bulk Terminal Report," and on Form EIA-816, "Monthly Natural Gas Liquids Report" for two facilities were combined with data reported for two refineries on Form EIA-810, "Monthly Refinery Re-
Table B1. New Basis Stocks
${ }^{1}$
(Million Barrels)

\begin{tabular}{|c|c|c|c|}
\hline Commodity & 1980 & 1982 & 1983 \\
\hline \multicolumn{4}{|l|}{ Crude Oil } \\
\hline $\begin{array}{l}\text { Total } \\
\text { Other Primary . . . . }\end{array}$ & $\begin{array}{l}488 \\
380\end{array}$ & $\begin{array}{l}645 \\
351\end{array}$ & $\begin{array}{l}723 \\
379\end{array}$ \\
\hline $\begin{array}{l}\text { Crude Oil and } \\
\text { Petroleum Products }\end{array}$ & 1,425 & 1,461 & 1,454 \\
\hline Motor Gasoline & & & \\
\hline $\begin{array}{l}\text { Total } \\
\text { Finished } \ldots\end{array}$ & $\begin{array}{l}263 \\
214\end{array}$ & $\begin{array}{l}244 \\
202\end{array}$ & $\begin{array}{l}222 \\
186\end{array}$ \\
\hline Distillate Fuel Oil . . . . & 205 & 186 & 140 \\
\hline $\begin{array}{l}\text { Residual Fuel Oil . . . } \\
\text { Jet Fuel }\end{array}$ & 91 & 69 & 49 \\
\hline $\begin{array}{l}\text { Total . . . . } \\
\text { Kerosene-type }\end{array}$ & $\begin{array}{l}42 \\
36\end{array}$ & $\begin{array}{l}39 \\
32\end{array}$ & $\begin{array}{l}39 \\
32\end{array}$ \\
\hline Propane/Propylene . & 69 & 57 & 55 \\
\hline $\begin{array}{l}\text { Liquefied } \\
\text { Petroleum Gases. }\end{array}$ & 128 & 102 & 108 \\
\hline Other Petroleum & & & \\
\hline Products ....... & 207 & 219 & 210 \\
\hline
\end{tabular}

${ }^{1}$ Stocks as of December 31.

port." The primary impact of this reporting change is on Table 18, "Stocks of Crude Oil and Petroleum Products by PAD District," of the Petroleum Supply Annual, 1986 which showed a decrease in natural gas liquids (NGL) stocks at bulk terminals and natural gas processing plants, and an increase in NGL stocks at refineries.

\section{Note 9. Descriptive Monthly Statistics}

The universe of each of the Petroleum Supply surveys (refinery, bulk terminal, pipeline, crude oil stock, import, etc.) is relatively small and ever-changing due to company

Table B2. Impact of New Respondents to December 1985 PSM Data

\begin{tabular}{|c|c|c|c|c|}
\hline \multirow[b]{2}{*}{ Product } & \multicolumn{2}{|c|}{$\frac{\text { Refinery Production }}{\text { (thousand barrels per day) }}$} & \multicolumn{2}{|c|}{$\frac{\text { Stocks }^{a}}{\text { (thousand barrels) }}$} \\
\hline & $\begin{array}{c}\text { Reported } \\
\text { by New } \\
\text { Respondents }\end{array}$ & $\begin{array}{l}\text { Published } \\
\text { U.S. Total }\end{array}$ & $\begin{array}{c}\text { Reported } \\
\text { by New } \\
\text { Respondents }\end{array}$ & $\begin{array}{l}\text { Published } \\
\text { U.S. Total }\end{array}$ \\
\hline Leaded Gasoline & 1.3 & 2,326 & 224 & 81,379 \\
\hline Unleaded Gasoline & 0.6 & 4,323 & 276 & 108,422 \\
\hline Distillate Fuel Oil & 0 & 3,174 & 1,217 & 143,911 \\
\hline Residual Fuel Oil & 0 & 1,055 & 1,747 & 50,671 \\
\hline NGLs \& LRGs & 0 & 393 & 409 & 80,898 \\
\hline Other Products & 0 & 3,302 & 1,413 & 239,158 \\
\hline Crude Oil (excl. SPR) & 一 & - & 2,314 & 318,695 \\
\hline
\end{tabular}

a Stocks as of December 31, 1985. 
formations, shutdowns, mergers and splits. The frequency distributions of the petroleum supply variables are nonnormal, highly variable, positive skewed and leptokurtic; that is, there are many small units and few large ones. Zeros often dominate the responses; that is, not all of the sampling units produce and/or store all products.

The statistics described in Table B3 were calculated from the 1996 monthly surveys and display the following petroleum supply variables:

(1) The number of active sampling units (respondents).

(2) The number of sampling units reporting nonzero values (nonzero respondents).

(3) The average of nonzero values reported in thousand barrels (average).

(4) The standard deviation of nonzero values reported in thousand barrels (standard deviation).

\section{Note 10. Practical Limitations of Data Collection Efforts}

\section{Crude Oil Lease Stock Adjustment}

End-of-month crude oil stocks held on leases are reported on the EIA-813, "Monthly Crude Oil Report." However, only those companies that store 1,000 barrels or more of crude oil are required to submit a report. Previous frames analysis has shown that crude oil stocks held on leases reported to the EIA are consistently lower than the lease stocks reported to individual states.

Up until 1983, monthly state government data on lease stocks were substituted for EIA data wherever possible in order to rectify the understatement of lease crude oil stocks. State data were available from three states Texas, New Mexico, and Montana. To calculate the "lease adjustment," a comparison between EIA reported data and the state government data was made and the difference added to the EIA data for the respective states.

In 1983, the EIA modified the Form EIA-813 to eliminate state data on crude oil stocks and began collecting crude oil stock data by Petroleum Administration for Defense (PAD) District. With this change, the "lease adjustment" could no longer be calculated on a state basis and was changed to a PAD District level.

\section{Trans Alaskan Pipeline System Adjustment}

Beginning with the January 1989 data, adjustments are made to refinery inputs and product supplied of natural gas liquids (NGLs) and refinery inputs of crude oil to account for refiner misreporting. Substantial volumes of NGLs are produced at natural gas processing plants in Alaska and injected into the crude oil moving in the Trans Alaska Pipeline System (TAPS). Refiners receiving any crude oil commingled with NGLs are instructed to report the NGL portion of that stream separately from the crude oil portion. This has not been done for Alaskan crude oil because refiners are unable to identify these volumes for accounting purposes. As a result, the NGL production in Alaska has been credited directly toward product supplied and also toward product supplied from refinery production when the refiner processes the crude oil-NGL mixture. In addition, the reporting of the commingled stream as crude oil by the refiner has overstated crude oil inputs and resulted in an increase in unaccounted for crude oil equal to the volume of NGL in the crude oil.

To offset this reporting error, an adjustment is made to refinery input in all states receiving Alaskan crude oil. The adjustment reduces the crude oil inputs and increases the NGL inputs by an equal amount. Each state adjustment is a portion of the known Alaskan-NGL production that is proportional to the state's share of Alaskan crude oil received at all refineries in the United States. The greatest impact occurs in PAD District V for butane and pentanes plus.

The reporting problem which began in 1987 grew as injections on NGLs into the TAPS increased. Data for 1988 was revised in the Petroleum Supply Annual to account for the adjustment.

\section{Finished Motor Gasoline Product Supplied Adjustment}

Beginning with the reporting of January 1993 data, adjustments were made to the product supplied series for finished motor gasoline. It was recognized that motor gasoline statistics published by the EIA through 1992 were underreported because the reporting system was not collecting all fuel ethanol and motor gasoline blending components being blended downstream from the refinery. The EIA was able to quantify these volumes and make corrective adjustments for 1992 in 1993 (refer to Table B4 in the 1994 PSA).

\section{Fuel Ethanol Adjustment}

Prior to 1993, an estimated 60 to 70 thousand barrels per day of fuel ethanol were added to motor gasoline to produce gasohol but were not included in the EIA finished motor gasoline production data. In 1992, the EIA attempted to collect these data from downstream fuel ethanol motor gasoline blenders but found that this effort was impractical and the results were inaccurate. 
Table B3. Descriptive Statistics for Selected Petroleum Supply Variables ${ }^{1}, 1997$

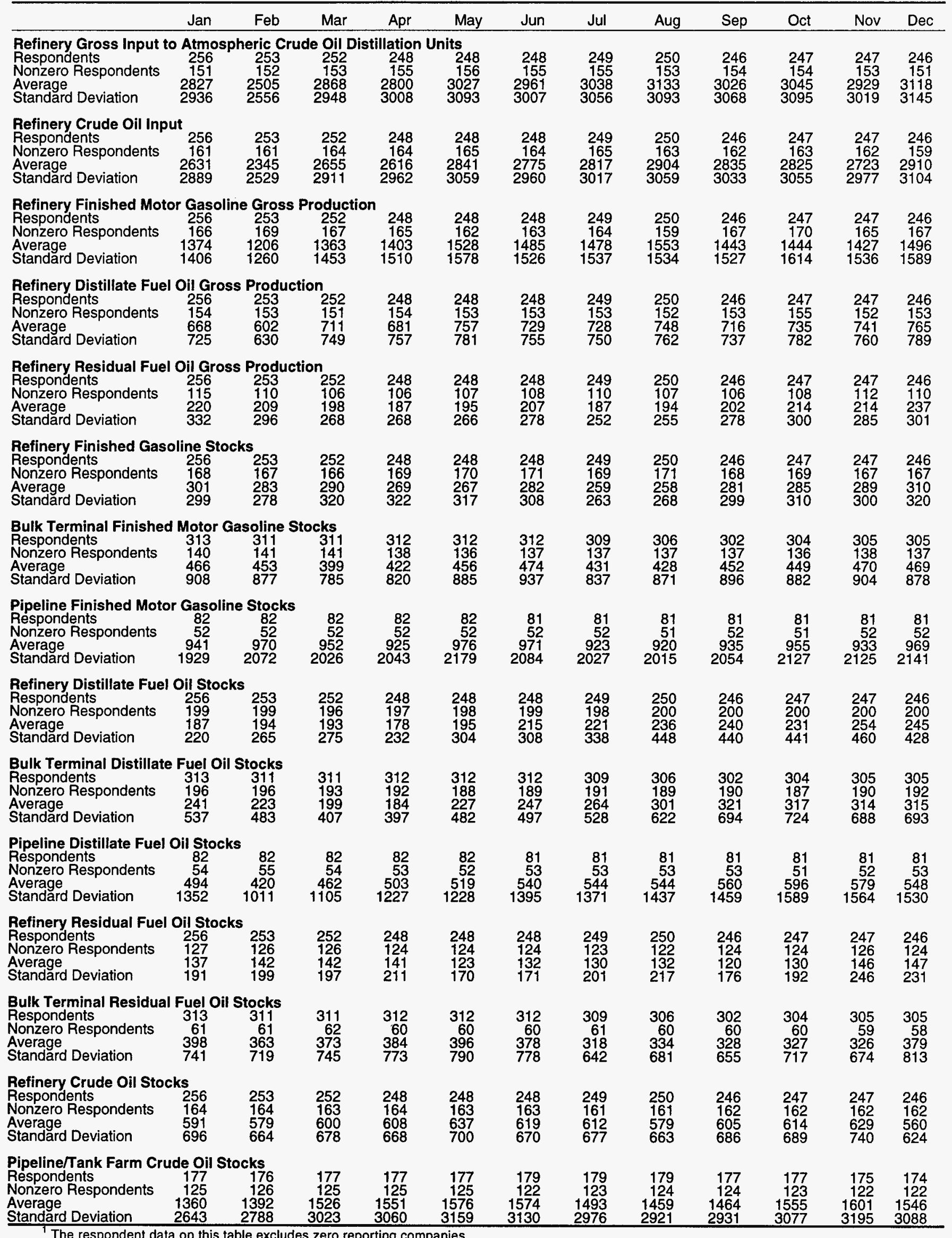


Table B4. Finished Motor Gasoline Product Supplied Adjustment, 1993 to Present (Thousand Barrels per Day)

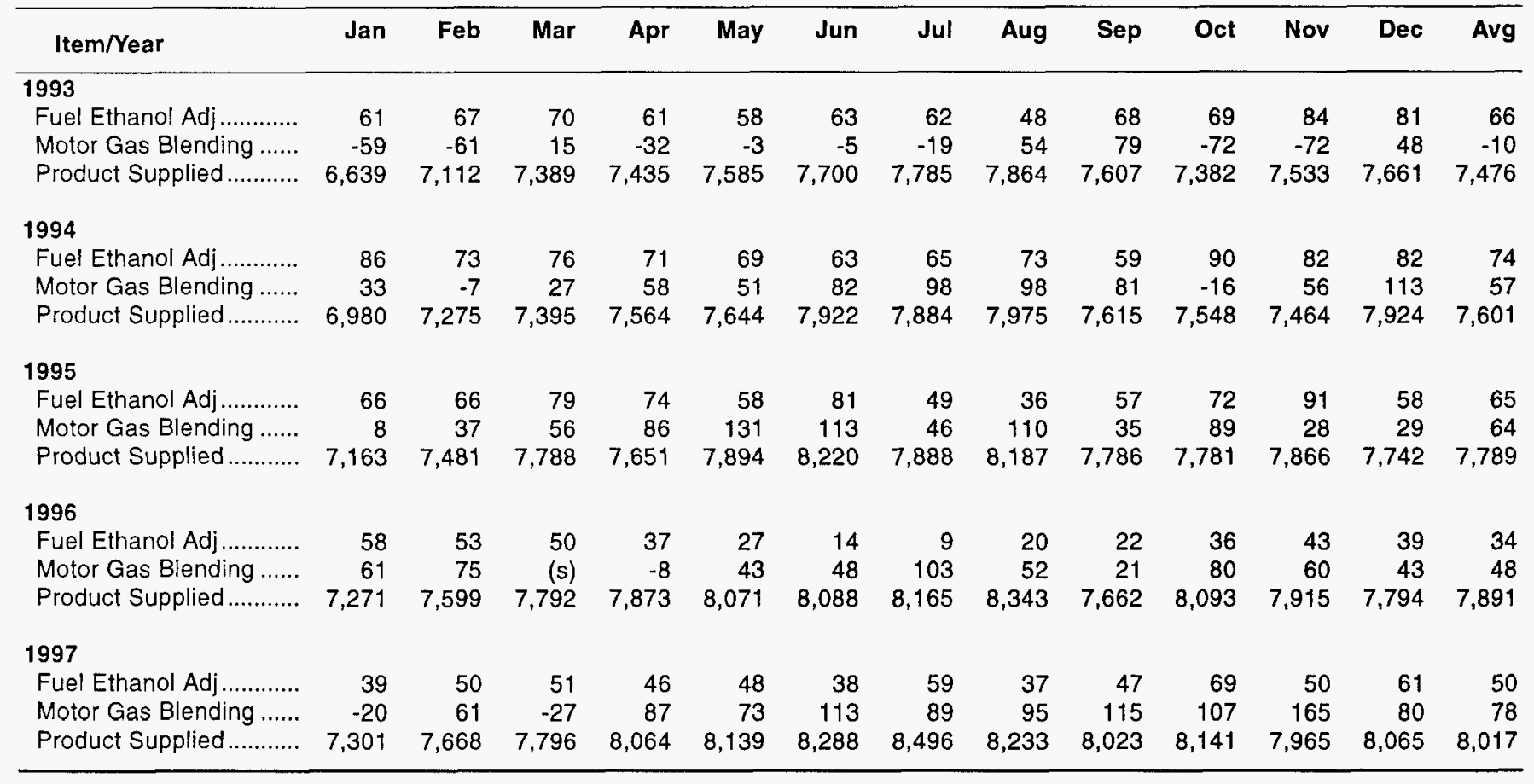

Note: Totals may not equal sum of components due to independent rounding.

Source: - Energy Information Administration. Petroleum Supply Annual, Volumes I and II.

Beginning in January 1993, an estimate for the missing fuel ethanol blended into motor gasoline was calculated (refer to Table B4). This estimate was calculated as production (from the EIA-819M, "Monthly Oxygenate Telephone Report"), plus imports (from the EIA-814, "Monthly Imports Report"), minus inputs at refineries (from the EIA-810, "Monthly Refinery Report"), plus or minus stock change (from the EIA-819M survey). This estimate for the amount of fuel ethanol blended into motor gasoline was added to Table 1 for Natural Gas Liquids Field Production (line 14) and in the Field Production column for finished motor gasoline in Tables 2 through 13 published in the PSA.

An estimate for the total amount of gasohol produced with the ethanol is given as 10 times the estimated fuel ethanol blended (this assumes a 10 percent ethanol blend). This amount is added to the column labeled field production of "oxygenated gasoline" and subtracted from the field production of "other" finished gasoline. The PAD District level detail was obtained by allocating the national level estimates according to the percent of gasohol sales from the U.S. Department of Transportation, Federal Highway Administration, Monthly Motor Fuel Reported by States, 1991.

\section{Motor Gasoline Blending Component Adjustment}

Prior to 1993, the EIA published a "product supplied" for motor gasoline blending components. Since these compo- nents are to be blended into finished motor gasoline, there is no actual demand for this intermediate product. The EIA corrected this series by including the quantity of "product supplied" for motor gasoline blending components with "other" finished motor gasoline. This change was accomplished in Tables 2 through 13 by adding product supplied for motor gasoline blending components to the column labeled field production of "other" motor gasoline, and subtracting it from the field production column for "motor gasoline blending components."

\section{Fuel Ethanol Stock Adjustment}

Total end-of-month stocks of fuel ethanol are underreported in the PSRS because of the inability to collect data from downstream fuel ethanol motor gasoline blenders. Total stocks of fuel ethanol are assumed to be those reported by ethanol producers on the Form EIA-819M, "Monthly Oxygenate Telephone Report." The difference between the stocks reported on the EIA-819M and the stocks reported in the PSRS (from refiners, bulk terminal and pipeline operators) is added to the stocks shown for bulk terminals. If the stocks for the PSRS are higher than those reported on the EIA-819M, no adjustment is made. 
Note 11. 1981 Changes in the Petroleum Supply Reporting System

Petroleum statistics for all years through 1980 were developed using definitions, concepts, reporting procedures, and aggregation methods that are consistent with those developed by the U.S. Bureau of Mines. Research conducted by the Energy Information Administration (EIA) in 1979 and 1980 indicated that changes had occurred in the petroleum industry that were not being adequately reflected in EIA's reporting system.

The EIA reporting forms, definitions, and procedures were modified beginning in January 1981 to describe industry operations more accurately. Unfortunately, empirical information is not available to precisely measure the data shortcomings through 1980. Estimates of the magnitudes of differences in the major data series are described below to form a basis for comparing 1979 , 1980 , and 1981 data.

\section{Motor Gasoline}

Prior to 1979, the EIA product-supplied series for motor gasoline was consistently about 2 percent lower than the Federal Highway Administration (FHWA) gasoline sales data series, which is derived from State tax receipts. The difference increased to about 3 percent in 1979 and 1980. There were two primary causes for this growing difference. First, refinery operations, particularly the flows of unfinished oils and the redesignation of some finished products, were not being accurately described on the EIA survey forms. Second, a large amount of gasoline was being produced away from refineries at "downstream blending stations" to take advantage of provisions in regulations governing the amount of lead that could be added. These blending stations were not reporting gasoline production to the EIA until the data system was changed in January 1981.

Quantitative estimates of the magnitude of the difference in EIA's gasoline product supplied data in 1979 and 1980 have been made by the EIA and the American Petroleum Institute (API). Table B5 provides 1979 and 1980 data as published in the Petroleum Statement, Annual, as well as EIA and API estimates of "recast" motor gasoline product supplied.

The EIA recast estimates were based upon preliminary monthly information in the Monthly Petroleum Statement. The ranges displayed in the EIA column reflect uncertainty in the estimates. Also shown are the FHWA motor gasoline sales statistics for those years.
Table B5. Finished Motor Gasoline Product Supplied

(Thousand Barrels per Day)

\begin{tabular}{ccccc}
\hline & $\begin{array}{c}\text { EIA } \\
\text { Reported }\end{array}$ & $\begin{array}{c}\text { API } \\
\text { Recast }\end{array}$ & $\begin{array}{c}\text { EIA } \\
\text { Recast }\end{array}$ & FHWA $^{\text {a }}$ \\
\hline $1979 \ldots .$. & 7,034 & 7,302 & $7,183-7,347$ & 7,258 \\
$1980 \ldots$. & 6,579 & 6,882 & $6,806-6,889$ & 6,792 \\
\hline
\end{tabular}

a FHW gasoline statistics based on data from Federal Highway Administration, Estimate of Total Gasoline Use, Table MF-21A published October 1980 and September 1981. Aviation gasoline (Table MF-24) has been subtracted from FHWA product supplied quantities to make data comparable.

\section{Distillate and Residual Fuel Oil}

Distillate and residual fuel oil refinery production statistics through 1980 were adjusted to account for an imbalance between unfinished oil supply and disposition. The reported quantities of refinery inputs of unfinished oils typically exceed the available supply of unfinished oils. It has been assumed that this occurs when distillate and residual fuel oils produced by a refinery are shipped to another refinery, where it is treated as unfinished oil. This oil is then reprocessed rather than used or sold as distillate or residual fuel oil.

For many years (including 1980), the difference between unfinished oil disposition and supply was subtracted from distillate and residual fuel oil production to adjust for this discrepancy. Two-thirds of the difference was applied to distillate fuel oil, and one-third to residual fuel oil.

Beginning in January 1981, this adjustment was discontinued because there was not sufficient empirical evidence to support it. Table B6 presents distillate and residual fuel oil refinery production in 1979 and 1980 as published (adjusted) and on the same basis as 1981 statistics (unadjusted) to permit comparison.

Table B6. Distillate and Residual Fuel Oil Production and Product Supplied

(Thousand Barrels per Day)

\begin{tabular}{|c|c|c|c|c|}
\hline & $\begin{array}{c}\text { Adjusted } \\
\text { Refinery } \\
\text { Production }\end{array}$ & $\begin{array}{c}\text { Unadjusted } \\
\text { Refinery } \\
\text { Production }\end{array}$ & Difference & $\begin{array}{c}\text { Unadjusted } \\
\text { Product } \\
\text { Supplied }\end{array}$ \\
\hline \multicolumn{5}{|l|}{$\begin{array}{l}\text { Distillate } \\
\text { Fuel Oil }\end{array}$} \\
\hline $1979 \ldots$ & 3,152 & 3,169 & 16 & 3,327 \\
\hline $1980 \ldots \ldots$ & 2,661 & 2,764 & 103 & 2,969 \\
\hline \multicolumn{5}{|l|}{$\begin{array}{l}\text { Residual } \\
\text { Fuel Oil }\end{array}$} \\
\hline $1979 \ldots \ldots$ & 1,687 & 1,695 & 8 & 2,834 \\
\hline $1980 \ldots \ldots$ & 1,580 & 1,634 & 54 & 2,562 \\
\hline
\end{tabular}


Adjusted distillate and residual fuel oil product supplied volumes differ from the unadjusted volumes by the same amounts as the adjusted and unadjusted production volumes.

\section{Total Petroleum Products}

The imbalance between the supply and disposition of unfinished oils and gasoline blending components is included with other products (line 35 ) in Table 1 . These imbalances are reported as negative product supplied in Table 2. Since these changes only involve redistribution of the volumes of finished motor gasoline, distillate and residual fuel oil, gasoline blending components, and unfinished oils, the total volume of petroleum products supplied remains unaffected by them.

\section{Alaskan In Transit Stocks}

Stocks of Alaskan crude oil in-transit were included for the first time in January 1981. The major impact of this change is on the reporting of stock change calculations. Using the expanded coverage (new basis), 1980 end-ofyear crude oil stocks would have been 488 million barrels (Total) and 380 million barrels (Other Primary).

\section{Note 12. 1983 Changes in the Petroleum Supply Reporting System}

January 1983 marked the implementation of recent changes in the collection, processing and availability of the Energy Information Administration's (EIA) petroleum supply data. Survey forms and definitions were made consistent; frames for bulk terminals, petroleum product pipelines and crude oil stock holders were updated, and the survey processing system was redesigned and incorporated into the new Petroleum Supply Reporting System (PSRS).

\section{Changes in Data Collection}

Changes in data collection can be grouped into five categories. Some were made to improve consistency, others to classify activity more precisely, and others to combine or eliminate information elements or to reduce the frequency of reporting in recognition of the trade-off between data value and reporting burden. The changes are itemized below.

- Motor gasoline was divided into three standard categories (finished leaded motor gasoline, finished unleaded motor gasoline and motor gasoline blending components).
- Aviation gasoline blending components were added to Form EIA-817.

- Crude oil burned as fuel on leases and by pipelines is reported as a single item on Form EIA-813. Previously it was reported as distillate or residual fuel oil consumption.

- Number 4 Fuel Oil is now included with distillate fuel oil.

- Gasohol was eliminated as a separate category and is now reported as either "finished leaded motor gasoline" or "finished unleaded motor gasoline."

- Waterborne movements of petrochemical feedstocks are now divided into naphtha-less than 401 degrees end-point and other-oils equal to or greater than 401 degrees end-point on Form EIA-817.

- Data aggregation for Petroleum Administration for Defense District (PADD) I was divided into three subdistricts on Forms EIA-812 and 817.

- Detailed categories of Gross Input to Crude Oil Distillation Units were eliminated, and only Total Gross Inputs are collected on Form EIA-810.

- Waterborne movements of crude oil and petroleum products between PADDs, on Form EIA-817, no longer reflect shipping and receiving States.

- Reporting of production and stocks of Number 4 Fuel Oil by sulfur levels were eliminated from Forms EIA$810,811,812$, and 817 .

- Crude oil stocks are collected at PADD levels rather than State levels on Form EIA-813.

- Shipments from natural gas processing plants no longer reflect destination by facility type on Form EIA-816.

- The four categories for unfinished oils were reduced to two on Form EIA-810.

- The five categories for sulfur content of residual fuel oil were reduced to three on Forms EIA-810, 811, and 817.

- Normal Butane and Other Butanes were combined into a single category on Forms EIA-810, 811, and 816.

- Three subcategories of lubricating oils (bright stock, neutral, and other) were combined into a single category on the Form EIA-810. 
- Three subcategories of waxes (microcrystalline, crystalline-fully refined, and crystalline-other) were combined into a single category on the Form EIA- 810 .

- Asphalt and Road Oil were combined into a single category on Forms EIA-810 and 811.

- Plant fuel use and Losses were combined on Form EIA-816.

- Natural Gasoline and Isopentane were combined on Form EIA-816.

\section{Change in Crude Oil Lease Stocks}

The end-of-month crude oil stocks held on leases are reported on the Form EIA-813, "Monthly Crude Oil Report." However, only those companies that store 1,000 barrels or more of crude oil are required to submit a report. Previous frames analysis has shown that crude oil stocks held on leases reported to the Energy Information Administration (EIA) are consistently lower than the lease stocks reported to individual states.

Up until 1983, monthly state government data on lease stocks were substituted for EIA data wherever possible in order to rectify the understatement of lease crude oil stocks. State data were available from three states Texas, New Mexico, and Montana. To calculate the "lease adjustment", a comparison between the EIA reported data and the state government data was made and the difference added to the EIA data for respective states.

In 1983, the EIA modified the Form EIA-813 to eliminate state data on crude oil stocks and began collecting crude oil stock data by PAD District. With this change, the "lease adjustment" could no longer be calculated on a state basis and was changed to a PAD District level.

\section{Note 13. 1984 Changes in the Petroleum Supply Reporting System}

In January 1984, a number of changes in the reporting of natural gas liquids (NGLs) were implemented. The modified system reflects supply and disposition of NGL on a component, rather than a product, basis.

From 1979 to 1983, the Energy Information Administration (EIA) collected and reported information on the supply and disposition of nine NGL products. Beginning with January 1984, NGL supply and disposition data were reported for 5 components to be consistent with record keeping practices used by the industry. Table B7 shows the product category under the new and old basis. Four Petro- leum Supply Reporting System surveys were modified beginning in January 1984. They were:
EIA- 810
"Monthly Refinery Report"
EIA-811
"Monthly Bulk Terminal Report"
EIA-812
EIA-816
"Monthly Product Pipeline Report"
"Monthly Natural Gas Liquids Report"

This change affected stocks reported and stock change calculations. Under the new basis, end-of-year 1983 stocks would have been 108 million barrels (Liquefied Petroleum Gases) and 210 million barrels (Other Petroleum Products).

Table B7. Product Basis vs. Component Basis Reporting

\begin{tabular}{|c|c|c|c|c|c|}
\hline \multirow[b]{2}{*}{$\begin{array}{l}\text { 1979-1983 } \\
\text { Product Basis }\end{array}$} & \multicolumn{5}{|c|}{$\begin{array}{c}1984 \text { Component } \\
\text { Basis }\end{array}$} \\
\hline & 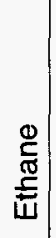 & 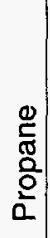 & 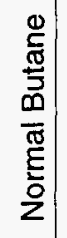 & 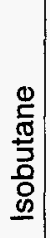 & $\begin{array}{l}\frac{0}{3} \\
\frac{0}{0} \\
0 \\
\frac{0}{\sigma} \\
\frac{\pi}{0} \\
0 \\
0\end{array}$ \\
\hline Ethane & $\cdot$ & & & & \\
\hline Ethane-Propane Mixtures & $\cdot$ & $\cdot$ & & & \\
\hline Propane & & $\cdot$ & & & \\
\hline Butane-Propane Mixtures & & $\cdot$ & $\cdot$ & & \\
\hline Butane & & & $\cdot$ & & \\
\hline Isobutane & & & & $\cdot$ & \\
\hline Unfractionated Stream & $\cdot$ & $\dot{-}$ & $\cdot$ & $\cdot$ & $\cdot$ \\
\hline Natural Gasoline and Isopentane & & & & & $\cdot$ \\
\hline Plant Condensate & & & & & $\cdot$ \\
\hline
\end{tabular}

A fifth survey, Form EIA-814, "Monthly Imports Report" (formerly Form ERA-60), was not modified. Therefore, in order to allocate imports and exports of mixed NGL streams to individual component parts, the EIA developed a statistical algorithm.

\section{Imports}

The imports algorithm was based on information gathered from the larger importers of NGL, who were asked to provide component analysis of the products they imported during the first 6 months of 1983. The percentages shown in Table B8 are derived from the weighted averages of the data provided by the importers.

\section{Exports}

The exports algorithm was based on information gathered from the larger exporters of NGL, who were asked to provide component analysis of the products they exported 


\begin{tabular}{|c|c|c|c|c|c|}
\hline \multirow{2}{*}{ Product } & \multicolumn{5}{|c|}{ EIA Component Slate } \\
\hline & Ethane & Propane & Normal Butane & Isobutane & Pentanes Plus \\
\hline \multicolumn{6}{|l|}{ Import Product } \\
\hline Natural Gasoline and Isopentane (EIA-814) & - & - & - & - & 100 \\
\hline Plant Condensate $(E \mid A-814) \ldots \ldots \ldots \ldots \ldots$ & - & - & - & - & 100 \\
\hline Ethane $(\mathrm{IM}-145) \ldots \ldots \ldots$ & 100 & - & - & - & - \\
\hline Propane $(I M-145) \ldots \ldots \ldots$ & - & 100 & - & - & - \\
\hline Butane (IM-145) & - & - & 65 & 35 & - \\
\hline Butane-Propane Mixtures (IM-145) & - & 40 & 35 & 20 & 5 \\
\hline Ethane-Propane Mixtures (IM-145) .. & 60 & 40 & - & - & - \\
\hline \multicolumn{6}{|l|}{ Export Product } \\
\hline Ethane (All PAD Districts) $\ldots \ldots \ldots \ldots \ldots \ldots$ & 100 & - & - & - & - \\
\hline 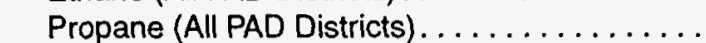 & - & 100 & - & - & - \\
\hline Butane (All PAD Districts) $\ldots \ldots \ldots \ldots \ldots \ldots$ & - & - & 100 & - & - \\
\hline \multicolumn{6}{|l|}{ Mixed Streams } \\
\hline PAD Districts I, IV, V . . . & - & 40 & 60 & - & - \\
\hline PAD District II . & 30 & 25 & 15 & 15 & 15 \\
\hline PAD District III. . & - & 80 & 20 & - & - \\
\hline
\end{tabular}

during 1983. The percentages shown in Table B8 are derived from the weighted averages of the data provided by the exporters. It was necessary to derive percentages by Petroleum Administration for Defense Districts of exportation, due to the wide variation of components included in the mixed streams.

\section{Note 14. 1985 Changes in the Petroleum Supply Reporting System}

Beginning in January 1985, inter-Petroleum Administration for Defense (PAD) District pipeline movements of crude oil were included in the crude oil supply balance at the PAD District level but did not affect National level statistics. As a result of including these movements, $\mathrm{Net}$ Receipts of crude oil and Unaccounted for Crude Oil at the PAD District level changed significantly. Also affected were crude oil imports and unfinished oil imports at the PAD District level which are provided by PAD District of Entry (Tables 4-8) and by PAD District of Processing (Table 14).

The tables in the Petroleum Supply Annual that were changed due to the inclusion of inter-PAD District pipeline movements of crude oil are listed below:
- Tables 4 through 8, "PAD Districts I to V, Supply and Disposition of Crude Oil and Petroleum Products."

- Effective January 1985, crude oil imports and unfinished oil imports in Tables 4 through 8 were reported at the PAD District of Entry rather than at the PAD District of Processing. Net Receipts now include movements by pipeline as well as by tanker and barge.

- Table 20, "Movements of Crude Oil and Petroleum Products by Pipeline, Tanker, and Barge Between PAD Districts."

- The crude oil line includes movements by pipeline as well as by tanker and barge.

- Table 21, "Movements of Crude Oil and Petroleum Products by Pipeline Between PAD Districts."

- A line was added to report crude oil movements.

- Table 23, "Net Movements of Crude Oil and Petroleum Products by Pipeline, Tanker, and Barge Between PAD Districts."

- The crude oil line includes movements by pipeline as well as by tanker and barge. 


\section{Note 15. 1986 Changes in the Petroleum Supply Reporting System}

Beginning in January 1986, several changes to the Petroleum Supply Reporting System (PSRS) went into effect. These changes affected the frame of operators of petroleum facilities required to complete the monthly surveys in the PSRS and resulted in some changes to the tables presented in the Petroleum Supply Monthly and were subsequently published in the Petroleum Supply Annual (PSA). Refer to Explanatory Note 8 for a detailed description of frames maintenance and updates.

\section{Changes in Data Collection}

- The unit of measure used on Form EIA-814, "Monthly Imports Report," has been changed from barrels to thousands of barrels.

- Unfinished oil imports data, previously reported as one product on the Form EIA-814, are now reported separately under four classifications. These classifications are:

- Naphthas and lighter

- Kerosene and light gas oils

- Heavy gas oils

- Residuum

- The number of categories for reporting natural gas liquids and liquefied petroleum gases data on Form EIA814 was reduced from 19 to 5 by eliminating the requirement to separately identify categories for further processing, petrochemical use, and fuel use.

- The requirements to report the type of processing facility and the applicable section of the oil import regulations were eliminated for the Form EIA-814.

- The requirement to report data for imports of crude oil, unfinished oils, and finished products on separate schedules of the Form EIA-814 was eliminated.

- The requirement to report two end-use categories, petrochemical use and other use, for still gas and liquefied refinery gases, was eliminated on Form EIA-810, "Monthly Refinery Report."

- Form EIA-815, "Monthly Shipments from Puerto Rico to the United States Report," was discontinued. The data previously reported on this form are now reported on Form-814.

\section{Changes in Publication Tables}

Several changes were also made to tables in the PSA either as a direct result of changes in reporting require- ments or to improve the usefulness of the publication. These changes were:

- Table 11, "Refinery Input of Crude Oil and Petroleum Products by PAD District."

- Alaskan crude oil receipts were shown separately.

- Table 12, "Refinery Production of Petroleum Products by PAD District."

- The breakout between "petrochemical feedstock use" and "other use" were no longer shown separately for still gas or for liquefied refinery gases.

- Table 14, "Imports of Crude Oil and Petroleum Products by PAD District."

- Imports of unfinished oils were separated into four categories: naphthas and lighter, kerosene and light gas oils, heavy gas oils, and residuum.

- Table 15, "Imports of Crude Oil and Petroleum Products by Source."

- Countries formerly included in the categories "Other Western Hemisphere" and "Other Eastern Hemisphere" were shown individually.

- Table 18, "Stocks of Crude Oil and Petroleum Products by PAD District."

- The breakout between "petrochemical feedstock use" and "other use" for each liquefied petroleum gas was eliminated.

\section{Note 16. 1987 Changes in the Petroleum Supply Reporting System}

Several changes to the Petroleum Supply Reporting System went into effect at the beginning of January 1987. These changes were made as part of the Energy Information Administration's (EIA's) continuing effort to provide pertinent, timely, and consistent energy information. These changes were subsequently reflected in the Petroleum Supply Annual (PSA).

\section{Changes in Data Collection}

Fresh feed input to catalytic cracking units, hydrocracking units, and cokers were added to the Form EIA-810, "Monthly Refinery Report." 


\section{Changes in Publication Tables}

- The "Appalachian No. 2" Refining District was combined with the "Indiana, Illinois, Kentucky," Refining District. This affected PSA Tables 10 through 13, 18, 24 , and 25 .

- Fresh feed inputs to catalytic cracking units, hydrocracking units, and cokers were added to Table 11, "Refinery Input of Crude Oil and Petroleum Products by PAD District."

\section{Clarification}

In 1986, several refineries and terminals in the United States applied for Foreign Trade Zone (FTZ) status and applications from three refineries were approved. Consequently, during 1986, some refineries with FTZ status were treated as if they were within the United States while the Hawaiian FTZ was considered outside.

Effective with the January 1987 data, all FTZ facilities located within the 50 United States are considered domestic entities and are included in PSA statistics. The principal differences in the PSA data series as a result of adding the Hawaiian FTZ was an approximate 1 percent increase in crude imports and a 3 percent decrease in product imports.

\section{Note 17. 1989 Changes in the Petroleum Supply Reporting System}

Several changes to the Petroleum Supply Reporting System (PSRS) went into effect at the beginning of January 1989. These changes were made to reduce respondent burden, to fulfill user requests for additional data, and to improve accuracy and consistency in reporting. To reflect these changes and to improve the usefulness of the Petroleum Supply Monthly (PSM) publication, the following changes were made in January 1989 and are subsequently reflected in the Petroleum Supply Annual (PSA) publication.

\section{Changes in Data Collection}

- Data on inputs and production of naphthenic and paraffinic lubricants were added to the Form EIA-810, "Monthly Refinery Report."

- Separate lines for the collection of inputs and production of olefins (ethylene, propylene, and butylene) were added to Form EIA-810, "Monthly Refinery Report."

- The collection of data on the movement of Liquefied Petroleum Gases (LPGs) and Liquefied Refinery Gases
(LRGs) on a component basis were added to the Forms EIA-812, "Monthly Product Pipeline Report," and the EIA-817, "Monthly Tanker and Barge Movement Report."

- Bonded imports of jet fuel and fuel oils and imports of LPGs previously published from data provided by the U.S. Bureau of the Census were discontinued. Data are now published from the data reported on the Form EIA-814, "Monthly Imports Report."

- Exports of butane/propane and ethane/propane mixtures were split in a ratio of 60 percent for the butane and ethane portions and 40 percent for the propane portion.

- The reporting of products other than Natural Gas Liquids (NGLs) by natural gas processing plants was eliminated on the Form EIA-816, "Monthly Natural Gas Liquids Report."

- Fractionators were required to report only end-ofmonth stocks of NGLs on the Form EIA-816, "Monthly Natural Gas Liquids Report."

\section{Changes in Natural Gas Liquids and Crude Oil Statistics}

Beginning with the January 1989 issue of the $P S M$, adjustments were made to refinery inputs and product supplied of NGLs and refinery inputs of crude oil to account for refiner misreporting. Substantial volumes of NGLs are produced at natural gas processing plants in Alaska and injected into the crude oil moving in the Trans Alaska Pipeline System (TAPS). Refiners receiving any crude oil commingled with NGLs are instructed to report the NGL portion of that stream separately from the crude oil portion. This has not been done for Alaskan crude oil because refiners are unable to identify these volumes for accounting purposes. As a result, the NGL production in Alaska has been credited directly toward product supplied and also toward product supplied from refinery production when the refiner processes the crude oil-NGL mixture. In addition, the reporting of the commingled stream as crude oil by the refiner has overstated crude oil inputs and resulted in an increase in unaccounted for crude oil equal to the volume of NGL in the crude oil.

To offset this reporting error, an adjustment was developed affecting refinery input in all Petroleum Administration for Defense (PAD) Districts receiving Alaskan crude oil. The adjustment reduces the crude oil inputs and increases the NGL inputs by an equal amount. Each PAD District adjustment is a portion of the known Alaskan NGL production that is proportional to the PAD District's share of Alaskan crude oil received at all refineries in the 


\begin{tabular}{ccccccccccc}
\hline \multicolumn{10}{c}{ Table Numbers } \\
\hline Old & New & Old & New & Old & New & Old & New & Old & New \\
\hline & & & & & & & & & & \\
1 & 1 & NA & 9 & 12,24 & 17 & 15 & 25 & 21 & 33 & 34 \\
2 & 2 & 7 & 10 & 18,25 & 18 & 27 & 26 & 22,26 & 35 & \\
3 & 3 & NA & 11 & 13 & 19 & 16 & 27 & 23 & \\
4 & 4 & 8 & 12 & 14,27 & 20 & 17 & 28 & & \\
NA & 5 & NA & 13 & 15 & 21 & NA & 29 & & \\
5 & 6 & 9 & 14 & 15 & 22 & 18,25 & 30 & & \\
NA & 7 & 10 & 15 & 15 & 23 & 19 & 31 & & \\
6 & 8 & 11 & 16 & 15 & 24 & 20 & 32 & & \\
\hline
\end{tabular}

NA $=$ Not Applicable

United States. The greatest impact occurs in PAD District $\mathrm{V}$ for butane and pentanes plus.

The reporting problem began in 1987 and has grown as injections of NGLs into the TAPS have increased. Data for 1988 was revised to account for the adjustment in the PSA.

\section{Changes in Publication Tables}

- "Stock Withdrawal" was renamed "Stock Change" and was moved from Supply to Disposition in Tables 2 through 13. A negative number indicates a decrease in stocks and a positive number indicates an increase in stocks.

- A jet fuel total line was added to Tables $2-13,17,18$, 20, 32-35.

- PAD District Supply and Disposition tables (Tables 4 through 13) now display liquefied petroleum gases on a component basis.

- A table showing net imports by country for the current month (Table 29) was added.

- Table numbers were changed as a result of data additions and table reorganization. Table B9 is provided to show the new to old table numbers for the detailed statistics tables.

- Table 15, "Natural Gas Plant Net Production and Stocks of Petroleum Products by PAD and Refining District."

- Stocks at natural gas processing plants by Refining District previously published on Table 10 was included with net production of petroleum products at natural gas plants.

- The reporting of products other than natural gas liquids by natural gas processing plants was eliminated.
- Table 17, "Net Refinery Production of Finished Petroleum Products by PAD and Refining District."

- Net production of olefins (ethylene, propylene, and butylene) was added.

- Net production of naphthenic and paraffinic lubricants was added.

- Net production of residual fuel oil by percent sulfur, previously published as Table 24 , was added.

- Table 18, "Refinery Stocks of Crude Oil and Petroleum Products by PAD and Refining District."

- Stocks at refineries by Refining District were added from Table 18.

- Stocks of residual fuel oil by percent sulfur content, previously published as Table 25 , were added.

- Tables 21 through 25, "Imports of Crude Oil and Petroleum Products by Country of Origin."

- Data previously included in the "Other Products" category were displayed separately for naphthas for petrochemical feedstock use, other oils for petrochemical feedstock use, lubricants, and asphalt and road oil.

- Table 20,"Imports of Crude Oil and Petroleum Products by PAD District."

- Sulfur content categories for residual fuel oil, previously published as Table 27 , were added.

- Table 28, "Exports of Crude Oil and Petroleum Products by Destination." 
- Data for exports by destination previously included in the Other Products category were displayed separately for pentanes plus, kerosene, naphthas for petrochemical feedstock use, and other oils for petrochemical feedstock use.

- Table 30, "Stocks of Crude Oil and Petroleum Products by PAD District."

- Refining District data were eliminated. Refinery stocks and natural gas processing plant stocks by Refining District were added to Table 18.

- Sulfur content categories for residual fuel oil, previously published as Table 25 , were added.

\section{Note 18. 1990 Changes in the Petroleum Supply Reporting System}

Beginning with the May 1990 issue of the Petroleum Supply Monthly (PSM), stocks of propane/propylene were added to Table 42, "Refinery, Bulk Terminal, and Natural Gas Plant Stocks of Selected Petroleum Products by State." This change is also reflected in the corresponding table in the Petroleum Supply Annual (PSA).

Beginning with the 1991 March issue of the PSM, several changes were made to the Petroleum Supply Reporting System to provide additional data and to improve the usefulness of the publication. Although these changes were made in 1991, these changes have been incorporated into the 1990 PSA to provide consistent energy information.

\section{Changes in Publication Tables}

\section{Summary Statistics Tables}

- A new table (Table S7) has been added to display jet fuel supply and disposition.

- Table S8, "Other Petroleum Products Supply and Disposition" has been redesignated as Table S9. Jet fuel data are no longer included. Historical data have been revised to exclude jet fuel.

- Table S3, "Crude Oil and Petroleum Product Imports" has been expanded to display all Organization of Petroleum Exporting Countries (OPEC) and additional NonOPEC countries. A separate column for crude oil imports has also been added for each country.

- Time periods have been included in table titles.

\section{Figures}

- Time periods have been included in figure titles.

- Sources have been provided for each figure.

- Bar graphs used to display end-of-month stocks have been replaced with line graphs.

\section{Sources}

The sources and explanatory notes for this section have been updated and are now located at the end of the Summary Statistics section.

\section{Detailed Statistics Tables}

- Table 1, "U.S. Petroleum Balance"

- A line has been added to display jet fuel as a separate category for Total Products Supplied and Total Stocks (Lines 34 and 44, respectively).

- Imports of Crude Oil and Petroleum Products by PAD District

- Residual fuel oil sulfur categories have been added.

- Imports of Crude Oil and Petroleum Products by Country of Origin

- Residual fuel oil sulfur categories by country of origin have been eliminated. These categories are now reported on a PAD District basis.

- Separate daily average columns have been added for crude oil and petroleum products.

\section{Note 19. 1993 Changes in the Petroleum Supply Reporting System}

In keeping with the Department of Energy's (DOE's) mandated responsibilities, the Energy Information Administration (EIA) made several changes to the Petroleum Supply Reporting System (PSRS) effective in January 1993. These changes were designed to accommodate the revisions to the Clean Air Act of 1990, and to reflect current and upcoming changes in the petroleum industry. These changes are subsequently reflected in the $1993 \mathrm{Pe}$ troleum Supply Annual.

\section{Changes in Data Collection}

- Motor gasoline categories have been revised to reflect the change in the type of fuels produced. The new 
categories are: reformulated gasoline, oxygenated gasoline, and other finished gasoline. These changes were made to Forms EIA-810, "Monthly Refinery Report," EIA-811, "Monthly Bulk Terminal Report," EIA812, "Monthly Product Pipeline Report," EIA-814, "Monthly Imports Report," and EIA-817, "Monthly Tanker and Barge Movement Report."

- Distillate Fuel Oil has been split into two sulfur categories to meet Environmental Protection Agency requirements effective in October 1993. The new categories for inputs, production, end-of-month stocks and movements are: $0.05 \%$ sulfur and under, and greater than $0.05 \%$ sulfur. These changes were made to Forms EIA810, "Monthly Refinery Report," EIA-811, "Monthly Bulk Terminal Report," EIA-812, "Monthly Product Pipeline Report," EIA-814, "Monthly Imports Report," and EIA-817, "Monthly Tanker and Barge Movement Report."

- Other hydrocarbons, hydrogen, and alcohol (Code 090) has been renamed "Other hydrocarbons, hydrogen, and oxygenates" on Form EIA-810, "Monthly Refinery Report." A new line has also been added to report Other hydrocarbons and hydrogen separately.

- Data on inputs and end-of-month stocks of oxygenates (i.e., fuel ethanol, ethyl tertiary butyl ether (ETBE), methanol, methyl tertiary butyl ether (MTBE), tertiary amyl methyl ether (TAME), tertiary butyl alcohol (TBA), and other oxygenates) has been added to Form EIA-810, "Monthly Refinery Report."

- Inputs and production of Isobutylene (Code 634) has been added as sub-categories to Isobutane (Code 615) on Form EIA-810, "Monthly Refinery Report."

- Data on inputs and production of military kerosenetype jet fuel and commercial kerosene-type jet fuel has been added to Form EIA-810, "Monthly Refinery Report."

- Liquefied Petroleum and Refinery Gases column headings for Ethane, Propane, Normal Butane, and Isobutane have been revised to include olefins (e.g., Ethane/Ethylene etc.) on Form EIA-811, "Monthly Bulk Terminal Report."

- Data on end-of-month stocks of oxygenates (i.e., fuel ethanol, ethyl tertiary butyl ether (ETBE), methyl tertiary butyl ether (MTBE), tertiary amyl methyl ether (TAME), tertiary butyl alcohol (TBA), and other oxygenates) have been added to Forms EIA-811, "Monthly Bulk Terminal Report," and EIA-812, "Monthly Product Pipeline Report." Data for methanol are not collected at this time but has been included on the form for future use.
- Imports of oxygenates (i.e., fuel ethanol, ethyl tertiary butyl ether (ETBE), methyl tertiary butyl ether (MTBE), tertiary amyl methyl ether (TAME), tertiary butyl alcohol (TBA), and other oxygenates) have been added to Form EIA-814, "Monthly Imports Report." Data for methanol are not requested at this time.

- Imports of olefins are collected separately from liquefied petroleum gases (i.e., ethylene, propylene, butylene, and isobutylene) on Form EIA-814, "Monthly Imports Report."

- Data on oxygenates blended into motor gasoline has been eliminated on the Form EIA-819M, "Monthly Oxygenate Telephone Report."

- Data on methanol is no longer required on the Form EIA-819M, "Monthly Oxygenate Telephone Report" but remains on the form for future use.

\section{Changes in Summary Statistics Tables}

- Table S1. Crude and Petroleum Products Overview

- History data for 1973 through 1980 has been dropped. The table title has been changed to reflect the change in time series.

- Table S2. Crude Oil Supply and Disposition

- History data for 1973 through 1980 has been dropped. The table title has been changed to reflect the change in time series.

- The Crude Used Directly column has been eliminated. This column is no longer applicable since the years 1973 through 1980 have been eliminated. The data for 1981 and 1982 are provided in a footnote.

- Table S3. Crude Oil and Petroleum Product Imports

- History data for 1973 through 1980 has been dropped. The table title has been changed to reflect the change in time series.

- The Former USSR has been renamed Russia. The remaining states that comprised the Former USSR have been included in the Other Non-OPEC column.

- Table S4. Finished Motor Gasoline Supply and Disposition

- History data for 1973 through 1980 has been dropped. The table title has been changed to reflect the change in time series. 
- Product supplied-unleaded and product suppliedunleaded (percent of Total) columns have been eliminated. A new column has been added to display end-of-month stocks of oxygenates. These stocks are not included in the Total Motor Gasoline end-of-month stocks.

- Table S5. Distillate Fuel Oil Supply and Disposition

- History data for 1973 through 1980 has been dropped. The table title has been changed to reflect the change in time series.

- Distillate fuel oil stocks have been separated into two sulfur categories $(0.05 \%$ sulfur and under and greater than $0.05 \%$ sulfur).

- The Crude Used Directly column has been eliminated. This column is no longer applicable since the years 1973 through 1980 have been eliminated. The data for 1981 and 1982 are provided in a footnote.

- Table S6. Residual Fuel Oil Supply and Disposition

- History data for 1973 through 1980 has been dropped. The table title has been changed to reflect the change in time series.

- The Crude Used Directly column has been eliminated. This column is no longer applicable since the years 1973 through 1980 have been eliminated. The data for 1981 and 1982 are provided in a footnote.

- Table S7. Jet Fuel Supply and Disposition

- History data for 1973 through 1980 has been dropped. The table title has been changed to reflect the change in time series.

- Table S8. Propane/Propylene Supply and Disposition

- A new summary table has been added to display supply and disposition data for propane/propylene. This information will continue to be included in the Liquefied Petroleum Gases Supply and Disposition table (renumbered as Table S9).

- Table S9. Liquefied Petroleum Gases Supply and Disposition

- Formerly numbered as Table $\mathbf{S} 8$.
- History data for 1973 through 1980 has been dropped. The table title has been changed to reflect the change in time series.

- Table S10. Other Petroleum Products Supply and Disposition

- Formerly numbered as Table S9.

- History data for 1973 through 1980 has been dropped. The table title has been changed to reflect the change in time series.

\section{Changes in Detailed Statistics Tables}

- Table 1. U.S. Petroleum Balance

- Line 14 includes fuel ethanol blended into finished motor gasoline. This quantity is comparable to the sum of field production of finished motor gasoline and natural gas liquids and LRGs on Table 2.

- Line 20 has been modified to read: Other Liquids New Supply (Field Production) to accommodate motor gasoline blending components field production.

- Tables 2 through 13. Supply and Disposition

- Isobutane has been renamed Isobutane/Isobutylene under Liquefied Petroleum Gases for clarification.

- Other Hydrocarbons/Hydrogen/Alcohol has been renamed Other_Hydrocarbons/Hydrogen/Oxygenates for clarification.

- Unleaded and leaded motor gasoline categories have been replaced with the new types of gasolines produced: reformulated, oxygenated, and other.

- Distillate fuel oil sulfur categories $(0.05 \%$ sulfur and under and greater than $0.05 \%$ sulfur) have been added.

- Table 16. Refinery Input

- Other Hydrocarbons/Hydrogen/Alcohol has been renamed Other Hydrocarbons/Hydrogen/Oxygenates for clarification. Sub-categories are displayed for Other Hydrocarbons/Hydrogen and for Oxygenates.

- Oxygenates are displayed separately for fuel ethanol, methanol, MTBE, and other oxygenates. Other oxygenates includes ethyl tertiary butyl ether (ETBE), tertiary amyl methyl alcohol (TAME), tertiary butyl alcohol (TBA), and other aliphatic alcohols and ethers intended for motor gasoline blending (e.g., isopropyl ether (IPE) or n-propanol). 
- Table 17. Refinery Net Production

- Isobutane has been renamed Isobutane/Isobutylene under Liquefied Petroleum Gases for clarification. Isobutylene is displayed as a sub-category to be consistent with the other liquefied gases.

- Unleaded and leaded motor gasoline categories have been replaced with the new types of gasolines produced: reformulated, oxygenated, and other.

- Military and commercial kerosene-type jet fuel has been added.

- Distillate fuel oil sulfur categories $(0.05 \%$ sulfur and under and greater than $0.05 \%$ sulfur) have been added.

- Table 18. Refinery Stocks

- Isobutane has been renamed Isobutane/Isobutylene under Liquefied Petroleum Gases for clarification.

- Other Hydrocarbons/Hydrogen/Alcohol has been renamed Other Hydrocarbons/Hydrogen/Oxygenates for clarification. Sub-categories are displayed for Other Hydrocarbons/Hydrogen and for Oxygenates.

- Oxygenates are displayed separately for fuel ethanol, methanol, MTBE, and other oxygenates. Other oxygenates includes ethyl tertiary butyl ether (ETBE), tertiary amyl methyl alcohol (TAME), tertiary butyl alcohol (TBA), and other aliphatic alcohols and ethers intended for motor gasoline blending (e.g., isopropyl ether (IPE) or n-propanol).

- Unleaded and leaded motor gasoline categories have been replaced with the new types of gasolines produced: reformulated, oxygenated, and other.

- Distillate fuel oil sulfur categories $(0.05 \%$ sulfur and under and greater than $0.05 \%$ sulfur) have been added.

- Table 20. Imports by PAD District

- Data on olefins are displayed separately from liquefied petroleum gases.

- Other Hydrocarbons/Hydrogen/Alcohol has been renamed Other Hydrocarbons/Hydrogen/Oxygenates for clarification. Sub-categories are displayed for Other Hydrocarbons/Hydrogen and for Oxygenates.

- Oxygenates are displayed separately for fuel ethanol, MTBE, and other oxygenates. Other oxygenates includes ethyl tertiary butyl ether (ETBE), tertiary amyl methyl alcohol (TAME), tertiary butyl alcohol (TBA), and other aliphatic alcohols and ethers intended for motor gasoline blending (e.g., isopropyl ether (IPE) or n-propanol).

- Unleaded and leaded motor gasoline categories have been replaced with the new types of gasolines produced: reformulated, oxygenated, and other.

- Distillate fuel oil sulfur categories $(0.05 \%$ sulfur and under and greater than $0.05 \%$ sulfur) have been added to both bonded ship bunkers and other.

- Tables 21-25. Imports by Country of Origin

- A new line has been added to appear below the Total line to show the sum of the Persian Gulf countries.

- Former USSR has been changed to read Russia. States formerly included in USSR are now included in the Other countries category under Non-OPEC.

- Table 27. Exports

- Isobutane has been renamed Isobutane/Isobutylene under Liquefied Petroleum Gases for clarification.

- Other Hydrocarbons/Oxygenates and Motor Gasoline Blending Components have been added as export products under the Other Liquids category.

- Table 28. Exports by Destination

- Miscellaneous products category has been renamed Other Products to accommodate exports of other hydrocarbons/ oxygenates and motor gasoline blending components.

- Table 29. Net Imports

- A new line has been added to appear below the Total line to show the sum of the Persian Gulf countries.

- Former USSR has been changed to read Russia. States formerly included in USSR are now included in the Other countries category under Non-OPEC.

- Table 30. Stocks

- Other Hydrocarbons/Hydrogen/Alcohol has been renamed Other Hydrocarbons/Hydrogen/Oxygenates for clarification. Sub-categories are displayed for Other hydrocarbons/hydrogen fuel ethanol, ETBE, methanol, MTBE, and other oxygenates.

- Other oxygenates includes tertiary amyl methyl alcohol (TAME), tertiary butyl alcohol (TBA), and other 
aliphatic alcohols and ethers intended for motor gasoline blending (e.g., isopropyl ether (IPE) or npropanol.

- Unleaded and leaded motor gasoline categories have been replaced with the new types of gasolines produced: reformulated, oxygenated, and other.

- Distillate fuel oil sulfur categories $(0.05 \%$ sulfur and under and greater than $0.05 \%$ sulfur) have been added.

- Table 31. Refinery, Bulk Terminal, and Natural Gas Plant Stocks

- Unleaded and leaded motor gasoline categories have been replaced with the new types of gasolines produced: reformulated, oxygenated, and other.

- Distillate fuel oil sulfur categories $(0.05 \%$ sulfur and under and greater than $0.05 \%$ sulfur) have been added.

- Table 32. Movements by Pipeline, Tanker, and Barge

- Unleaded and leaded motor gasoline categories have been replaced with the new types of gasolines produced: reformulated, oxygenated, and other.

- Distillate fuel oil sulfur categories $(0.05 \%$ sulfur and under and greater than $0.05 \%$ sulfur) have been added.

- Table 33. Movements by Pipeline

- Unleaded and leaded motor gasoline categories have been replaced with the new types of gasolines produced: reformulated, oxygenated, and other.

- Distillate fuel oil sulfur categories $(0.05 \%$ sulfur and under and greater than $0.05 \%$ sulfur) have been added.

- Table 34. Movements by Tanker and Barge

- Unleaded and leaded motor gasoline categories have been replaced with the new types of gasolines produced: reformulated, oxygenated, and other.

- Distillate fuel oil sulfur categories $(0.05 \%$ sulfur and under and greater than $0.05 \%$ sulfur) have been added.

\section{- Table 35. Net Movements}

- Isobutane has been renamed Isobutane/Isobutylene under Liquefied Petroleum Gases for clarification.
- Unleaded and leaded motor gasoline categories have been replaced with the new types of gasolines produced: reformulated, oxygenated, and other.

- Distillate fuel oil sulfur categories $(0.05 \%$ sulfur and under and greater than $0.05 \%$ sulfur) have been added.

\section{Changes in Appendix C (PSM)}

- Inputs

- Other hydrocarbons has been renamed Other Hydrocarbons/Oxygenates for clarification.

- Production

- Isobutane has been renamed Isobutane/Isobutylene under Liquefied Petroleum Gases for clarification.

- Unleaded and leaded motor gasoline categories have been replaced with the new types of gasolines produced: reformulated, oxygenated, and other.

- A new line has been added to display field production of motor gasoline blending components.

- Imports

- Isobutane has been renamed Isobutane/Isobutylene under Liquefied Petroleum Gases for clarification.

- Unleaded and leaded motor gasoline categories have been replaced with the new types of gasolines produced: reformulated, oxygenated, and other.

- Stocks

- Other hydrocarbons has been renamed Other Hydrocarbons/Oxygenates for clarification.

- Isobutane has been renamed Isobutane/Isobutylene under Liquefied Petroleum Gases for clarification.

- Unleaded and leaded motor gasoline categories have been replaced with the new types of gasolines produced: reformulated, oxygenated, and other.

- Product Supplied

- Isobutane has been renamed Isobutane/Isobutylene under Liquefied Petroleum Gases for clarification.

- Unleaded and leaded motor gasoline categories have been replaced with the new types of gasolines produced: reformulated, oxygenated, and other. 


\section{Changes in Appendix D}

- Table D1. U.S. Summary Table

- Data on oxygenates blended into motor gasoline has been eliminated. This information is no longer collected on the survey EIA-819M, "Monthly Oxygenate Telephone Report."

- Table D2. Monthly Fuel Ethanol Production and Ending Stocks

- Data for the previous year as well as current year are displayed.

- Data on oxygenates blended into motor gasoline has been eliminated. This information is no longer collected on the survey EIA-819M, "Monthly Oxygenate Telephone Report."

- Data for fuel ethanol imports has been dropped due to small volumes reported by respondents.

- Table D3. Monthly MTBE Production and Ending Stocks

- Data for the previous year as well as current year are displayed.

- Data on oxygenates blended into motor gasoline has been eliminated. This information is no longer collected on the survey EIA-819M, "Monthly Oxygenate Telephone Report."

- Data on MTBE imports has been dropped from the table due to small volumes reported by respondents.

\section{Note 20. 1994 Changes in the Petroleum Supply Reporting System}

Effective with January 1994 data, several enhancements were made to the tables to reflect changes in the petroleum industry and to provide more meaningful petroleum statistics. These changes primarily affect data reported for imports, exports, and product supplied.
- On December 31, 1992, Ecuador withdrew as a member of the Organization of Petroleum Exporting Countries (OPEC). As of January 1994, imports of petroleum from Ecuador now appear under imports from Non-OPEC sources. No revision was made to 1993 data. This change is evident in Tables S3 and 35 through 44, 49 and 50.

- Exports data are now published for oxygenates and the sub-categories of finished motor gasoline (reformulated, oxygenated, and other) and distillate fuel oil $(0.05 \%$ sulfur and under, and greater than $0.05 \%$ sulfur).

- Product supplied is now calculated for reformulated, oxygenated, and other finished motor gasoline as well as the sulfur categories of distillate fuel oil $(0.05 \%$ sulfur and under, and greater than $0.05 \%$ sulfur).

\section{Note 21. 1995 Changes in the Petroleum Supply Reporting System}

- Annual U.S. refinery capacity data collection and publication normally presented each year in Volume 1 of the PSA has been moved to a biennial schedule (every other year). Collection and publication of January 1, 1996 refinery capacity data did not occur.

- Annual U.S. oxygenate production capacity data collection and publication normally presented each year in Volume 1 of the PSA has been eliminated. This information was first collected by EIA to effectively monitor the transition of reformulated motor gasoline into the market.

\section{Note 22. 1997 Changes in the Petroleum Supply Reporting System}

- During 1997, Zaire became the Democratic Republic of the Congo. Zaire has been changed to read Congo (Kinshasa). This change is evident in Tables 21 through 25, and Table 29.

- The next year collection of refinery capacity data will occur is 1999 and will present refinery capacity data as of January 1, 1999. 


\section{Note 23. Motor Gasoline Blending Plants Operating During 1997}

Amoco Oil Co.

Forest View, IL

Milwaukee, WI

Ashland Oil Inc.

Clarksville, IN

Covington, $\mathrm{KY}$

Clark Refg. \& Mktg. Inc.

Blue Island, IL

Milwaukee, WI

Citgo Petroleum Corp.

East Chicago, IN

Linden, NJ

Milwaukee, WI

Mt. Prospect, IL

Richmond, VA

Selma, NC

GATX Terminals Corp.

Carteret, NJ

Pasadena, TX

Staten Island, NY
Getty Petro. Corp.

East Providence, RI

New Haven, CT

Newark, NJ

Rensselaer, NY

Harrisburg, PA

Global Petroleum Corp.

Revere, MA

Golden West Refining Co.

Sante Fe Springs, CA

Hartford/WoodRiver Term. Hartford, IL

Int'l Matex Tank Term.

Bayonne, NJ

Itochu International Inc.

Sewaren, NJ

Marathon Oil Co.

Hammond, IN

Mt. Prospect, IL

Willow Springs, IL

Mobil Oil Corp.

Arlington Heights, IL

Hammond, IN

Lockport, IL
New Haven Term, Inc.

East Haven, CT

Northville Industries Corp.

Linden, NJ

Oiltanking Houston Inc.

Houston, TX

Phillips 66 Co.

Forsythe, IL

East Chicago, IN

Phillips Pipeline Co.

Denver, CO

East Saint Louis, IL

Santa Fe Pacific Pipeline Phoenix, AZ

Shell Oil Co.

Argo, IL

Carson, CA

Des Plaines, IL

Sinclair Oil Corp.

Denver, CO
Stolthaven Inc.

Perth Amboy, NJ

Texaco Inc.

Phoenix, AZ

Tucson, AZ

Unocal Corp.

Beaumont, TX

Westec Petro. Inc.

Denver, CO

Westfrac Inc. Blending

Grand Junction, $\mathrm{CO}$

Williams Pipeline Co.

Des Moines, IA

Iowa City, IA 


\section{Appendix C}

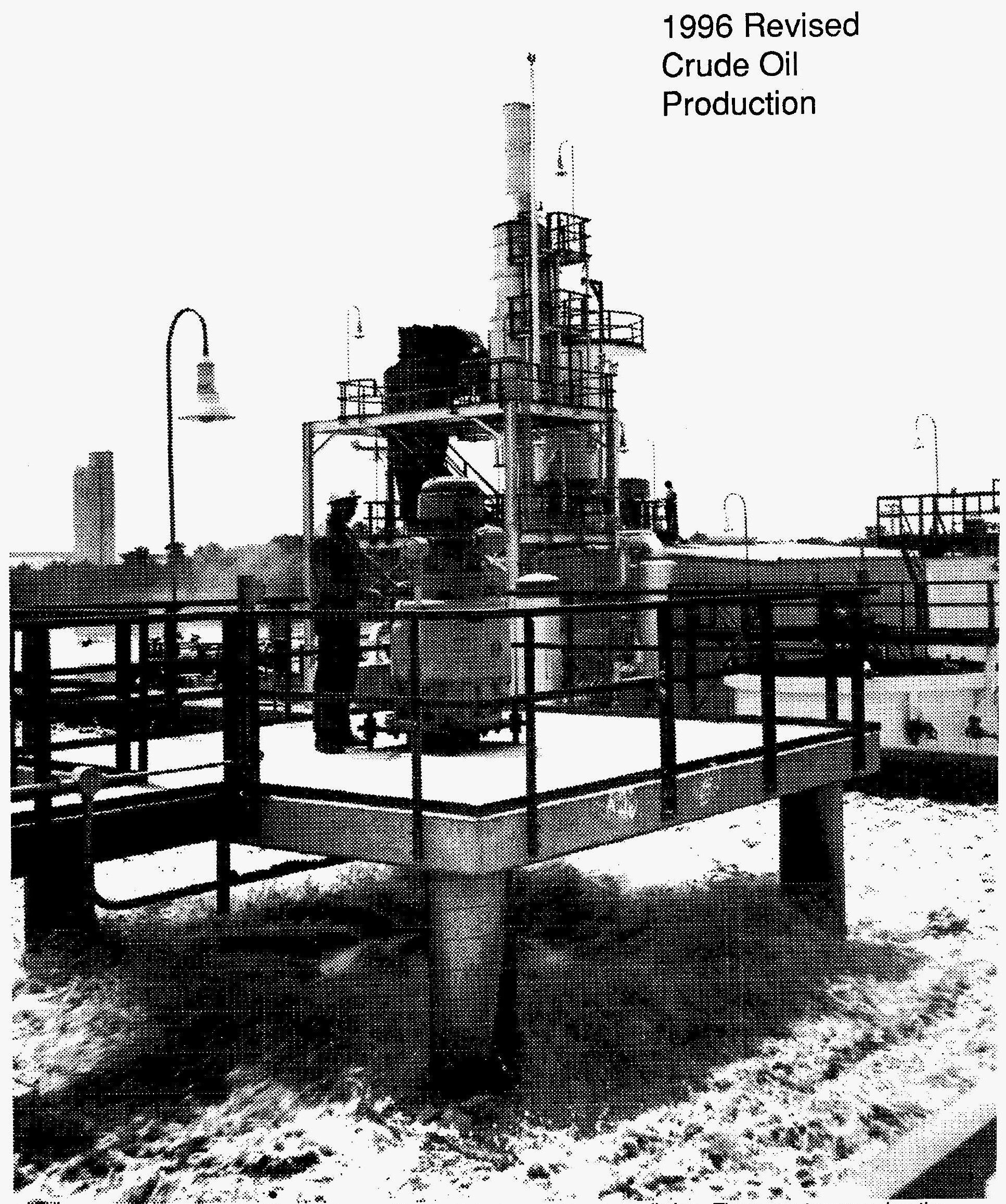

Surface aerators are used at U.S. petroleum refineries to help prevent water pollution. These aerators speed up the oxidation process by beating air into water. 
Table C1. Revised ${ }^{a}$ Crude Oil Production by PAD District and State, 1996 (Thousand Barrels)

\begin{tabular}{|c|c|c|c|c|c|c|c|}
\hline PAD District and State & January & February & March & April & May & June & July \\
\hline 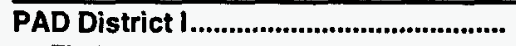 & 701 & 649 & 769 & 868 & 874 & 844 & 892 \\
\hline Florida & 447 & 385 & 474 & 553 & 560 & 544 & 579 \\
\hline 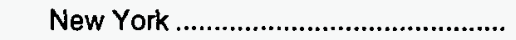 & 20 & 19 & 23 & 28 & 26 & 25 & 28 \\
\hline 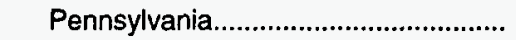 & 119 & 125 & 136 & 149 & 148 & 137 & 142 \\
\hline 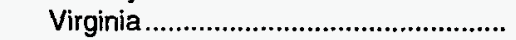 & 1 & 1 & 1 & 2 & 1 & 1 & 1 \\
\hline 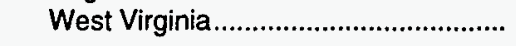 & 113 & 119 & 135 & 137 & 139 & 136 & 142 \\
\hline 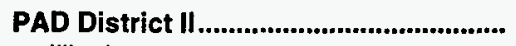 & 17,355 & 16,394 & 16,627 & 18,270 & 17,488 & 16,815 & 17,555 \\
\hline 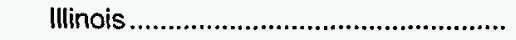 & 1,225 & 1,110 & 1,375 & 1,260 & 1,310 & 1,295 & 1,310 \\
\hline 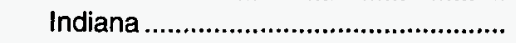 & 212 & 201 & 202 & 216 & 196 & 194 & 224 \\
\hline 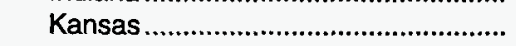 & 3,468 & 3,382 & 3,425 & 3,546 & 3,619 & 3,409 & 3,573 \\
\hline 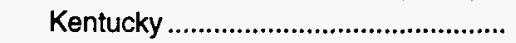 & 328 & 383 & 84 & 463 & 276 & 272 & 389 \\
\hline 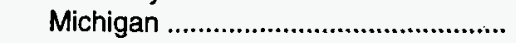 & 1,016 & 902 & 214 & 1,592 & 877 & 924 & 1,052 \\
\hline 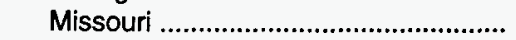 & 10 & 8 & 11 & 10 & 10 & 10 & 10 \\
\hline 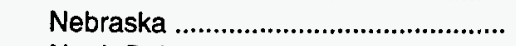 & 298 & 284 & 306 & 293 & 298 & 296 & 300 \\
\hline 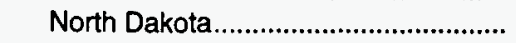 & 2,565 & 2,419 & 2,663 & 2,618 & 2,708 & 2,674 & 2,807 \\
\hline 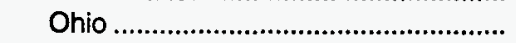 & 673 & 678 & 727 & 778 & 712 & 611 & 654 \\
\hline 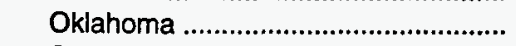 & 7,424 & 6,894 & 7,480 & 7,354 & 7,341 & 6,996 & 7,096 \\
\hline 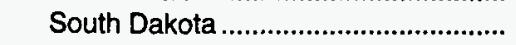 & 104 & 101 & 110 & 107 & 107 & 103 & 107 \\
\hline 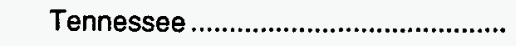 & 31 & 32 & 30 & 35 & 32 & 31 & 34 \\
\hline PAD District III.......................................... & 98,389 & 92,476 & 99,385 & 96,076 & 98,714 & 98,605 & 97,512 \\
\hline 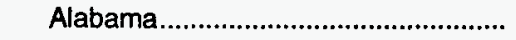 & 1,461 & 1,305 & 1,500 & 1,449 & 1,459 & 1,394 & 1,394 \\
\hline 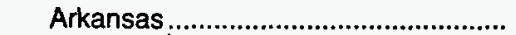 & 821 & 731 & 806 & 782 & 796 & 763 & 747 \\
\hline 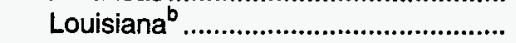 & 10,323 & 10,375 & 11,538 & 11,628 & 11,438 & 11,015 & 11,204 \\
\hline 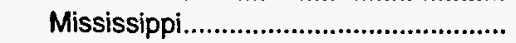 & 1,638 & 1,522 & 1,708 & 1,627 & 1,617 & 1,532 & 1,620 \\
\hline 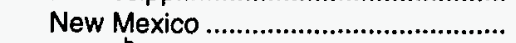 & 6,434 & 5,952 & 5,933 & 6,124 & 6,175 & 5,816 & 5,990 \\
\hline 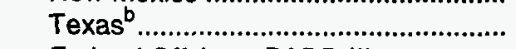 & 46,721 & 43,421 & 47,044 & 45,295 & 46,331 & 44,336 & 45,425 \\
\hline Federal Offshore PADD III ................... & 30,991 & 29,170 & 30,857 & 29,171 & 30,898 & 33,751 & 31,131 \\
\hline PAD District IV & 11,412 & 10,819 & 11,220 & 10,944 & 11,018 & 10,720 & 11,053 \\
\hline 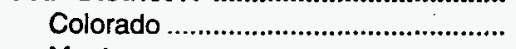 & 2,108 & 1,984 & 2,062 & 2,062 & 2,010 & 1,990 & 2,077 \\
\hline Montana & 1,289 & 1,240 & 1,355 & 1,331 & 1,377 & 1,329 & 1,385 \\
\hline Utah & 1,669 & 1,555 & 1,647 & 1,602 & 1,635 & 1,564 & 1,623 \\
\hline Wyoming & 6,345 & 6,041 & 6,155 & 5,948 & 5,995 & 5,837 & 5,968 \\
\hline 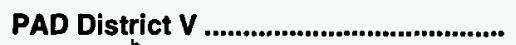 & 74,373 & 70,983 & 75,219 & 69,445 & 71,067 & 71,479 & 70,169 \\
\hline Alaska $\mathrm{b}$ & 44,767 & 42,988 & 45,086 & 41,014 & 41,573 & 42,581 & 40,821 \\
\hline 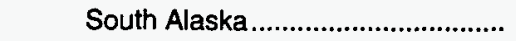 & 1,380 & 1,225 & 1,294 & 1,228 & 1,262 & 1,201 & 1,243 \\
\hline 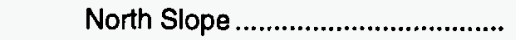 & 43,387 & 41,763 & 43,792 & 39,786 & 40,311 & 41,380 & 39,578 \\
\hline 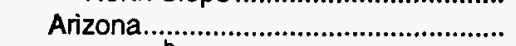 & 6 & 5 & 6 & 6 & 6 & 8 & 8 \\
\hline California $^{b}$ & 23,740 & 22,245 & 23,968 & 22,753 & 24,068 & 23,325 & 23,865 \\
\hline 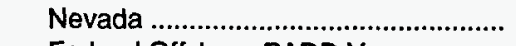 & 99 & 91 & 91 & 90 & 89 & 86 & 87 \\
\hline Federal Offshore PADD V.................... & 5,761 & 5,654 & 6,067 & 5,582 & 5,330 & 5,479 & 5,388 \\
\hline 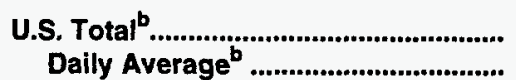 & $\begin{array}{r}202,230 \\
6,524\end{array}$ & $\begin{array}{r}191,321 \\
6,833\end{array}$ & $\begin{array}{r}203,219 \\
6,555\end{array}$ & $\begin{array}{r}195,603 \\
6,520\end{array}$ & $\begin{array}{r}199,161 \\
6,425\end{array}$ & $\begin{array}{r}198,463 \\
6,615\end{array}$ & $\begin{array}{r}197,181 \\
6,361\end{array}$ \\
\hline
\end{tabular}

This table contains updates on 1996 crude oil production statistics published in the Petroleum Supply Annual (PSA), 1996.

Statistics on crude oil production for States and for Federal offshore areas are reported to the Energy Information Administration (EIA) by State government agencies and by the Minerals Management Service, U.S. Department of the Interior. These data are updated periodically by the reporting agencies and are received by the EIA on an ongoing basis. At the time of publication of the 1996 PSA, the EIA had not received complete and/or updated statistics on crude oil production for several States. This table is provided to inform the user of updated monthly and annual crude oil production statistics for 1996, and are not subject to further revision by the EIA. 
Table C1. Revised ${ }^{\mathrm{a}}$ Crude Oil Production by PAD District and State, 1996 (Continued) (Thousand Barrels)

\begin{tabular}{|c|c|c|c|c|c|c|c|}
\hline PAD District and State & August & September & October & November & December & Total & $\begin{array}{c}\text { Daily } \\
\text { Average }\end{array}$ \\
\hline PAD District I & 861 & 823 & 867 & 835 & 866 & 9,849 & 27 \\
\hline Florida & 565 & 537 & 541 & 539 & 569 & 6,292 & 17 \\
\hline New York & 25 & 31 & 30 & 25 & 28 & 309 & 1 \\
\hline 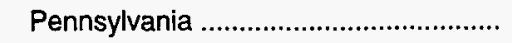 & 148 & 139 & 156 & 146 & 145 & 1,692 & 5 \\
\hline Virginia & 1 & 1 & 1 & 1 & 1 & 13 & (s) \\
\hline 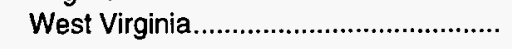 & 122 & 114 & 139 & 124 & 123 & 1,544 & 4 \\
\hline PAD District II & 17,049 & 17,006 & 17,459 & 16,272 & 17,246 & 205,538 & 563 \\
\hline |llinois & 1,400 & 1,390 & 1,360 & 1,350 & 1,290 & 15,675 & 43 \\
\hline Indiana & 218 & 214 & 226 & 197 & 223 & 2,523 & 7 \\
\hline 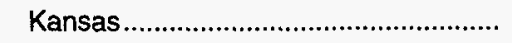 & 3,497 & 3,432 & 3,607 & 3,144 & 3,499 & 41,599 & 114 \\
\hline 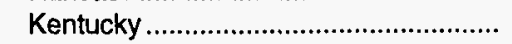 & 1 & 431 & 279 & 261 & 280 & 3,448 & 9 \\
\hline 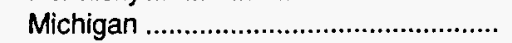 & 822 & 837 & 743 & 1,024 & 736 & 10,740 & 29 \\
\hline 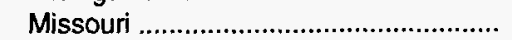 & 9 & 9 & 10 & 10 & 9 & 115 & (s) \\
\hline 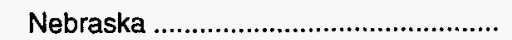 & 297 & 291 & 302 & 287 & 290 & 3,541 & 10 \\
\hline 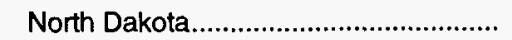 & 2,820 & 2,761 & 2,872 & 2,674 & 2,734 & 32,317 & 89 \\
\hline Ohio & 654 & 698 & 744 & 662 & 714 & 8,305 & 23 \\
\hline 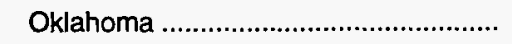 & 7,194 & 6,810 & 7,178 & 6,532 & 7,336 & 85,636 & 235 \\
\hline 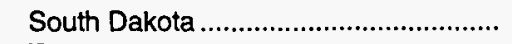 & 105 & 104 & 104 & 102 & 103 & 1,257 & 3 \\
\hline 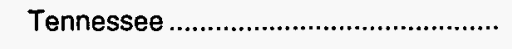 & 31 & 30 & 34 & 29 & 32 & 381 & 1 \\
\hline PAD District III & 98,737 & 95,826 & 97,348 & 96,476 & 99,562 & $1,169,108$ & 3,203 \\
\hline 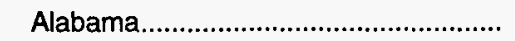 & 1,446 & 1,364 & 1,417 & 1,334 & 1,343 & 16,868 & 46 \\
\hline 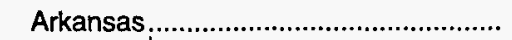 & 762 & 728 & 716 & 721 & 717 & 9,088 & 25 \\
\hline Louisianab & 12,011 & 11,148 & 11,264 & 10,961 & 11,457 & 134,363 & 368 \\
\hline 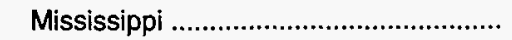 & 1,623 & 1,593 & 1,740 & 1,646 & 1,668 & 19,535 & 54 \\
\hline 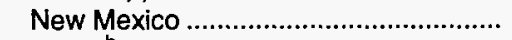 & 6,083 & 5,905 & 5,652 & 5,886 & 6,054 & 72,004 & 197 \\
\hline Texas $^{b}$ & 45,249 & 44,112 & 45,879 & 44,492 & 45,885 & 544,191 & 1,491 \\
\hline Federal Offshore PADD III ..................... & 31,562 & 30,975 & 30,680 & 31,435 & 32,438 & 373,059 & 1,022 \\
\hline PAD District IV & 11,308 & 11,109 & 11,268 & 11,070 & 11,273 & 133,213 & 365 \\
\hline 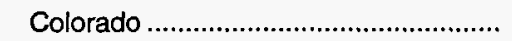 & 1,954 & 1,967 & 2,032 & 1,952 & 1,971 & 24,170 & 66 \\
\hline 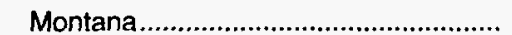 & 1,393 & 1,354 & 1,427 & 1,344 & 1,350 & 16,173 & 44 \\
\hline 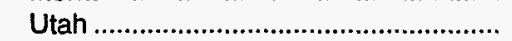 & 1,636 & 1,638 & 1,696 & 1,616 & 1,622 & 19,503 & 53 \\
\hline 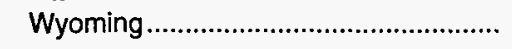 & 6,325 & 6,151 & 6,113 & 6,158 & 6,331 & 73,368 & 201 \\
\hline PAD District V & 70,463 & 70,382 & 72,612 & $\mathbf{7 0 , 4 4 5}$ & 72,099 & 858,736 & 2,353 \\
\hline Alaska $^{\mathrm{b}}$ & 41,126 & 42,019 & 43,521 & 42,104 & 43,166 & 510,766 & 1,399 \\
\hline 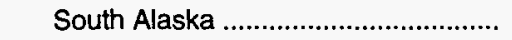 & 1,168 & 1,140 & 1,178 & 1,119 & 1,148 & 14,586 & 40 \\
\hline 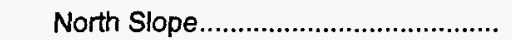 & 39,958 & 40,879 & 42,343 & 40,985 & 42,018 & 496,179 & 1,359 \\
\hline Arizona & 8 & 8 & 8 & 8 & 6 & 84 & (s) \\
\hline California $^{b} \ldots \ldots \ldots \ldots \ldots$ & 24,082 & 23,235 & 23,888 & 23,379 & 23,862 & 282,409 & 774 \\
\hline Nevada & 86 & 89 & 90 & 78 & 82 & 1,058 & 3 \\
\hline Federal Offshore PADD V....................... & 5,161 & 5,031 & 5,106 & 4,877 & 4,983 & 64,419 & 176 \\
\hline $\begin{array}{l}\text { U.S. Total } \\
\text { Daily Average }\end{array}$ & $\begin{array}{r}198,418 \\
6,401\end{array}$ & $\begin{array}{r}195,147 \\
6.505\end{array}$ & $\begin{array}{r}199,554 \\
6,437\end{array}$ & $\begin{array}{r}195,099 \\
6,503\end{array}$ & $\begin{array}{r}201,047 \\
6,485\end{array}$ & $\begin{array}{r}2,376,444 \\
6.511\end{array}$ & 6,511 \\
\hline
\end{tabular}

\footnotetext{
${ }^{a}$ Data are based upon revisions received as of April 1998.

b Includes the following offshore production (thousand barrels): Alaska: State - 96,122; California: State - 20,032; Louisiana: State - 22,774; Texas: State - 1,$081 ;$ U.S. Total, including Federal Offshore - 577,488.

Note: • Production data are revised from those published in the Petroleum Supply Annual 1996 for the following States: Alabama, Alaska, Arizona, Arkansas, California, Colorado, Federal Offshore PAD District III, Federal Offshore PAD District V, Florida, Illinois, Indiana, Kansas, Kentucky, Louisiana, Michigan, Mississippi, Missouri, Montana, Nebraska, Nevada, New Mexico, New York, North Dakota, Ohio, Oklahoma, South Dakota, Texas, Utah, West Virginia, and Wyoming. - Annual crude oil production for New York, and Pennsylvania was prorated by month based on first purchaser monthly crude oil volumes collected on Form EIA-182, "Domestic Crude oil First Purchase Report." Pennsylvania data are based on EIA estimate from form EIA-182. - Totals may not equal sum of components due to independent rounding.

Source: State Conservation agencies, U.S. Department of the Interior, Minerals Management Service and the Conservation Committee of California Oil and Gas Producers.
} 


\section{Glossary}

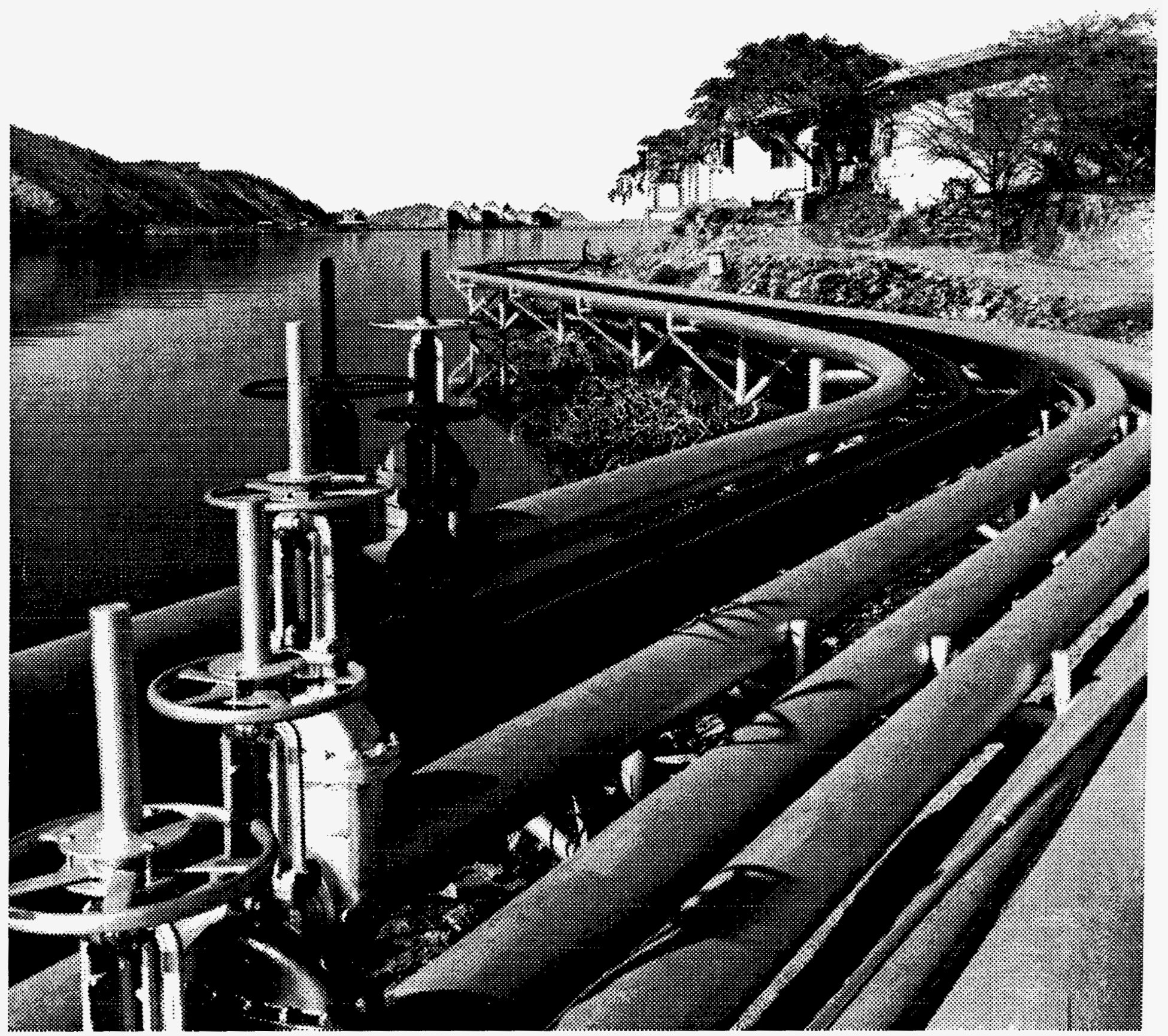

\footnotetext{
Pipelines carry natural gas across geographic regions.
} 


\section{Definitions of Petroleum Products and Other Terms}

Alcohol. The family name of a group of organic chemical compounds composed of carbon, hydrogen, and oxygen. The series of molecules vary in chain length and are composed of a hydrocarbon plus a hydroxyl group; $\mathrm{CH}_{3}-$ $\left(\mathrm{CH}_{2}\right)$ n-OH (e.g., methanol, ethanol, and tertiary butyl alcohol).

Alkylate. The product of an alkylation reaction. It usually refers to the high octane product from alkylation units. This alkylate is used in blending high octane gasoline.

Alkylation. A refining process for chemically combining isobutane with olefin hydrocarbons (e.g., propylene, butylene) through the control of temperature and pressure in the presence of an acid catalyst, usually sulfuric acid or hydrofluoric acid. The product, alkylate, an isoparaffin, has high octane value and is blended with motor and aviation gasoline to improve the antiknock value of the fuel.

API Gravity. An arbitrary scale expressing the gravity or density of liquid petroleum products. The measuring scale is calibrated in terms of degrees API; it may be calculated in terms of the following formula:

$$
\text { Degrees } A P I=\frac{141.5}{s p . g r .60^{\circ} \mathrm{F} / 60^{\circ} \mathrm{F}}-131.5
$$

The higher the API gravity, the lighter the compound. Light crudes generally exceed 38 degrees API and heavy crudes are commonly labeled as all crudes with an API gravity of 22 degrees or below. Intermediate crudes fall in the range of 22 degrees to 38 degrees API gravity.

Aromatics. Hydrocarbons characterized by unsaturated ring structures of carbon atoms. Commercial petroleum aromatics are benzene, toluene, and xylene (BTX).

Asphalt. A dark-brown-to-black cement-like material containing bitumens as the predominant constituent obtained by petroleum processing. The definition includes crude asphalt as well as the following finished products: cements, fluxes, the asphalt content of emulsions (exclusive of water), and petroleum distillates blended with asphalt to make cutback asphalts. The conversion factor for asphalt is 5.5 barrels per short ton.

ASTM. The acronym for the American Society for Testing and Materials.
Atmospheric Crude Oil Distillation. The refining process of separating crude oil components at atmospheric pressure by heating to temperatures of about $600^{\circ}$ to $750^{\circ}$ $F$ (depending on the nature of the crude oil and desired products) and subsequent condensing of the fractions by cooling.

Aviation Gasoline (Finished). All special grades of gasoline for use in aviation reciprocating engines, as given in ASTM Specification D910 and Military Specification MIL-G-5572. Excludes blending components which will be used in blending or compounding into finished aviation gasoline.

Aviation Gasoline Blending Components. Naphthas which will be used for blending or compounding into finished aviation gasoline (e.g., straight-run gasoline, alkylate, reformate, benzene, toluene, and xylene). Excludes oxygenates (alcohols, ethers), butane, and pentanes plus. Oxygenates are reported as other hydrocarbons, hydrogen, and oxygenates.

Barrel. A volumetric unit of measure for crude oil and petroleum products equivalent to 42 U.S. gallons. This measure is used in most statistical reports. Factors for converting petroleum coke, asphalt, still gas and wax to barrels are given in the definitions of these products.

Barrels Per Calendar Day. The maximum number of barrels of input that can be processed during a 24-hour period after making allowances for the following limitations:

the capability of downstream facilities to absorb the output of crude oil processing facilities of a given refinery. No reduction is made when a planned distribution of intermediate streams through other than downstream facilities is part of a refinery's normal operation;

the types and grades of inputs to be processed;

the types and grades of products expected to be manufactured;

the environmental constraints associated with refinery operations;

the reduction of capacity for scheduled downtime such as routine inspection, mechanical problems, maintenance, repairs, and turnaround; and 
the reduction of capacity for unscheduled downtime such as mechanical problems, repairs, and slowdowns.

Barrels Per Stream Day. The amount a unit can process running at full capacity under optimal crude oil and product slate conditions.

Benzene $\left(\mathrm{C}_{6} \mathrm{H}_{6}\right)$. An aromatic hydrocarbon present in small proportion in some crude oils and made commercially from petroleum by the catalytic reforming of naphthenes in petroleum naphtha. Also made from coal in the manufacture of coke. Used as a solvent, in manufacturing detergents, synthetic fibers, and petrochemicals and as a component of high-octane gasoline.

Blending Components. See Motor or Aviation Gasoline Blending Components.

Blending Plant. A facility which has no refining capability but is either capable of producing finished motor gasoline through mechanical blending or blends oxygenates with motor gasoline.

Bonded Petroleum Imports. Petroleum imported and entered into Customs bonded storage. These imports are not included in the import statistics until they are: (1) withdrawn from storage free of duty for use as fuel for vessels and aircraft engaged in international trade; or (2) withdrawn from storage with duty paid for domestic use.

$B T X$. The acronym for the commercial petroleum aromatics benzene, toluene, and xylene. See individual categories for definitions.

Bulk Station. A facility used primarily for the storage and/or marketing of petroleum products which has a total bulk storage capacity of less than 50,000 barrels and receives its petroleum products by tank car or truck.

Bulk Terminal. A facility used primarily for the storage and/or marketing of petroleum products which has a total bulk storage capacity of 50,000 barrels or more and/or receives petroleum products by tanker, barge, or pipeline.

Butane $\left(\mathrm{C}_{4} \mathrm{H}_{10}\right)$. A normally gaseous straight-chain or branch-chain hydrocarbon extracted from natural gas or refinery gas streams. It includes isobutane and normal butane and is designated in ASTM Specification D1835 and Gas Processors Association Specifications for commercial butane.

Isobutane $\left(\mathrm{C}_{4} \mathrm{H}_{10}\right)$. A normally gaseous branch-chain hydrocarbon. It is a colorless paraffinic gas that boils at a temperature of $10.9^{\circ} \mathrm{F}$. It is extracted from natural gas or refinery gas streams.
Normal Butane $\left(\mathrm{C}_{4} \mathrm{H}_{10}\right)$. A normally gaseous straightchain hydrocarbon. It is a colorless paraffinic gas that boils at a temperature of $31.1^{\circ} \mathrm{F}$. It is extracted from natural gas or refinery gas streams.

Butylene $\left(\mathrm{C}_{4} \mathrm{H}_{8}\right)$. An olefinic hydrocarbon recovered from refinery processes.

Captive Refinery Oxygenate Plants. Oxygenate production facilities located within or adjacent to a refinery complex.

Catalytic Cracking. The refining process of breaking down the larger, heavier, and more complex hydrocarbon molecules into simpler and lighter molecules. Catalytic cracking is accomplished by the use of a catalytic agent and is an effective process for increasing the yield of gasoline from crude oil. Catalytic cracking processes fresh feeds and recycled feeds.

Fresh Feeds. Crude oil or petroleum distillates which are being fed to processing units for the first time.

Recycled Feeds. Feeds that are continuously fed back for additional processing.

Catalytic Hydrocracking. A refining process that uses hydrogen and catalysts with relatively low temperatures and high pressures for converting middle boiling or residual material to high-octane gasoline, reformer charge stock, jet fuel, and/or high grade fuel oil. The process uses one or more catalysts, depending upon product output, and can handle high sulfur feedstocks without prior desulfurization.

Catalytic Hydrotreating. A refining process for treating petroleum fractions from atmospheric or vacuum distillation units (e.g., naphthas, middle distillates, reformer feeds, residual fuel oil, and heavy gas oil) and other petroleum (e.g., cat cracked naphtha, coker naphtha, gas oil, etc.) in the presence of catalysts and substantial quantities of hydrogen. Hydrotreating includes desulfurization, removal of substances (e.g., nitrogen compounds) that deactivate catalysts, conversion of olefins to paraffins to reduce gum formation in gasoline, and other processes to upgrade the quality of the fractions.

Catalytic Reforming. A refining process using controlled heat and pressure with catalysts to rearrange certain hydrocarbon molecules, thereby converting paraffinic and naphthenic type hydrocarbons (e.g., low-octane gasoline boiling range fractions) into petrochemical feedstocks and higher octane stocks suitable for blending into finished gasoline. Catalytic reforming is reported in two categories. They are: 
Low Pressure. A processing unit operating at less than 225 pounds per square inch gauge (PSIG) measured at the outlet separator.

High Pressure. A processing unit operating at either equal to or greater than 225 pounds per square inch gauge (PSIG) measured at the outlet separator.

Charge Capacity. The input (feed) capacity of the refinery processing facilities.

Coal. A black or brownish-black solid combustible substance formed by the partial decomposition of vegetable matter without access to air. The rank of coal, which includes anthracite, bituminous coal, subbituminous coal, and lignite, is based on fixed carbon, volatile matter, and heating value. Coal rank indicates the progressive alteration, or coalification, from lignite to anthracite. Lignite contains approximately 9 to 17 million BTU per ton. The heat contents of subbituminous and bituminous coal range from 16 to 24 million BTU per ton, and from 19 to 30 million BTU per ton, respectively. Anthracite contains approximately 22 to 28 million BTU per ton.

\section{Commercial Kerosene-Type Jet Fuel. See Kerosene- Type Jet Fuel.}

Crude Oil (Including Lease Condensate). A mixture of hydrocarbons that exists in liquid phase in underground reservoirs and remains liquid at atmospheric pressure after passing through surface-separating facilities. Included are lease condensate and liquid hydrocarbons produced from tar sands, gilsonite, and oil shale. Drip gases are also included, but topped crude oil (residual oil) and other unfinished oils are excluded. Liquids produced at natural gas processing plants and mixed with crude oil are likewise excluded where identifiable. Crude oil is considered as either domestic or foreign, according to the following:

Domestic. Crude oil produced in the United States or from its "outer continental shelf" as defined in 43 USC 1331 .

Foreign. Crude oil produced outside the United States. Imported Athabasca hydrocarbons (tar sands from Canada) are included.

Crude Oil, Refinery Receipts. Receipts of domestic and foreign crude oil at a refinery. Includes all crude oil in transit except crude oil in transit by pipeline. Foreign crude oil is reported as a receipt only after entry through customs. Crude oil of foreign origin held in bonded storage is excluded.

Crude Oil Losses. Represents the volume of crude oil reported by petroleum refineries as being lost in their operations. These losses are due to spills, contamination, fires, etc. as opposed to refinery processing losses.

Crude Oil Production. The volume of crude oil produced from oil reservoirs during given periods of time. The amount of such production for a given period is measured as volumes delivered from lease storage tanks (i.e., the point of custody transfer) to pipelines, trucks, or other media for transport to refineries or terminals with adjustments for (1) net differences between opening and closing lease inventories, and (2) basic sediment and water (BS\&W).

Crude Oil Qualities. Refers to two properties of crude oil, the sulfur content and API gravity, which affect processing complexity and product characteristics.

Delayed Coking. A process by which heavier crude oil fractions can be thermally decomposed under conditions of elevated temperatures and pressure to produce a mixture of lighter oils and petroleum coke. The light oils can be processed further in other refinery units to meet product specifications. The coke can be used either as a fuel or in other applications such as the manufacturing of steel or aluminum.

Disposition. The components of petroleum disposition are stock change, crude oil losses, refinery inputs, exports, and products supplied for domestic consumption.

Distillate Fuel Oil. A general classification for one of the petroleum fractions produced in conventional distillation operations. It is used primarily for space heating, on-andoff-highway diesel engine fuel (including railroad engine fuel and fuel for agricultural machinery), and electric power generation. Included are products known as No. 1 , No. 2, and No. 4 fuel oils; No. 1, No. 2, and No. 4 diesel fuels. Distillate fuel oil is reported in the following sulfur categories: $0.05 \%$ sulfur and under, for use in on-highway diesel engines which could be described as meeting EPA regulations; and greater than $0.05 \%$ sulfur, for use in all other distillate applications.

No. 1 Distillate. A petroleum distillate which meets the specifications for No. 1 heating or fuel oil as defined in ASTM D 396 and/or the specifications for No. 1 diesel fuel as defined in ASTM Specification D 975 with distillation temperatures of $420^{\circ} \mathrm{F}$ at the 10-percent recovery point and $550^{\circ} \mathrm{F}$ at the 90 -percent recovery point, and kinematic viscosities between 1.4 and 2.2 centistokes at $100^{\circ} \mathrm{F}$.

No. 2 Distillate. A petroleum distillate which meets the specifications for No. 2 heating or fuel oil as defined in ASTM D 396 and/or the specifications for No. 2 diesel fuel as defined in ASTM Specification D 975 with distillation temperatures of $540^{\circ}$ and $640^{\circ} \mathrm{F}$ at the 90 - 
percent recovery point, and kinematic viscosities between 2.0 and 4.3 centistokes at $100^{\circ} \mathrm{F}$.

No. 4 Fuel Oil. A fuel oil for commercial burner installations not equipped with preheating facilities. It is used extensively in industrial plants. This grade is a blend of distillate fuel oil and residual fuel oil stocks that conforms to ASTM Specification D396 or Federal Specification VV-F-815C; with minimum and maximum kinematic viscosities between 5.8 and 26.4 centistokes at $100^{\circ} \mathrm{F}$. Also included is No. 4-D, a fuel oil for low and medium-speed diesel engines that conforms to ASTM Specification D975.

Electricity (Purchased). Electricity purchased for refinery operations that is not produced within the refinery complex.

Ending Stocks. Primary stocks of crude oil and petroleum products held in storage as of 12 midnight on the last day of the month. Primary stocks include crude oil or petroleum products held in storage at (or in) leases, refineries, natural gas processing plants, pipelines, tank farms, and bulk terminals that can store at least 50,000 barrels of petroleum products or that can receive petroleum products by tanker, barge, or pipeline. Crude oil that is in-transit by water from Alaska, or that is stored on Federal leases or in the Strategic Petroleum Reserve is included. Primary Stocks exclude stocks of foreign origin that are held in bonded warehouse storage.

ETBE (Ethyl tertiary butyl ether) $\left(\mathrm{CH}_{3}\right)_{3} \mathrm{COC}_{2} \mathrm{H}_{5}$. An oxygenate blend stock formed by the catalytic etherification of isobutylene with ethanol.

Ethane $\left(\mathrm{C}_{2} \mathrm{H}_{6}\right)$. A normally gaseous straight-chain hydrocarbon. It is a colorless paraffinic gas that boils at a temperature of $-127.48^{\circ} \mathrm{F}$. It is extracted from natural gas and refinery gas streams.

Ether. A generic term applied to a group of organic chemical compounds composed of carbon, hydrogen, and oxygen, characterized by an oxygen atom attached to two carbon atoms (e.g., methyl tertiary butyl ether).

Ethylene $\left(\mathrm{C}_{2} \mathrm{H}_{4}\right)$. An olefinic hydrocarbon recovered from refinery processes or petrochemical processes.

Exports. Shipments of crude oil and petroleum products from the 50 States and the District of Columbia to foreign countries, Puerto Rico, the Virgin Islands, and other U.S. possessions and territories.

Field Production. Represents crude oil production on leases, natural gas liquids production at natural gas processing plants, new supply of other hydrocarbons/ oxygenates and motor gasoline blending components, and fuel ethanol blended into finished motor gasoline.

Flexicoking. A thermal cracking process which converts heavy hydrocarbons such as crude oil, tar sands bitumen, and distillation residues into light hydrocarbons. Feedstocks can be any pumpable hydrocarbons including those containing high concentrations of sulfur and metals.

Fluid Coking. A thermal cracking process utilizing the fluidized-solids technique to remove carbon (coke) for continuous conversion of heavy, low-grade oils into lighter products.

Fresh Feed Input. Represents input of material (crude oil, unfinished oils, natural gas liquids, other hydrocarbons and oxygenates or finished products) to processing units at a refinery that is being processed (input) into a particular unit for the first time.

\section{Examples:}

(1) Unfinished oils coming out of a crude oil distillation unit which are input into a catalytic cracking unit are considered fresh feed to the catalytic cracking unit.

(2) Unfinished oils coming out of a catalytic cracking unit being looped back into the same catalytic cracking unit to be reprocessed are not considered fresh feed.

Fuel Ethanol $\left(\mathrm{C}_{2} \mathrm{H}_{5} \mathrm{OH}\right)$. An anhydrous denatured aliphatic alcohol intended for gasoline blending as described in Oxygenates definition.

Fuels Solvent Deasphalting. A refining process for removing asphalt compounds from petroleum fractions, such as reduced crude oil. The recovered stream from this process is used to produce fuel products.

Gas Oil. A liquid petroleum distillate having a viscosity intermediate between that of kerosene and lubricating oil. It derives its name from having originally been used in the manufacture of illuminating gas. It is now used to produce distillate fuel oils and gasoline.

Gasohol. A blend of finished motor gasoline and alcohol (generally ethanol but sometimes methanol), limited to 10 percent by volume of alcohol.

Gasoline Blending Components. Naphthas which will be used for blending or compounding into finished aviation or motor gasoline (e.g., straight-run gasoline, alkylate, reformate, benzene, toluene, and xylene). Excludes oxygenates (alcohols, ethers), butane, and pentanes plus.

Gross Input to Atmospheric Crude Oil Distillation Units. Total input to atmospheric crude oil distillation units. 
Includes all crude oil, lease condensate, natural gas plant liquids, unfinished oils, liquefied refinery gases, slop oils, and other liquid hydrocarbons produced from tar sands, gilsonite, and oil shale.

Heavy Gas Oil. Petroleum distillates with an approximate boiling range from $651^{\circ}$ to $1000^{\circ} \mathrm{F}$.

Hydrogen. The lightest of all gases, occurring chiefly in combination with oxygen in water; exists also in acids, bases, alcohols, petroleum, and other hydrocarbons.

Idle Capacity. The component of operable capacity that is not in operation and not under active repair, but capable of being placed in operation within $\mathbf{3 0}$ days; and capacity not in operation but under active repair that can be completed within 90 days.

Imported Crude Oil Burned As Fuel. The amount of foreign crude oil burned as a fuel oil, usually as residual fuel oil, without being processed as such. Imported crude oil burned as fuel includes lease condensate and liquid hydrocarbons produced from tar sands, gilsonite, and oil shale.

Imports. Receipts of crude oil and petroleum products into the 50 States and the District of Columbia from foreign countries, Puerto Rico, the Virgin Islands, and other U.S. possessions and territories.

\section{Isobutane. See Butane.}

Isobutylene $\left(\mathrm{C}_{4} \mathrm{H}_{8}\right)$. An olefinic hydrocarbon recovered from refinery processes or petrochemical processes.

Isohexane $\left(\mathrm{C}_{6} \mathrm{H}_{14}\right)$. A saturated branch-chain hydrocarbon. It is a colorless liquid that boils at a temperature of $156.2^{\circ} \mathrm{F}$.

Isomerization. A refining process which alters the fundamental arrangement of atoms in the molecule without adding or removing anything from the original material. Used to convert normal butane into isobutane $\left(\mathrm{C}_{4}\right)$, an alkylation process feedstock, and normal pentane and hexane into isopentane $\left(\mathrm{C}_{5}\right)$ and isohexane $\left(\mathrm{C}_{6}\right)$, high-octane gasoline components.

Isopentane. See Natural Gasoline and Isopentane.

Kerosene. A petroleum distillate that has a maximum distillation temperature of $401^{\circ} \mathrm{F}$ at the 10 -percent recovery point, a final boiling point of $572^{\circ} \mathrm{F}$, and a minimum flash point of $100^{\circ} \mathrm{F}$. Included are the two grades designated in ASTM D3699: No. 1-K and No. 2-K, and all grades of kerosene called range or stove oil. Kerosene is used in space heaters, cook stoves, and water heaters and is suitable for use as an illuminant when burned in wick lamps.

Kerosene-Type Jet Fuel. A quality kerosene product with a maximum distillation temperature of $400^{\circ} \mathrm{F}$ at the $10-$ percent recovery point and a final maximum boiling point of $572^{\circ} \mathrm{F}$. The fuel is designated in ASTM Specification D1655 and Military Specifications MIL-T-5624R and MIL-T-83133D (Grades JP-5 and JP-8). A relatively lowfreezing point distillate of the kerosene type used primarily for turbojet and turboprop aircraft engines.

Commercial. Kerosene-type jet fuel intended for use in commercial aircraft.

Military. Kerosene-type jet fuel intended for use in military aircraft.

Lease Condensate. A natural gas liquid recovered from gas well gas (associated and non-associated) in lease separators or natural gas field facilities. Lease condensate consists primarily of pentanes and heavier hydrocarbons.

Light Gas Oils. Liquid petroleum distillates heavier than naphtha, with an approximate boiling range from $401^{\circ} \mathrm{F}$ to $650^{\circ} \mathrm{F}$.

Liquefied Petroleum Gases (LPG). Ethane, ethylene, propane, propylene, normal butane, butylene, isobutane, and isobutylene produced at refineries or natural gas processing plants, including plants that fractionate raw natural gas plant liquids.

Liquefied Refinery Gases (LRG). Liquefied petroleum gases fractionated from refinery or still gases. Through compression and/or refrigeration, they are retained in the liquid state. The reported categories are ethane/ethylene, propane/propylene, normal butane/butylene, and isobutane/isobutylene. Excludes still gas.

Lubricants. A substance used to reduce friction between bearing surfaces or as process materials either incorporated into other materials used as processing aids in the manufacturing of other products, or as carriers of other materials. Petroleum lubricants may be produced either from distillates or residues. Other substances may be added to impart or improve certain required properties. Do not include byproducts of lubricating oil refining such as aromatic extracts derived from solvent extraction or tars derived from deasphalting. "Lubricants" includes all grades of lubricating oils from spindle oil to cylinder oil and those used in greases. Reporting categories include:

Paraffinic. Includes all grades of bright stock and neutrals with a Viscosity Index $>75$. 
Naphthenic. Includes all lubricating oil base stocks with a Viscosity Index $<75$.

Note: The criterion for categorizing the lubricants is based solely on the Viscosity Index of the stocks and is independent of crude sources and type of processing used to produce the oils.

Exceptions: Lubricating oil base stocks that have been historically classified as naphthenic or paraffinic by a refiner may continue to be so categorized irrespective of the Viscosity Index criterion.

Example:

(1) Unextracted paraffinic oils that would not meet the Viscosity Index test.

Merchant Oxygenate Plants. Oxygenate production facilities that are not associated with a petroleum refinery. Production from these facilities is sold under contract or on the spot market to refiners or other gasoline blenders.

Methanol $\left(\mathrm{CH}_{3} \mathrm{OH}\right)$. A light, volatile alcohol intended for gasoline blending as described in Oxygenate definition.

Middle Distillates. A general classification of refined petroleum products that includes distillate fuel oil and kerosene.

Military Kerosene-Type Jet Fuel. See Kerosene-Type Jet Fuel.

Miscellaneous Products. Includes all finished products not classified elsewhere (e.g., petrolatum, lube refining byproducts (aromatic extracts and tars), absorption oils, ram-jet fuel, petroleum rocket fuels, synthetic natural gas feedstocks, and specialty oils).

Motor Gasoline (Finished). A complex mixture of relatively volatile hydrocarbons, with or without small quantities of additives, that has been blended to form a fuel suitable for use in spark-ignition engines. Motor gasoline, as given in ASTM Specification D-4814 or Federal Specification VV-G-1690C, includes a range in distillation temperatures from 122 degrees to 158 degrees $\mathrm{F}$ at the 10-percent recovery point and from 365 degrees to 374 degrees $F$ at the 90 -percent recovery point. "Motor gasoline" includes reformulated gasoline, oxygenated gasoline, and other finished gasoline. Blendstock is excluded until blending has been completed.

Reformulated Gasoline. Gasoline formulated for use in motor vehicles, the composition and properties of which meet the requirements of the reformulated gasoline regulations promulgated by the U.S. Environmental
Protection Agency under Section $211 \mathrm{~K}$ of the Clean Air Act. Includes oxygenated fuels program reformulated gasoline (OPRG). Excludes reformulated gasoline blendstock for oxygenate blending (RBOB).

Oxygenated Gasoline. Gasoline formulated for use in motor vehicles that has an oxygen content of 1.8 percent or higher, by weight. Includes gasohol. Excludes reformulated gasoline, oxygenated fuels program reformulated gasoline (OPRG) and reformulated gasoline blendstock for oxygenate blending (RBOB).

OPRG. "Oxygenated Fuels Program Reformulated Gasoline" is reformulated gasoline which is intended for use in an oxygenated fuels program control period.

Other Finished or Conventional Gasoline. Motor gasoline not included in the oxygenated or reformulated gasoline categories. Excludes reformulated gasoline blendstock for oxygenate blending (RBOB).

Motor Gasoline Blending. Mechanical mixing of motor gasoline blending components and oxygenates to produce finished motor gasoline. Mechanical mixing of finished motor gasoline with motor gasoline blending components or oxygenates which results in increased volumes of finished motor gasoline, and/or changes in the classification of finished motor gasoline (e.g., other finished motor gasoline mixed with MTBE to produce oxygenated motor gasoline), is considered motor gasoline blending.

Motor Gasoline Blending Components. Naphthas which will be used for blending or compounding into finished motor gasoline (e.g., straight-run gasoline, alkylate, reformate, benzene, toluene, xylene) and includes reformulated gasoline blendstock for oxygenate blending (RBOB). Excludes oxygenates (alcohols, ethers), butane, and pentanes plus. Oxygenates are reported as individual components and included in the total for other hydrocarbons, hydrogens, and oxygenates.

MTBE (Methyl tertiary butyl ether) $\left(\mathrm{CH}_{3}\right)_{3} \mathrm{COCH}_{3}$. An ether intended for gasoline blending as described in Oxygenate definition.

Naphtha. A generic term applied to a petroleum fraction with an approximate boiling range between $122^{\circ}$ and $400^{\circ}$ F.

Naphtha Less Than 401 $1^{\circ}$. See Petrochemical Feedstocks.

Naphtha-Type Jet Fuel. A fuel in the heavy naphtha boiling range. ASTM Specification D1655 specifies for this fuel maximum distillation temperatures of $290^{\circ} \mathrm{F}$ at the 20 -percent recovery point and $470^{\circ} \mathrm{F}$ at the 90 -percent point, meeting Military Specification MIL-T-5624L 
(Grade JP-4). JP-4 is used for turbojet and turboprop aircraft engines, primarily by the military. Excludes ramjet and petroleum rocket fuels.

Natural Gas. A mixture of hydrocarbons and small quantities of various nonhydrocarbons existing in the gaseous phase or in solution with crude oil in underground reservoirs.

Natural Gas Field Facility. A field facility designed to process natural gas produced from more than one lease for the purpose of recovering condensate from a stream of natural gas; however, some field facilities are designed to recover propane, normal butane, pentanes plus, etc., and to control the quality of natural gas to be marketed.

Natural Gas Plant Liquids. Natural gas liquids recovered from natural gas in gas processing plants, and in some situations, from natural gas field facilities. Natural gas liquids extracted by fractionators are also included. These liquids are defined according to the published specifications of the Gas Processors Association and the American Society for Testing and Materials and are classified as follows: ethane, propane, normal butane, isobutane, and pentanes plus.

Natural Gas Processing Plant. A facility designed (1) to achieve the recovery of natural gas liquids from the stream of natural gas which may or may not have been processed through lease separators and field facilities, and (2) to control the quality of the natural gas to be marketed. Cycling plants are classified as gas processing plants.

Natural Gasoline and Isopentane. A mixture of hydrocarbons, mostly pentanes and heavier, extracted from natural gas, that meets vapor pressure, end-point, and other specifications for natural gasoline set by the Gas Processors Association. Includes isopentane which is a saturated branch-chain hydrocarbon, $\left(\mathrm{C}_{5} \mathrm{H}_{12}\right)$, obtained by fractionation of natural gasoline or isomerization of normal pentane.

Net Receipts. The difference between total movements into and total movements out of each PAD District by pipeline, tanker, and barge.

\section{Normal Butane. See Butane.}

OPEC. The acronym for the Organization of Petroleum Exporting Countries, that have organized for the purpose of negotiating with oil companies on matters of oil production, prices and future concession rights. Current members are Algeria, Indonesia, Iran, Iraq, Kuwait, Libya, Nigeria, Qatar, Saudi Arabia, United Arab Emirates, and Venezuela. The Neutral Zone between Kuwait and Saudi Arabia is considered part of OPEC. Prior to
January 1, 1993, Ecuador was a member of OPEC. Prior to January 1995, Gabon was a member of OPEC.

OPRG. "Oxygenated Fuels Program Reformulated Gasoline" is reformulated gasoline which is intended for use in an oxygenated fuels program control area during an oxygenated fuels program control period.

Operable Capacity. The amount of capacity that, at the beginning of the period, is in operation; not in operation and not under active repair, but capable of being placed in operation within 30 days; or not in operation but under active repair that can be completed within 90 days. Operable capacity is the sum of the operating and idle capacity and is measured in barrels per calendar day or barrels per stream day.

Operating Capacity. The component of operable capacity that is in operation at the beginning of the period.

Operable Utilization Rate. Represents the utilization of the atmospheric crude oil distillation units. The rate is calculated by dividing the gross input to these units by the operable refining capacity of the units.

Operating Utilization Rate. Represents the utilization of the atmospheric crude oil distillation units. The rate is calculated by dividing the gross input to these units by the operating refining capacity of the units.

\section{Other Finished. See Motor Gasoline (Finished).}

Other Hydrocarbons. Materials received by a refinery and consumed as a raw material. Includes hydrogen, coal tar derivatives, gilsonite, and natural gas received by the refinery for reforming into hydrogen. Natural gas to be used as fuel is excluded.

Other Oils Equal To or Greater Than $401^{\circ} \mathrm{F}$. See Petrochemical Feedstocks.

Other Oxygenates. Other aliphatic alcohols and aliphatic ethers intended for motor gasoline blending (e.g., isopropyl ether (IPE) or n-propanol).

\section{Oxygenated Gasoline. See Motor Gasoline (Finished).}

Oxygenates. Any substance which, when added to gasoline, increases the amount of oxygen in that gasoline blend. Through a series of waivers and interpretive rules, the Environmental Protection Agency (EPA) has determined the allowable limits for oxygenates in unleaded gasoline. The "Substantially Similar" Interpretive Rules (56 FR (February 11, 1991)) allows blends of aliphatic alcohols other than methanol and aliphatic ethers, provided the oxygen content does not exceed 2.7 percent by weight. The "Substantially Similar" Interpretive Rules 
also provides for blends of methanol up to 0.3 percent by volume exclusive of other oxygenates, and butanol or alcohols of a higher molecular weight up to 2.75 percent by weight. Individual waivers pertaining to the use of oxygenates in unleaded gasoline have been issued by the EPA. They include:

Fuel Ethanol. Blends of up to 10 percent by volume anhydrous ethanol (200 proof) (commonly referred to as the "gasohol waiver").

Methanol. Blends of methanol and gasoline-grade tertiary butyl alcohol (GTBA) such that the total oxygen content does not exceed 3.5 percent by weight and the ratio of methanol to GTBA is less than or equal to 1 . It is also specified that this blended fuel must meet ASTM volatility specifications (commonly referred to as the "ARCO" waiver).

Blends of up to 5.0 percent by volume methanol with a minimum of 2.5 percent by volume cosolvent alcohols having a carbon number of 4 or less (i.e., ethanol, propanol, butanol, and/or GTBA). The total oxygen must not exceed 3.7 percent by weight, and the blend must meet ASTM volatility specifications as well as phase separation and alcohol purity specifications (commonly referred to as the "DuPont" waiver).

MTBE (Methyl tertiary butyl ether). Blends up to 15.0 percent by volume MTBE which must meet the ASTM D4814 specifications. Blenders must take precautions that the blends are not used as base gasolines for other oxygenated blends (commonly referred to as the "Sun" waiver).

Pentanes Plus. A mixture of hydrocarbons, mostly pentanes and heavier, extracted from natural gas. Includes isopentane, natural gasoline, and plant condensate.

Persian Gulf. The countries that comprise the Persian Gulf are: Bahrain, Iran, Iraq, Kuwait, Qatar, Saudi Arabia, and the United Arab Emirates.

Petrochemical Feedstocks. Chemical feedstocks derived from petroleum principally for the manufacture of chemicals, synthetic rubber, and a variety of plastics. The categories reported are "Naphtha Less Than $401^{\circ} \mathrm{F}$ " and "Other Oils Equal To or Greater Than $401^{\circ} \mathrm{F}$."

Naphtha Less Than $401^{\circ} \mathrm{F}$. A naphtha with a boiling range of less than $401^{\circ} \mathrm{F}$ that is intended for use as a petrochemical feedstock.

Other Oils Equal To or Greater Than $401^{\circ} \mathrm{F}$. Oils with a boiling range equal to or greater than $401^{\circ} \mathrm{F}$ that are intended for use as a petrochemical feedstock.
Petroleum Administration for Defense (PAD) Districts. Geographic aggregations of the 50 States and the District of Columbia into five districts by the Petroleum Administration for Defense in 1950. These districts were originally defined during World War II for purposes of administering oil allocation.

Petroleum Coke. A residue, the final product of the condensation process in cracking. This product is reported as marketable coke or catalyst coke. The conversion factor is $\mathbf{5}$ barrels per short ton.

Marketable Coke. Those grades of coke produced in delayed or fluid cokers which may be recovered as relatively pure carbon. This "green" coke may be sold as is or further purified by calcining.

Catalyst Coke. In many catalytic operations (e.g., catalytic cracking) carbon is deposited on the catalyst, thus deactivating the catalyst. The catalyst is reactivated by burning off the carbon, which is used as a fuel in the refining process. This carbon or coke is not recoverable in a concentrated form.

Petroleum Products. Petroleum products are obtained from the processing of crude oil (including lease condensate), natural gas, and other hydrocarbon compounds. Petroleum products include unfinished oils, liquefied petroleum gases, pentanes plus, aviation gasoline, motor gasoline, naphtha-type jet fuel, kerosene-type jet fuel, kerosene, distillate fuel oil, residual fuel oil, petrochemical feedstocks, special naphthas, lubricants, waxes, petroleum coke, asphalt, road oil, still gas, and miscellaneous products.

Pipeline (Petroleum). Crude oil and product pipelines used to transport crude oil and petroleum products respectively, (including interstate, intrastate, and intracompany pipelines) within the 50 States and the District of Columbia.

Plant Condensate. One of the natural gas liquids, mostly pentanes and heavier hydrocarbons, recovered and separated as liquids at gas inlet separators or scrubbers in processing plants.

Processing Gain. The volumetric amount by which total output is greater than input for a given period of time. This difference is due to the processing of crude oil into products which, in total, have a lower specific gravity than the crude oil processed.

Processing Loss. The volumetric amount by which total refinery output is less than input for a given period of time. This difference is due to the processing of crude oil into products which, in total, have a higher specific gravity than the crude oil processed. 
Product Supplied, Crude Oil. Crude oil burned on leases and by pipelines as fuel.

Production Capacity. The maximum amount of product that can be produced from processing facilities.

Products Supplied. Approximately represents consumption of petroleum products because it measures the disappearance of these products from primary sources, i.e., refineries, natural gas processing plants, blending plants, pipelines, and bulk terminals. In general, product supplied of each product in any given period is computed as follows: field production, plus refinery production, plus imports, plus unaccounted for crude oil, (plus net receipts when calculated on a PAD District basis), minus stock change, minus crude oil losses, minus refinery inputs, minus exports.

Propane $\left(\mathrm{C}_{3} \mathrm{H}_{8}\right)$. A normally gaseous straight-chain hydrocarbon. It is a colorless paraffinic gas that boils at a temperature of $-43.67^{\circ} \mathrm{F}$. It is extracted from natural gas or refinery gas streams. It includes all products designated in ASTM Specification D1835 and Gas Processors Association Specifications for commercial propane and HD-5 propane.

Propylene $\left(\mathrm{C}_{3} \mathrm{H}_{6}\right)$. An olefinic hydrocarbon recovered from refinery processes or petrochemical processes.

$\boldsymbol{R} B \boldsymbol{B}$. "Reformulated Gasoline Blendstock for Qxygenate Blending" is a motor gasoline blending component which, when blended with a specified type and percentage of oxygenate, meets the definition of reformulated gasoline.

Refinery. An installation that manufactures finished petroleum products from crude oil, unfinished oils, natural gas liquids, other hydrocarbons, and oxygenates.

Refinery Input, Crude Oil. Total crude oil (domestic plus foreign) input to crude oil distillation units and other refinery processing units (cokers, etc.).

Refinery Input, Total. The raw materials and intermediate materials processed at refineries to produce finished petroleum products. They include crude oil, products of natural gas processing plants, unfinished oils, other hydrocarbons and oxygenates, motor gasoline and aviation gasoline blending components and finished petroleum products.

Refinery Production. Petroleum products produced at a refinery or blending plant. Published production of these products equals refinery production minus refinery input. Negative production will occur when the amount of a product produced during the month is less than the amount of that same product that is reprocessed (input) or reclas- sified to become another product during the same month. Refinery production of unfinished oils, and motor and aviation gasoline blending components appear on a net basis under refinery input.

Refinery Yield. Refinery yield (expressed as a percentage) represents the percent of finished product produced from input of crude oil and net input of unfinished oils. It is calculated by dividing the sum of crude oil and net unfinished input into the individual net production of finished products. Before calculating the yield for finished motor gasoline, the input of natural gas liquids, other hydrocarbons and oxygenates, and net input of motor gasoline blending components must be subtracted from the net production of finished motor gasoline. Before calculating the yield for finished aviation gasoline, input of aviation gasoline blending components must be subtracted from the net production of finished aviation gasoline.

Reformulated Gasoline. See Motor Gasoline (Finished).

Residual Fuel Oil. The heavier oils that remain after the distillate fuel oils and lighter hydrocarbons are distilled away in refinery operations and that conform to ASTM Specification D396. Included are No. 5, a residual fuel oil of medium viscosity; Navy Special, for use in steam-powered vessels in government service and in shore power plants; No. 6, which includes Bunker $\mathrm{C}$ fuel oil, and is used for commercial and industrial heating, electricity generation and to power ships.

Residuum. Residue from crude oil after distilling off all but the heaviest components, with a boiling range greater than $1000^{\circ} \mathrm{F}$.

Road Oil. Any heavy petroleum oil, including residual asphaltic oil used as a dust pallative and surface treatment on roads and highways. It is generally produced in six grades from 0 , the most liquid, to 5 , the most viscous.

Shell Storage Capacity. The design capacity of a petroleum storage tank which is always greater than or equal to working storage capacity.

Special Naphthas. All finished products within the naphtha boiling range that are used as paint thinners, cleaners, or solvents. These products are refined to a specified flash point. Special naphthas include all commercial hexane and cleaning solvents conforming to ASTM Specification D1836 and D484, respectively. Naphthas to be blended or marketed as motor gasoline or aviation gasoline, or that are to be used as petrochemical and synthetic natural gas (SNG) feedstocks are excluded. 
Steam (Purchased). Steam, purchased for use by a refinery, that was not generated from within the refinery complex.

Still Gas (Refinery Gas). Any form or mixture of gases produced in refineries by distillation, cracking, reforming, and other processes. The principal constituents are methane, ethane, ethylene, normal butane, butylene, propane, propylene, etc. Still gas is used as a refinery fuel and a petrochemical feedstock. The conversion factor is 6 million BTU's per fuel oil equivalent barrel.

Stock Change. The difference between stocks at the beginning of the month and stocks at the end of the month. A negative number indicates a decrease in stocks and a positive number indicates an increase in stocks.

Strategic Petroleum Reserve (SPR). Petroleum stocks maintained by the Federal Government for use during periods of major supply interruption.

Sulfur. A yellowish nonmetallic element, sometimes known as "brimstone".

Supply. The components of petroleum supply are field production, refinery production, imports, and net receipts when calculated on a PAD District basis.

TAME (Tertiary amyl methyl ether) $\left(\mathrm{CH}_{3}\right)_{2}\left(\mathrm{C}_{2} \mathrm{H}_{5}\right) \mathrm{COCH}_{3}$. An oxygenate blend stock formed by the catalytic etherification of isoamylene with methanol.

Tank Farm. An installation used by gathering and trunk pipeline companies, crude oil producers, and terminal operators (except refineries) to store crude oil.

Tanker and Barge. Vessels that transport crude oil or petroleum products. Data are reported for movements between PAD Districts; from a PAD District to the Panama Canal; or from the Panama Canal to a PAD District.

TBA (Tertiary butyl alcohol) $\left(\mathrm{CH}_{3}\right)_{3} \mathrm{COH}$. An alcohol primarily used as a chemical feedstock, a solvent or feedstock for isobutylene production for MTBE; produced as a co-product of propylene oxide production or by direct hydration of isobutylene.

Thermal Cracking. A refining process in which heat and pressure are used to break down, rearrange, or combine hydrocarbon molecules. Thermal cracking includes gas oil, visbreaking, fluid coking, delayed coking, and other thermal cracking processes (e.g., flexicoking). See individual categories for definition.

Toluene $\left(\mathrm{C}_{6} \mathrm{H}_{5} \mathrm{CH}_{3}\right)$. Colorless liquid of the aromatic group of petroleum hydrocarbons, made by the catalytic reforming of petroleum naphthas containing methyl cyclohexane. A high-octane gasoline-blending agent, solvent, and chemical intermediate, base for TNT.

Unaccounted for Crude Oil. Represents the arithmetic difference between the calculated supply and the calculated disposition of crude oil. The calculated supply is the sum of crude oil production plus imports minus changes in crude oil stocks. The calculated disposition of crude oil is the sum of crude oil input to refineries, crude oil exports, crude oil burned as fuel, and crude oil losses.

Unfinished Oils. Includes all oils requiring further processing, except those requiring only mechanical blending. Includes naphthas and lighter oils, kerosene and light gas oils, heavy gas oils, and residuum. See individual categories for definition.

Unfractionated Streams. Mixtures of unsegregated natural gas liquid components excluding those in plant condensate. This product is extracted from natural gas.

United States. The United States is defined as the 50 States and the District of Columbia.

Vacuum Distillation. Distillation under reduced pressure (less the atmospheric) which lowers the boiling temperature of the liquid being distilled. This technique with its relatively low temperatures prevents cracking or decomposition of the charge stock.

Visbreaking. A thermal cracking process in which heavy atmospheric or vacuum-still bottoms are cracked at moderate temperatures to increase production of distillate products and reduce viscosity of the distillation residues.

Wax. A solid or semi-solid material derived from petroleum distillates or residues by such treatments as chilling, precipitating with a solvent, or de-oiling. It is light-colored, more-or-less translucent crystalline mass, slightly greasy to the touch, consisting of a mixture of solid hydrocarbons in which the paraffin series predominates. Includes all marketable wax whether crude scale or fully refined. The three grades included are microcrystalline, crystalline-fully refined, and crystalline-other. The conversion factor is 280 pounds per 42 U.S. gallons per barrel.

Microcrystalline Wax. Wax extracted from certain petroleum residues having a finer and less apparent crystalline structure than paraffin wax and having the following physical characteristics: penetration at $77^{\circ} \mathrm{F}$ (D1321)-60 maximum; viscosity at $210^{\circ} \mathrm{F}$ in Saybolt Universal Seconds (SUS); (D88)-60 SUS (10.22 centistokes) minimum to 150 SUS (31.8 centistokes) maximum; oil content (D721)-5 percent minimum. 
Crystalline-Fully Refined Wax. A light-colored paraffin wax having the following characteristics: viscosity at $210^{\circ} \mathrm{F}$ (D88)-59.9 SUS (10.18 centistokes) maximum; oil content (D721)-0.5 percent maximum; other +20 color, Saybolt minimum.

Crystalline-Other Wax. A paraffin wax having the following characteristics: viscosity at $210^{\circ} \mathrm{F}$ (D88)-59.9 SUS (10.18 centistokes) maximum; oil content (D721)0.51 percent minimum to 15 percent maximum.
Working Storage Capacity. The difference in volume between the maximum safe fill capacity and the quantity below which pump suction is ineffective (bottoms).

Xylene $\left(\mathrm{C}_{6} \mathrm{H}_{4}\left(\mathrm{CH}_{3}\right)_{2}\right)$. Colorless liquid of the aromatic group of hydrocarbons made the catalytic reforming of certain naphthenic petroleum fractions. Used as high-octane motor and aviation gasoline blending agents, solvents, chemical intermediates. Isomers are metaxylene, orthoxylene, paraxylene. 


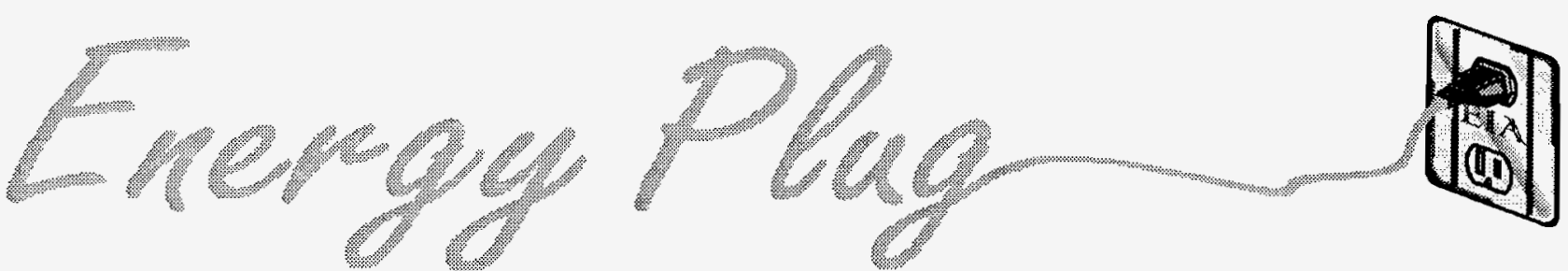

Assessment of Summer 1997 Motor Gasoline Price Increase

Gasoline prices fluctuate seasonally (see figure), but the last couple of years have seen unusual variations. A sharp increase occurred in spring 1996, driven mainly by an upturn in crude oil prices just as gasoline prices began their normal seasonal rise. A year later, however, crude oil prices fell and pulled gasoline prices down with them. In Assessment of Summer 1997 Motor Gasoline Price Increase, the Energy Information Administration (EIA) explains how the price surge of August 1997 differed from the earlier swings. This time, gasoline market dynamics underlay the changes and crude oil prices were essentially immaterial.

Weekly Retail Regular U.S. Gasoline Prices 1992-1997

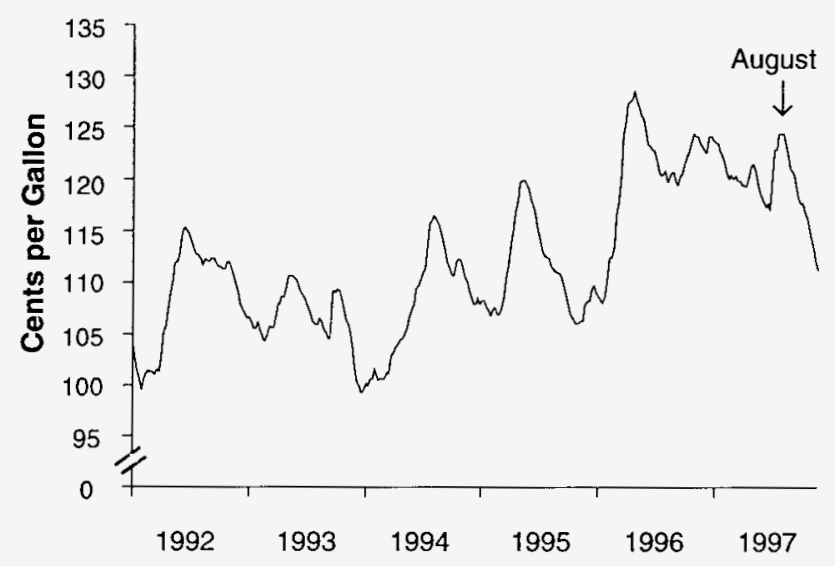

Source: Energy Information Administration.
The August increase was unusually large, with prices at the pump rising 7 or more cents per gallon, depending on type. There were several reasons. Demand rose in July to unexpected levels, ending the month higher by 4 percent than in July 1996. Domestic gasoline production and net imports, both strong earlier in the summer, were undermined by softening U.S. prices and declined during July. Refinery problems here and abroad in late July and early August restrained supply increases from production and imports. The imbalance between demand and new supplies caused gasoline stocks to plummet in July and continue to fall in August; they ended the month at the lowest level recorded since 1981. Prices rose in response. New York Harbor spot-market prices peaked at nearly 77 cents per gallon, 20 cents above the prices of late July.

Retail prices soon followed, and did not soften until Labor Day signaled the end of the summer driving season.

The report details the fundamental supply and demand forces behind the summer 1997 gasoline price increase. It also closely analyzes gasoline markets on the West Coast, U.S. refinery production capacity last summer, gasoline imports, the interrelationships among gasoline prices at various levels in the distribution system, and the behavior of futures markets.

This is the third EIA report discussing significant changes in gasoline prices since 1996. In contrast to the current report, the previous two reports found that shifts in crude oil prices were a major factor behind fluctuating gasoline prices in spring 1996 and a year later. Readers wishing to compare market conditions during those periods may access all three reports via the EIA Web site (http://www.eia.doe.gov). Select "Petroleum" and then "Feature Articles and Special Reports."

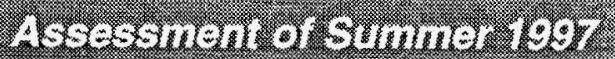

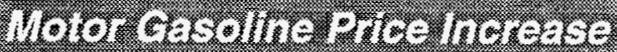

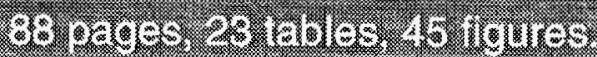

To order a hard copy of the report, use the form in the back of this publication. To access the report via the Internet, go to http://www.eia.doe.gov and click on "Petroleum" and then "Feature Articles and Special Reports." For help with problems accessing the report, call 202-586-1130 or contact wmaster@eia.doe.gov. If you have technical questions about the content, contact Charles Dale, Office of Oil and Gas, at 202-586-1805 or charles.dale@ eia.doe.gov. For general information about energy, contact the National Energy Information Center at 202-586-8800 or infoctr@eia.doe.gov. 
Energy Information Administration

U.S. Department of Energy

Forrestal Building, EI-30

Washington, DC 20585

OFFICIAL BUSINESS

Do Not Forward, Do Not Return

Address Correction Requested

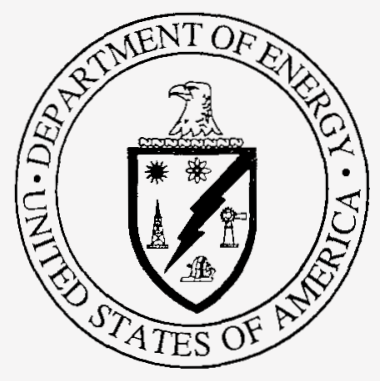


M98005573

Report Number (14)DOE/EIA--0340 (97)/1

Publ. Date (11) 199806

Sponsor Code (18) DOEIEI, XF
UC Category (19) UC-900, DOE/ER

19980702020

DTTC QUALTTY INOPECTED 1

DOE 